UCRL-CR-125620

P.O. B291867

\title{
Estimated Use of Explosives in the Mining Industries of Egypt, Jordan, Syria, Tunisia, and Turkey
}

David R. Wilburn

Carl Di Francesco

Donald I. Bleiwas

U.S. Geological Survey, Golden, CO

This report was prepared for the U.S. Department of Energy's

Office of Non-Proliferation and National Security

November 1996

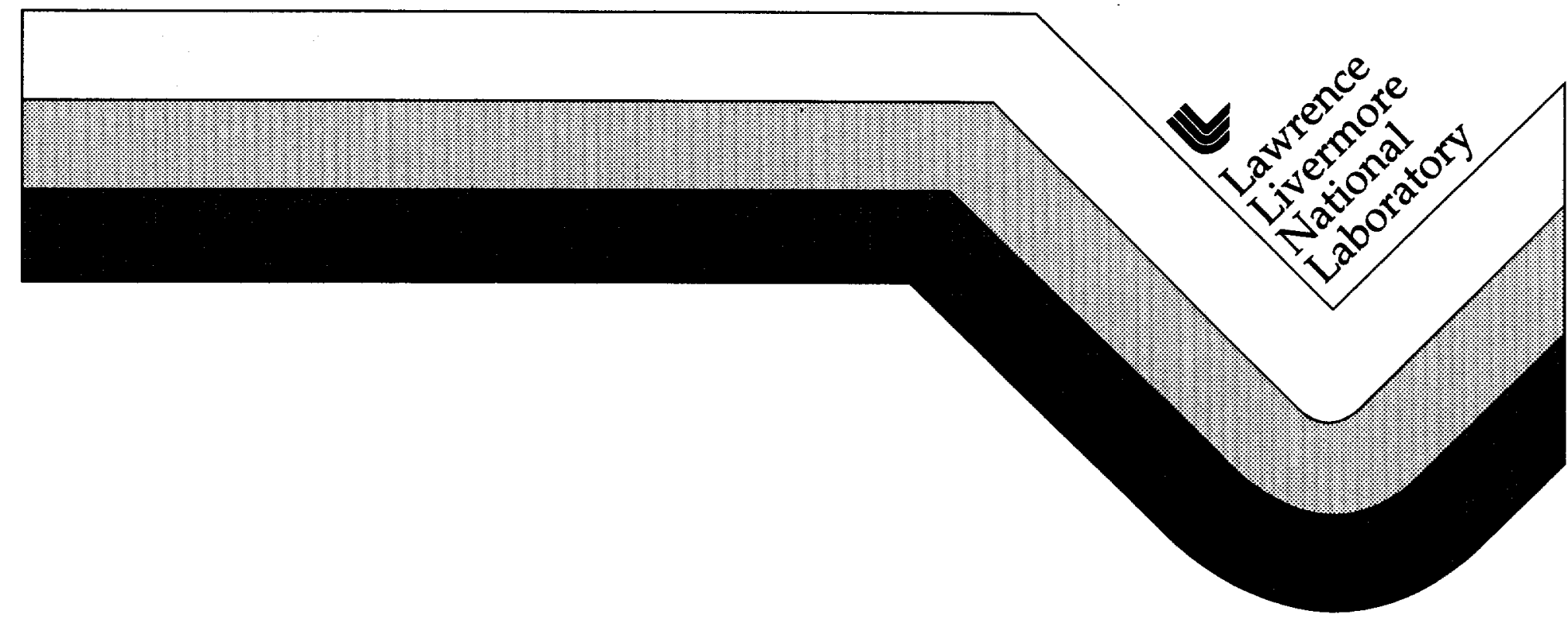




\section{DISCLAIMER}

This document was prepared as an account of work sponsored by an agency of the United States Government. Neither the United States Government nor the University of California nor any of their employees, makes any warranty, express or implied, or assumes any legal liability or responsibility for the accuracy, completeness, or usefulness of any information, apparatus, product, or process disclosed, or represents that its use would not infringe privately owned rights. Reference herein to any specific commercial product, process, or service by trade name, trademark, manufacturer, or otherwise, does not necessarily constitute or imply its endorsement, recommendation, or favoring by the United States Government or the University of California. The views and opinions of authors expressed herein do not necessarily state or reflect those of the United States Government or the University of California, and shall not be used for advertising or product endorsement purposes.

Work performed under the auspices of the U.S. Department of Energy by Lawrence Livermore National Laboratory under Contract W-7405-ENG-48. 


\title{
Best Available Quality
}

\author{
for original report \\ call \\ Reports Library
}

x37097 



\section{Foreword}

This work was performed under Memorandum of Agreement B 291867 between the Lawrence Livermore National Laboratory (LLNL), and the U.S. Geological Survey (USGS) in Golden, $\mathrm{CO}$.

The USGS authors were formerly with the U.S. Bureau of Mines' Minerals Availability Field Office in Denver, CO. Earlier, they performed for LLNL a similar study concerning Algeria, Iran, Iraq, and Lybia. The corresponding report is UCRL-CR-122186, dated September 1995.

The study was initiated and directed by F. Heuze, at LLNL. It is part of the activities under the LLNL Comprehensive Test Ban (CTB) Treaty Program. J. Zucca was the Program Leader.

This work was supported by the LLNL CTB Seismic Project under contract W-7405-ENG48 between LLNL and the U.S. Department of Energy (DOE). D. Harris was the Seismic Project Leader.

The Program Monitor at DOE was L. Casey of the Office of Non-Proliferation and National Security (NN-20). 
Egypt 



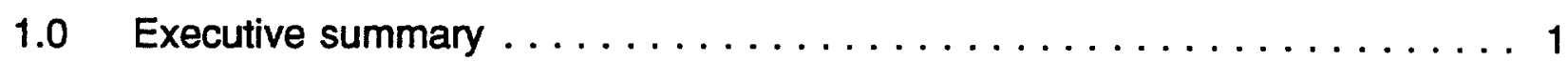

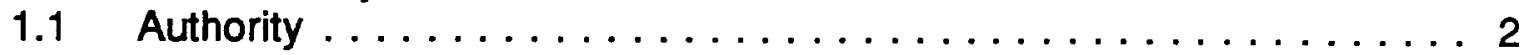

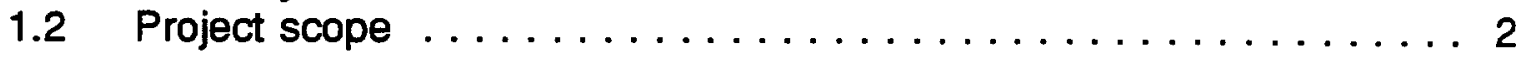

2.0 Sources of information $\ldots \ldots \ldots \ldots \ldots \ldots \ldots \ldots \ldots$

3.0 The mining industry of Egypt $\ldots \ldots \ldots \ldots \ldots \ldots \ldots \ldots \ldots$

3.1 Industrial minerals $\ldots \ldots \ldots \ldots \ldots \ldots \ldots \ldots \ldots \ldots$

3.1.1 Phosphate $\ldots \ldots \ldots \ldots \ldots \ldots \ldots \ldots \ldots \ldots \ldots \ldots \ldots$

3.1.2 Limestone \& cement . . . . . . . . . . . . . . . . . 9

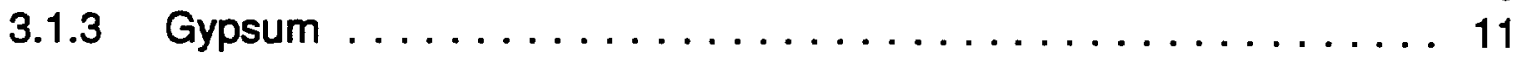

3.1.4 Other industrial minerals $\ldots \ldots \ldots \ldots \ldots \ldots \ldots \ldots \ldots \ldots \ldots 11$

3.2 Metals ...................... 13

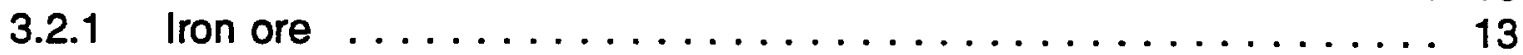

3.2.2 Manganese ....................... 14

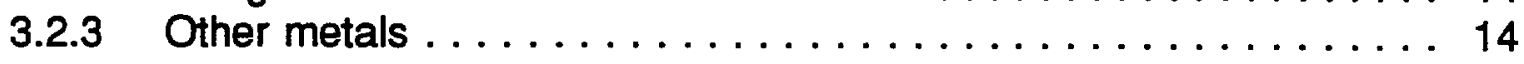

$4.0 \quad$ Mine-related explosives use $\ldots \ldots \ldots \ldots \ldots \ldots \ldots \ldots \ldots \ldots$

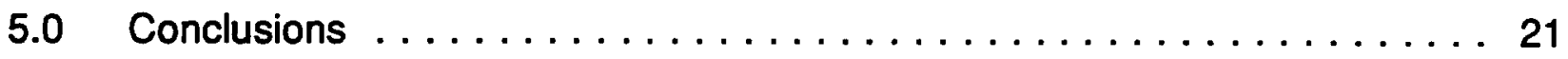

Appendix A: Producing and developing mineral properties in Egypt . . . . . . 23 Appendix B: Past producing mineral properties in Egypt . . . . . . . . . . . 30 Appendix C: Prospects and undeveloped mineral properties in Egypt . . . . . 44 Appendix D: Public sources of information . . . . . . . . . . . . 60

\section{TABLES}

3.1 Reported mineral production in Egypt, 1993 and $1994 \ldots \ldots \ldots \ldots \ldots$. . . . . 6

4.1 Estimated explosives usage at the main Egyptian mines used in this study in order of estimated ANFO consumption . . . . . . . . . 19

\section{FIGURES}

4.1 Selected Egyptian mines and estimated maximum blasting events . . . . . 20

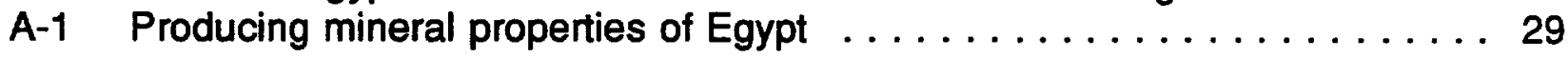

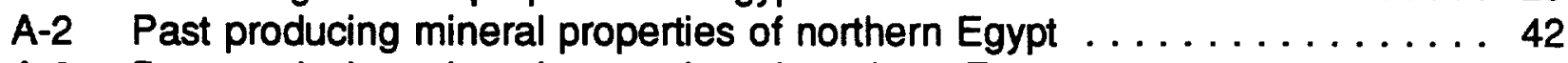

A-3 Past producing mineral properties of southern Egypt . . . . . . . . . 43

A-4 Prospects and undeveloped mineral properties of northern Egypt . . . . . . 58

A-5 Prospects and undeveloped mineral properties of southern Egypt . . . . . 59 



\subsection{EXECUTIVE SUMMARY}

This report was prepared under Memorandum of Agreement B291867 between Lawrence Livermore National Laboratory (LLNL) and the U.S. Geological Survey (USGS). It provides LLNL information on the mining industry of Egypt, and quantitative information on the blasting potential of this industry. The USGS identified mining activities through the use of the Minerals Availability data base, its data collection and analytic capabilities, and an extensive network of information sources.

While a wide variety of minerals may be found in some areas of Egypt, large scale mining is limited to a few mineral commodities, notably iron ore, phosphate rock, limestone and cement, and gypsum. Most mineral production comes from small scale mines; production of over 25 minerals in Egypt has historically come from more than $\mathbf{6 0 0}$ mines, quarries, and evaporite basins. Mining is concentrated in the Nile Valley and Eastern Desert regions of Egypt, with some mining occurring on the Sinai Peninsula. Research conducted for this study resulted in the identification of 644 mineral properties. Most properties require minimal blasting. The blasting potential for 20 properties is reported.

Egypt possesses the raw materials, technology and facilities to manufacture blasting agents and detonation systems suitable for mining applications. Blasting is generally conducted on a small scale but may occur frequently where mine geology requires explosive use. Ammonium nitrate-fuel oil (ANFO) is the most commonly used explosive agent, but dynamite or ANFO/gel mixtures are also used. Estimates for daily ANFO consumption from the largest mines in Egypt range from 10-50 metric tons of ANFO equivalent. 


\subsection{Authority}

This report was prepared under Memorandum of Agreement B291867 between Lawrence Livermore National Laboratory (LLNL) and the U.S. Geological Survey, Minerals Availability Team, currently the Minerals \& Materials Analysis Section of the Minerals Information Team (MIT).

\subsection{Project Scope}

As part of this agreement, MIT is to identify mining activities in Egypt, Jordan, Syria, Tunisia, and Turkey as they relate to monitoring/verifying compliance of the Comprehensive Test Ban Treaty. The MIT is to use the Minerals Availability and the Mineral Resources Data System data bases, its data collection and analytic capabilities, and an extensive network of information sources to provide background information focusing on the use of explosives by the mining industry of these countries. This information is of particular interest because the normal blasting activities of mining can cause false alarms during monitoring and disguise nuclear blasting events.

Reports with accompanying figures and tables summarize location, type of mining method, commodity(ies), estimated frequency and size of mine blasts, operational status, and distribution to foreign or internal markets for the specified countries.

Once country data were collected and verified, the explosive use at selected sites was evaluated. Focus was placed on locations that consume large quantities of conventional chemical explosives. Undeveloped sites and small scale mines which consume minimal amounts of explosive material (included in Appendices A-C of this report) were not analyzed in terms of the site's anticipated use of explosives. Mineral prospects generally make only small use of explosives and small mines (some of which are operated on an intermittent basis) are assumed to require minimal blasting. Appendix $A$ lists producing and developing mineral properties in Egypt, Appendix $B$ lists known past producers, and Appendix $C$ lists identified prospects and undeveloped 
properties. These listings provided the basis from which the principal Egyptian mines with the greatest explosive consumption potential were selected.

Based upon known site information (geological conditions, mine technology, production capacity, and current blasting practices), the blasting potential for significant mining sites was evaluated. Where site-specific data were not available, estimates for representative, important properties were developed based upon accepted industry practice, knowledge of the Egyptian mining industry, and regional geologic characteristics.

\subsection{SOURCES OF INFORMATION}

Data for this report were derived from published sources, unpublished documents, and personal communications through an extensive network of public and private contacts. Public sources of information are listed in Appendix D. Much of the industry summary was drawn from data reported by the U.S. Bureau of Mines Mineral Yearbook chapter on Egypt. from the years 1992-1994. Information for 1995 was obtained from the U.S. Geological Survey, Minerals Information Team, International Minerals Section, Reston, VA (formerly the U.S. Bureau of Mines, Division of International Minerals).

Principal agencies contacted include, but were not limited to the U.S. Geological Survey,

the U.S. Department of State, Central Intelligence Agency, Defense Intelligence Agency, the United Nations, the World Bank, World Resources Institute, and International Studies of Minerals Issues (ISMI). In addition, selected academic and industry contacts, explosives manufacturers and suppliers, and trade groups were contacted.

\subsection{THE MINING INDUSTRY OF EGYPT}

Mineral deposits in. Egypt, particularly sites containing gold, copper, and gemstones, have been known and exploited by the ancient Egyptians for thousands of years. Such minerals were found in complex form in hard rock deposits. In contrast, the main economic minerals exploited in Egypt today include iron ore, phosphate rock, gypsum, 
limestone, and salt. These typically come from sedimentary sequences as native elements or simple compounds. Tin is the only mineral that has been recently produced from hard rock sources; gold and copper mineralization is widespread, but not of high enough grade to be economically viable at present prices'.

While a relatively wide variety of minerals may be found in the Eastern Desert and Nile Valley regions of Egypt, mining occurs on a limited scale, except for some iron ore, phosphate rock, gypsum, and salt deposits. Egyptian mining operations have produced approximately 25 commodities from more than 600 mines, quarries, and salt pans, most of which operate on a very small scale. Exploration and production on the Sinai Peninsula has been limited since 1967, when hostilities broke out in the region between the Arabs and Israelis. Large amounts of low grade phosphate have been identified on the Abu Tartur plateau, northwest of El Kharga in the Western Desert. Development of this resource is ongoing, although full production is not expected until $1997^{2}$. Similarly, the Marghara coal mine in northern Sinai southwest of El Arish is in the process of beginning production. Production of 2 of the 5 planned longwall faces is not scheduled until 1998. Production is planned to feed the Ain Musa power station. Israel has expressed interest in importing Marghara coal to reduce the high transport costs associated with delivering coal to Israeli power generating plants ${ }^{2}$.

While the hydrocarbon sector accounted for more than $15 \%$ of the gross domestic product (GDP) in 1994, non-fuel minerals contributed only a small portion of industrial production. Non-fuel production levels remained relatively low when compared with global competitors. Egypt's economy is heavily reliant upon oil sales, tourism, and revenues from both the Suez Canal and the SUMED oil pipeline. Revenues from tourism dropped in 1994 as a result of threats of terrorist activity.

\footnotetext{
1 A.M.A. Wali. Industrial Minerals of Egypt. Paper 90-77 presented at SME annual meeting, Salt Lake City, 1990.
}

2 Mining Annual Review 1996. Mining Journal, Sept. 1996, p. 159. 
Mineral trade operating via the Suez Canal continues to be critical to the Egyptian economy. Industrial mineral exports of phosphate rock, aluminum manufactured goods, coke and semicoke, fertilizers and salt accounted for approximately $25 \%$ of total export value in 1994. Mineral imports accounted for $8.8 \%$ of total import value in $1994^{3}$. Mineral imports included chromite, copper, iron and steel products, lead, nickel, silver, tin, titanium, tungsten, and zinc. Industrial mineral imports included asbestos, barite, coal, graphite, pumice, and sulfur. Nations of the European Union are the largest trading partners with Egypt, although trade with neighboring Middle Eastern countries is increasing.

Virtually all mining and mineral processing in Egypt is carried out by Government-owned mining companies. The parastatal Mining and Refractories Corp (Maric) controls the mining and refractories industries and also controls five major companies that dominate the Egyptian mining industry. The companies are the El Nasr Phosphate Co., Red Sea Phosphate Co, Misr Phosphate Co., the Sinai Manganese Co., and the EI Nasr Saline $\mathrm{Co}^{4}$. Administration and mining legislation is conducted by the Egyptian Geological Survey and Mining Authority (EGSMA). Foreign investors in the mining industry coordinate exploration activities through EGSMA. In 1993, EGSMA completed a draft unified law for all quarries, mines, and salt operations, which is currently under review.

Egyptian mineral production estimates for 1993 and 1994 are provided in table 3.1. Summaries of mineral site data are provided in Appendices A-C. Data on significant producing sites, past producers, prospects, and undeveloped mineral occurrences are provided in tabular form. Maps showing mineral property locations are provided in Appendix map sets A-C. It should be noted that not all mineral occurrences are reported in this study. Sites with unverifiable information or lacking specific site locations may not be included. Data are reported for 87 producers, 222 past producers,

\footnotetext{
${ }^{3}$ U.S. Bureau of Mines. Egypt, Ch. in Mineral Industry Surveys 1994, by T. P. Dolley.

Ibid.
} 
Table 3.1 -- Reported Mineral Production in Egypt, 1993 and 1994 (Metric tons, except as noted)

\begin{tabular}{|c|c|c|}
\hline Commodity (1) & $\begin{array}{r}1993 \\
\text { Production } \\
\end{array}$ & $\begin{array}{r}1994 \\
\text { Production (e) }\end{array}$ \\
\hline \multicolumn{3}{|l|}{ METALS } \\
\hline Aluminum metal & 180,000 & 180,000 \\
\hline Chromite & 600 & 600 \\
\hline Copper, refined (secondary) & 4,000 & 4,000 \\
\hline Iron ore and concentrate & $2,190,000$ & $2,100,000$ \\
\hline Ferromanganese & 30,000 & 30,000 \\
\hline Manganese & 15,000 & 15,000 \\
\hline \multicolumn{3}{|l|}{ INDUSTRIAL MINERALS } \\
\hline Asbestos & 436 & 400 \\
\hline Barite & 4,090 & 4,000 \\
\hline Cement, hydraulic & $16,000,000$ & $16,000,000$ \\
\hline Clay, bentonite & 15,000 & 14,000 \\
\hline Clay, fire clay & 421,000 & 420,000 \\
\hline Clay, kaolin & 157,000 & 156,000 \\
\hline Feldspar, crude & 38,900 & 39,000 \\
\hline Fluorspar & 773 & 800 \\
\hline Gypsum and anhydrite, crude & $1,200,000$ & $1,200,000$ \\
\hline Lime & 748,000 & 750,000 \\
\hline $\mathrm{N}$ content of ammonia & 941,000 & 900,000 \\
\hline Phosphate rock & $1,590,000$ & $1,600,000$ \\
\hline Salt, marine & 972,000 & $1,000,000$ \\
\hline Soda ash & 51,000 & 50,000 \\
\hline Sodium sulfate & 26,500 & 26,000 \\
\hline \multicolumn{3}{|l|}{ Stone products: } \\
\hline Basalt (cubic meters) & 551,000 & 600,000 \\
\hline Dolomite & 952,000 & $1,000,000$ \\
\hline Granite, dimension (cubic meters) & 12,900 & 13,000 \\
\hline Gravel (cubic meters) & $7,180,000$ & $7,200,000$ \\
\hline
\end{tabular}




\begin{tabular}{||l|r|r|}
\hline Limestone (cubic meters) & $18,100,000$ & $18,000,000$ \\
\hline Marble blocks (cubic meters) & 15,800 & 16,000 \\
\hline Sand, glass & 743,000 & 740,000 \\
\hline Sand, construction & $21,700,000$ & $22,000,000$ \\
\hline Sulfur, elemental and byproduct & 4,100 & 4,000 \\
\hline Talc and related products & 2,100 & 2,000 \\
\hline
\end{tabular}

Source: U.S. Bureau of Mines. Egypt. Ch. in Mineral Industry Survey series, 1994, by T. P. Dolley.

(e) Estimated

(1) Table includes data through March 1995. Previously published and 1994 estimates are rounded to three significant digits; may not add to totals shown. In addition to the commodities listed, pig iron, ferrosilicon, crude steel, sandstone, sulfuric acid, and vermiculite are produced for local consumption. Data on coke, natural gas and petroleum products are not reported here. 
and 335 mineral deposits. It is believed that all sites with significant potential have been reported.

\subsection{Industrial minerals}

While metallic minerals from Egypt were mined thousands of years ago, industrial minerals today provide a greater source of raw materials and revenue than do metals. Domestic feed sources for the Egyptian cement and fertilizer industries are abundant, and raw materials needed for the local building and construction industry are readily available.

\subsubsection{Phosphate}

Phosphate has been a principal mineral commodity in Egypt since the beginning of the 20th Century. There are two main sources of production: (1) the Red Sea coast between Quseir and Safaga, and (2) along the banks of the Nile River near Sebaiya. Production from the Nile Valley deposits normally feeds the local fertilizer industry while production from the Red Sea region is directed to exportation. During the 1970's, exploration work revived phosphate production in the Quseir area, at a time when most of the old mines were thought to be nearing exhaustion.

At about the same time, exploration further defined large reserves on the Abu Tartur plateau, located in the Western Desert between Kharga and Dakhla. Since that time, the area has undergone various stages of exploration and preliminary development. Reserves are estimated to exceed $988 \mathrm{Mmt} @ 23 \%$ P2O55. Development has been hindered by the site's remote location, estimated high production costs, and lack of available funding. This project would be the largest industrial mineral project in Egypt.

Plans call for a capital expenditure of over $\$ 400$ million for a mining and beneficiation facility with a production capacity of $4.5 \mathrm{Mmt}$ of phosphate ore (2.2 Mmt per year of

\footnotetext{
5 Mining Annual Review 1995. Mining Journal, Nov. 1995, pp. 151-152.
} 
concentrate), directed initially for domestic production of fertilizer ${ }^{6}$. Surplus production would be used to generate export revenues. Infrastructure would include a $400-\mathrm{km}$ railway to transport the phosphate from Abu Tartur to the port of Safaga. After full production is reached, it is initially estimated that about $30 \%$ of the total phosphate produced would be exported. Egypt's sole phosphoric acid plant is at Abu Zaabal, northeast of Cairo. Uranium has the potential to be a significant byproduct of Egyptian phosphate production, but is not currently recovered.

Mining of the Red Sea coast phosphates began in 1910 for export to the Far East. The phosphate rock requires calcination to upgrade the product. Presently, the largest mine operating is the Hamrawein underground site, operated by Misr Phosphates, with a production capacity of $1.2 \mathrm{Mmt} / \mathrm{yr}$. Recent production has dropped to $592 \mathrm{Kmt}$ due to a lower phosphate price and high mine costs. As with all Egyptian mines on the Red Sea coast, ore is extracted from carbonates by room \& pillar and shortwall mining methods, processed, then marketed to Europe and the Far East. Other mines in the area include the Safaga and Quseir complexes, both of which consist of multiple small mines operated by the Red Sea Phosphate Co.

Domestic needs for phosphate rock as feed for fertilizer production are supplied from the Nile Valley deposits at Sebaiya. The ore-bearing Duwi formation includes three phosphate units interbedded with marls and limestones. The Abu Zaabel Fertilizer Chemical Co. owns and operates the East and West Sebaiya mines. While older areas were mined by underground methods, newer areas are most commonly mined by surface methods using draglines from open pits. Most of the Nile Valley output is delivered to the phosphate fertilizer plants of the Nile delta.

\subsubsection{Limestone \& cement}

Egypt has sufficient raw materials to meet domestic needs for limestone to feed its building and construction industries and for cement production. The Eocene limestones

6 Mining Annual Review 1996. Mining Journal, Sept. 1996, p. 159. 
and clays exposed near Helwan and south of Suez are extensively quarried for the cement industry. In addition, the sintering plant of the Helwan Steel Complex uses limestone for sintering ore fines, and as a fluxing agent. Certain limestones in the region are also suitable for making concrete blocks.

Lime production needs are met from limestones in the Cairo-Suez district. Limestones west of Alexandria are also used for production of lime and soda ash.

The extensive Eocene limestone exposures located near Cairo satisfy the needs of the Egyptian building and road construction industry. Cretaceous limestone exposed at Abu Roash is extensively quarried for road construction materials, while dimensional limestone blocks are obtained from the Mukkatam deposit for building needs in Cairo. West of Alexandria, limestone ridges provide raw material for building purposes. High grade material is quarried from the Eocene limestone at Samalut. The product is highly valued and is used in many industries.

The Egyptian cement industry has the capacity to produce up to $20 \mathrm{Mmt} / \mathrm{yr}$ of cement, requiring approximately $36 \mathrm{Mmt}$ of raw material feed (limestone, clay, etc). Egypt continues to be virtually self sufficient in the mineral commodities needed to manufacture cement. The industry, however, is under increasing pressure to address environmental problems. All cement and associated raw material production is currently under the control of the Egyptian Government, by means of various parastatal cement companies. The Government unsuccessfully tried to privatize several cement companies in 1994. A second round of privatization talks were being conducted in 1995.

The Helwan Portland Cement Company mines near Helwan are representative of quarries in Egypt. The quarry complex produces 15,000 tons of limestone per day. The site operates a 3-shift operation using a 200 man crew. Blasted rock is loaded and transported during the day shift. Blasting operations, repairs, and maintenance are done with smaller crews on the second and third shifts. Ammonium nitrate mixed with fuel oil 
(ANFO) is used in blasting, generally twice a week. The company extracts both clay and limestone feed for the cement plant from separate open pits located near the cement plant. Feed to the kiln is approximately $85 \%$ limestone and $15 \%$ clay. Up to $3.5 \%$ gypsum may be added to the raw material feed, depending upon required cement composition and market requirements.

\subsubsection{Gypsum}

Egypt is one of the world's largest gypsum producers $(1.2 \%$ of world production in 1994), producing approximately 1.2Mmt/yr of gypsum. Much of this gypsum is found in deposits on the Sinai Peninsula and the Mediterranean coast area of Egypt. Extra pure gypsum for exportation principally came from the Sinai until 1967. Gypsum for local consumption in construction, as an additive in agriculture, and in the cement industry comes from several small quarries west of Alexandria and from Girza. Mining is conducted by means of small quarries requiring limited blasting.

\subsubsection{Other industrial minerals}

Sinai used to be the main source of kaolinitic clay consumed by the porcelain industry until hostilities broke out in the Sinai in 1967. The Egyptian Geological Survey had discovered an important deposit at Kalabsha, which had been put into production at a rate of $50 \mathrm{kt} / \mathrm{yr}$. It is believed that the site resumed production in the 1990s, but this has not been confirmed.

White sand for local glass manufacture also came principally from the Sinai until 1967. Other sources of lower quality glass sand from sites on the Gulf of Suez (Zaafarana) and at a site east of Cairo could not substitute for Sinai ore.

Common salt is extracted from several evaporite basins along the Mediterranean coast near Alexandria, Port Said and Damietta. Additional production comes from small operations at Mersa Matruh, Idku, Baltim and Rosetta. Three grades of salt are produced: industrial $(85 \% \mathrm{NaCl})$, washed $(98 \% \mathrm{NaCl})$ and refined $(99 \% \mathrm{NaCl})$. Natural 
rock salt deposits about $300 \mathrm{~m}$ thick occur at Ras Gharib, Gabal al Zeit and Ras Gemsa on the Gulf of Suez. The upper layers often are rich in potassium salts, containing $4-6 \%$ $\mathrm{K} 2 \mathrm{O}$. Salt production currently is approximately $1 \mathrm{Mmt}$, most of which is used domestically. Salt is most commonly extracted without the use of explosives by surface methods.

Among ornamental stones, marble, alabaster, serpentine, breccia, and the famous pink granite of Aswan are all quarried. Building stones are cut from small quarries scattered along the scarps bordering the Nile River. Most quarries are small operations mined intermittently as building needs require. Beryl is present in appreciable amounts in quartz veins associated with tin-tungsten deposits, as well as other areas of the Eastern Desert. Gem quality beryl was mined during Greco-Roman times, but has since been exhausted.

Other ores are being exploited for diversified needs. Talc and soapstone are mined from Darheib and Atshan in the southeastern desert as filling material in local industries. Production typically averages 5 t/yr. Bentonitic clays from Kasr el Sagha is quarried (5 $\mathrm{kt}$ in 1979) for local consumption. Diatomite from Kom Oushim is likewise quarried at a small rate for local needs. Feldspar and fluorspar are mined from many sites in the Eastern Desert. Feldspar is exported to Turkey and Saudi Arabia. Asbestos, vermiculite, magnesite, chromite, barite, and graphite have also been mined at various times for local industries. Native sulfur and potassium salts occur near Gemsa ang other localities bordering the Gulf of Suez.

Exploration for mineral fuels in Egypt took place since the early 1950s. Carbonaceous shales were discovered outcropping at Bedaa and Thora; coal was discovered at Ain Mousa at depths ranging between $420 \mathrm{~m}$ and $620 \mathrm{~m}$. With the discovery of the Maghara coal deposit in 1959, focus was shifted to developing this significant reserve. The first longwall face is reported to have commenced operation in November 1995 , 
although production of two faces is not scheduled until $1998^{7}$ Production will likely be tailored to market demand, but it is expected that the primary markey will be the domestic power industry.

\subsection{Metals}

Egypt produces few metals. Its largest production in the metals sector is primary aluminum metal, produced at one plant from imported ore, and iron production used in the domestic steel industry. Egypt mines iron ore and recovers ferrosilicon and ferromanganese byproducts for its domestic steel industry. Manganese, chromite, and refined copper from secondary sources are also produced.

\subsubsection{Iron ore}

Egyptian iron ore is currently mined primarily from the Bahariya area in the Western Desert. The ore occurs in sedimentary deposits as lenticular bands alternating with sandstone, limestone, and clay beds. While both high grade and low grade ores are present in this area, only high grade ore $(52-55 \% \mathrm{Fe})$ is presently mined by surface methods at the El Gedida mine. Production capacity of the region is $3 \mathrm{Mmt} / \mathrm{yr}$, but current production is estimated at approximately $2.2 \mathrm{Mmt}$. Iron ores of the Bahariya area are sufficient to meet the needs of the Helwan steel plant for the immediate future.

In addition to the Bahariya iron deposits, Egypt has traditionally produced iron ore from mines east of Aswan, which supplied the Helwan furnaces prior to the discovery of Bahariyan deposits. Ore is a oolitic hematite interbedded with limestones and clays. The average ore thickness is $1 \mathrm{~m}$; production in the region was $500 \mathrm{kt} / \mathrm{yr}$ in the 1980's. Other potential sources of iron include 14 sites of metamorphosed iron ore in the Eastern Desert south of Quseir. While these sites have not produced, they are considered resources for the future. Ore has the potential to produce a concentrate contaịning $58.5 \% \mathrm{Fe}$ and $14 \% \mathrm{SiO}$, and achieve an $87 \%$ recovery.

\footnotetext{
${ }^{7}$ Mining Annual Review 1996. Mining Journal, Sept. 1996, p. 159.
} 


\subsubsection{Manganese}

Manganese oxides were used by the ancient Egyptians in the ceramic industry; more recently, manganese was used in steel making, and for paints and pigments. Deposits of potential economic interest are found at Um Bogma in the Sinai Peninsula and at Halaib in the Eastern Desert. The Um Bogma deposits, discovered in 1898, represent the largest occurrence of manganese in Egypt. The region produced for exportation during the period 1911-1967, and achieved a maximum production rate of $300 \mathrm{kt} / \mathrm{yr}$. Rehabilitation of the facility was completed in 1990, but significant production has not been achieved as of 1995. Minor production is reported to occur at the Halaib deposit in the Eastern Desert and in the southwestern desert between Hamata and Elba. Manganese ore occurs as lenticular bodies filling fractures in sediments.

Manganese ore is generally lower grade than those mined in other countries, and ranges from $25-40 \% \mathrm{Mn}$. Ore is transported to the $40 \mathrm{kt}$ Abu Zenema steel plant, where it is blended with imported material to produce high carbon ferromanganese and slag. More than $50 \%$ of the ferromanganese produced is used in Egypt's steel industry. Export markets include Germany, Japan, Libya, Taiwan. and several Persian Gulf states.

\subsubsection{Other metals}

Egypt produces aluminum metal at one $180 \mathrm{kt} / \mathrm{yr}$ facility from imported material. While there is no bauxite in Egypt, there are deposits of nepheline syenite, which has the potential to be used as a raw material in the aluminum industry. Deposits at Abu Khrug occur as ring inclusions in alkaline rocks.

Chromite occurs in the Eastern Desert at eight sites. It occurs as lenses associated with serpentine and talc-carbonate rocks. Production of chromite ore in 1994 was limited to 600 tons.

Gold mineralization is recorded at more than 95 locations in the Eastern Desert, 
practically all were worked in ancient times at shallow depths. Gold quartz veins cut various basement rocks of Precambrian age. Deposits are small, total production for the 1902-1958 period is reported to be about seven tons. Remaining ore is located at a depth requiring underground mining methods. Metal content of in-situ ore reached 28 $\mathrm{g} / \mathrm{t}$, while tailings areas at Sokari, Barramiya, and Um Gariat average $5 \mathrm{~g} / \mathrm{t}^{8}$.

Copper deposits in Egypt were exploited on a small scale by the ancient Egyptians. Copper ores occur in two regions, the Sinai Peninsula and the Eastern Desert. Sinai copper deposits occur associated with sandstones and clays or as fracture fillings in Precambrian rocks. Eastern Desert copper deposits occur as fracture fillings (Al Atawi), gold bearing quartz veins (Hamash), and as impregnations in sandstone. Copper content is generally less than $1 \% \mathrm{Cu}$.

Copper is also found associated with sulfide mineralization in the Eastern Desert. The Abu Suwayel copper-nickel deposit was exploited by the ancient Egyptians, while the Akarem copper-nickel deposit, discovered in 1972, hosts disseminated and massive sulfide ore. Lead-zinc-copper mineralization occurs at Um Samiuki and Um Gheig in the Eastern Desert. A number of smaller ore zones have also been identified in the Eastern Desert.

Small deposits of other metals have also been identified. Deposits of tin, tungsten, tantalum, niobium, and molybdenum occur in the Eastern Desert of Egypt. Nickel is found on St. Johns Island in the Red Sea. Ore is associated with gem-quality peridotite, which as been mined in the past. Uranium and thorium is found is selected localities in the Eastern and Western Deserts, and in the Sinai.

\subsection{MINE-RELATED EXPLOSIVES USE}

Egypt uses both internally produced explosives and imports raw materials for explosive

B F. Habashi and F.A. Bassyouni. Mineral Resources of the Arab Countries. Chemecon Pub. (Quebec), 1982, pp. 18-27. 
manufacture. It possesses raw materials, technology and facilities to manufacture blasting agents and detonation systems suitable for mining applications. Most surface and underground mines in Egypt utilize ammonium nitrate-fuel oil (ANFO) blasting agents.

Some mines use explosives to fragment or loosen rock and consolidated material prior to excavation. Bulk or packaged explosives and blasting agents are detonated after emplacement in material to be excavated. Minor quantities of sachet (bagged powder exploșives) and shaped charges may be used for secondary breakage and other special applications.

The type and amount of explosives used are influenced by the nature of the rock or ore, the mining methods employed, the production rate of the mine, the type and availability of explosives and detonation systems, hydrologic conditions, mining equipment, drilling equipment, mine geometry, level of technical expertise, and external constraints such as the proximity of residences and costs. At almost any mine, the size of each blast can vary significantly due to local conditions, production schedules, weather, etc.

Surface mines typically shoot much larger blasts than underground operations and tend to have higher production rates than underground mines. In addition, limitations of working room, limited free faces, type of mineralization, ventilation requirements, and drilling limitations may constrain maximum blast sizes in underground mines.

Where blasting is required, most Egyptian mines use ANFO blasting agents. This may include some of the newer emulsion and/or aluminum boosted products presently available. While an ANFO/dynamite mixture is believed to be in use at some mines in Egypt, ANFO systems are preferred in most mining applications due to their ease of manufacture, low cost, inherent safety, and bulk loading advantages. High explosives, however, would be preferable for small underground operations that use drill sizes that are below the critical diameter needed for emplacing ANFO blasting agents, or under 
wet conditions, in methane-rich atmospheres, and conditions that require higher detonation velocities and/or convenience of packaged explosives. Many small mines in Egypt are labor intensive and employ non-mechanized ore recovery methods, where blasting is not used.

In most cases, site-specific blasting information was unaccessible. Consequently, estimates were based upon estimated production rates, mine geology, and typical mining practices. Experience, engineering judgement, and available data were incorporated into calculations and estimates. Explosive use can vary considerably as mining conditions change. ANFO consumption was assumed to be dependent upon mine production rate, average stripping ratio, specific gravity of the host rock, assumed powder factor limits, and mining method. Only a small number of mines in Egypt require significant blasting, primarily because of the small size of most sites.

For each site, a stripping ratio (quantity of overburden or waste removed per metric ton of ore mined) and powder factor (quantity of rock blasted per unit of ANFO blasting agent equivalent) limits were estimated. A range of ANFO consumption was calculated for both daily blasting requirements and for an assumed maximum blasting event. Daily ANFO requirements were estimated assuming a 300 day production schedule ${ }^{9}$. Consumption estimates for all sites were calculated in a similar manner. The lower consumption value applies a minimum powder factor while the higher value assumes a maximum powder factor. Unlike daily consumption estimates, a maximum blasting,event would not take place on a daily basis. For this study, it was assumed that a maximum blasting event (an estimated technical upper limit of cumulative explosive usage) would use 10 days worth of explosives for a surface mine and 5 days worth for an underground mine. Such events are designed to account for such factors as blasting delays, geological irregularities, and mining method variations that require a higher ANFO consumption than the typical blasting event. Mine development or pillar country to country.

Production schedule determined by available country information. Schedule may vary from 
extraction conditions, for example, often require larger blasts.

The following examples illustrate typical blasting calculations using the estimation procedure described above:

Bahariya ANFO daily consumption lower limit (L):

$\mathrm{L}=$ Production rate ${ }^{*}\left[1+\right.$ (stripping ratio * (specific gravity of ore + waste)) ${ }^{*}$ [Low powder factor

$/ 1000$ (converts $\mathrm{kg}$ to $\mathrm{mt}$ )] / Operating days per year

$L=3,000,000 *[1+(0.1 * 2.7)] *[0.13 / 1000] / 300$

$L=1.652 \mathrm{mt}$ ANFO equivalent (rounded to nearest unit)

Bahariya ANFO daily consumption higher limit $(H)$ :

$H=$ Production rate ${ }^{*}[1+($ stripping ratio * (specific gravity of ore + waste) $)]$ * [High powder factor / 1000 (converts $\mathrm{kg}$ to $\mathrm{mt}$ )] / Operating days per year

$L=3,000,000 \cdot[1+(0.1 \cdot 2.7)] *[0.23 / 1000] / 300$

$\mathrm{L}=2.92 \quad 3 \mathrm{mt}$ ANFO equivalent (rounded to nearest unit)

Bahariva maximum blasting event ANFO consumption (M):

$M=$ Production rate ${ }^{*}[1+$ (stripping ratio * (specific gravity of ore + waste) $\left.)\right]^{*}$ [High powder factor $/ 1000$ (convents $\mathrm{kg}$ to $\mathrm{mt}$ )] / Operating days per year * maximum blast cycle time (working days between blasting events)

$M=[3,000,000 \cdot[1+(0.1 \cdot 2.7)] *[0.23 / 1000] / 300] * 10$

$M=29.2 \quad 30 \mathrm{~m}$ ANFO equivalent (rounded)

Table 4.1 provides the corresponding blasting range estimates for the main Egyptian mines identified in this study. Figure 4.1 shows site locations for the mines reported in table 4.1. Symbols reflect mine type (surface or underground) and maximum ANFO consumption for a given blasting event.

There were no mine sites that had calculated maximum blasting events greater than $50 \mathrm{mt}$ ANFO equivalent, and only nine sites with an estimated explosive potential in the range of $10-50 \mathrm{mt}$ of ANFO equivalent for a maximum blasting event. Several 
Table 4.1-Estimated Explosives Usage at the Main Egyptian Mines Used in this Study in Order of Esttmated ANFO Consumption

\begin{tabular}{|c|c|c|c|c|c|c|c|c|c|}
\hline \multirow[t]{2}{*}{ Mine } & \multirow[t]{2}{*}{ Latitude } & \multirow[t]{2}{*}{ Longitude } & \multirow[t]{2}{*}{$\begin{array}{l}\text { Primary } \\
\text { Product }\end{array}$} & \multirow[t]{2}{*}{$\begin{array}{l}\text { Mine } \\
\text { Type } \\
\text { (1) }\end{array}$} & \multirow[t]{2}{*}{$\begin{array}{r}\text { Production } \\
\text { (Mmtlyr) } \\
\text { (2) }\end{array}$} & \multicolumn{2}{|c|}{$\begin{array}{l}\text { Daily consumpion } \\
\text { (mi ANIFo) } \\
\text { (3) (4) }\end{array}$} & \multirow[t]{2}{*}{$\begin{array}{l}\text { Maximum } \\
\text { Blast Cycle } \\
\text { Time (days) } \\
\text { (5) }\end{array}$} & \multirow[t]{2}{*}{$\begin{array}{l}\text { Maximum } \\
\text { Blasting } \\
\text { Event } \\
\text { (mt ANFO) }\end{array}$} \\
\hline & & & & & & Low & High & & \\
\hline Assiut & N $27^{\prime} 15^{\prime}$ & $E 3111$ & Cement feed (6) & $s$ & 7.020 & 3 & 5 & 10 & 50 \\
\hline etwan area & N $29^{\circ} 31^{\prime}$ & E $31^{\circ} 20^{\prime}$ & feed (6) & $\mathbf{s}$ & 6.8 & 3 & 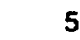 & 10 & 48 \\
\hline ahariya & $N 28^{\circ} 28^{\prime}$ & E $28^{\circ} 58^{\prime}$ & Iron & $\mathbf{s}$ & 3.000 & 2 & 3 & 10 & 30 \\
\hline neriyah & N $31^{\circ} 02^{\prime}$ & E $29^{\circ} 53^{\prime}$ & Cement feed (6) & $\mathbf{S}$ & 3.780 & 2 & 3 & 10 & 27 \\
\hline mm Bogma (n) & $N 29^{\circ} 00^{\circ}$ & E $33^{\circ} 21^{\circ}$ & Manganese & $\mathbf{S}$ & 0.300 & 1 & 2 & 10 & 22 \\
\hline bu Tartur (8) & $N 25^{\circ} 26^{\circ}$ & E $30^{\circ} 02$ & Phosphate & UG & 4.500 & 2 & 3 & 5 & 15 \\
\hline Beni Suef & N $29^{\circ} 01^{\circ}$ & E $31^{\circ} 09^{\prime}$ & Coment feed (6) & $\mathbf{S}$ & 1.800 & 1 & 1 & 10 & 13 \\
\hline Wadi Qena & $N 28^{\circ} 02$ & E $32^{\circ} 34^{\prime}$ & Cement feed (6) & $\mathbf{S}$ & 1.800 & 1 & 1 & 10 & 13 \\
\hline & $N 31^{\circ} 07^{\circ}$ & E $29^{\circ} 51^{\prime}$ & Cement feed (6) & $S$ & 1.440 & 1 & 1 & 10 & 10 \\
\hline Sebaiya & $N 25^{\circ} 10^{\prime}$ & E $32^{\circ} 40^{\prime}$ & Phosphate & $\mathbf{S}$ & 0.481 & 0 & 1 & 10 & 9 \\
\hline Hamrawein (9) & $N 26^{*} 17^{\prime}$ & E $3^{\circ} 4^{\circ} 03^{\prime}$ & Phosphate & UG & 1.200 & 1 & 1 & 5 & \\
\hline Rifaie & N 29. $55^{\circ}$ & E $31^{\circ} 22^{\prime}$ & Limestone & $\mathbf{S}$ & 0.750 & 0 & I & 10 & \\
\hline Beni Khalid & N $28^{\circ} 16^{\prime}$ & E $30^{\circ} 49^{\prime}$ & Limestone & $\mathbf{S}$ & 0.750 & 0 & 1 & 10 & \\
\hline hamid & $N 25^{\circ} 09^{\circ}$ & E 32' 55' & Phosphate & $\mathbf{s}$ & 0.099 & 0 & 0 & 10 & 2 \\
\hline Wadi Alaki & N 22. $09^{\prime}$ & E $33^{\circ} 12^{\prime}$ & Martile & $\mathbf{s}$ & 0.150 & 0 & 0 & 10 & \\
\hline aqa & $N 29^{\circ} 54^{\prime}$ & E $32^{*} 27$ & Dolomite & $\mathbf{S}$ & 0.100 & 0 & 0 & 10 & \\
\hline Sataga & N $26^{\circ} 32^{\circ}$ & E $\mathbf{3 3}^{\circ} \mathbf{5 8 ^ { \circ }}$ & Phosphate & UG & 0.170 & 0 & 0 & 5 & \\
\hline Adabiya & N $29^{\circ} 52$ & E $32^{*} 28^{\circ}$ & Dolomite & $\mathbf{s}$ & 0.093 & 0 & 0 & 10 & \\
\hline Maghara (8) & N $30^{\circ} 42$ & E $33^{\circ} 23^{\circ}$ & Coal & UG & 0.125 & 0 & 0 & 5 & \\
\hline all & $N 26^{\circ} 10^{\circ}$ & E $34^{\circ} 20^{\circ}$ & Phosphate & UG & 0.200 & 0 & 0 & 5 & 1 \\
\hline
\end{tabular}

(1) S-Surface; UG-Underground

(2) Mintyr-Million metric tons per year

(3) $m$ t ANFO-Metric tons of Ammonium Nitrate/Fuel Oil blasting agent equivalent. Estimate based on equations reported on pages 11-12.

(4) Assumed production schedule for all sites is 300 days/yr.

(5) Assumed maximum blasting cycle time for surface operation - 10 working days; underground operation - 5 working days.

(6) Typical raw material feed for cement plant is $85 \%$ limestone and $15 \%$ clay; minor amounts of gypsum and other materials may also be required. Production rate reflects total feed required to feed plant; raw materials assumed to come from local quarries near plant.

(7) Rehabilitation of the site was completed in 1990, but significant production had not been achieved by 1995.

(8) Sites are in the final development stage. Production at Abu Tartur began in 1995, but production halted by fire. Reported production rate reflects rate when in full production. Sites to use longwall mining, requiring only limited blasting.

(9) Reported production rate reflects full production capacity. In 1994, site produced 592ktyr, well below production capacity. 


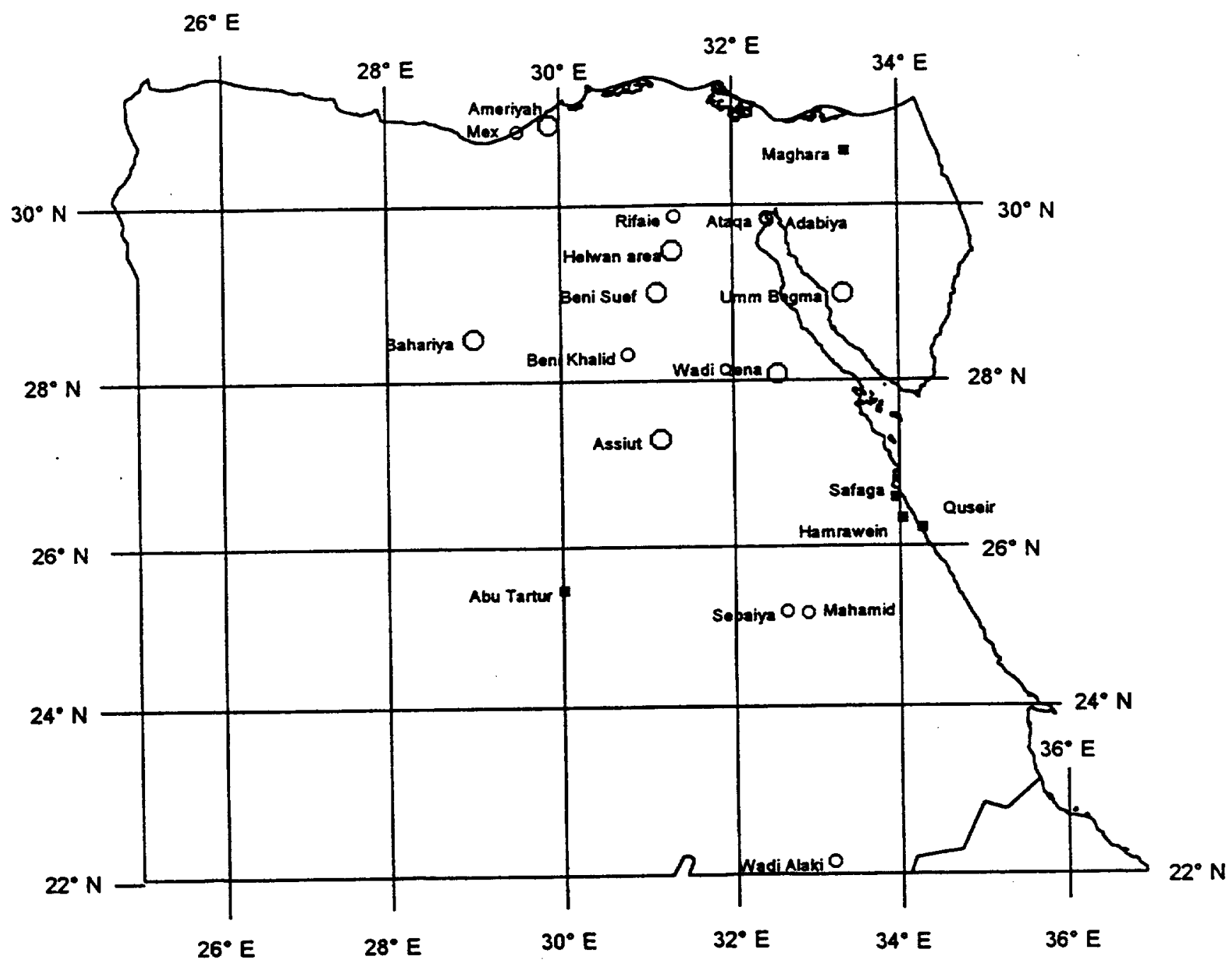

\section{LEGEND}

Underground Surface Maximum Blasting Event*

\begin{tabular}{lll}
$0 \quad 200 \quad 400$ & Kilometers \\
\hline
\end{tabular}

Not Applicable $0 \quad 10$ to 50 metric tons
- $0 \quad$ Less than 10 metric tons
ANFO Equivalent

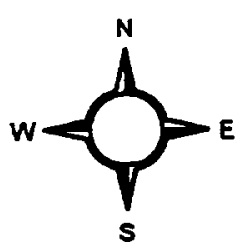

FIGURE 4.1 - Selected Egyptian Mines and Estimated Maximum Blasting Events 
sites included in this range provide limestone/clay feed material for cement production. It should be noted that production from such sites often comes from numerous small quarries feeding the nearby plant, rather that one large mining operation, so individual site explosive consumption would be smaller that the aggregated estimates reported in Table 4.1. Several sites were not producing in 1995, but were included in Table 4.1 because of their significant potential. Developing mines such as the Abu Tartur and Marghara mines use mechanized mining technology that requires only limited blasting. Production levels reported in the table reflect maximum capacities; in several cases actual production is well below that reported here.

\subsection{CONCLUSIONS}

While Egypt has potential to produce a wide variety of minerals, current production centers around several industrial minerals. Most mines are small, and few are world class mining operations. The largest mining units in Egypt have an estimated maximum explosive consumption potential of less than $50 \mathrm{mt}$ ANFO equivalent. Most mines require less than $10 \mathrm{mt}$ of ANFO equivalent per day. Mining is centered in two areas, the Nile Valley and Eastern Desert regions of Egypt. 
APPENDICES

1 
APPENDIX A: PRODUCINO MINERAL PROPERTIES IN EGYPT.

\begin{tabular}{|c|c|c|c|c|c|c|c|c|c|c|c|}
\hline \multicolumn{2}{|c|}{$\begin{array}{l}\text { MAP } \\
\text { KEY } \\
(1)\end{array}$} & \multirow{2}{*}{$\begin{array}{l}\text { NAME } \\
\text { (2) } \\
\text { Manzolo } \\
\text { Manzallan }\end{array}$} & \multicolumn{2}{|c|}{$\begin{array}{l}\text { COORDINATES } \\
\text { LAT. LONG. }\end{array}$} & COMMODTYY(IES) & $\begin{array}{l}\text { DATA } \\
\text { SOURCES } \\
\text { (3) }\end{array}$ & \multirow{2}{*}{$\begin{array}{l}\text { DATA } \\
\text { RELLANCE } \\
\text { (4) } \\
\text { General }\end{array}$} & \multirow{2}{*}{$\begin{array}{l}\text { 8TATU8 } \\
\text { (5) } \\
\text { Producer }\end{array}$} & \multirow{2}{*}{$\begin{array}{l}\text { MINE } \\
\text { TYPE } \\
\text { Surface }\end{array}$} & \multirow{2}{*}{$\begin{array}{l}\text { MARKETS } \\
\text { (8) } \\
\text { Domestle }\end{array}$} & COMMENTS \\
\hline & 1 & & $N 31^{\circ} 00^{\circ}$ & E $32^{\circ} 00^{\circ}$ & Gypsum & $2,4,13,16,30$ & & & & & $\begin{array}{l}\text { Ore h lake bash. } \\
\text { Frlable beds about } 80 \mathrm{~cm} \text { thlck overtaln by sall. }\end{array}$ \\
\hline & 2 & Matartya & N 31' or & E $31^{*} 65^{\prime}$ & sell & 22 & Genoral & Producer & Surface & Domeslic & Produced in 1979. \\
\hline & 3 & Abu Khachaba & $N 28^{\circ} 11^{\circ}$ & E $33^{\bullet} 10^{\circ}$ & Thenlum & 4 & Gonoral & Producer & Surface & Domestle & $\begin{array}{l}\text { Upper } 12 \mathrm{~m} \text { contalns } 7.5 \mathrm{Mmt} \text { sand with } 707 \mathrm{kt} \\
\text { oconomle minorals. }\end{array}$ \\
\hline & 4 & Ambaul & $N 24^{\circ} 61^{\prime}$ & E $34^{\circ} 40^{\circ}$ & Chromtte & 22 & Goneral & Producer & Unknown & Domostlo & Produced In 1979. \\
\hline & 5 & Benl Hassan & $N 27^{\circ} 57$ & $E 3^{\circ} 57$ & Limestone & 22 & General & Producer & Surface & Domestle & Producer h 1979. \\
\hline & 6 & Benl Khalld & N 28* $16^{\prime}$ & E $30^{\circ} 48^{\circ}$ & Limestone & 22,31 & General & Producer & Surface & Domestlc & $\begin{array}{l}\text { Resources for cement } 10.5 \mathrm{Mmm} \text {. } \\
\text { Annual production } 750 \mathrm{k} \text {. }\end{array}$ \\
\hline & 7 & Br Hafaft & $N 24^{\circ} 29^{\prime}$ & E $34 \cdot 4 r$ & Anthophyllate & 4 & General & Producer & Surface & Domestic & Mined shce 1944500 tpy. \\
\hline & 8 & $\begin{array}{l}\text { Darhalb } \\
\text { Darhib } \\
\text { Um Sololmat } \\
\text { Elgat }\end{array}$ & N $23^{\circ} 59^{\circ}$ & $E 5^{\circ} 05^{\circ}$ & $\begin{array}{l}\text { Talc } \\
\text { sampalono }\end{array}$ & $4,22,30$ & General & Producer & $\begin{array}{l}\text { Surface } \\
\text { Underground }\end{array}$ & Domeslle & $\begin{array}{l}\text { Produced in } 1979 . \\
\text { Used as filler in local industries. } \\
\text { Lonses confined to } 40 \mathrm{~m} \text { thick shear zone in rhyolite. } \\
\text { Annual produclion } 5 \mathrm{kt} \text {. }\end{array}$ \\
\hline & 9 & $\begin{array}{l}\text { El Alshan area } \\
\text { Hugban } \\
\text { Hololi } \\
\text { Khashir } \\
\text { Mashim } \\
\text { Bir Disis } \\
\text { Anguria }\end{array}$ & $N 24^{\circ} 15^{\prime}$ & E $35^{\circ} 12^{\prime}$ & $\begin{array}{l}\text { Tole } \\
\text { soapsiono }\end{array}$ & $4,22,30$ & Genoral & Producer & Underground & Domestic & $\begin{array}{l}\text { Tale neat besalls, ihyolites in association with } \\
\text { sulfides. } \\
\text { Tale in groenstone. } \\
\text { Annual production Skt. }\end{array}$ \\
\hline B & 10 & Gabal Duwn & $N 26^{*} 15^{\circ}$ & E $33^{*} 58^{\circ}$ & Phosphate & 22 & General & Producer & Surface & Domestle & Produced in 1979. \\
\hline \multirow[t]{3}{*}{ A } & 11 & Glran EI Ful & N 29.59 & E $31^{\circ} 03^{\prime}$ & $\begin{array}{l}\text { Dolomile } \\
\text { Limetione }\end{array}$ & 4,22 & General & Producor & Surface & Dorneslle & Quarry producls. \\
\hline & 12 & Halaft & $N 24^{\circ} 50^{\circ}$ & E $34^{\circ} 30^{\circ}$ & $\begin{array}{l}\text { Asbestos } \\
\text { Foldspar }\end{array}$ & 22,30 & General & Producer & Surface & Domesile & $\begin{array}{l}\text { Produced in } 1970 . \\
\text { Annual produclion } 440 \text { ipy. }\end{array}$ \\
\hline & 13 & Halaib & $N 23^{\circ} 30^{\circ}$ & E $35^{\circ} 00^{\prime}$ & Manganese & 1 & Goneral & Producer & Unknown & Domestic & $\begin{array}{l}\text { Ore occurs In belf } 70 \mathrm{~km} \times 7 \mathrm{~km} \text { replacing } \\
\text { conglomaralos or limestones. } \\
\text { Mined in } 1882 \text { al } 500 \mathrm{lpy} \text {. }\end{array}$ \\
\hline B & 14 & $\begin{array}{l}\text { Hamraweln } \\
\text { El Hamra } \\
\text { Kuelk }\end{array}$ & N 26. 17 & E $\mathbf{3}^{\circ} 03^{\circ}$ & Phosphale & $5,16,30$ & General & Producer & Underground & $\begin{array}{l}\text { Exportad } \\
\text { Europerfar East }\end{array}$ & $\begin{array}{l}\text { Hamrawah mhne is } 1.2 \mathrm{M} \text { lpy using UG methods. } \\
\text { Minng begen in } 1978.1990 \text { production } 592 \mathrm{kt} \text {. } \\
\text { Ore grade } 28 \mathrm{X} \text { P2O5. }\end{array}$ \\
\hline \multirow[t]{2}{*}{ B } & 15 & Haramiya & $N 20^{\circ} 11^{\circ}$ & E $33^{\circ} 55^{\prime}$ & Takc & 22 & General & Producer & Surface & Domesile & Produced h 1979. \\
\hline & 16 & Homi Mukbid & $N 24^{\circ} 09^{\circ}$ & $E 3^{\circ} 21^{\prime}$ & Fluorspar & 22 & Goneral & Producer & Surface & Domesice & Produced in 1979. \\
\hline B & 17 & Kab Um El Abas & $N 28^{\circ} 19^{\circ}$ & E $33^{\circ} 18^{\circ}$ & $\begin{array}{l}\text { Tak, } \\
\text { magnestite }\end{array}$ & 22 & General & Producer & Surface & Domestlc & Produced in 1979. \\
\hline
\end{tabular}


APPENDIX A: PRODUCING MINERAL PROPERTIES IN EOYPT.

\begin{tabular}{|c|c|c|c|c|c|c|c|c|c|c|c|}
\hline $\begin{array}{l}M \\
K E \\
\text { (1) }\end{array}$ & & $\begin{array}{l}\text { NAME } \\
\text { (2) }\end{array}$ & $\begin{array}{l}\text { COORDIN } \\
\text { LAT. }\end{array}$ & $\begin{array}{l}\text { ATES } \\
\text { LONG. }\end{array}$ & COMMODITY(IES) & $\begin{array}{l}\text { DATA } \\
\text { BOURCES } \\
\text { (3) }\end{array}$ & $\begin{array}{l}\text { DATA } \\
\text { RELIANCE } \\
\text { (4) }\end{array}$ & $\begin{array}{l}\text { STATUS } \\
\text { (5) }\end{array}$ & $\begin{array}{l}\text { MINE } \\
\text { TYPE }\end{array}$ & $\begin{array}{l}\text { MARKETS } \\
\text { (e) }\end{array}$ & COMMENTS \\
\hline & 18 & Khasm EI Galata & $N 28^{\circ} 42$ & E $32 * 22$ & Limestone & 2 & General & Producer & Surtace & Domealle & $\begin{array}{l}\text { Total production of crystalline llmestion in Egypt } \\
\text { reported to be } 9000 \mathrm{~m} 3 \mathrm{yr} \text { in } 1984 \text { from at sthes. }\end{array}$ \\
\hline & 19 & $\begin{array}{l}\text { Modinol Nogrús } \\
\text { Sakalt }\end{array}$ & $N 24^{\circ} 40^{\circ}$ & $E 34^{*} 47$ & Beny & $1,4,16,22$ & Oeneral & Producer & Surface & Domestic & $\begin{array}{l}\text { Dump sthos visibie, worked in ancient times. } \\
\text { Production from Roman timas in schists. } \\
\text { intermittont producer. }\end{array}$ \\
\hline B & 20 & $\begin{array}{l}\text { Ousok aroa } \\
\text { Hamadal } \\
\text { Alshan } \\
\text { Duw } \\
\text { Anz } \\
\text { Abu Tundub } \\
\text { Abu Snogella } \\
\text { Gohoha }\end{array}$ & $N 20^{*} 10^{\circ}$ & E $34^{\circ} 20^{\circ}$ & Phosphate & $\begin{array}{l}1,2,4,5,16 \\
17,22,28,30\end{array}$ & Confrmed & Producer & Underground & Exportod & 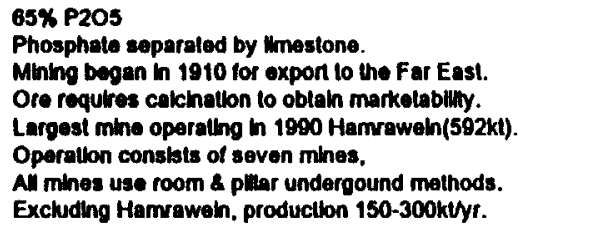 \\
\hline & 21 & $\begin{array}{l}\text { Res Shukek } \\
\text { Res Shujatr }\end{array}$ & N 28. $05^{\circ}$ & E $33^{\circ} 18^{\circ}$ & Sall & 22,28 & General & Producer & Surrace & Domestlc & Used in maunfacture of driting mud for petroloum indusiry. \\
\hline $\mathbf{B}$ & 22 & $\begin{array}{l}\text { Saraga area } \\
\text { Um El Howellat } \\
\text { Gasus } \\
\text { Wast } \\
\text { Monamed Rabah }\end{array}$ & $N 26^{*} 32$ & E $33^{\circ} 58^{\circ}$ & Phosphate & $\begin{array}{l}1,2,4,5,16 \\
17,22,28,30\end{array}$ & Confrmed & Producer & Underground & Exported & 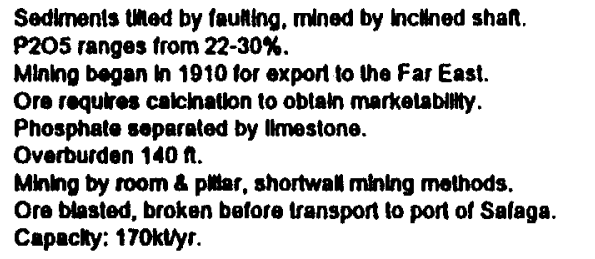 \\
\hline B & 23 & Sagla & $N 26^{\circ} \mathbf{2 0}$ & E $33^{\circ} 43^{\circ}$ & Magneske & 22 & General & Producer & Surface & Domestle & Produced in 1979. \\
\hline B & 24 & Soloumet & N 26. $16^{\circ}$ & E $33^{\circ} 40^{\circ}$ & Talc & 22 & Goneral & Producor & Surface & Domestle' & Produced in 1979. \\
\hline & 25 & Um Gerlfal & N 25*36 & $E \mathbf{3 4}^{\circ} \mathbf{3 4}$ & Ochro & 4 & General & Producer & Surface & Domostic & $\begin{array}{l}\text { Mined Intermittantty on a small scalo. } \\
\text { Res: } 0.4 \text { Mml. }\end{array}$ \\
\hline & 26 & Um Hobal & $N 23^{\circ} 45^{\prime}$ & E $33^{\circ} 11^{\circ}$ & Kaolln & 4 & General & Producer & Surface & Domesilic & Produced in 1979. \\
\hline & 27 & Um Kabu & $N 24^{\circ} 36^{\circ}$ & E $34^{\circ} 52$ & $\begin{array}{l}\text { Emeralds } \\
\text { Beryl }\end{array}$ & 16,22 & General & Producor & Surface & Domesile & $\begin{array}{l}\text { Anclent workings. } \\
\text { intermitent producer. }\end{array}$ \\
\hline & 28 & Unnamed & $N 25^{\circ} 00$ & E $33^{*} 40$ & Dolomine & 4 & General & Producer & Surface & Domesllo & Low grade glase sand. \\
\hline & 29 & Wadl Alakd & N $22^{\circ} 09^{\circ}$ & $E 33^{\circ} 12$ & $\begin{array}{l}\text { Marble } \\
\text { dotormino }\end{array}$ & 2,22 & General & Producer & Surrace & $\begin{array}{l}\text { Domesile } \\
\text { Exported }\end{array}$ & $\begin{array}{l}\text { In assoclatlon whit limestone \& dolomille. } \\
\text { Opened in } 1982 \text {. CaO conlent 33-42\%. } \\
\text { Largest marble quarry in Egypt. }\end{array}$ \\
\hline B & 30 & Wadi Alalla & $N 28^{\circ} 12$ & E $33^{\circ} 28^{\circ}$ & Stona & 2 & General & Producer. & Surface & Domestle & Serponitine ornarnental stone. \\
\hline B & 31 & Wadl Binl Abu Guraly & N 26 $16^{\circ}$ & E $34^{\circ} 02$ & Graphno & 22 & General & Producer & Surface & Domesilc & Small producer in 1979. \\
\hline & 32 & Wad EI Tom & N 23. 33' & E $\mathbf{3 3}^{\circ} \mathbf{1 3}$ & Barko & 22 & General & Producer & Surface & Domestle & Produced In 1979. \\
\hline
\end{tabular}


APPENDIX A: PRODUCING MINERAL PROPERTIES IN EOYPT.

\begin{tabular}{|c|c|c|c|c|c|c|c|c|c|c|c|}
\hline \multicolumn{2}{|c|}{$\begin{array}{l}\text { MAP } \\
\text { KEY } \\
\text { (1) }\end{array}$} & \multirow{2}{*}{$\begin{array}{l}\text { NAME } \\
\text { (2) } \\
\text { Wadl Hamamal }\end{array}$} & \multicolumn{2}{|c|}{$\begin{array}{l}\text { COORDINATES } \\
\text { LAT. LONG. }\end{array}$} & СоммоDITY(IES) & \multirow{2}{*}{$\begin{array}{l}\text { DATA } \\
\text { SOURCES } \\
\text { (3) } \\
2,22\end{array}$} & \multirow{2}{*}{$\begin{array}{l}\text { DATA } \\
\text { RELLANCE } \\
\text { (A) } \\
\text { General }\end{array}$} & \multirow{2}{*}{$\begin{array}{l}\text { STATUS } \\
\text { (6) } \\
\text { Producer }\end{array}$} & \multirow{2}{*}{$\begin{array}{l}\text { MINE } \\
\text { TYPE } \\
\text { Surface }\end{array}$} & \multirow{2}{*}{$\begin{array}{l}\text { MARKETS } \\
\text { (6) } \\
\text { Domestic } \\
\text { Exported }\end{array}$} & \multirow{2}{*}{$\begin{array}{l}\text { COMMENTS } \\
\text { Breccle verde omamental stone. } \\
\text { Produced intermitlanty from anclent times. }\end{array}$} \\
\hline B & 33 & & $N 25^{\circ} 59^{\circ}$ & E $33^{\circ} 35^{\prime}$ & Stone & & & & & & \\
\hline & 34 & Wadi Sannur & $N 28^{\circ} 5 z$ & E $31^{\circ} 05^{\circ}$ & Alabaster & $2,4,22$ & General & Producer & Surface & Domestle & $\begin{array}{l}\text { Quarny production was } 400 \text { m3yyr in small blocks. } \\
\text { Mined since Pharaonlc umes. } \\
\text { Ore in Wmestone iractures. }\end{array}$ \\
\hline & 35 & $\begin{array}{l}\text { Wadl SKreln } \\
\text { Wadl Solroln }\end{array}$ & $N 25^{\circ} 06^{\circ}$ & E $34^{\circ} 43$ & Chromite & 4,22 & General & Producer & Unknown & Domestke & $\begin{array}{l}2 \text { lenses, } 250 \text { tons, 35\% Cr2O3. } \\
\text { Operalling in 1979. }\end{array}$ \\
\hline & 36 & Wadi SHra & $N 25^{\circ} 31^{\circ}$ & E $34^{\circ} 18^{\circ}$ & Graphlto & 22 & General & Producer & Surface & Domestic & Produced al $500 \mathrm{lpy}$. \\
\hline & 37 & Zabara & $N 24^{\circ} 45^{\circ}$ & E $34^{\circ} 41^{\circ}$ & $\begin{array}{l}\text { Bond, tin } \\
\text { Emeralds }\end{array}$ & $4,16,22$ & Goneral & Producer & Surface & Domeslic & $\begin{array}{l}\text { Zono } 1-20 \mathrm{~m} \text { thlck in mlca echist. } \\
\text { Intermiltent producer. }\end{array}$ \\
\hline & 38 & $\begin{array}{l}\text { Zaglarana } \\
\text { Wadl Dakhal }\end{array}$ & $N 28^{\circ} 43^{\prime}$ & E $32 * 32$ & $\begin{array}{l}\text { Lmeston } \\
\text { Glass zand }\end{array}$ & 2.4 & General & Producor & Surface & Domestle & 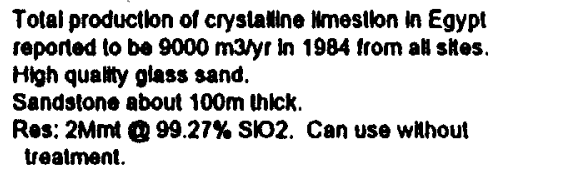 \\
\hline & 39 & Alam EI Margab & $\mathbf{N} 30^{\circ} \mathbf{4 3 ^ { \circ }}$ & E $30^{\circ} 08^{\circ}$ & Limesione & 22 & Gonoral & Producar & Surface & Domestic & Assay: $99.2 \%$ S1O2, $0.02 \%$ Fe2O3. \\
\hline & 40 & Idku & $N 31^{\circ} 15^{\circ}$ & E $30^{*} 15^{*}$ & San & 4.22 & General & Producer & Surfece & Domestic & Produced in 1979. \\
\hline & 41 & Harrafa & $N 30^{\circ} 53^{\circ}$ & E $30^{\circ} 19^{\circ}$ & $\begin{array}{l}\text { Sall, } \\
\text { natron }\end{array}$ & $1,22,30$ & General & Producer & Surface & Domestlc & $\begin{array}{l}\text { Ore doposititod by evaporation. } \\
\text { Two shatiow lakes. }\end{array}$ \\
\hline & 42 & $\begin{array}{l}\text { Rashid } \\
\text { Rosotta } \\
\text { Damielta }\end{array}$ & $\mathrm{N} 31^{*} 25^{\circ}$ & E $30^{\circ} \mathbf{2 3 ^ { \circ }}$ & $\begin{array}{l}\text { Sall } \\
\text { Iron }\end{array}$ & $4,17,22$ & General & Producer & Surface & Domestic & $\begin{array}{l}\text { Produces both sodlum carbonate and sulfale. } \\
\text { Ore deposited by ovaporation. } \\
\text { Produces salt, no iron production. }\end{array}$ \\
\hline \multirow[t]{3}{*}{ A } & 43 & Unnamed & $\mathbf{N} \mathbf{3 0}^{*} \mathbf{1 3}$ & $E 30^{\circ} 48^{\circ}$ & Bentonile & 22 & General & Producer & Surface & Domestic & Produced In 1979. \\
\hline & 44 & Wadi Nalrun & N $30^{\circ} 21^{\prime}$ & E $30^{\circ} 18^{\circ}$ & $\begin{array}{l}\text { Sall, } \\
\text { natron }\end{array}$ & $1,22,28,30$ & General & Producer & Surface & Domestle & $\begin{array}{l}\text { Oldest occurrence of natural soda. } \\
\text { Serles of ovaporke lakes. } \\
\text { Nalrun ts used in domestic soap, oll, and glass industrles. }\end{array}$ \\
\hline & 45 & $\begin{array}{l}\text { Bahartya area } \\
\text { EI Godlda } \\
\text { Ghorabl } \\
\text { Nasser } \\
\text { El Harra }\end{array}$ & N 28. $28^{\circ}$ & E $28^{\circ} 58^{\circ}$ & $\begin{array}{l}\text { Iron } \\
\text { barlle }\end{array}$ & $\begin{array}{l}2,4,6,16 \\
17,18,19,22 \\
27,30,31\end{array}$ & Confirmed & Producer & Surface & Domestle & 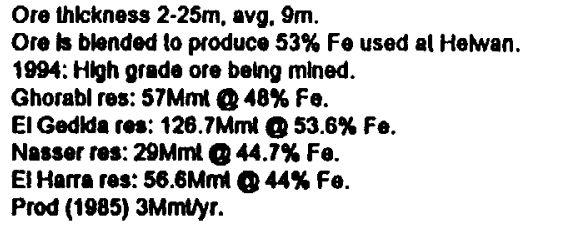 \\
\hline \multirow[t]{2}{*}{$A$} & 46 & Unnamod & N 200 $52^{\circ}$ & E $30^{\circ} 40^{\circ}$ & Basall & 22 & Gonomal & Producer & Surface & Domeatlo & Produced in 1979. \\
\hline & 47 & $\begin{array}{c}\text { Amoripah } \\
\text { Amoryla }\end{array}$ & N 31'02' & E 29॰53' & LImesione & 22 & General & Producer & Surface & Domestle & Produced in 1979. \\
\hline
\end{tabular}


APPENDIX A: PRODUCING MINERAL PROPERTIES IN EGYPT.

\begin{tabular}{|c|c|c|c|c|c|c|c|c|c|c|c|}
\hline \multirow{2}{*}{\multicolumn{2}{|c|}{$\begin{array}{l}\text { MAP } \\
\text { KEY } \\
\text { (1) }\end{array}$}} & \multirow{2}{*}{$\begin{array}{l}\text { MAME } \\
\text { (2) } \\
\text { MeX }\end{array}$} & \multicolumn{2}{|c|}{$\begin{array}{l}\text { COOROINATES } \\
\text { LAT. LONG. }\end{array}$} & COMMODITY(IES) & $\begin{array}{l}\text { DATA } \\
\text { SOURCES } \\
\text { (3) }\end{array}$ & $\begin{array}{l}\text { DATA } \\
\text { RELIANCE } \\
\text { (4) }\end{array}$ & $\begin{array}{l}\text { STATUS } \\
\text { (5) }\end{array}$ & $\begin{array}{l}\text { MINE } \\
\text { TYPE }\end{array}$ & $\begin{array}{l}\text { MARKETS } \\
\text { (6) }\end{array}$ & \multirow[b]{2}{*}{ 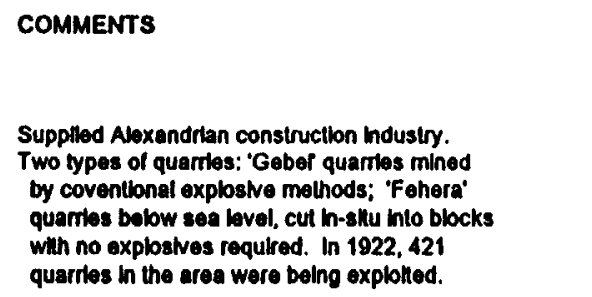 } \\
\hline & 48 & & $N 31^{\circ}$ or & E $29^{\circ} 50^{\circ}$ & $\begin{array}{l}\text { Dimension stone } \\
\text { lime } \\
\text { Soda ash } \\
\text { Sal }\end{array}$ & $1,4,22,28$ & General & Producer & Surface & Domesic & \\
\hline & 49 & $\begin{array}{l}\text { Kom Oushim } \\
\text { Kom Aoushelm }\end{array}$ & $\mathrm{N} 30^{\circ} \mathbf{3 2}$ & E $30^{\circ} 55^{\circ}$ & Dtatomito & 22 & Goneral & Producer & Surface & Domesilc & Produced in 1979 al $5 \mathrm{ktyr}$. \\
\hline & 50 & $\begin{array}{l}\text { El Menya } \\
\text { Idmu }\end{array}$ & N $28^{\circ} 08^{\circ}$ & E $30^{\circ} 36^{\circ}$ & Lmeatone & $\mathbf{2 , 2 2}$ & General & Producer & Surface & Domestle & $\begin{array}{l}\text { Tolal production of crystathe Hriestion in Egypt } \\
\text { reported to be } 9000 \mathrm{~m} 3 \mathrm{yr} \text { in } 1984 \text { Irom all shes. }\end{array}$ \\
\hline & 51 & Samalun & N $28^{\circ} 18^{\circ}$ & E $30^{\circ} 42$ & Limeatone & 4 & Gonoral & Producer & Surface & $\begin{array}{l}\text { Domostio } \\
\text { Exportod }\end{array}$ & $\begin{array}{l}\text { Hl-grade producls with varied usos. } \\
\text { Grado 96.4-98.8\% MgCO3. }\end{array}$ \\
\hline & 52 & Tona EI Gabel & N $27^{\circ} 45^{\prime}$ & E $30^{\circ} 40^{\circ}$ & Limedione & 22 & Genoral & Producer & Surface & Domestle & Producer ln 1979. \\
\hline A & 53 & Helwan area & $N 29^{\circ} 51^{\circ}$ & E $31^{\circ} 20^{\circ}$ & $\begin{array}{l}\text { Llmostone } \\
\text { Clay }\end{array}$ & $1,4,18,22,31$ & Confrmed & Producer & Surface & Domesile & $\begin{array}{l}\text { Quarties used for comont production. } \\
\text { Plant has a capaclyy of } 4.4 \mathrm{ML} \text {. } \\
\text { Blasting done on shins } 2 \text { and } 3 \text {; twice a week. } \\
\text { Blast holes on } 5 \mathrm{~m} \text { centers, } 6 \mathrm{~m} \text { Irom face, depth } 30-55 \mathrm{~m} \text {. } \\
\text { ANFO used as oxplosive. } \\
15000 \text { ipe minod. }\end{array}$ \\
\hline A & 54 & Rilale & $N 29^{\circ} 55^{\circ}$ & E $31^{*} 22$ & Limestone & 22,31 & General & Producer & Surface & Domesilc & $\begin{array}{l}\text { Produced in } 1985 . \\
\text { Production } 750 \mathrm{k} \text { tyr. }\end{array}$ \\
\hline A & 55 & $\begin{array}{l}\text { Tura } \\
\text { Tourah }\end{array}$ & E $29^{\circ} 56^{\circ}$ & E $31^{\circ} 16^{\prime}$ & Limestone & 1,18 & General & Producer & Surface & Domesilc & $\begin{array}{l}\text { Old quarry sthe reported to be closed in } 1992 . \\
\text { Plant has a capacty of } 1.4 \mathrm{Mt} \text {. }\end{array}$ \\
\hline A & 56 & Abu Zaabal & N $30^{\circ} 11^{\circ}$ & E $31^{\bullet} 25^{\prime}$ & Basalt & 22 & Genorat & Producer & Surface & Domestic & Large scale producer. \\
\hline A & 57 & $\begin{array}{l}\text { El Anmar } \\
\text { Gabal EI Anmar }\end{array}$ & $N 30^{\circ} 03^{\circ}$ & E $31^{\circ} 10^{\circ}$ & $\begin{array}{l}\text { Limestone } \\
\text { Quartzlite } \\
\text { Sendsione }\end{array}$ & $1,4,22$ & Genoral & Producer & Surfaco & Domestic & Old quarry she. \\
\hline A & 58 & EI Oigla & $N 30^{\circ} 00^{\circ}$ & E $31^{\circ} 00^{\circ}$ & Dolomite & 4 & Gonoral & Producer & Surface & Domestic & Selective quarrying methodz used. \\
\hline A & 59 & Gabal Mukkatam & $\mathrm{N} 30^{\circ} \mathbf{0 0}^{\circ}$ & E $31^{\circ} 10$ & Limeatone & 4 & General & Producer & Surrace & Domestle & Dimensional thmestone blocks for construclion. \\
\hline A & 60 & Maadl & N $29^{\circ} 68^{\circ}$ & $E 31^{\circ} 15^{*}$ & Glass sand & 2,4 & General & Producer & Surface & Domestlc & $\begin{array}{l}\text { Production has occurred for al bast } 50 \text { yrs. } \\
\text { Low qually (97\% S1O2، 1\%fe2O3) glass sand. }\end{array}$ \\
\hline & 61 & $\begin{array}{l}\text { Ballah } \\
\text { El Baliah }\end{array}$ & N $30^{\circ} 43^{\prime}$ & E $32^{\circ} 20^{\circ}$ & Gypsum & $\begin{array}{l}2,4,13 \\
16,22,30\end{array}$ & General & Producer & Surface & Dormestle & $\begin{array}{l}\text { Oro in lake bash. } \\
\text { Res: } 11 \text { Mml. } \\
\text { Frtable beds about } 80 \mathrm{~cm} \text { thlck overlaln by satt. }\end{array}$ \\
\hline & 62 & Pont Said & $N 31^{\bullet} 13^{\prime}$ & E $32^{\bullet} 22^{\prime}$ & Salt & 22 & Gonoral & Producar & Surface & 'Domostle & Produced In 1979. \\
\hline & 63 & Dokhan & $N 31^{\circ} 21^{\prime}$ & E $25^{*} 25^{\prime}$ & Stone & 2 & General & Producer & Surface & Exported & Impertal porphyry ormamental stone. \\
\hline & 64 & Morsa Mairuh & $N 31^{\circ} 21^{\circ}$ & E $27^{*} 1 T$ & Sall & 4,22 & General & Producor & Surface & Domestic & Ore doposilod by ovaporalion. \\
\hline
\end{tabular}


APPENDIX A: PRODUCING MINERAL PROPERTIES IN EOYPT.

\begin{tabular}{|c|c|c|c|c|c|c|c|c|c|c|}
\hline $\begin{array}{l}\operatorname{MAP} \\
\text { (1) }\end{array}$ & $\begin{array}{l}\text { NAME } \\
\text { (2) }\end{array}$ & $\begin{array}{l}\text { COORDIN } \\
\text { LAT. }\end{array}$ & $\begin{array}{l}\text { ITES } \\
\text { LONO. }\end{array}$ & COMMODITY(IES) & $\begin{array}{l}\text { DATA } \\
\text { SOURCES } \\
\text { (3) }\end{array}$ & $\begin{array}{l}\text { DATA } \\
\text { RELLANCE } \\
\text { (4) }\end{array}$ & $\begin{array}{l}\text { STATUS } \\
\text { (s) }\end{array}$ & $\begin{array}{l}\text { MINE } \\
\text { TYPE }\end{array}$ & 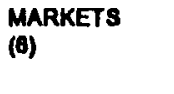 & COMMENTS \\
\hline 65 & $\begin{array}{l}\text { Kharga area } \\
\text { Abu Tartur }\end{array}$ & N 25" 26' & E 30.02 & $\begin{array}{l}\text { Phosphale } \\
\text { Alumina salks } \\
\text { Magneatum salts }\end{array}$ & $\begin{array}{l}1.2,4,5,14 \\
17,19,30\end{array}$ & General & Plenned (7) & Underground & $\begin{array}{l}\text { 50\% Dornestlc } \\
50 \% \text { Exported }\end{array}$ & 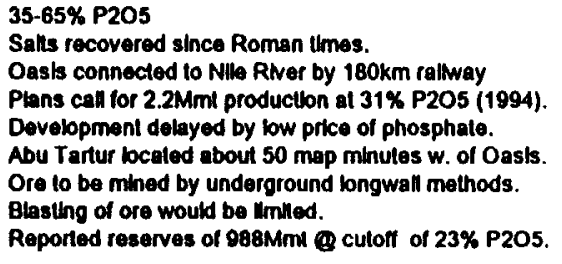 \\
\hline 66 & Kharga & $N 25^{\circ} 20^{\circ}$ & E $30^{\circ} 30^{\circ}$ & Ochre ciays & 1,22 & General & Producer & Surface & Domestle & Intermiltant production. \\
\hline 67 & Adablya & $N 29^{\circ} 52$ & E $32^{\circ} 28^{\circ}$ & Dolomite & $22,27,30$ & General & Producer & Surtace & Domestlc & $\begin{array}{l}\text { Operated by Egyptlan iron \& Sleel. } \\
\text { Production } 1985 \text { 93kt. }\end{array}$ \\
\hline 68 & Abu Er-Darag & N $29^{\circ} 22$ & E $32^{*} 34^{\circ}$ & $\begin{array}{l}\text { Limostone } \\
\text { Glass sand }\end{array}$ & 2,4 & General & Producer & Surface & Domestlc & $\begin{array}{l}\text { Tolal production of crystalinge Hrmasilon in Egypt } \\
\text { reported to be } 9000 \text { m3yr in } 1984 \text { from at sties. } \\
\text { Low qually glass send. }\end{array}$ \\
\hline 69 & Alaq" & $N 29^{\circ} 54^{\prime}$ & E $32^{\circ} 27$ & Dolomite & $4,22,30$ & Goneral & Producer & Surface & Domestle & $\begin{array}{l}\text { Producls used in glass, coramic, and stool. } \\
\text { Annual production } 1982 \text { 100kt. }\end{array}$ \\
\hline 70 & Aswan & N $24^{\circ} 04^{\circ}$ & E $32^{\circ} 34^{\circ}$ & Grankle & 2 & Goneral & Producer & Surface & $\begin{array}{l}\text { Domeslle } \\
\text { Exported }\end{array}$ & $\begin{array}{l}22 \text { sthes in arou. } \\
\text { Reglonal production in } 1984 \text { reported as } 4200 \mathrm{m3} .\end{array}$ \\
\hline 11 & Kom Ombo & $\mathbf{N} 24^{\circ} 30^{\prime}$ & E 32' $51^{\circ}$ & Limestone & 22 & General & Producer & Surfece & Domestlc & Produced in 1979. \\
\hline 72 & Kom Ombo & N 24' $31^{\prime}$ & E 33. $04^{\circ}$ & Clay & 22 & General & Producer & Surface & Domestle & Produced in 1979. \\
\hline 73 & $\begin{array}{l}\text { Mahamid } \\
\text { East Sebalya }\end{array}$ & N 25' $09^{\prime}$ & E 32 $55^{\prime}$ & Phosphale & 22.28 & General & Producer & $\begin{array}{l}\text { Surface } \\
\text { Underground }\end{array}$ & Domesilc & $\begin{array}{l}\text { Mahamld mine worked by UG room \& pllar tin 1980's. } \\
\text { Mahamid open ph and Wost Sebalya mlned by open phn } \\
\text { and draglines. }\end{array}$ \\
\hline 14 & $\begin{array}{l}\text { Sebals } \\
\text { Sharawne } \\
\text { Oweinlya } \\
\text { Qurayal } \\
\text { Heganta } \\
\text { Mosilah Yassin } \\
\text { Wost Sobalya }\end{array}$ & N 25" $10^{\circ}$ & E $32^{\circ} 40^{\circ}$ & Phosphale & $\begin{array}{l}1,2,5,16,17 \\
22,26,28 \\
30,31\end{array}$ & General & Producer & $\begin{array}{l}\text { Surface } \\
\text { Underground }\end{array}$ & Domestlc & 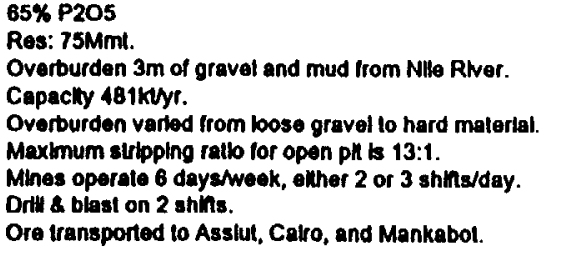 \\
\hline 75 & Kalabsha & N $23^{\circ} 32$ & E $32^{\circ} 40^{\circ}$ & Kaollin & $4,16,22,30$ & General & Producor & Surface & Domestic & $\begin{array}{l}\text { Res: } 16.5 M m \text { m. } \\
\text { Produced 20-50ktyr. }\end{array}$ \\
\hline 76 & $\begin{array}{l}\text { Asslut } \\
\text { Wadl EI Asshity } \\
\text { Basra } \\
\text { Asyut }\end{array}$ & N $27^{\circ} 15^{\prime}$ & E $31^{\circ} 11^{\circ}$ & $\begin{array}{l}\text { Lrneslone } \\
\text { Alabastor }\end{array}$ & $2,4,22$ & General & Producer & Surfaco & Domestle & $\begin{array}{l}\text { Total production of crystalline limestion in Egypi } \\
\text { reported to be } 9000 \mathrm{~m} 3 / \mathrm{yr} \text { in } 1984 \text { from all sthes. }\end{array}$ \\
\hline 77 & El Kharsha & N 2933 & E $30^{\circ} 38^{\prime}$ & Dlatomio & 22 & General & Producer & Surtace & Domestlc & Produced ln 1979. \\
\hline 78 & Kasi El Sagha & $N 29^{\circ} 35^{\circ}$ & $E 30^{\circ} 40^{\circ}$ & $\begin{array}{l}\text { Glass sand. } \\
\text { bentonito }\end{array}$ & 22 & General & Producer & Surlace & Domestic & $\begin{array}{l}\text { Producod In } 1979 . \\
\text { Ros: 4.8Mml. }\end{array}$ \\
\hline
\end{tabular}


APPENDIX A: PRODUCINO MINERAL. PROPERTIES IN EOYPT.

\begin{tabular}{|c|c|c|c|c|c|c|c|c|c|c|c|}
\hline \multicolumn{2}{|c|}{$\begin{array}{l}\text { MAP } \\
\text { KEY } \\
\text { (1) }\end{array}$} & \multirow{2}{*}{$\begin{array}{l}\text { NAME } \\
\text { (2) } \\
\text { Damiotia }\end{array}$} & \multicolumn{2}{|c|}{$\begin{array}{l}\text { COORDINATES } \\
\text { LAT. LONG. }\end{array}$} & COMMODITY(IES) & \multirow{2}{*}{$\begin{array}{l}\text { DATA } \\
\text { SOURCES } \\
\text { (3) } \\
22\end{array}$} & \multirow{2}{*}{$\begin{array}{l}\text { DATA } \\
\text { RELLANCE } \\
\text { (4) } \\
\text { Gonoral }\end{array}$} & \multirow{2}{*}{$\begin{array}{l}\text { 8TATUS } \\
\text { (5) } \\
\text { Producor }\end{array}$} & \multirow{2}{*}{$\begin{array}{l}\text { MINE } \\
\text { TYPE } \\
\text { Surface }\end{array}$} & \multirow{2}{*}{$\begin{array}{l}\text { MARKETS } \\
\text { (6) } \\
\text { Domostio }\end{array}$} & \multirow{2}{*}{$\begin{array}{l}\text { COMMENTS } \\
\text { Producod in } 1979 .\end{array}$} \\
\hline A & 79 & & $N 30^{\circ} 13^{\prime}$ & $E 30^{\circ} 48^{\circ}$ & $\begin{array}{l}\text { Salt } \\
\text { Black sande }\end{array}$ & & & & & & \\
\hline & 80 & Baltim & $\mathbf{N} 31^{*} 33^{*}$ & $E 31^{\circ} 05^{\prime}$ & $\operatorname{san}$ & 4,22 & Ganeral & Producer & Surface & Domastic & Ore deposilled by evaporation. \\
\hline & 81 & 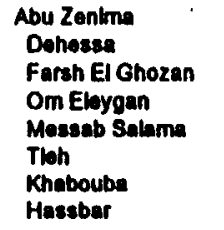 & N 29, $03^{\circ}$ & E 33" 28' & $\begin{array}{l}\text { Glass sand } \\
\text { Kaolln }\end{array}$ & $2,4,22,30$ & General & Producer & Surface & $\begin{array}{l}\text { Exported } \\
\text { Domestic }\end{array}$ & $\begin{array}{l}500,000 \text { tons sulkable for tableware and sheel. } \\
\text { Kaollin beds } 2-2.5 \mathrm{~m} \text { thikk under } 30-40 \mathrm{~m} \text { sandstone. }\end{array}$ \\
\hline & 82 & $\begin{array}{l}\text { Maghare } \\
\text { Magharah } \\
\text { Sala }\end{array}$ & $N 30^{\circ} 42$ & E $33^{\circ} \mathbf{2 3}$ & Coal & $\begin{array}{l}2,4,9,14,15 \\
22,28,30,31\end{array}$ & Confirmod & Planned (7) & Underground & Domesilc & 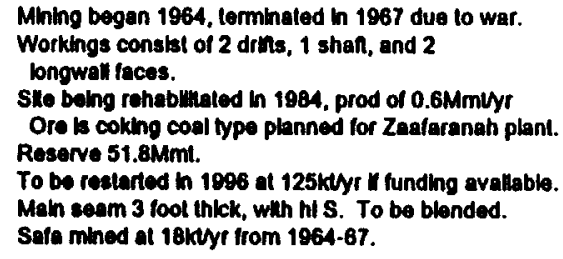 \\
\hline & 83 & $\begin{array}{l}\text { Serabll of Khadem } \\
\text { Sarabell El Khadlm }\end{array}$ & N 28, $55^{\circ}$ & E $33^{\circ} 28^{\prime}$ & Turquatse & 1,22 & General & Producer & Surface & Domosilic & Intermitent producer since anclont times. \\
\hline & 84 & $\begin{array}{l}\text { Umm Bogma } \\
\text { Wadl Nassib } \\
\text { Wadl El Nosman } \\
\text { Wadl Shallal } \\
\text { Abu Qafas } \\
\text { Wadl EI Hussonl } \\
\text { El Adklya }\end{array}$ & $\mathbf{N} \mathbf{2 0}^{\circ} \mathbf{0 0}^{\circ}$ & E $33^{\circ} 21^{\prime}$ & $\begin{array}{l}\text { Mangenese } \\
\text { Copper }\end{array}$ & $\begin{array}{l}1,4.16 .22 \\
30,31\end{array}$ & General & $\begin{array}{l}\text { Plonned (7) } \\
\text { Past producer }\end{array}$ & Surfaco & Domestle & 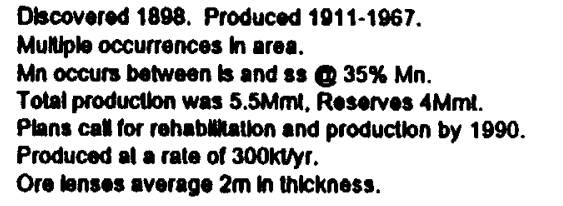 \\
\hline & 85 & Elba area & N 22* 25' & $E \mathbf{3 0 ^ { \circ }} \mathbf{1 0}$ & $\begin{array}{l}\text { Manganose } \\
\text { Chromite }\end{array}$ & 16,22 & General & Producer & Unknown & Domeslle & $\begin{array}{l}\text { Mined since the } 1860^{\prime} \mathrm{s} \text {. } \\
\text { Ore from } 11 \text { sthes. }\end{array}$ \\
\hline & 86 & Bent Suef & N $29^{\circ} 01^{\circ}$ & E 31'09' & $\begin{array}{l}\text { LImosione } \\
\text { Gypsum }\end{array}$ & 22 & General & Producer & Surface & Domesile & Began production in 1991. \\
\hline & 87 & Wadl Qona & N 28, 02 & E $32^{*} 3^{*}$ & $\begin{array}{l}\text { Limestone } \\
\text { Glass sand }\end{array}$ & 22 & Goneral & Producer & Surface & Domestle & Production began in 1891. \\
\hline & & $\begin{array}{l}\text { (1) Represents prop } \\
\text { (2) Due to solnware I } \\
\text { (3) Complete lat of d } \\
\text { (4) General - denote: } \\
\text { (5) Baceuse of the v } \\
\text { (6) Renacts whother } \\
\text { (7) Stes are not curr }\end{array}$ & ary & of lor prodi & $\begin{array}{l}\text { denotes deposil inf } \\
\text { ation, the slatus of } \\
\text { t i mlernal or for ox }\end{array}$ & $\begin{array}{l}\text { Nollon confim } \\
\text { Nolual sties } \\
\text { t. }\end{array}$ & $\begin{array}{l}\text { oral source } \\
\text { current. }\end{array}$ & nes vany & & & \\
\hline
\end{tabular}




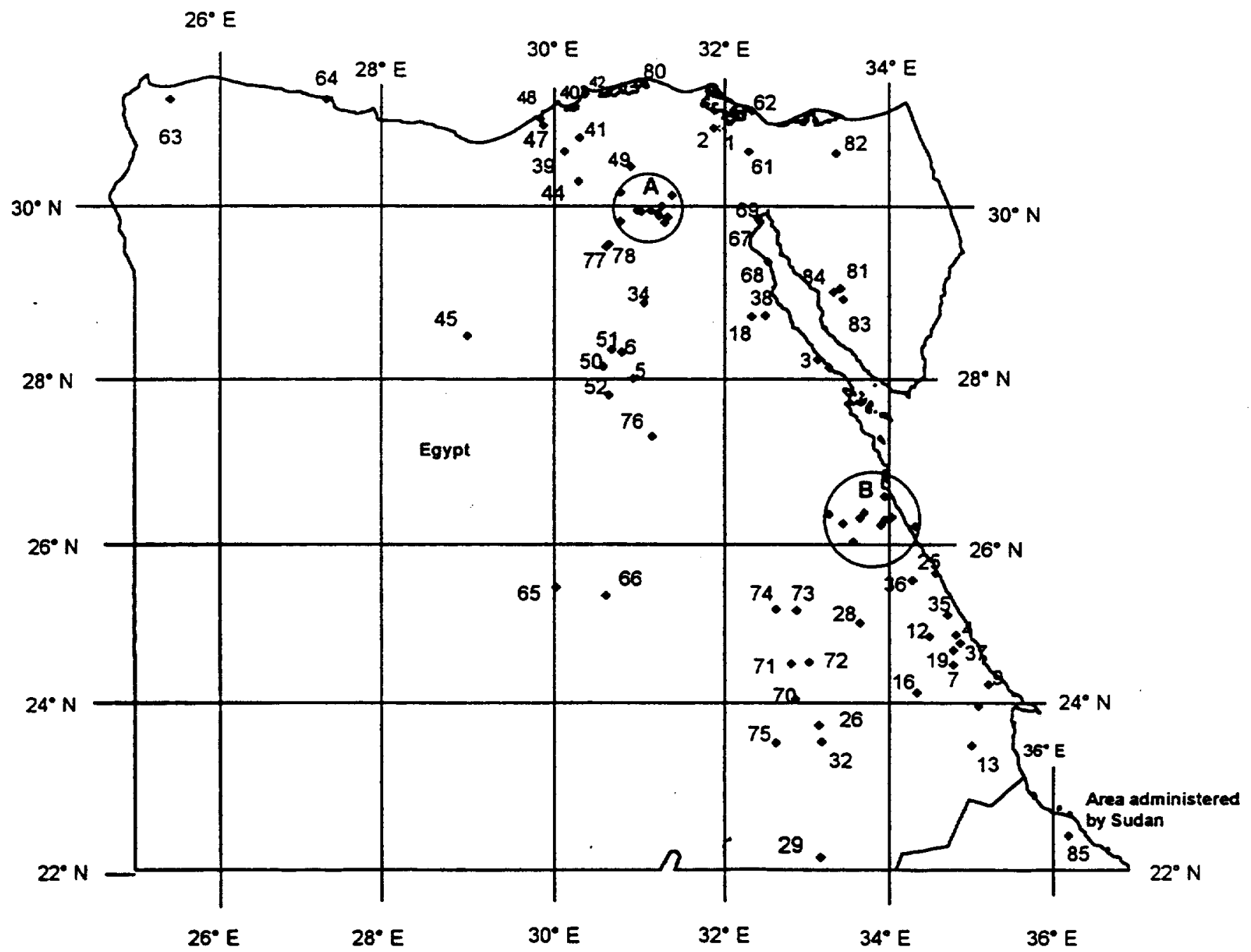

\section{LEGEND}

Producing or Developing Mineral Property*
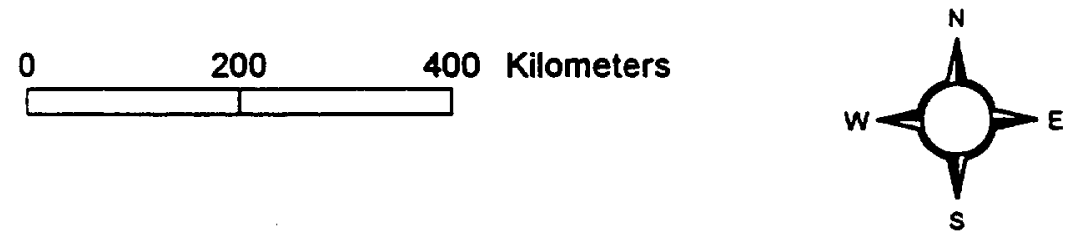

Letter represents property grouping as defined in appendix. 
APPENDIX B: PAST PRODUCING MINERUL PROPERTIES IN EOYPT.

\begin{tabular}{|c|c|c|c|c|c|c|c|c|c|c|}
\hline $\begin{array}{l}\text { MA } \\
\text { KE) } \\
(1)\end{array}$ & & $\begin{array}{l}\text { NAME } \\
\text { (2) }\end{array}$ & $\begin{array}{l}\text { COORDIN } \\
\text { LAT. }\end{array}$ & $\begin{array}{l}\text { ATES } \\
\text { LONO. }\end{array}$ & COMMODITY(IE8) & $\begin{array}{l}\text { DATA } \\
\text { SOURCES } \\
\text { (3) }\end{array}$ & $\begin{array}{l}\text { DATA } \\
\text { RELANCE } \\
\text { (4) }\end{array}$ & $\begin{array}{l}\text { 8TATUS } \\
\text { (5) }\end{array}$ & $\begin{array}{l}\text { MINE } \\
\text { TYPE }\end{array}$ & COMMENTS \\
\hline & 1 & Dakhla & N $25^{\circ} 30^{\circ}$ & E $20^{\circ} 10^{\circ}$ & $\begin{array}{l}\text { Phouphate } \\
\text { Alumina salts } \\
\text { Magnealum sells }\end{array}$ & $1,4,22,30$ & Confirmed & Pest producer & Surface & $\begin{array}{l}\text { 35-65\% P205 } \\
\text { Salls recovered since Roman thes. } \\
\text { Mhing coased at beginaling of the } 20 \text { th cenlury. }\end{array}$ \\
\hline & 2 & $\begin{array}{l}\text { Umm Rus } \\
\text { Om Rus }\end{array}$ & N $25^{\circ} 28$ & E $34^{\prime} 35^{\prime}$ & Gold & $\begin{array}{l}1,2,3,4 \\
16,22\end{array}$ & Confimad & Past producer & Surtug & 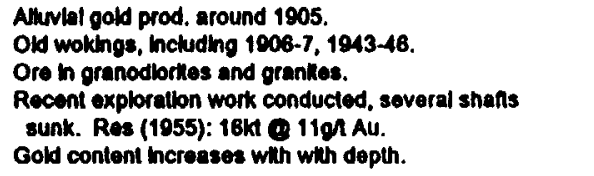 \\
\hline M & 3 & Umm Garalat & N $22^{\circ} 30^{\circ}$ & E $33^{\circ} 28^{\prime}$ & Gold & $1,3.16,22,30$ & General & Past producer & Surface & 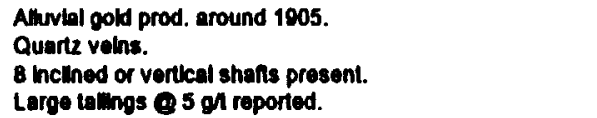 \\
\hline H & 4 & $\begin{array}{c}\text { Barramiyah } \\
\text { Barramiya }\end{array}$ & N 22, $20^{\circ}$ & E $33^{\circ} 4 r$ & Gold & $\begin{array}{l}1,3,4,16 \\
22,30\end{array}$ & Confirmed & Pasl producer & Surface & 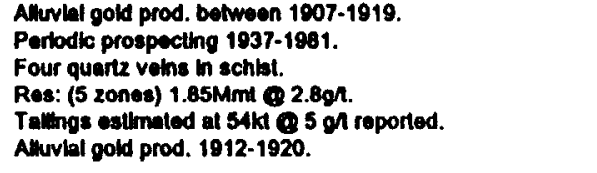 \\
\hline $\mathbf{k}$ & 6 & Sukeri & $N 24^{\circ} 5 r$ & $E 3^{\circ} 42$ & Gold & $1,3,4,22,30$ & Conilumed & Post producer & Underground & 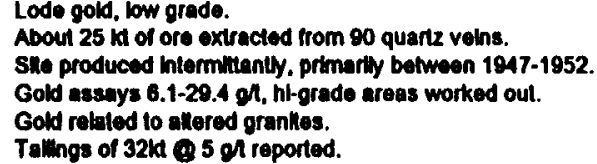 \\
\hline G & 7 & $\begin{array}{l}\text { Alallah } \\
\text { Alatila }\end{array}$ & $N 28^{\circ} 06^{\circ}$ & $E 3^{\prime} 2 r$ & $\begin{array}{l}\text { Gold } \\
\text { silver }\end{array}$ & $1,3,16,22$ & General & Past producer & Unknown & Worked 1914-1918. \\
\hline G & 8 & Semna & $N 26^{\circ} 2 T^{2}$ & E $3^{\circ} 3^{\circ}$ & Gold & $1,3,16,22$ & General & Past producer & Underground & $\begin{array}{l}\text { Low grade occurrence } \\
\text { Anclent workhgs. }\end{array}$ \\
\hline & 9 & Halmuir & N $22^{\circ} 35^{\prime}$ & $E 3^{\circ} 1 r$ & Gold & $1,3,16,22$ & General & Past producer & Surface & $\begin{array}{l}\text { Low grade occurrence } \\
\text { Ouart' volns. } \\
\text { Worked 1904-1907. }\end{array}$ \\
\hline K & 10 & $\begin{array}{l}\text { Gobel Rossas } \\
\text { Gobel El Rusas }\end{array}$ & $N 25^{\circ} 10^{\circ}$ & E $34^{\circ} 44^{\circ}$ & Lead & $1,2,11,16,22$ & Conifrmed & Past producer & Underground & $\begin{array}{l}\text { Worked } 1912-1916 . \\
\text { Sundes } \mathrm{h} \text { bedded is. } \\
\text { 1984 reserves reported al } 100,000 \text { lons } 10 \% \mathrm{Zn} \text {. } \\
\text { 1981 res: 375kt Q } 7 \% \mathrm{Zn}, 16.2 \% \mathrm{~Pb} \text {. }\end{array}$ \\
\hline $\mathbf{K}$ & 11 & Ranga & $N 24^{\circ} 25^{\circ}$ & E $35^{\circ} 08^{\prime}$ & Lead & 1,22 & General & Past producer & Unknown & $\begin{array}{l}\text { Small deposkl, low grade. } \\
\text { In gypsum assoctated whih sulur and pyrtie. }\end{array}$ \\
\hline & 12 & $\begin{array}{l}\text { Zebligel } \\
\text { Zoberel }\end{array}$ & $N 23^{\circ} 3 r$ & E $36^{\circ} 12^{\circ}$ & Perldol & 1 & General & Past producer & Surface & $\begin{array}{l}\text { No production atice } 1914 . \\
\text { Gem quallty stones. }\end{array}$ \\
\hline$M$ & 13 & $\begin{array}{l}\text { Abu Soyal } \\
\text { Abu Swayol }\end{array}$ & N $22^{\circ} 48^{\circ}$ & E $33^{\circ} 45^{\circ}$ & $\begin{array}{l}\text { Copper } \\
\text { nilkel } \\
\text { coball }\end{array}$ & $\begin{array}{l}1,4,11,16 \\
22,30\end{array}$ & Goneral & Past producer & Surface & $\begin{array}{l}\text { Worked } \mathrm{h} \text { anclent thmes. } \\
\text { Workings Inctudo } 69 \mathrm{~m} \text { shan, } 1205 \mathrm{~m} \text { drill core. } \\
\text { Messive \& disseminaled ore in amphlbolite. } \\
\text { Lens } 500 \mathrm{~m} \times 30 \mathrm{~m} \text {. } \\
\text { Res: } 85 \mathrm{kt} \quad 2.8 \% \text { Cu. } 1.53 \% \text { NI. }\end{array}$ \\
\hline
\end{tabular}


APPENDIX B: PAST PRODUCING MINERAL PROPERTIES IN EOYPT.




APPENOIX B: PAST PRODUCINO MINERAL PROPERTIES IN EGYPT.

\begin{tabular}{|c|c|c|c|c|c|c|c|c|c|c|}
\hline $\begin{array}{l}\mathrm{MA} \\
\mathrm{KE} \\
\text { (1) }\end{array}$ & & $\begin{array}{l}\text { NAME } \\
\text { (2) }\end{array}$ & $\begin{array}{l}\text { COORDIN } \\
\text { UAT. }\end{array}$ & $\begin{array}{l}\text { ATES } \\
\text { LONG. }\end{array}$ & COMMODITY(IES) & $\begin{array}{l}\text { DATA } \\
\text { BOURCES } \\
\text { (3) }\end{array}$ & $\begin{array}{l}\text { DATA } \\
\text { RELLANCE } \\
\text { (4) }\end{array}$ & $\begin{array}{l}\text { STATUS } \\
\text { (5) }\end{array}$ & $\begin{array}{l}\text { MINE } \\
\text { TYPE }\end{array}$ & COMMENTS \\
\hline G & 37 & Kubanlya & $N 26^{\circ} 00^{\circ}$ & E $33^{\circ} 32$ & $\begin{array}{l}\text { Granne } \\
\text { Quartz } \\
\text { Clay }\end{array}$ & 1 & General & Past producer & Surface & Old quarry sile. \\
\hline B & 38 & Maisara & N $30^{\circ} 00^{\circ}$ & E $31^{\circ} 10$ & Lumestone & 1 & General & Past producer & Surface & Old quarry slle. \\
\hline B & 39 & Haysum & $\mathrm{N} 30^{\circ} 00^{\circ}$ & E $31^{\circ} 10^{\circ}$ & Limestone & 1 & General & Past producer & Surface & Old quany she. \\
\hline B & 40 & Muse & E $30^{\circ} 01^{\circ}$ & E $31^{\circ} 19^{\circ}$ & Limestone & 1 & Generel & Past producer & Surface & Old quarry slle. \\
\hline $\mathbf{B}$ & 41 & El Rane & E $30^{\circ} 12$ & E $31^{\circ} 02$ & Limestone & 1 & General & Past producer & Surface & ONd quarry she. \\
\hline D & 12 & El Harr & $N 29^{\circ} 50^{\circ}$ & E 32' 02 & Linnestone & 1.22 & General & Past producer & Surface & Old quarry she. \\
\hline c & 43 & Rimal el Basatin & E $29^{\circ} 12$ & E $31^{\circ} 28^{\circ}$ & Limestone & 1 & General & Past producer & Surface & Old quarry she. \\
\hline B & 44 & El Tablina & N $30^{\circ} 00^{\circ}$ & E $31^{\circ} 10$ & Limosione & 1 & General & Past producer & Surface & ONd quarry she. \\
\hline B & 45 & El Amara & $E 30^{\circ} 21^{\circ}$ & E $31^{\circ} 08^{\circ}$ & Limestone & 1 & General & Past producer & Surface & Old quarry slle. \\
\hline B & 14 & Athar ol Nabl & $N 30^{\circ} 00^{\circ}$ & E $31^{\circ} 10^{\circ}$ & Limestone & 1 & General & Past producer & Surface & Old quarry sille. \\
\hline B & 44 & Batn el Bagara & N $30^{\circ} 00^{\circ}$ & E $31^{\circ} 10^{\circ}$ & Limestone & 1 & Generel & Past producer & Surface & Old quarry she. \\
\hline $\mathbf{\theta}$ & 4 & Eln ol Stra & $N 30^{\circ} 00^{\circ}$ & E $31^{\circ} 10^{\circ}$ & Limestone & 1 & General & Past producer & Surface & Old querry alke. \\
\hline $\mathbf{B}$ & 44 & Zawbl Nasara & $N 30^{\circ} 00$ & E $31^{\circ} 10^{\circ}$ & Limestone & 1 & Goneral & Past producer & Surtace & Ord quarty sthe. \\
\hline $\mathbf{\theta}$ & 44 & Elablad & $N 30^{\circ} 00^{\circ}$ & E $31^{\circ} 10^{\circ}$ & Lumesione & 1 & General & Pest producer & Surface & Old quarry slle. \\
\hline $\mathbf{8}$ & 44 & Moedissa & $N 30^{\circ} \infty^{\circ}$ & E $31^{\circ} 10^{\circ}$ & Llmeslone & 1 & Genoral & Past producer & Surface & Old quarry stie. \\
\hline 8 & 4 & Dowaleqa & $N 30^{\circ} 00^{\circ}$ & $E 31^{\circ} 10^{\circ}$ & Limestone & 1 & General & Past producer & Surface & Old quarry she. \\
\hline & 46 & Rimal el Abbaslya & $N 30^{\circ} 32$ & $E 31^{\circ} 43^{\circ}$ & Lumeslone & 1 & General & Pasi producer & Surface & Old querry sthe. \\
\hline B & 47 & Rimal el Anmar & N $30^{\circ} 03^{\circ}$ & E $31^{\circ} 18^{\prime}$ & Limestone & 1 & General & Past producer & Surface & Old quarry she. \\
\hline B & 48 & Gebol of Mounayar & $N 30^{\circ} 30^{\circ}$ & E $31^{\circ} 00^{\circ}$ & Llmestone & 1 & General & Past producer & Surface & Ond quarry alle. \\
\hline & 49 & lemallis & N $30^{\circ} 35^{\circ}$ & E $32^{\circ} 16^{\circ}$ & $\begin{array}{l}\text { Sand \& graval } \\
\text { Gypaum }\end{array}$ & 1 & Genera: & Past producer & Surface & Old quarry ske. \\
\hline J & so & $\begin{array}{l}\text { Aswan } \\
\text { Lake Nasser }\end{array}$ & N $24^{\circ} 05^{\circ}$ & E $32^{\circ} 56^{\circ}$ & Iron & $\begin{array}{l}2,4,6,18,17 \\
22,30\end{array}$ & Confirmod & Past producer & Surface & 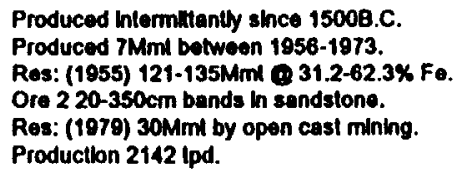 \\
\hline
\end{tabular}


APPENDIX B: PAST PRODUCING MINERAL PROPERTIES IN EOYPT.

\begin{tabular}{|c|c|c|c|c|c|c|c|c|c|c|}
\hline $\begin{array}{l}\text { MA } \\
\text { KE } \\
(1)\end{array}$ & & $\begin{array}{l}\text { NAME } \\
\text { (2) }\end{array}$ & $\begin{array}{l}\text { COOROINA } \\
\text { LAT. }\end{array}$ & $\begin{array}{l}\text { ATEs } \\
\text { LONG. }\end{array}$ & COMMODITY(IES) & $\begin{array}{l}\text { DATA } \\
\text { SOURCES } \\
\text { (3) }\end{array}$ & $\begin{array}{l}\text { DATA } \\
\text { RELIANCE } \\
\text { (4) }\end{array}$ & $\begin{array}{l}\text { STATUS } \\
\text { (5) }\end{array}$ & $\begin{array}{l}\text { MINE } \\
\text { TYPE }\end{array}$ & COMMENTS \\
\hline $\mathbf{K}$ & 51 & Umm Oud & $N 24^{\circ} 51^{\prime}$ & E $3^{*} 38^{\circ}$ & Gold & $2,3,16,22,30$ & General & Past producer & Underground & $\begin{array}{l}\text { Disseminaled ore in lentlcutar zones. } \\
14,000 \text { lons o } 25 \mathrm{gm} / \mathrm{m} \text {. } \\
\text { Explolled durthg } 1947-1953 \text {. }\end{array}$ \\
\hline G & 52 & El SWd & N $28^{\circ} 00^{\circ}$ & E $33^{\circ} 35^{\circ}$ & Gold & $2,4,16,22$ & General & Past producer & Underground & 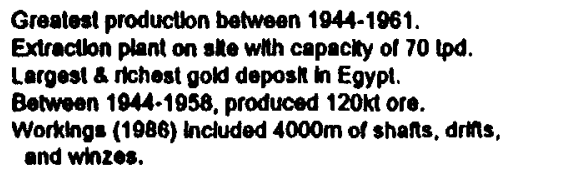 \\
\hline & 53 & Fatire & $N 26^{\circ} 42$ & E $33^{\circ} 17$ & Gold & $2,3,16,22$ & Goneral & Past producer & Underground & $\begin{array}{l}\text { Gold-bearing dikes of loblille porphyry. } \\
\text { Anclent mine. }\end{array}$ \\
\hline K & 54 & $\begin{array}{l}\text { Umm Samiuld } \\
\text { High } \\
\text { El Maskl }\end{array}$ & $N 24^{\circ} 14^{\circ}$ & E $34^{\circ} 40^{\circ}$ & $\begin{array}{l}\text { 2he, Copper } \\
\text { Lead, Gold } \\
\text { siver }\end{array}$ & $\begin{array}{l}1,4,11,16,17 \\
22,23,30\end{array}$ & Contrmed & Past producer & Underground & 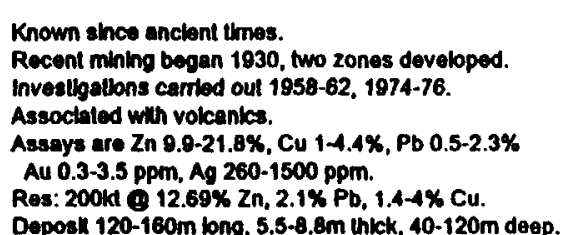 \\
\hline к & 55 & $\begin{array}{c}\text { Hamata } \\
\text { Alshan }\end{array}$ & N $24^{\circ} 22$ & E $35^{\circ}$ or & $\begin{array}{l}\text { Zlnc, Copper } \\
\text { Lead, Talc }\end{array}$ & 2,22 & Genoral & Past producor & Underground & $\begin{array}{l}\text { Gossans and sullide lenses in lake. } \\
\text { Ore grades } 42 \% \mathrm{Zn}, 2.5 \% \mathrm{Cu} \text {, and } 0.08 \% \mathrm{~Pb} \text {. }\end{array}$ \\
\hline A & 56 & $\begin{array}{l}\text { Gharbanylal } \\
\text { EI Ghortantal }\end{array}$ & $N 30^{\circ} 53^{\circ}$ & E $20^{\circ} 30^{\circ}$ & $\begin{array}{l}\text { Gypsum } \\
\text { ineslone }\end{array}$ & $2,4,16,22$ & General & Pest producer & Surface & $\begin{array}{l}\text { Salls in deproseton elong const. } \\
\text { Reserves reported al } 17 \mathrm{Mm} \text {. }\end{array}$ \\
\hline A & 57 & Omayld & N $30^{\circ} \mathbf{A T}$ & E $29^{\circ} 12$ & Gypsum & 2,4.16.22 & General & Past producer & Surface & $\begin{array}{l}\text { Sells in deproselon along coast. } \\
\text { Reserves reported at } 17 \mathrm{Mmm} \text {. }\end{array}$ \\
\hline A & 58 & Hermman & N $30^{\circ} 48^{\prime}$ & E $29^{\circ} 23^{\circ}$ & Gypsum & $2,13,16,22,30$ & Gonoral & Past producer & Surface & $\begin{array}{l}\text { Saks in depression slong coast. } \\
\text { Frlable beds aboul } 80 \mathrm{~cm} \text { thick overtain by sall. }\end{array}$ \\
\hline c & 59 & Quarrel EIFarass & $N 29^{\circ} 35^{\circ}$ & E $30^{\circ} 58^{\circ}$ & Gypaum & 2 & General & Past producer & Surface & Aluuvhum deposks. \\
\hline c & 60 & Girza & $N 29^{\prime} 2 r$ & E $31^{*} 12$ & Gypsum & 2,16 & General & Past producer & Surface & Alurvlum dopostis. \\
\hline A & 61 & $\begin{array}{c}\text { Burqan } \\
\text { Barkan }\end{array}$ & N 30' $36^{\circ}$ & E $29^{\circ} 30^{\circ}$ & Gypsum & 2,22 & General & Past producer & Surface & Large extent, $7-10 \mathrm{~m}$ In thickness. \\
\hline D & 62 & Ras Maland & N 29, $13^{\circ}$ & E $32^{\circ} 56^{\prime}$ & $\begin{array}{l}\text { Gypsum } \\
\text { Anhydrtte }\end{array}$ & $\begin{array}{l}2,4,13,16, \\
22,30\end{array}$ & General & Past producer & Surface & 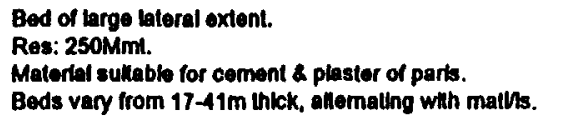 \\
\hline $\mathbf{K}$ & 63 & $\begin{array}{l}\text { Abu Ghalage } \\
\text { Abu Ghalaga }\end{array}$ & $N 24^{\circ} 21^{\prime}$ & E $35^{\circ} 00^{\circ}$ & $\begin{array}{l}\text { TKankum, Iron } \\
\text { Vanadbum }\end{array}$ & $2,4,16,22,30$ & Confrmed & Past producer & Unknown & 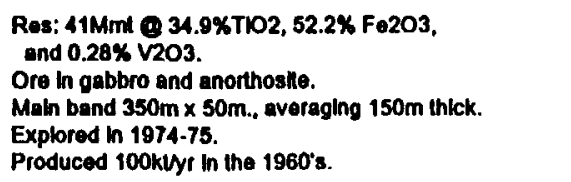 \\
\hline
\end{tabular}


APPENDIX B: PAST PRODUCINO MINERAL PROPERTIES IN EGYPT.

\begin{tabular}{|c|c|c|c|c|c|c|c|c|c|c|}
\hline \multicolumn{2}{|c|}{$\begin{array}{l}\text { MAP } \\
\text { KEY } \\
\text { (1) }\end{array}$} & \multirow{2}{*}{$\begin{array}{l}\text { NAME } \\
\text { (2) } \\
\text { Wadi EI Mirah }\end{array}$} & \multicolumn{2}{|c|}{$\begin{array}{l}\text { COORDINATES } \\
\text { LAT. LONG. }\end{array}$} & \multirow{2}{*}{$\begin{array}{l}\text { COMMODITY(IES) } \\
\text { Marble }\end{array}$} & \multirow{2}{*}{$\begin{array}{l}\text { DATA } \\
\text { SOURCES } \\
\text { (3) } \\
2.22\end{array}$} & \multirow{2}{*}{$\begin{array}{l}\text { DATA } \\
\text { RELIANCE } \\
\text { (d) } \\
\text { General }\end{array}$} & \multirow{2}{*}{$\begin{array}{l}\text { STATUS } \\
\text { (5) } \\
\text { Pasl producer }\end{array}$} & \multirow{2}{*}{$\begin{array}{l}\text { MINE } \\
\text { TYPE } \\
\text { Surface }\end{array}$} & COMMENTS \\
\hline H & 64 & & N 25. $11^{\circ}$ & E $33^{\circ} 45^{\circ}$ & & & & & & Producllon reached 1200 m3yr; mtermittanl $1980 \mathrm{~s}$. \\
\hline & 65 & Umm Ghelg & $N 25^{\circ} 43^{\circ}$ & E $3 A^{\cdot} \cdot 2 T$ & $\begin{array}{l}\text { Loed, Zinc } \\
\text { copper }\end{array}$ & $\begin{array}{l}2,4,11,16,17 \\
22,30\end{array}$ & Confrmed & Past producer & Unknown & 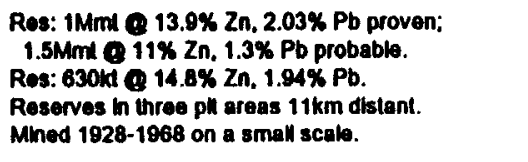 \\
\hline H & 66 & Alud & N $25^{\circ} 02$ & E $34^{\circ} 24^{\prime}$ & $\begin{array}{l}\text { Gold } \\
\text { barke }\end{array}$ & $\begin{array}{l}2,3,4,16,17 \\
22,30\end{array}$ & Conflrmed & Pasl producer & Underground & 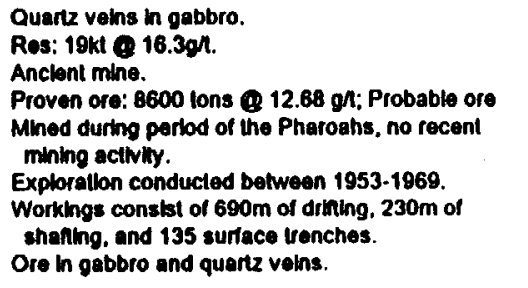 \\
\hline $\mathrm{L}$ & 67 & Umm Elelge & $N 233 r$ & E $34^{\circ} 59^{\circ}$ & Gold & 3.22 & General & Past producer & Surface & 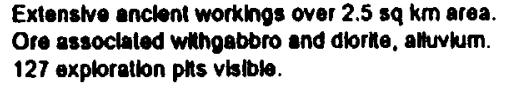 \\
\hline L & 68 & Hutk & $N 23^{\circ} 2 T$ & E $35^{\circ} 11^{\circ}$ & Gold & $2,3,16,22$ & Goneral & Past producer & SurfacervG & $\begin{array}{l}\text { Mined by anclents and early } 1900 \text { s. } \\
\text { Ore in molavolcanic nissures niling quartz velns. }\end{array}$ \\
\hline L & 69 & $\begin{array}{l}\text { Urga El Raran } \\
\text { Ourga Rayan } \\
\text { Argaryan }\end{array}$ & $N 23^{\circ} 20^{\circ}$ & E $35^{\circ} 04^{\circ}$ & Gold & 3.22 & General & Past producer & Unknown & $\begin{array}{l}\text { Ore in molevolcank fulfs cul by quartz velns. } \\
\text { Anclent workling. }\end{array}$ \\
\hline L & 70 & Gobel El Anbal & $N 23^{\circ} 06^{\circ}$ & E $35^{\circ} 10^{\circ}$ & Gold & 3 & General & Pasi producer & Surface & $\begin{array}{l}\text { Anclent ruins vissible. } \\
\text { Ore in molavolcanics. }\end{array}$ \\
\hline K & 71 & $\begin{array}{l}\text { Umm Tundaba } \\
\text { Umm Tanolaba }\end{array}$ & $N 24^{\circ} 56^{\circ}$ & E $3^{\circ} \mathbf{4 4}^{\circ}$ & Gold & $\mathbf{3 , 2 2}$ & General & Past producer & Underground & $\begin{array}{l}\text { Ore in quartz vein culling molavolcanlc lufls. } \\
\text { Workhgs constat of } 4 \text { shans and dumps. }\end{array}$ \\
\hline $\mathbf{L}$ & 72 & Bolan & $\mathbf{N} 23^{\prime} 3 r$ & E $35^{\circ} 02^{\circ}$ & Gold & 3,22 & General & Past producar & Unknown & Anclenl mlne. \\
\hline L & 73 & Umm Kalib & $N 2320^{\circ}$ & E $35^{\circ} 10^{\circ}$ & Gold & 3,22 & General & Pasl producer & Unknown & Anclent working. \\
\hline G & 75 & Hammamal & N 26. 15' & E $33^{\circ} 24^{\prime}$ & Gold & 3,30 & General & Past producar & Unknown & $\begin{array}{l}\text { Ore In granodlorke slock. } \\
\text { Ore mined ln ancient limes, 1944-1958. } \\
\text { Ore apparenily mined oul. } \\
\text { Ore grade range was } 11-26 \mathrm{gh} \text {. } \\
\text { Anclent workhg. }\end{array}$ \\
\hline G & 76 & Abu Moroiwal & N 26. $34^{\circ}$ & $E$ 33. $42^{\circ}$ & Gold & 22 & Genoral & Pasi producer & Unknown & Ancionl working. \\
\hline 1 & 71 & Kunlunos & $\mathbf{N} 23^{*} 59^{\prime}$ & E $33^{\circ} 00^{\circ}$ & Gold & 3,22 & General & Past producer & Unknown & Anclent worklng. \\
\hline J & 78 & El Hudl & $N 23^{\circ} 57^{\prime}$ & E $33^{\circ} 08^{\prime}$ & $\begin{array}{l}\text { Gold } \\
\text { Barlte }\end{array}$ & $3,4,22,30$ & Genaral & Pasl producet & Unknown & $\begin{array}{l}\text { Anclent mine. } \\
\text { Barfe velns In metamorphlc rocks. }\end{array}$ \\
\hline & 79 & Umm Ashira & $N 23^{\circ} 08^{\prime}$ & E $33^{\circ} 15^{\prime}$ & Gold & 3.22 & General & Pas! producer & Surface & $\begin{array}{l}\text { Quartz voln and placer. } \\
\text { Anclent working. }\end{array}$ \\
\hline
\end{tabular}


APPENDIX B: PAST PRODUCINO MINERAL PROPERTIES IN EGYPT.

\begin{tabular}{|c|c|c|c|c|c|c|c|c|c|c|}
\hline \multicolumn{2}{|c|}{$\begin{array}{l}\text { MAP } \\
\text { KEY } \\
\text { (1) }\end{array}$} & \multirow{2}{*}{$\begin{array}{l}\text { NAME } \\
\text { (2) } \\
\text { Hertart }\end{array}$} & \multicolumn{2}{|c|}{$\begin{array}{l}\text { COORDINATES } \\
\text { LAT. LONO. }\end{array}$} & COMMODITY(IES) & \multirow{2}{*}{$\begin{array}{l}\text { DATA } \\
\text { BOURCES } \\
\text { (3) } \\
3,22\end{array}$} & \multirow{2}{*}{$\begin{array}{l}\text { DATA } \\
\text { RELLANCE } \\
\text { (4) } \\
\text { General }\end{array}$} & \multirow{2}{*}{$\begin{array}{l}\text { 8TATUS } \\
\text { (5) } \\
\text { Past producer }\end{array}$} & \multirow{2}{*}{$\begin{array}{l}\text { MINE } \\
\text { TYPE } \\
\text { Unknown }\end{array}$} & \multirow{2}{*}{$\begin{array}{l}\text { COMMENTS } \\
\text { Anclent working. }\end{array}$} \\
\hline$M$ & 80 & & N 22* $59^{\circ}$ & E $33^{\circ} 26^{\circ}$ & Gold & & & & & \\
\hline M & 81 & $\begin{array}{l}\text { Neklb } \\
\text { Neqlb }\end{array}$ & $N 22^{\circ} 40^{\circ}$ & E $33^{\circ} 43^{\prime}$ & Gold & 3,22 & General & Pest producer & Unknown & $\begin{array}{l}\text { Occurrence of placer and alleralton zones. } \\
\text { Anclent working. }\end{array}$ \\
\hline M & 82 & Block E & N $222^{\circ} 36^{\circ}$ & E $33^{\circ} 20^{\circ}$ & Gold & 3,22 & General & Pest producer & Unknown & $\begin{array}{l}\text { Occurrence of placers on alluvial terraces. } \\
\text { Worked before WWI. }\end{array}$ \\
\hline M & 83 & $\begin{array}{l}\text { Marahk } \\
\text { Marahb }\end{array}$ & N 22, 30' & E $33^{\circ} 2 r$ & Gold & $\mathbf{3 , 2 2}$ & General & Past producer & Unknown & $\begin{array}{l}\text { Occurrence of quentz velns. } \\
\text { Anclent working. }\end{array}$ \\
\hline $\mathbf{M}$ & 84 & Atshant & N 22: 34' & E $33^{\circ} 33^{\prime}$ & Gold & 3,22 & General & Pasl producer & Unknown & $\begin{array}{l}\text { Occurrence of quartz valus and elleratton zones. } \\
\text { Ancloni working. }\end{array}$ \\
\hline$M$ & 85 & Fllal & $N 22^{\circ} \mathbf{2 4 ^ { \prime }}$ & E 33' 36' & Gold & 3,22 & General & Past producer & Unknown & $\begin{array}{l}\text { Occurrence of quartz velns. } \\
\text { Anclent working. }\end{array}$ \\
\hline $\mathbf{M}$ & 86 & Murra & $N 22^{\circ} 34^{\prime}$ & E $33^{\circ} 55^{\prime}$ & Gold & 3,22 & General & Past producer & Unknown & $\begin{array}{l}\text { Occurrence of placer and alleralton zones. } \\
\text { Anclent working. }\end{array}$ \\
\hline$M$ & 87 & Abu Fass & N 22'08' & E $33^{\circ} 52$ & Gold & 3.22 & General & Pasl producer & Unknown & $\begin{array}{l}\text { Occurrence of quartz velins. } \\
\text { Anctent working. }\end{array}$ \\
\hline K & 88 & Hangallya & $N 24^{\circ} 51^{\prime}$ & E $34^{\circ} 32$ & Gold & $3,16.22,30$ & General & Past producar & Undarground & $\begin{array}{l}\text { Explohed durting 1938-1952. } \\
\text { Ore in granlite. }\end{array}$ \\
\hline H & 89 & Dungash & $N 24^{\circ} 5 T$ & E $33^{\circ} 52$ & Gold & 3,22 & Goneral & Past producer & Unknown & Anctont mino. \\
\hline $\mathbf{H}$ & 90 & $\begin{array}{l}\text { Talal Gadalla } \\
\text { Flle Godella }\end{array}$ & $N 25^{\circ} 24^{\circ}$ & E 33' $36^{+}$ & Gold & 3,22 & General & Past producer & Unknown & $\begin{array}{l}\text { Occurrence in dlorlle. } \\
\text { Anclent working. }\end{array}$ \\
\hline H & 91 & Umm Samn & $N 25^{\circ} 19^{\circ}$ & E $34^{\circ} 06^{\prime}$ & Gold & 3.22 & General & Past producer & Unknown & Anclent working. \\
\hline H & 92 & Umm Hugab & $N 25^{\circ} 00^{\circ}$ & E 34 $05^{\circ}$ & Gold & 3.22 & General & Past producer & Unknown & Anclant worklng. \\
\hline & 93 & Umm Mongul & N 27 $50^{\circ}$ & E 33. $00^{\circ}$ & Gold & 3,22 & General & Past producer & Unknown & Anclont working. \\
\hline & 94 & Umm Balad & $N 27^{\circ} \mathbf{2 0}$ & E $32^{\circ} 46^{\circ}$ & Gold & 3,22 & General & Past producer & Unknown & Anclent working. \\
\hline & 95 & Wadl Dlb & $N 27^{\circ} 48^{\circ}$ & E $32^{\circ} 59^{\prime}$ & Gold & 3,22 & General & Past producor & Unknown & Anclont working. \\
\hline $\mathbf{G}$ & 96 & $\begin{array}{l}\text { Abu Qarahish } \\
\text { Abu Drahish }\end{array}$ & $N 26^{\circ} 24^{\circ}$ & E $33^{\circ} 35^{\circ}$ & Gold & 3,22 & General & Past producer & Unknown & Anclent workling. \\
\hline G & 97 & Kab Andrl & $N 28^{\circ} 22^{\prime}$ & E $33^{\circ} 35^{\prime}$ & Gold & $\mathbf{3 , 2 2}$ & General & Past producer & Unknown & Anclent working. \\
\hline $\mathbf{G}$ & 98 & Sagl & $N 26^{\circ} 21^{\circ}$ & E $33^{\circ} 49^{\circ}$ & Gold & 3.22 & General & Past producer & Unknown & Anclont working. \\
\hline $\mathbf{G}$ & 99 & Gldam! & $N 26^{\circ} 24^{\circ}$ & E $33^{\circ} 26^{\circ}$ & Gold & 3.22 & Oeneral & Past producer & Unknown & Anclent worklng. \\
\hline G & 100 & Abu Had & $N 28^{\circ} 16^{\circ}$ & E $\mathbf{3 3}^{\circ} \mathbf{3 1 ^ { \circ }}$ & Gold & 3,22 & General & Past producer & Unknown & Anclent working. \\
\hline G & 101 & $\begin{array}{l}\text { EIRebshl } \\
\text { Gabal Rabshl }\end{array}$ & $N 26^{\circ} 12$ & E $33^{\circ} 42$ & Gold & 3,22 & Goneral & Past producer & Unknown & Anclent working. \\
\hline
\end{tabular}


APPENDIX B: PAST PRODUCING MINERAL PROPERTIES IN EOYPT.

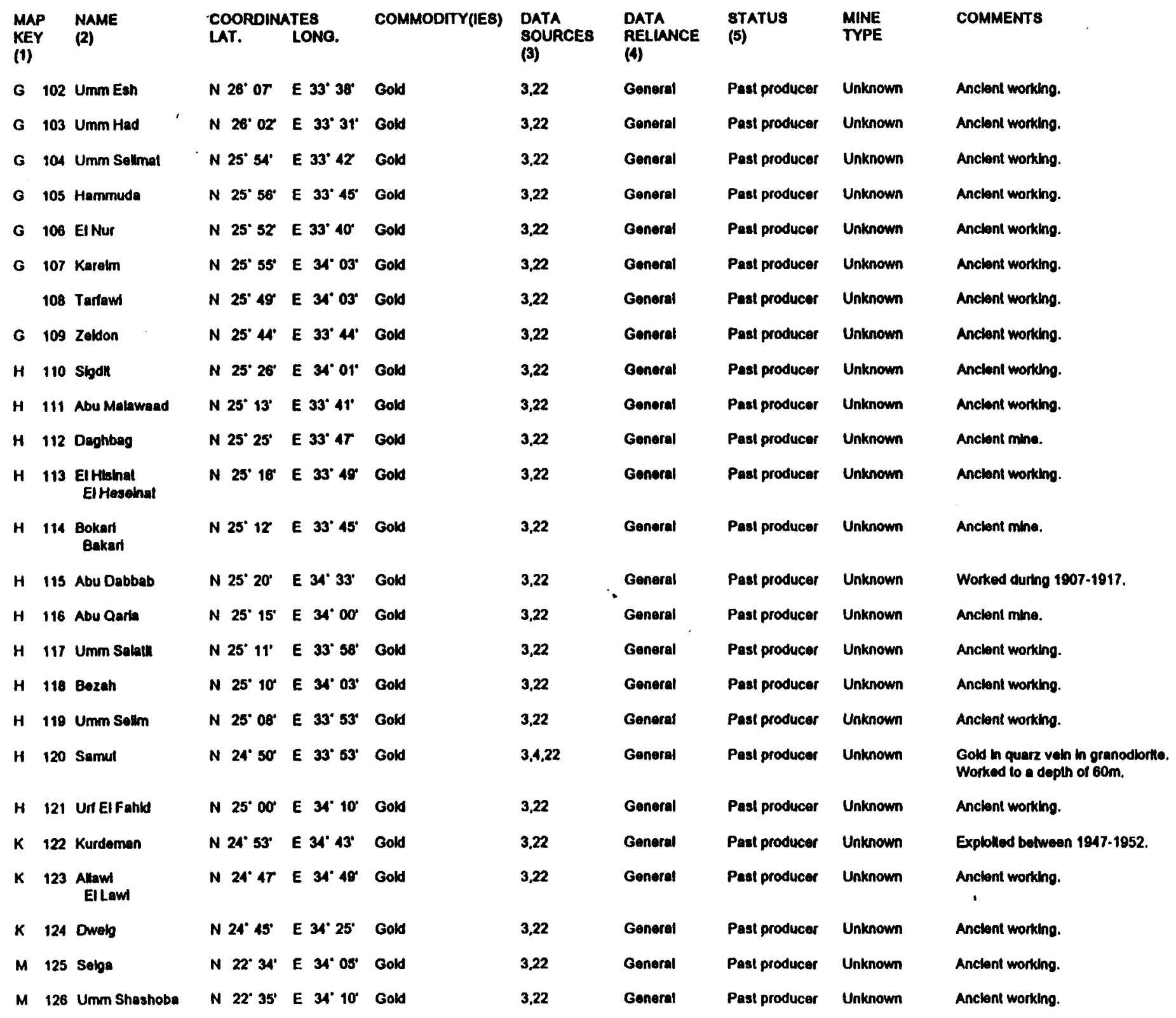


APPENDIX B: PAST PRODUCING MINERAL PROPERTIES IN EGYPT.

\begin{tabular}{|c|c|c|c|c|c|c|c|c|c|c|}
\hline \multicolumn{2}{|c|}{$\begin{array}{l}\operatorname{MAP} \\
\text { KEY } \\
\text { (1) }\end{array}$} & \multirow{2}{*}{$\begin{array}{l}\text { NAME } \\
\text { (2) } \\
\text { Botam }\end{array}$} & \multicolumn{2}{|c|}{$\begin{array}{l}\text { COORDINATES } \\
\text { LAT. LONG. }\end{array}$} & COMMODITY(IES) & \multirow{2}{*}{$\begin{array}{l}\text { DATA } \\
\text { SOURCES } \\
\text { (3) } \\
3.22\end{array}$} & \multirow{2}{*}{$\begin{array}{l}\text { DATA } \\
\text { RELAANCE } \\
\text { (4) } \\
\text { General }\end{array}$} & \multirow{2}{*}{$\begin{array}{l}\text { STATUS } \\
\text { (5) } \\
\text { Past producer }\end{array}$} & \multirow{2}{*}{$\begin{array}{l}\text { MINE } \\
\text { TYPE } \\
\text { Unknown }\end{array}$} & \multirow{2}{*}{$\begin{array}{l}\text { COMMENTS } \\
\text { Anclent working. }\end{array}$} \\
\hline & 127 & & $N 22^{\circ}+r$ & E $34^{*} 31^{\prime}$ & Gold & & & & & \\
\hline & 128 & Umm Egal & N $22^{\circ} 03^{\prime}$ & E $3^{\prime} \mathbf{5 H}^{\prime}$ & Gold & 3,22 & General & Past producer & Unknown & Anclent workhng. \\
\hline & 129 & Wadl Zeldon & N $25^{\circ} 38^{\circ}$ & E 33* 5T & Gold & 3,22 & General & Past producer & Unknown & Anclonl workhng. \\
\hline K & 130 & Gell & N 24: $31^{\prime}$ & E $34^{*} 42$ & Gold & 3.22 & General & Past producer & Unknown & Anclont working. \\
\hline K & 131 & Qulan & $N 24^{*} 18^{\prime}$ & E $35^{\circ} 08^{\prime}$ & Gold & 3,22 & General & Past producer & Unknown & Anclent working. \\
\hline K & 132 & Shelellik & $N 24^{\circ} 11^{\circ}$ & E $35^{\circ} 1 T$ & Gold & 3,22 & General & Past producer & Unknown & Anclent workhing. \\
\hline K & 133 & Abu Rahaya & $N 24^{\circ} 10^{\circ}$ & E $35^{\circ} 10^{\circ}$ & Gold & 3.22 & General & Past producer & Unknown & Anclent workhng. \\
\hline \multirow[t]{2}{*}{$\mathbf{L}$} & 134 & Korblal & N $22^{\circ} 49^{\circ}$ & E $35^{\circ} 09^{\circ}$ & Gold & 3,22 & General & Past producer & Surface & Alluvlal doppsk explothed belore WW!. \\
\hline & 135 & Romk & $N 22^{\circ} 19^{\circ}$ & E $35^{\circ} 49^{\circ}$ & Gold & 3.22 & General & Past producer & Unknown & Anctent mlne. \\
\hline M & 136 & Gabal Moqassem & N $22^{\circ} 08^{\circ}$ & E $3^{\circ} 5^{\prime}$ & Chromilo & 4 & General & Past producer & Unknown & Sxx lanses containing 180 tons of med. gr ore. \\
\hline \multirow[t]{2}{*}{ M } & 137 & Um Doml & $N 22^{\circ} 12$ & E 34. 10 & Chromite & 4 & General & Past producer & Unknown & Nine kenses of small tonnage and med. grade. \\
\hline & 136 & Um El Tyur & $N 22,1 T$ & E $34^{\circ} 35^{\prime}$ & $\begin{array}{l}\text { Chromlle } \\
\text { gold }\end{array}$ & $4,22,30$ & General & Pest producer & Unknown & $\begin{array}{l}14 \text { lonses in talc-cartonate rocks. } \\
\text { Worked during the 1920's. }\end{array}$ \\
\hline M & 139 & Sol Hamkd & N 22: 15' & E $33^{\circ} 40^{\circ}$ & Chromile & 4 & General & Past producar & Unknown & 13 lonses, 630 lons, $48 \%$ Cr203. \\
\hline M & 140 & Um Knush & N $22^{\circ} 40^{\circ}$ & E $33^{\circ} 48^{\circ}$ & Chromile & 1,22 & General & Past producer & Unknown & $\begin{array}{l}\text { Wadl Alleql aree. } \\
16 \text { lenses, } 1100 \text { lons, } 49 \% \text { Cr2O3. }\end{array}$ \\
\hline M & 141 & Dynyat EI Guelelb & N 22* $40^{\prime}$ & E $33^{\circ} 48^{\prime}$ & Chromlite & 4,22 & Generat & Past producer & Unknown & $\begin{array}{l}\text { Wadl Allaql area. } \\
6 \text { small lenses ol high grade ore. }\end{array}$ \\
\hline M & 142 & Wadl Halmour & N $22^{\circ} 40^{\circ}$ & E $33^{\circ} 48^{\circ}$ & Chromile & 4.22 & General & Past producer & Unknown & $\begin{array}{l}\text { Wadl Allaql area. } \\
1 \text { lens. } 550 \text { lons of high grade ore. }\end{array}$ \\
\hline L & 143 & Wadl Arayes & N 23* $35^{\prime}$ & E 34. 51' & Chromile & 4 & General & Pasl producer & Unknown & 33 small lenses enclosed in takc-carbonate rocks. \\
\hline $\mathbf{L}$ & 144 & Abu Dahr & $N 23^{\circ} 3 r$ & E $35^{\circ} 00^{\circ}$ & Chromilo & 4,22 & General & Pasi producer & Unknown & 1 lans, $2-10 \mathrm{~m}$ thlck, $53.8 \%$ Cr2O3. \\
\hline $\mathbf{K}$ & 145 & Wadi Ghadir & $N 24^{\circ} 49^{\circ}$ & E $34^{\circ} 49^{\circ}$ & Chromile & $\$, 22$ & General & Past producer & Unknown & $\begin{array}{l}\text { 8 lenses, } 1800 \text { lons medlum grade ore. } \\
\text { Produced in } 1979 .\end{array}$ \\
\hline K & 146 & Wadl Umm Hegart & N 24. $44^{\prime}$ & E $34^{\circ} 42$ & Chromke & 4 & General & Past producer & Unknown & 1 lens, low grade ore. \\
\hline H & 147 & Abu Mirelwa & $N 25^{\circ} 01^{\circ}$ & E $3^{\circ} 52$ & Chromte & 4 & General & Past producer & Unknown & 4 lonses, 195 tons, 36\% Cr2O3. \\
\hline H & 148 & Barramba & N $25^{\circ} 06^{\circ}$ & E $33^{\circ} 50^{\prime}$ & Chromite & $4,22,25$ & Goneral & Past producar & Unknown & $\begin{array}{l}\text { OS lonses over } 9 \text { shes in talc-carbonato. } \\
\text { Smal scale minhing. } \\
\text { Ore h podllorm deposhs in ultrabastc rocks. } \\
\text { Ore grades } 35-38 \% \mathrm{Cr} \text {. }\end{array}$ \\
\hline \multirow[t]{2}{*}{ H } & 149 & Barramlya II & N $25^{\circ}$ or & E $33^{\circ} 22^{*}$ & Chromite & 22 & General & Past producer & Unknown & Small scale mining. \\
\hline & 150 & Kolet Um Hornr & $N 25^{\circ} 45^{\circ}$ & E $34^{\circ} 15^{\circ}$ & Chromite & 4 & General & Past producer & Unknown & 17 lenses, small reserves, $42 \%$ Cr2O3. \\
\hline
\end{tabular}


APPENDIX 8: PAST PRODUCINO MINERAL PROPERTIES IN EOYPT.

\begin{tabular}{|c|c|c|c|c|c|c|c|c|c|c|}
\hline \multirow[t]{2}{*}{$\begin{array}{l}\text { MAP } \\
\text { KEY } \\
\text { (1) }\end{array}$} & & \multirow{2}{*}{$\begin{array}{l}\text { NAME } \\
\text { (2) } \\
\text { Gabal EI Rabshl }\end{array}$} & \multicolumn{2}{|c|}{$\begin{array}{l}\text { COOROINATES } \\
\text { LAT. LONG. }\end{array}$} & COMMODIYY(IES) & \multirow{2}{*}{$\begin{array}{l}\text { DATA } \\
\text { SOURCES } \\
\text { (3) } \\
1.22\end{array}$} & \multirow{2}{*}{$\begin{array}{l}\text { DATA } \\
\text { RELIANCE } \\
\text { (4) } \\
\text { General }\end{array}$} & \multirow{2}{*}{$\begin{array}{l}\text { STATUS } \\
\text { (5) } \\
\text { Past producer }\end{array}$} & $\begin{array}{l}\text { MINE } \\
\text { TYPE }\end{array}$ & COMMENTS \\
\hline & 151 & & $N 26^{\circ} 11^{\circ}$ & E $33^{\circ} 40^{\circ}$ & Chromine & & & & Unknown & $\begin{array}{l}16 \text { sllos, } 100 \text { lenses, } 2700 \text { tons, } 44 \% \text { Cr2O3. } \\
\text { Small scale explollation. }\end{array}$ \\
\hline K & 152 & Ras Shall & $N 24^{\circ} 51^{\prime}$ & E $34^{\circ} 34^{\prime}$ & Chromilo & 4.22 & General & Past producer & Unknown & Small scais expiollation. \\
\hline K & 153 & Um EI Abas & $N 21: 31^{\prime}$ & E $35^{\circ} 08^{\prime}$ & Magneskto & 4 & Goneral & Past producer & Unknown & Old producer. \\
\hline & 154 & Sagla & $N 30^{\circ} 56^{\circ}$ & E $32^{\circ} 49^{\prime}$ & Megnosite & 4 & Goneral & Past producor & Unknown & Old producer. \\
\hline & 155 & Umm Salalk & $N 25^{\circ} 10^{\circ}$ & E $33^{\circ} 56^{*}$ & Magneske & 4,22 & General & Pasl producer & Unknown & Old producer. \\
\hline & 156 & St. John's island & $N 23^{\circ} 3 r$ & E $36^{\circ} 12$ & $\begin{array}{l}\text { Nickel } \\
\text { Perkdot }\end{array}$ & $4,16,22$ & General & Past producer & Surface & $\begin{array}{l}\text { Two velns } 4.86 \% \text { NI, } 12.25 \% \text { Fe. } \\
\text { Res: } 5 \text {-6kt of ofe. } \\
\text { Produced in 1937-38. }\end{array}$ \\
\hline H & 157 & Hamash & $N 24^{\circ} 41^{\prime}$ & E $34^{\circ} 05^{\prime}$ & $\begin{array}{l}\text { Copper } \\
\text { Gold }\end{array}$ & $4,16,22,30$ & General & Past producer & Unknown & $\begin{array}{l}\text { Porphyry copper noled at } 4 \text { shos. } \\
\text { Ros: 60Mm } 0.3-0.4 \% \mathrm{Cu} \text {. }\end{array}$ \\
\hline H & 158 & $\begin{array}{l}\text { Muelhe } \\
\text { Muellha }\end{array}$ & $N 24^{\circ} 54^{\circ}$ & E $33^{\circ} 55^{\circ}$ & $\begin{array}{l}\text { Th } \\
\text { rungaten }\end{array}$ & $4,22,30$ & Goneral & Past producer & Surtace & $\begin{array}{l}\text { Ore from quartz volns and alluvium. } \\
\text { Mined in the } 1940 \text { 's. } \\
\text { Zone of } 70 \text { velns. }\end{array}$ \\
\hline K & 159 & Igla & N $25^{\circ} 06^{\circ}$ & E $34^{\circ} 39^{\circ}$ & $\begin{array}{l}\text { Th } \\
\text { Tungsion } \\
\text { Tentulum, nboblum }\end{array}$ & $4,22,30$ & General & Past producer & Surface & 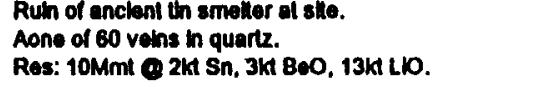 \\
\hline G & 160 & Maghrablya & $N 26^{\circ} 22^{\prime}$ & E $33^{\circ} \mathbf{2 T}$ & Tungaton & 4,22 & Goneral & Pasl producer & Surface & $\begin{array}{l}\text { Throe voins in granlte. } 150 \mathrm{~m} \times 30 \mathrm{~cm} \text {. } \\
180 \text { lons of WO3 conconlralo producod in } 1938 \text {. }\end{array}$ \\
\hline $\mathbf{L}$ & 161 & Zargat Naam & N 23. 16 & E $34^{\circ} \mathbf{4 1}^{\circ}$ & $\begin{array}{l}\text { Tungsten } \\
\text { Chromile }\end{array}$ & $4,22,30$ & - Goneral & Past producer & Surface & Explohed in the 1940's and 1950's. \\
\hline H & 162 & $\begin{array}{l}\text { Gabal Inolgl } \\
\text { Enoigi }\end{array}$ & $N 25^{\circ} 13^{\prime}$ & E $34^{\circ} 09^{\circ}$ & Fluorte & $4,22,30$ & Gonoral & Past producer & Surface & Voins roportedly $2-3 \mathrm{~m}$ in thickness, worked out. \\
\hline 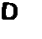 & 163 & Abu Dareg & $\mathbf{N} 29^{\circ} 23^{\circ}$ & E $32^{\circ} 33^{\prime}$ & Kaolin & 4,22 & Goneral & Pest producer & Surface & Low grade kaollnite, production limiled. \\
\hline & 164 & Gabal Um Solim & N $25^{\circ} 08^{\circ}$ & E $33^{\circ} 58^{\prime}$ & Graphite & 4 & Goneral & Past producer & Unknown & Discovered in 1938. \\
\hline & 165 & Rosetta & N 31 $11^{\circ}$ & E $30^{*} 30^{\circ}$ & $\begin{array}{l}\text { Black sand } \\
\text { REO }\end{array}$ & $17,22,30$ & Goneral & Past producer & Surface & $\begin{array}{l}\text { Exploitod during 1929-1968. No present production. } \\
\text { Reserves estimalod at 47Mml (1982). } \\
\text { Produced at 20klyr. }\end{array}$ \\
\hline 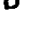 & 866 & Aou Rinnam & N 29 in & $=0280$ & Coppoit & 22,30 & Gontral & rastprodocot & Onicromin & Ancletil Working. \\
\hline D & 167 & Wadl Bikhell & N $29^{\circ} 08^{*}$ & E $32^{\bullet} \mathbf{2 4 ^ { * }}$ & Copper & 22,30 & General & Past producer & Unknown & Anclont morking. \\
\hline E & 168 & Sarabil EI Khadim & N $29^{\circ} 00^{\prime}$ & E $33^{*} 2 T$ & Copper & 22 & General & Past producer & Unknown & Anclenl working. \\
\hline & 169 & El Agma & N 29० $34^{\prime}$ & E $34^{\circ} 45^{\circ}$ & Copper & 22 & Goneral & Past producer & Unknown & Ancient worklng. \\
\hline & 170 & Regeila & N 28 $38^{\circ}$ & E $34^{\circ} 05^{\prime}$ & Copper & 22,30 & Gonoral & Past producer & Unknown & Ancient working. \\
\hline & 171 & Rahaba & N 28*21' & E $34^{\circ} 04^{\circ}$ & Copper & 22 & Goneral & Past producer & Unknown & Ancienl working. \\
\hline & 172 & Samra & $N 28^{\circ} 13^{\prime}$ & E $34^{\circ} 21^{\circ}$ & Copper & $16,22,30$ & General & Past producer & Unknown & Ancionl working. \\
\hline
\end{tabular}


APPENOIX B: PAST PRODUCING MINERAL. PROPERTIES IN EOYPT.

\begin{tabular}{|c|c|c|c|c|c|c|c|c|c|c|}
\hline \multirow[t]{2}{*}{$\begin{array}{l}\text { MAP } \\
\text { KEY } \\
\text { (1) }\end{array}$} & \multirow{2}{*}{\multicolumn{2}{|c|}{$\begin{array}{l}\text { NAME } \\
\text { (2) } \\
\text { Gabal Darah }\end{array}$}} & \multicolumn{2}{|c|}{$\begin{array}{l}\text { COORDINATES } \\
\text { LAT. LONO. }\end{array}$} & COMMODIYY(IES) & $\begin{array}{l}\text { DATA } \\
\text { 8OURCES } \\
\text { (3) }\end{array}$ & \multirow{2}{*}{$\begin{array}{l}\text { DATA } \\
\text { RELIANCE } \\
\text { (4) } \\
\text { Goneral }\end{array}$} & \multirow{2}{*}{$\begin{array}{l}\text { STATUS } \\
\text { (5) } \\
\text { Past producar }\end{array}$} & \multirow{2}{*}{$\begin{array}{l}\text { MINE } \\
\text { TYPE } \\
\text { Unknown }\end{array}$} & COMMENTS \\
\hline & 173 & & N 28* or & E $33^{\circ} 05^{\prime}$ & Copper & 22 & & & & Anclent working. \\
\hline G & 174 & Mammamá & $N 28^{\circ} 31^{\circ}$ & E $33^{\circ} 14^{\prime}$ & Gold & 22 & Goneral & Past producor & Unknown & Anclent worling. \\
\hline $\mathbf{G}$ & 175 & Eroldiya & N 26• $20^{\circ}$ & E $33^{\circ} 31^{\prime}$ & Gold & 22,30 & Gonaral & Past producar & Unknown & Worked in 1904, then in the 1940's. \\
\hline G & 176 & Atallah EI Mur & $N 26^{\circ} 11^{\circ}$ & $E 3^{\circ} 25^{\circ}$ & Gold & 22 & Genoral & Pael producer & Unknown & Anclont working. \\
\hline $\mathbf{G}$ & 177 & Aalla & N 26• $10^{\circ}$ & E $33^{\circ} 30^{\circ}$ & Gold & 22,30 & General & Paat producer & Unknown & Worked during 1914-1916. \\
\hline G & 178 & Fannakhir & N 26.02' & E $33^{*} 33^{\prime}$ & Gold & 22 & Goneral & Past producar & Unknown & Worked during 1946-1954. \\
\hline & 179 & El Masaara & N 25० 30 & E $29^{\circ} 01^{\circ}$ & Ochie & 22 & General & Past producor & Surface & Explotted on a small scale. \\
\hline H & 180 & Gabal Dungesh & $N 24^{\circ} 40^{\circ}$ & E $33^{\circ}$ s8' & Chromlie & 22 & General & Past producer & Unknown & Small scalo mining. \\
\hline $\mathbf{H}$ & 181 & Zug EI Bohar & $N 24^{*} 57$ & E $34^{\circ} \mathbf{2 0}$ & $\begin{array}{l}\text { Lead } \\
\text { zino }\end{array}$ & 18.22 & General & Past producer & Unknown & Anclent mine. Load contont $1.6-2.5 \% \mathrm{~Pb}$. \\
\hline H & 182 & Wadi Easel & $N 24^{\circ} 55^{\circ}$ & E $34^{\circ} 21^{*}$ & $\begin{array}{l}\text { Loed } \\
\text { zino }\end{array}$ & 16.22 & Gonoral & Past producar & Unknown & Anclont mino. \\
\hline $\mathbf{H}$ & 183 & El Alawl & N $25^{\circ} 40^{\circ}$ & E $34^{\circ} 10^{\circ}$ & Copper & $16,22,30$ & Goneral & Past producar & Unknown & Anclent worting. \\
\hline & 184 & Wadi El Gomal & N 24" 36" & E $34^{\circ} 52^{\circ}$ & $\begin{array}{l}\text { Copper } \\
\text { lead, xinc }\end{array}$ & 16.22 & Genoral & Past producer & Unknown & $\begin{array}{l}\text { Anclonl working. } \\
\text { Four ailes. }\end{array}$ \\
\hline $\mathbf{K}$ & 185 & Maialolk & N $24^{\circ} 13^{\prime}$ & E $35^{\circ} \mathbf{1 3 ^ { \circ }}$ & Manganose & $16,22,30$ & General & Past producer & Unknown & $\begin{array}{l}\text { Oporaled in } 1956 . \\
\text { Voin of } 300 \mathrm{~m} \text { longth. }\end{array}$ \\
\hline K & 186 & Khushab & N 24० 15 & E $34^{\circ} 23^{\circ}$ & Gold & 22 & Gonoral & Pasil producer & Unknown & Anclent working. \\
\hline J & 187 & Ras Banas & N 23. $59^{\circ}$ & E $32^{*} 36^{\circ}$ & $\begin{array}{l}\text { Copper, load } \\
\text { Zine }\end{array}$ & $16,22,30$ & General & Past producer & Unknown & Anclent working. \\
\hline $\boldsymbol{\theta}$ & 188 & Mukattom & N 20.5 $58^{\prime \prime}$ & E $31^{\circ} 18$ & Lmestone & 22 & General & Past producer & Surtace & Old quarry slle. \\
\hline C & 189 & Kair EIEWw & $N \mathbf{2 9 ^ { * }} 45^{t}$ & E $31^{\circ} 20^{\circ}$ & Clay & 22 & General & Past producer & Surtece & Old quany stie. \\
\hline & 190 & ElYahmum & $N 29^{\circ} 5 r$ & E $31^{\circ} 40^{\circ}$ & Basalk & 22 & General & Pest producer & Surface & OVd quarry site. \\
\hline D & 191 & El Shatl & $N 20^{\circ} 5 T$ & E $32^{\circ} 42$ & Gypsum & 22 & General & Past producer & Surface & Ord quarry stive. \\
\hline D & 192 & Wadi Rayana & N 28* 51' & E $32^{\circ} 52^{\prime}$ & Gypsum & 16.22 & Genoral & Past producer & Surface & Old quamy she. \\
\hline D & 193 & Sadal & N 20 $46^{\circ}$ & E 32' $23^{\circ}$ & Lmestone & 22 & General & Past producer & Surface & Old quarry stho. \\
\hline D & 194 & Um Zolla & N 20, $40^{\circ}$ & E $32^{\prime} 12$ & Limestone & 22 & General & Past producer & Surface & Old quarry sthe. \\
\hline o & 195 & Axheldar & $N 29^{\circ} 41^{\circ}$ & E $32^{\circ} 15^{\circ}$ & Utrnestone & 22 & General & Past producer & Surface & Old quarty sthe. \\
\hline
\end{tabular}


APPENDIX B: PAST PRODUGINO MINERAL PROPERTIES IN EOYPT.

\begin{tabular}{|c|c|c|c|c|c|c|c|c|c|c|}
\hline \multicolumn{2}{|c|}{$\begin{array}{l}\text { MAP } \\
\text { KEY } \\
\text { (1) }\end{array}$} & \multirow{2}{*}{$\begin{array}{l}\text { NAME } \\
\text { (2) } \\
\text { Abu Sondouk }\end{array}$} & \multicolumn{2}{|c|}{$\begin{array}{l}\text { COORDINATES } \\
\text { LAT. LONG. }\end{array}$} & \multirow{2}{*}{$\begin{array}{l}\text { COMMODITY(IES) } \\
\text { KaOln }\end{array}$} & \multirow{2}{*}{$\begin{array}{l}\text { DATA } \\
\text { SOURCES } \\
\text { (3) } \\
22\end{array}$} & \multirow{2}{*}{$\begin{array}{l}\text { DATA } \\
\text { RELLANCE } \\
\text { (4) } \\
\text { General }\end{array}$} & \multirow{2}{*}{$\begin{array}{l}\text { STATUS } \\
\text { (5) } \\
\text { Pasl producer }\end{array}$} & \multirow{2}{*}{$\begin{array}{l}\text { MINE } \\
\text { TYPE } \\
\text { Surtace }\end{array}$} & \multirow{2}{*}{$\begin{array}{l}\text { COMMENTS } \\
\text { ON quarry stie. }\end{array}$} \\
\hline D & 196 & & $N 20^{\circ} 21^{\circ}$ & E $32^{*} 21^{\prime}$ & & & & & & \\
\hline $\mathbf{E}$ & 197 & Gharandal & $N \cdot 20^{\circ} 25^{\circ}$ & E $33^{\circ} 06^{\circ}$ & Gypsum & 22 & Generat & Past producer & Surfece & Old quarry she. \\
\hline D & 198 & Gabal Thimmal. & N 2000 00 & E $32^{\circ} 3 r$ & Llmestone & 22 & General & Past producer & Surface & Old quarry stle. \\
\hline $\mathbf{E}$ & 199 & Farsh El Ghozlan & $N 29^{\circ}$ or & E $33^{\circ} 22$ & Kaoln & 16,22 & Genoral & Past producer & Surface & Old quarry slle. \\
\hline $\mathbf{E}$ & 200 & El Khaboubs & N 20, 04 & E $33^{\circ} 25^{\circ}$ & Glass sand & 22 & General & Past producer & Surface & Old quarry slle. \\
\hline $\mathbf{E}$ & 201 & Musabbe Selame & $N 20^{\circ} 04^{\circ}$ & E $33^{\circ} 20^{\circ}$ & Keoln & 16.22 & Goneral & Pasl producar & Surface & Old quarry ske. \\
\hline $\mathbf{E}$ & 202 & Budra & $N 28^{\circ} 56^{\circ}$ & $E 33^{\circ} 18^{\prime}$ & Keolln & $16,22,30$ & General & Past producer & Surface & Ond quarry stie. \\
\hline $\mathbf{E}$ & 203 & Abu Natash & N 28 $58^{\circ}$ & E $33^{\prime} 22$ & Knaln & $16,22,30$ & General & Past producer & Surface & Old quarry sthe. \\
\hline \multirow[t]{2}{*}{$\mathbf{E}$} & 204 & Wadi Sidnt & $N 28^{\circ} 51^{\circ}$ & $E 3^{*} 13^{\prime}$ & Gypsum & 22 & General & Past producer & Surface & Oxd quamy sine. \\
\hline & 205 & Gabal El Dokhan & N $27^{\circ} 15^{\prime}$ & E $33^{\circ} 19^{\circ}$ & Stons & 22 & General & Past producer & Surface & Sllo of old ples. \\
\hline G & 206 & Um Esh El Hamra & N 26. 02 & E $33^{\circ} 40^{\circ}$ & Take & 22 & General & Past producer & Surface & Produced h 1979. \\
\hline 1 & 207 & ElKelh. & N 25. $05^{\circ}$ & E $32^{\circ} 58^{\prime}$ & Lmestone & 22 & General & Past producer & Surtace & Produced in 1979. \\
\hline 1 & 208 & El Atwanl & N 25. $03^{\prime}$ & E $32^{\circ} 59^{\prime}$ & Sendstone & 22 & General & Paat producer & Surface & Produced in 1979. \\
\hline 1 & 209 & Redysle North & $N 24^{\circ} 5 T$ & E $32^{\circ} 58^{*}$ & Sendstone & 22 & General & Past producer & Surrace & Produced h 1979. \\
\hline I & 210 & EI Sirag & $N 24^{\circ} 54^{\prime}$ & E $32^{\circ} 59^{\prime}$ & Sandstone & 22 & General & Past producer & Surrace & Produced h 1979. \\
\hline 1 & 211 & SIlwa North & $N 24^{\circ} 45^{\circ}$ & E $33^{\circ} 00^{\circ}$ & Sandstone & 22 & Goneral & Past producor & Surface & Produced in 1979. \\
\hline 1 & 212 & Farts & $N 24^{*} 33^{\prime}$ & E 32' $51^{\prime}$ & Sandatone & 22 & General & Pest producer & Surface & Produced h 1979. \\
\hline J & 213 & Unnamed & N 24. 02 & E $32^{\circ} 43^{\prime}$ & Croy & 16,22 & Gonoral & Pest producer & Surface & Reserves of 8.5Mmn. \\
\hline J & 214 & Unnamed & N 24. 01' & E $32^{\circ} 5 r$ & Foldoper & 22 & General & Past producer & Surface & Produced in 1979. \\
\hline J & 215 & EIMksalia & N 24. $01^{\prime}$ & E $33^{\circ} 00^{\circ}$ & Granke & 22 & Gonoral & Pasl producer & Surfoce & Produced in 1979. \\
\hline$H$ & 216 & Wadl Um Huqab & $N 24^{\circ} 58^{\circ}$ & E 34 01' & Talc & 22 & Genoral & Past producer & Surface & Produced in 1979. \\
\hline$H$ & 217 & Gabal Urt Abu Hama & IN $24^{\circ} 45^{\circ}$ & E $34^{\circ} 01^{\prime}$ & Barte & 22 & Goneral & Pasi producer & Surface & Produced in 1979. \\
\hline K & 210 & Gabel Zabara & $N 24^{\circ} 45^{\circ}$ & E $34^{\circ} 41^{\circ}$ & Emeralds & 22 & Goneral & Past producer & Surface & Anctent workings. \\
\hline K & 219 & Gabal Slkel & $N 24^{\circ} 42$ & E $34^{\circ} 49^{\circ}$ & $\begin{array}{l}\text { Fhorspar } \\
\text { Emeralds }\end{array}$ & 22 & General & Past producer & Surface & Anclent workings. \\
\hline $\mathrm{K}$ & 220 & Ranga & $N 24^{\circ} 25^{\circ}$ & E $35^{*} 10^{*}$ & $\begin{array}{l}\text { Surur, } \\
\text { gypsum }\end{array}$ & 22 & General & Past producer & Surtace & Anclent worklng. \\
\hline
\end{tabular}


APPENDIX B: PAST PRODUCINO MINERAL PROPERTIES IN EOYPT.

\begin{tabular}{|c|c|c|c|c|c|c|c|c|c|}
\hline $\begin{array}{l}\text { MAP } \\
\text { KEY } \\
\text { (1) }\end{array}$ & $\begin{array}{l}\text { NAME } \\
\text { (2) }\end{array}$ & $\begin{array}{l}\text { COORDIR } \\
\text { LAT. }\end{array}$ & $\begin{array}{l}\text { ATES } \\
\text { LONG. }\end{array}$ & COMMODITY(IES) & $\begin{array}{l}\text { DATA } \\
\text { SOURCES } \\
\text { (3) }\end{array}$ & $\begin{array}{l}\text { DATA } \\
\text { RELUACE } \\
\text { (4) }\end{array}$ & $\begin{array}{l}\text { STATUS } \\
\text { (J) }\end{array}$ & $\begin{array}{l}\text { MINE } \\
\text { TrPE }\end{array}$ & COMMENTS \\
\hline 15221 & Br El Shab & N 22 $21^{\prime}$ & E 29.45 & Alum & $\mathbf{2 2}$ & Generet & Pest producest & surteces & $\begin{array}{l}\text { Explollod shce enclent umes. } \\
\text { Reserves 200kt (1979). }\end{array}$ \\
\hline 15222 & Cheiren & N 22, 52 & E $31^{\circ} 16^{\circ}$ & $\begin{array}{l}\text { Gnebs, faldsper. } \\
\text { emethyst }\end{array}$ & 22 & Genoret & Pest producer & Surfoce & Ancionl wonkings. \\
\hline
\end{tabular}

(1) Represents property ox property grouping as defined in Appendte maps A-2 and A-3.

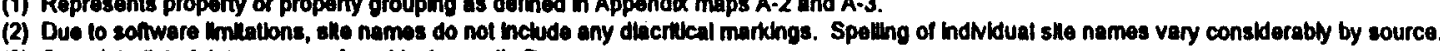

(3) Complete list of datu sourcess found in Appendbx D.

(4) General - denotes linited data; Confimed - denoles deposkl informatton confumed by several sources.

(5) Because of the verying age of source inlormalton, the status of howldual sllos may not be current. 
$26^{\circ} \mathrm{E}$

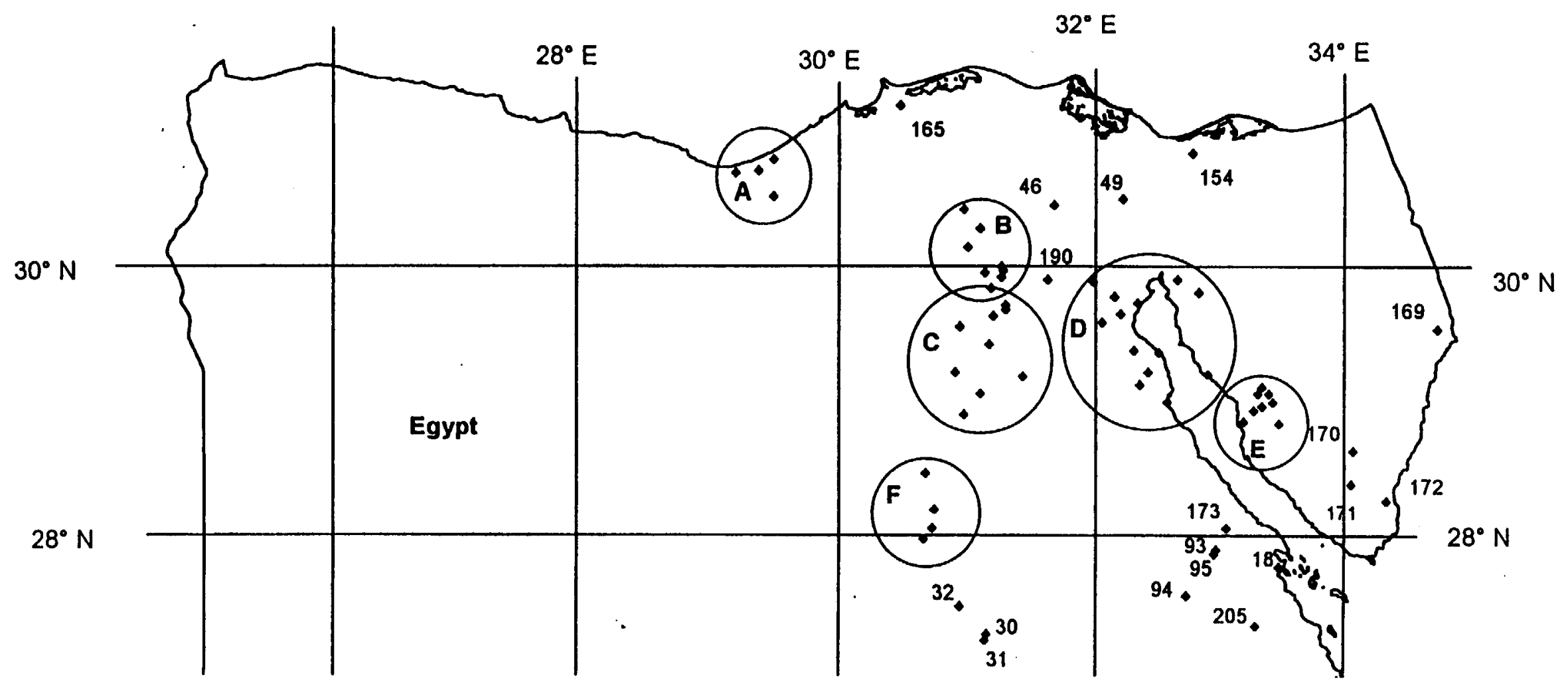

LEGEND
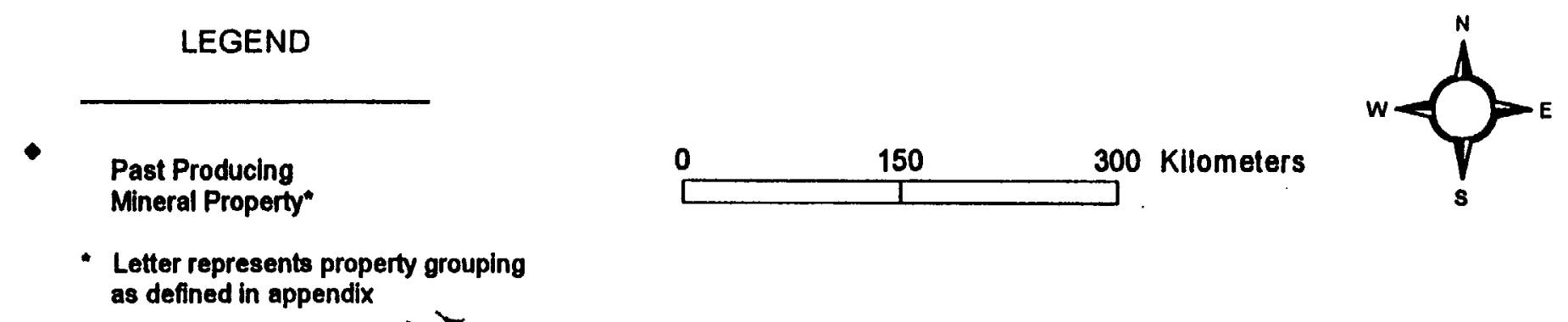

as defined in appendix

APPENDIX MAP A-2: PAST PRODUCING MINERAL PROPERTIES OF NORTHERN EGYPT 


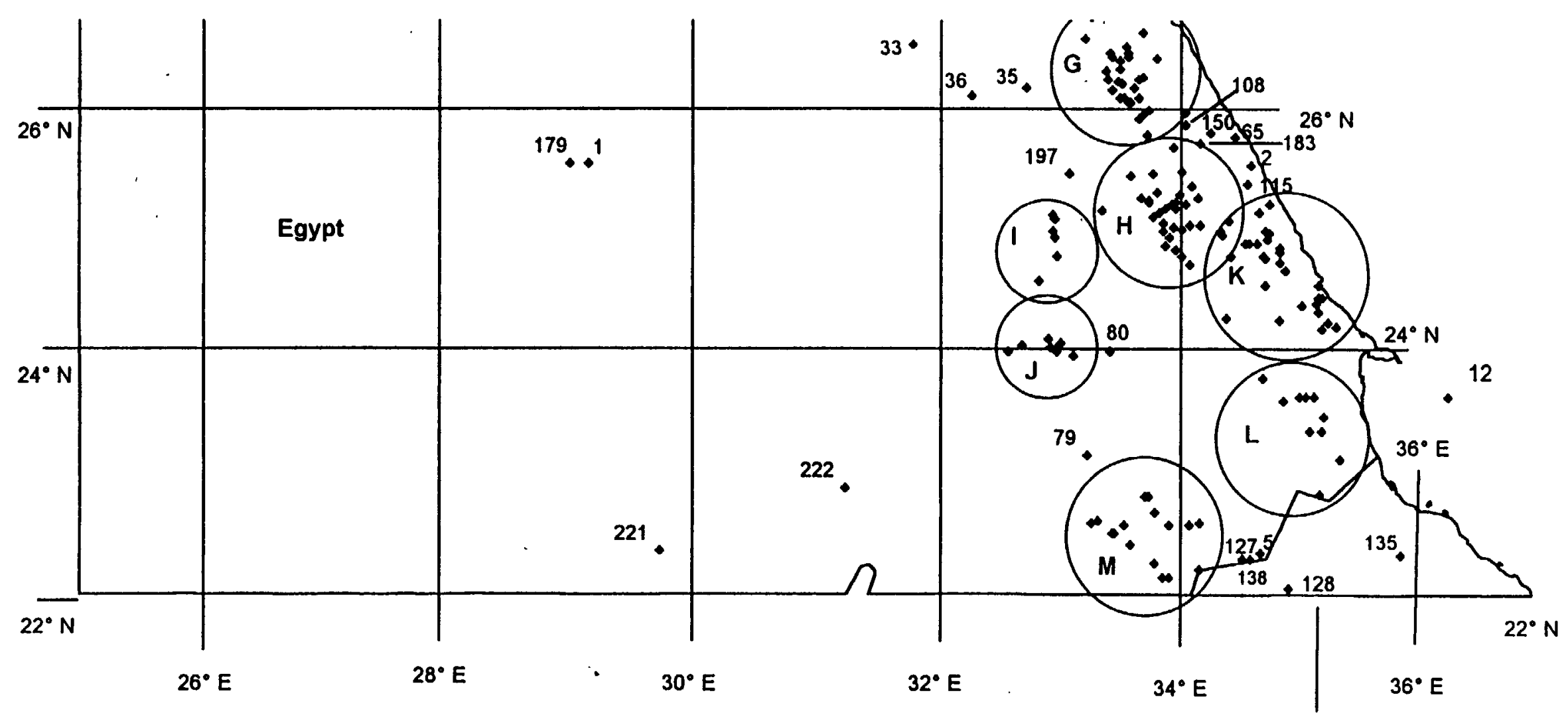

Area administered by Sudan

LEGEND
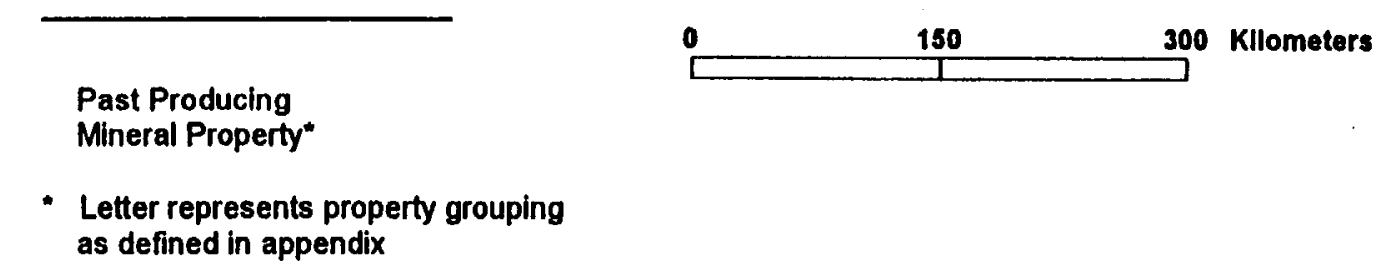

Kllometers

Past Producing

Letter represents property grouping as defined in appendix

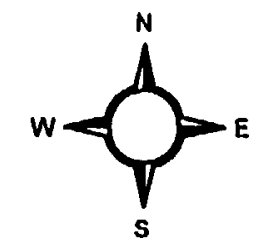

APPENDIX MAP A-3: PAST PRODUCING MINERAL PROPERTIES OF SOUTHERN EGYPT 
APPENDIX C: PROSPECTS AND NON PRODUCING MINERAL PROPERTIES IN EOYPT.

\begin{tabular}{|c|c|c|c|c|c|c|c|c|c|c|}
\hline \multicolumn{2}{|c|}{$\begin{array}{l}\text { MAP } \\
\text { KEY } \\
\text { (1) }\end{array}$} & \multirow{2}{*}{$\begin{array}{l}\text { NAME } \\
\text { (2) } \\
\text { Qum } \\
\text { Wadl EI Mashash }\end{array}$} & \multicolumn{2}{|c|}{$\begin{array}{l}\text { COORDINATES } \\
\text { LAT. LONO. }\end{array}$} & COMMODITY(IES) & $\begin{array}{l}\text { DATA } \\
\text { SOURCES } \\
\text { (3) }\end{array}$ & $\begin{array}{l}\text { DATA } \\
\text { RELLANCE } \\
\text { (A) }\end{array}$ & $\begin{array}{l}\text { STatus } \\
\text { (5) }\end{array}$ & $\begin{array}{l}\text { MINE } \\
\text { TYPE }\end{array}$ & COMMENTS \\
\hline & 1 & & $N 25^{\circ} 45^{\prime}$ & $E$ E $33^{\circ} 03^{\circ}$ & Phosphato & 1.2 & General & Deposk & Surface & 35-65\% P2O5 \\
\hline & 2 & Hamama, & $N 25^{\circ} 42$ & $E \quad 33^{\circ} 45^{\circ}$ & Phosphate & 1.2 & Genoral & Depost & Surface & 35-65X P2O5 \\
\hline G & 3 & Wadl Gasus & N $26^{\circ} 34^{\prime}$ & E $34^{\circ} 02$ & Lead & 1 & Generet & Depont: & Unknown & $\begin{array}{l}\text { Small deposh, low grade. } \\
\text { Most rock is limestone. }\end{array}$ \\
\hline J & 4 & Wadl Hamr & N $24^{\circ} 00^{\circ}$ & E $33^{\circ} 00$ & Lend & 1 & Oenerat & Oeposil & Unknown & $\begin{array}{l}\text { Small doposh, low grade. } \\
\text { Host rock is schist. }\end{array}$ \\
\hline & 5 & Zeblrget & $N 23^{\circ} 3 T$ & E $38^{\circ} 12$ & Nickol & 1 & Genoral & Deposk & Surface & $\begin{array}{l}\text { Veln whth averages 2-3 feel, exposed for } 50 \mathrm{~m} \text {. } \\
\text { Ni contonl 5-9\%. }\end{array}$ \\
\hline K & 6 & Abu Hermamid & $N 24^{\circ} 10^{\circ}$ & E $30^{\circ} \mathbf{3 0}$ & Copper & 1 & Genoral & Deposk & Surface & Occurrence. \\
\hline & 7 & Both & $N 32^{\circ} 52$ & E $27^{\circ} 05$ & Molybdentle & 1 & General & Deposh & Surtace & Occurrence. \\
\hline G & 8 & Fatra & $N 3^{\circ} 03^{\circ}$ & E $20^{\circ} \mathbf{5 0}$ & Take & 1 & General & Deposk & Surface & Occurrance. \\
\hline & 9 & Unt & $N 27^{\circ} 50^{\circ}$ & E $32^{\prime} 40^{\circ}$ & Berte & 1 & General & Depoesk & Surtace & Occurrence. \\
\hline & 10 & ElHelz & $N 28^{\circ} 03^{\circ}$ & E $28^{\circ} 3 r$ & Iron & $2,16,22,30$ & General & Doposkt & Surface & $\begin{array}{l}\text { El Holz res: } 39 \mathrm{Mmt} \text { C } 25 \% \text { Fe, S1O2 up } 1040 \% \text {. } \\
\text { Part of Bahartya Oasts. } \\
\text { Ferruginous sandstono. }\end{array}$ \\
\hline $\mathbf{F}$ & 11 & Zek & $N 27^{\circ} 56^{\circ}$ & E $33^{\circ} 30^{\prime}$ & Polesh & 2 & General & Deposk & Surface & 250 sq. km. on west coast of Gull of Suez. \\
\hline $\mathbf{F}$ & 12 & Abu Ngar & N $27^{\circ} 40^{\circ}$ & E $33^{\circ} 20^{\circ}$ & Potesh & 2 & General & Deposk & Surfaco & $100 \mathrm{~km}$. south of Zell \\
\hline G & 13 & El Eredlye & N $26^{\circ} 20^{\circ}$ & E $33^{\circ} 28^{\prime}$ & Urankum & 2,4 & General & Deposk & Underground & Ore in grentilc fraclure zones. \\
\hline & 14 & El Mbsakat & $N 26^{\circ} 24^{\circ}$ & E $33^{\circ} 24^{\circ}$ & Urantum & 2,4 & General & Deposk & Underground & $\begin{array}{l}\text { Ore h granilic fracture zones. } \\
\text { Discovered in } 1982 \text { by geophysical aomaly. }\end{array}$ \\
\hline & 15 & $\begin{array}{l}\text { Damib } \\
\text { Darhelb }\end{array}$ & N 24. $01^{\circ}$ & E $35^{\circ} 01^{\circ}$ & $\begin{array}{l}\text { Zlnc, Copper } \\
\text { Lond }\end{array}$ & $2,16,22$ & General & Doposk & Underground & $\begin{array}{l}\text { Ore in basak, andeshle, and inyollo. } \\
\text { Assay of } 0.3-6.3 \% \mathrm{Zn}, 0.1-9.5 \% \mathrm{Cu}, 0.08-2.5 \% \mathrm{~Pb} \text {. }\end{array}$ \\
\hline & 16 & Abu Gurdl & N $23^{\circ} 59$ & E $35^{\circ} 05^{\prime}$ & $\begin{array}{l}\text { Zlnc, Coppar } \\
\text { Lead }\end{array}$ & 2,22 & Goneral & Deposk & Underground & $\begin{array}{l}\text { Ore in basak, andeske, and thyolie. } \\
\text { Assay of 3.2-21\% } \mathrm{Zn}, .3-3.7 \times \mathrm{Cu}, 0.3-2.8 \% \mathrm{~Pb} \text {. }\end{array}$ \\
\hline & 17 & $\begin{array}{l}\text { Egat } \\
\text { Egat }\end{array}$ & N $24^{\circ} 02$ & E $35^{\circ} \mathbf{0 3}$ & $\begin{array}{l}\text { Zlnc, Copper } \\
\text { Lead }\end{array}$ & $\mathbf{2 , 2 2}$ & General & Deposk & Underground & $\begin{array}{l}\text { Ore in basak, andeske, and thyollo. } \\
\text { Assay of 0.1-9.2\% Zn,0.7-7.2\% Cu, 0.02-1.1\% Pb. }\end{array}$ \\
\hline & 18 & Gebal Alaw & N 25 $3 T$ & E $34^{*} 10^{\circ}$ & $\begin{array}{l}\text { Zinc, Copper } \\
\text { Load. Nloblum }\end{array}$ & $\mathbf{2 , 2 2}$ & General & Deposin & Underground & Occurrence. \\
\hline & 19 & Abu Dabbab & $N 25^{\circ} 20^{\circ}$ & E $34^{*} 32$ & $\begin{array}{l}\text { Nioblum } \\
\text { Tanilumum } \\
\text { Th. Tungsten }\end{array}$ & $2,4,17,22,30$ & Confirmed & Deposil & Surface & 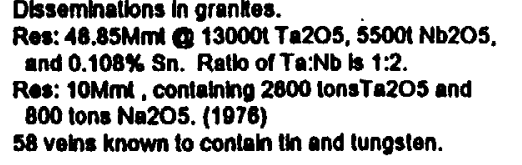 \\
\hline
\end{tabular}


APPENDIX C: PROSPECTS AND NON PRODUGING MINERAL PROPERTIES IN EOYPT.

\begin{tabular}{|c|c|c|c|c|c|c|c|c|c|c|}
\hline $\begin{array}{l}\text { MA } \\
\text { KEY } \\
\text { (1) }\end{array}$ & & $\begin{array}{l}\text { NAME } \\
\text { (2) }\end{array}$ & $\begin{array}{l}\text { COORDIN } \\
\text { LAT. }\end{array}$ & $\begin{array}{l}\text { ATES } \\
\text { LONO. }\end{array}$ & COMMODITY(IES) & $\begin{array}{l}\text { DATA } \\
\text { 8OURCES } \\
\text { (3) }\end{array}$ & $\begin{array}{l}\text { DATA } \\
\text { RELLANCE } \\
\text { (4) }\end{array}$ & $\begin{array}{l}\text { STATUS } \\
\text { (S) }\end{array}$ & $\begin{array}{l}\text { MINE } \\
\text { TYPE }\end{array}$ & COMMENTS \\
\hline & 20 & Nuwetbl & $N 25^{\circ} 12$ & E $34^{\circ} 30^{\circ}$ & $\begin{array}{l}\text { Nloblum } \\
\text { Tantolum } \\
\text { un. lungston }\end{array}$ & $2,4,17,22,30$ & Confrmed & Deposkt & Surface & 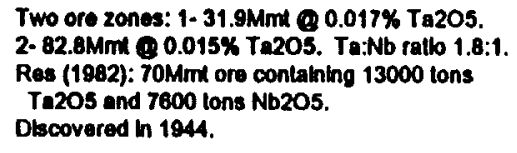 \\
\hline & 21 & Hams Waggad & $N 25^{\circ} 11^{\circ}$ & E 34. $21^{\circ}$ & $\begin{array}{l}\text { Th. nloblum } \\
\text { Tantalum }\end{array}$ & $2,4,22$ & General & Doposk & Unknown & Occurrence. \\
\hline & 22 & Um Neggat & N $25^{\circ} 30^{\circ}$ & E 34' $11^{\circ}$ & $\begin{array}{l}\text { Noblum } \\
\text { Tantalum }\end{array}$ & 2,22 & General & Deposn & Unknown & $\begin{array}{l}\text { Ore in granites contaln } 0.022 \% \text { Ta2O5\& } 0.02 \% \\
\text { Nb2OS. }\end{array}$ \\
\hline & 23 & Maryut & N $31^{\circ} 12$ & E 30" 02 & Gypsum & $2,4,13,22,30$ & General & Deposh & Surface & $\begin{array}{l}\text { Ore in lake basin. } \\
\text { Frlable bods aboul } 80 \mathrm{~cm} \text { thick overtain by sall. }\end{array}$ \\
\hline & 24 & Maryut & N 31" 02 & E $20^{\circ} 46^{\circ}$ & Linestone & 22 & Generat & Deposk & Surface & Occurrence. \\
\hline & 25 & Bardawall & $N 31^{\circ} 10^{\circ}$ & E $33^{\circ} 10^{\circ}$ & Gypsum & 2 & General & Deposk & Surface & Ore in lake bash. \\
\hline & 28 & Alameh & $\mathbf{N} 30^{\circ} \mathbf{5 0}$ & E $28^{\circ} 5 T$ & Gypsum & 2 & General & Deposk & Surface & Salts in depresston atong coast. \\
\hline & 27 & Mersa Matruh & $N 31^{\circ} 12$ & E $27^{7} 12$ & Gypsum & 2,22 & Goneral & Depoall & Surface & Salls in deprosston along coast. \\
\hline B & 28 & $\begin{array}{l}\text { Buquirat } \\
\text { Bugeirat }\end{array}$ & $N 29^{\circ} \mathbf{2 3}$ & E $30^{\circ} 22$ & Gypsum & $2,16,22$ & General & Dopoall & Surface & Alluvium deposils. \\
\hline & 29 & Abu El Daraz & $N 29^{\circ} 38^{\prime}$ & E 3217 & Glass sand & 2,4 & Genoral & Doposk & surface & $\begin{array}{l}\text { Res: 4.1Mmt. } \\
\text { Assay 98.5\% siO2, 0.3-0.5\% Fe203. }\end{array}$ \\
\hline & 30 & Elartsh & N $31^{\circ} 08^{\circ}$ & E $33^{\circ} 48^{\circ}$ & Glass sand & 2 & General & Deposkl & Surface & Locallon approxtmate. \\
\hline & 31 & Wadl El Shaghab & $N 25^{\circ} 20^{\circ}$ & E $33^{\circ} 2 r$ & Phosphale & 2.22 & Genoral & Deposhl & Surface & $\begin{array}{l}\text { Nibe Valloy Phosphate distrlet conlalns 1.5Bmt. } \\
\text { Sile reserves } 495 \mathrm{Mml} \text { (1979). }\end{array}$ \\
\hline $\mathbf{G}$ & 32 & $\begin{array}{l}\text { Gobel EI GV } \\
\text { Qena }\end{array}$ & $N 26^{\circ} 06$ & E $33^{\circ} 09^{\prime}$ & Phosphale & $2,17,22$ & General & Deposin & Surface & $\begin{array}{l}\text { Nite Valloy Phosphate district conlains } 1.5 \mathrm{Bm} \text {. } \\
\text { She reserves } 44 \mathrm{Mml} \text { (1979). }\end{array}$ \\
\hline $\mathbf{G}$ & 33 & Wadt Semt & N $26^{\circ} 12$ & $E 33^{\circ} 11^{\prime}$ & Phosphate & 2,22 & General & Deposk & Surface & $\begin{array}{l}\text { Nillo Velloy Phosphate distinct conlains } 1.5 \mathrm{Bmt} \text {. } \\
\text { Stre reserves } 50 \mathrm{Mml} \text { (1979). }\end{array}$ \\
\hline $\mathbf{G}$ & 34 & Gobel Abu Had & N 26. $30^{\prime}$ & E $33^{\circ} 14^{\circ}$ & Phosphale & 2.22 & General & Deposht & Surface & $\begin{array}{l}\text { Nile Valley Phosphate dislincl contains } 1.58 \mathrm{mt} \text {. } \\
\text { SHe reserves } 317 \mathrm{Mmt} \text { (1979). }\end{array}$ \\
\hline & 35 & $\begin{array}{l}\text { Wadl Buda } \\
\text { Wad Thora }\end{array}$ & N $29^{\circ} 50^{\circ}$ & E $32^{\prime} \mathbf{4 5 ^ { \prime }}$ & $\begin{array}{l}\text { Coal } \\
\text { Sant }\end{array}$ & $2,4,28,30$ & General & Depost & Underground & 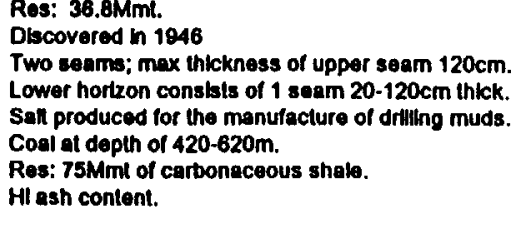 \\
\hline & 37 & Umm Ashire & $\mathbf{N} 23^{\circ} 30^{\circ}$ & E $35^{\circ} 03^{\circ}$ & Gold & 3 & General & cosk & own & Occurrence. \\
\hline
\end{tabular}


APPENDIX C: PROSPECTS AND NON PRODUCINO MINERAL PROPERTIES IN EOYPT.

\begin{tabular}{|c|c|c|c|c|c|c|c|c|c|c|}
\hline \multicolumn{2}{|c|}{$\begin{array}{l}\text { MAP } \\
\text { KEY } \\
\text { (1) }\end{array}$} & \multirow{2}{*}{$\begin{array}{l}\text { NAME } \\
\text { (2) } \\
\text { Bonlog }\end{array}$} & \multicolumn{2}{|c|}{$\begin{array}{l}\text { COORDIMATES } \\
\text { LAT. LONO. }\end{array}$} & COMMODITY(IES) & \multirow{2}{*}{$\begin{array}{l}\text { DATA } \\
\text { SOURCES } \\
\text { (3) } \\
3\end{array}$} & \multirow{2}{*}{$\begin{array}{l}\text { DATA } \\
\text { RELUNCE } \\
\text { (4) } \\
\text { General }\end{array}$} & \multirow{2}{*}{$\begin{array}{l}\text { STATUS } \\
\text { (6) } \\
\text { Deposh }\end{array}$} & \multirow{2}{*}{$\begin{array}{l}\text { MINE } \\
\text { TYPE } \\
\text { Unknown }\end{array}$} & \multirow[t]{2}{*}{ COMMENTS } \\
\hline G & 38 & & N $26^{\circ} 25^{\circ}$ & E $33^{\circ} 28^{\prime}$ & Gold & & & & & \\
\hline & 39 & Erodia ' & N $26^{\circ} 22$ & E $33^{\circ} 24^{\prime \prime}$ & Gold & 3,16 & General & Deposk & Unknown & Occurrence. \\
\hline G & 40 & Alata EIMort & $N 26^{\circ} 11^{\circ}$ & E $33^{\circ} 30^{\circ}$ & Gold & 3 & General & Deposin & Unknown & Occurrence. \\
\hline G & 41 & Kab El Abyad & N $26^{\circ} 12$ & E $33^{\circ} 35^{\prime}$ & Gold & 3 & General & Deposk & Unknown & Occurrence. \\
\hline \multirow[t]{3}{*}{$G$} & 42 & Abu Manwat & $N 26^{\circ} 30^{\circ}$ & E $33^{\circ} 10^{\circ}$ & $\begin{array}{l}\text { Gold, alver } \\
\text { copper, zlnc }\end{array}$ & 3,24 & Gonoral & Deposh & Unknown & 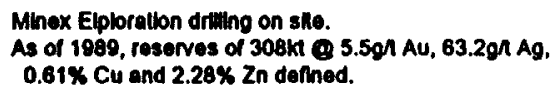 \\
\hline & 43 & Sabahla & N $24^{\circ} 51^{\circ}$ & E $34^{*} 45$ & Gold & 3 & Generat & Deposn & Unknown & Occurrence. \\
\hline & 44 & $\begin{array}{l}\text { Hamagh } \\
\text { Um Hagatg } \\
\text { Um Hams }\end{array}$ & $N 24^{\circ} 40^{\circ}$ & E $3^{\circ}$ O4 & Gold & 3,4 & General & Deposin & Unknown & $\begin{array}{l}\text { Occurrence in granodlortlo. } \\
\text { Quartz veln several hundied m long, } 50-70 \mathrm{~cm} \text { inlck. } \\
\text { Workings to depth of } 60 \mathrm{~m} \text {. }\end{array}$ \\
\hline \multirow[t]{8}{*}{$\mathbf{G}$} & 45 & Wadl Gasus & N 26: 32 & E 33' 54 & Gold & 3 & General & Deposn & Unknown & Occurrence. \\
\hline & 46 & Sherm El Bahar & N $25^{\circ} 48^{\circ}$ & E $34^{\circ} 16^{\circ}$ & Gold & 3.22 & Goneral & Deposk & Unknown & Occurrence. \\
\hline & 47 & Umm Tundebe & $N 24^{\circ} 55^{\circ}$ & E $34^{\circ} 45^{\circ}$ & Gold & 3 & Goneral & Deposin & Unknown & Occurrence. \\
\hline & 48 & Lowowt & N $24^{\circ} 46^{\prime}$ & E $34^{\circ} 45^{\circ}$ & Gold & 3 & Gonoral & Deposll & Unknown & Occurrence. \\
\hline & 49 & Kab EI Rayan & N 24. 210 & E $35^{\circ}$ or & Gold & 3 & General & Deposill & Unknown & Occurrence. \\
\hline & 50 & Wadl Law & N $24^{\circ} 50^{\circ}$ & E $34 \cdot 4 r$ & Chromive & 4.22 & General & Deposin & Unknown & 10 lenses h lelc-carbonate, med. grade ore. \\
\hline & $\mathbf{5 1}$ & Wadl Um Kharlga & N 25, 02 & $E 34^{\circ} 42$ & Chromilo & 4,22 & Gonoral & Deposk & Unknown & 4 bnses, 350 tons, $35 \% \mathrm{Cr} 203$. \\
\hline & 52 & Wadl EI Nakarl & $N 24^{\circ} 31^{\circ}$ & E $\mathbf{3 4}^{\circ} \mathbf{5 0 ^ { \circ }}$ & Chromile & 4 & General & Deposk & Unknown & Occurrence. \\
\hline \multirow[t]{5}{*}{ K } & 53 & Wadl Khashab & N $24^{\circ} 22$ & E 34: 22 & Chromilo & 4 & Goneral & Deposin & Unknown & Occurrences. \\
\hline & 54 & Umm Kabu & $N 24^{\circ} 34^{\circ}$ & E $34^{*} 56^{*}$ & Chromile & 4 & General & Deposh & Unknown & Occurrence. \\
\hline & 55 & Wadl Gert & $N 24^{\circ} 5 r$ & E $34 \cdot 49$ & Chromile & 4,22 & Goneral & Deposin & Unknown & Occurrence. \\
\hline & 56 & ElGenehe & $N 23^{\circ} 6 r$ & E $34^{\circ} 3 r$ & $\begin{array}{l}\text { Copper } \\
\text { Nickel }\end{array}$ & 4,22 & Genaral & Doposk & Unknown & Gossen whin copper and nkckel discovered in 1973. \\
\hline & 57 & Abu Nkrur & N $24^{\circ} 36$ & E $34^{*} 46^{\prime}$ & Conundum & 1 & Goneral & Deposn & Surtace & Occurrence. \\
\hline \multirow[t]{3}{*}{ K } & 58 & Gabbro Akarom & $N 24^{\circ} 00^{\circ}$ & $E 3^{\circ} 11^{\circ}$ & $\begin{array}{l}\text { Copper } \\
\text { Nikel }\end{array}$ & $4,22,23,30$ & Goneral & Deposk & Unknown & 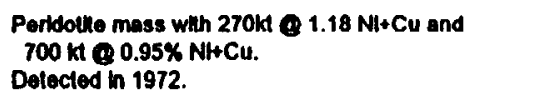 \\
\hline & 59 & WedI EI Mlyah & $N 25^{\circ} 17$ & E $34^{\circ} 00^{\circ}$ & $\begin{array}{l}\text { Thanlum } \\
\text { Iron }\end{array}$ & 4,16 & General & Deposh & Unknown & Two tenses in gabbro \\
\hline & 60 & Abu Khrug & $N 24^{\circ} 39^{\circ}$ & E $34^{*} 16^{\circ}$ & $\begin{array}{l}\text { Nephellno Syenke } \\
\text { (AI,Na,K,Cernent) }\end{array}$ & $4,18,22$ & General & Deposh & Surface & Ros: 28Mml 21.63\% ARO3. \\
\hline
\end{tabular}


APPENDIX C: PROSPECTS AND NON PRODUCING MINERAL PROPERTIES IN EOYPT.

\begin{tabular}{|c|c|c|c|c|c|c|c|c|c|c|}
\hline $\begin{array}{l}\text { MA } \\
\text { (1) } \\
\text { (1) }\end{array}$ & & $\begin{array}{l}\text { NAME } \\
\text { (2) }\end{array}$ & $\begin{array}{l}\text { COORDIN } \\
\text { LAT. }\end{array}$ & $\begin{array}{l}\text { ATES } \\
\text { LONO. }\end{array}$ & COMMODITY(IES) & $\begin{array}{l}\text { DATA } \\
\text { SOURCES } \\
\text { (3) }\end{array}$ & $\begin{array}{l}\text { DATA } \\
\text { RELAANCE } \\
\text { (4) }\end{array}$ & $\begin{array}{l}\text { STATUS } \\
\text { (Б) }\end{array}$ & $\begin{array}{l}\text { MINE } \\
\text { TYPE }\end{array}$ & COMMENTS \\
\hline $\mathbf{N}$ & 61 & $\begin{array}{l}\text { Um Garayat } \\
\text { Helmur }\end{array}$ & N $22^{\circ} 40^{\circ}$ & E $33^{\circ} 29^{\circ}$ & Copper & 4.22 & General & Doposk & Unknown & $\begin{array}{l}3 \mathrm{~km} \text { sourtheast of Umm Gartal gold mlne. } \\
\text { Porphyry copper } \mathrm{h} \text { granodiorte. }\end{array}$ \\
\hline $\mathbf{F}$ & 62 & Gabal Galtar & N $27^{\circ}$ os & E $33^{\circ} 16^{\circ}$ & Molybdonilo & $4,22,30$ & Goneral & Deposx & Underground & $\begin{array}{l}\text { Zone of velns } 25 \mathrm{~m} \text { wdde and } 500 \mathrm{~m} \text { long. } \\
\text { Ore hn quartz velns and disseminated in grantie. } \\
\text { Res: } 2500 \text { lons } 2.25 \% \text { Mo. } \\
\text { Prospected durthg the 1930's and } 1940 \text { 's. }\end{array}$ \\
\hline $\mathbf{F}$ & 63 & Abu Marwa & N $27^{\circ} 20^{\circ}$ & E $33^{\circ} 09^{\circ}$ & Molybdentite & $4,22,30$ & Goneral & Dopost & Underground & Occurrence. \\
\hline $\mathbf{F}$ & 64 & ADu Haba & $N 27^{\circ} 18^{\prime}$ & $E \quad 33^{\circ} 13^{\prime}$ & Moyybdenlice & $4,22,30$ & General & Deposil & Underground & $\begin{array}{l}\text { Occurrence. } \\
\text { Prospected durng the } 1930 \text { 's and } 1940 \text { 's. }\end{array}$ \\
\hline $\mathbf{F}$ & 65 & Um Dbs & $N 27^{\circ} 00^{\circ}$ & E $33^{\circ} 31^{\prime}$ & Mobybdentito & $4,22,30$ & General & Deposk & Underground & Occurrence. \\
\hline K & 66 & Hormr Akarem & $N 24^{\circ} 11^{*}$ & E $3^{\circ} 05^{\circ}$ & $\begin{array}{l}\text { Th, Molybdente } \\
\text { Blsmuth, Copper } \\
\text { Nloblum, Beryl }\end{array}$ & $4,22,30$ & General & Deposin & Underground & $\begin{array}{l}\text { Ora In zone } 1100 \mathrm{~m} \times 800 \mathrm{~m} \text { m grantle. } \\
\text { Ore low grade } 0.031 \% \mathrm{Mo} \text {. } \\
\text { Res (1982) 8Mml } 0.3 \% \text { Mo, but } 300 \mathrm{~m} \text { below surface. }\end{array}$ \\
\hline & 67 & $\begin{array}{l}\text { Wadl Hafla } \\
\text { Wadl Um Barh }\end{array}$ & N $24^{\circ} 53^{\prime}$ & E $3^{\circ} 08^{\prime}$ & $\begin{array}{l}\text { Th, Molybdenlie } \\
\text { Blamuth, Copper } \\
\text { Beryillum }\end{array}$ & 4,22 & Goneral & Deposh & Underground & $\begin{array}{l}\text { Ore hn zone } 500 \mathrm{~m} \times 300 \mathrm{~m} \text { hi granke. } \\
\text { Ore low grade } \mathrm{B} \text { 0.03\% Mo, 0.008\% Sn, \& } 0.02 \% \\
\text { Cu, Be, and BI. }\end{array}$ \\
\hline $\mathbf{F}$ & 68 & Abu Hamad & N $27^{\circ} 29^{\circ}$ & $E 33^{\circ} 15^{\circ}$ & Tungston & $4,22,30$ & Gonerat & Deposh & Surnace & $\begin{array}{l}\text { 14 velne assoclated with granile. } \\
\text { Dlecovered in } 1931 \text {. }\end{array}$ \\
\hline G & 69 & Fallra Ei Bolda & $N 26^{\circ} 46^{\circ}$ & E $33^{\circ} 20^{\circ}$ & Tungsten & $4,22,30$ & Goneral & Doposit & Surface & $\begin{array}{l}\text { Large number ov velns up } 10500 \mathrm{~m} \text { long. } 10-30 \mathrm{~cm} \\
\text { thick. }\end{array}$ \\
\hline G & 70 & Abu Kharif & $N 26^{\circ} 48^{\prime}$ & E $33^{\circ} 25^{\prime}$ & Tungston & $4,22,30$ & Goneral & Deposil & Surface & Two sols of quartz vains associaled with granito. \\
\hline G & 71 & $\begin{array}{l}\text { El Dob } \\
\text { Um 8lsilla }\end{array}$ & $\begin{array}{l}\text { N } 26^{\circ} 27 \\
N 25^{\circ} 21^{\circ}\end{array}$ & $\begin{array}{l}\text { E } 33^{\circ} 28^{\circ} \\
\text { E } 34^{\circ} 01^{\circ}\end{array}$ & $\begin{array}{l}\text { Tungsion } \\
\text { Molybdenilo } \\
\text { Tungaten }\end{array}$ & $\begin{array}{l}4,22,30 \\
4,22,30\end{array}$ & $\begin{array}{l}\text { Gonoral } \\
\text { Goneral }\end{array}$ & $\begin{array}{l}\text { Doposh } \\
\text { Dopostt }\end{array}$ & $\begin{array}{l}\text { Surface } \\
\text { Surface }\end{array}$ & Sorlos of small volns assoclated with granite. \\
\hline 0 & 73 & Gash Amer & N $22^{\circ} 18^{\circ}$ & E $36^{*} 12^{\prime}$ & Tungaten & $4,22,30$ & Genoral & Doposit & Surfece & Occurrence. \\
\hline & 74 & Abu Rusheld & N $24^{\circ} 37$ & $E 34^{\circ} 46^{\circ}$ & $\begin{array}{l}\text { Niobłum } \\
\text { Tantalum }\end{array}$ & 4.22 & Goneral & Doposff & Unknown & $\begin{array}{l}\text { Nb2O5:Ta2O5 rallo 5:1. } \\
\text { Thleknose and grade decroateas with depth. } \\
\text { Rea: DOK Nb2O5 and 13kt Ta2OS } 0.02 \% \text { Ta2O5. }\end{array}$ \\
\hline K & 75 & Homrol Mikbld & $N 24^{\circ} 10^{\circ}$ & E $34^{\circ} \mathbf{2 3}$ & Bond & 4 & Genaral & Deposil & Surface & $\begin{array}{l}\text { Ore as alteratlons in granille and small pegmatilo } \\
\text { voins. }\end{array}$ \\
\hline G & 76 & ElAishan & $\mathrm{N} 26^{\circ} \mathrm{Or}$ & E $34^{\circ} 05^{\circ}$ & Uranlum & 4,30 & General & Doposit & Unknown & Earty occurrence. \\
\hline & 77 & Um Dowolla & N 22, $1 T$ & E $33^{\circ} 26^{\circ}$ & Uranlum & 4 & Gonoral & Deposil & Unknown & Dike oxtends $10.6 \mathrm{~km}$, is $2-20 \mathrm{~m}$ thick. \\
\hline & 78 & Ranga & N $24^{\circ} 2 T$ & $E 35^{\circ} 12$ & Suliur & 4 & General & Deposil & Unknown & Oecumence. \\
\hline & 79 & Bonl Abu Goralya & N $30^{\circ} 26^{\circ}$ & E $34^{\circ} 01^{\circ}$ & Graphltor & 4 & Goneral & Deposht & Unknown & ceurrence \\
\hline
\end{tabular}


APPENDIX C: PROSPECTS AND NON PRODUCINO MINERAL. PROPERTIES IN EOYPT.

\begin{tabular}{|c|c|c|c|c|c|c|c|c|c|c|}
\hline \multirow{2}{*}{$\begin{array}{l}\text { MAF } \\
\text { KEY } \\
\text { (1) }\end{array}$} & \multirow{2}{*}{\multicolumn{2}{|c|}{$\begin{array}{ll}\text { NAME } \\
\text { (2) } \\
\text { Wadl Sitra }\end{array}$}} & \multicolumn{2}{|c|}{$\begin{array}{l}\text { COORDINATES } \\
\text { UAT. LONO. }\end{array}$} & \multirow{2}{*}{$\begin{array}{l}\text { COMMODITY(IES) } \\
\text { Graphlio }\end{array}$} & \multirow{2}{*}{$\begin{array}{l}\text { DATA } \\
\text { SOURCES } \\
\text { (3) } \\
4\end{array}$} & \multirow{2}{*}{$\begin{array}{l}\text { DATA } \\
\text { RELIANCE } \\
\text { (4) } \\
\text { General }\end{array}$} & \multirow{2}{*}{$\begin{array}{l}\text { STATUS } \\
\text { (5) } \\
\text { Deposit }\end{array}$} & \multirow{2}{*}{$\begin{array}{l}\text { MINE } \\
\text { TYPE } \\
\text { Unknown }\end{array}$} & \multirow{2}{*}{$\begin{array}{l}\text { COMMENTS } \\
\text { Occurrenco. }\end{array}$} \\
\hline & & & $N 28^{\circ} 42^{\prime}$ & E $26^{\circ} 54$ & & & & & & \\
\hline G & 81 & Abu Marewat & $N 26^{\circ} 31^{\prime}$ & $E 3^{*} 39^{\circ}$ & Iron & $4,16,22$ & General & Deposith & Unknown & $\begin{array}{l}\text { Banded Iron formation. } \\
\text { Oro bands 3-17m thick in tuff. } \\
\text { Res: } 6.5 \mathrm{Mml} \otimes 44.4 \% \mathrm{Fo} \text {. }\end{array}$ \\
\hline & 82 & $\begin{array}{l}\text { Wadl Karlm } \\
\text { Wadi Kareem }\end{array}$ & $N 25^{\circ} 54^{\prime}$ & E $34^{\circ} 09^{\circ}$ & Iron & $4,16,22,30$ & General & Deposit & Unknown & $\begin{array}{l}\text { Bandod iron formalion. } \\
\text { Four seams in motasedimentary } 130 \mathrm{~m} \text { section. } \\
\text { Minable ore } 17.8 \mathrm{Mmt} \text {. 14-45\% Fo. }\end{array}$ \\
\hline & 83 & Wadi El Dabbah & N $25^{\circ} 45^{\prime}$ & $E 3^{\circ} 13^{\prime}$ & Iron & $4,16,22,30$ & Genoral & Doposit & Unknown & $\begin{array}{l}\text { Banded lron formallon. } \\
\text { Ore bande up to tom thick. } \\
\text { Minable ore } 6.1 \mathrm{Mmt} \text { 39-13.8+\% Fo. }\end{array}$ \\
\hline & 84 & $\begin{array}{l}\text { Um Khamis El Zarga } \\
\text { Um Chamis }\end{array}$ & N $25^{\circ} 34^{\prime}$ & E $34^{*} 1 T$ & Iron & $4,16,22,30$ & Goneral & Doposit & Unknown & $\begin{array}{l}\text { Bandod iron formallon. } \\
\text { Small }(<1.5 \mathrm{~m}) \text { bands in groonschisis. } \\
\text { Res: } 3 M m t Q 40-41 \% \mathrm{Fe} \text {. }\end{array}$ \\
\hline & 85 & Gabal El Hadid & N $25^{\circ} 21^{\prime}$ & E $34^{\circ} 08^{\circ}$ & Iron & $4,16,22$ & General & Deposilf & Unknown & $\begin{array}{l}\text { Banded iron formallion. } \\
\text { Ore bands in chert. } \\
\text { Minablo reserves } 2.15 \mathrm{Mm} \text { @ } 43-47 \% \mathrm{Fe} \text {. }\end{array}$ \\
\hline & 86 & Um Nar & $N 25^{\circ} 18^{\circ}$ & E $34^{*} 15^{\prime}$ & Iron & $4,16,22$ & General & Deposit & Unknown & $\begin{array}{l}\text { Banded iron formallon. } \\
\text { Nine groups of bands in achist. } \\
\text { Minable ore reserves } 13.7 \mathrm{Mmi} 10.51-45.5 \% \mathrm{Fe} \text {. }\end{array}$ \\
\hline & 87 & Damietla & N 31' $32^{\prime}$ & E $31^{*} 51^{\prime}$ & $\begin{array}{l}\text { Black sand } \\
\text { REO }\end{array}$ & 22,30 & General & Deposht & Surface & Occurrence. \\
\hline & 88 & El Arish & N 31•09 & E $33^{\circ} 40^{\circ}$ & $\begin{array}{l}\text { Black sand } \\
\text { REO }\end{array}$ & 22,30 & General & Deposit & Surface & Prelliminary fold work done in 1970 's. \\
\hline B & $\begin{array}{l}89 \\
90\end{array}$ & $\begin{array}{l}\text { Gabal Calrani } \\
\text { Tabaket All }\end{array}$ & $\begin{array}{l}\text { N } 29^{\circ} 30^{\prime} \\
\text { N } 29^{\circ} 20^{\circ}\end{array}$ & $\begin{array}{l}\text { E } 30^{\circ} 37 \\
\text { E } 32^{*} 25^{\circ}\end{array}$ & $\begin{array}{l}\text { Uranlum } \\
\text { Iron }\end{array}$ & $\begin{array}{l}22,30 \\
22\end{array}$ & $\begin{array}{l}\text { General } \\
\text { Genoral }\end{array}$ & $\begin{array}{l}\text { Deposit } \\
\text { Deposit }\end{array}$ & $\begin{array}{l}\text { Surface } \\
\text { Surface }\end{array}$ & $\begin{array}{l}\text { Uranlum in } 3 \text { lorms; black shales, phospatle deposils, } \\
\text { and sandstone. } \\
\text { Occurrence. }\end{array}$ \\
\hline & 91 & Wadl Arabah & N 29॰ or & E $32^{*} 39^{\prime}$ & $\begin{array}{l}\text { Manganese } \\
\text { Copper }\end{array}$ & 22,30 & General & Deposil & Unknown & Res: 4kt 20.65\% Mn. \\
\hline E & 92 & Abu Sumoira & $N 28^{\circ} 56^{\prime}$ & E $\mathbf{3 3}^{\circ} \mathbf{3 8 ^ { \prime }}$ & Copper & 22 & Goneral & Deposit & Unknown & Occurrence. \\
\hline $\mathbf{E}$ & 93 & Abu Rudeib & N 28. $55^{\prime}$ & E $33^{*} 35^{\prime}$ & Coppor & 22 & General & Deposit & Unknown & Occurrence. \\
\hline E & 94 & Rashadla & $N 28^{\circ} 54^{\circ}$ & $E 3^{\circ} 31^{\circ}$ & Copper & 22 & General & Doposit & Unknown & Occurtence. \\
\hline E & 95 & Abu Zagatan & $N 28^{\circ} 51^{\circ}$ & E $33^{\circ} \mathbf{3} 0^{\circ}$ & Copper & 22 & Goneral & Doposit & Unknown & Occurrence. \\
\hline E & 96 & Tawilleh & N 28. 50" & E $\mathbf{3 3}^{\circ} \mathbf{3 2}^{\prime}$ & Coppor & 22 & Genoral & Deposiff & Unknown & Occurrence. \\
\hline E & 97 & Abu EI Nimrah & $N 28^{\circ} 48^{\circ}$ & $E 3^{\circ} 36^{\circ}$ & Copper & 22,30 & General & Deposit & Unknown & Occurrence. \\
\hline $\mathbf{E}$ & 98 & Tarfa & $N 28^{\circ} 50^{\circ}$ & E $33^{\circ} 38^{\prime}$ & Coppor & 22 & Genoral & Doposit & Unknown & Occurrence. \\
\hline$E$ & 99 & Tart & $N 28^{\circ} 45^{\prime}$ & E $33^{\circ} 36^{\circ}$ & Copper & 22 & General & Deposit & Unknown & Occurrence. \\
\hline$E$ & 100 & Felran & $N 28^{\circ} 42^{\prime}$ & E $33^{\circ} 39^{\circ}$ & Copper & 22 & General & Deposit & Unknown & Occurrence. \\
\hline
\end{tabular}


APPENDIX C: PROSPECTS AND NON PRODUCING MINERAL PROPERTIES IN EGYPT.

\begin{tabular}{|c|c|c|c|c|c|c|c|c|c|c|}
\hline $\begin{array}{l}\text { MA } \\
\text { KE } \\
\text { (4) }\end{array}$ & & $\begin{array}{l}\text { NAME } \\
\text { (2) }\end{array}$ & $\begin{array}{l}\text { COORDIN } \\
\text { LAT. }\end{array}$ & $\begin{array}{l}\text { ATES } \\
\text { LONG. }\end{array}$ & COMMODITY(IES) & $\begin{array}{l}\text { DATA } \\
\text { SOURCES } \\
\text { (3) }\end{array}$ & $\begin{array}{l}\text { DATA } \\
\text { RELIANCE } \\
\text { (d) }\end{array}$ & $\begin{array}{l}\text { 8TATUS } \\
\text { (5) }\end{array}$ & $\begin{array}{l}\text { MINE } \\
\text { TYPE }\end{array}$ & COMMENTS \\
\hline $\mathbf{F}$ & 101 & Wadl Dib & $N 27^{\circ} 50^{\circ}$ & $E 3^{*} 15^{\prime}$ & Molybdenum & 22,30 & General & Deposin & Unknown & Occurrence. \\
\hline $\mathbf{F}$ & 102 & Wadl Abu Triof & N $27^{\circ} 26^{\circ}$ & E $33^{*} 33^{\prime}$ & Manganese & 22 & Goneral & Deposh & Unknown & Small reserves. \\
\hline $\mathbf{F}$ & 103 & $\begin{array}{l}\text { Wadi Abu Shagar } \\
\text { El Quibll }\end{array}$ & $\begin{array}{c}\mathbf{N} 27^{-22} \\
-\end{array}$ & E $\mathbf{3 3}^{\circ} \mathbf{3 4 ^ { \prime }}$ & Manganose & 22 & General & Doposil & Unknown & Dlscovered in 1912. Small reserves. \\
\hline $\mathbf{F}$ & 104 & $\begin{array}{l}\text { Wadl Abu Dholss } \\
\text { El Baharl }\end{array}$ & N $27^{\circ} 20^{\circ}$ & $E \mathbf{3 3}^{\circ} \mathbf{3 3 ^ { \prime }}$ & Manganese & 22 & General & Deposil & Unknown & Occurrence. \\
\hline $\mathbf{F}$ & 105 & Wadl Hammad & $N 27^{\circ}$ or & $E \mathbf{3 2} 2^{*} 9^{\circ}$ & Lead, zinc & 22 & General & Doposth & Unknown & Occurrence. \\
\hline G & 106 & Barrud & $N 26^{*} 51^{*}$ & E $33^{*} 36^{*}$ & Copper & 22 & General & Deposil & Unknown & Occurrence. \\
\hline $\mathbf{G}$ & 107 & EI Blelda & $N 20^{\circ} 44^{\prime}$ & $E 33^{\circ} 24^{\prime}$ & Molybdenum & 22 & Goneral & Deposit & Unknown & Occurrence. \\
\hline $\mathbf{G}$ & 108 & Gabal Dob & $N 20^{\circ} 44^{\circ}$ & E $\mathbf{3 3 ^ { \circ }} \mathbf{3 3 ^ { \prime }}$ & Molybdenum & 22 & Gonoral & Deposht & Unknown & Occurrence. \\
\hline $\mathbf{G}$ & 109 & $\begin{array}{l}\text { Umm Taghir } \\
\text { Um Tagher }\end{array}$ & N 26. $43^{\circ}$ & $E 3^{\circ} 37$ & Coppor & 22 & General & Deposil & Unknown & Occurrence. \\
\hline G & 110 & Mabart & $N 26^{\circ} 42^{\prime}$ & $E 33^{\circ} 48^{\prime}$ & Copper & 22 & Goneral & Deposit & Unknown & Occurrence. \\
\hline G & 111 & $\begin{array}{l}\text { Abu Furad } \\
\text { Abu Fanad }\end{array}$ & $N 26^{\circ} 40^{\circ}$ & E $33^{\circ} 37$ & Iron & 22 & General & Deposilt & Unknown & Oecurrence. \\
\hline $\mathbf{G}$ & 112 & EI Wahera & $N 26^{\circ} 28^{\prime}$ & $E 33^{\circ} 44^{\prime}$ & Copper & 22 & General & Deposit & Unknown & Occurrence. \\
\hline $\mathbf{G}$ & 113 & Kab Um El Ab & $N 26^{\circ} 21^{\circ}$ & E $33^{*} 40^{\prime}$ & Iron & 22 & Genoral & Deposit & Unknown & Occurrence. \\
\hline G & 114 & Abu Haya & N 26. $20^{\circ}$ & $E \mathbf{3 3}^{\circ} \mathbf{5 0 ^ { \circ }}$ & Coppor & 22 & General & Deposit & Unknown & Occurrence. \\
\hline $\mathbf{G}$ & 115 & Kab Amln & N 26*17 & E $33^{\circ} 38^{\circ}$ & $\begin{array}{l}\text { Nloblum } \\
\text { Tin }\end{array}$ & 22 & Gonoral & Deposit & Unknown & Rare earth conlent of Nb $0.02 \% \mathrm{Nb}, 0.02 \% \mathrm{Sn}$. \\
\hline G & 116 & Talel EI Gr & $N 26^{\circ} 17$ & E $33^{\circ} 42^{\circ}$ & Iron & 22 & Goneral & Deposit & Unknown & Occurrence. \\
\hline $\mathbf{G}$ & 117 & Um Selimat & $N 26^{\circ} 15^{\circ}$ & $E 33^{\circ} 43^{\circ}$ & Copper & 22 & General & Doposit & Unknown & Occurrence. \\
\hline G & 118 & Wadi Abu Diwan & N $26^{*} 10^{\circ}$ & E $33^{\circ} 53^{\circ}$ & Iron & 22 & General & Deposil & Unknown & Reserves: $36 \mathrm{Mml}$ 37\% Fa. \\
\hline $\mathbf{G}$ & 119 & Sodmein & N $26^{\circ} 10^{\circ}$ & E $33^{\circ} 48^{\circ}$ & Chromllo & 22 & Goneral & Doposit & Unknown & Occurrence. \\
\hline $\mathbf{G}$ & 120 & Abu Ziran & $N 26^{\circ} 04^{\circ}$ & E $33^{\circ} 56^{\circ}$ & $\begin{array}{l}\text { Nloblum } \\
\text { Tanlalum }\end{array}$ & 22 & Goneral & Doposit & Unknown & Occurrence. \\
\hline & 121 & Sharm EI Sholkh & N $27^{\circ} 55^{\circ}$ & E $34^{\circ} 15^{\prime}$ & Manganese & 22,30 & General & Deposit & Unknown & $\begin{array}{l}\text { Small reserves. } \\
\text { Bod thickness 4m. }\end{array}$ \\
\hline & 122 & Abu Marwa & N 25* 03' & E $33^{\circ} 43^{\prime}$ & Chromile & 22 & Oeneral & Deposit & Unknown & Occurrence. \\
\hline & 123 & Abu Rakeb & N 24. 55 & E $34^{\circ} 10^{\circ}$ & Iron & 22 & General & Deposit & Unknown & Occurronce. \\
\hline & 124 & Wadi EI Hindusl & $N 24^{\circ} 53^{\prime}$ & E $34^{\circ} 10^{\circ}$ & Iron & 22 & General & Deposit & Unknown & Occurrence. \\
\hline
\end{tabular}


APPENDIX C: PROSPECTS AND NON PRODUCING MINERAL PROPERTIES IN EOYPT.

\begin{tabular}{|c|c|c|c|c|c|c|c|c|c|}
\hline $\begin{array}{l}\text { MAP } \\
\text { KEY } \\
\text { (1) }\end{array}$ & $\begin{array}{l}\text { NAME } \\
\text { (2) }\end{array}$ & $\begin{array}{l}\text { COORDIN } \\
\text { LAT. }\end{array}$ & $\begin{array}{l}\text { LTE8 } \\
\text { LONG. }\end{array}$ & COMMODITY(IES) & $\begin{array}{l}\text { DATA } \\
\text { SOURCES } \\
\text { (3) }\end{array}$ & $\begin{array}{l}\text { DATA } \\
\text { RELIANCE } \\
\text { (4) }\end{array}$ & $\begin{array}{l}\text { STATUS } \\
\text { (5) }\end{array}$ & $\begin{array}{l}\text { MINE } \\
\text { MYPE }\end{array}$ & COMMENTS \\
\hline 125 & Wadl Wizr & $N 25^{\circ} 45^{\circ}$ & E $34^{\circ} 25^{\circ}$ & $\begin{array}{l}\text { Lead } \\
\text { zinc }\end{array}$ & 16,22 & General & Deposit & Unknown & Occurranca. \\
\hline 128 & Abu Chorban & N 25॰ 38' & E $34^{\circ} 29^{\circ}$ & Ochre & 22 & General & Deposh & Surface & Occurrence. \\
\hline 127 & Abu Anz & $N 25^{\circ} 34^{\circ}$ & $E 34^{\circ} 33^{\circ}$ & $\begin{array}{l}\text { Lead } \\
\text { zinc }\end{array}$ & 16,22 & Gonoral & Deposit & Unknown & Res: $0.3 \mathrm{Mml}$. \\
\hline 128 & Wadl Um Shaddad & $N 25^{\circ} 40^{\circ}$ & E $34^{\circ} 18^{\circ}$ & Iron & 22 & Goneral & Deposil & Unknown & Occurrence. \\
\hline 129 & Wadi Stira & N 25* $36^{\circ}$ & E $34^{\circ} 12^{\circ}$ & Chromlte & 22 & Genoral & Doposil & Unknown & Occurrence. \\
\hline 130 & El Alawi & $N 25^{\circ} 27$ & E $34^{\circ} 10^{\circ}$ & $\begin{array}{l}\text { Nloblum } \\
\text { Tantalum }\end{array}$ & 22 & General & Deposil & Unknown & Occurrence. \\
\hline 131 & $\begin{array}{l}\text { Wadi Sitra } \\
\text { Gobel El Hadid }\end{array}$ & $N 25^{\circ} 2 T$ & $E 34^{*} 15^{\circ}$ & Iron & 16,22 & General & Doposil & Unknown & Docurrence. \\
\hline 132 & Siwiqal EI Soda & $N 25^{\circ} 08^{\prime}$ & E $34^{\circ} 45^{\prime}$ & Lead, zino & 22 & General & Deposh & Unknown & Occurrence. \\
\hline 133 & Gabal Mudargag & $N 24^{\circ} 55^{\prime}$ & E $\mathbf{3 4 ^ { \circ }} \mathbf{2 0 ^ { \circ }}$ & Chromtte & 22 & Gonoral & Deposil & Unknown & Oecurronce. \\
\hline 134 & Um Hamr & $N 24^{\circ} 41^{\prime}$ & E $34^{\circ} 00^{\circ}$ & Copper & 22 & Gonoral & Deposil & Unknown & Occurrence. \\
\hline 135 & Wadi Um El Khoiran & $N 24^{\circ} 3 T$ & E $34^{\circ} 50^{\circ}$ & $\begin{array}{l}\text { Nloblum } \\
\text { Tantalum }\end{array}$ & 22 & General & Doposit & Unknown & Occurrence. \\
\hline 136 & Wadl El Sharm & $N 24^{\circ} 43^{\circ}$ & E $35^{\circ} 00^{\circ}$ & $\begin{array}{l}\text { Nloblum } \\
\text { Tantalum }\end{array}$ & 22 & General & Deposil & Unknown & Occurrence. \\
\hline 137 & Wadi Um Karolm & $N 24^{\circ} 26^{\circ}$ & $E 3^{\circ} 00^{\circ}$ & Copper & 22 & General & Deposil & Unknown & Occurrenco. \\
\hline 138 & Wadl Um Salama & $N 24^{\circ} 23^{\prime}$ & E $35^{\circ} 03^{\circ}$ & Copper & 22 & General & Doposit & Unknown & Occurronce. \\
\hline 139 & Wadi Helelin & $N 24^{\circ} 21^{\circ}$ & E 35* $08^{\prime}$ & Copper & 22 & General & Deposil & Unknown & Occurrence. \\
\hline 140 & Wadl Sarabl & $N 24^{\circ} 20^{\circ}$ & E $3^{\circ}$ or & Copper & 22 & General & Deposilf & Unknown & Occurrence. \\
\hline 141 & Wadi Abu Ghusun & $N 24^{*} 20^{\prime}$ & E 34` 53' & Copper & 22 & General & Deposil & Unknown & Occurrence. \\
\hline 142 & Um Effoln & N 24.08' & E $35^{\circ} 04^{\circ}$ & Thanlum & $16,22,30$ & General & Deposil & Unknown & Occurrence. \\
\hline 143 & El Aswad & N 23" $10^{\circ}$ & E $27^{\circ} 30^{\circ}$ & Iron & 22 & Goneral & Deposill & Unknown & Occurrence. \\
\hline 144 & Ef Arbaoln & N 23*06 & $E 3^{\circ} 11^{\circ}$ & Iron & 22 & General & Deposit & Unknown & Occurrence. \\
\hline 145 & Kalabsha & N 23' 32' & E $32^{*} 49^{\prime}$ & Iron & 22 & General & Doposil & Unknown & Submerged by Aswan Hlgh Dam.waler. \\
\hline 146 & Gart Hussoin. & N $23^{\circ} \mathbf{2 0 ^ { \prime }}$ & E $32^{\circ} 50^{\circ}$ & Iron & 22 & Genoral & Doposit & Unknown & Submerged by Aswan Hlgh Dam waler. \\
\hline 147 & South Kalabsha & N 23- 20' & E $32^{*} 22^{\circ}$ & Iron & 22 & Goneral & Deposit & Unknown & Submerged by Aswan Hlgh Dam waler. \\
\hline 148 & Korosko & N 22* 35' & E $32^{\circ} 25^{\prime \prime}$ & Iron & 22 & General & Deposit & Unknown & Submerged by Aswan High Dam water. \\
\hline 149 & Abu SImbel & N $22^{\circ} 20^{\circ}$ & E $31^{\bullet} 47$ & Iron & 22 & General & Deposh & Unknown & Submerged by Aswan High Dam waler. \\
\hline 150 & Bir Hassin & N $23^{\circ} 05^{*}$ & E $31^{*} 14^{\prime}$ & Iron & 22 & Gonoral & Deposil & Unknown & Discovered In 1966. \\
\hline
\end{tabular}


APPENDIX C: PROSPECTS AND NON PRODUCING MINERAL PROPERTIES IN EOYPT.

\begin{tabular}{|c|c|c|c|c|c|c|c|c|c|c|}
\hline \multicolumn{2}{|c|}{$\begin{array}{l}\text { MAP } \\
\text { KEY } \\
\text { (1) }\end{array}$} & \multirow{2}{*}{$\begin{array}{l}\text { NAME } \\
\text { (2) } \\
\text { Gabal Tupur }\end{array}$} & \multicolumn{2}{|c|}{$\begin{array}{l}\text { COORDINATES } \\
\text { LAT. LONG. }\end{array}$} & \multirow{2}{*}{$\begin{array}{l}\text { COMMODITY(IES) } \\
\text { Manganose }\end{array}$} & \multirow{2}{*}{$\begin{array}{l}\text { DATA } \\
\text { SOURCES } \\
\text { (3) } \\
22\end{array}$} & \multirow{2}{*}{$\begin{array}{l}\text { DATA } \\
\text { RELIANCE } \\
\text { (4) } \\
\text { Goneral }\end{array}$} & \multirow{2}{*}{$\begin{array}{l}\text { STATUS } \\
\text { (s) } \\
\text { Deposit }\end{array}$} & \multirow{2}{*}{$\begin{array}{l}\text { MINE } \\
\text { TYPE } \\
\text { Unknown }\end{array}$} & \multirow{3}{*}{$\begin{array}{l}\text { COMMENTS } \\
\text { Occurrence. } \\
\text { Occurrence. }\end{array}$} \\
\hline 0 & 151 & & $N 22^{\circ} 42^{\prime}$ & E $35^{\circ} 43^{\prime}$ & & & & & & \\
\hline 0 & 152 & Hamra Dome & N 22 $36^{\circ}$ & E $35^{\circ} 48^{\circ}$ & Tltanlum & $16,22,30$ & General & Deposit & Unknown & \\
\hline \multirow[t]{4}{*}{0} & 153 & Dilib & N $22^{\circ} 28^{\prime}$ & E $35^{\circ} 59^{\prime}$ & Manganese & 22 & General & Deposit & Unknown & Occurrence. \\
\hline & 154 & Morsa Malruh & $N 31^{\circ} 12^{\prime}$ & E $27^{\circ} 19^{\circ}$ & Limestone & 22 & Oeneral & Deposit & Surface & Occurrence. \\
\hline & 155 & Abu Slr & N $30^{\circ} 58^{\prime}$ & E $29^{\circ} 46^{\circ}$ & Limestone & 22 & General & Deposilt & Surface & Occurrence. \\
\hline & 156 & lamallia & N 30' 28' & E 32* 19' & Salt & 22 & Goneral & Deposit & Surface & Occurrence. \\
\hline A & 157 & Dar El Bolda & N $30^{\circ} 03^{\prime}$ & E $31^{\circ} 51^{\prime}$ & Basalt & 22 & General & Deposit & Surface & Occurrence. \\
\hline A & 158 & Unnamed & N 30 $03^{*}$ & $E 3^{\circ} \mathbf{3 6}$ & Basalt & 22 & General & Doposit & Surface & Occurrence. \\
\hline \multirow[t]{2}{*}{ A } & 159 & Unnamed & N 30 06 & E $31^{\circ} 30^{\circ}$ & Olass sand & 22 & General & Deposil & Surface & Occurrence. \\
\hline & 160 & Minkat El Munkhafad & N 29* $19^{\prime}$ & E $28^{\prime} \mathbf{3 9}$ & Colestite & 22 & General & Doposill & Surface & Occurrence. \\
\hline B & 161 & Dlmiya & N 29. $31^{\circ}$ & E $30^{\circ} \mathbf{3 4}^{\prime}$ & Dlatomne & 22 & General & Deposh & Surface & Occurrence. \\
\hline B & 162 & Abu Gandr & $N 29^{\circ} 14^{\prime}$ & $E 30^{\circ} 41^{\prime}$ & Sand & 22 & General & Deposk & Surface & Occurrence. \\
\hline 8 & 163 & El Masakhi & N 29* $10^{\circ}$ & E $30^{\circ} \mathbf{3 0}$ & $\begin{array}{l}\text { Llmestone, } \\
\text { dlatomile }\end{array}$ & 22 & General & Deposh & Surface & Occurrence. \\
\hline $\mathbf{B}$ & 164 & Dindtal & N 29. $10^{\circ}$ & E 30 $50^{\circ}$ & Limestone & 22 & General & Deposit & Surface & Occurrence. \\
\hline B & 165 & Gabal EI Nolon & N 29.06' & E $30^{\circ} \mathbf{5 1 ^ { \circ }}$ & Limestone & 22 & General & Deposit & Surface & Occurrence. \\
\hline B & 166 & Dashasha & N 28. $58^{\prime}$ & E $30^{\circ} 47$ & LImestone & 22 & General & Deposh & Surface & Occurrence. \\
\hline B & 167 & El Fashn & $N 28^{\circ} 49^{\prime}$ & $E 30^{\circ} 54^{\circ}$ & $\begin{array}{l}\text { Limestone } \\
\text { Clay }\end{array}$ & 22 & General & Deposn & Surface & Occurrence. \\
\hline c & 168 & Gabal Karara & $N 28^{\circ} 37$ & E $30^{\circ} 56^{\circ}$ & Limestone & 22 & General & Depost & Surface & Occurrence. \\
\hline C & 169 & ElEdwa & N 28' 40 & E $30^{\circ} 35^{\circ}$ & Llmestone & 22 & General & Deposit & Surface & Occurrence. \\
\hline C & 170 & El Bahnasa & $N 28^{\circ} 30^{\circ}$ & $E \mathbf{3 0 ^ { \circ }} 33^{\prime}$ & $\begin{array}{l}\text { Llmestone, } \\
\text { basait }\end{array}$ & 22 & General & Deposk & Surface & Occurrence. \\
\hline C & 171 & Nazlol Amer & N 28 $28^{\circ}$ & $E 30^{\circ} 54^{\circ}$ & Limestone & 22 & General & Deposit & Surface & Occurrence. \\
\hline c & 172 & Shelkh Hassan & $N 28^{\circ} 25^{\circ}$ & $E 30^{\circ} 54^{\prime}$ & Alabaster & 22 & Goneral & Deposit & Surface & Occurrence. \\
\hline C & 173 & Gabal El Telr & N $28^{\circ} 12$ & $E \mathbf{3 0} 0^{\circ} 50^{\circ}$ & Limestone & 22 & General & Deposk & Surface & Occurrence. \\
\hline c & 174 & Shousha & N 28. $15^{\prime}$ & $E$ 30 $31^{\circ}$ & Limestone & 22 & General & Deposh & Surface & Occurrence. \\
\hline c & 175 & Abu Rouh & N 28 $18^{\circ}$ & E $30^{\circ} 24^{\circ}$ & Limestone & 22 & General & Deposh & Surface & Occurrence. \\
\hline C & 178 & Zawyat Sultan & N 28 $08^{\circ}$ & E $30^{\circ} 52$ & Limeslone & 22 & General & Depos" & Surface & Surrence. \\
\hline
\end{tabular}


APPENDIX C: PROSPECTS AND NON PRODUCINO MINERAL PROPERTIES IN EGYPT.

\begin{tabular}{|c|c|c|c|c|c|c|c|c|c|c|}
\hline $\begin{array}{l}\text { MAI } \\
\text { KEI } \\
\text { (1) }\end{array}$ & & $\begin{array}{l}\text { NAME } \\
\text { (2) }\end{array}$ & $\begin{array}{l}\text { COORDIN } \\
\text { LAT. }\end{array}$ & $\begin{array}{l}\text { ATES } \\
\text { LONG. }\end{array}$ & СOMMODITY(IES) & $\begin{array}{l}\text { DATA } \\
\text { SOURCES } \\
\text { (3) }\end{array}$ & $\begin{array}{l}\text { DATA } \\
\text { RELIANCE } \\
\text { (4) }\end{array}$ & $\begin{array}{l}\text { STATUS } \\
\text { (5) }\end{array}$ & $\begin{array}{l}\text { MINE } \\
\text { TYPE }\end{array}$ & $\begin{array}{c}\text { COMMENTS } \\
.\end{array}$ \\
\hline c & 177 & El Shourala & N 28. $03^{\prime}$ & E $30^{\circ} 56^{\circ}$ & Alabaster & 22 & General & Deposh & Surface & Occurrence. \\
\hline A & 178 & Tibbln & $N 29^{\circ} 45$ & E 31' $25^{\prime}$ & Limestone & 22 & General & Deposk & Surface & Oecurrance. \\
\hline \multirow[t]{3}{*}{ A } & 179 & Orr ElFahm & N 29"54' & E $31^{\circ} 30^{\prime}$ & Clay & 22 & General & Deposk & Surface & Occurrence. \\
\hline & 180 & El Sukhna & N 20: $30^{\circ}$ & E $32^{\circ} 21^{\prime}$ & $\begin{array}{l}\text { Limestone, } \\
\text { alabaster }\end{array}$ & 22 & General & Deposhl & Surface & Occurrence. \\
\hline & 181 & Ras Melarma & 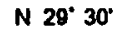 & E $32^{\circ} 50^{\circ}$ & Gypsum & 22 & General & Deposh & Surface & Occurrence. \\
\hline $\mathbf{E}$ & 182 & El Dohelsese & $N 28^{\circ} 55^{\circ}$. & E $33^{\circ} 20^{\circ}$ & Kaolln & 22 & General & Depost & Surface & Occurrence. \\
\hline \multirow[t]{6}{*}{ E } & 183 & GlnI & $N 28^{*} 53^{\circ}$ & E $33^{\circ} 22$ & Kaolln & 22 & General & Deposit & Surface & Occurrence. \\
\hline & 184 & Abu Durba & $N 28^{\prime} 28^{\prime}$ & E $33^{\circ} 22$ & Sullur & 22 & General & Deposk & Surface & Occurtence. \\
\hline & 185 & Ras Gharlb & $N 28^{\circ} 18^{\circ}$ & E $33^{\circ} 03^{\circ}$ & Polasstum salis & 22 & General & Deposk & Surface & Occurrence. \\
\hline & 188 & Abu Swora & N 28. $20^{\prime}$ & E $33^{\circ} 3 T$ & Gypsum & 22 & General & Deposk & Surface & Occurrence. \\
\hline & 187 & Wadl Avaba & N 28. $59^{\circ}$ & E $31^{\circ} 58^{\prime}$ & $\begin{array}{l}\text { Limestono } \\
\text { Clay }\end{array}$ & 16,22 & General & Deposk & Surface & Occurrence. \\
\hline & 188 & Unnamod & N 29. $05^{\circ}$ & E $31^{\circ} 55^{\circ}$ & LImestone & 22 & General & Deposk & Surface & Occurrence. \\
\hline B & 189 & El Sall & N 29* $29^{\circ}$ & E 31' 28' & Llmestone & 22 & General & Deposk & Surface & Occurrence. \\
\hline $\mathbf{B}$ & 190 & Gerza & $N 29^{\circ} 24^{\circ}$ & E $31^{\circ}$ OT & Gypsum & 22 & General & Deposh & Surface & Occurrence. \\
\hline B & 191 & Kremat & N 29. $19^{\circ}$ & E 31' 27 & $\begin{array}{l}\text { Alabaster, } \\
\text { gypsum }\end{array}$ & $22=$ & General & Deposh & Surface & Occurtence. \\
\hline B & 192 & 2 Gabal Tarboul & $N 29^{\circ} 1 T$ & E $31^{\circ} 18^{\prime}$ & Lmestone & 22 & General & Doposh & Surface & Occurrence. \\
\hline B & 193 & Abu Str El Malaa & N 29. $13^{\prime}$ & E 31. 04: & $\begin{array}{l}\text { Clay } \\
\text { Almestone }\end{array}$ & 22 & General & Deposi & Surface & Occurrence. \\
\hline B & 194 & Humrat Shybon & $N 29^{\circ}$ or & E $31^{*} 20^{\prime}$ & Limestone & 22 & General & Deposk & Surface & Occurrence. \\
\hline B & 195 & Gabal Ghorab & $N 28^{\circ} 56^{\prime}$ & $E 31^{\circ} 15^{\prime \prime}$ & Limestone & 22 & General & Deposk & Surface & Occurrence. \\
\hline B & 196 & Gabal Mashash & $N 28^{\circ} 53^{\prime}$ & E $31^{\circ} 11^{\circ}$ & Limestone & 22 & General & Deposk & Surface & Occurrence. \\
\hline B & 197 & Wadl Ghayada & N $28^{\circ} 53^{\prime}$ & E $31^{\circ} 01^{\circ}$ & Limestone & 22 & General & Deposin & Surface & Occurrence. \\
\hline D & 198 & 3 ElAmama & $N 27^{\circ} 40^{\circ}$ & E $30^{\circ} 58^{\circ}$ & Limestone & 22 & General & Deposh & Surface & Occurrence. \\
\hline D & 199 & ElAoula & N 27 $37^{\circ}$ & E $30^{\circ} 40^{\circ}$ & Limestone & 22 & General & Depos" & Surface & Occurrence. \\
\hline D & 200 & Benl Korra & $N 27^{\circ} 25^{\prime}$ & E $30^{\circ} 56^{\circ}$ & Limestone & 22 & General & Deposin & Surface & Occurrence. \\
\hline D & 201 & Khashaba & $N 27^{\circ} 18^{\circ}$ & E $30^{\circ} \mathbf{4 2}$ & Llmestone & 22 & General & Deposil & Surface & Occurrence. \\
\hline D & 202 & Benl Adl & $N 27^{\circ} 12$ & E $30^{\circ} 53^{\prime}$ & Llmestone & 22 & General & Deposin & Surface & Occurrence. \\
\hline
\end{tabular}


APPENDIX C: PROSPECTS AND NON PRODUCINO MINERAL PROPERTIES IN EGYPT.

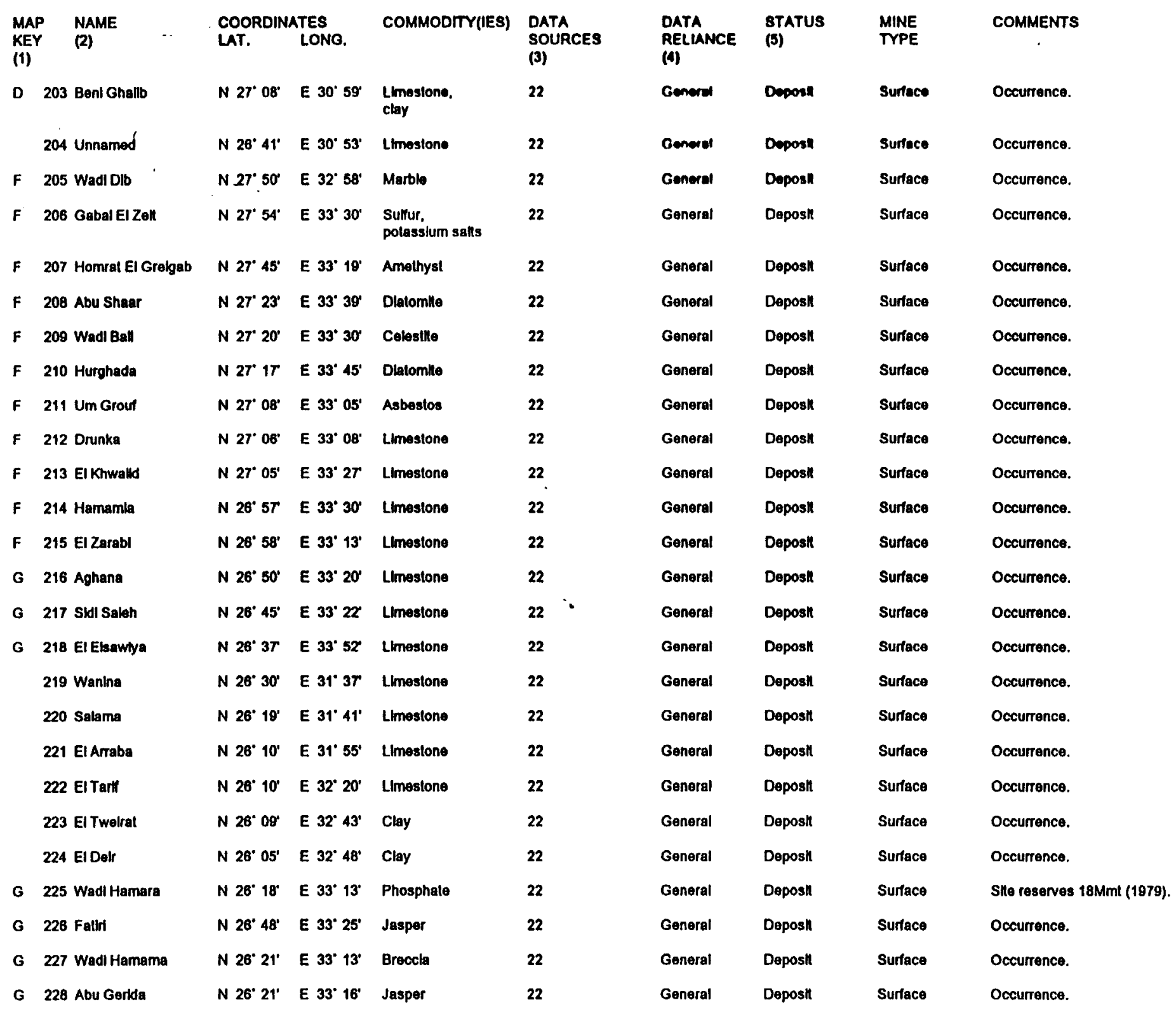


APPENDIX C: PROSPECTB ANO NON PRODUCING MINERAL PROPERTIES IN EOYPT.

\begin{tabular}{|c|c|c|c|c|c|c|c|c|c|}
\hline $\begin{array}{l}\text { MAP } \\
\text { KEY } \\
\text { (1) }\end{array}$ & $\begin{array}{l}\text { NAME } \\
\text { (2) }\end{array}$ & $\begin{array}{l}\text { COOROIN } \\
\text { LAT. }\end{array}$ & $\begin{array}{l}\text { ATEB } \\
\text { LONG. }\end{array}$ & COMMODITY(IE8) & $\begin{array}{l}\text { DATA } \\
\text { BOURCES } \\
\text { (3) }\end{array}$ & $\begin{array}{l}\text { DATA } \\
\text { RELIANCE } \\
\text { (4) }\end{array}$ & $\begin{array}{l}\text { STATUS } \\
\text { (5) }\end{array}$ & $\begin{array}{l}\text { MINE } \\
\text { TYPE }\end{array}$ & COMMENTS \\
\hline G & 229 Sodmeln & N 28. $09^{\prime}$ & $E 3^{\circ} 40^{\prime}$ & Asbestos & 22 & Gonoral & Deposth & Surfaco & Occurrence. \\
\hline $\mathbf{G}$ & 230 Um Esh EI Zarga & N 26. $09^{\circ}$ & $E 33^{\circ} 35^{\prime}$ & Fluorspar & 22 & General & Deposh & Surface & Occurrence. \\
\hline o & 231 Wadi Alallah & N 26. $10^{\prime}$ & E $33^{\circ} 30^{\circ}$ & Serpenline & 22 & General & Deposit & Surtace & Occurrence. \\
\hline G & 232 Fawakhlir & N $28^{\circ} 02^{\prime}$ & E $33^{\circ} 30^{\circ}$ & Fluorepat & 22 & General & Depostt & Surface & Occurrence. \\
\hline \multirow[t]{2}{*}{ G } & 233 Fawakhlr & N 26. $02^{\prime}$ & E $33^{\circ} 43^{\prime}$ & Tale & 22 & General & Deposit & Surface & Occurrence. \\
\hline & 234 Unnamod & $N 26^{\circ} 00^{\circ}$ & E $34^{\circ} 18^{\prime}$ & Gypsum & 22 & General & Deposin & Surface & Occurrence. \\
\hline \multirow[t]{4}{*}{ G } & 235 Harmadal & N 26. $04^{\prime}$ & E $34^{\circ} 02^{\prime}$ & Talc & 22 & General & Deposh & Surface & Occunence. \\
\hline & 236 Baris oasls & $N 24^{\circ} 42^{\prime}$ & E 30 $30^{\circ} \mathbf{3 3 ^ { \prime }}$ & Clay & 22 & General & Deposł & Surface & Occurrence. \\
\hline & 237 Abu EI Nur & $N 25^{\circ} 56^{\circ}$ & $E 32^{\circ} 05^{\circ}$ & Limeslone & 22 & Genora! & Deposit & Surface & Occurronce. \\
\hline & 238 Gabal Garns & N 25. 59' & E $32^{\circ} 45^{\circ}$ & Llmostone & 22 & Genoral & Doposit & Surface & Occurrenco. \\
\hline o & 239 Abu Fannanl & N $25^{\circ} 58^{\prime}$ & $E 3^{\circ} 45^{\prime}$ & Talc & 22 & General & Deposit & Surface & Occurrence. \\
\hline \multirow[t]{4}{*}{$\mathbf{G}$} & 240 Harmmuds & $N 25^{\circ} 56^{\circ}$ & E $33^{\circ} 48^{\prime}$ & Tak & 22 & General & Deposin & Surface & Occurrence. \\
\hline & 241 Abu Ankhor & $N 25^{\circ} 26^{\prime}$ & E $33^{\circ} 56^{\prime}$ & Foldspar & 22 & General & Deposil & Surface & Occurrence. \\
\hline & 242 Bir Minoih & N $25^{\circ} 33^{\circ}$ & E 33' 51' & $\begin{array}{l}\text { Magnestle, } \\
\text { fuorspar }\end{array}$ & 22 & Goneral & Deposil & Surface & Occurrence. \\
\hline & 243 Mashash & $N 25^{\circ} 34^{\prime}$ & E $33^{\circ} 19^{\prime}$ & Phosphate & 22 & General & Deposit & Surface & Resorves estimaled al 360Mmi (1979). \\
\hline I & 244 Hagazah & N 25 30" & $E 32^{\circ} 42^{\prime}$ & Limestone & 22 & Goneral & Deposin & surface & Occurrence. \\
\hline ' & 245 El Ghantra & $N 25^{\circ} 29^{\circ}$ & E $32^{\circ} 30^{\prime}$ & Clay & 22 & Gonoral & Deposit & Surface & Occurrence. \\
\hline 1 & 246 El Mataana & N 25' $28^{\prime}$ & $E 32^{\circ} 46^{\circ}$ & Limestone & 22 & Gonoral & Deposit & Surface & Occurtence. \\
\hline I & 247 Hemoldal & $N 25^{\circ} 24^{\prime}$ & E $32^{\circ} 44^{\prime}$ & Limestone & 22 & Goneral & Deposin & Surface & Occurrence. \\
\hline 1 & 248 El Sharawna & $N 25^{\circ} 17^{\prime}$ & E $32^{\circ} 48^{\prime}$ & Limestone & 22 & Goneral & Deposh & Surface & Estimaled reserves 35Mmt (1979). \\
\hline \multirow[t]{8}{*}{1} & 249 El Shemakhla & N 25" $09^{\prime}$ & E $32^{\circ} 44^{\circ}$ & Sandstone & 22 & General & Depostt & Surface & Occurrence. \\
\hline & 250 Batur & $N 25^{\circ} 17^{\prime}$ & E $3^{\circ} \mathbf{1 3}^{\prime}$ & Phosphato & 2,22 & General & Deposit & Surface & Occurrence. \\
\hline & 251 El Bakriya & $N 25^{\circ} 17^{\circ}$ & $E 3^{\circ} 35^{\prime}$ & $\begin{array}{l}\text { Feldspar, } \\
\text { nuorepar }\end{array}$ & 22 & Genera: & Deposit & Surlace & Oecurrence. \\
\hline & 252 Wadi El Himeri & $N 25^{\circ} 17^{\circ}$ & E $33^{\circ} 57^{\prime}$ & Graphilo & 22 & Goneral & Deposh & Surface & Occurnence. \\
\hline & 253 Sowoigat El Zarga & N 25' $09^{\prime}$ & E $33^{\circ}$ 52' & Foldspar & 22 & General & Deposit & Surface & Occurtence. \\
\hline & 254 Barramiya & $N 25^{\circ} 05^{\circ}$ & E $33^{\circ} 50^{\circ}$ & Asbeslos & 22 & General & Deposit & Surface & Occurrence. \\
\hline & 255 Um Sewelgat & $N 25^{\circ} 02^{\circ}$ & E $33^{\circ} 51^{\circ}$ & Talc & 22 & General & Deposh & Surface & Occurrence. \\
\hline & 256 Um Higlig & N $25^{\circ} 01^{\prime}$ & E 33' 58' & Quark & 22,30 & General & Deposit & Surface & $\begin{array}{l}\text { Occurrence. } \\
\text { Large slock Intruding volcanles. }\end{array}$ \\
\hline
\end{tabular}


APPENDIX C: PROSPECTS AND NON PRODUCING MINERAL PROPERTIES IN EOYPT.

\begin{tabular}{|c|c|c|c|c|c|c|c|c|c|}
\hline $\begin{array}{l}\text { MAP } \\
\text { KEY } \\
\text { (1) }\end{array}$ & $\begin{array}{l}\text { NAME } \\
\text { (2) }\end{array}$ & $\begin{array}{l}\text { COORDINA } \\
\text { LAT. }\end{array}$ & $\begin{array}{l}\text { ATES } \\
\text { LONG. }\end{array}$ & COMMODTY(IES) & \multirow{2}{*}{$\begin{array}{l}\text { DATA } \\
\text { SOURCES } \\
\text { (3) } \\
22\end{array}$} & \multirow{2}{*}{$\begin{array}{l}\text { DATA } \\
\text { RELIANCE } \\
\text { (4) } \\
\text { General }\end{array}$} & \multirow{2}{*}{$\begin{array}{l}\text { STATUS } \\
\text { (5) } \\
\text { Deposk }\end{array}$} & \multirow{2}{*}{$\begin{array}{l}\text { MINE } \\
\text { TYPE } \\
\text { Surface }\end{array}$} & $\begin{array}{c}\text { COMMENTS } \\
.\end{array}$ \\
\hline & 257 Um Dalalit & $N 24^{\circ} 58^{\prime}$ & E $33^{\circ} \mathbf{5 8}^{\circ}$ & Talc & & & & & Occurrence. \\
\hline & 259 Wadl Khark & N 24' 22 & E 33" 44' & Talc & 22 & General & Deposk & Surface & Occurrence. \\
\hline J & 260 Rughama El Beld & N 24. $35^{\circ}$ & E 33* 02 & Lmestone & 22 & General & Deposk & Surface & Occurrence. \\
\hline J & 262 Wadl El LaW & N $24^{\circ} 21^{\prime}$ & $E 3^{\circ} 15^{\prime}$ & Llmestone & 22 & General & Deposit & Surface & Occurrence. \\
\hline J & 263 El Aaqab & N 24. 18' & E $33^{\circ} 00^{\circ}$ & Sandstone & 22 & General & Deposh & Surface & Occurrence. \\
\hline J & 264 El Aqaba & N $24^{\circ} 15^{\prime}$ & E $33^{\circ} 00^{\circ}$ & Clay & 22 & General & Deposk & Surface & Occurrence. \\
\hline J & 265 Abu El Relsh & $N 24^{\circ} 09^{\prime}$ & E $32^{\circ} 58^{\circ}$ & Clay & 22 & General & Deposit & Surface & Occurrence. \\
\hline J & 269 Unnamed & N 24. $01^{\circ}$ & E $33^{\circ} 10^{\circ}$ & Barlite & 22 & General & Deposk & Surface & Occurrence. \\
\hline & 270 Wadl Essal & N 25' 56' & E $\mathbf{3 4} 4^{\circ} \mathbf{3 0}$ & Celesthe & 22 & General & Deposil & Surface & Estilnaled reserves 2.3Mml (1979). \\
\hline & 271 Wadl Sharm & N 25. 52 & E $34^{\circ} 28^{\prime}$ & $\begin{array}{l}\text { Asbeslos, } \\
\text { lalc }\end{array}$ & 22 & General & Deposil & Surface & Occurrence. \\
\hline & 272 Gabal Abu Tuyur & N 25. 45' & E $34^{\circ} 25^{\prime}$ & Asbestos & 22 & General & Deposil & Surface & Occurrence. \\
\hline & 273 Um Grolg & $N 25^{\circ} 4 r$ & E $\mathbf{3}^{*} \mathbf{2 6}$ & Celestte & 22 & General & Deposk & Surface & Occurrence. \\
\hline & 274 Abu Ghorban & N $25^{\circ} 45^{\circ}$ & E 34' 29' & Colestlte & 22 & General & Deposhl & Surface & Estmatod reserves 5.6Mmt (1979). \\
\hline & 275 Kadabora Hamra & N $25^{\circ} 32$ & E $34^{\circ} 26^{\prime}$ & Asbestos & 22 & General & Deposk & Surface & Occurrence. \\
\hline & 276 Um Naggat & N 25. $30^{\circ}$ & E $34^{\circ} 11^{\circ}$ & Fluorspar & 22 & General & Depost & Surface & Occurrence. \\
\hline & 277 Um Diwan & N 25. $30^{\circ}$ & E $34^{\circ} 06^{\circ}$ & Talc & 22 & General & Deposn & Surtace & Occurrence. \\
\hline & 278 Rod Ashab & N 25'09' & E $34^{\circ} 05^{\circ}$ & Foldspar & 22 & General & Deposk & Surface & Occurrence. \\
\hline & 279 Gabal El Maylet & N 25* $18^{\prime}$ & E $34^{\circ} 19^{\circ}$ & Magneshe & 22 & Generat & Deposk & Surface & Occurrence. \\
\hline & 280 Nekhella & N 25" 15' & E $34^{\circ} 32^{\prime}$ & Talc & 22 & General & Deposk & Surface & Occurrence. \\
\hline & 281 Homr Waggad & N $25^{\circ} 11^{\prime}$ & E $34^{\circ} 20^{\prime}$ & Fluorspar & 22 & General & Deposil & Surface & Occurrence. \\
\hline & 282 Rod Um El Farag & N 25. $06^{\circ}$ & E 34. 19. & Muscovlie & 22 & General & Deposn & Surface & Occurrence. \\
\hline & 283 El Tallaa & N 25' 08' & $E 34^{\circ} 15^{\circ}$ & Feldspar & 22 & General & Deposil & Surtace & Occurrence. \\
\hline
\end{tabular}


APPENDIX C: PROSPECTS AND NON PRODUCING MINERAL PROPERTIES IN EGYPT.

\begin{tabular}{|c|c|c|c|c|c|c|c|c|c|}
\hline \multirow{2}{*}{$\begin{array}{l}\text { MAP } \\
\text { KEY } \\
\text { (1) }\end{array}$} & \multirow{2}{*}{$\begin{array}{l}\text { NAME } \\
\text { (2) } \\
284 \text { Rod El Buram }\end{array}$} & \multicolumn{2}{|c|}{$\begin{array}{l}\text { COORDINATES } \\
\text { LAT. LONG. }\end{array}$} & \multirow{2}{*}{$\begin{array}{l}\text { COMMODITY(IES) } \\
\text { Feldspar }\end{array}$} & \multirow{2}{*}{$\begin{array}{l}\text { DATA } \\
\text { SOURCES } \\
\text { (3) } \\
22\end{array}$} & \multirow{2}{*}{$\begin{array}{l}\text { DATA } \\
\text { RELIANCE } \\
\text { (4) } \\
\text { Genoral }\end{array}$} & \multirow{2}{*}{$\begin{array}{l}\text { STATUS } \\
\text { (5) } \\
\text { Deposn }\end{array}$} & \multirow{2}{*}{$\begin{array}{l}\text { MINE } \\
\text { TYPE } \\
\text { Surface }\end{array}$} & \multirow{2}{*}{$\begin{array}{l}\text { COMMENTS } \\
\text { Occurrence. }\end{array}$} \\
\hline & & $N 25^{\circ} 03^{\prime}$ & E $34^{\prime} 04^{\prime}$ & & & & & & \\
\hline & 285 Um Dalalh, & N $24^{\circ} 55^{\prime}$ & E $34^{\circ} 0 Z$ & Fluorspaf & 22 & Gonere & Deposk & Surface & Occurrence. \\
\hline & 286 Gabal Ur El Fand & $N 24^{\circ} 51^{\prime}$ & E $34^{*} 09^{\circ}$ & Foldspar & 22 & Goneret & Deponell & Surfece & Occurrence. \\
\hline & 287 Wadl Um Tundub & $N 24^{\circ} 58^{\prime}$ & E $34^{*} 20^{\prime}$ & Asbestos & 22 & Generel & Deposil & Surfece & Occurrence. \\
\hline & 288 Wadl Um Kharkga & N 25. 02 & E $34^{\circ} 40^{\circ}$ & Bartlo & 22 & Gonorel & Deposil & Surfece & Occurrence. \\
\hline & 289 Nugrus & $N 24^{\circ} 59^{\prime}$ & E $34^{*} 32$ & Fhuorspar & 22 & General & Deposn & Surface & Occurrence. \\
\hline & 290 Wedl Hormral Mastura & $\mathrm{N} 24^{\circ} 51^{\prime}$ & E $34^{\circ} 19^{\circ}$ & Talc & 22 & General & Deposh & Surface & Occurrence. \\
\hline & 291 Wadi Nalesh & N $24^{\circ} 38^{\prime}$ & $E 3^{*} 12$ & Bartite & 22 & General & Deposh & Surface & Occurrence. \\
\hline & 292 Darb El Shugelra & $N 24^{\circ} 40^{\circ}$ & E $34^{*} 25$ & Conundum & 22 & General & Deposh & Surface & Occurrence. \\
\hline & 293 Gabal Mlgl & N 24. $48^{\prime}$ & E $\mathbf{3 1}^{\circ} \mathbf{2 8 ^ { \prime }}$ & Foldspar & 22 & General & Deposk & Surface & Occurrence. \\
\hline & 294 Wadl El Duwolg & $N 24^{\circ} 49^{\circ}$ & E 34' 24' & Asbestos & 22 & General & Deposh & Surface & Occurrence. \\
\hline & 295 Wadl Arak & $N 24^{\circ} 43^{\circ}$ & E 34' 32 & Conundum & 22 & General & Deposin & Surface & Occurrence. \\
\hline & 296 Wadl Hengallya & $\mathrm{N} 24^{\circ} 50^{\circ}$ & E $34^{\prime} 36^{\prime}$ & Asbestos & 22 & General & Deposk & Surtace & Occurrence. \\
\hline & 297 Ambaout & N $24^{\circ} 53^{\circ}$ & $E 34^{\circ} 45^{\circ}$ & Magnesilo & 22 & General & Deposit & Surface & Occurrence. \\
\hline & 298 Gabal Ghadlr & N $24^{\circ} 50^{\circ}$ & E $3^{\circ} 4^{\prime}$ & Graphito & 22 & General & Deposit & Surface & Occurrence. \\
\hline & 299 Gabal Um Seyal & $N 24^{\circ} 36^{\prime}$ & $E 3^{\circ} 58^{*}$ & Asbestos & 22 & General & Deposk & Surface & Occurrence. \\
\hline & 300 Wadl Erolr & N $24^{\circ} 31^{\prime}$ & E $35^{\circ} 02$ & Sulfur & 22 & General & Deposh & Surface & Occurrence. \\
\hline & 301 Abu Ghusun & N 24. $30^{\prime}$ & E $35^{\circ} 09^{\prime}$ & Gypsum & 22 & General & Deposh & Surface & Occurrence. \\
\hline & 302 Wadl Um Ghazal & $N 24^{\circ} 11^{*}$ & E $35^{\circ} 20^{\circ}$ & Bartle & 22 & General & Deposh & Surface & Occurrence. \\
\hline K & 303 Wadl Antar & $N 24^{\circ} 21^{\circ}$ & E $34^{\circ} 19^{\circ}$ & Bartle & 22 & General & Deposh & Surface & Occurrence. \\
\hline K & 304 Gabal El Kahla & $N 24^{\circ} 09^{\prime}$ & E $34^{*} 3 r$ & Nepheline syentle & 22 & General & Deposh & Surface & Occurrence. \\
\hline & 305 Gabal Sharshar & N 23. 55' & E $30^{\circ} 21^{\circ}$ & $\begin{array}{l}\text { Clay, } \\
\text { Hinestono }\end{array}$ & 22 & General & Deposk & Surface & Occurrence. \\
\hline $\mathbf{J}$ & 306 Um Tehalwal & N 23. $58^{\prime}$ & $E 33^{\circ} 00^{\circ}$ & Quartz & 22 & General & Deposh & Surface & Occurrence. \\
\hline & 307 Kertas & N 23. $45^{\circ}$ & E $32^{\circ} 58^{\circ}$ & Sandstone & 22 & Oeneral & Deposil & Surface & Occurrence. \\
\hline & 308 Br Um Hobal & $N 23^{\circ} 40^{\circ}$ & E $33^{\circ} 14^{\circ}$ & Marble & 22 & General & Depos\| & Surface & Occurrence. \\
\hline & 309 Gara El Soda & N $23^{\circ} 22$ & $E 3^{\circ} 18^{\circ}$ & Bartio & 22 & General & Deposh & Surface & Occurrence. \\
\hline $\mathbf{N}$ & 310 Gabal Um Araqa & N 23. $00^{\circ}$ & E $33^{\circ} 2 T$ & Tale & 22 & General & Deposil & Surface & Occurrence. \\
\hline $\mathbf{N}$ & 311 Abu Swayell & N 22 $50^{\circ}$ & E $33^{\circ} 40^{\prime}$ & Marble & 22 & General & Deposin & Surface & Occurrence. \\
\hline
\end{tabular}


APPENDIX C: PROSPECTS AND NON PRODUCING MINERAL PROPERTIES IN EOYPT.

\begin{tabular}{|c|c|c|c|c|c|c|c|c|c|}
\hline $\begin{array}{l}\text { MAP } \\
\text { KEY } \\
\text { (1) }\end{array}$ & $\begin{array}{l}\text { NAME } \\
\text { (2) }\end{array}$ & $\begin{array}{l}\text { COORDINA } \\
\text { LAT. }\end{array}$ & $\begin{array}{l}\text { ITES } \\
\text { LONO. }\end{array}$ & COMMODTYY(IES) & $\begin{array}{l}\text { DATA } \\
\text { SOURCES } \\
\text { (3) }\end{array}$ & $\begin{array}{l}\text { DATA } \\
\text { RELIANCE } \\
\text { (4) }\end{array}$ & $\begin{array}{l}\text { STATUS } \\
\text { (5) }\end{array}$ & $\begin{array}{l}\text { MINE } \\
\text { TYPE }\end{array}$ & COMMENTS \\
\hline N & 312 Halmur & N 22 $48^{\circ}$ & E 33' 38' & Talc & 22 & General & Deposin & Surtace & Occurrence. \\
\hline $\mathbf{N}$ & 313 Halmur & N 22' 42 & E $33^{\circ} 40^{\circ}$ & Graphte & 22 & General & Deposit & Surface & Occurrence. \\
\hline \multirow[t]{10}{*}{$\mathbf{N}$} & 314 Wadl Klelb. & N $22^{\circ} 46^{\prime}$ & E $33^{\circ} \mathbf{2 0}$ & Talc & 22 & General & Deposin & Surface & Occurrence. \\
\hline & 315 Abu Gurdl & N 23. $53^{\prime}$ & E 35'00' & Talc & 22 & General & Deposn & Surface & Occurrence. \\
\hline & 316 Wadl Bhan & N 23. $45^{\prime}$ & E $34^{\circ} 56^{\prime}$ & Asbestos & 22 & General & Deposin & Surface & Occurrence. \\
\hline & 317 Zargat Naam & $N 23^{\prime} 45^{\prime}$ & $E 34^{\circ} 36^{\circ}$ & Magneshe & 22 & General & Deposh & Surtace & Occurrence. \\
\hline & 318 Um Karaba & N 23' $39^{\circ}$ & E $35^{\circ} 04^{\circ}$ & Talc & 22 & General & Deposil & Surface & Occurrence. \\
\hline & 319 Abu Dahr & N 23. 35' & E $35^{\circ} 06^{\circ}$ & $\begin{array}{l}\text { Asboslos } \\
\text { Thanlum }\end{array}$ & $\mathbf{2 2 , 3 0}$ & General & Deposn & Surface & Occurrence. \\
\hline & 320 El Rohaba & N 23. $18^{\prime}$ & E $3518^{\prime}$ & Tak & 22 & General & Weposin & Sunger & Occurrence. \\
\hline & 321 Gabal Nugrub & N 220 $51^{\circ}$ & E $3^{*} 56^{*}$ & Nephelline syentite & 22 & General & Deposk & Surface & Occurrence. \\
\hline & 322 Gabal Mlshbl & N 22, $44^{\prime}$ & $E 34^{\circ} 41^{\prime}$ & Nephelline syentle & 22 & General & Deposin & Surface & Occurrence. \\
\hline & 323 Gabal EI Naga & N 22' 43' & E 3' $2 T$ & Nephelline syentite & 22 & General & Deposh & Surface & Occurrence. \\
\hline 0 & 324 Wadl El Dirdira & N 220 30 & E $35^{*} 59^{\circ}$ & Bartho & 22 & General & Deposil & Surface & Occurrence. \\
\hline 0 & $\begin{array}{l}325 \text { Girara } \\
\text { Halalb } \\
326 \text { Wadl Elkwan }\end{array}$ & $\begin{array}{l}\text { N 22: } 2 r \\
\text { N } 22^{\circ} 23^{\prime}\end{array}$ & $\begin{array}{l}\text { E } 35^{*} 58^{\prime} \\
\text { E } 36^{\circ} 10^{\circ}\end{array}$ & $\begin{array}{l}\text { Bartle } \\
\text { Magneslle }\end{array}$ & $\begin{array}{l}22,30 \\
22\end{array}$ & $\begin{array}{l}\text { General } \\
\text { General }\end{array}$ & $\begin{array}{l}\text { Deposin } \\
\text { Deposin }\end{array}$ & $\begin{array}{l}\text { Surface } \\
\text { Surface }\end{array}$ & $\begin{array}{l}16 \text { bartle velns in serpenthes and sandstones. } \\
\text { Occurrence. }\end{array}$ \\
\hline 0 & 327 Sol Hamld & N 22' $20^{\prime}$ & E $36^{*} 10^{\circ}$ & Asbestos & 22 & General & Deposit & Surface & Occurrence. \\
\hline \multirow[t]{3}{*}{0} & 328 Wadl Dllab & N 22 $19^{\prime}$ & E $36^{\circ} 26^{\circ}$ & Bartho & 22 & General & Deposit & Surface & Occurrence. \\
\hline & 329 Gabel El Hagr & N $30^{\circ} 32^{\circ}$ & E $29^{\circ} 26^{\circ}$ & Gypsum & 16 & General & Deposin & Surface & Occurrence. \\
\hline & 330 El Alameln & N $30^{\circ} 50^{\circ}$ & E $28^{\circ} 55^{\circ}$ & Gypsum & 16 & General & Depostt & Surface & Occurrence. \\
\hline \multirow[t]{2}{*}{ A } & 331 Maadl Kalamla & $N 30^{\circ} 00^{\circ}$ & E 31' or & Gypsum & 16 & General & Deposin & Surface & Occurrence. \\
\hline & 332 Wadl Um Gerflat & N $24^{\circ} 52$ & E $34^{*} 22$ & Iron $^{\circ}$ & 16 & General & Deposit & Surface & Occurrence. \\
\hline \multirow[t]{4}{*}{ G } & 333 DJebel Alda & $N 26^{\circ} 40^{\circ}$ & E $33^{\circ} 00^{\circ}$ & Manganese & 16 & General & Deposil & Surface & Occurrence. \\
\hline & 334 Sherm El Shelkh & $N 27^{\circ} 45^{\circ}$ & E 34. 20 & Manganese & 16 & Goneral & Deposin & Surface & Occurrence. \\
\hline & 335 Agroud & $N 30^{\circ} 05^{\prime}$ & E $32^{\circ} 25^{\prime}$ & Clay & 10 & General & Deposil & Surface & Occurtence. \\
\hline & $\begin{array}{l}\text { (1) Represents pr } \\
\text { (2) Due lo sortwa } \\
\text { (3) Complete tst } \\
\text { (4) General - den } \\
\text { (5) Because of th }\end{array}$ & yling age of & source infon & $\begin{array}{l}\text { defined in Appe } \\
\text { ol helude any die } \\
\text { ndlu D. } \\
\text { denoles deposh } \\
\text { catton, the status }\end{array}$ & $\begin{array}{l}\text { aton connor } \\
\text { vldual skes }\end{array}$ & $\begin{array}{l}\text { eral sourc } \\
\text { current. }\end{array}$ & & rably & \\
\hline
\end{tabular}




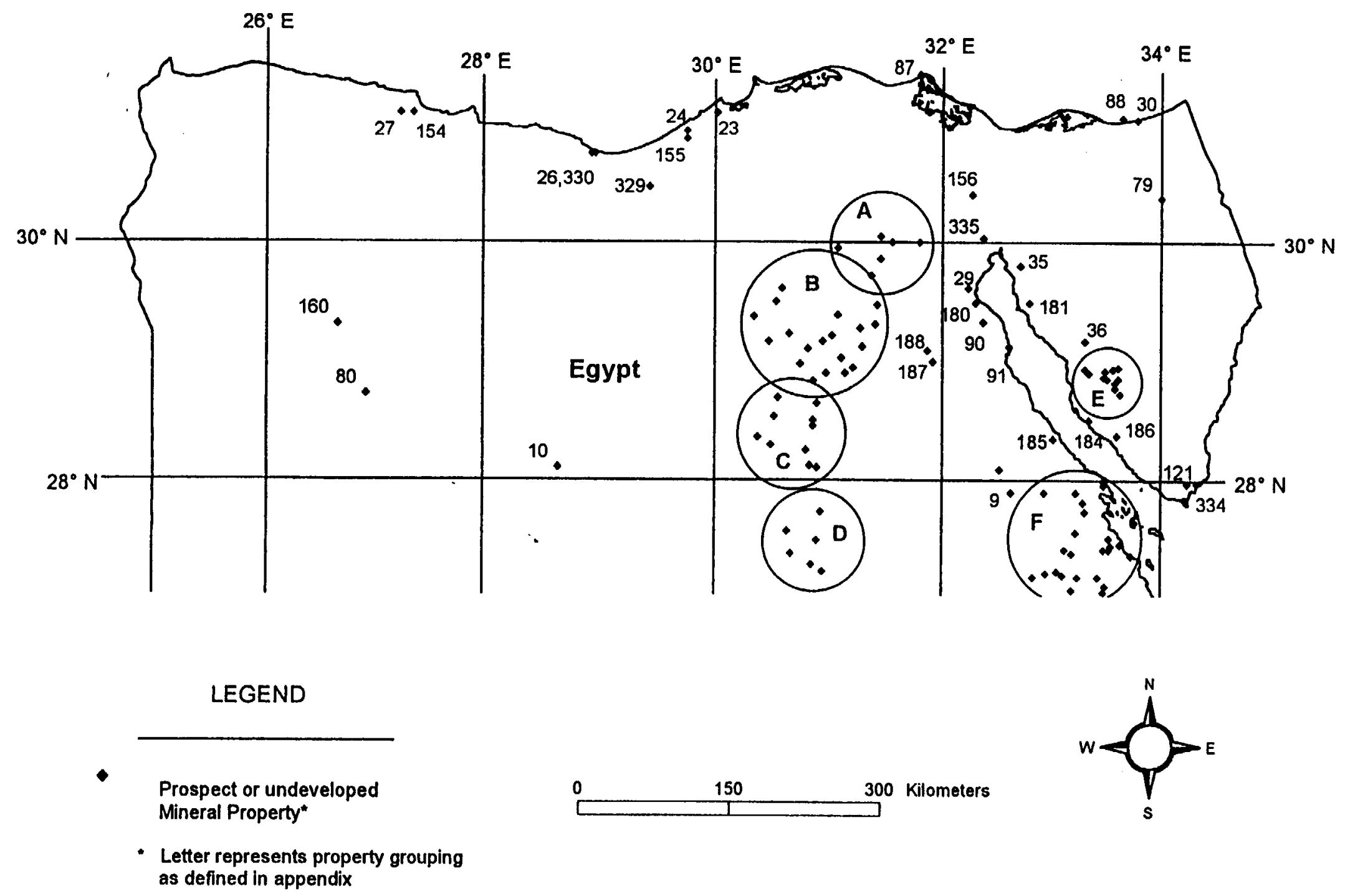

APPENDIX MAP A-4: PROSPECTS AND UNDEVELOPED MINERAL PROPERTIES OF NORTHERN EGYPT. 


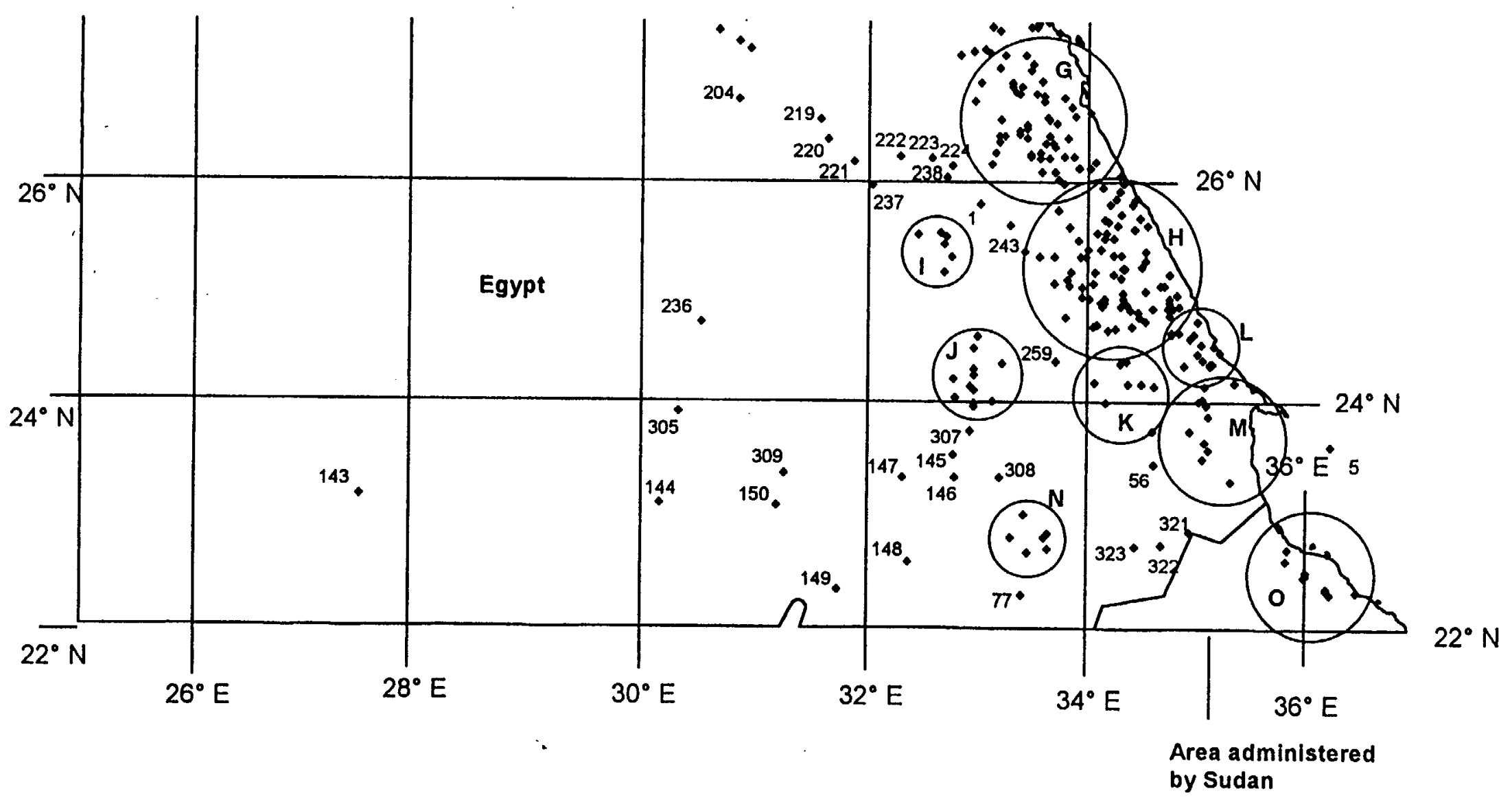

LEGEND

Prospect or undeveloped

0

150

300 Kilometers

Mineral Property
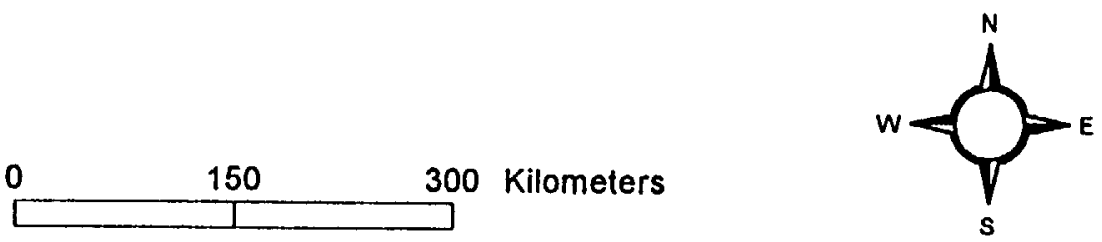

- Letter represents property grouping as defined in appendix 
APPENDIX D: PUBLIC SOURCES OF INFORMATION

1. Report on the Mineral Industry of Egypt. Egyptian Ministry of Finance. Cairo, 1922, $50 \mathrm{pp}$.

2. Potential Mineral Resources of Egypt. Egyplian Geological Survey and Mining Authority. 1984, 144 pp.

3. Gold in Egypt - A Commodity Package. Ed. by S. Z. Gabra, Egyptian Geological Survey \& Mining Authority. USAID Project 263-0105, 1986. 86

4. R. Said. The Geology of Egypt. A.A. Balkerna (Rotterdam). 1990, pp. 511-566.

5. British Sulphur, Ltd. World Survey of Phosphate Deposits. Sth Ed., 1987, pp. 161-162.

6. Roskill Publication Services. Inc. Iron. 1988.

7. Cambridge Information and Research Services Limited. World Directory of Energy Information. Vol. 2. 1994. Selected country profiles.

8. Europa Publications Limited. The Middle East and North Africa 1994. 40th Ed. pp. 411-421.

9. U. S. Library of Congress. Egypt, A Country Study. Dec. 1990, pp. 195-209.

10. U. S. Energy information Administration. The Petroleum Resources of the Middle East. Mar. 1984, 137 pp.

11. Arab Mining Joumal. Apr. 1981, vol. 1. No. 3, pp. 21, 47.

12 U. S. Board on Geographic Names. Gazetteer No. 45 - Egypl and the Gaza Strip, 1959.

13. British Sulphur, Lid. World Survey of Sulphur Resources. 3rd Ed., 1985, pp. 156-57.

14. Annual Mining Review 1995. Mining Joumal, Nov. 1995. pp. 151-152.

15. Library of Congress. Egypt, a country study. 1990, pp. 197-209.

16. U. S. Geological Survey. Mineral Resources Data System (MRDS), 1996.

17. U. S. Bureau of Mines. Minerals Availability Data Base, June 1995.

18. U. S. Bureau of Mines. The Mineral Industry of Egypt. T. P. Doliey. Ch. in Minerals Yearbook 1993, pp. 41-45.

19. U. S. Bureau of Mines. Egypt. T. P. Dolley. Mineral Industry Surveys series, ‘1994, 8 pp.

20. Hussein. H.A. T.A. Sayyah and H.M. Shatoury. Metallogeny of Uranium and Thorium Occurrences in Egypt 1989.

21. World Cement Diroctory 1991. Cimourope s.a.r.l., pp. 180-183.

22. Mineral Map of Egypt. Egyptian Geological Survey and Mining Authority. 1979. 44 pp.

23. Roskill Publication Services, Inc. Copper. 1992.

24. Roskill Publication Services, Inc. Gold. 1991.

25. Roskill Publication Services, Inc. Chromium. 1991.

26. Industrial Minerats Directory. Metals Bulletin. ist Ed. 1987.

27. Metal Bulletin Monthly June, 1992.

28. A.M.A. Wali. Industrial Minerals of Egypt. Paper 90-77 presented at SME annual meeting. Salt Lake City, 1990.

29. S. Omara. Phosphatic Deposits in Syria and Salaga District, Egypt. In Economic Geology. Vol. 60, 1965, pp. $214-227$.

30. F. Habeshi and F.A. Bassyouni. Mineral Resources of the Arab Countries. Chemecon Pub. (Quebec). 1982. pp. $18-27$.

31. B. A. Komhauser. Trip Report-Field Evaluation of the Mineral Industries of Egypt \& israel. Sept. 2-22, 1985. 
Jordan 
Table of Contents

Page

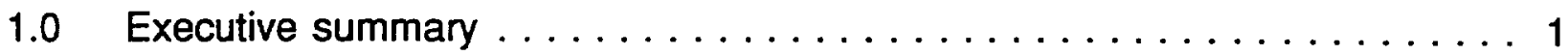

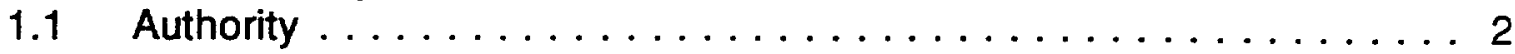

1.2 Project scope $\ldots \ldots \ldots \ldots \ldots \ldots \ldots \ldots \ldots \ldots \ldots \ldots \ldots \ldots$

2.0 Sources of information $\ldots \ldots \ldots \ldots \ldots \ldots \ldots \ldots \ldots \ldots \ldots$

3.0 The mining industry of Jordan $\ldots \ldots \ldots \ldots \ldots \ldots \ldots \ldots \ldots$

3.1 Industrial minerals $\ldots \ldots \ldots \ldots \ldots \ldots \ldots \ldots \ldots \ldots \ldots$

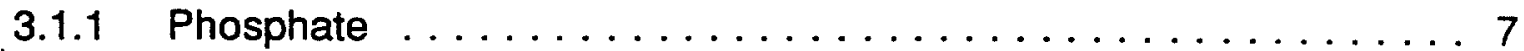

3.1.2 Potash . . . . . . . .

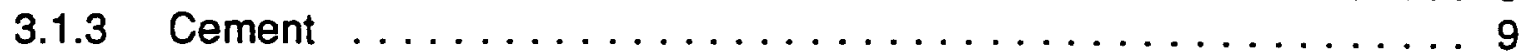

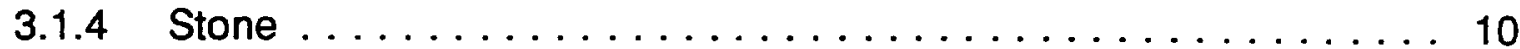

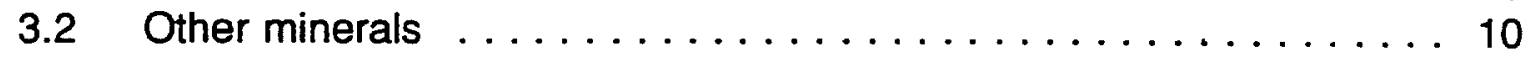

4.0 Mine-related explosives use $\ldots \ldots \ldots \ldots \ldots \ldots \ldots \ldots \ldots \ldots \ldots$

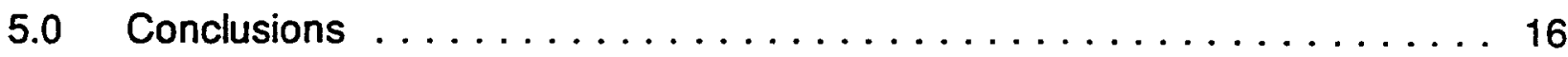

Appendix A: Producing and developing mineral properties in Jordan ... . . . 18 Appendix B: Past producers, prospects and undeveloped mineral properties

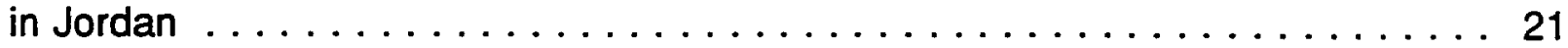

Appendix C: Public sources of information ................. 24

\section{TABLES}

3.1 Estimated mineral production in Jordan, 1993 and $1994 \ldots \ldots \ldots \ldots 6$

4.1 Estimated explosives usage at the main Jordanian mines used in this study in order of estimated ANFO consumption . . . . . . . . . 14

\section{FIGURES}

4.1 Selected Jordanian mines and estimated blasting events . . . . . . . 15

A-1: Producing mineral properties of Jordan . . . . . . . . . . . . 20

B-1: Non-producing mineral properties of Jordan $\ldots \ldots \ldots \ldots \ldots \ldots$ 



\subsection{EXECUTIVE SUMMARY}

This report was prepared under Memorandum of Agreement B291867 between Lawrence Livermore National Laboratory (LLNL) and the U.S. Geological Survey, Minerals Availability Team (MAT). It provides LLNL information on the mining industry of Jordan, and quantitative information on the blasting potential of its mining industry. MAT identified mining activities through the use of the Minerals Availability data base, its data collection and analytic capabilities, and an extensive network of information sources.

Much of Jordan's known mineral potential is associated with the Wadi al Arabah-Jordan rift zone in western Jordan. The Dead Sea, centrally located along this rift, is the source for much of Jordan's mineral salt wealth. Jordan is known for its industrial minerals, primarily phosphate and potash. Only two mines, however, have an estimated maximum blasting event larger than $100 \mathrm{mt}$ ANFO equivalent. One of these mines is nearing the end of its life, however. Research activities for this study resulted in the identification of $\mathbf{5 2}$ significant mineral properties. Most properties operate on a small scale and require minimal blasting. The blasting potential for 6 properties is reported. 


\subsection{Authority}

This report was prepared under Memorandum of Agreement B291867 between Lawrence Livermore National Laboratory (LLNL) and the U.S. Geological Survey, Minerals Availability Team (MAT).

\subsection{Project Scope}

As part of this agreement, MAT is to identify mining activities in Egypt, Jordan, Syria, Tunisia, and Turkey as they relate to monitoring/verifying compliance of the Comprehensive Test Ban Treaty. MAT is to use the Minerals Availability and the Mineral Resources Data System data bases, its data collection and analytic capabilities, and an extensive network of information sources to provide background information focusing on the use of explosives by the mining industry, which can cause false alarms during monitoring and hide nuclear events.

Reports with accompanying figures and tables summarize location, type of mining method, commodity(ies), estimated frequency and size of mine blasts, operational status, and mine product distribution to foreign or internal markets for the specified countries.

Once country data were collected and verified, the explosive use at selected sites was evaluated. Focus was placed on a limited number of larger operations, or those reported to have potential for short-term development. Undeveloped sites and small scale mines, which may be included in Appendices A-B of this report, were not analyzed in terms of the site's anticipated use of explosives. Mineral prospects generally make only small use of explosives and small mines (some of which are operated on an intermittent basis) are assumed to require minimal blasting.

Based upon known site information (geological conditions, mine technology, production capacity, and current blasting practices), the blasting potential for significant mining sites was evaluated. Where site-specific data were not available, estimates for 
representative, important properties were developed based upon accepted industry practice, knowledge of the Jordanian mining industry, and regional geologic characteristics.

\subsection{SOURCES OF INFORMATION}

Data for this report were derived from published sources, unpublished documents, and personal communications through an extensive network of public and private contacts. Public sources of information are listed in Appendix $\mathrm{C}$. Much of the industry summary was drawn from data reported by the U.S. Bureau of Mines Mineral Yearbook chapter on Jordan, from the years 1992-1994. Information on 1995 was obtained from the U.S. Geological Survey, International Minerals Section, Reston, VA (formerly the U.S. Bureau of Mines, Division of International Minerals).

Principal agencies contacted include, but were not limited to the U.S. Geological Survey, the U.S. State Department, Central Intelligence Agency, Defense Intelligence Agency, the United Nations, the World Bank, World Resource Institute, and International Studies of Minerals Issues (ISMI). In addition, academic and industry contacts, explosives manufacturers and suppliers, and trade groups were contacted.

\subsection{THE MINING INDUSTRY OF JORDAN}

Jordan covers an area of approximately $96,500 \mathrm{~km}^{2}$, of which $60,000 \mathrm{~km}^{2}$ was reportedly unexplored for minerals or fuels in 1988. Much of its known mineral potential is associated with the Wadi al Arabah-Jordan rift zone which extends the length of Jordan from the Mediterranean Sea to the Gulf of Aqaba. The Dead Sea, centrally located along this rift, is the source for much of Jordan's mineral salt wealth.

The minerals sector continues to play an important role in the Jordanian economy. In 1994, Jordan ranked within the top five in global phosphate rock production and was a major world producer of potash. Revenues for Jordanian bulk phosphate and fertilizer 
exports typically account for almost one-third of the nation's total export revenues'. Jordan also is a major regional producer of fertilizer products, limestones and building products, and mineral salts. It also produces significant quantities of cement and kaolin clay which are used in domestic construction. Jordan does not have significant petroleum production. Output of energy minerals was modest, and oil shale and natural gas potential is uncertain.

The Provisional Law of Natural Resources 37 of 1966, amended, is the basic mining law of Jordan. The law allowed for private Jordanian or foreign national ownership of a mine or quarry with the provision that mine management be conducted by a Jordanian operator. The Jordan Natural Resources Authority (NRA) is the Government agency responsible for all activities related to the exploration and development of minerals and mineral fuels.

The manufacturing sector of Jordan has two tiers. On one level are the large-scale, wholly or partially state-owned industrial establishments (parastatals) such as the Jordan Phosphate Mines Company (JPMC), the Jordan Fertilizer Industries Company (JFIC), the Arab Potash Company (APC), and the Jordan Cement Factories Company (JCFC). On the other level are small to medium sized entities, most privately owned, that individually account for insignificant mineral production, but when aggregated, make up a sizeable portion of the minerals industry. Exploitation of the major mineral commodities of Jordan--cement, kaolin, phosphates, potash, and rock wool--are all controlled by parastatals. Building materials such as aggregates, basalt, calcium carbonate, dimensional stone, glass sand, and natural sand is produced by private sector firms.

Since independence, Jordan has imported far more than it has exported, primarily due to a dependence on imported petroleum products. It had not attempted to achieve a trade balance with any major trading partner. The United States and Western Europe supplied about half of Jordan's imports in 1988, but bought less than $10 \%$ of Jordanian

1 U.S. Geological Survey. Jordan. Ch. in Mineral Industry Survey series. 1994. 
exports. Arab imports were minor, but Arab nations purchased nearly half of the country's exports. Prior to the U.N. trade embargo with Iraq, this nation has received about $20 \%$ of Jordan's exports ${ }^{2}$. JPMC is the largest exporter in Jordan, and APC is the country's second largest exporter.

Shitting international markets for phosphates and phosphatic fertilizers in 1993 placed pressure on that sector of the Jordanian mining industry. In the late 1980's, about $30 \%$ of Jordan's phosphate products were exported to Eastern Europe. Newer Asian markets have helped offset the decrease in exports to Eastern Europe since 1991. JPMC is also developing new markets in Australia and New Zealand, and expanding markets in Arab countries. The potash industry has benefitted from increased demand from India and the Far East. There are presently no United Nations sanctions against Jordan.

Jordanian mineral production estimates for 1993 and 1994 are provided in table 3.1. Summaries of mineral site data are provided in Appendices A-B. Data on significant producing sites, past producers, prospects, and undeveloped mineral occurrences are provided in tabular form. Maps showing mineral property locations are also provided in Appendix maps A-B. It should be noted that not all mineral occurrences are reported in this study. Sites with unverifiable information or lacking specific site locations may not be included. Data are reported for 17 producers, and 35 past producers or mineral deposits. While data on small, private sector mining operations are limited, it is believed that all sites with significant potential have been reported. 
Table 3.1 -- Estimated Mineral Production in Jordan, 1993 and $1994^{1}$ (Metric tons)

\begin{tabular}{|lrr|}
\hline Commodity & & 1993 \\
& $\begin{array}{r}1994 \\
\text { Cement, hydraulic }\end{array}$ & Production $^{3}$ \\
Clays & $3,400,000$ & $6,600,000$ \\
Gypsum & 47,200 & 47,200 \\
Lime & 195,000 & 195,000 \\
Phosphate rock, gross weight & 7,270 & 7,270 \\
Potash, crude salts & $4,280,000$ & $4,220,000$ \\
Salt & $1,370,000$ & $1,500,000$ \\
Stone, limestone & 26,000 & 26,000 \\
Stone, marble & 5,340 & 5,340 \\
\hline
\end{tabular}

SOURCE: U. S. Bureau of Mines and U. S. Geological Survey. The Mineral Industry of Jordan. Mineral Industry Survey series 1992-1994. Chapters prepared by Thomas P. Dolley.

1 Includes data available through July 1995.

2 In addition to the commodities listed, crude steel and petroleum products are produced, but not reported here.

3 Estimated. 


\subsection{Industrial minerals}

Jordan is an important producer of several industrial minerals, notably phosphate products, potash and other mineral salts, cement, and building and construction stone products. While phosphate and potash are principally exported, cement and construction products are produced primarily to meet domestic needs. Decreased demand from Western Europe, coupled with a significant decline in exports to Eastern Europe have forced Jordan to look toward new markets in India, Asia, and Oceana for industrial mineral products. Jordan is also looking to increase trade with its Middle Eastern neighbors.

\subsubsection{Phosphate}

Phosphate deposits are Jordan's primary natural resource and a major source of export income. Revenues generated from products derived from this industry accounted for about one third of Jordan's export revenue in 1993. Jordan accounted for about $3 \%$ of world phosphate in $1994^{3}$, in spite of decreased market demand for Jordanian phosphate from the former Soviet Union and Eastern Europe.

The most important industrial company in Jordan, JPMC, controls phosphate production in the country. Phosphate rock is mined by JPMC from three mines; the Al Hasa and Wadi Al Abyad mines in central Jordan and the new Ash Shidiya mine in southern Jordan. The Ash Shidiya mine is scheduled to replace declining ore reserves at the other mines by the year 2000. In 1994, active mining at Al Hasa was limited; activity was confined to the removal of stockpiled ore. Reported 1994 phosphate rock production was approximately $4.3 \mathrm{Mt}$ from an industry with a production capacity of about $8.1 \mathrm{Mt}$.

Phosphate mining in Jordan began at Ruseifa in 1943. Surface and underground production at Ruseifa ended in 1985. While production in the Al Hasa/Al Abyad area first began by underground methods, the relatively low overburden to ore ratio in the

3 U.S. Geological Survey and U.S. Bureau of Mines. Mineral Commodity Summaries 1996. 
area permitted extraction by highly mechanized open cast mining in which overburden is removed by scrapers, rippers and bulldozers; blasting is limited to selected areas. Ore is extracted in a similar manner. A walking dragline is used to remove overburden and ore in some areas. Blasting of ore is also limited.

The Esh Shidiyah began operations in 1988 using modern draglines to recover phosphate from beneath $40 \mathrm{~m}$ of limestone/marl overburden. The operation is designed to replace production from the Al Hasa and Al Abyad sites by 2000. Present production capacity is $2.8 \mathrm{Mt} / \mathrm{yr}$. Reserves at this site are extensive and it is expected that this mine will be the primary source of Jordanian phosphate well into the next century.

Most of the phosphate rock production is exported through the port of Aqaba. In 1992, the latest year for which such data were available, about half was exported to India, Indonesia, and the Netherlands. The rest was exported to various countries, including neighboring Arab states, India, and Australia. About $1 \mathrm{Mt} / \mathrm{yr}$ are used domestically by the fertilizer complex at Aqaba, where an expansion program for the phosphoric acid facilities was completed in 1994.

\subsubsection{Potash}

Potash is the second major component of Jordan's mining sector. Jordan produced $11 \%$ of the world's potash in $1994^{2}$. Production is conducted by the Arab Potash Company (APC), which was established in 1956 by a combination of Arab and private interests. In 1958, the APC was granted a 100 year concession to mine and produce chemicals from the Jordanian portion of the Dead Sea. The mine and extraction plant are located near Safi at the southern end of the Dead Sea, south of the Lisan Peninsula and east of the truce line with Israel.

Production began in 1982. Since 1985, APC has sustained levels greater than $90 \%$ of the plant's $1.8 \mathrm{Mt} \mathrm{KCl}$ production capacity (1995). Potash production from Dead Sea brine requires solar evaporation to concentrate the brine and produce sylvite $(\mathrm{KCl})$ and 
carnallite, a hydrated mineral containing $\mathrm{KCl}$ and $\mathrm{MgCl}_{2}$, and subsequent refining to produce agricultural grade potash $\left(\mathrm{KCl}\right.$ at $60-62 \% \mathrm{~K}_{2} \mathrm{O}$ equivalent) from the carnallite.

Extraction at the full production rate requires pumping 288Mt/yr of brines from the Dead Sea to a series of evaporation basins (salt pans) where the brine is concentrated and salt is removed. Blasting is not required in this process. APC was considering plans in 1995 to increase surface area for evaporation and to purchase a bucketwheel suction dredge to mine salt. The Jordanian Dead Sea Minerals Company was created in 1994 to study methods for recovery of bromine and other non-potash mineral salts from the Dead Sea, both from the Safi site and from the Lisan Peninsula to the north.

Potash is also exported. Principal export destinations in 1992 were India, China, and Indonesia. Currently APC is directing its marketing efforts to increase sales to third world countries and expand sales to its Arab customers.

\subsubsection{Cement}

Cement production within Jordan is primarily for domestic consumption, with some available for export to neighboring countries such as Egypt. The Jordan Cement Factories Company (JCFC) is the primary cement producer in Jordan, with a 3.4Mt plant at Fuheis near Amman.

Cement in Jordan is derived primarily from nodular limestones east of the Rift in northern Jordan. While reserves are practically unlimited, most of the production occurs in the industrialized area around Amman. As with most countries in this area, mining of cement raw materials comes from small pits or quarries located near destination markets. Because the limestone deposits are often located near the surface, little overburden removal is required. Typical small scale quarrying techniques are most often employed. Mining requires limited and infrequent blasting.

The cement industry in Jordan is becoming increasingly important as solutions for Jordan's water shortage require increased demand for cement. Approximately $60 \%$ of 
Jordan's water supplies come from aquifers in the eastern part of the country. In recent years, however, much of Jordan's population has migrated to the west while intermittent rainfall has not recharged the aquifers sufficiently to meet growing domestic needs. Jordan is in the process of building several major dams in an attempt to make maximum use of limited water supplies. While not all dams are made of cement, dam construction requires increased quantities of cement, and the Jordanian industry has increased production in an attempt to meet that demand. In addition, several major pipeline projects and increased building construction have impacted the demand for cement. Cement production is expected to increase significantly by 2000 .

\subsubsection{Stone}

Due to a lack of timber, many different rock types have been used as building stones in Jordan since ancient times. Near Petra, in southwestern Jordan, the Nabateans chiseled stone tombs with impressive facades from local sandstone. Basalts are used for construction purposes in northeast Jordan. Oolitic limestones from the western Jordan valley supplies the construction industry in the Amman area. In the Jerusalem and Amman area, polishable limestone "marble" is often used as ornamental building stone.

Unlike the major mining industries, construction aggregates, basalt, limestone, dimensional stone, glass sand and natural sand exploitation is controlled by small, private sector firms, often operating very small pits or quarries requiring a small number of personnel to operate. As a result, information on the number and size of these operations is difficult to ascertain. While information on significant deposits is available, often it is difficult to verify the operating status of these sites. Consequently, data reported in Appendices $A-B$ for stone products may be incomplete or assigned an inappropriate status.

\subsection{Other minerals}

Other industrial minerals produced include clays, gypsum, lime, and salt. Jordan also 
has a small steel plant near Zarqa, which processes imported raw material and scrap for domestic use. Jordan possesses some barite, chromite, copper, manganese, and nickel resources, none of which is considered economic. Iron was produced in ancient times from the Warda deposit, but no production has occurred recently. Uranium could be recovered as a byproduct of phosphate production. Jordan also has the potential to produce energy from its bituminous rocks and oil shale.

\subsection{MINE-RELATED EXPLOSIVES USE}

Almost all mines use explosives to fragment or loosen rock and consolidated material prior to excavation. Bulk or packaged explosives and blasting agents are detonated after emplacement in material to be excavated. Minor quantities of sachet and shaped charges may be used for secondary breakage and other special applications.

The type and amount of explosives used are influenced by the geotechnical nature of the rock, the mining methods employed, the production rate of the mine, the type and availability of explosives and detonation systems, hydrologic conditions, mining equipment, drilling equipment, mine geometry, level of technical expertise, and external constraints such as the proximity of residences, availability of explosives, and available funding. At almost any mine, the size of each blast can vary significantly due to local conditions, production schedules, weather, etc.

Surface mines typically shoot much larger blasts than underground operations and tend to have higher production rates than underground mines. In addition, limitations of working room, limited free faces, type of mineralization, ventilation requirements, and drilling limitations may constrain maximum blast sizes in underground mines.

Jordan is not limited to domestically produced explosives, and has sufficient raw materials to produce what is needed. Jordan has the military-related technology and facilities to manufacture blasting agents and detonation systems suitable for most mining applications. 
Where blasting is required, most Jordanian mines use ammonium nitrate-fuel oil (ANFO) blasting agents. ANFO systems are preferred in most mining applications due to their ease of manufacture, low cost, inherent safety, and bulk loading advantages. High explosives, however, may be preferable for small underground operations that use drill sizes that are below the critical diameter needed for emplacing ANFO blasting agents, or under wet conditions, in methane-rich atmospheres, and conditions that require higher detonation velocities and/or convenience of packaged explosives. Few such underground mining sites operate in Jordan, however.

In most cases, site-specific blasting information was unavailable. Consequently, estimates were based upon known or estimated production rates, mine geology, and typical mining practices. Experience, engineering judgement, and available data were incorporated into calculations and estimates. Explosive use can vary considerably as mining conditions change. ANFO consumption was assumed to be dependent upon mine production rate, average stripping ratio, specific gravity of the host rock, assumed powder factor limits, and mining method. Only a small number of mines in Jordan require significant blasting, primarily because of their small size.

For each site, a stripping ratio (Quantity of overburden or waste removed per $\mathrm{mt}$ of ore mined) and powder factor (Quantity of rock blasted per unit of ANFO blasting agent equivalent) limits were estimated. A range of ANFO consumption was calculated for both daily blasting requirements and for an assumed maximum blasting event. Daily ANFO requirements were estimated assuming a 330 day production schedule. Consumption estimates for all sites were calculated in a similar manner. The lower consumption value applies a minimum powder factor while the higher value assumes a maximum powder factor. Unlike daily consumption estimates, a maximum blasting event would not take place on a daily basis. For this study, it was assumed that a maximum blasting event could occur every 10 working days for a surface mine and every 5 working days for an underground mine. Such events are designed to account for such factors as blasting delays, geological irregularities, and mining method variations that require a higher ANFO consumption than the typical blasting event. Mine development 
or pillar extraction conditions, for example, often require larger blasts.

The following examples illustrate typical blasting calculations using the estimation procedure described above:

Esh Shidiyah ANFO daily consumption lower limit (L):

$L=$ Production rate * $[1+\text { (stripping ratio * specific gravity of waste) }]^{*}$ [Low powder factor

/ 1000 (converts $\mathrm{kg}$ to $\mathrm{mt}$ )] / Operating days per year

$L=2,800,000 *[1+(2.0 * 2.5)] *[0.11 / 1000] / 330$

$L=5.60 \quad 6 \mathrm{mt}$ ANFO equivalent (rounded)

Esh Shidiyah ANFO daily consumption higher limit $(H)$ :

$H=$ Production rate * $[1+($ stripping ratio * specific gravity of waste) $]$ * [High powder factor

$/ 1000$ (converts $\mathrm{kg}$ to $\mathrm{mt})] /$ Operating days per year

$L=2,800,000 *[1+(2.0 * 2.5)] *[0.20 / 1000] / 330$

$L=10.18 \quad 10 \mathrm{mt}$ ANFO equivalent (rounded)

Esh Shidivah maximum blasting event ANFO consumption (M):

$M=$ Production rate * $[1+$ (stripping ratio * specific gravity of waste) $]$ " [High powder factor

$/ 1000$ (converts $\mathrm{kg}$ to $\mathrm{mt}$ )] / Operating days per year * maximum blast cycle time (working days between blasting events)

$M=[2,800,000 *[1+(2.0 * 2.5)] *[0.20 / 1000] / 330] * 10$

$M=101.82 \quad 102 \mathrm{mt}$ ANFO equivalent (rounded)

Table 4.1 provides the corresponding blasting range estimates for the main Jordanian mines identified in this study. Figure 4.1 shows site locations for the mines reported in table 4.1. Symbols reflect mine type (surface or underground) and maximum ANFO consumption for a given blasting event. Where a mine produces using both underground and surface methods, the predominant method is shown on figure 4.1.

Only two mining sites, the El Hasa and Esh Shidiyah phosphate mines, had calculated maximum blasting events that exceeded $100 \mathrm{mt}$ ANFO equivalent. The El Hasa (as 
Table 4.1-Estimated Explosives Usage at the Main Jordanian Mines

Used in this Study in Order of Estimated ANFO Consumption

\begin{tabular}{|c|c|c|c|c|c|c|c|c|c|}
\hline \multirow[t]{2}{*}{ Mine" } & \multirow[t]{2}{*}{ Latitude } & \multirow[t]{2}{*}{ Longitude } & \multirow[t]{2}{*}{$\begin{array}{l}\text { Primary } \\
\text { Product }\end{array}$} & \multirow{2}{*}{$\begin{array}{l}\text { Mlne } \\
\text { Type } \\
\text { (1) }\end{array}$} & \multirow{2}{*}{$\begin{array}{r}\text { Production } \\
(\mathrm{Mm} t / y) \\
(2)\end{array}$} & \multicolumn{2}{|c|}{$\begin{array}{c}\text { Daily Consumption } \\
\text { (mt ANFO) } \\
\text { (3) (4) }\end{array}$} & \multirow{2}{*}{$\begin{array}{c}\text { Maximum } \\
\text { Blast Cycle } \\
\text { Time (days) } \\
\text { (5) }\end{array}$} & \multirow{2}{*}{$\begin{array}{c}\text { Maximum } \\
\text { Blasting } \\
\text { Event } \\
\text { (mt ANFO) }\end{array}$} \\
\hline & & & & & & Low & High & & \\
\hline El Hasa (6) & N $30^{\circ} 49^{\circ}$ & $E 36^{\circ} 00^{\prime}$ & Phosphate & $s$ & 3.800 & 10 & 18 & 10 & 184 \\
\hline Esh Shidiyah (6) & N $29^{\circ} 46^{\prime}$ & $E 35^{\circ} 54^{\prime}$ & Phosphate & $\mathbf{s}$ & 2.800 & 6 & 10 & 10 & 102 \\
\hline Wadi al Abyad (6) & $N 30^{\circ} 58^{\prime}$ & $E 36^{\circ} 00^{\prime}$ & Phosphate & $\mathbf{s}$ & 1.500 & 4 & 7 & 10 & 73 \\
\hline Fuheis (7) & N $32^{\circ} 01^{\prime}$ & E $35^{\circ} 46^{\prime}$ & Limestone & $s$ & 3.400 & 1 & 2 & 10 & 21 \\
\hline Dead Sea (8) & $N 31^{*} 06^{\prime}$ & $E 35^{\circ} 31^{\prime}$ & Potash & $\mathbf{s}$ & 1.800 & 0 & 0 & 10 & 2 \\
\hline Wadi Mujib & N $31^{\circ} 28^{\prime}$ & $E 35^{\circ} 34^{\prime}$ & Sulfur & $S$ & 0.045 & 0 & 0 & 10 & 1 \\
\hline
\end{tabular}

(1) S-Surface

(2) Mmtlyr--Million metric tons per year

(3) $\mathrm{mt}$ ANFO-Metric tons of Ammonium Nitrate/Fuel Oil blasting agent equivalent. Estimate based on equations reported on pages 11-12.

(4) Assumed production schedule for all sites is 330 days/yr.

(5) Assumed maximum blasting cycle time for surface operation - 10 working days; underground operation - 5 working days.

(6) Assumes both overburden and ore removed on a daily basis. In actual practice, overburden removed by contractor prior to mining a site. Blasting of ore is limited to selected areas, therefore figures reported here represent maximum consumption if both overburden and ore were blasted concurrently. The El Hasa and Wadi al Abyad mines are nearing the end of their mining life, and most overburden has been removed. It is expected that current blasting is minimal.

(7) Limestone for cement production is recovered from local quarries. Capacity is total for region, so ANFO consumption at an individual site should be lower.

(8) Potash and salt production from Dead Sea sites is conducted by solution mining which generally does not require blasting. Production is total for the complex. 


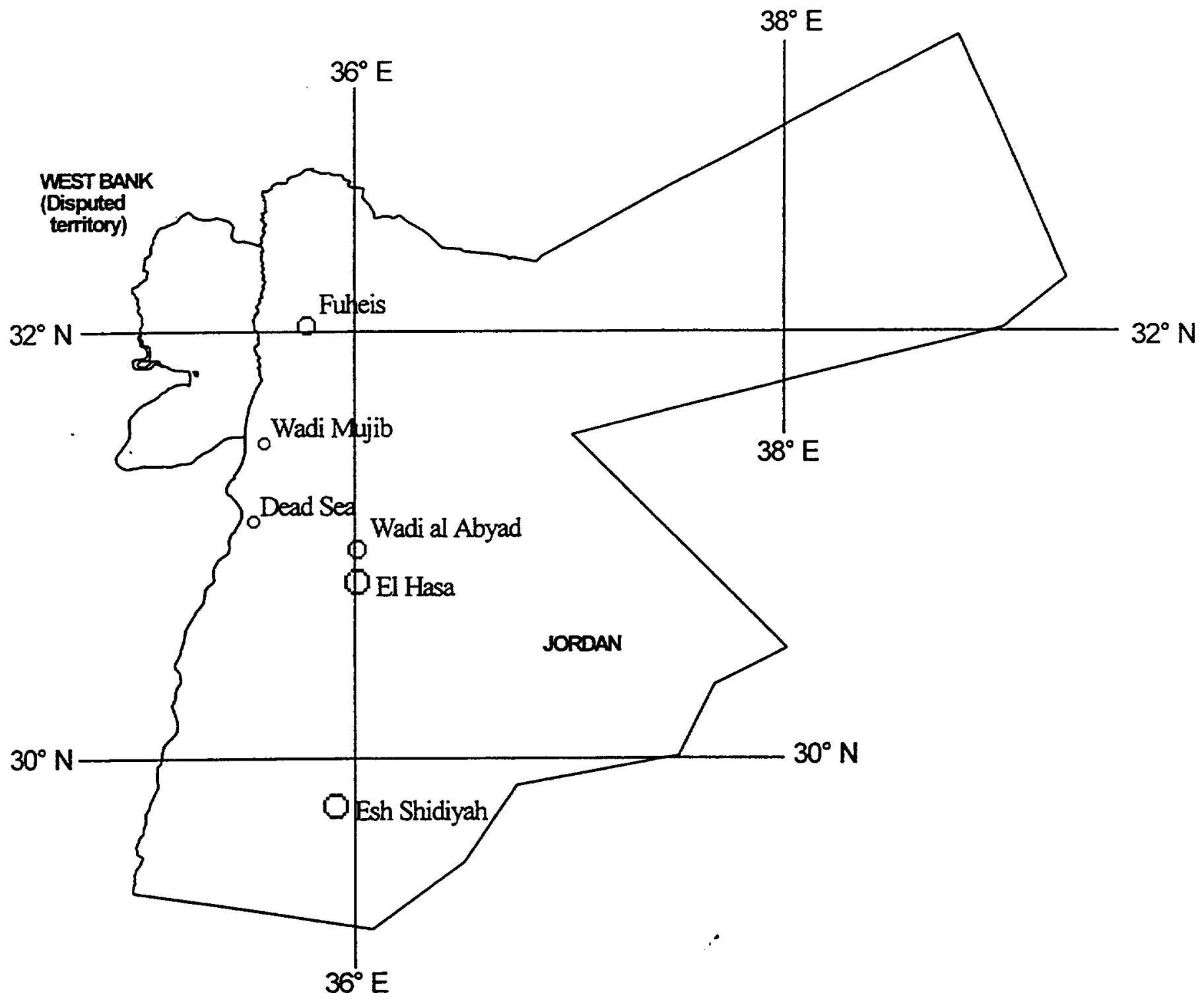

LEGEND

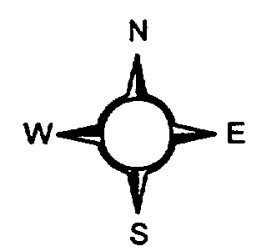

Size

Greater than $100 \mathrm{mt}$ ANFO*

O 10 to $100 \mathrm{mt}$ ANFO

$0 \quad 100 \quad 200$ Kilometers

- Less than $10 \mathrm{mt}$ ANFO'

FGURE 4.1-Selected Jordanian Mines and Estimated Maximum Blasting Events 
well as the Al Abyad site) were only recovering stockpiled ore in 1994, due to limited reserves. The Esh Shidiyah mine is being developed to replace the other sites. The While both sites have the potential for a maximum blasting event of over $100 \mathrm{mt}$ ANFO equivalent, under normal circumstances this is unlikely. Jordanian phosphate mines employ open cast mining techniques and require selected blasting. Overburden is commonly removed by contract prior to ore mining, so mining often requires only limited blasting of hard ore or separate blasting of ore or overburden. On a daily basis, however, ANFO consumption for both overburden and ore is given, although blasts may occur in separate areas.

In addition to the two sites previously mentioned, there are several sites that have an estimated explosive potential of less than $25 \mathrm{mt}$ of ANFO equivalent for a maximum blasting event. While mining of Dead Sea salts and potash typically does not require blasting, the Table 4.1 blasting consumption estimate assumes that a minor quantity of blasting will be required for ancillary operations. Limestone production feeding the Fuheis cement plant comes from numerous small quarries feeding the nearby plant, rather that one large mining operation, as suggested in Table 4.1.

\subsection{CONCLUSIONS}

While the mining industry of Jordan is an important regional producer accounting for about one third of Jordan's export revenue, it is not significant on a world scale, except for its production of phosphate and potash. Identified resources are associated with the rift zone in western Jordan. Resource potential in eastern Jordan has not been fully explored, but is not thought to be important. Explosive consumption by the mining industry of Jordan is low, particularly when its largest phosphate mines face depleted resources and reduced production. 


\section{APPENDICES}


APPENDIX A: PRODUGINO AND DEVELLPING MINERAL PROPERTIES IN JORDAN

\begin{tabular}{|c|c|c|c|c|c|c|c|c|c|c|}
\hline $\begin{array}{l}\text { MAP } \\
\text { KEY } \\
\text { (1) }\end{array}$ & $\begin{array}{l}\text { NAME } \\
\text { (2) }\end{array}$ & $\begin{array}{l}\text { COORDIN } \\
\text { LAT. }\end{array}$ & $\begin{array}{l}\text { TES } \\
\text { LONO. }\end{array}$ & COMMODITY(IES) & $\begin{array}{l}\text { DATA } \\
\text { SOURCES } \\
\text { (3) }\end{array}$ & $\begin{array}{l}\text { DATA } \\
\text { RELIANCE } \\
\text { (4) }\end{array}$ & STATUS & $\begin{array}{l}\text { MINE } \\
\text { TYPE }\end{array}$ & MARKETS & COMMENTS \\
\hline 1 & Amman & $N 31^{\circ} 5 T$ & E $35^{\circ} 56^{\prime}$ & 'Stone & 1,2 & General & Producer & Surface & Domestle & Limestone used for bullding slone. \\
\hline 1 & Qasr al Mammann & N 31' 32 & E $36^{\circ} 11^{\circ}$ & Martle & 2 & General & Producer & Surtace & Domestle & Pollshable ltmestone used for bullding stone. \\
\hline 1 & Unnamed & N 31' 43' & $E 3^{\circ} 05^{\circ}$ & Stone & 1,2 & General & Producer & Surface & Domestle & Oyster shell llmestone used for bulliding stone. \\
\hline 2 & Klrbal as Samra & N $31^{\circ} 39^{\prime}$ & E $35^{\circ} 10^{\circ}$ & Stone & 1,2 & General & Producer & Surface & Domestic & Oalliti limestone used for bulding stone. \\
\hline 3 & Jabal ar Rutayn & $N 32^{\circ}$ or & $E 36^{\circ} 53^{\prime}$ & Tuff & 1,2 & General & Producer & Surface & Domestle & Used in thermal insulation and road making. \\
\hline 3 & Jarash & $\mathrm{N} 32 \cdot 17$ & $E 35^{\circ} 54^{\prime}$ & Stone & 1.2 & General & Producer & Surface & Domestlo & Callche used for bulldilng stone. \\
\hline 6 & Deir Alla & $N$ 32 $00^{\circ}$ & E $35^{\circ} 37$ & Travertine & 1.2 & General & Producer & Surface & Domestle & Beds of $10-20 \mathrm{~m}$ thick occur. \\
\hline 6 & $\begin{array}{l}\text { Fuhels } \\
\text { Amman dtstrict }\end{array}$ & N 32' 01' & $E 35^{\circ} 46^{\circ}$ & Comont & $1,2,15$ & General & Producer & Surface & Domestle & $\begin{array}{l}\text { Porlland cement produced from area llmestones. } \\
\text { Area quarrles supplled aboul } 74 \% \text { of Jordanlan cement } \\
\text { In } 1992 \text {. }\end{array}$ \\
\hline 7 & $\begin{array}{l}\text { Dead Sea } \\
\text { Sant } \\
\text { Ghor as Safl }\end{array}$ & $N 31^{\circ} 06^{*}$ & E $35^{\circ} 3 t^{\prime}$ & $\begin{array}{l}\text { Polash } \\
\text { Sall } \\
\text { Bromine } \\
\text { lodine }\end{array}$ & $\begin{array}{l}1,6,10 \\
14,15,16\end{array}$ & Confrmod & Producer & Surface & $\begin{array}{l}\text { Domestle } \\
\text { Export }\end{array}$ & 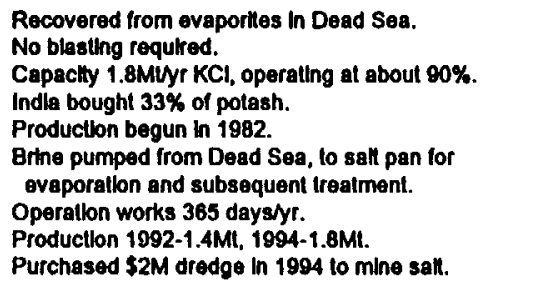 \\
\hline 7 & $\begin{array}{l}\text { EI Hasa district } \\
\text { A Hasa }\end{array}$ & N $30^{\circ} 49^{\circ}$ & E $36^{\circ} 00^{\circ}$ & $\begin{array}{l}\text { Phosphate } \\
\text { Urantum } \\
\text { Gypsum }\end{array}$ & $\begin{array}{l}1,2,5,10 \\
14,15,16\end{array}$ & General & Producer & $\begin{array}{l}\text { Surface } \\
\text { Underground }\end{array}$ & $\begin{array}{l}\text { Export } \\
\text { Domestle }\end{array}$ & 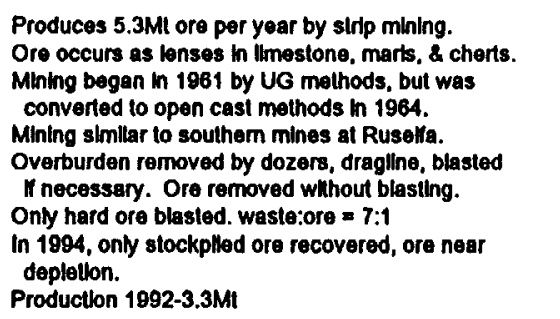 \\
\hline 7 & ElLusan & N $31 * 17$ & E $35^{\circ} 28^{\prime}$ & $\begin{array}{l}\text { Sakt } \\
\text { Potash } \\
\text { Bromine } \\
\text { lodine }\end{array}$ & $1,6,7$ & General & Producer & Surface & $\begin{array}{l}\text { Domesile } \\
\text { Export }\end{array}$ & $\begin{array}{l}\text { Recovered from ovaporties in Dead Sea. } \\
\text { No blasting required. } \\
\text { Potash presently not rocovered from thls locallon. } \\
\text { Potash beds at depths of } 149-165,347-351 \mathrm{~m} \text {. }\end{array}$ \\
\hline 7 & Qatrana & $N 3^{\circ} 15^{\circ}$ & E $36^{\circ} 02^{\prime}$ & Stone & 1 & General & Producer & Surface & Domestle & Quarted for decorative stone and floor thes. \\
\hline 7 & $\begin{array}{l}\text { Wadl al Abyad } \\
\text { Wadl al Abluth } \\
\text { Wadi Abu Ubaydah }\end{array}$ & N $30^{\circ} 58^{\prime}$ & E $36^{\circ} 00^{\circ}$ & Phosphate & $\begin{array}{l}2,5,10 . \\
14,15,16\end{array}$ & Goneral & Producer & Surface & $\begin{array}{l}\text { Export } \\
\text { Domestic }\end{array}$ & $\begin{array}{l}\text { Came on stream in } 1979 \text { whin design capactly of } \\
1.5 \mathrm{M} y \mathrm{y} \text {. Considered part of El Hasa complex. } \\
\text { Walking dragline used to sirip ore, overburden. }\end{array}$ \\
\hline
\end{tabular}


APPENDIX A: PRODUCING AND DEVELOPINO MINERAL PROPERTIES IN JORDAN

\begin{tabular}{|c|c|c|c|c|c|c|c|c|c|c|}
\hline \multirow[t]{2}{*}{$\begin{array}{l}\text { MAP } \\
\text { KEY } \\
\text { (1) }\end{array}$} & \multirow[t]{2}{*}{$\begin{array}{l}\text { NAME } \\
\text { (2) }\end{array}$} & $\begin{array}{ll} & \text { COORDIN } \\
\text {-. LAT. }\end{array}$ & $\begin{array}{l}\text { ATES } \\
\text { LONG. }\end{array}$ & \multirow[t]{2}{*}{ COMMODITY(IES) } & \multirow[t]{2}{*}{$\begin{array}{l}\text { DATA } \\
\text { SOURCES } \\
\text { (3) }\end{array}$} & \multirow[t]{2}{*}{$\begin{array}{l}\text { DATA } \\
\text { RELIANCE } \\
\text { (4) }\end{array}$} & \multirow[t]{2}{*}{ STATUS } & \multirow[t]{2}{*}{$\begin{array}{l}\text { MINE } \\
\text { TYPE }\end{array}$} & \multirow[t]{2}{*}{ MARKETS } & COMMENTS \\
\hline & & & & & & & & & & $\begin{array}{l}\text { In 1994, only stockplled ore recovered, ore near } \\
\text { depletion. } \\
\text { Production 1992-3Mt }\end{array}$ \\
\hline 7 & $\begin{array}{l}\text { Wadl Mullb } \\
\text { Al Moogob }\end{array}$ & N 31' 28' & E 35' $34^{\prime}$ & $\begin{array}{l}\text { Gypsum } \\
\text { Sultur }\end{array}$ & $1,2.4$ & General & Producer & Surface & Domestle & $\begin{array}{l}\text { Thlckness of } 15 \mathrm{~m} \text { reported. } \\
\text { Reported to produce } 45 \mathrm{kt} \text { of gypsum per year. } \\
\text { No byproduct sulfur belng produced. } \\
\text { Used in domesitc bulling and construction Industry }\end{array}$ \\
\hline 8 & Esh Shldyah & $N 29^{\circ} 46^{\circ}$ & E $35^{\circ} 54^{\prime}$ & Phosphate & $\begin{array}{l}5,10,14 \\
15,16\end{array}$ & General & Producer & Surface & $\begin{array}{l}\text { Export } \\
\text { Domestle }\end{array}$ & $\begin{array}{l}\text { Mine belng developed to replace El Hasa/Al Abyad. } \\
\text { Full production expected by } 2000 \text {. } \\
\text { Production capachy } 2.8 \mathrm{MUyr} \text {; produced } 1.9 \mathrm{Mt} 1994 \text {. } \\
\text { Average depth to mineralization } 40 \mathrm{~m} \text {, thickness 8m. } \\
\text { Production } 1992-1.5 \mathrm{Mt}\end{array}$ \\
\hline
\end{tabular}

(1) Represents property or property groupling as defined on Appendbe Map A.

(2) Due to sonware Hmllatlons, she names do not inctude any dlacritleal markings. Spelling of indlvidual names vary considerably by source.

(3) Complete ist of data sources lound in Appendlx C.

(4) General - denoles Hinlted dala; Confrmed - denotes deposin informallon confirmed by several sources. 


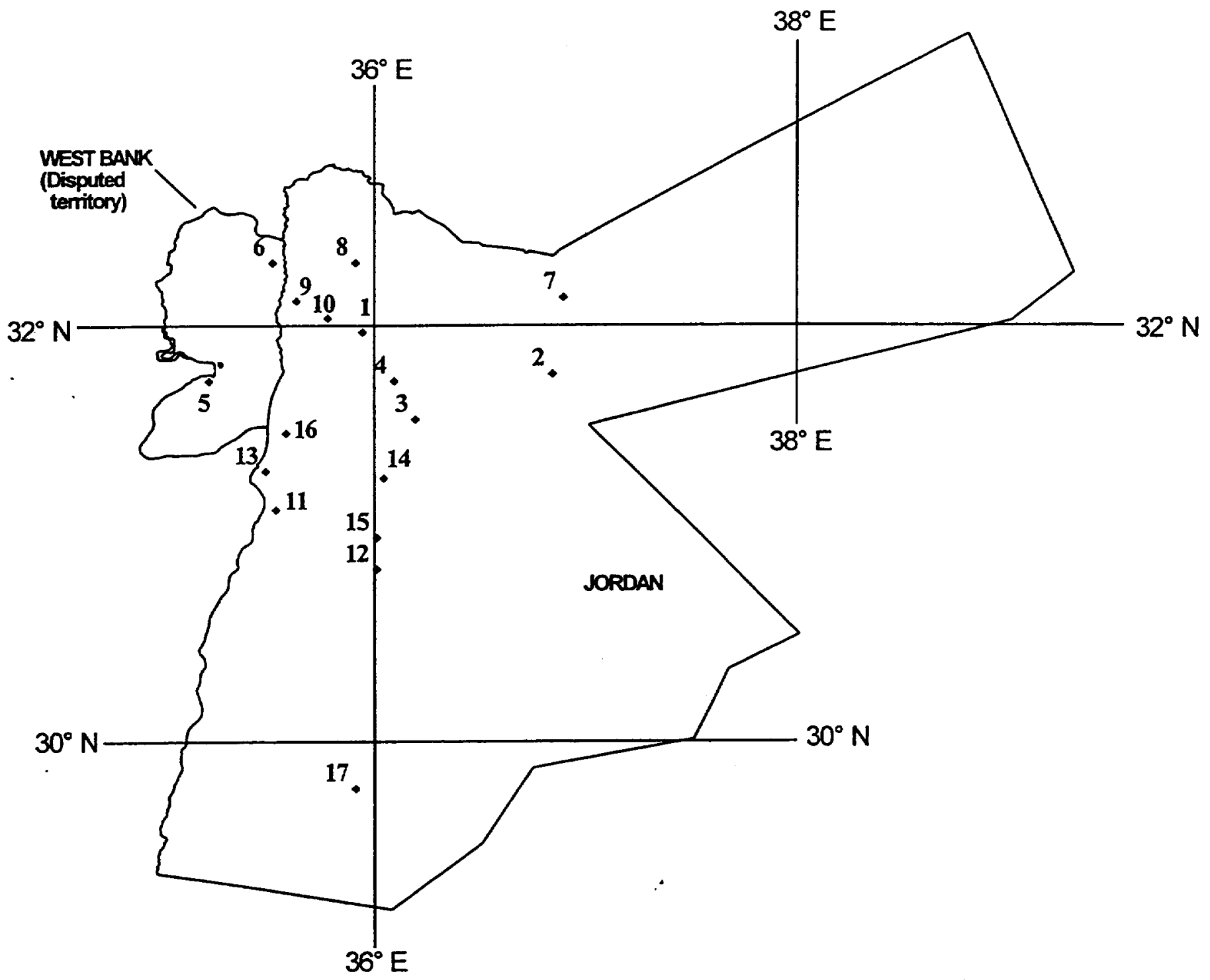

LEGEND

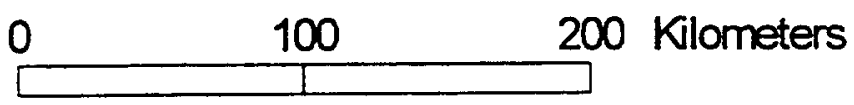

Producing or Developing

- Mineral Property

- Letter represents property grouping as defined in appendix 
APPENDIX B: PAST PRODUCERS, PROSPECTS AND UNDEVELOPED MINERAL PROPERTIES IN JORDAN

\begin{tabular}{|c|c|c|c|c|c|c|c|c|c|c|}
\hline $\begin{array}{l}\text { MAP } \\
\text { KEY } \\
\text { (1) }\end{array}$ & $\begin{array}{l}\text { NAME } \\
\text { (2) }\end{array}$ & $\begin{array}{l}\text { COORDIN } \\
\text { LAT. }\end{array}$ & $\begin{array}{l}\text { ATES } \\
\text { LONO. }\end{array}$ & COMMODITY(IES) & $\begin{array}{l}\text { DATA } \\
\text { SOURCES } \\
\text { (3) }\end{array}$ & $\begin{array}{l}\text { DATA } \\
\text { REL.JANCE } \\
\text { (4) }\end{array}$ & status & $\begin{array}{l}\text { MINE } \\
\text { TYPE }\end{array}$ & MARKETS & COMMENTS \\
\hline 1 & Humrat Ma'n & $N 31^{\circ} 40^{\circ}$ & E $35^{*} 36^{\prime}$ & Ochre & 1,2 & General & Deposk & Surface & NAp(5) & Wodgling lenses about $1 \mathrm{~m}$ thick in sedlenents. \\
\hline 1 & $\begin{array}{l}\text { Ruserra disirict } \\
\text { North mine } \\
\text { Ras al An } \\
\text { Zarqa A } \\
\text { Zarqa B } \\
\text { Far South Area }\end{array}$ & $N 32^{\circ} 01^{\circ}$ & E $36^{\circ} 02$ & $\begin{array}{l}\text { Phosphale } \\
\text { Uranlum }\end{array}$ & $\begin{array}{l}1,2,5 \\
10,13,14\end{array}$ & General & Past producer & $\begin{array}{l}\text { Surface } \\
\text { Underground }\end{array}$ & $\begin{array}{l}\text { Expont } \\
\text { Domestlc }\end{array}$ & 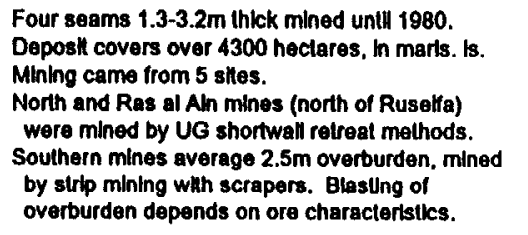 \\
\hline 1 & Unnamed & N 34 52 & E $35^{\circ} 47$ & Glass sand & 1,2 & General & Deposh & Surface & NAP & Good qualliy glass sand occurrence. \\
\hline 2 & Bell Sahur & $\mathrm{N} 31^{\circ} 41^{\circ}$ & E $35^{\circ} 16^{\circ}$ & Bantio & 1,2 & General & Deposit & Unknown & NAp & Ore zone $30 \mathrm{~m} \times 15 \mathrm{~m} \times 12 \mathrm{~m} \mathrm{~m}$ marts, Ulmestone. \\
\hline 2 & Haturim & $N 31^{\circ} 48^{\circ}$ & E $35^{\circ} \mathbf{2 0}$ & Chromlum & 1,2 & General & Deposk & Unknown & NAP & Traces in chalk, marls; ore is nol economlc. \\
\hline 2 & Llsan lake & $N 31^{\circ} 4 r$ & E $35^{\circ} 32^{\prime}$ & $\begin{array}{l}\text { Sulfur } \\
\text { Potash }\end{array}$ & 1,2 & General & Deposit & Surface & NAP & Assoclated whin evaporites, shales, and marts. \\
\hline 2 & Nabl Musa & $N 31^{\circ} 47^{*}$ & E $35^{\circ} 26^{\circ}$ & Bllumen & 1 & General & Past producer & Surface & Domestlc & $\begin{array}{l}\text { Bhuminous marks, ltmestones quarrited for local } \\
\text { consumplion. }\end{array}$ \\
\hline 3 & Suwvilith & N $32^{\circ} 02^{\prime}$ & E 35 $50^{\circ}$ & Phosphate & 1,2 & General & Deposn & Surface & NAp & Phosphorte beds $35-40 \mathrm{~m}$ thikk ldentifled. \\
\hline 3 & Suwellih & $\mathbf{N} 32^{\circ} 03^{\circ}$ & E $35^{\circ} 4 r$ & Apaltio & 1,2 & General & Deposk & Surface & NAp & $\begin{array}{l}\text { Apatte allered from phosphorite. } \\
\text { Occurs along } 2200 \mathrm{~m} \text { strke length, up to } 8.3 \mathrm{~m} \text { thlck. } \\
\text { Reserves (1974) } 300 \mathrm{kt} \text { proven, } 650 \mathrm{kl} \text { possible. }\end{array}$ \\
\hline 3 & Unnamed & N 32* 51' & E $37^{\circ} 54^{\prime}$ & Barlte & 1,2 & General & Deposn & Unknown & NAP & Bartle dikes $0.6 \mathrm{~m}$ thlck about $60 \mathrm{~m} \mathrm{~m}$ length. \\
\hline 3 & Wadl Hunl & N $32^{\circ} 11^{\circ}$ & E $35^{\circ} 44^{\circ}$ & Gypsum & 1,2 & General & Deposit & Surface & NAP & Layer 2-3m thlck in marts. \\
\hline 3 & $\begin{array}{l}\text { Warda } \\
\text { Tell Ekwoder }\end{array}$ & N $32^{*} 13^{*}$ & E $35^{\circ} 43^{\prime}$ & Iron & $1,2,3$ & General & Pasi producer & Underground & Domestle & $\begin{array}{l}\text { Minlng dates back to Crusades, no recent mining. } \\
\text { Ore body about } 200 \mathrm{~m} \text { wlde, } 300 \mathrm{~m} \text { long, and } 9.8 \mathrm{~m} \\
\text { thlck. } \\
\text { Ore surrounded by chally llmestone. } \\
\text { Reserves (1962) } 561 \mathrm{kT} \text { 67. } 6 \% \text { Fe. }\end{array}$ \\
\hline${ }^{3}$ & Yarmuk River & N $32^{\circ} 38^{\circ}$ & E $35^{\circ} 34^{\circ}$ & Bhumen & 1 & General & Pasl producer & Surface & Domestle & $\begin{array}{l}\text { Biluminous marts, Umestones to produce qulck llme. } \\
\text { Reserves for sufilclent for oil shale production. }\end{array}$ \\
\hline 4 & Zorqa River & N 32*06' & E $35^{\circ} 32^{\circ}$ & Pyrte & 1 & General & Depostt & Surface & NAP & Crystals In sandy Hmestone, non-commerclal. \\
\hline 6 & Ghor Kabld & N $32^{\circ} 00^{\prime}$ & E 35' 33' & Clay & 1,2 & General & Deposit & Surface & NAP & $\begin{array}{l}\text { Clay layer of } 2.8 \mathrm{~m} \text { suthable for ceramles. } \\
\text { 1975 reserves: } 47 \mathrm{kt} @ 32-37 \% \text { ALO3. }\end{array}$ \\
\hline B & Mahis & N $31^{\circ} 59^{\circ}$ & E $35^{\circ} 46^{\circ}$ & Clay & 1,2 & General & Deposht & Surface & NAp & $\begin{array}{l}\text { Clay layer of } 4.2 \mathrm{~m} \text { suttable for ceramics. } \\
1975 \text { reserves: } 46 \mathrm{kt} @ 32.37 \% \text { A12O3. }\end{array}$ \\
\hline 7 & Al Hasa & N $30^{\circ} 48^{\prime}$ & E $35^{\circ} 59^{\circ}$ & Bltumen & 1 & General & Deposit & Unknown & NAP & Btuminous ilmestones, shates up to $30 \mathrm{~m}$ thick. \\
\hline 7 & Al Tufelila & $\mathrm{N} 30^{\circ} 50^{\circ}$ & $E 3^{\circ} 36^{\circ}$ & Gypsum & 4 & General & Deposh & Surface & NAP & Occurrence. \\
\hline 7 & $\begin{array}{l}\text { Lallun } \\
\text { ElLajun }\end{array}$ & N 31' 14' & E 35' $52^{\prime}$ & Bitumen & 1 & General & Depost & Unknown & NAp & Oll shale with reserves of $420 \mathrm{M}$ barrels 2 \\
\hline
\end{tabular}


APPENDIX B: PAST PRODUCERS, PROSPECTS AND UNDEVELOPED MINERAL PROPERTIES IN JORDAN

\begin{tabular}{|c|c|c|c|c|c|c|c|c|c|c|}
\hline $\begin{array}{l}\text { MAP } \\
\text { KEY } \\
\text { (1) }\end{array}$ & $\begin{array}{l}\text { NAME } \\
\text { (2) }\end{array}$ & $\begin{array}{l}\text { COOROIN } \\
\text { LAT. }\end{array}$ & $\begin{array}{l}\text { ATES } \\
\text { LONO. }\end{array}$ & COMMODTY(IES) & $\begin{array}{l}\text { DATA } \\
\text { SOURCES } \\
\text { (3) }\end{array}$ & $\begin{array}{l}\text { DATA } \\
\text { RELIANCE } \\
\text { (4) }\end{array}$ & STATUS & $\begin{array}{l}\text { MINE } \\
\text { TYPE }\end{array}$ & MARKETS & COMMENTS \\
\hline 7 & Qalrana & $N 31^{\circ} 15^{-}$ & E 36*03' & Chromlum & 1,2 & General & Deposh & Unknown & NAp & Occurrence. \\
\hline 7 & $\begin{array}{l}\text { Wadl Kerak } \\
\text { Al Kark }\end{array}$ & N $31^{\circ} 17$ & E $35^{\circ} 31^{\circ}$ & Gypsum & $1,2,4$ & General & Deposn & Surface & NAp & Layer 2-3m thick in marls. \\
\hline 8 & Aqaba & N 29. $29^{\prime}$ & E 35' 03' & Glass sand & 1,2 & General & Depostt & Surface & NAp & Good qualliy glass sand occurrence. \\
\hline 8 & Bir Madhkur & N $30^{\circ} 24^{\prime}$ & E $35^{\circ} 21^{\circ}$ & Copper & 1 & General & Depost & Surface & NAp & Mineralizallon in dolomie, Cu content less than $1 \%$. \\
\hline 8 & Folnan & N 30'38' & E $35^{\circ} 26^{\prime}$ & Nlcket & 1,2 & General & Depostt & Unknown & NAp & Reported to contaln $0.2-0.7 \% \mathrm{NI}$. \\
\hline 8 & Gharandal & N $30^{\circ} 12$ & E $35^{\circ} 14^{*}$ & Bartio & 1 & General & Doposh & Unknown & NAP & Occurrence of barthe dlkes. \\
\hline 8 & Hatlya & N 29'36' & $E 35^{*} 54^{\prime}$ & Phosphate & 1,2 & General & Deposk & Surface & NAp & Phosphorthe beds abundant but less than $1 \mathrm{~m}$ inkck. \\
\hline B & Quwera & $N 29^{\circ} 42$ & E $35^{*} 1 T$ & Foldspar & 1.2 & General & Deposn & Unknown & NAp & Occurrence in granlie pegmalime. \\
\hline 8 & Ram & N 29: 35' & E $35^{\circ} 24^{\prime}$ & Manganese & 1 & Generat & Deposh & Surface & NAp & Minerallzetton in fointhg of granlle over $60 \mathrm{~m}$ length. \\
\hline 8 & Ras on Naqb & $N 29^{\circ} 58^{\prime}$ & E 35* 33' & Glass sand & 1.2 & General & Deposin & Surface & NAp & Good quallity glass sand occurrence. \\
\hline B & $\begin{array}{l}\text { Wadl Abu Khushelba } \\
\text { Nabatean }\end{array}$ & $\mathrm{N} 30^{\circ} 1 r$ & E $35^{\circ} 20^{\prime}$ & Copper & 1.2 & General & Deposil & Surface & NAp & $\begin{array}{l}\text { Copper in sandstone. Ore low grade. } \\
\text { Some sthes mined intermittenty in the past. }\end{array}$ \\
\hline 8 & Wadl Araba & $\mathrm{N} 30^{\circ} 10^{\circ}$ & E $35^{\circ} 10^{\prime}$ & $\begin{array}{l}\text { Copper } \\
\text { Silver }\end{array}$ & 1.7 & General & Deposit & Surface & NAp & 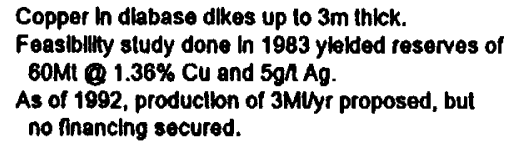 \\
\hline 8 & Wadl Dana & $\begin{array}{l}N 3^{\circ} \quad 3^{\circ} \\
N \quad 31^{\circ} \\
N\end{array}$ & $\begin{array}{c}\text { E } 35^{\circ} 32^{\prime} \\
\text { E } 35^{\circ} 22^{\prime}\end{array}$ & $\begin{array}{l}\text { Manganesse } \\
\text { Copper }\end{array}$ & $1,2,14$ & Generaf & Deposn & Surface & NAp & 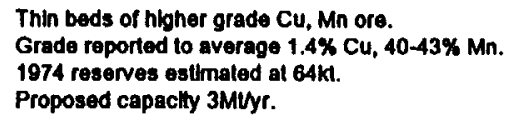 \\
\hline 8 & Wadl es Sik & N $30^{\circ} 00^{\circ}$ & $E 3^{\circ} 15^{\prime}$ & Glass sand & 1,2 & General & Deposk & Surtace & NAp & Good qualty glass sand occurrence. \\
\hline 8 & Wadl Ghuwelr & N $30^{\circ} 38^{\circ}$ & E $35^{\circ} 29^{\circ}$ & Copper & 1 & General & Depos! & Surface & NAP & Mineralization in sandslone-ore low grade. \\
\hline 8 & Wadl Halld & N 30* $36^{\prime}$ & $E$ 35' 30' & Copper & 1,2 & General & Deposit & Surface & NAP & Minerallzation in sandstone-ore low grade. \\
\hline 8 & Zakimal El Hasa & N $30^{\circ} 32$ & E $37^{*} 10^{*}$ & Barke & 1,2 & General & Deposk & Unknown & NAP & Occurrence of barte roses in sendstone. \\
\hline
\end{tabular}



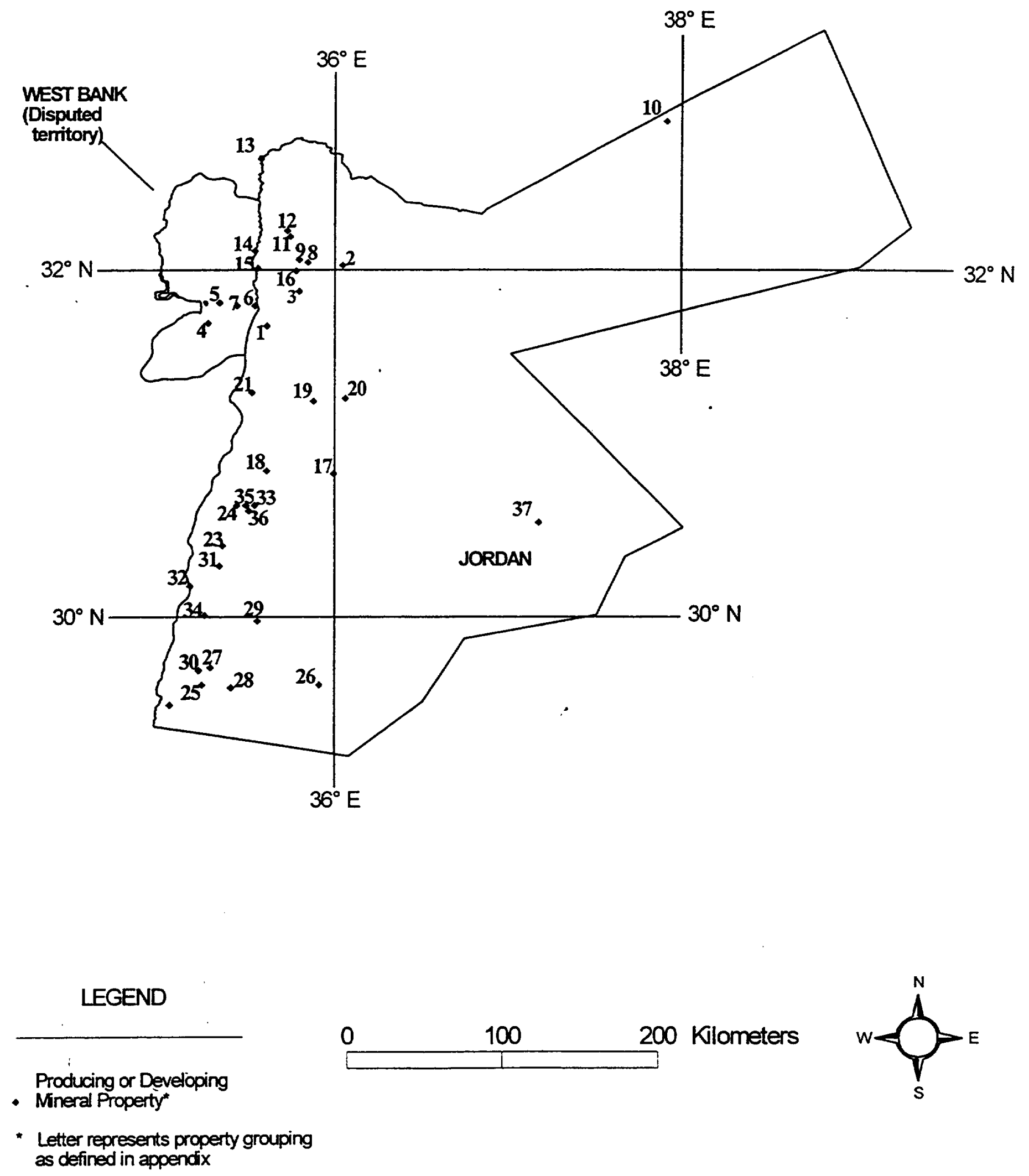

APPENDIX MAP B-1: NON PRODUCING MINERAL PROPERTIES OF JORDAN 
1. F. Bender. Geology of Jordan. Gebruder Borntraeger Press (Berlin). English Ediltion. 1974, 196 pp.

2. F. Bender. Geology of the Arablan Peninsula: Jordan. USGS Prot. Paper 560-1, 1975, $136 \mathrm{pp}$.

3. A. Zltzmann. The Iron Ores in Jordan. Ch. In The Iron Ore Deposits of Europe, Vol. 1, 1977, p. 219.

4. Brillsh Sulphur, Lid. World Survey of Sulphur Resources. 3rd Ed., 1985, p. 197.

5. Brillsh Sulphur, Lid. World Survey of Phosphate Deposits. 5th Ed., 1987, pp. 165-167.

6. $\quad$ Britlsh Sulphur, Ltd. World Survey of Potash Resources. 3rd Ed., 1979, pp. 57-61.

7. Rosklll Pubilication Services, Inc. The Economics of Potash, 2nd Ed. 1989, pp. 38-39.

8. Cambridge Informatlon and Research Services Limlted. World Directory of Energy Informatton. Vol. 2, 1994. Selected country proflles.

9. Europa Publlcatlons Limited. The Middle East and North Africa 1994. 40th Ed. pp. 411-421.

10. U. S. Llbrary of Congress. Jordan, A Country Study. Dec. 1989, pp. 148-173.

11. U. S. Energy Information Administration. The Petroleum Resources of the Mlddle East. Mar. 1984, $137 \mathrm{pp}$.

12. U. S. Board on Geographlc Names. Gazelteer No. 37 - Iraq, 1957.

13. U. S. Geological Survey. Mineral Resources Data System (MRDS), 1994.

14. U. S. Bureau of Mines. Minerals Avallability Data Base, July 1996.

15. U. S. Bureau of Mines. The Mineral Industry of Jordan. T. P. Dolley. Ch. In Minerals Yearbook 1992, Vol. III, pp. 45-54, 1993.

16. U. S. Bureau of Mines. The Mineral Industry of Jordan. T. P. Dolley. Ch. In Minerals Yearbook 1993, Vol. III, pp. 37-45, 1994.

17. U. S. Geological Survey. Jordan. Mineral Industry Survey serles. 1994, 5 pp.

18. World Cement Directory 1991. Clmeurope s.a.r.l., pp. 180-183.

19. Mining Annual Revlew - 1995. Mining Journal (London). P. 165. 
Syria 



\section{Table of Contents}

\section{Page}

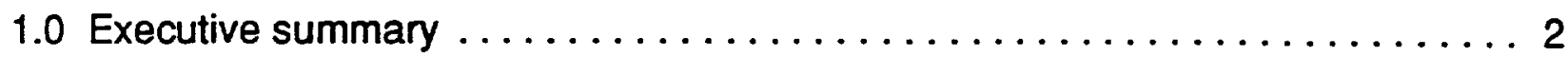

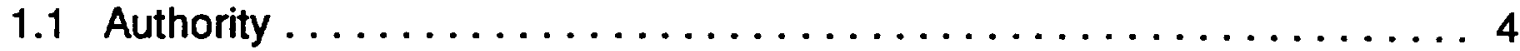

1.2 Project scope $\ldots \ldots \ldots \ldots \ldots \ldots \ldots \ldots \ldots \ldots \ldots \ldots \ldots \ldots$

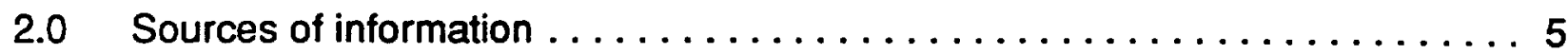

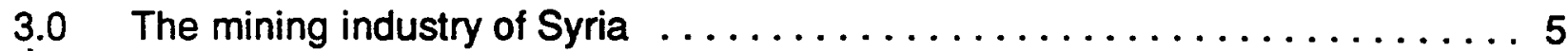

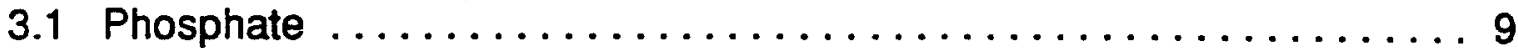

3.2 Other industrial minerals $\ldots \ldots \ldots \ldots \ldots \ldots \ldots \ldots \ldots \ldots \ldots \ldots$

3.3 Other minerals $\ldots \ldots \ldots \ldots \ldots \ldots \ldots \ldots \ldots \ldots \ldots \ldots \ldots$

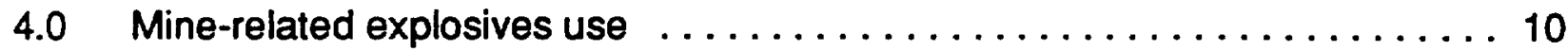

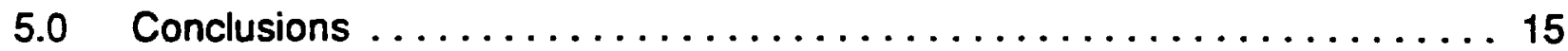

Appendix A: Producing mineral properties in Syria . . . . . . . . . . . 17 Appendix B: Prospects and past producing mineral properties in Syria . . . . . . 19

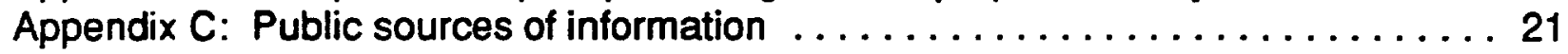

\section{TABLES}

3.1 Estimated mineral production in Syria, 1993 and 1994 . . . . . . . . . . . . . 8

4.1 Estimated explosives usage at the main Syrian mines used in this study in order of estimated ANFO consumption.

FIGURES

Fig. 4.1: Selected Syrian mines and estimated maximum blasting events ... . . . 14 Map A-1: Producing mineral properties in Syria . . . . . . . . . . . . . . 18 Map B-1: Non-producing mineral properties in Syria .............. 20 
• 


\subsection{EXECUTIVE SUMMARY}

This report was prepared under Memorandum of Agreement B291867 between Lawrence Livermore National Laboratory (LLNL) and the U.S. Geological Survey, Minerals Information Team (MIT) (formerly the Office of Minerals Information). This report provides LLNL information on the mining industry of Syria, and quantitative information on the blasting potential of this industry. MIT identified mining activities through the use of its Minerals Availability System data base, its data collection and analytic capabilities, and an extensive network of information sources.

Crude petroleum and phosphate rock production account for the largest share of Syria's income ${ }^{1}$. Ferrous, non-ferrous and industrial minerals also occur and add to the mineral wealth of the country. Research activities for this report resulted in the identification of $\mathbf{2 6}$ mineral properties in Syria, however, most properties operate on a small scale and require minimal blasting. The blasting potential for 13 main Syrian mines is reported, with only 4 operating mines having the potential to produce blasts of over $\mathbf{3 5}$ metric tons of ammonium nitrate-fuel-oil (ANFO) equivalent at any given time as part of normal mining activities.

\footnotetext{
${ }^{1}$ The Mining Journal. Mining Annual Review 1995. London, U.K. PP. 164.
} 
The largest mining sector is phosphate mining, which is generally centered around the deposits of the Khadir Al Hamar region. The region is comprised of 3 operating surface mines, the Khneifess mine and the Shakira $A$ and $B$ which are in the immediate Al Sawanat Al Hamra proximity. These have maximum blasting events in the 35-50 metric ton of ANFO equivalent range.

Syria uses internally produced explosives or imports and possesses the technology and facilities to manufacture blasting agents and detonation systems suitable for mining applications. Most surface mines in Syria utilize ANFO blasting agents. 


\subsection{Authority}

This report was prepared under Memorandum of Agreement B291867 between Lawrence Livermore National Laboratory (LLNL) and the U.S. Geological Survey, MIT.

\subsection{Project Scope}

As part of this agreement, MIT is to identify mining activities in Egypt, Jordan, Syria, Tunisia and Turkey. This information will be used for monitoring/verifying compliance of the Comprehensive Test Ban Treaty. MIT will use the Minerals Availability System data base, the Mineral Resource Data System, its data collection and analytic capabilities, and an extensive network of information sources to provide background information on the use of explosives by the mining industry of these countries. This information is of particular interest because the normal blasting activities of mining can cause false alarms during monitoring and disguise nuclear blast events.

Reports with accompanying figures and tables summarize location, type of mining method, commodity(ies), estimated frequency and size of mine blasts, operational status, and distribution of products to foreign or internal markets for Syria.

Once country data were collected and verified, the explosive use at selected sites was evaluated. Focus was placed on locations that consume large quantities of conventional chemical explosives. Undeveloped sites and small scale mines which consume minimal amounts of explosives (included in Appendix A-B of this report), were not analyzed in terms of the site's anticipated use of explosives. Mineral prospects generally make only small use of explosives and small mines (some of which are operated on an intermittent basis) are assumed to require minimal blasting. Appendix A lists all the known producing mineral properties in Syria, while Appendix B lists all the known non producing properties (past producers and undeveloped deposits) in Syria. These listings were used as the basis from which the main Syrian mines consuming explosives were selected. It should be noted that available data indicated the potential of additional mineral occurrences in Syria, but confirmation of site data 
was not possible. Information on these potential sites was not included in this study.

Based upon known site information (geological conditions, mine technology, production capacity, and current blasting practices), the blasting potential for significant mining sites was evaluated. Where site-specific data were not available, estimates for important properties were developed based upon accepted industry practice, knowledge of the Syrian mining industry, and related geologic characteristics.

\subsection{SOURCES OF INFORMATION}

Data for this report were derived from published sources, unpublished documents, and personal communications through an extensive network of public and private contacts. Public sources of information are listed in Appendix C. Much of the industry summary was drawn from data reported by the U.S. Bureau of Mines Mineral Yearbook chapter on Syria, from the years 1992-1994. Information for 1995 was obtained from the U.S. Geological Survey, Minerals Information Team, International Minerals Section, Reston, Virginia (formerly the U.S. Bureau of Mines, Division of International Minerals).

Principal agencies contacted include, but were not limited to, the U.S. Geological Survey, the U.S. Department of State, the Central Intelligence Agency, the Defense Intelligence Agency, the United Nations, the World Bank, the World Resources Institute, and the International Strategic Minerals Institute (ISMI). In addition, selected academic and industry contacts, explosives manufacturers and suppliers, and trade groups were contacted.

\subsection{THE MINING INDUSTRY OF SYRIA}

The mining industry of Syria is minor when compared to the minerals fuels industry; the leading industrial sector. The estimated gross domestic product (GDP)-purchasing power parity- for Syria in 1994 was $\$ 74.4$ billion (est) ${ }^{2}$. In 1993 , the mineral industry

\footnotetext{
${ }^{2}$ United States Central Intelligence Agency. Syria. CIA-95 facts-Government publications. 10 pp.
} 
accounted for about $\$ 6$ billion of the nation's $\$ 30$ billion gross domestic product ${ }^{3}$. As of 1995 , Syria's credit was in poor condition. Syria has been working to resolve its $\$ 700$ million arrears on debts to foreign states with the individual creditors and with the Paris Club (an institution to assist the severely indebted low-income countries with special repayment terms for debt relief). It was also trying to resolve its $\$ 400$ million debt to the World Bank.

Phosphate rock is the only mineral product beside hydrocarbons which is being produced on a worldwide scale (in 1994 it produced $1 \%$ of world production). Syria increased its export volumes of phosphate rock by $24 \%$ in 1994 . Estimated output was up from a 1993 level of 931,000 metric tons $(\mathrm{mt})$ to $1,200,000 \mathrm{mt}$ in 1994 , but did not reach the 1992 level of $1,270,000 \mathrm{mt}$. Local sales of phosphate rock were not up due to a refitting of the Homs phosphoric acid plant. A proposed chemical plant in the desert could increase mine production potential ${ }^{4}$.

Syria has a socialist economic system but has permitted the emergence of a private sector. The mining industry (mining-processing-distribution) is still owned and controlled by the Government. Legislation adopted in 1994 encouraged domestic and foreign private participation by lifting restrictions on foreign exchange transactions, as well as providing tax and customs concessions. Most private investment in 1994 was directed toward agricultural and tourism industries. Petroleum refining and petrochemical ventures were expected to be the next large industrial segments to utilize private investment.

As shown by table 3.1, other industrial minerals produced include gypsum, hydraulic cement, marble, salt, and sand and gravel. As shown by the table, in 1994 phosphate production was estimated to increase by $261,000 \mathrm{mt}$ from 1993. This 1994 production

\footnotetext{
${ }^{3}$ Michalski, Bernadette. The Minerals Industry of Syria. USBM Yearbook Chapter-1993. PP. 74-77.

${ }^{4}$ The Mining Joumal. Mining Annual Review 1995. London, U.K. PP. 164.
} 
level was not, however, as high as in 1992, which was $70,000 \mathrm{mt}$ higher, for a total production of $331,000 \mathrm{mt}$ in 1992. During the same time the estimated production of crude oil showed a slight increase, from 208,000 barrels to 211,000 barrels in $1994^{5}$. Syrian exports have been dominated by purchasing from the United States for petroleum products and mainly Europe for phosphate rock consumption. Some phosphate rock is exported to India.

${ }^{5}$ Michalski, Bernadette. Syria. Mineral Industry Survey series. USBM. 1994. 
Table 3.1 -- Estimated Mineral Production in Syria, 1993 and 1994 (Metric tons)(1)(2)

\begin{tabular}{|l|r|r|}
\hline \hline Commodity (3) & $\begin{array}{r}1993 \\
\text { Production }\end{array}$ & $\begin{array}{r}1994 \\
\text { Production(e) }\end{array}$ \\
\hline Cement, hydraulic & $4,500,000$ & $4,500,000$ \\
\hline Gypsum & 235,000 & 235,000 \\
\hline - Nitrogen content of ammonia & 66,700 & 66,700 \\
\hline Nitrogen, urea & 75,000 & 75,000 \\
\hline Phosphate rock, gross weight & 931,000 & $1,200,000$ \\
\hline Salt & 130,000 & 130,000 \\
\hline Stone, dimension marble (cu m) & 18,000 & 18,000 \\
\hline Stone, sand \& gravel & $4,200,000$ & $4,200,000$ \\
\hline Sulfur, byproduct of petroleum and natural gas & 30,000 & 30,000 \\
\hline
\end{tabular}

Source: U.S. Bureau of Mines. Minerals Yearbook, 1994.

(e) Estimated

(1) Previously published and 1994 data are rounded by the U.S. Bureau of Mines to three significant digits; may not add to totals shown.

(2) Table includes data available through June 1, 1995.

(3) Limestone quarried for cement manufacture is substantial; however, information is inadequate to make accurate estimates of output. 


\subsection{Phosphate}

Phosphate is mainly produced from the Khadir Al Hamar region of Syria (see Appendix A) from the Shakira A and B mines. The other area of phosphate deposits and mining is near Khneifess, which has one mine currently in operation. Syria exports most of its phosphate rock to countries in Europe where it is further processed. In 1994, local sales of phosphate rock were at a stand still until the chemical plant located at Homs was revamped. A major growth in capacity to the present mines or development of additional mines could occur if a proposed chemical complex in the desert is completed.

\subsection{Other industrial minerals}

Limestone and clays for cement feed are quarried from sites near the plants for usage at the ten cement plants operating in Syria. Total cement production in 1995 was. estimated to be 4.5 million $\mathrm{mt}$. While limestone production was substantial, information is inadequate to make accurate estimates of output.

Gypsum facilities are located at Damascus and Latakia, but specific mine locations are not available.

Salt mines are located south east of Aleppo and near Dayr az-Zawr, with specific locations not available.

Clay, dimension stone, sand and gravel, and sulfur (byproduct of petroleum and natural gas) were also recovered in 1994.

\subsection{Other minerals}

Syria also possesses mineral resources of bituminous rock (natural or rock asphalt), chromium, copper, iron, lead, and sulfur. Presently, these commodities are not being 
mined.

\subsection{MINE RELATED EXPLOSIVES USE}

Syria uses internally produced explosives or imports, and possesses the technology and facilities to manufacture blasting agents and detonation systems suitable for mining applications. Most surface mines in Syria utilize ammonium nitrate-fuel oil (ANFO) blasting agents.

Most mines utilize explosives to loosen or fragment rock and other consolidated material prior to excavation. Bulk or packaged explosives are detonated either electrically or thermally after being placed in the material to be excavated. Minor quantities of sachet (bagged powder explosives) and shaped charges may be used for secondary breakage and other special applications.

The type and amount of explosives used are influenced by the nature of the rock or ore, the mining methods employed, the production rate of the mine, the type and availability of explosives and detonation systems, hydrologic conditions, mining equipment, drilling equipment, mine geometry, level of technical expertise, and external constraints such as the proximity of residences and costs. At almost any mine, the size of each blast can vary significantly due to local conditions, production schedules, weather, etc.

Most Syrian mines use ANFO blasting agents where blasting is required. ANFO systems are preferred in most mining applications due to their ease of manufacture, low cost, inherent safety, and bulk loading advantages.

In most cases, site-specific blasting information was unaccessible: Consequently; estimates were based upon estimated production rates, mine geology, mining systems, and typical mining practices. Experience, engineering judgement, and available data were incorporated into calculations and estimates. Explosive use can vary considerably as mining conditions change. ANFO consumption was assumed to be 
dependent upon mine production rate, average stripping ratio, specific gravity of the host rock, assumed powder factor limits, and mining method.

For each site, a stripping ratio (quantity of overburden or waste removed per metric ton of ore mined) and powder factor (quantity of rock blasted per unit of ANFO blasting agent equivalent) limits were estimated. A range of ANFO consumption was calculated for both daily blasting requirements and an assumed maximum blasting event. Daily ANFO requirements were estimated using a 312 day/year production schedule. Consumption estimates for all sites were calculated in a similar manner. The lower consumption value applies a minimum powder factor while the higher value assumes a maximum powder factor. Unlike daily consumption estimates, a maximum blasting event would not take place on a daily basis. For this study, it was assumed that the maximum blasting event (an estimated technical upper limit of cumulative explosive usage) would consume 10 days worth of explosives for a surface mine and.5.days worth for an underground mine. Such events are designed to account for such factors as blasting delays, geological irregularities, and mining method variations that require a higher ANFO consumption than the typical blasting event. Mine development or pillar extraction conditions, for example, often require larger blasts than normal production.

The following examples illustrate typical blasting calculations for the Sharkaya A mine using the estimation procedure described above:

Sharkaya A mine ANFO daily consumption, lower limit (L):

$L=$ Production rate * $[1+$ (stripping ratio * (combined specific gravity of ore and waste))]

* [Low powder factor / 1000 (converts $\mathrm{kg}$ to $\mathrm{mt}$ )] / 312 (assumed operating days per year)

$L=790,000 *[1+(2.8 * 2.5)]^{*}[0.11 / 1000] / 312$

$\mathrm{L}=2: 232 \mathrm{mt}$ ANFO equivalent (rounded to nearest unit)

Sharkaya A mine ANFO daily consumption higher limit (H): 
$\mathrm{H}=$ Production rate * $[1+$ (stripping ratio *(combined specific gravity of ore and waste))] * [High powder factor / 1000 (converts $\mathrm{kg}$ to $\mathrm{mt}$ )] / 312 (assumed operating days per year)

$H=790,000 *[1+(2.8 * 2.5)] *[0.20 / 1000] / 312$

$\mathrm{H}=4.054 \mathrm{mt}$ ANFO equivalent (rounded to nearest unit)

Sharkaya A mine maximum blasting event ANFO consumption (M):

$M=$ Production rate * $[1+$ stripping ratio * (combined specific gravity of ore and waste))] - [High powder factor / 1000 (converts $\mathrm{kg}$ to $\mathrm{mt}$ )] / 312 (assumed operating days per year) * maximum blast cycle time (working days between blasting events) $M=[790,000 *[1+(2.8 * 2.5)] *[0.2 / 1000]] / 312 * 10$ $M=40.541 \mathrm{mt}$ ANFO equivalent (Rounded to nearest unit)

Table 4.1 provides the corresponding blasting range estimates for the main Syrian mines identified in this study. Figure 4.1 shows site locations for the mines reported in Table 4.1. Symbols reflect the maximum ANFO consumption for a given blasting event. 
Table 4.1-Estimated Explosives Usage at the Main Syrian Mines Used in this Study in Order of Estimated ANFO Consumption

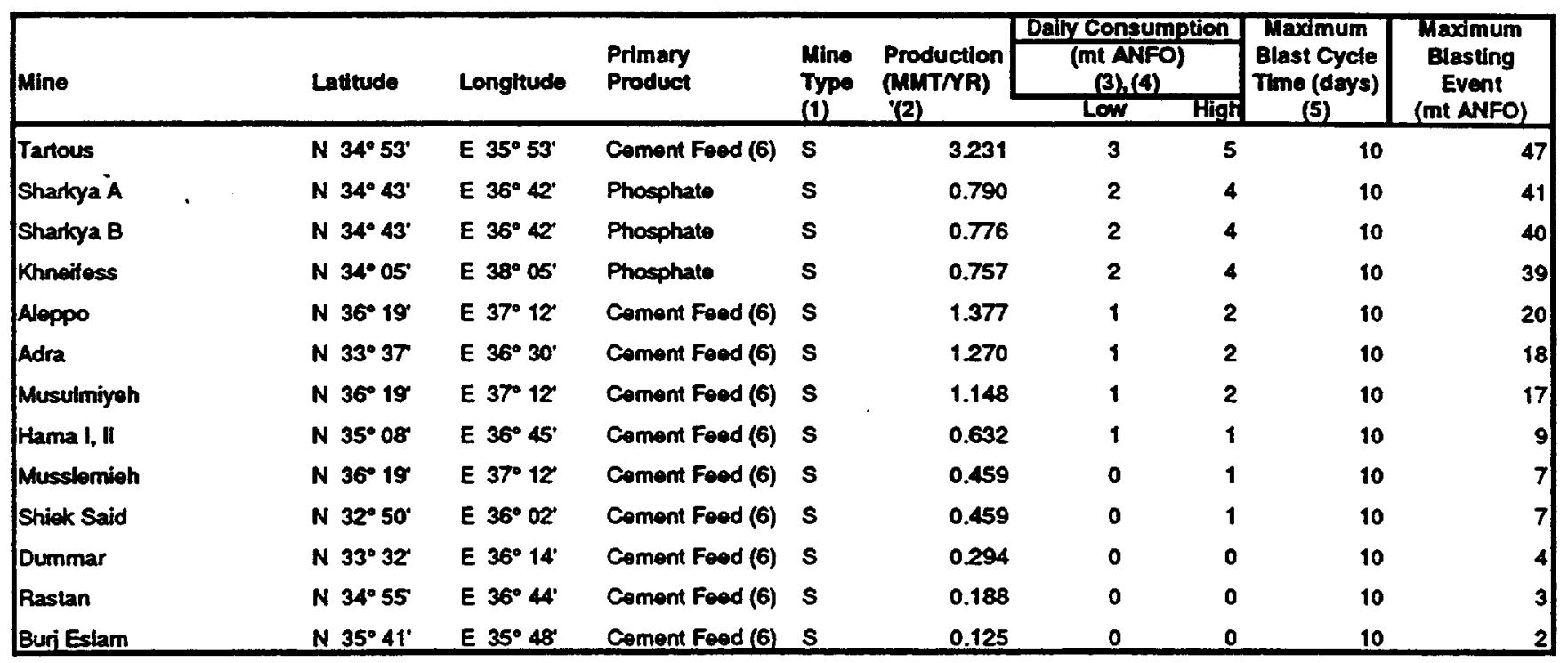

(1) S- Surtace

(2) Mmetyr-Mition motric tons per year

(3) MT ANFO-Motric tons of Ammonium Nitrate/Fuel Oil blasting agent equivalent

(4) Assumed annual production schodule of 312 days per yoar, results may differ if another production schodule used.

(5) Assumed maximum blasting cycle time for surface operation - 10 working days.

(6) Typical raw material foed for coment plart is $85 \%$ limestone and $15 \%$ clay, minor amounts of gypsum and other materials may also be required. 


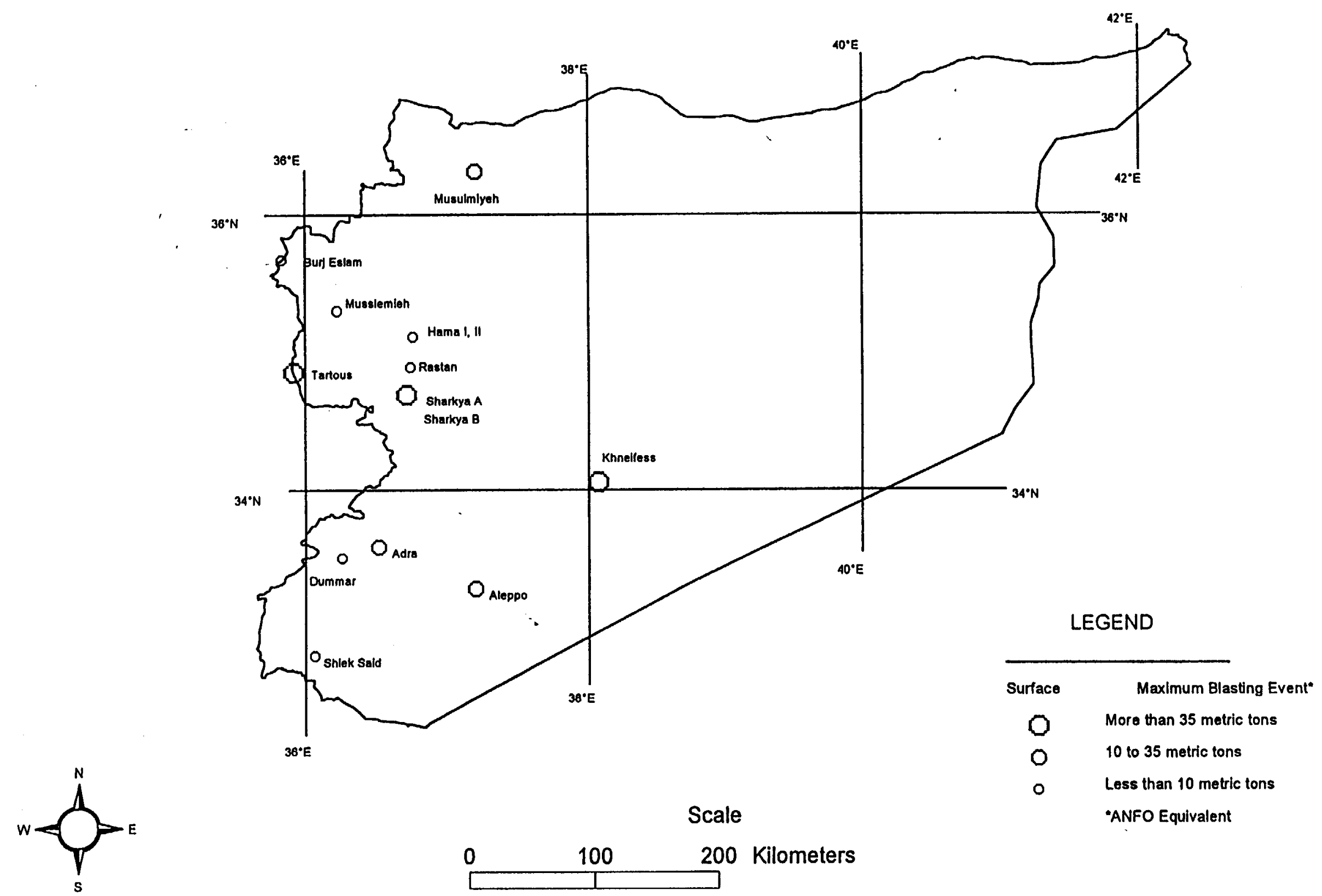

FIGURE 4.1--Selected Syrian Mines and Estimated Maximum Blasting Events 


\subsection{CONCLUSIONS}

Mining in Syria is dominated in the Khadir Al Hamal region (Sharkaya A and B Mines) by phosphate rock mining. These are primarily surface operations. In the Khadir AI Hamal region, maximum blasting events for surface operations are in the 35-50 metric ton of ANFO equivalent range.

While only a few individual sites possess the capacity to produce regularly scheduled blasts over the 35 metric ton (ANFO equivalent) range, there are several areas where multiple surface mines are operating. Hence, it could be feasible for operators to fire several individual mine blasts at the same time, thus effectively creating a larger event. 
APPENDICES 
APPENDIX A: PRODUCING MINERAL PROPERTIES IN SYRIA

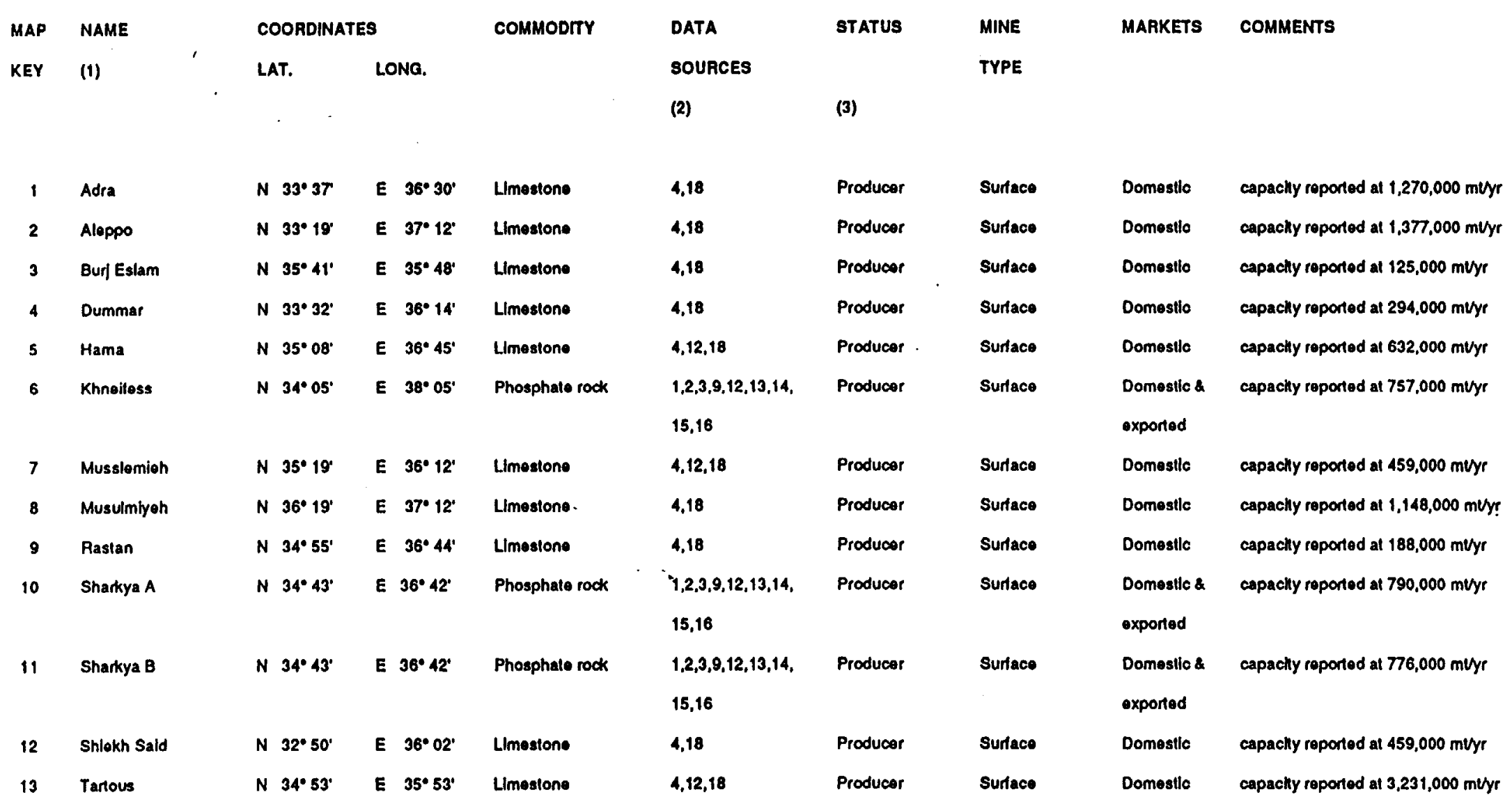

(1) Due lo soltware limhations, she names do not include any diactilical markings. Spollings ol Individual stte names vary considerably by source.

(2) Complete list of data sources is shown in Appendix C.
(3) Becauso of the varying age of source inlormation, the status at Individual shes may not be current. 


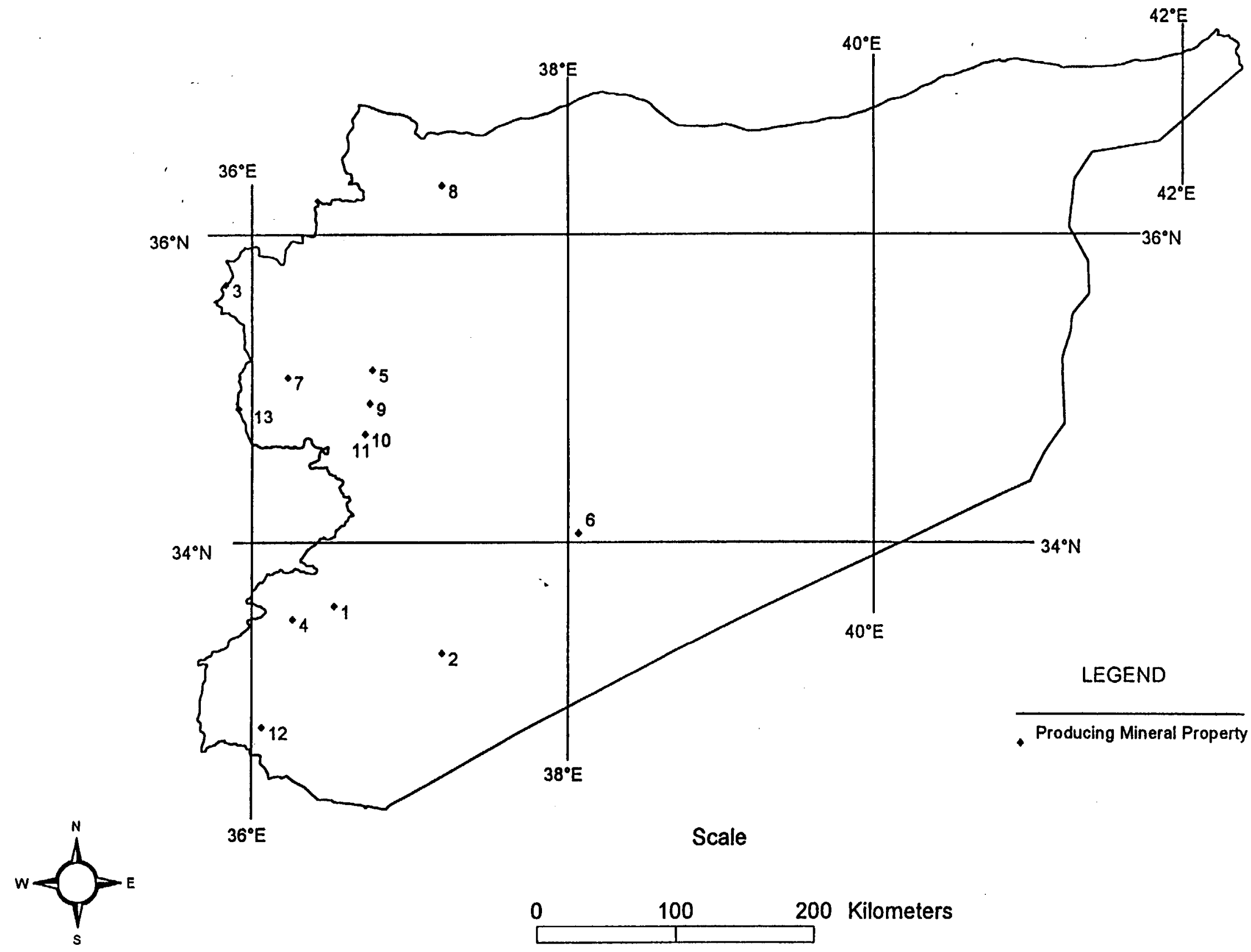

APPENDIX MAP A-1: PRODUCING MINERAL PROPERTIES IN SYRIA 
APPENDIX B: NON PRODUCING MINERAL PROPEATIES IN SYAIA

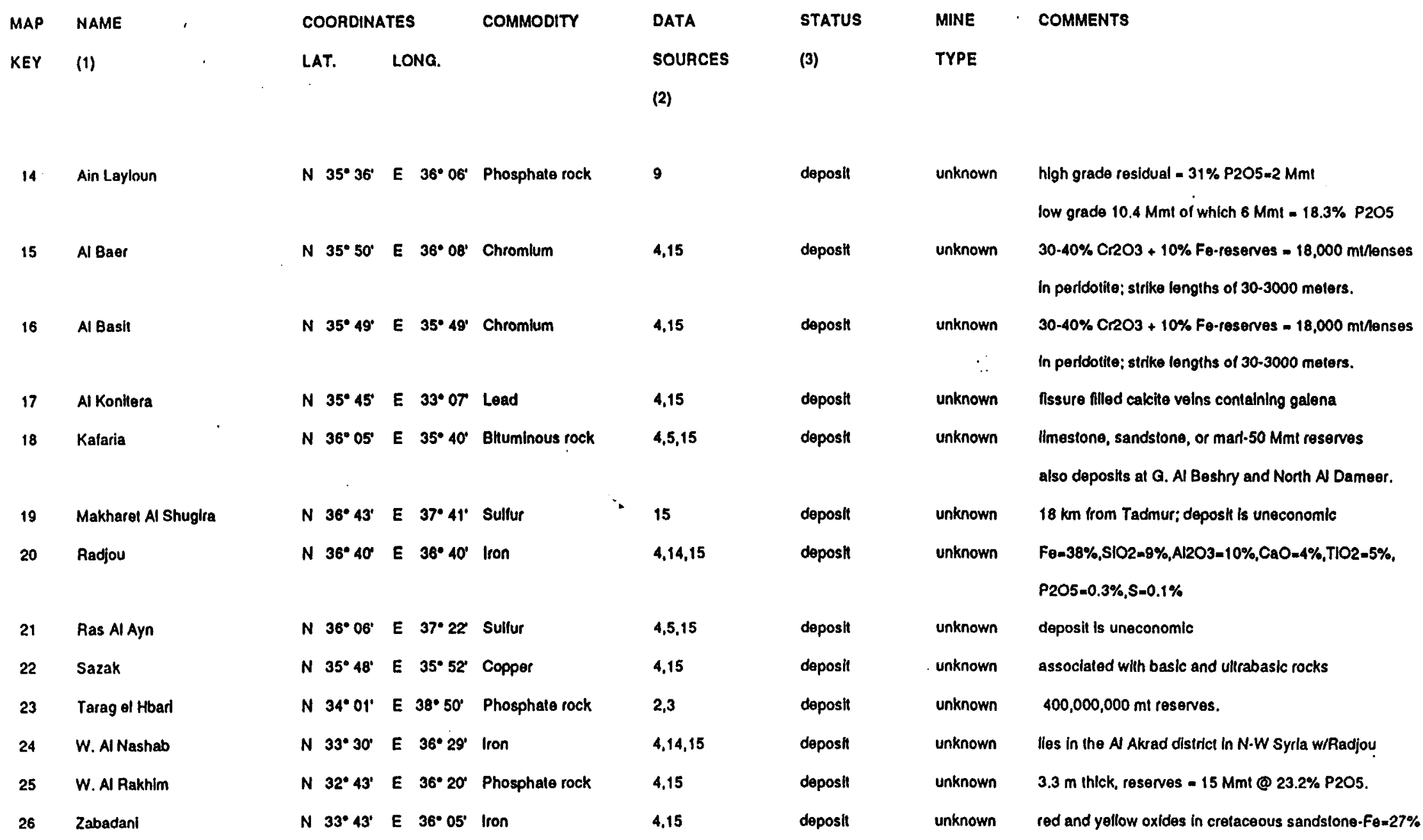

(1) Due to soltware limitations, site names do not Include any diacrillcal markings. Spellings of individual site names vary considerably by source. (2) Complete list of data sources is shown in Appendix C.

(3) Because of the varying age of source inlormation, the slatus at individual sites may nol be current. 


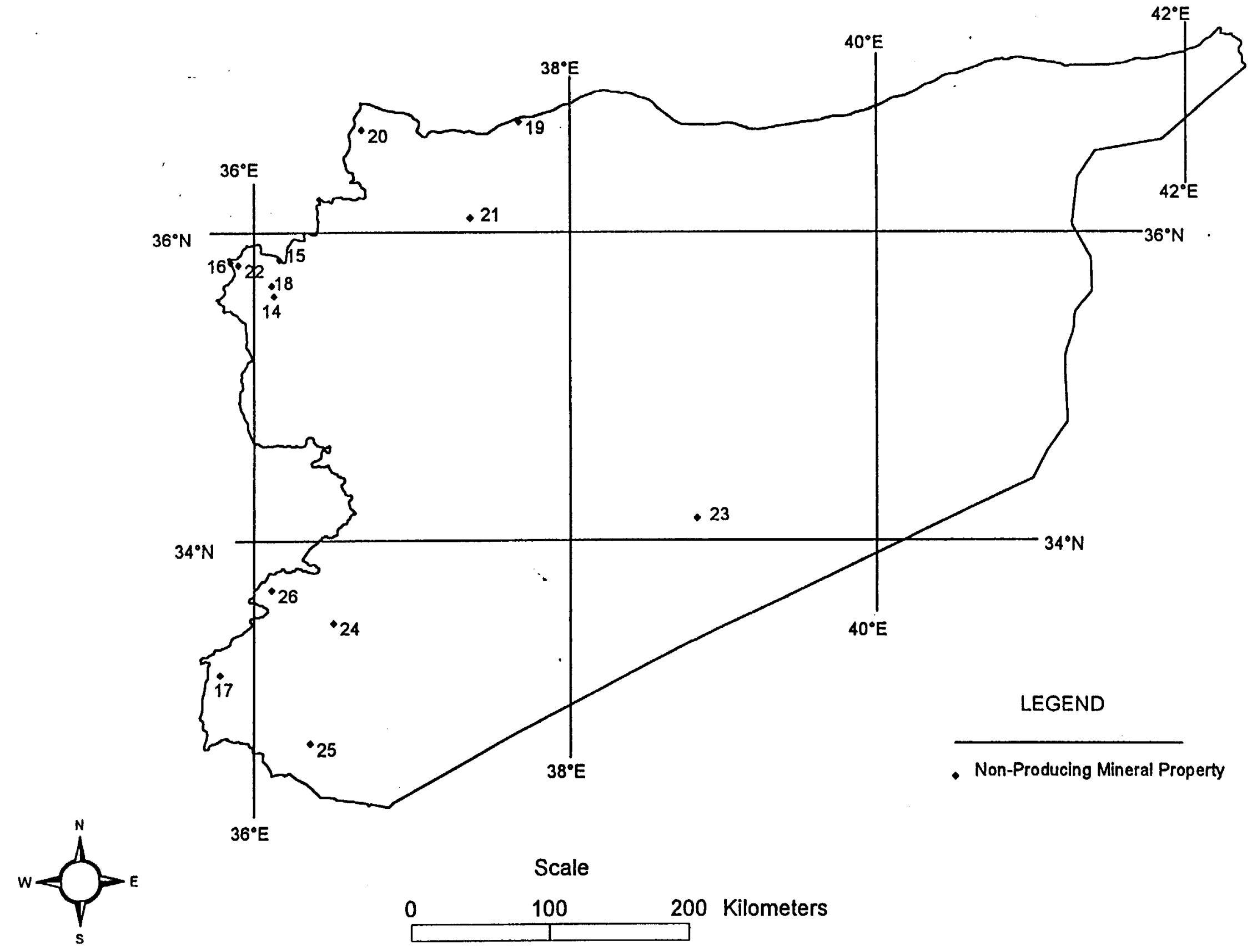

APPENDIX MAP B-1: NON-PRODUCINIG MINERAL PROPERTIES IN SYRIA 


\section{APPENDIX C: PUBLIC SOURCES OF INFORMATION}

1.--Omara, S. Phosphatic Deposits in Syria and Safaga District, Egypt. Economic Geology. Vol. 60. 1965. PP. 214-227.

2.--U.S. Geological Survey. MRDS The Mineral Resource Data System. (1996)

3.--U.S. Geological Survey. MAS The Minerals Availability System. (1996)

4.---Defense Mapping Agency. Gazateer of Syria-Second Edition. Washington, D.C. 1983.

5.--Defense Mapping Agency. Operational Navigation Chart-1:1,000,000. ONC G-4. 1974.

6.--Michalski, Bemadette. Syria. Mineral Industry Survey series. USBM. 1994.

7.--Michalski, Bernadette. The Minerals Industry of Syria. USBM Yearbook Chapter-1993. PP. 74-77.

8.--Collelo, Thomas. ed. Syria-A Country Study. Foreign Area Studies The American University. 1986. PP. 146-153, 160-163.

9..---The British Sulfur Corporation Limited. Syria. World Survey of Phosphate Deposits. 1987. PP. 1731.78.

10.--The Mining Journal. Mining Annual Review 1994. London, U.K. P. 161.

11.- The Mining Journal. Mining Annual Review 1995. London, U.K. PP. 164.

12.-Michalski, Bernadette. The Mineral Industry of Tunisia. USBM Yearbook Chapter 1992. PP. 90-98.

13.--Atteh, S. The Phosphorite Resources of Syria. Phosphate Deposits of the World Vol 2. Ed. A. G. J. Northolt, R.P. Sheldon, D.F. Davidson. Cambridge University Press 1989. PP. 357-362.

14.--Habashi, Fathi and Foad A. Bassyouni. Mineral Resources of the Arab Countries. Department of Mining \& Metallurgy-Laval University, Quebec City, Canada 1978. PP. 100-102.

15.--Habashi, F. and F. A. Bassyouni. Mineral Resources of the Arab Countries-2nd ed. Department of Mining \& Metallurgy-Laval University, Quebec City, Canada 1982. PP. 49-51.

16.--Khawlie, M. R. Beyond the Oil Era-Arab mineral resources and future development. Mansell

Publishing Ltd. London. 1990. 132 pp.

17.-United States Central Intelligence Agency. Syria. ClA-95 facts-Government Publications. 6 pp.

18.-World Cement Directory 1991. Cimeurop s.a.r.l., 4 pp. 
Tunisia 

Table of Contents

Page

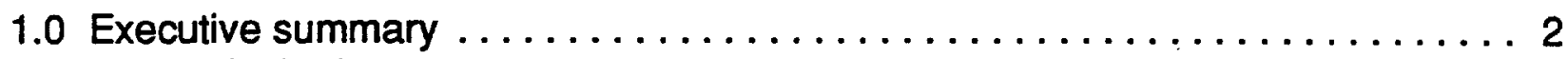

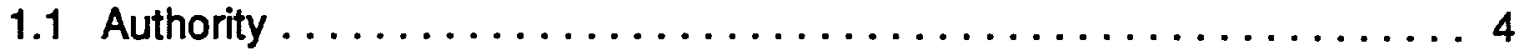

1.2 Project scope $\ldots \ldots \ldots \ldots \ldots \ldots \ldots \ldots \ldots \ldots \ldots \ldots \ldots \ldots$

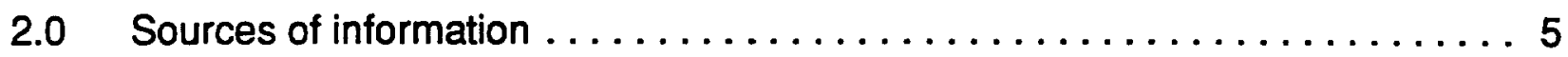

3.0 The mining industry of Tunisia $\ldots \ldots \ldots \ldots \ldots \ldots \ldots \ldots \ldots \ldots \ldots \ldots \ldots \ldots$

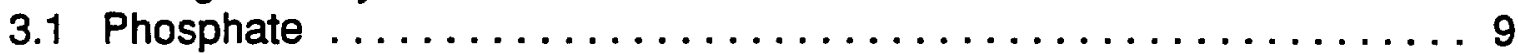

3.2 Other industrial minerals $\ldots \ldots \ldots \ldots \ldots \ldots \ldots \ldots \ldots \ldots \ldots$

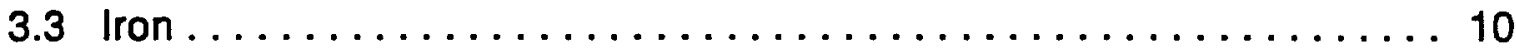

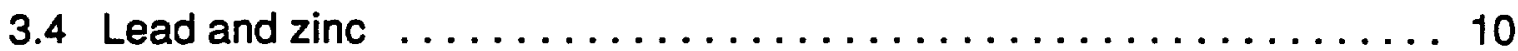

3.5 Other minerals $\ldots \ldots \ldots \ldots \ldots \ldots \ldots \ldots \ldots \ldots \ldots \ldots \ldots \ldots \ldots$

4.0 Mine-related explosives use $\ldots \ldots \ldots \ldots \ldots \ldots \ldots \ldots \ldots \ldots \ldots \ldots \ldots \ldots$

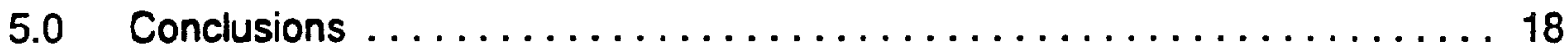

Appendix A: Producing mineral properties in Tunisia $\ldots \ldots \ldots \ldots \ldots \ldots \ldots 20$

Appendix B: Prospects and past producing mineral properties in Tunisia . . . . . 22

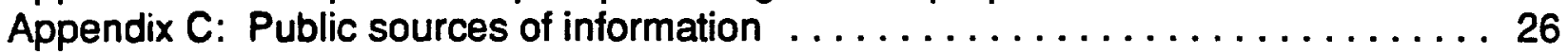

\section{TABLES}

3.1 Estimated mineral production in Tunisia, 1993 and $1994 \ldots \ldots \ldots \ldots$. . . . . 8

4.1 Estimated explosives usage at the main Tunisian mines used in this study in order of estimated ANFO consumption $\ldots \ldots \ldots \ldots \ldots \ldots \ldots \ldots \ldots \ldots \ldots \ldots \ldots \ldots$

FIGURES

Fig. 4.1: Selected Tunisian mines and estimated maximum blasting events . . . . 17 Map A: Producing mineral properties of Tunisia ................. 21

Map B: Prospects and undeveloped mineral properties of Tunisia $\ldots \ldots \ldots \ldots 25$ 


\subsection{EXECUTIVE SUMMARY}

This report was prepared under Memorandum of Agreement B291867 between Lawrence Livermore National Laboratory (LLNL) and the U.S. Geological Survey, Minerals Information Team (MIT)( formerly the Office of Minerals Information). It provides LLNL information on the mining industry of Tunisia, and quantitative information on the blasting potential of this industry. MIT identified mining activities through the use of its Minerals Availability System data base, its data collection and analytic capabilities, and an extensive network of information sources.

Crude petroleum and phosphate rock production accounts for the largest share of Tunisia's income '. Ferrous, non-ferrous and industrial minerals also add to the mineral wealth of the country. Research activities resulted in the identification of 135 mineral properties in Tunisia, however, most properties operate on a small scale and require minimal blasting. The blasting potential for 21 main Tunislan mines is reported, with only 4 operating mines having the potential to produce blasts of over 75 metric tons of ANFO equivalent at any given time as part of normal mining activities.

The largest mining sector is phosphate mining, which is generally centered

\footnotetext{
${ }^{1}$ The Mining Journal. Mining Annual Review 1995. London, U.K. PP. 154-155.
} 
around the low grade deposits of the Gafsa region. The region is comprised of 9 operating mines, 8 of which are in the Immediate Gafsa proximity and the Kalaa Khasba mine, which is further to the north.

Tunisia uses internally produced explosives or imports and possesses the technology and facilities to manufacture blasting agents and detonation systems suitable for mining applications. Most surface and underground mines in Tunisia utilize ammonium nitrate-fuel oil (ANFO) blasting agents. High explosives may be used in a few small underground operations where geologic conditions and characteristics require its use. 


\subsection{Authority}

This report was prepared under Memorandum of Agreement B291867 between Lawrence Livermore National Laboratory (LLNL) and the U.S. Geological Survey, MIT.

\subsection{Project Scope}

As part of this agreement, MIT is to identify mining activities in Egypt, Jordan, Syria, Tunisia and Turkey. This information will be used for monitoring/verifying compliance of the Comprehensive Test Ban Treaty. MIT will use the Minerals Availability System data base, its data collection and analytic capabilities, and an extensive network of information sources to provide background information on the use of explosives by the mining industry of these countries. This information is of particular interest because the normal blasting activities of mining can cause false alarms during monitoring and disguise nuclear blast events.

Reports with accompanying figures and tables summarize location, type of mining method, commodity(ies), estimated frequency and size of mine blasts, operational status, and distribution to foreign or internal markets for the specified countries.

Once country data were collected and verified, the explosive use at selected sites was evaluated. Focus was placed on locations that consume large quantities of conventional chemical explosives. Undeveloped sites and small scale mines which consume minimal amounts of explosives (included in Appendix A-B of this report), were 
not analyzed in terms of the site's anticipated use of explosives. Mineral prospects generally make only small use of explosives and small mines (some of which are operated on an intermittent basis) are assumed to require minimal blasting. Appendix A lists all the producing and developing mineral properties in Tunisia, while Appendix B lists all the known past producers and undeveloped deposits in Tunisia. These listings were used as the basis from which the main Tunisian mines consuming explosives were selected.

Based upon known site information (geological conditions, mine technology, production capacity, and current blasting practices), the blasting potential for significant mining sites was evaluated. Where site-specific data were not available, estimates for representative, important properties were developed based upon accepted industry practice, knowledge of the Tunisian mining industry, and related geologic characteristics.

\subsection{SOURCES OF INFORMATION}

Data for this report were derived from published sources, unpublished documents, and personal communications through an extensive network of public and private contacts. Public sources of information are listed in Appendix C. Much of the industry summary was drawn from data reported by the U.S. Bureau of Mines Mineral Yearbook chapter on Tunisia, from the years 1992-1994. Information for 1995 was obtained from the U.S. Geological Survey, Minerals Information Team, International Minerals Section, Reston, 
VA (formerly the U.S. Bureau of Mines, Division of International Minerals).

Principal agencies contacted include, but were not limited to, the U.S. Geological Survey, the U.S. Department of State, Central Intelligence Agency, Defense Intelligence Agency, the United Nations, the World Bank, World Resources Institute, and the International Strategic Minerals Institute (ISMI). In addition, selected academic and industry contacts, explosives manufacturers and suppliers, and trade groups were contacted.

\subsection{THE MINING INDUSTRY OF TUNISIA}

The mining industry of Tunisia is minor compared to the minerals fuels industry, the leading industrial sector. The estimated gross domestic product (GDP)-purchasing power parity- for Tunisia in 1994 was $\$ 37.1$ billion (est) ${ }^{2}$. In 1994 , the mining industry activity accounted for about $\$ 300$ million in foreign exchange ${ }^{3}$. Of the minerals and metals produced in Tunisia, only phosphate is produced on a scale to be considered important by world standards. In 1994 , approximately $80 \%$ of the 12,000 people employed in the mining sector in Tunisia were employed in the phosphate industry4. The Government parastatal Compagnie des Phosphates de Gafsa (CPG), founded in 1896 entirely controls phosphate production.

\footnotetext{
${ }^{2}$ United States Central Intelligence Agency. Tunisia. CIA-95 facts-Government Publications. 6 pp.

${ }^{3}$ Dolley, Thomas P. The Minerals Industry of Tunisia. MIS Mineral Industry Survey series. USBM 1994. 5 pp.

${ }^{4}$ lbid.
} 
As shown by table 3.1, other industrial minerals produced include barite, hydraulic cement, construction clays, gypsum, lime and marine salt. Metal production includes iron ore and pig iron, lead and zinc, but production is not large on a worldwide basis. As shown by the table, in 1994 phosphate production increased slightly from 1993. During the same time however, the production of crude oil showed a slight decline ${ }^{5}$. The production of acid grade fluorspar ceased in 1992. Tunisian trade has been dominated by the European Union (EU) (75\%) - generally exporting mostly to France ( $30 \%$ of total trade) and importing mostly from Italy. India has, however, accounted for about $50 \%$ of Tunisia's phosphoric acid exports. In 1994 phosphate rock exports increased from 1.1 Mmt in 1993 to $1.3 \mathrm{Mmt}$. In Tunisia, most mining and mineral processing plants are state owned, while cement plants were in the process of being privatized in the Tunisian economy. The Tunisian legal system, which is based on French civil law, had a unified investment code introduced in 1993 to help facilitate foreign investment in the minerals industry, with further legislation being planned. Tunisia has also determined that its industry should take a leadership role in the environment, among north African nations. The Government's plan includes a forestry program; a 10-year action plan for water and soil conservation; a national strategy to combat desertification; and a national action program to combat pollution, preserve the country's archaeological heritage, protect the marine environment and beaches and preserve the quality of life of the urban and rural populations.

${ }^{5}$ Dolley, Thomas P. The Minerals Industry of Tunisia. MIS Mineral Industry Survey series. USBM 1994. 5 pp. 
Table 3.1 -- Estimated Mineral Production in Tunisia, 1993 and 1994 (Metric tons)

\begin{tabular}{||l|r|r|}
\hline Commodity (1) & $\begin{array}{r}1993 \\
\text { Production }\end{array}$ & $\begin{array}{r}1994 \\
\text { Production(e) }\end{array}$ \\
\hline INDUSTRIAL MINERALS & & \\
\hline Barite & 15,300 & 15,700 \\
\hline Cement, hydraulic & $3,300,000$ & $3,300,000$ \\
\hline Clay, construction & 350,000 & 350,000 \\
\hline Gypsum & 100,000 & 100,000 \\
\hline Lime & 600,000 & 600,000 \\
\hline Phosphate rock, gross weight & $5,500,000$ & $5,660,000$ \\
\hline Salt, marine & 435,000 & 414,000 \\
\hline METALS & & \\
\hline Iron ore \& concentrate, gross weight & 299,000 & 240,000 \\
\hline Iron, pig iron & 140,000 & 140,000 \\
\hline Lead mine output, Pb content & 863 & 4610 \\
\hline Zinc mine output. Zn content & 1,350 & 15,000 \\
\hline
\end{tabular}

Source: U.S. Bureau of Mines. Minerals Yearbook, 1994. E/Estimated

(1) Previously published and 1994 data are rounded by the U.S. Bureau of Mines to three significant digits; may not add to totals shown.

(2) In addition to the commodities listed, a variety of crude construction materials (common clays, sand and gravel, and stone) is produced, but output is not reported, and available information is inadequate to make reliable estimates of output levels. Limestone quarried for cement manufacture is substantial; however, information is inadequate to make accurate estimates of output. 


\subsection{Phosphate}

Phosphate is mainly produced from the Gafsa region of Tunisia (see Appendix A) which has operating mines. The other area of phosphate deposits and mining is the TebessaThala area, which has two mines in operation. Tunisia exports most of its phosphates as phosphoric acid to countries in Europe, Asia and Africa. These phosphoric acid plants are located near the ports of Stax, La Skhira and Gabes. In 1994, Tunisia was the world's fifth largest producer of phosphate in the world with a $4 \%$ share of the world's output. Making up only $5 \%$ of world reserves (3.5 to 4 billion metric tons), and $1 \%$ of Tunisia's

's GDP in 1994, phosphates are still the most important mineral commodity in Tunisia's economy.

CPG has proposed the development of another open pit at Moulares by 1996.

\subsection{Other industrial minerals}

Limestone and other cement feed is quarried for usage at the seven cement plants operating in Tunisia. Total cement capacity in 1996 is 5.29 million metric tons per year. The government has recently announced that the cement industry will be sold to the private sector in the coming months. Since 1977 , four new cement plants have come on line. This, added to the diversification of products, the start up of hydraulic lime (sets and hardens under water) factories at Thala in 1987 and introduction of a white cement (Portland cement with a low iron content) work at Feriana in 1988, and advanced technology has provided for a rapid progression of the overall profitability of 
the industry. An important element of Tunisia's cement profitability is the distribution of factories throughout the country, assuring each plant an appropriate market share. In 1995, a quarter of national production of clinker and cement was sent as exports, earning US\$60 Million in hard currency.

Salt is also recovered near Sousse, although at a small rate and primarily for local usage. Barite, clay and gypsum were also recovered in 1994.

\subsection{Iron}

The four main areas of iron deposition in Tunisia are 1) Djerissa, (Djerissa mine) near the Algerian frontier; 2) Tamera, near the coast and the Algerian frontier; 3) Douaria, near Tamera (Tamera-Douaria mine), and 4) Djebel Ank in the Gafsa region. The Tamera-Douaria mine is currently being operated as a surface-underground operation. The Djerissa mine is operated as an underground operation, and was scheduled to close in 1997. However the operators, Societe du Djebel Djerissa (SDD), stated that it could have an extended mine life by utilizing modern mining equipment.

\subsection{Lead and Zinc}

Lead and zinc are mined in Tunisia on a small scale at the 3 Compagnie Miniere du Nord-Ouest (COMINO) mines. The Bougrine Mine, which came into production in 1994 and has an expected production life of 15 years at 350,000 metric tons per year, is the largest producer of $\mathrm{Pb}-\mathrm{Zn}$ in Tunisia. It employs about 300 people and has reported 
minable reserves of 5.3 million metric tons grading $11.7 \%$ zinc and $2.6 \%$ lead. It utilizes drift and fill and sublevel mining. It was the only private sector mine in Tunisia in 1995. Construction took 18 months and included two mine entrances, twin declines, underground drifts and raises, the installation of fixed mining equipment, an underground explosives magazine, and electrical facilities. The ore is disseminated in the micron-size range within metasomatized galena and sphalerite. The other operating mines are small, and production only amounts to about $13,000 \mathrm{mt}$ combined contained lead and zinc output. The owner, Metall Mining Corp., and the Government are continuing to explore for other carbonate or sediment-hosted-lead-zinc deposits similar to Bougrine.

\subsection{Other minerals}

Tunisia possesses additional mineral resources of copper, fluorspar, barite, mercury, lignite, uranium and vanadium. Presently, these commodities are not being mined. Copper exploration is underway at the Ghardimaou-Cap Serrat fault in northern Tunisia.

\subsection{MINE RELATED EXPLOSIVES USE}

Tunisia uses internally produced explosives or imports and possesses the technology and facilities to manufacture blasting agents and detonation systems suitable for mining applications. Most surface and underground mines in Tunisia utilize ammonium nitratefuel oil (ANFO) blasting agents. High explosives may be used in a few small 
underground operations where geologic conditions and characteristics require its use.

Most mines utilize explosives to loosen or fragment rock and other consolidated material prior to excavation. Bulk or packaged explosives are detonated either electrically or thermally after being placed in the material to be excavated. Minor quantities of sachet (bagged powder explosives) and shaped charges may be used for secondary breakage and other special applications.

The type and amount of explosives used are influenced by the nature of the rock or ore, the mining methods employed, the production rate of the mine, the type and availability of explosives and detonation systems, hydrologic conditions, mining equipment, drilling - equipment, mine geometry, level of technical expertise, and external constraints such as the proximity of residences, and costs. At almost any mine, the size of each blast can vary significantly due to local conditions, production schedules, weather, etc.

Surface mines typically shoot much larger blasts than underground operations and tend to have higher production rates than underground mines. "Tn addition, limitations of working room, limited free faces, ventilation requirements, and drilling limitations may constrain maximum blast sizes in underground mines.

Most Tunisian mines use ANFO blasting agents where blasting is required. ANFO systems are preferred in most mining applications due to their ease of manufacture, low 
cost, inherent safety, and bulk loading advantages.

In most cases, site-specific blasting information was unaccessible. Consequently, estimates were based upon estimated production rates, mine geology, mining systems, and typical mining practices. Experience, engineering judgement, and available data were incorporated into calculations and estimates. Explosive use can vary considerably as mining conditions change. ANFO consumption was assumed to be dependent upon mine production rate, average stripping ratio, specific gravity of the host rock, assumed powder factor limits, and mining method.

For each site, a stripping ratio (quantity of overburden or waste removed per metric ton of ore mined) and powder factor (quantity of rock blasted per unit of ANFO blasting agent equivalent) limits were estimated. A range of ANFO consumption was calculated for both daily blasting requirements and an assumed maximum blasting event. Daily ANFO requirements were estimated using a 330 day/year production schedule. Consumption estimates for all sites were calculated in a similar manner. The lower consumption value applies a minimum powder factor while the higher value assumes a maximum powder factor. Unlike daily consumption estimates, a maximum blasting event would not take place on a daily basis. For this study, it was assumed that the maximum blasting event (an estimated technical upper limit of cumulative explosive usage) would use 10 days worth of explosives for a surface mine and 5 days worth for an underground mine. Such events are designed to account for such factors as 
blasting delays, geological irregularities, and mining method variations that require a higher ANFO consumption than the typical blasting event. Mine development or pillar extraction conditions, for example, often require larger blasts than normal production.

The following examples illustrate typical blasting calculations for the Djellabia mine using the estimation procedure described above:

Djellabia mine ANFO daily consumption, lower limit (L):

$L=$ Production rate * $[1+\text { (stripping ratio * ( specific gravity of ore + waste)) }]^{*}$ [Low powder factor / 1000 (converts $\mathrm{kg}$ to $\mathrm{mt}$ )] / 330 (assumed operating days per year) $L=1,478,000 *[1+(4.0 * 2.8)] *[0.11 / 1000] / 330$ $L=6.016 \mathrm{mt}$ ANFO equivalent (rounded to nearest unit)

Diellabia mine ANFO daily consumption higher limit (H): $\mathrm{H}=$ Production rate * $[1+$ (stripping ratio *(specific gravity of ore + waste) $)]$ * $[$ High powder factor / 1000 (converts $\mathrm{kg}$ to $\mathrm{mt}$ )] / 330 (assumed operating days per year) $H=1,478,000 *[1+(4.0 * 2.8)] *[0.20 / 1000] / 330$ $\mathrm{H}=10.9311 \mathrm{mt}$ ANFO equivalent (rounded to nearest unit)

Djellabia mine maximum blasting event ANFO consumption (M): $M=$ Production rate * $[1+$ stripping ratio *(specific gravity of ore + waste $))]^{*}[$ High powder factor / 1000 (converts $\mathrm{kg}$ to $\mathrm{mt}$ )] / 330 (assumed operating days per year) * 
maximum blast cycle time (working days between blasting events)

$M=[1,478,000 *[1+(4.0 * 2.8)] *[0.2 / 1000]] / 330 * 10$

$M=109.3109 \mathrm{mt}$ ANFO equivalent (Rounded to nearest unit)

Table 4.1 provides the corresponding blasting range estimates for the main Tunisian mines identified in this study. Figure 4.1 shows site locations for the mines reported in Table 4.1. Symbols reflect the mine type (surface or underground) and maximum ANFO consumption for a given blasting event. 
Table 4.1-Estimated Explosives Usage at the Main Tunisian Mines Used in this Study in Order of Estimated ANFO Consumption

\begin{tabular}{|c|c|c|c|c|c|c|c|c|c|}
\hline Mine & Lathude & Longltude & $\begin{array}{l}\text { Primary } \\
\text { Product }\end{array}$ & $\begin{array}{l}\text { Mlne } \\
\text { Type } \\
\text { (1) }\end{array}$ & $\begin{array}{r}\text { Production } \\
\text { (Mmthy) } \\
\text { (Z) }\end{array}$ & $\begin{array}{r}\text { Dalty Con } \\
\text { (mt } 1 \mathrm{x} \\
\text { (3). } \\
\text { Low }\end{array}$ & Tlon: & $\begin{array}{c}\text { Maximum } \\
\text { Blast Cyele } \\
\text { Thme (days) } \\
\text { (5) }\end{array}$ & $\begin{array}{l}\text { Taxdmum } \\
\text { Blasting } \\
\text { Event } \\
\text { (mt ANFO) }\end{array}$ \\
\hline Djellabla & N $36^{\circ} 09^{\prime}$ & E $08^{\circ} 25^{\prime}$ & Phosphate & $\mathbf{s}$ & 1.478 & 6 & 11 & 10 & 109 \\
\hline Kel Eddour & $\mathrm{N} 34^{\circ} 01^{\circ}$ & E $08^{\circ} 25^{\prime}$ & Phosphate & $\mathbf{S}$ & 1.393 & 6 & 10 & 10 & 103 \\
\hline Kel Eschatalr ' & $\mathbf{N} 34^{\circ} 23^{\prime}$ & E $08^{\circ} 28^{\circ}$ & Phosphate & $\mathbf{S}$ & 1.293 & 5 & 10 & 10 & 96 \\
\hline Sra Ouertane & N $36^{\circ} 30^{\circ}$ & E $0^{\circ} 30^{\prime}$ & Phosphate & $\mathbf{s}$ & 1.178 & 5 & $\mathbf{9}$ & 10 & 87 \\
\hline Redeyer & $N 34^{\circ} 22$ & E $00^{\circ} 25^{\prime}$ & Phosphate & $\mathbf{S}$ & 0.921 & 4 & 7 & 10 & 68 \\
\hline Sousse & $N 35^{\circ} 42^{\circ}$ & E $10^{\circ} 38^{\prime}$ & Cement Feed (7) & $\mathbf{s}$ & 1.530 & 1 & 2 & 10 & 22 \\
\hline Jebel Oust & N $36^{\circ} 28^{\circ}$ & E $10^{\circ} 14^{\circ}$ & Cement Feed (7) & $\mathbf{s}$ & 1.354 & 1 & 2 & 10 & 19 \\
\hline Tadjerouine & $N 35^{\circ} 54^{\prime}$ & E $08^{\circ} 34^{\prime}$ & Cement Foed (7) & $\mathbf{S}$ & 1.148 & 1 & 2 & 10 & 16 \\
\hline Gabes & $N 33^{\circ} 53^{\prime}$ & E $10^{\circ} 06^{\circ}$ & Cement Feed (n) & $\mathbf{S}$ & 0.979 & 1 & 1 & 10 & 14 \\
\hline Djerissa & $N 5^{\circ} 50^{\circ}$ & E $08^{\circ} 39^{\circ}$ & Iron & $\mathbf{S}$ & 0.240 & 1 & 1 & 10 & 11 \\
\hline Jebel-Jelloud & $N 36^{\circ} 55^{\circ}$ & E $10^{\circ} 10^{\circ}$ & Cement Feed (7) & $\mathbf{s}$ & 0.612 & 0 & 1 & 10 & 9 \\
\hline Tamera-Douarla(6) & $N 36^{\circ} 49^{\circ}$ & $E 08^{\circ} 41^{\circ}$ & Iron & S-UG & 0.246 & 1 & 1 & 10 & 5 \\
\hline Elzerte & N $37^{\circ} 10^{\circ}$ & E $09^{\circ} 50^{\circ}$ & Cement Feed (7) & $\mathbf{s}$ & 0.383 & 0 & 1 & 10 & 5 \\
\hline Ferlano & $\mathbf{N} 34^{\circ} 53^{\prime}$ & E $08^{\circ} 30^{\circ}$ & Cement Feed (7) & $\mathbf{s}$ & 0.306 & 0 & 0 & 10 & 4 \\
\hline Sehib (Sector 10) & $N 34^{\circ} 12^{\circ}$ & E $08^{\circ} 41^{\prime}$ & Phosphate & UG & 1.397 & 0 & 1 & 5 & 4 \\
\hline MrRata & $N 34^{\circ} 28^{\circ}$ & E $08^{\circ} 08^{\circ}$ & Phosphate & UG & 1.280 & 0 & 1 & 5 & 4 \\
\hline Bougrine (8) & N $36^{\circ} 30^{\circ}$ & E $08^{\circ} 30^{\circ}$ & Leed, Zlnc & UG & 0.628 & 0 & 0 & 5 & 2 \\
\hline Moulares & $\mathbf{N} 34^{\circ} 29^{\prime}$ & E $08^{\circ} 16^{\prime}$ & Phosphate & UG & 0.732 & 0 & 0 & 5 & 2 \\
\hline MOilla & $N 34^{\circ} 18^{r}$ & $E 08^{\circ} 46^{\prime}$ & Phosphate & UG & 0.490 & 0 & 0 & 5 & 1 \\
\hline Motalaoui & $N 34^{\circ} 19$ & E $08^{\circ} 25^{\prime}$ & Phosphate & UG & 0.475 & 0 & 0 & 5 & 1 \\
\hline Kalaa Mrasba & N $35^{\circ} 40^{\circ}$ & E $08^{\circ} 40^{\prime}$ & Phosphate & UG & 0.233 & 0 & 0 & 5 & 1 \\
\hline
\end{tabular}

(1) S-Surtace; UG-Underground

(2) Mrntyr-Milion metric lons per yeer

(3) mt ANFO-Metic cons of Ammonlum Nibrateffuel Oll blasting aoent equival en

(4) Assumed annual production schedule of 330 days per year, results may differ $\mathrm{H}$ another production schedule used.

(5) Assumed maxhum blasting cycle time tox surtace operation - 10 working days; underground operation - 5 worktng days.

(6) Site presently operates using surtace methods only, atthough underground methods were used in the past.

(7) Typical raw materiad feed tor cement plant is $85 \%$ limestone and $15 \%$ clay; minor amounts of gypsum and other materlals may also be required.

(8) As of Cotober 1996, Sodete Mnlere Bougrine has Indefinitely suspended operations at Bougrine due to current zinc price levels. 


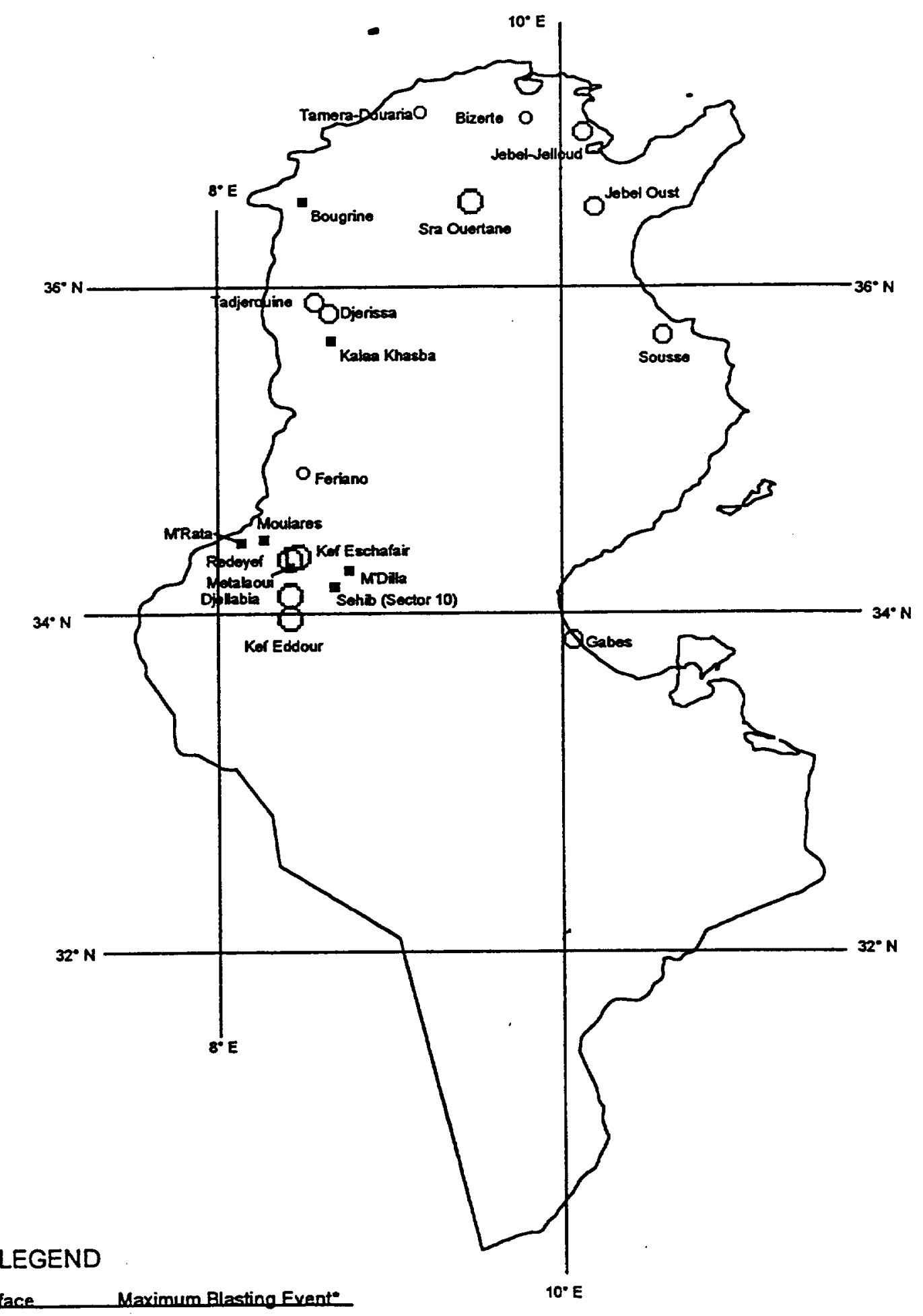

Underaround Sudace

Maximum Blastino Event*

(D) Mote than 50 metric tons

O 9 to 50 metric tons

-
- Less than 9 metric tons
-ANFO Equivalent
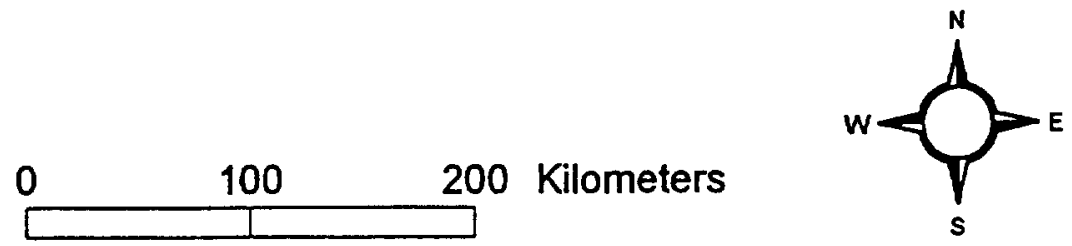

FIGURE 4.1--Selected Tunisian Mines and Estimated Maximum Blasting Events 


\subsection{CONCLUSIONS}

Mining in Tunisia occurs primarily in the Gafsa region(circle $G$ on Map A) for phosphate extraction. These are primarily surface operations. Most underground operations have maximum blasting events under 5 metric tons of ANFO equivalent per event at an individual site. Surface mining operations are generally on a larger scale. In the Gafsa region, maximum blasting events for surface operations are in the 65-110 metric ton of ANFO equivalent range.

While only a few individual sites possess the capacity to produce regularly scheduled blasts over 75 metric tons (ANFO equivalent), there are several areas where multiple surface mines are operating. Hence it could be feasible for operators to fire several individual mine blasts at the same time, thus effectively creating a larger event. 


\section{APPENDICES}


APPENDIX A: PRODUCINO OR DEVELOPINO MINERAL PROPERTIES IN TUNISIA

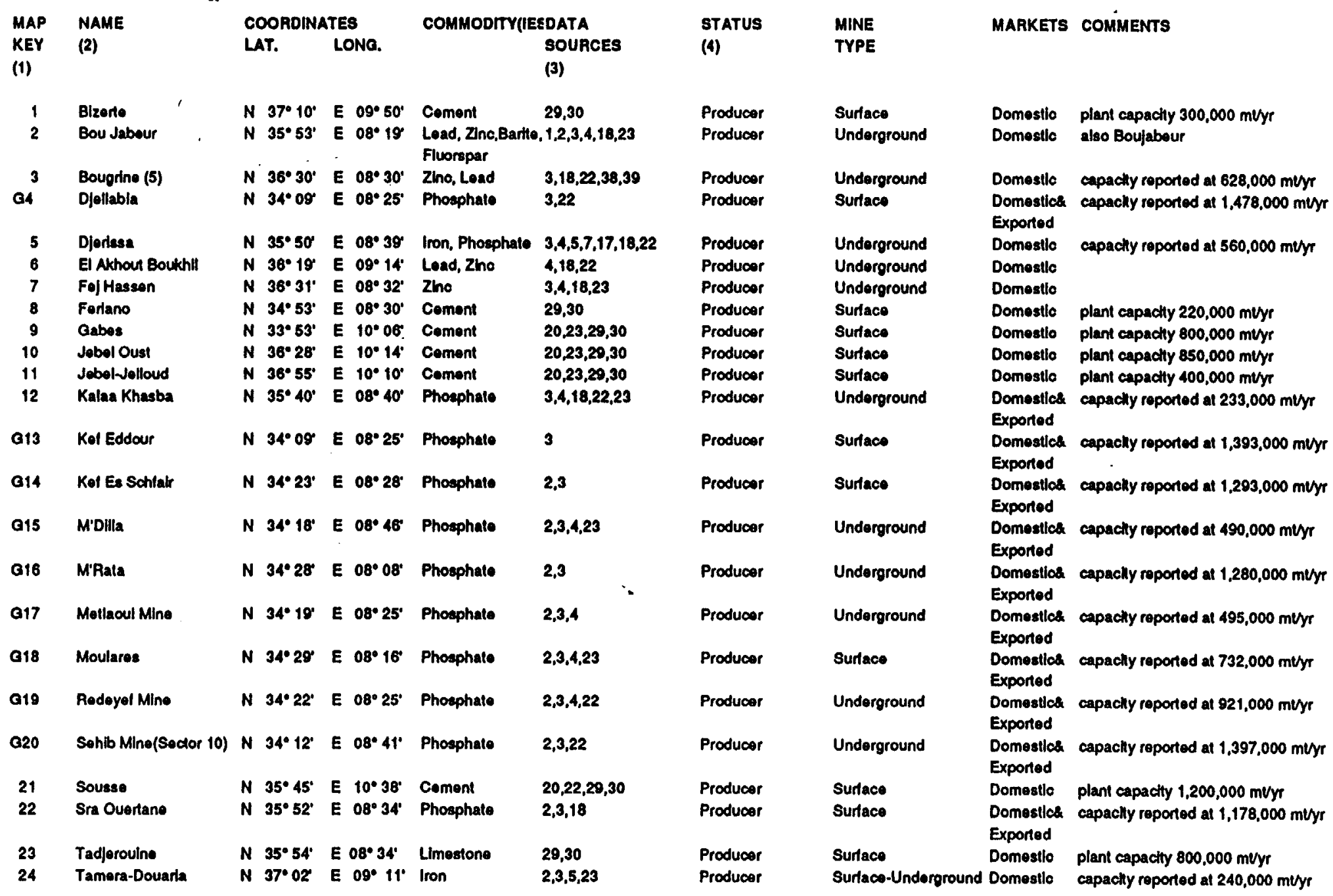

(1) Represents property or property grouping as defined on Appendix map A.

(2) Due to software limitations, site names do not include any diacritical markings. Spellings of individual site names vary considerably by source.

(3) Complete list of data sources found in Appendix C.

(4) Because of the varying age of source information, the status at individual sites may not be current.

(5) As of October 1996, Societe Miniere Bougrine has indefinitely suspended operations at Bougrine due to current zine price levels. 


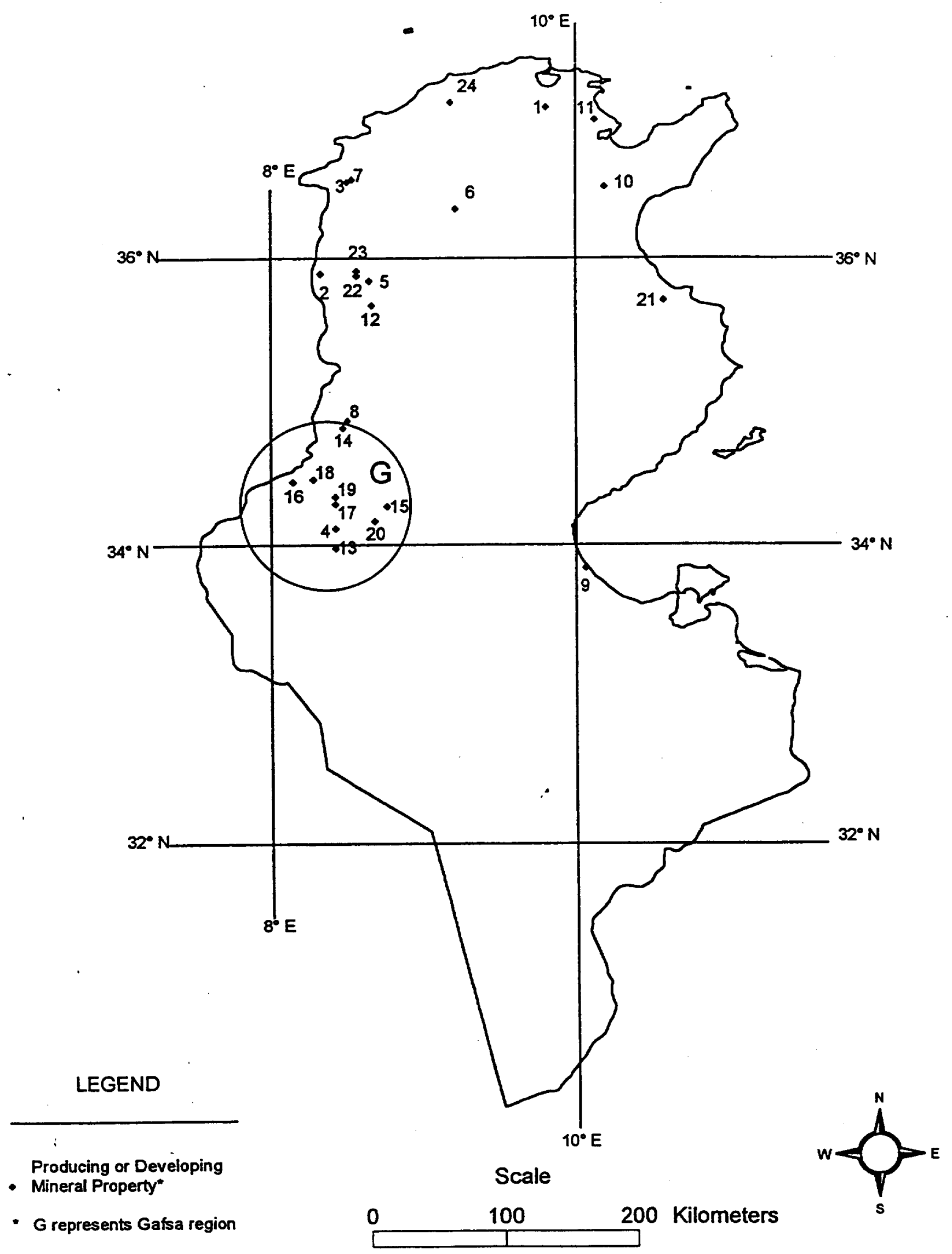

APPENDIX MAP A-1: PRODUCING MINERAL PROPERTIES OF TUNISIA 
APPENDIX B: PAST PRODUCERS, UNDEVELOPED DEPOSITS AND UNKNOWN MINERAL PROPERTES OF TUNISIA

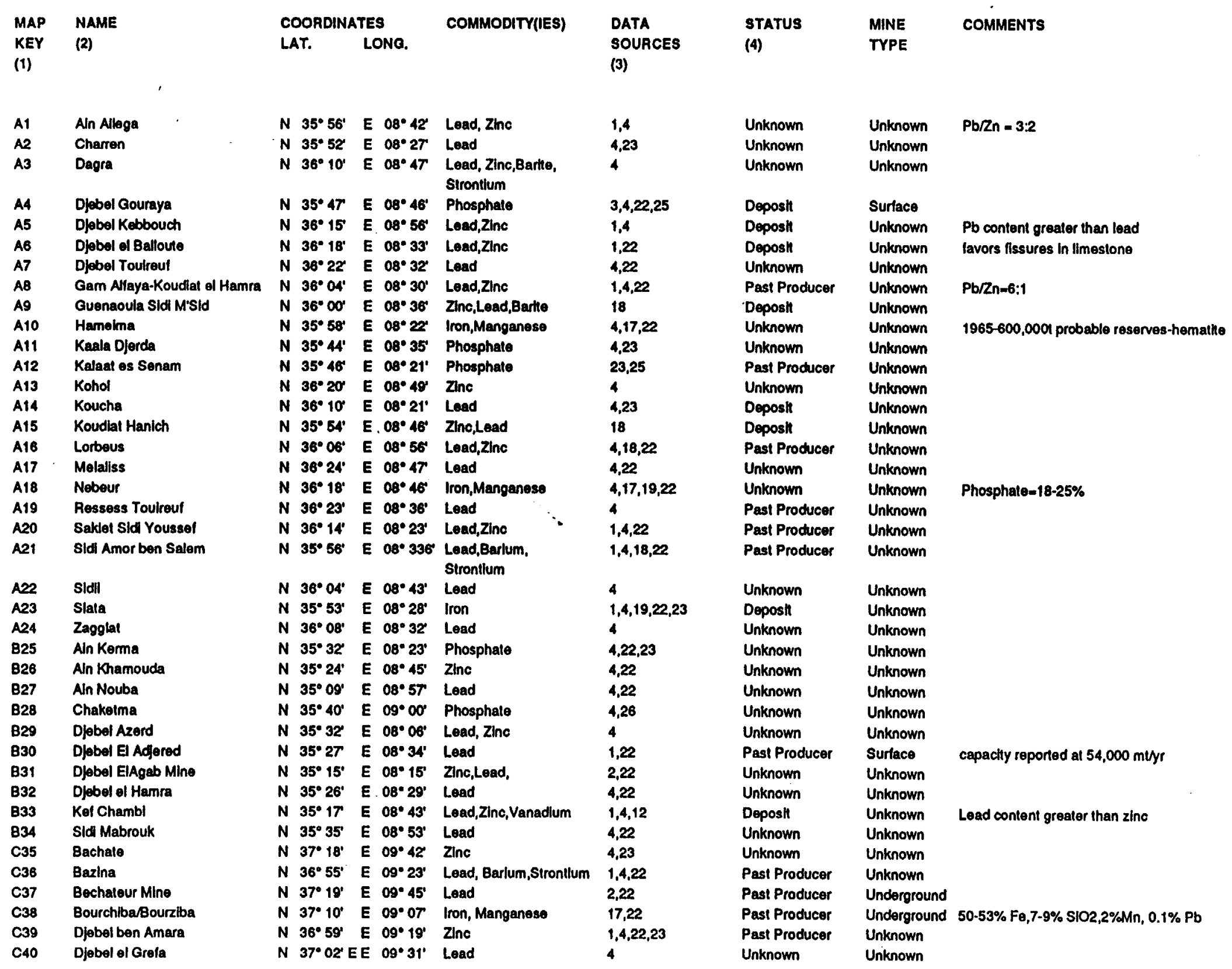


APPENDIX B: PAST PRODUCERS, UNDEVELOPED DEPOSITS AND UNKNOWN MINERAL PROPERTIES OF TUNISIA

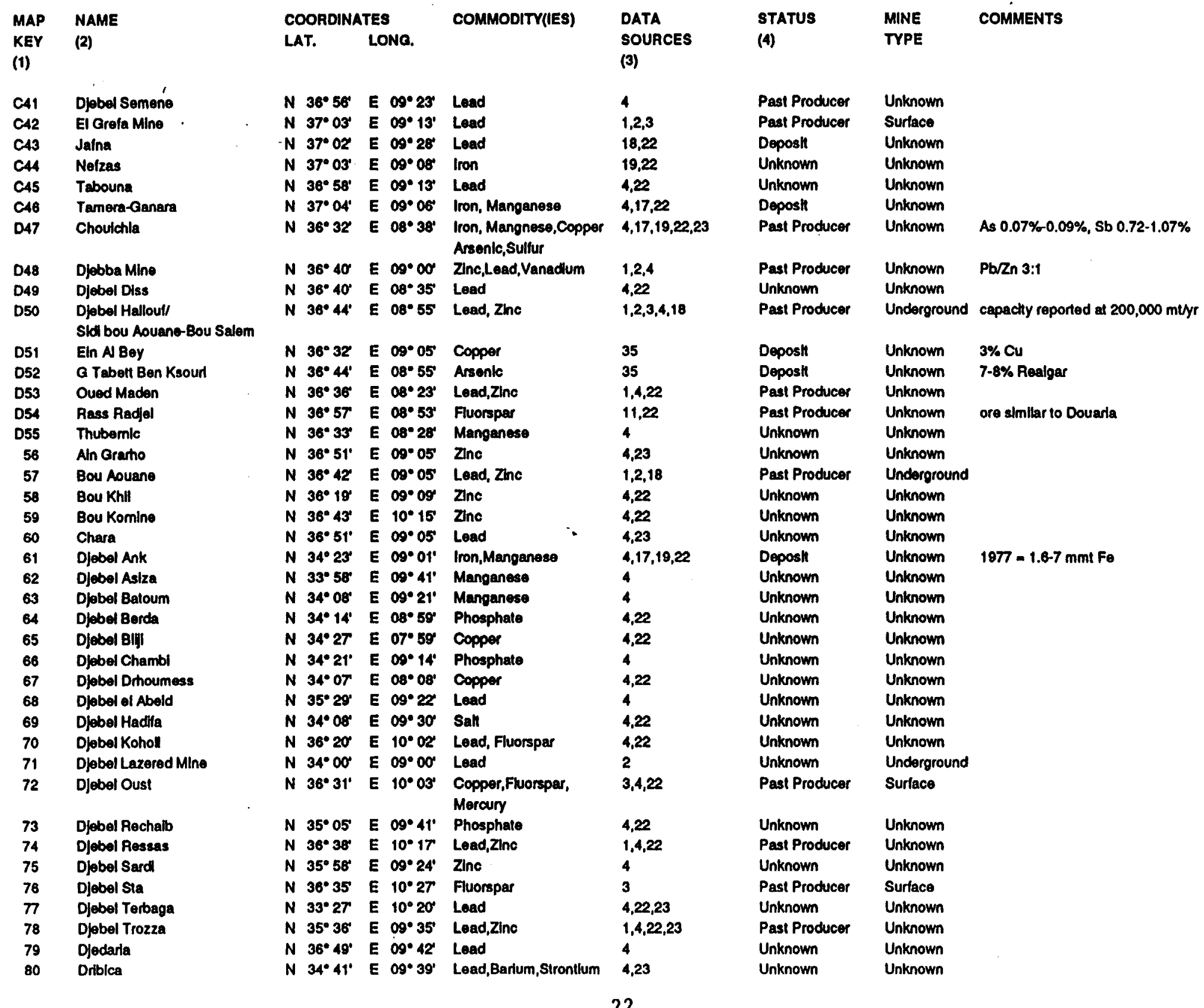


APPENDIX B: PAST PRODUCERS, UNDEVELOPED DEPOSITS AND UNKNOWN MINERAL PROPERTIES OF TUNISIA

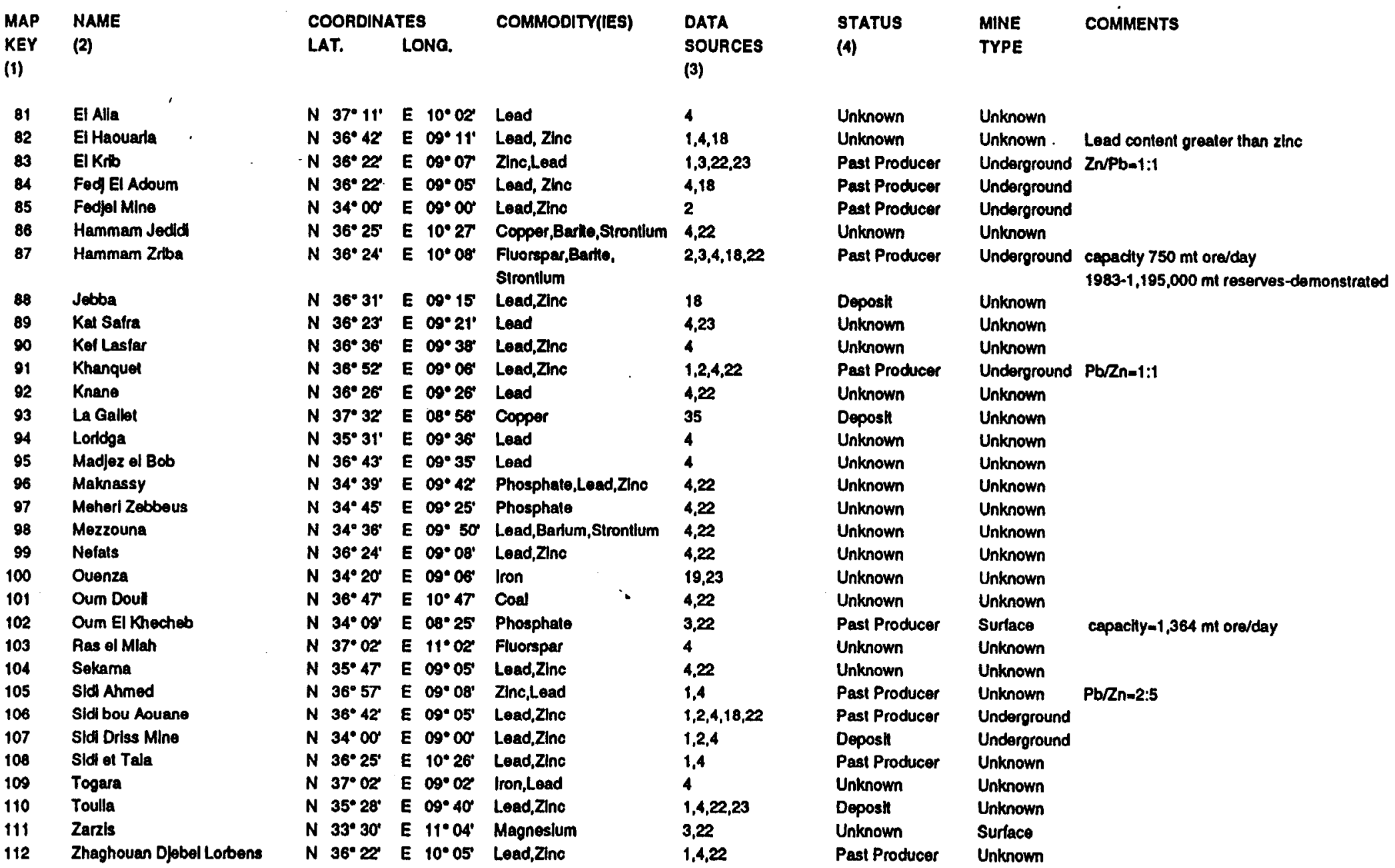

(1) Represents property or property grouplng as defined on Appendix map B.

(2) Due to software limitations, site names do not include any diacritical markings. Spellings of individual site names vary considerably by source.

(3) Complete list of data sources found in Appendix C.

(4) Because of the varying age of source Information, the status at individual sites may not be current. 


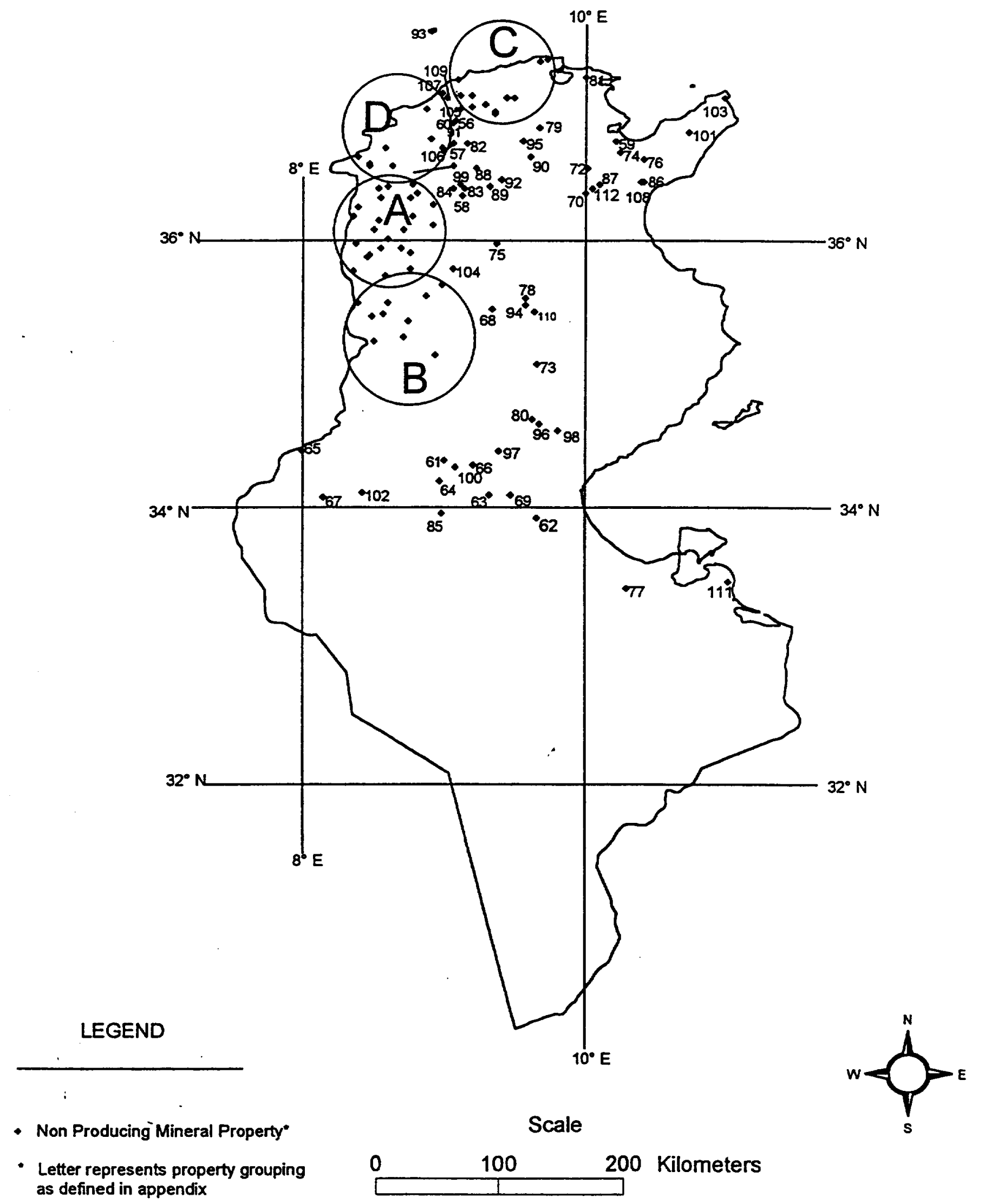




\section{APPENDIX C: PUBLIC SOURCES OF INFORMATION}

1.--The Mineral Resources of Africa. 1965. PP. 385-387.

2.--U.S. Geological Survey. MRDS The Mineral Resource Data System.

3.--U.S. Geological Survey. MAS The Minerals Availability System.

4.--Sainfeld, Paul. The Lead-Zinc bearing Deposits of Tunisia. Economic Geology. 1956. PP. 150-177.

5.-United Nations. Survey of World Iron Resources-Occurrence and Appraisal. New York, 1970. PP. 62-67

6.---Slim-Shimi, Najet and Said Tlig. Mixed type sulfide deposits in Northern Tunisia, regenerated in relation to paleogeography and tectonism. Journal of African Earth Sciences, Vol. 16, No. 3, 1993. PP.287-307.

7.-Pohl, W., M. Amouri, O. Kolli, and D. Zachmann. A new genetic model for the North African metasomatic siderite deposits. Mineralium Deposita 21, 1986. PP. 228-233.

8.-Burkhart-Bauman, I., J. Ottenman and P. Nicolini. Mineralogische Untersuchungen an Jordanit, Semseyit und Cesarolith von drei tunesischen Blei-Zink-Lagerstaetten. Chemie der Erde 1967. PP 256259.

9.-Reese, Howard C., Thomas D. Roberts, Barbara Bamford, Sharon Camp, Grant V. Mc Lanahan and Taine Tompkins. Area Handbook for the Republic of Tunisia-1970. PP. 19-21, 276-281.

10.-Duwaji, Ghazi. Economic Development in Tunisia-The Impact and Course of Government Planning. Fr. Praeger, Publishers.

11.-Kuhn, Olin R. Africa's Iron Ore--An Unknown Quantity. May 14, 1927. P. 803.

12.-Shea. John C. Guidebook to the Geology and History of Tunisia. Petroleum Exploration Society of Libya-Ninth Annual Field Conference-1967-Map.

13.-Svoboda, K. Phosphates of Tunisia. Industrial Minerals. December 1984. PP. 37-47.

14.-Dolley. Thomas P. The Minerals Industry of Tunisia. USBM Yearbook Chapter-1993. PP. 189-193.

15. - Belayouni, Habib, Maurice Slanski and Jean Trichet. A study of the organic matter in Tunisian phosphate series: Relevance to phosphorite genesis in the Gafsa Basin (Tunisia). Organic Geochemistry, Vol. 15. No. 1. 1990. PP. 47-51.

16. - Ognar. S. Geothermometrie des Concentrations Plombo-Zinciferes Dans le Cretace de Tunise Centrale. Bull, Centre Rech. Pau - SPNA, December 31, 1969. PP. 377-399.

17.-Walther, H. W., and A. Zitzman ed. The Iron Ore Deposits of Europe and Adjacent Areas. International Geological Conference, Hanover 1977. PP. 303-308.

18. - Orgeval, Jean-Jaques, Denis Giot, Jalia Karoui, Jaques Monthel, and Rachel Sahli. Le Gisement de $\mathrm{Zn}$-Pb de Bou Grine (Atlas tunisien). Description et hitorique'de la decouverte. Chron. Rech. Min. No. 482, March 1986. PP. 5-32.

19.- Massin, J. M. Le champ ferrifere de Nebeur. Contribution a Vinventaire des resources minieres de la Tunisie. Bulletin du B.R.G.M. (Deuxieme serie) Section II, no. 4, 1970. PP. $29-33$.

20.-Dolley. Thomas P. The Minerals Industry of Tunisia. MIS Mineral Industry Survey series. USBM 1994. 5 pp.

21.-Nelson, Harold D. Tunisia-A Country Study. Foreign Area Studies. The American University. 1986. PP. 148-149, 180-195.

22.--Defense Mapping Agency. Tunisia-Official Standard Names Gazateer No. 81. 1964.

23.--Defense Mapping Agency. Operational Navigation Chart-1:1,000,000. ONC G-2. Edition G 1967.

24.--Power, Tim. Tunisia-Developing Downstream Industries. International Minerals. July 1986. PP. 20-

27.

25.--Tig, S. Rare earth redistribution and fractionation during secondary mineral formation: examples from reef carbonate karst (Jebel Stah, Tunisia) and from oceanic basalts (site 417A, Nothem Atlantic). Journal of African Earth Sciences, Vol. 13, No. 2, 1991. PP. 173-182.

26..- The British Sulfur Corporation Limited. Tunisia. World Survey of Phosphate Deposits. 1987. PP. 108-114

27. - The Mining Journal. Mining Annual Review 1994. London, U.K. P. 155.

28.--The Mining Journal. Mining Annual Review 1995. London, U.K. PP. 154-155. 
29.--Industrial Minerals. Mahgreb cement where next? July 1996. PP. 11-14.

30.--World Cement Directory 1991. Cimeurop s.a.r.I., PP. 40-43.

31.-Dolley, Thomas P. MIS-The Mineral Industry of Tunisia. Mineral Industry Survey series. USBM. 1992.

32.--Svoboda, K. The Lower Tertiary Phosphate Deposits of Tunisia. Phosphate Deposits of the World Vol 2. Ed. A. G. J. Northolt, R.P. Sheldon, D.F. Davidson. Cambridge University Press 1989. PP. 284-288. 33.-Grogan, Robert M. And G. Montgomery. Fluorspar and Cryolite. In Industrial Minerals and Rocks. 1975. AlME. P. 666.

34.--Habashi, Fathi and Foad A. Bassyouni. Mineral Resources of the Arab Countrie. Department of Mining \& Metallurgy-Lavall University, Quebec City, Canada 1978. PP. 103-109.

35.--Habashi, F. and F. A. Bassyouni. Mineral Resources of the Arab Countries-2nd ed. Department of Mining \& Metallurgy-Lavall University, Quebec City, Canada 1982. PP. 51-53.

36.-Khawlie, M. R. Beyond the Oil Era-Arab mineral resources and future development. Mansell Publishing Ltd. London. 1990. $132 \mathrm{pp.}$

37.-United States Central Intelligence Agency. Tunisia. ClA-95 facts-Government Publications. 6 pp. 38.--American Metal Market. Inmet unit suspends work at Tunisia mine. New York. Oct. 21, 1996. P. 1. 39.--The Mining Journal. Bougrine Suspended. London. October 25, 1996. P. 327. 
Turkey 



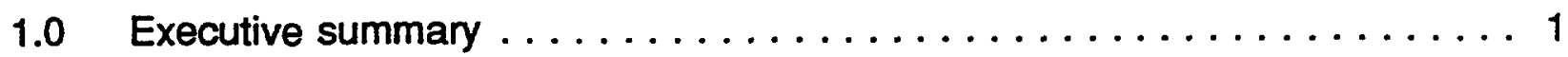

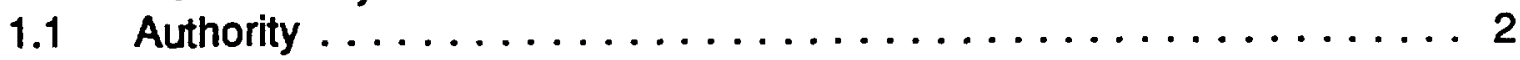

1.2 Project scope $\ldots \ldots \ldots \ldots \ldots \ldots \ldots \ldots \ldots \ldots \ldots \ldots \ldots$

2.0 Sources of information $\ldots \ldots \ldots \ldots \ldots \ldots \ldots \ldots \ldots \ldots$

3.0 The mining industry of Turkey $\ldots \ldots \ldots \ldots \ldots \ldots \ldots \ldots \ldots \ldots \ldots \ldots \ldots \ldots$

3.1 Industrial minerals $\ldots \ldots \ldots \ldots \ldots \ldots \ldots \ldots \ldots \ldots \ldots$

3.1.1 Boron . . . . . . . . . . . . . .

3.1.2 Magnesite . . . . . . . . . . . .

3.1.3 Perlite ....................... 11

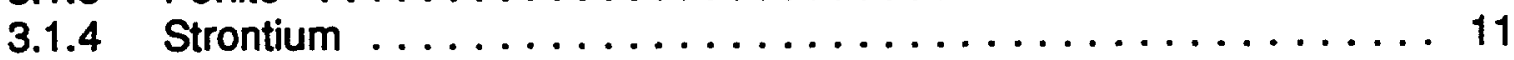

3.1.5 Other industrial minerals $\ldots \ldots \ldots \ldots \ldots \ldots \ldots \ldots \ldots \ldots \ldots 11$

3.2 Metals ........................ 14

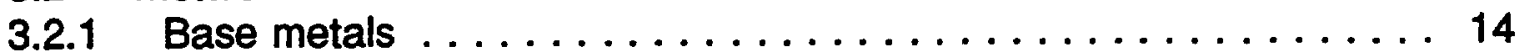

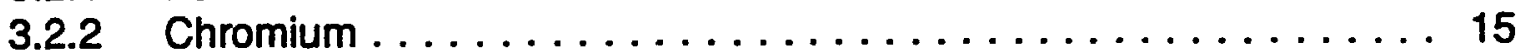

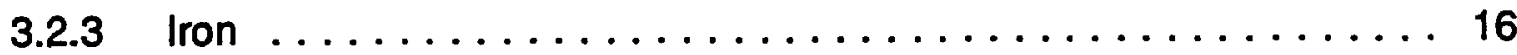

3.2.4 Precious metals $\ldots \ldots \ldots \ldots \ldots \ldots \ldots \ldots \ldots \ldots \ldots$

4.0 Mine-related explosives use $\ldots \ldots \ldots \ldots \ldots \ldots \ldots \ldots \ldots \ldots \ldots$

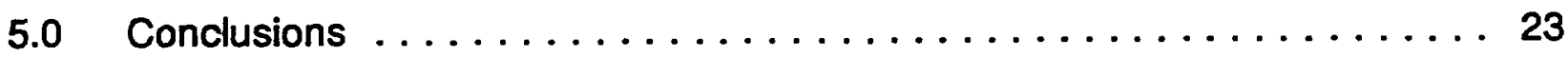

Appendix A: Producing and developing mineral properties in Turkey . . . . . 25

Appendix B: Past producing mineral properties in Turkey . ........... 38

Appendix C: Prospects and undeveloped mineral properties in Turkey . . . . . 62

Appendix D: Public sources of information . . . . . . . . . . . . 87

\section{TABLES}

3.1 Estimated mineral production in Turkey, 1993 and $1994 \ldots \ldots \ldots \ldots .6$

4.1 Estimated explosives usage at the main Turkish mines used in this study in order of estimated ANFO consumption . . . . . . . . 21

\section{FIGURES}

4.1 Selected Turkish mines and estimated blasting events . . . . . . . . . 22 Map set A: Producing mineral properties . . . . . . . . . . . . . . 36 Map set B: Past producing mineral properties . . . . . . . . . . . 59 Map set C: Prospects and undeveloped mineral properties . . . . . . . 84 



\subsection{EXECUTIVE SUMMARY}

This report was prepared under Memorandum of Agreement B291867 between Lawrence Livermore National Laboratory (LLNL) and the U.S. Geological Survey, Minerals Availability Team (MAT). It provides LLNL information on the mining industry of Turkey, and quantitative information on the blasting potential of its mining industry. MAT identified mining activities through the use of the Minerals Avallability data base, its data collection and analytic capabilities, and an extensive network of information sources.

The geology of Turkey is complex and this is reflected in the diversity of its mineral deposits. While best known for its industrial minerals, a variety of metallic minerals are produced, although output is not large by world standards. Much of Turkey's mineral production originates from a large number of small mines spread across the country; there are very few world class producers. Research conducted for this study resulted in the identification of 1,150 mineral properties. Most properties operate on a small scale and require minimal blasting. The blasting potential for 32 properties is reported.

Turkey possesses the raw materials, technology and faclities to manufacture blasting agents and detonation systems suitable for mining applications, and has established trade relations with neighboring Middle Eastern and former Soviet republics. While the mining industry most commonly uses ammonium nitrate-fuel oil (ANFO), ANFO/gel mixtures are used for selected applications. Blasting is generally small scale, but may occur frequently where mine geology requires explosive use. Estimates for daily ANFO consumption rarely exceed $\mathbf{4 0}$ metric tons of ANFO equivalent. The mining area in Turkey with the greatest blast size potential is the Murgul copper district, with an estimated maximum blasting event of $93 \mathrm{mt}$ of ANFO equivalent. 


\subsection{Authority}

This report was prepared under Memorandum of Agreement B291867 between Lawrence Livermore National Laboratory (LLNL) and the U.S. Geological Survey, Minerals Availability Team (MAT).

\subsection{Project Scope}

As part of this agreement, MAT is to identify mining activities in Egypt, Jordan, Syria, Tunisia, and Turkey as they relate to monitoring/verifying compliance of the Comprehensive Test Ban Treaty. MAT is to use the Minerals Availability and the Mineral Resources Data System data bases, its data collection and analytic capabilities, and an extensive network of information sources to provide background information focusing on the use of explosives by the mining industry, which can cause false alarms during monitoring and hide nuclear blasting events.

Reports with accompanying figures and tables summarize location, type of mining method, commodity(ies), estimated frequency and size of mine blasts, operational status, and mine product distribution to foreign or internal markets for the specified countries.

Once country data were collected and verified, the explosive use at selected sites was evaluated. Focus was placed on a limited number of larger operations, or those reported to have potential for short-term development. Undeveloped sites and small scale mines, which may be included in Appendices A-C of this report, were not analyzed in terms of the site's anticipated use of explosives. Mineral prospects generally make only small use of explosives and small mines (some of which are operated on an intermittent basis) are assumed to require minimal blasting.

Based upon known site information (geological conditions, mine technology, production capacity, and current blasting practices), the blasting potential for significant mining sites was evaluated. Where site-specific data were not available, estimates for 
representative, important properties were developed based upon accepted industry practice, knowledge of the Turkish mining industry, and regional geologic characteristics.

\subsection{SOURCES OF INFORMATION}

Data for this report were derived from published sources, unpublished documents, and personal communications through an extensive network of public and private contacts. Public sources of information are listed in Appendix D. Much of the industry summary was drawn from data reported by the U.S. Bureau of Mines Mineral Yearbook chapter on Turkey, from the years 1992-1994. Information for 1995 was obtained from the U.S. Geological Survey, International Minerals Section, Reston, VA (formerly the U.S. Bureau of Mines, Division of International Minerals).

Principal agencies contacted include, but were not limited to the U.S. Geological Survey, the U.S. State Department, Central Intelligence Agency, Defense Intelligence Agency, the United Nations, the World Bank, World Resource Institute, and International Studies of Minerals issues (ISMI). In addition, academic and industry contacts, explosives manufacturers and suppliers, and trade groups were contacted.

\subsection{THE MINING INDUSTRY OF TURKEY}

The geology of Turkey is extremely complex and is reflected in the diversity of its mineral deposits. Best known for its industrial minerals, Turkey was the world's largest producer of boron minerals in 1995, and was a major producer of barite ( $3 \%$ of world production), celestite (a source of strontium; $22 \%$ ), emery ${ }^{1}$, feldspar ( $\left.8 \%\right)$, cement $(2 \%)$, magnesite (12\%), marble, perlite (16\%), and pumice (9\%) ${ }^{2}$. Among secondary. industrial mineral commodities, Turkey continued to be a major world producer of refined borates, cement, ceramics, and glass. A wide variety of metallic minerals was produced

\section{U.S. Bureau of Mines.}

Turkey is one of the few producers of emery. Worldwide production data is not compiled by

2 U.S. Geological Survey and U.S. Bureau of Mines. Mineral Commodity Summaries 1996. 
as well, but output was not considered large by world standards. Chromite was the most șignificant ( $8 \%$ of world production) of the metals produced in Turkey. Except for lignite, output of energy minerals was modest.

Turkey's primary mineral industry contributed slightly more than $1 \%$ of the gross domestic product (GDP) in 1994. Turkey's economy in 1994 reflected a period of public sector debt, unemployment, inflation, tight credit, and rapid devaluation of the Turkish lira ${ }^{3}$. Overall, primary plus secondary mineral sector revenues were estimated at about $15 \%$ of GDP. Secondary mineral commodities, such as refined petroleum products, steel, cement, glass, and certain chemicals, accounted for about $70 \%$ of the value of the nation's manufacturing output.

Much of Turkey's mineral production comes from a large number of small mines. The Government continued to be a major participant in most sectors of the Turkish mineral industry through various state-owned industrial corporations (parastatals), banks, and shareholdings in a number of private companies. Etibank, the largest mining-related parastatal, was created in 1935 to develop and operate mines, generate and distribute electricity, and provide the raw materials needed for industry. Etibank produced all of Turkey's aluminum, blister copper, boron minerals, and ferrochrome; it remains the largest producer of chromite. The Government is in the process of transferring its shares in certain parastatals to a privatization administration for sale. The private sector component has grown since a new mining law was enacted in 1985, and is expected to increase if the privatization process, begun in 1989, gains momentum. Privatization has to date been delayed by political infighting and challenges from trade unions.

Turkey has a well-developed trade network for its industrial minerals and refined metals, but is a net exporter of only a limited amount of crude metallic ore. It is one of the largest exporters of boron, cement, glass, and ceramics. Turkey has maintained strong trade relations with neighboring countries in the Middle East and Central Asia. It has

3 U.S. Bureau of Mines. Turkey. Ch. in Minerals Industry Surveys 1994, by H. G. van Oss. 
been reported that a significant volume of diesel fuel (a component of ANFO) was brought across the border from Iraq in $1995^{4}$.

Turkey's minerals trade was traditionally with OECD countries, the European Economic Community (EEC) in particular. West Germany was Turkey's most important trading partner. Trade with Middle Eastern countries increased considerably after 1970. Trade with Iran and Iraq was important because these countries provided petroleum in exchange for food and minerals. Since the collapse of the Soviet Union, Turkey has traded heavily with the neighboring Central Asian republics. Trade with the United States has decreased substantially since the 1950's and 1960's.

The Turkish mining sector is reported to have about 790 mining establishments, roughly equivalent to 'company' or 'corporate division', which owned or operated about 3,000 mines in 1993. Of these, many are intermittent operations below cutoff size for this report, about which little information is available. In addition, the minerals industry also had 46 cement plants, 22 steel mills, 5 petroleum refineries, and a number of base metals refineries, glass factories, and fertilizer plants. The mining sector of Turkey possesses both the raw materials and technology to produce most types of blasting components.

Turkish mineral production estimates for 1993 and 1994 are provided in table 3.1. Summaries of mineral site data are provided in Appendices A-C. Data on significant producing sites, past producers, prospects, and undeveloped mineral occurrences are provided in tabular form. Maps showing mineral property locations are provided in Appendix map sets A-C. It should be noted that not all mineral occurrences are reported in this study. Sites with unverifiable information or lacking specific site 1996.

Personal communication. Bernadette Michalski. U.S. Geological Survey. July 17, 
Table 3.1 - Reported Mineral Production in Turkey, 1993 and 1994 (Metric tons, except as noted)

\begin{tabular}{|c|c|c|}
\hline Commodity (1) & $\begin{array}{r}1993 \\
\text { Production }\end{array}$ & $\begin{array}{r}1994 \\
\text { Production }\end{array}$ \\
\hline \multicolumn{3}{|l|}{ METALS } \\
\hline Aluminum, bauxite (2) & 538,439 & 445,020 \\
\hline Antimony mine output, gross $w t$. & 2,100 & 1,415 \\
\hline Cadmium & 31 & 22 \\
\hline Chromite, gross wt. & 767,313 & $1,270,431$ \\
\hline Copper mine output, gross wt. & $3,343,532$ & $3,346,490$ \\
\hline Gold, kilograms (3)(e) & 1,110 & 996 \\
\hline Iron ore, gross wt. & $6,480,000$ & $5,755,000$ \\
\hline Lead mine output, gross wt. & 179,731 & 232,140 \\
\hline Manganese ore, gross wt. (4) & 37,491 & 34,500 \\
\hline Silver mine output, Ag cont. $\mathrm{kg}(5)(e)$ & 103,000 & 65,000 \\
\hline Zinc mine output, gross wt. & 231,756 & 297,252 \\
\hline \multicolumn{3}{|l|}{ INDUST'RIAL MINERALS } \\
\hline Abrasive, emery & 10,988 & 12,000 \\
\hline Barite, run of mine & 118,367 & 116,220 \\
\hline Boron, run of mine & $1,892,356$ & $2,092,032$ \\
\hline Cement, hydraulic & $31,241,000$ & $29,493,000$ \\
\hline Clay, bentonite & 456,597 & 516,187 \\
\hline Clay, kaolin & 210,356 & 179,775 \\
\hline Clay, other & 665,351 & 956,012 \\
\hline Feldspar, run of mine & 366,166 & 502,608 \\
\hline Fluorspar (e) & 4,000 & 6,671 \\
\hline Glass, crude (e) & $1,300,000$ & $1,400,000$ \\
\hline Graphite, run of mine (e) & 20,000 & 20,000 \\
\hline Gypsum, other than for cement & 492,705 & (e) 500,000 \\
\hline Lime (6)! & $1,767,000$ & $(\theta) 1,800,000$ \\
\hline Magnesite, run of mine & 525,640 & $1,279,614$ \\
\hline Nitrogen, $\mathrm{N}$ content of ammonia & 325,800 & (e) 350,000 \\
\hline
\end{tabular}




\begin{tabular}{|c|c|c|}
\hline Perlite, run of mine & 147,864 & 164,582 \\
\hline Phosphate rock & 77,671 & - \\
\hline Pumice (7) & $1,224,114$ & 947,174 \\
\hline Pyrite, gross wt. (e) & 50,000 & 50,000 \\
\hline Salt, all types & $1,426,000$ & $1,353,000$ \\
\hline Silica sand (e) & 350,000 & 415,000 \\
\hline Soda ash (trona) (e) & 385,000 & 385,000 \\
\hline Sodium sulfate & 170,680 & 307,049 \\
\hline Stone, dolomite & 376,518 & 378,004 \\
\hline Stone, limestone, other than cement(e) & $10,852,000$ & $11,000,000$ \\
\hline Stone, marble (e) & 730,000 & 750,000 \\
\hline Stone, quartzite & $1,205,694$ & $1,350,299$ \\
\hline Strontium, celestite (e) & 68,000 & 45,000 \\
\hline Sulfur, native, other than Frasch & $(e) 17,400$ & 16,673 \\
\hline Sulfur, byproduct of petroleum & 21,000 & $(e) 25,000$ \\
\hline Talc (e) & 4,000 & 4,000 \\
\hline \multicolumn{3}{|l|}{ ENERGY MINERALS } \\
\hline Asphalt, natural & 309,348 & 108,364 \\
\hline Hard coal, nun of mine & $4,609,000$ & $4,211,000$ \\
\hline Lignite, run of mine & $51,359,000$ & $55,038,000$ \\
\hline
\end{tabular}

Source: U.S. Bureau of Mines. Middle East. Ch. in Mineral Industry Survey series, 1995, (Dratt) Ed. by P. M. Mobbs

(e) Estimated

(1) Table includes data through May 16, 1996. In addition to the commodities listed, aluminum, copper, iron, lead, and zinc metals are produced. Quantities of pyrite, meerschaum and alunite are also produced for local consumption. Construction clay, sand, and gravel are quarried, as are limestone and gypsum for cement manufacture; However, information is inadequate to make estimates of output levels.

(2) Data are for public sector only. Data for private sector production are not available, but production is believed to be about 30kt/yr only.

(3) Data are estimated content of Turkish copper refinery tankhouse slimes.

(4) Does not include manganiferous iron ore from the Deveci Mine, production of which amounts to several hundred thousand tons annually and has a manganese content of $3 \%$ to $5 \%$.

(5) Includes estimated content of base metals refinery tankhouse slimes.

(6) Data represent lime produced for steel production and do not include the widespread artisanal production of lime for whitewash and for sanitation purposes.

(7) Turkish pumice production is officially reported in cubic meters and has a density reported to range from 0.5 to 1.0 ton per cubic meter. Values in this table have been converted using 1 cubic meter=0.75 ton. 
locations may not be included; producers whose output was less that $10 \mathrm{kt} / \mathrm{yr}$ may also be omitted. Data are reported for 163 producers, 407 past producers, and 480 mineral deposits. It is believed that all sites with significant potential have been reported.

\subsection{Industrial minerals}

Turkey is endowed with a wide range of industrial minerals. Production of industrial minerals has been maintained at or near normal levels in recent years. The breakup of the Soviet Union and UN trade sanctions on Iran and Iraq have had some impact on Turkish industrial mineral trade, as these countries have traditionally been strong trading partners with Turkey. Increased competition from Chinese and Indian barite and magnesium have also had a damaging effect. Borates continue to dominate exports and the boron industry has maintained stable production levels. As a partial result of privatization, the cement industry is attracting increased foreign investment. It is expected that cement output and exports may increase as foreign companies assume a larger ownership role in the industry. Turkey has developed a significant amount of export trade in feldspar with Italy and Spain. Improving efficiency and modernization of the industrial minerals sector continues to be high on the agenda for the Turkish Government.

\subsubsection{Boron}

Turkey is the largest producer of boron minerals in the world. Approximately $42 \%$ of all crude ore production for boron comes from Turkey. Extensive resources are found in lacustrine deposits primarily in the Balıkesir/Eskişehir/Kũtahya areas of west-central Anatolia. Borates are generally found in distinct horizons, in clay or marl forming part of a flat-lying sedimentary series several hundred meters thick. Ore zones are typically overlain by limestone. Surface exposures are altered to calcite by ground waters, making detection by surface geologic surveys difficult. Drilling, trenching, and adit exploration are used to define ore zones.

Borate mining dates back to the 13th century, but annual production was less than 50kt 
until the 1850's. Current Turkish mine capacity is estimated near 2Mt run-of-mine ore, mines are operating near the full production rate to yield approximately $1.25 \mathrm{Mt}$ of $\mathrm{B}_{2} \mathrm{O}_{3}$ content in the boron concentrate. Mining equipment is old, and some mines are facing higher stripping ratios in ore recovery. Presently, all borate production is under the control of Etibank, a parastatal corporation controlled by the Turkish Government, except for a small amount of colemanite recovered from the reworking of dumps by private companies.

Gurrently, production is centered in three mining areas. The Bigadiç area produces colemanite and ulexite by both surface and underground methods. The Emet area has produced colemanite by both underground and surface methods, although most mines use open pit methods today. The Kirka area produces borax by surface methods. Surface mining consists of drilling, blasting, and removing overburden, then the ore is mined by open pit methods, prior to transport to the local concentrator for crushing, screening, washing, and export. Underground ore, where employed, is drilled, blasted, mined, then transported for crushing, screening and washing at local sites prior to export. Some ore is processed to boron chemicals at the Bandirma plant.

As with most Turkish mineral production, capacity utilization is closely tied to demand and market structure. Both boron minerals and boron derivatives (chemicals) are exported. Starting in 1968, Turkey constructed facilities to produce derivatives such as boric acid, borax decahydrate, and borax pentahydrate.

\subsubsection{Magnesite}

Turkey produces about $12 \%$ of the world's magnesite. Mining of magnesite in Turkey has been ongoing for many years. Most production has been conducted by State-run companies; although some production comes from numerous small private producers. In recent years, many of the smaller producers have been driven out of business by Chinese magnesium and lack of markets for raw magnesite. The largest verticallyintegrated dead-burned magnesite producer in Turkey is Kumaş Kũtahya Manyezit 
(Kumaş), and was sold to Zeytinoğlu Holding A.Ş. in 1995. The other large parastatal company, Konya Krom Magnesit, is slated to be privatized.

There are over 300 known magnesite occurrences in Turkey, related to ophiolitic sequences that have undergone alteration and faulting. Like boron minerals, magnesite deposits tend to occur beneath limestone and volcanic tuff in the Balıkesir/Eskişehir/Kûtahya region of west-central Turkey and in the Konya region of central Turkey. Ore occurs as pods or veins within altered serpentine, in areas of broad lateral extent. Ore is mined primarily by surface methods. As many as $\mathbf{4 0}$ small pits may be mined at one time as part of a mining operation; with ore being transported by truck up to $200 \mathrm{~km}$ to a company-operated mill.

The largest magnesite mining complex is near Kưtahya and is operated by Kumaş (now privatized). The complex consists of a number of small, scattered pits and a centralized processing plant. For the purpose of this report, all pits have been treated as one mining unit with a location based on the centralized mill. Overburden is removed prior to mining; a stripping ratio of waste to ore is reported to be 5:1. Ore is drilled and is then blasted using an ANFO/gel explosive mixture. The ore is crushed and screened, then sent to the centralized concentration plant where the dead-burned magnesite product (essentially periclase) is produced by calcination:

Mining by Konya Krom Magnesit near Konya is very similar. Magnesite is mined from stockwork ore from several pits. While underground mining was conducted on a limited basis in the past as a means of eliminating the need for overburden stripping, it proved to be costly; presently all magnesite mining is by surface methods. Ore is processed at a centralized plant in Konya that produces a variety of dead-burned magnesite products, mainly refractory brick.

Turkey's third-major dead-burned magnesia producer, Magnesit AŞ (MAŞ), operates open pit mines west of Eskişehir. This private Austrian company uses similar methods 
to mine the ore, which is then crushed, screened, and hand sorted at the mine before going directly to the sintering plant. Much of the sinter output is shipped to Austria for brick manufacture.

COMAG is Turkey's sole producer of caustic-calcined magnesite, from mines near Tavsanlı and Eskişehir. While mining methods are similar to other sites, overburden depth in this region is less, requiring less stripping prior to mining. Most of COMAG's output is supplied to fused magnesia producers, mainly in Europe.

\subsubsection{Perlite}

Turkey produces about $15 \%$ of the world's perlite. Reserves are located in the Aegean region, in central Anatolia, and in eastern Anatolia near Erzincan. Production is mainly by Etibank and three small, private sector companies, usually from small surface mines in the capacity range of $5-35 \mathrm{kt} / \mathrm{yr}$. Total production capacity is about $300 \mathrm{kt}$, but production has been running about $50 \%$ in recent years, in part due to a decrease in domestic construction activity. About $130 \mathrm{kt} / \mathrm{yr}$ is exported.

\subsubsection{Strontium}

While Turkey has been an important producer and exporter of celestite $\left(\mathrm{SrSO}_{4}\right)$, production has fallen in recent years due mainly to competition from Spain and Mexico. Turkey was estimated to produce about $21 \%$ of the world's strontium in 1994 . The mineral is mined by surface methods by one, privately-owned company, Barit Maden, at Sivas in central Anatolia. Annual production reaches $150 \mathrm{kt}$ of raw celestite ore per year. Only a small quantity is used domestically, the remainder is exported. Reserves are reported to be very extensive.

\subsubsection{Other industrial minerals}

Other industrial minerals produced include barite, bentonite, limestone for cement, emery, pumice, sodium sulfate, sulfur, and lignite. While production for some is important on a world scale, individual mining units generally are numerous but small in 
size.

Barite is produced from a number of small mines, located across Turkey. Individual mine production capacities can reach up to $150 \mathrm{kt} / \mathrm{yr}$; production varies strongly with market conditions. The largest mine is located in the Sivas region of central Turkey. Barite is also recovered in small quantities as a byproduct of lead-zinc mining. Exports are principally to the former Soviet Union republics, the Middle East, and Europe.

Bentonite production increased dramatically in 1993 to about 500kt/yr in response to strong demand in the domestic ceramic and paper industries. Large deposits are in Ankara, Edirne, Konya, Ordu, and Tokat Provinces, and a number of smaller occurrences are found in Çankırı Province. Production comes from small surface mines individually producing up to 20kt/yr. Bentonite supplies domestic and foreign refractory plants and is used as drilling mud.

Cement manufacture utilizes the abundant limestone and clay deposits found in Turkey. Turkey presently has 46 cement plants, located primarily near large cities along the Aegean and Mediterranean coasts, where cement would find a ready market. Cement plants reach a capacity of $2.75 \mathrm{Mt} / \mathrm{yr}$, cumulatively within the top 10 world cement producers. Cement production has increased in recent years due to increased domestic demand, resulting in part from a major population shift in recent years to cities, as well as several major ongoing dam construction projects (not all use concrete, however). Turkey is one of the world's largest exporters of cement. Turkish trade in cement and clinker has been affected by regional rather than overall supply imbalances. Exports accounted for about $10 \%$ of cement production, mainly to Spain and Greece. Cement production is presently being privatized. Of the 46 plants in operation, only nine were Government-controlled in 1993. If foreign companies assume ownership, it is quite possible that exports of Turkish cement will increase.

As with other countries in the region, limestone extraction is generally conducted at 
small-scale open pit mining operations requiring minimal blasting. For the purpose of this study, it is assumed that each cement plant listed in the appendices is fed from nearby limestone deposits. Specific information on limestone occurrences is often not available.

Turkey produces more than $80 \%$ of the world's supply of emery. Emery has gradually been supplanted as an abrasive by electric-furnace abrasives. Deposits are located in Antalya, Aydın, Izmir, Manisa, and Muğla provinces of western Turkey. Production at individual sites in Aydın and Muğla provinces is small, with capacities up to $8 \mathrm{kt} / \mathrm{yr}$.

Turkey supplied about $9 \%$ of world pumice from deposits in central Anatolia, near Kayseri and Nevsehir, in 1994. Individual production is small, using quarrying techniques. Limited blasting is required. Pumice exports have increased following a dramatic rise in the use of the stone for textile washing. Turkey exported about $15 \%$ of its pumice produced in 1992, mainly to Europe and the U.S.

Of the mineral fuels, Turkey relies on abundant supplies of lignite. Hard coal reserves are limited to the area around Zonguldak. Lignite deposits are scattered across Turkey. Many lignite operations require additional capital to modernize equipment and process technology. In 1993, Tũrkiye Kömũr Işletmesi Kurumu (TKi) operated 38 mines throughout Turkey with a combined annual capacity of $45 \mathrm{Mt}$, while private sector producers operated about 200 small mines across Turkey. Mines feed domestic power plants.

A large trona deposit was discovered in 1979 at Beypazari near Ankara. This deposit is important because nearby European countries have no known natural soda deposits. Funding to develop this deposit has not yet been secured.

Turkey is basically an agricultural country and requires much phosphate to meet its fertilizer needs. There is only one phosphate producer in Turkey, in the Mazıdağı area. 
Production at this site decreased to very low levels in 1995, and is believed to have ceased altogether. Turkey does produce some sulfur from mines and as a byproduct of petroleum refining to support the fertilizer industry, but much sulfur and phosphate are extensively imported.

\subsection{Metals}

Turkey produced a wide variety of metals as well, but output generally is not considered large by world standards. Among the primary metallic minerals, chromite production is the most significant on a world scale, producing $8 \%$ of the world's chromium. Turkey is also a significant producer of processed ferrochromium and steel.

Much of Turkey's metallic mineral production was from a large number of mostly small mines. Capacity expansion projects continued at many secondary mineral production facilities, particularly those in the private sector. Mineral exploration by foreign companies in Turkey is primarily for gold, copper, and zinc.

\subsubsection{Base metals}

The history of copper and lead/zinc mining dates back to about 7,000 B.C. Over 600 copper occurrences have since been identified. There are four main copper and leadzinc metallogenic provinces:

1. Eastern Black Sea region - Kuroko-type mineralization related to calcalkaline volcanics.

-2. Bursa Province - Skarn and vein-type mineralization along limestone-acidic intrusives.

3. Southeastern Turkey - Sulfide mineralization related to ophiolites.

4. Central Taurus Mountains of southern Turkey.

Over $99 \%$ of the copper industry in Turkey is controlled by Etibank and its subsidiary, Black Sea Copper Works (KBi). Etibank operates open pit mines at Küre and Ergani (idle in 1995), KBI has open pit mines at Murgul and Kutlular and an underground mine 
at Küre.

The country's main lead/zinc resource occurs in the Çanakkale region in northwestern Turkey, where numerous small deposits are reported. Six private companies are also involved with mining lead/zinc.

The largest copper mining operation occurs at Murgul, near Artvin, with the capacity to recover $3.85 \mathrm{Mt} / \mathrm{yr}$ of copper ore to produce $175 \mathrm{kt} / \mathrm{yr}$ of copper concentrate. Capacity has not been reached in recent years. Ore occurs in volcanics; mining is open pit with an average stripping ratio of 3:1 waste to ore. The mine and adjacent concentrator were modernized in 1987. Most other Turkish base metal mines use similar mining methods, as sulfide mineralization occurs at relatively shallow depths.

The most significant development in the base metals sector in recent years was the development of the Çayeli copper-zinc underground mine near Rize, on the Black Sea coast: The mine reached full production capacity of $600 \mathrm{kt} / \mathrm{yr}$ in 1995; concentrates were exported and consequently did not affect domestic output of refined copper or zinc. Prospecting in the region is ongoing. Regional exploration has identified several potential target areas; the most notable is the Cerattepe copper-silver-gold property which is being developed by Cominco. Much of the copper ore in Turkey contains significant gold values (about $2 \mathrm{ppm}$ ) which is recovered from refinery slimes after transport to Europe.

Most Turkish copper ore is concentrated locally. Copper concentrates are either exported, as in the case of the Çayeli mine, or processed domestically, producing either blister (Samsun only) or refined copper. Some refined copper is exported, primarily to Europe. Turkey has one small (34kt) zinc refiner at Kayseri.

\subsubsection{Chromium}

Turkey produced $8 \%$ of the world's chromium in 1994. Large areas of Turkey are 
covered with serpentine, the host rock for chromite. Since its discovery in Turkey in 1848, chromite has played an important part in the Turkish economy. Turkey's chromite reserves are not well defined and are spread out over several hundred occurrences across the country. Deposits can be grouped in six major areas:

1. Gûleman/Elazığ area - eastern Turkey.

2. Fethiye/Koycegiz/Denizli area - southwestern Turkey.

3. Bursa/Eskişehir area - northwestern Turkey.

4. Kayseri/Adana/Mersin area - southern Turkey.

5. Kopdağ area - eastern Turkey.

6. İskenderun/islahiye/Maraş area - southern Turkey.

In 1990, about 100 chromite mines were producing, but most individual sites produce less than 20kt/yr. Total Turkish chromite production is significant, however. The Güleman complex near Elazığ is the largest producing group of mines in Turkey, with a production capacity of about $150 \mathrm{kt}$ of ore per year. Ore occurs in peridotite complexes and is generally lenticular, tabular, or irregular in form. The Gũleman complex is actually a group of mines, each mining a separate lens of ore. Mining employs either open pit, underground cut \& fill, or longwall methods. Blasting may be required, depending upon mining method selected. Most smaller mines require significant blasting to remove the hard ore, as longwall mining methods are not appropriate for this șize operation.

All chromite output is directly or indirectly export-driven. The only domestic consumption of chromite is by Etibank, which converts all of its own and some privately produced ore into ferrochromium at its two domestic smelters. Additional domestic consumption is restricted to very small amounts for the manufacture of refractories and leather tanning chemicals.

\subsubsection{Iron -}

Iron ore deposits in Turkey feed the Turkish steel industry, one of the fastest growing 
steel industries in the world. There are two main metallogenic provinces for iron ore in Turkey; one in the Sivas/Malatya area of central Turkey, the other extending from Kayseri to Adana, south of the first district. Ore occurs in limestone related to metasomatism and hydrothermal activities.

The largest iron producer is the Divriği area mines northwest of Elazığ. The complex has the capacity to produce $4.5 \mathrm{Mt} / \mathrm{yr}$ of ore from several sites. Ore is produced mainly by open pit methods.

\subsubsection{Precious metals}

In recent years, Turkey's precious metal's output has all been derived from credits in base metal refinery slime output, or as smelted metals exported to Europe. Most Turkish copper ores contain 1-2g/t gold, and variable amounts of silver. As a result of revised mining regulations favoring foreign exploration in Turkey, precious metals exploration has increased and several precious metals deposits are either beginning production or being developed. The Aktepe silver mine northwest of Kûtahya has been in production since 1987 . The mine has a production capacity of $1 \mathrm{Mt} / \mathrm{yr}$.

In 1995, there were three gold deposits awaiting approvals for development. The most advariced property was the Ovacık deposit, an epithermal vein deposit in Tertiary andesite, which is planned to produce at a rate of 300kt of ore per year by surface/underground methods. The Kũçũkdere deposit east of Edremit and the Kaymaz gold deposit southwest of Ankara received environmental permits for mining in 1995, but additional permitting is required before production can begin. All three projects face strong local opposition over the proposed use of sodium cyanide for gold extraction. Gold exploration is continuing, mainly in the Aegean and eastern Black Sea coastal regions.

\subsection{MINE-RELATED EXPLOSIVES USE}

Almost all mines use explosives to fragment or loosen rock and consolidated material 
prior to excavation. Bulk or packaged explosives and blasting agents are detonated after emplacement in material to be excavated. Minor quantities of sachet and shaped charges may be used for secondary breakage and other special applications.

The type and amount of explosives used are influenced by the geotechnical nature of the rock, the mining methods employed, the production rate of the mine, the type and availability of explosives and detonation systems, hydrologic conditions, mining equipment, drilling equipment, mine geometry, level of technical expertise, and external constraints such as the proximity of residences, availability of explosives, and available funding. At almost any mine, the size of each blast can vary significantly due to local conditions, production schedules, weather, etc.

Surface mines typically shoot much larger blasts than underground operations and tend to have higher production rates than underground mines. In addition, limitations of working room, limited free faces, type of mineralization, ventilation requirements, and drilling limitations may constrain maximum blast sizes in underground mines.

Turkey is not limited to domestically produced explosives, but has ample raw materials to produce what is needed. It traditionally has exported materials that could be utilized for explosive manufacture to neighboring countries, including countries currently under UN sanctions. Turkey has the military-related technology and facilities to manufacture blasting agents and detonation systems suitable for most mining applications.

Where blasting is required, most Turkish mines use ANFO blasting agents. This may include some of the newer emulsion and/or aluminum boosted products presently available. While an ANFO/gel mixture is believed to be in use at some mines in Turkey, ANFO systems are preferred in most mining applications due to their ease of manufacture, low cost, inherent safety, and bulk loading advantages. High explosives, however, would be preferable for small underground operations that use drill sizes that are below the critical diameter needed for emplacing ANFO blasting agents, or under 
wet conditions, in methane-rich atmospheres, and conditions that require higher detonation velocities and/or convenience of packaged explosives. Many underground mines in Turkey are very small, are labor intensive, and employ non-mechanized methods.

In most cases, site-specific blasting information was unavailable. Consequently, estimates were based upon known or estimated production rates, mine geology, and typical mining practices. Experience, engineering judgement, and available data were incorporated into calculations and estimates. Explosive use can vary considerably as mining conditions change. ANFO consumption was assumed to be dependent upon mine production rate, average stripping ratio, specific gravity of the host rock, assumed powder factor limits, and mining method. Only a small number of mines in Turkey require significant blasting, primarily because of their small size. Many mines do not use the latest blasting techniques, and commonly use more explosives than is needed, resulting in shattered ore.

For each site, a stripping ratio (Quantity of overburden or waste removed per metric ton of ore mined) and powder factor (Quantity of rock blasted per unit of ANFO blasting agent equivalent) limits were estimated. A range of ANFO consumption was calculated for both daily blasting requirements and for an assumed maximum blasting event. Daily ANFQ requirements were estimated assuming a 330 day production schedule. Consumption estimates for all sites were calculated in a similar manner. The lower consumption value applies a minimum powder factor while the higher value assumes a maximum powder factor. Unlike daily consumption estimates, a maximum blasting event would not take place on a daily basis. For this study, it was assumed that a maximum blasting event could occur every 10 working days for a surface mine and every 5 working days for an underground mine. Such events are designed to account for such factors as blasting delays, geological irregularities, and mining method variations that require a higher ANFO consumption than the typical blasting event. Mine development or pillar extraction conditions, for example, often require larger blasts. 
The following examples illustrate typical blasting calculations using the estimation procedure described above:

\section{Mürgul ANFO daily consumption lower limit (L):}

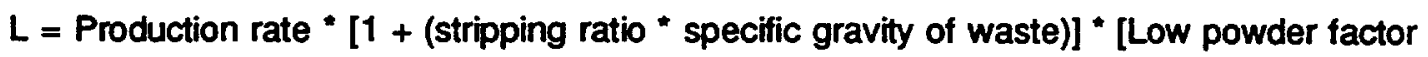

$/ 1000$ (converts $\mathrm{kg}$ to $\mathrm{mt}$ )] / Operating days per year

$L=3,850,000 *[1+(1.1 * 2.7)] *[0.11 / 1000] / 330$

$L=5.09 \quad 5.1 \mathrm{mt}$ ANFO equivalent (rounded)

Mürgut ANFO daily consumption higher limit (H):

$H=$ Production rate * $\left[1+\right.$ (stripping ratio * specific gravity of waste) ${ }^{*}$ [High powder factor

$/ 1000$ (converts $\mathrm{kg}$ to $\mathrm{mt})] /$ Operating days per year

$L=3,850,000 *[1+(1.1 * 2.7)] *[0.20 / 1000] / 330$

$L=9.26 \quad 9.3 \mathrm{mt}$ ANFO equivalent (rounded)

\section{Mürgul maximum blasting event ANFO consumption (M):}

$M=$ Production rate * $[1+\text { (stripping ratio * specific gravity of waste) }]^{*}$ [High powder factor

/ 1000 (converts $\mathrm{kg}$ to $\mathrm{mt}$ )] / Operating days per year * maximum blast cycle time (working days between blasting events)

$M=[3,850,000 *[1+(1.1 * 2.7)] *[0.20 / 1000] / 330] * 10$

$M=92.63 \quad 92.6 \mathrm{mt}$ ANFO equivalent (rounded)

Table 4.1 provides the corresponding blasting range estimates for the main Turkish mines identified in this study. Figure 4.1 shows site locations for the mines reported in table 4.1. Symbols reflect mine type (surface or underground) and maximum ANFO consumption for a given blasting event. Where a mine produces using both underground and surface methods, the predominant method is shown on figure 4.1.

Only two mining sites, the Mũrgul copper mine and the Mazıdağı phosphate mine, had calculated maximum blasting events that exceeded $50 \mathrm{mt}$ ANFO equivalent, and the Mazıdağı site reportedly closed in 1995. Both mines employ typical open pit mining techniques and requires blasting of both ore and overburden. A maximum blasting 
Table 4.1-Estimated Explosives Usage at the Main Turkish Mines Used in this Study in Order of Estimated ANFO Consumption

\begin{tabular}{|c|c|c|c|c|c|c|c|c|c|}
\hline Mine & Lettude & Longlitude & $\begin{array}{l}\text { Prtmery } \\
\text { Product }\end{array}$ & $\begin{array}{l}\text { Mine } \\
\text { Type } \\
\text { (1) }\end{array}$ & $\begin{array}{r}\text { Production } \\
\text { Pimlsy } \\
\text { (2) }\end{array}$ & 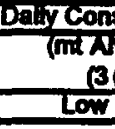 & ion & $\begin{array}{c}\text { Mnomum } \\
\text { Blad Cycto } \\
\text { Thme (days) } \\
\text { (5) }\end{array}$ & $\begin{array}{c}\text { Maxdmum } \\
\text { Blasting } \\
\text { Event } \\
\text { (mi ANFO) }\end{array}$ \\
\hline Murgul - & $N 41^{\circ} 15^{\circ}$ & E $41^{\circ} 33^{\prime}$ & Copper & $\mathbf{s}$ & 3.850 & 5 & 9 & 10 & 93 \\
\hline Mazidagl (6) & $N 3 T^{\circ} 3 t^{\circ}$ & E $40^{\circ} 30^{\prime}$ & Phosphate & $\mathbf{s}$ & 1.000 & 3 & 6 & 10 & 76 \\
\hline Buyukcekmece & $N 41^{\circ} 01^{\prime}$ & E $28^{\circ} 34^{\circ}$ & Cement clay & $\mathbf{s}$ & 2.750 & 2 & 4 & 10 & 38 \\
\hline Divrigi Area & N $39^{\circ} 25$ & E 38.05 & tron & $\mathbf{s}$ & 4.500 & 3 & 4 & 10 & 36 \\
\hline$|c|$ & N $36^{\circ} 48^{\circ}$ & E $34^{\circ} 38^{*}$ & Cement clay & $\mathbf{s}$ & 2.500 & 2 & 3 & 10 & 34 \\
\hline \multirow[t]{2}{*}{ Kure $(7)$} & $N 41^{\circ} 48^{\circ}$ & E $33^{\circ} 43^{\prime}$ & Pyrite/Copper & $\mathbf{s}$ & 1.100 & 2 & 3 & 10 & 28 \\
\hline & & & PyrtedCopper & UG & 0.095 & 0 & 0 & 5 & 1 \\
\hline Ezhe & $N 39^{\circ} 47$ & E $26^{\circ} 20^{\circ}$ & Cement clay & $\mathbf{s}$ & 2.000 & 2 & 3 & 10 & 27 \\
\hline Saray & $N 39^{\circ} 42$ & E $34^{\circ} 40^{\circ}$ & Cement ctay & $\mathbf{s}$ & 2.000 & 2 & 3 & 10 & 27 \\
\hline Tumurtalk & $N 36^{\circ} 49^{\circ}$ & $E 35^{\circ} 45^{\prime}$ & Cernent clay & $\mathbf{s}$ & 1.850 & 1 & 3 & 10 & 25 \\
\hline Ermadag & N 39" $58^{\circ}$ & E $33^{\circ} 08^{\circ}$ & Coment clay & $\mathbf{s}$ & 1.300 & 1 & 2 & 10 & 18 \\
\hline Diskelesi & $N 40^{\circ} 4 T$ & E $29^{\circ} 31^{\prime}$ & Cement ctay & $\mathbf{s}$ & 1.300 & 1 & 2 & 10 & 18 \\
\hline Ergani & $N 38^{\circ} 30^{\circ}$ & E $39^{\circ} 45^{\circ}$ & Copper & $\mathbf{s}$ & 0.724 & 1 & 2 & 10 & 16 \\
\hline Hektmhan & N $38^{\circ} 59$ & E 3752 & Iron & $\mathbf{s}$ & 0.750 & 1 & 2 & 10 & 15 \\
\hline Kumas & N $39^{\circ} 29^{\circ}$ & E $30^{\circ} 04^{\circ}$ & Magnesite & $\mathbf{s}$ & 0.846 & 1 & 2 & 10 & 15 \\
\hline Axtepe & N $38^{\circ} 58^{\circ}$ & E $29^{\circ} 29^{\circ}$ & Sitver & $\mathbf{s}$ & 1.000 & 1 & 1 & 10 & 14 \\
\hline Emet Area & N $39^{\circ} 13^{\prime}$ & E $29^{\circ} 09^{\circ}$ & Boron & $\mathbf{s}$ & 0.650 & 1 & 1 & 10 & 14 \\
\hline Seydiseht & $N 3 r 25$ & E $31^{\circ} 51^{\circ}$ & Bauxle & $\mathbf{s}$ & 0.681 & 1 & 1 & 10 & 12 \\
\hline \multirow[t]{2}{*}{ Bigadic Area (7) } & N $39^{\circ} 25$ & E $28^{\circ} 08^{\prime}$ & Boron & $\mathbf{s}$ & 0.281 & 1 & 1 & 10 & 10 \\
\hline & & & & UG & 0.242 & 0 & 0 & 5 & 1 \\
\hline Ovackk (8) & N 39. 05 & E $26^{\circ} 52^{\circ}$ & Gold & $\mathbf{s}$ & 0.300 & 1 & 1 & 10 & 9 \\
\hline Karadiken & $N 37^{\circ} 00$ & E $34^{*} 42$ & Llmestone & $\mathbf{s}$ & 0.550 & 0 & 1 & 10 & 8 \\
\hline Karka & $N 39^{\circ} 17$ & E $30^{\circ} 33^{\prime}$ & Boron & $\mathbf{s}$ & 0.815 & 0 & 1 & 10 & 7 \\
\hline \multirow[t]{2}{*}{ Cerattepe $(7,8)$} & $N 41^{\circ} 12$ & E $41^{\circ} 48^{\prime}$ & Copper & UG & 2.280 & 1 & 1 & 5 & 7 \\
\hline & & & Gold/sliver & $\mathbf{s}$ & 0.355 & 0 & 0 & 10 & 4 \\
\hline Margi & N 39. 55 & E $\mathbf{3 0}^{\circ} 54^{\circ}$ & Magnesile & $\mathbf{s}$ & 0.400 & 0 & 0 & 10 & 3 \\
\hline Sivas & N $39^{\circ} 40^{\circ}$ & E $37^{\circ} 00^{\circ}$ & Strontium & $\mathbf{s}$ & 0.150 & 0 & 0 & 10 & 2 \\
\hline Konya & N $37^{\circ} 50^{\circ}$ & E $32^{\circ} 38^{\circ}$ & Magnesium & $\mathbf{s}$ & 0.318 & 0 & 0 & 10 & 2 \\
\hline Cayell & $N 41^{\circ} 04^{\circ}$ & E $40^{\circ} 48^{\circ}$ & Znnc & UG & 0.600 & 0 & 0 & 5 & 2 \\
\hline \multirow[t]{2}{*}{ Uludag (n) } & $N 40^{\circ} 03^{\circ}$ & E $29^{\circ} 10^{\circ}$ & Tungsten & UG & 0212 & 0 & 0 & 5 & 2 \\
\hline & & & & $\mathbf{s}$ & 0.038 & 0 & 0 & 10 & 2 \\
\hline Tavsanli & N $39^{\circ} 33^{\circ}$ & E $29^{\circ} 30^{\circ}$ & Magnesium & $\mathbf{s}$ & 0.265 & 0 & 0 & 10 & 1 \\
\hline \multirow[t]{2}{*}{ Keabortu (7) } & $N 3 r 5 r$ & E $30^{\circ} 18^{\circ}$ & Sulfur & $\mathbf{s}$ & 0.017 & 0 & 0 & 10 & 1 \\
\hline & & & & UG & 0.128 & o & 0 & 5 & 1 \\
\hline Clrakman & $N 3^{\circ} \uparrow T$ & E $32^{\circ} 25^{\prime}$ & Mercury & UG & 0.100 & 0 & 0 & 5 & 1 \\
\hline Guleman & N $38^{\circ} 30^{\circ}$ & $E 39^{\circ} 46^{\circ}$ & Chromile & UG & 0.250 & 0 & 0 & 5 & 1 \\
\hline Halkoy & N $38^{\circ} 05$ & E $28^{\circ} 10^{\circ}$ & Mercury & UG & 0.190 & 0 & 0 & 5 & 1 \\
\hline
\end{tabular}

(1) S-Sintace; UG-Underground

(2) Mmtyr-Mition metric tons per year

(3) mt ANFO-Metrk tons of Ammonlum Nitrate/Fuel Ol blasting agent equivalent. Estimate based on equations reported on page 18.

(4) Assumed annual production schodule of 330 days per year, resulls may difier II another production schedule used.

(5) Assumed maxtmum blasting cycle time for surface operation - 10 workthg days; underground operation - 5 working days.

(6) The Mazidagi phosphate mine produced very wile in 1995. It is thought to be closed.

(7) Slle uses both surface and underground methods. Explostve usage for each method reported separately.

(8) Slle is currently being developed for production. 


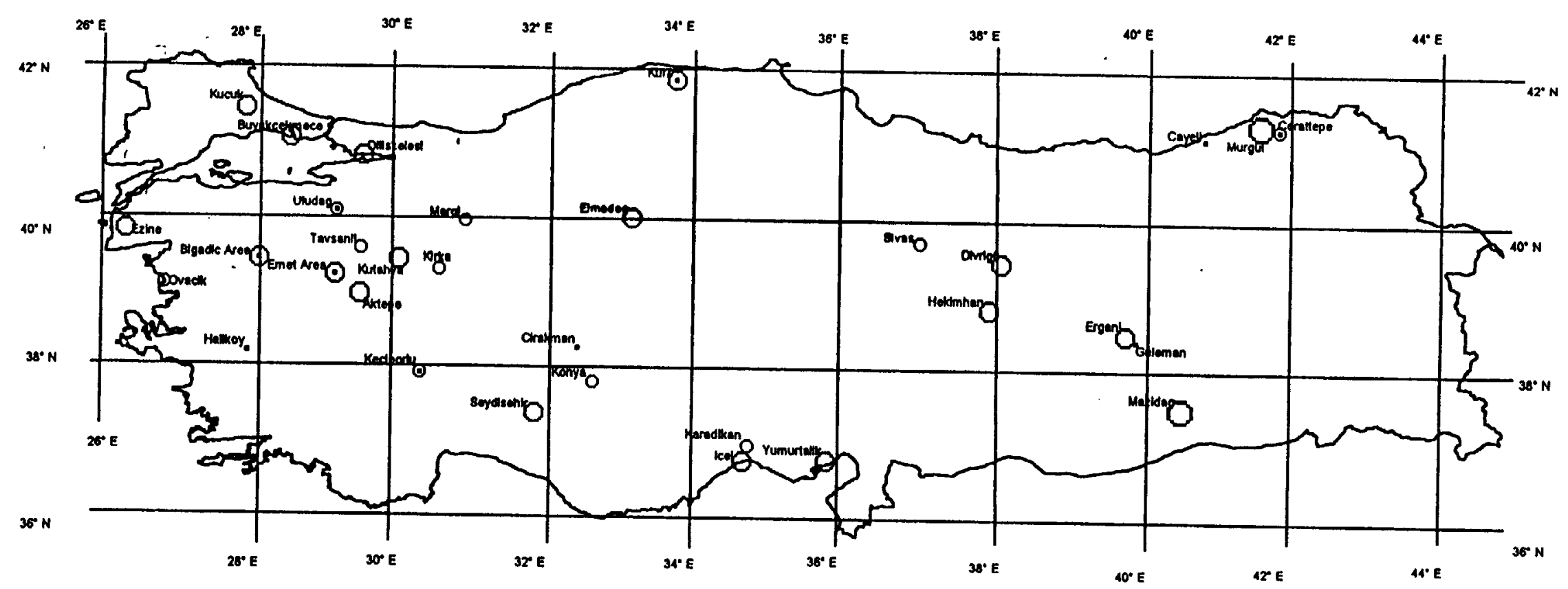

\section{LEGEND}

Underground Surface Maximum Blasting Event*

Not Applicable

More than 75 metrle tons

Not Applicablo

O 10 to 75 metric tons

0

200

400 Kilometers

- Less then 10 metric tons

"ANFO Equivalent

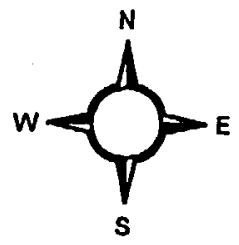

FIGURE 4.1--Selected Turkish Mines and Estimated Maximum Blasting Events 
event of $93 \mathrm{mt}$ of ANFO equivalent was estimated for the Mũrgul mine and a maximum blasting event of $76 \mathrm{mt}$ ANFO equivalent was estimated for the Mazıdağı mine.

In addition to the two sites previously mentioned, there are 17 sites that have an estimated explosive potential in the range of $10-40 \mathrm{mt}$ of ANFO equivalent for a maximum blasting event. Several of these relate to limestone production feeding cement plants. It should be noted that production from such sites comes from numerous small quarries feeding the nearby plant, rather that one large mining operation, so individual site explosive consumption would be much smaller that the aggregated estimates reported in Table 4.1.

\subsection{CONCLUSIONS}

The mining industry of Turkey is diverse, industrial minerals production is most important. While there are many mines, most mines are small and only require small amounts of explosives. The largest maximum blasting event in Turkey should be less than $100 \mathrm{mt}$ ANFO equivalent. Most mines require less than $10 \mathrm{mt}$ of ANFO equivalent per day. 


\section{APPENDICES}


APPENDIX A PRODUCINO AND DEVELOPINO MINERNL PROPERTES IN TURKEY

\begin{tabular}{|c|c|c|c|c|c|c|c|c|c|c|c|}
\hline \multicolumn{2}{|c|}{$\begin{array}{l}\text { MAP } \\
\mathrm{KEY} \\
\text { (1) }\end{array}$} & $\begin{array}{l}\text { NAME } \\
\text { (2) }\end{array}$ & \multicolumn{2}{|c|}{$\begin{array}{l}\text { COORDINATES } \\
\text { LAT. LONO. }\end{array}$} & COMMODITY(IES) & $\begin{array}{l}\text { DATA } \\
\text { SOURCES } \\
\text { (3) }\end{array}$ & $\begin{array}{l}\text { DATA } \\
\text { RELIANCE } \\
\text { (A) }\end{array}$ & $\begin{array}{l}\text { 8TATUS } \\
\text { (s) }\end{array}$ & $\begin{array}{l}\text { MINE } \\
\text { TYPE }\end{array}$ & MARKETS & \multirow{2}{*}{$\begin{array}{l}\text { COMMENTS } \\
\text { Res: 100Mt. } \\
\text { Production of th reponted in } 1986 .\end{array}$} \\
\hline A & 1 & $\begin{array}{l}\text { Adana } \\
\text { Gordibl }\end{array}$ & N $37^{\circ} 33^{\circ}$ & E $35^{\circ} 16^{\prime}$ & Chromlum & 19,21 & General & Producer & Surface & Domeatle & \\
\hline A & 2 & Adana & N $37^{\circ} 01^{\circ}$ & $E 3^{\circ} 18^{\circ}$ & Quanzito & 17 & General & Producer & Surface & Domestle & Rea: 1.2Bt (1985). \\
\hline A & 3 & Foke & $N 37^{\circ} 48^{\prime}$ & E $35^{\circ} 65^{\circ}$ & Bartto & 21 & General & Producar & Underground & Unknown & Mine output: sokt bartte. \\
\hline A & 4 & Kanantl & N. $37^{*} 33^{\circ}$ & E 35' 22' & Chromlum & 19 & Gonoral & Producer & Surface & Domestle & Small operation, private owner. \\
\hline A & 5 & Yumuntallk & $N 36^{\circ} 49^{\circ}$ & E $35^{\circ} 45^{\circ}$ & Cemmm loedatock & 17 & Ceneral & Producer & Surface & Domestle & $\begin{array}{l}\text { Res: } 210 \mathrm{MM} \text { clay (1982). } \\
\text { Plant produces 1.85Mt cerment annually. }\end{array}$ \\
\hline A & 6 & Yenlce & $N 36^{\prime} 4 r$ & E $35^{\circ} 1 T$ & Salt & 21 & Goneral & Producer & Underground & Unknown & Annual output $700 \mathrm{kt} \mathrm{NaCl}$ brine by solution mining. \\
\hline B & 7 & Dedell & $N 39^{\circ} 11^{\circ}$ & E $43^{\circ} 04^{\prime}$ & Pumico & 17 & General & Producar & Surface & Unknown & $\begin{array}{l}\text { Producing in } 1989 . \\
\text { Res: } 28 \mathrm{M} \text { cuble moters. }\end{array}$ \\
\hline B & 8 & Ortakent & $N 39^{\circ} 57$ & E $43^{\circ} 18^{\prime}$ & Pumles & 17 & General & Producer & Surface & Unknown & $\begin{array}{l}\text { Producing in } 1989 . \\
\text { Res: } 60 \mathrm{M} \text { cuble moters. }\end{array}$ \\
\hline c & 9 & $\begin{array}{l}\text { Somadoglu } \\
\text { Gurmushaclkoy }\end{array}$ & $N 40^{\circ} 53^{\circ}$ & E $35^{\circ} 35^{\prime \prime}$ & Clay & 2.17 & General & Producer & Surtace & Dometile & $\begin{array}{l}\text { Moderato size fullers earth deposit. } \\
\text { Ree: } 32.5 \text { sit for refractory/ceramles. }\end{array}$ \\
\hline c & 10 & Beypazar & $N 40^{\circ} 51^{\prime}$ & E $35^{\circ} 41^{\circ}$ & Llgnilio & 17 & General & Producor & Unknown & Unknown & Res: 408Mt Q 2.0-2.8Kcalkg from 3 sthes. \\
\hline D & 11 & Beypazan & $N 40^{\circ} 10^{\circ}$ & E $31^{\circ} 66^{\prime}$ & Trona & 10,17 & Ceneral & Dev. Depostt & Unknown & Unknown & $\begin{array}{l}\text { Rea: } 178 \mathrm{MM} \text { 45.5\% Na2CO3 (1993). } \\
\text { Mine has not yet opened, negotlatlons in progress. }\end{array}$ \\
\hline D & 12 & Cankaya & $N 40^{\circ} 10^{\circ}$ & E $32^{\circ} 49^{\circ}$ & Llgnile & 17 & Goneral & Producer & Unknown & Unknown & $\begin{array}{l}\text { Res: 30Mt Q 2.0Kcalkg. } \\
\text { Producling in } 1989 .\end{array}$ \\
\hline D & 13 & Caylman & $N 40^{\circ} 00^{\circ}$ & E $31^{\circ} 3 T$ & Sodium sulfate & 17 & Ceneral & Producer & Brine pumplng & Unknown & Ret: 193Mt O 44-79\% Na2SO4 (1988). \\
\hline 0 & 14 & Elmadag & $\mathbf{N} 39^{\circ} \mathbf{5 8 ^ { \prime }}$ & E 33' 08' & Comont foedetock & 17 & General & Producer & Surface & Domeatle & 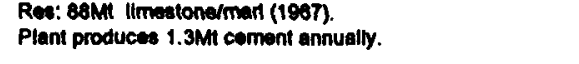 \\
\hline E & 15 & Deminas & $N 36^{\circ} 25^{\prime}$ & E $32^{*} 13^{\prime}$ & Bartite & 11.17 & General & Producer & Unknown & Unknown & $\begin{array}{l}\text { Reserves from } 5 \text { sttes: 1.5Mt } 929-99 \% \text { BeSO4 (1978). } \\
\text { Plant produces 120kt ground bartle. }\end{array}$ \\
\hline $\mathbf{F}$ & 16 & Cerattepe & $\mathrm{N} 41^{\circ} 12^{\prime}$ & E $41^{\circ} 40^{\circ}$ & $\begin{array}{l}\text { Copper, gold, } \\
\text { silvor }\end{array}$ & 19 & General & Dev. depositt & $\begin{array}{l}\text { Surtace } \\
\text { Underground }\end{array}$ & Expon & 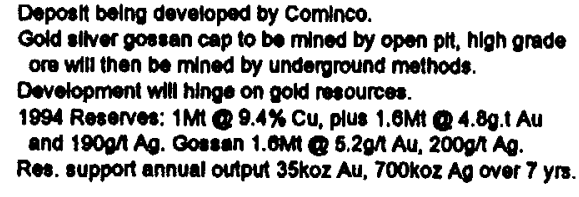 \\
\hline $\mathbf{F}$ & 17 & $\begin{array}{l}\text { Murgul } \\
\text { Goltes }\end{array}$ & $N 41^{\prime} 15^{\prime}$ & E $41^{\circ} 33^{\prime}$ & $\begin{array}{l}\text { Coppor, Iron, } \\
\text { sulfur, alver, } \\
\text { gold }\end{array}$ & $\begin{array}{l}2,4,11,15,16,19 \\
23\end{array}$ & Confirmed & Producer & surface & $\begin{array}{l}\text { Domostit } \\
\text { Export }\end{array}$ & 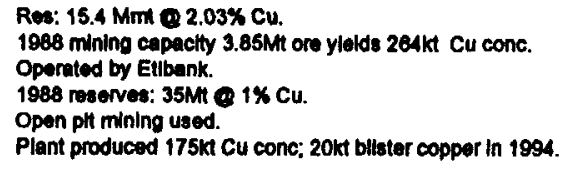 \\
\hline $\mathbf{F}$ & 18 & Savsat & $N 41^{\prime} 15^{\prime}$ & E $42^{\circ} 20^{\circ}$ & Foldepar & 17 & Goneral & Producer & Surface & Unknown & Res: 369Mt (1980). \\
\hline
\end{tabular}


APPENDIX A PRODUCINO AND DEVELOPINO MINERCL PROPERTIES IN TURKEY

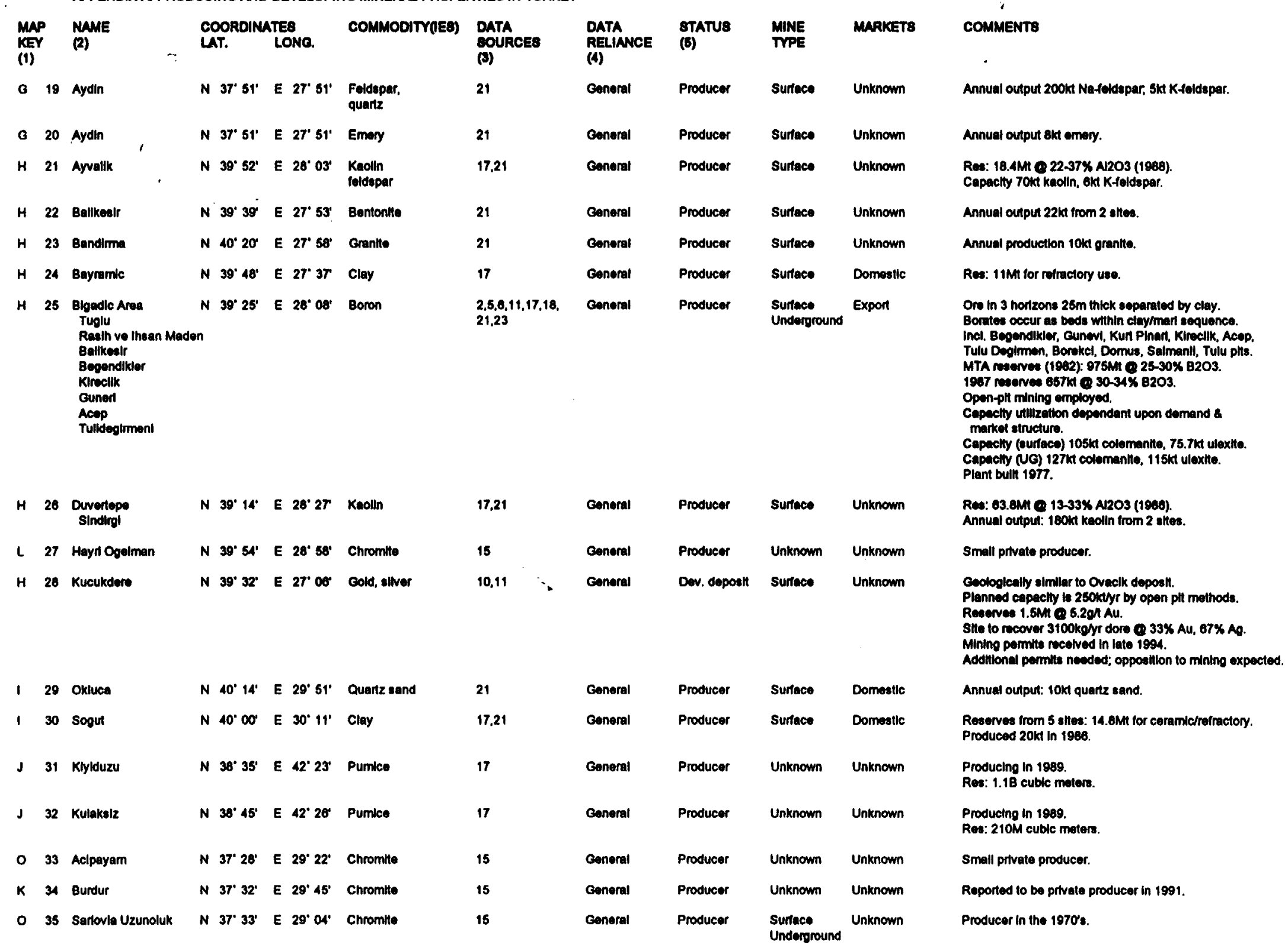


APPENDIX A' PRODUCING ANO DEVELOPINO MINERNL PROPERTES IN TURKEY

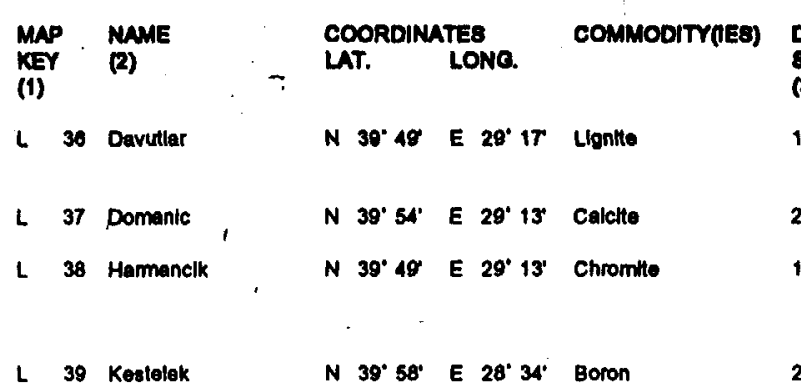

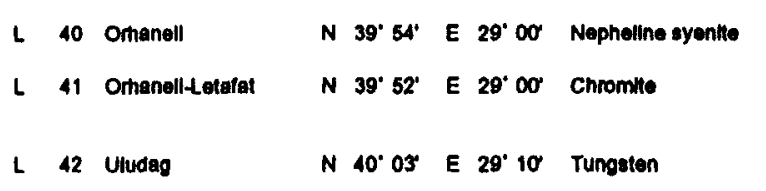

DATA
8OURCES
(3)

$(3)$

17

21

$15,17,23$

$2,5,6,11,17,10$

DATA

(A)

onoral

(6)

conating

Producor

General

Producer

General

Producer

Beneral

Producer

(n)

Surface
Underground

21

$2.15,23$

Conoral

Producer

Surface Unknown

Goneral

Producer

Undergnound Unknown

$2,16.17,23$

Ceneral

Producer (6)

Surtace

Export

Goneral

Producer

Unknown

Unknown

Yonuculer

N 39" $52^{\circ}$ E $28^{\circ} 59^{\circ}$ Chromite

15

Cenoral

Producer

Surface

Domestic

$N 45$ Conkli

N $40^{\circ} 36^{\circ}$ E $33^{\circ} 3 T^{\circ}$ Sall

2,17,23 $\because \quad$ Cenoral

Producor

Surface

Domestle

- 46 Den|zi|

N 37 46 E 29.00 comont focdatock

17

Coneral

Producer

surface

Dornestlc

- 47 SIAnkoy

N 37' 46 E 29, 00 Quartzho.

17,21

Coneral

Producer

Surfaco

Domostle

N 37 34' E 28' O4' Manganese

$2,17,20,23$

Ooneral

Uluken

P 49 Erganl

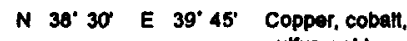
sulfur, gold,
sitver, iron

$2,4,11,15$

General

Producer

Surfaco

Unknown

Producar (6) Surface Damestlo

Export

P $50 \begin{array}{r}\text { Guloman } \\ \text { Sortdag }\end{array}$

N $38^{\circ} 30^{\circ}$ E $39^{\circ} 46^{\circ}$ Chromite

$2,4,14,15,17,23$

General
Producer
Underground Export Surface Domestle
' commants

.

Res: 32Mm 2103 Cankg

Producing in 1989.

Annual production $20 k$ calchts.

Explored by Eltbank pritor to 1990.

Res: (Hlign) 32.5kt $30-40 \%$ Cr2O3: (10-9n) 103kt $20 \%$ MIII has capactiy of $25 \mathrm{k}$ yr.

Cotemanne occune in 3 horlzone over om Interval.

Ore grade of selected specimens $40-44 \%$ B2O3.

Eilbenk mentres (1982): $7 \mathrm{Mt}$ 4 45\% B2O3.

1987 resonves okt a 30-35\% 8203

Plant bulk in 1979

Capactly 50.6kt (eurtaco); 32.0kt (UO) colemantrayr.

Annual output cokt nophelline syentio.

sino of Egemetars Bursa area oporationa.

County reserves 30K $38-48 \% \mathrm{Cr} 2 \mathrm{O}$

Ore in marthe, thicknose up to $100 \mathrm{~m}$

Mining begen in 1971; Currently 85\% Uo, 15\% surnece. Operating bollow cap of $550 k$ due to weel herleapt. problems. Res: 12.0Mm 0.5\% WO3 (1995)

1994 production 37kt cone. trom stocks, mine closed in 1993.

Small prtvelto producer.

Produces 2Meyr coment from local limestones and clays.

Oporated in 1950's as a Siato Enterprise. Res: 1.75MR Q 60-95\% NaCl (1981).

Res: 13Mt coment raw matertals (1984). Plant capachy $704 \mathrm{xt}$ coment.

Res: 72.5MI (1987)

1986

Produced t01kt between 1950-55, hlgh grade.

AMt 4 33.80\% Mn (1988).

Dopostit in ectritat, limestone, and tut

1994 Reasves: 11.4Mt Q 1.03\% Cu, 0.5gh Au, 3.2gn Ag.

Second zone 1.45Mm @ 1.01\% Cu.

Smathed on elle to produce 17,000 bister

Cu (e) 8.32 oz Ag and 0.68902 Aunon.

Largent Cr producer in the 1950'

Rosorvos from aros: 9.6Mi O 22-50\% Cr2O3 (1987).

Producod $150 \mathrm{kt}$ lump ore, 7 okt concentrate in 1900 .

Mins reserves (1991): $2.84 t$ Q 44-48\% CR2O3.

Ferrochrome is exported. 
APPENDIX A. PRODUCINO MND DEVELOPINO MINERAL PROPERTES IN TURKEY

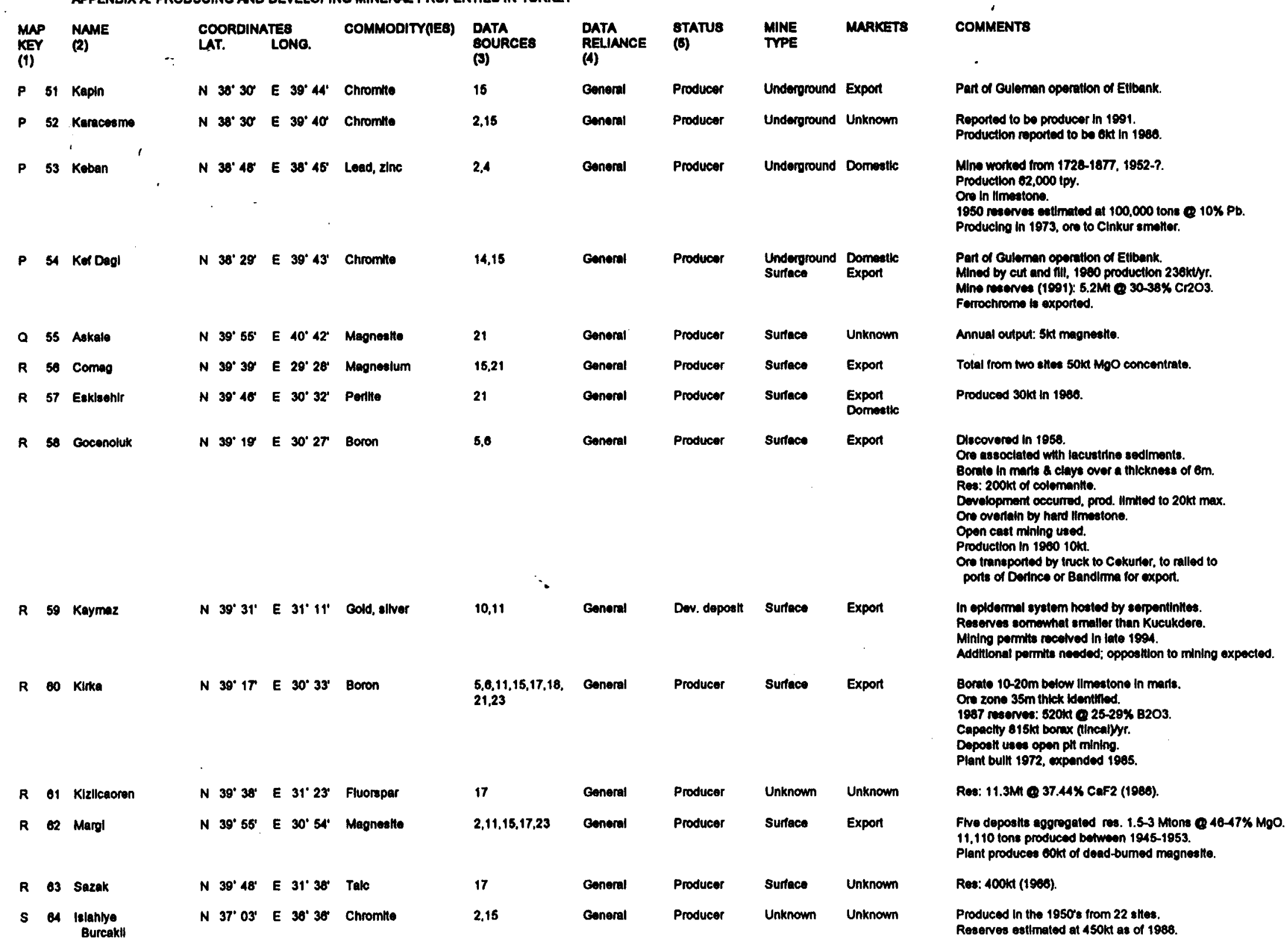


APPENDIX A PRODUCINO AND DEVELOPINO MINERAL PROPERTES IN TURKEY

\begin{tabular}{|c|c|c|c|c|c|c|c|c|c|c|c|}
\hline $\operatorname{man}_{\mathrm{K}}^{\operatorname{MN}}$ & & $\begin{array}{l}\text { NAME } \\
\text { (2) }\end{array}$ & $\begin{array}{l}\text { COOROINA } \\
\text { LAT. }\end{array}$ & $\begin{array}{l}\text { TES } \\
\text { LONO. }\end{array}$ & COMMODITY(IES) & $\begin{array}{l}\text { DATA } \\
\text { SOURCES } \\
\text { (3) }\end{array}$ & $\begin{array}{l}\text { DATA } \\
\text { RELIANCE } \\
\text { (4) }\end{array}$ & $\begin{array}{l}\text { STATUS } \\
(6)\end{array}$ & $\begin{array}{l}\text { MINE } \\
\text { TYPE }\end{array}$ & MARKETS & COMMENTS \\
\hline T & 65 & $\begin{array}{l}\text { Lahamos } \\
\text { Eaplyy }\end{array}$ & $N 40^{\circ} 5 T$ & E $30^{\circ} 44^{\circ}$ & Copper, zlne & 17 & Gonoral & Producer & Unknown & Unknown & Res: $2.3 \mathrm{Mt}$ 3.59\% Cu, 2.30\% Zn. \\
\hline u & $\infty$ & Bozuyuk , & $N 30^{\circ} 10^{\circ}$ & $E 30^{\circ} 15^{\prime}$ & $\begin{array}{l}\text { Clay, } \\
\text { sllica cand, } \\
\text { magneatto }\end{array}$ & 21 & Goneral & Producer & Surtace & Unknown & $\begin{array}{l}\text { Annual production: 10kt clay, 10kt slilica send, 10kt } \\
\text { magnesno. }\end{array}$ \\
\hline u & 67 & Iakondorun & N. $30^{\circ} 32^{2}$ & E $30^{\circ} 11^{\prime}$ & Chromito & 2.16 & Ganeral & Producer & Unknown & Unknown & $\begin{array}{l}\text { Produced in the 1950's from two stles. } \\
1955 \text { county roserves } 250 \mathrm{kt} \text {. }\end{array}$ \\
\hline v & $\infty$ & Icol & $N 36^{\circ} 18^{\circ}$ & E 34' 38' & Coment loodatock & 17 & General & Producer & Surrace & Domestlc & $\begin{array}{l}\text { Ros: } 117 \mathrm{M} \text { IImestone/marl (1983). } \\
\text { Plant produces 2.5Mt comont from lcel and Nlgdo plts. }\end{array}$ \\
\hline v & 09 & Karadiken & $N 37^{\circ} 00^{\circ}$ & $E 3^{\circ} 42^{\prime}$ & Limeatone & 21 & General & Producer & Surraco & Unknown & Annual output: 550kt calclum carbonate. \\
\hline v & 70 & Taraus & $N 37^{\circ} 09^{\circ}$ & E $34^{\circ} 30^{\circ}$ & Llgnite & 17 & General & Producor & Unknown & Unknown & $\begin{array}{l}\text { Res: } 2.6 \mathrm{Mt} \text { Q } 3000 \mathrm{Calkg} \text {. } \\
\text { Productng in } 1989 \text {. }\end{array}$ \\
\hline w & 71 & Karaagac & $N 30^{\circ}$ on & E $31^{\circ} 23^{\prime}$ & Bartte & 21 & General & Producer & $\begin{array}{l}\text { Surtace } \\
\text { Underpround }\end{array}$ & Unknown & In 1988, reported to produce 15kt surface, 5k UG. \\
\hline $\mathbf{w}$ & 72 & $\begin{array}{l}\text { Keclborlu } \\
\text { Kukuntoere } \\
\text { Deglimendere }\end{array}$ & N $37^{\circ} 57$ & E $30^{\circ} 18^{\prime}$ & Suliur & $2,11,15,17,21,23$ & Gonoral & Producer & $\begin{array}{l}\text { Surface } \\
\text { Underground }\end{array}$ & Unknown & 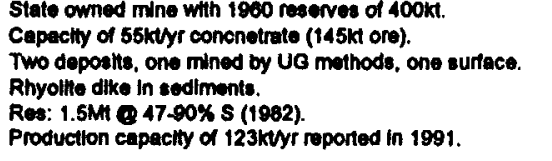 \\
\hline$x$ & 73 & Buyukcokmece & $\mathrm{N} / 1^{\circ} 01^{\circ}$ & E 28' 33' & Coment loedatock & 17 & Ganeral & Producer & Surface & Domettlc & $\begin{array}{l}\text { Res: 1 177M coment raw malerlals (1970). } \\
\text { Plant produces } 2.75 \mathrm{M} \text { coment annually. }\end{array}$ \\
\hline$x$ & 74 & letanbul & $N 41^{\circ} \infty 0^{\circ}$ & E 29'02' & Clay & 21 & General & Producer & Surtace & Domestle & Annual production: 10kt rofractory clay; 40ke coramic clay. \\
\hline$x$ & 75 & Sile & $N 41^{\circ} 11^{\circ}$ & E 29' 36' & Quartz cand & 17 & Goneral & Producer & Surface & Domestic & Res: 435MM (1980). \\
\hline$x$ & 70 & Sinekll & $N 41^{\circ} 14^{\prime}$ & E $26^{\circ} 12^{\prime}$ & Llgnite & 17 & Goneral & Producer & Unknown & Unknown & $\begin{array}{l}\text { Res: } 60 \text { Mt Q } 1950 \text { Callkg. } \\
\text { Produelng in } 1989 .\end{array}$ \\
\hline $\mathbf{Y}$ & 77 & Bomova & $N 30^{+} 27$ & E $27^{\circ} 14^{\prime}$ & Cement leodatock & 17 & General & Producer & Surtace & Domestle & Ros: 10Mt IImestone/clay (1909). \\
\hline $\mathbf{Y}$ & 78 & CumaovasI & N $38^{\prime} 15^{\prime}$ & E $2700^{\circ}$ & Penlte & 21 & Goneral & Producer & Suriace & $\begin{array}{l}\text { Domestle } \\
\text { Expont }\end{array}$ & $\begin{array}{l}\text { Annual production 26kt pertike. } \\
\text { Production from several plie. }\end{array}$ \\
\hline Y & 79 & Hallkoy & N 36. $13^{\circ}$ & E $27^{\circ} 59^{\circ}$ & Antlmony & 21 & Ceneral & Producer & Underground & Unknown & Annual output 7980 lons stlbnlle. \\
\hline $\mathbf{Y}$ & 80 & Hallkoy & $N 3^{\circ} 05^{\prime}$ & E $20^{\circ} 10^{\circ}$ & Morcuny & $2,11,15,17,23$ & Conoral & Producer & Underground & Expont & 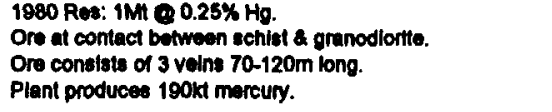 \\
\hline Y & 81 & Karaburun & N 38 $8^{\circ} 18^{\circ}$ & E $20^{\circ} 19^{\circ}$ & Dolomitto & 17 & General & Producer & urrace & Enown & Res: $2.88 t] 17.21 \% \mathrm{MgO}$ (1982). \\
\hline
\end{tabular}


APPENDIX A PRODUCING AND DEVELOPINO MINERAL PROPERTIES IN TURKEY

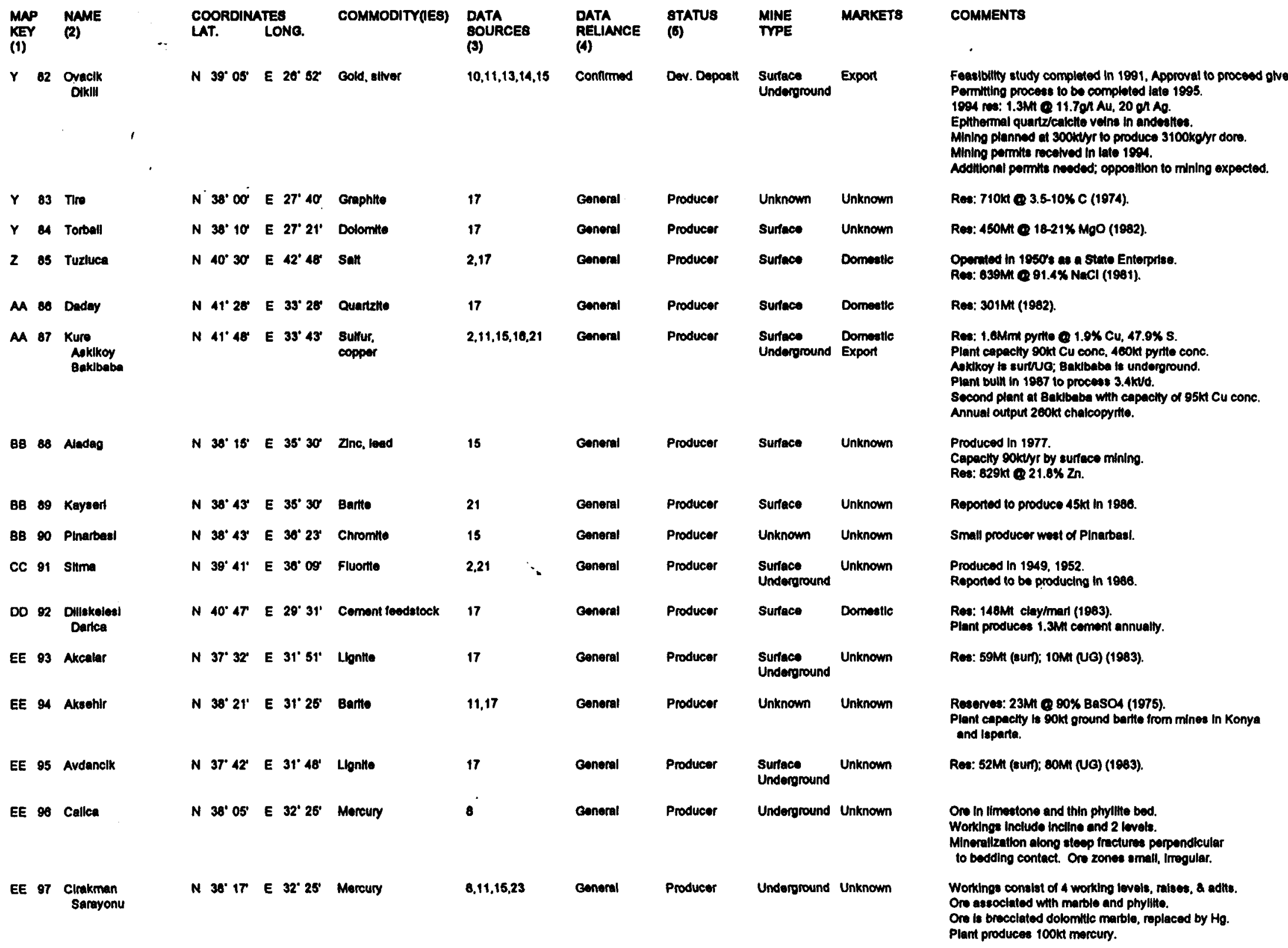


APPENDIX A: PRODUCINO AND DEVELOPINO MINERAL PROPERTIES IN TURKEY

\begin{tabular}{|c|c|c|c|c|c|c|c|c|c|c|c|c|}
\hline $\begin{array}{l}\text { MAP } \\
\text { KEY } \\
(1)\end{array}$ & & $\begin{array}{l}\text { MAME } \\
\text { (2) }\end{array}$ & $\because$ & $\begin{array}{l}\text { COORDINA } \\
\text { LAT. }\end{array}$ & $\begin{array}{l}\text { ATES } \\
\text { LONO. }\end{array}$ & COMMODITYRES) & $\begin{array}{l}\text { OATA } \\
\text { BOURCES } \\
\text { (כ) }\end{array}$ & $\begin{array}{l}\text { DATA } \\
\text { RELIANCE } \\
\text { (A) }\end{array}$ & $\begin{array}{l}\text { 8TATus } \\
\text { (B) }\end{array}$ & $\begin{array}{l}\text { MINE } \\
\text { MPE }\end{array}$ & MARKETS & COMMENTS \\
\hline EE & 98 & $\begin{array}{l}\text { Hoyuk } \\
\text { Beyeohit } \\
\text { Huyuk }\end{array}$ & & $N 37^{\prime} 41^{\prime}$ & E $31^{\circ} 43^{\prime}$ & Barto & $11,17,21$ & Canorat & Producer & Sunteces & Unknown & $\begin{array}{l}\text { Roservos: } 13 M \text { Q } 95.5 \% \text { BaSOA (1974). } \\
\text { Plent produces } 70 k \text { ground barte. } \\
\text { Muyuk mine produces 5kt crude bartte. }\end{array}$ \\
\hline EE & 99 & $\begin{array}{l}\text { 'Konya ' } \\
\text { Holvacibaba } \\
\text { Caylibagl } \\
\text { Konya Krom }\end{array}$ & & $\begin{array}{c}\text { N } 37^{\circ} 50^{\prime} \\
-\end{array}$ & E $32^{\circ} 38^{\circ}$ & Megneathe & 11.15 .21 & andur & Prosucer & sumece & $\begin{array}{l}\text { Domoutle } \\
\text { Expont }\end{array}$ & $\begin{array}{l}\text { Capactly } 48 \mathrm{kt} \text { dead-burned magnesla and } 38 \mathrm{kt} \text { brlcks. } \\
\text { Holveclogba plt is closed. }\end{array}$ \\
\hline EE & & Medrese & & $N 38^{\circ} 03^{\prime}$ & E $32^{\prime} 23^{\circ}$ & Mercuny & 8 & General & Producer & Underground & Unknown & $\begin{array}{l}\text { Nower mine in } 1970 \text {. } \\
\text { Workings consist of } 4 \text { drtis and crossculs. }\end{array}$ \\
\hline EE & 101 & Mortas & & $N 37^{\circ} 16^{\circ}$ & E $31^{\circ} 52^{\prime}$ & Bauxito & $11.15,23$ & General & Producer & Surface & Domestlc & 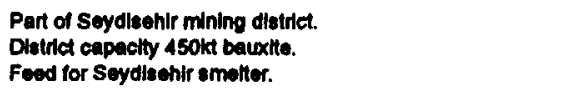 \\
\hline EE & & $\begin{array}{l}\text { Seydisahir } \\
\text { Mortas } \\
\text { Dogankuzu }\end{array}$ & & N $37^{\prime} 26^{\prime}$ & E $31^{\circ} 61^{\prime}$ & Bauxht: & $10,11,16,16,17,21$ & Goneral & Producer & Surrace & $\begin{array}{l}\text { Domostlc } \\
\text { Expont }\end{array}$ & 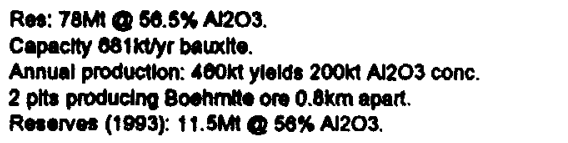 \\
\hline EE & & $\begin{array}{l}\text { Slzma-Ladik } \\
\text { Buyuk }\end{array}$ & & N $38^{\circ}$ or & E $32^{\circ} 25^{\prime}$ & Mercury & $2,8,15,17,23$ & General & Producer & $\begin{array}{l}\text { Surface } \\
\text { Underground }\end{array}$ & Export & 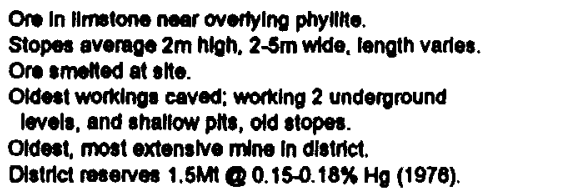 \\
\hline $\mathbf{F F}$ & & $\begin{array}{l}\text { Aktepe } \\
\text { Gumuskoy }\end{array}$ & & N $30^{\circ} 58^{\prime}$ & E $29^{\circ} 29^{\prime}$ & Silvor & $\begin{array}{r}10,11,14,16 \\
\ddots\end{array}$ & General & Producer & Surface & Expont & 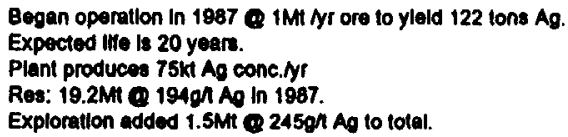 \\
\hline FF & 105 & Emol Area & & N $39^{\circ} 13^{\prime}$ & E $29^{\circ} 09^{\circ}$ & Boron & $\begin{array}{l}2,5,6,11,15,17 \\
18,21,23\end{array}$ & General & Producer & $\begin{array}{l}\text { Surface } \\
\text { Underground }\end{array}$ & Export & 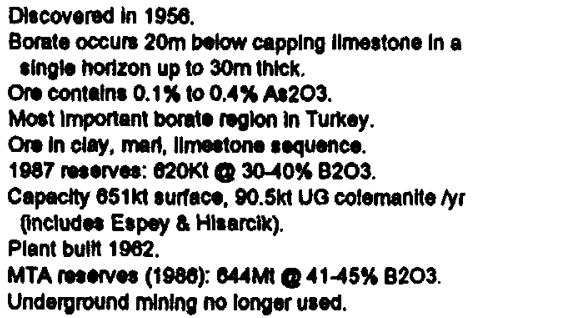 \\
\hline
\end{tabular}


APPENDIX A. PROOUCINO AND DEVELOPINO MINERUL PROPERTIES IN TURKEY

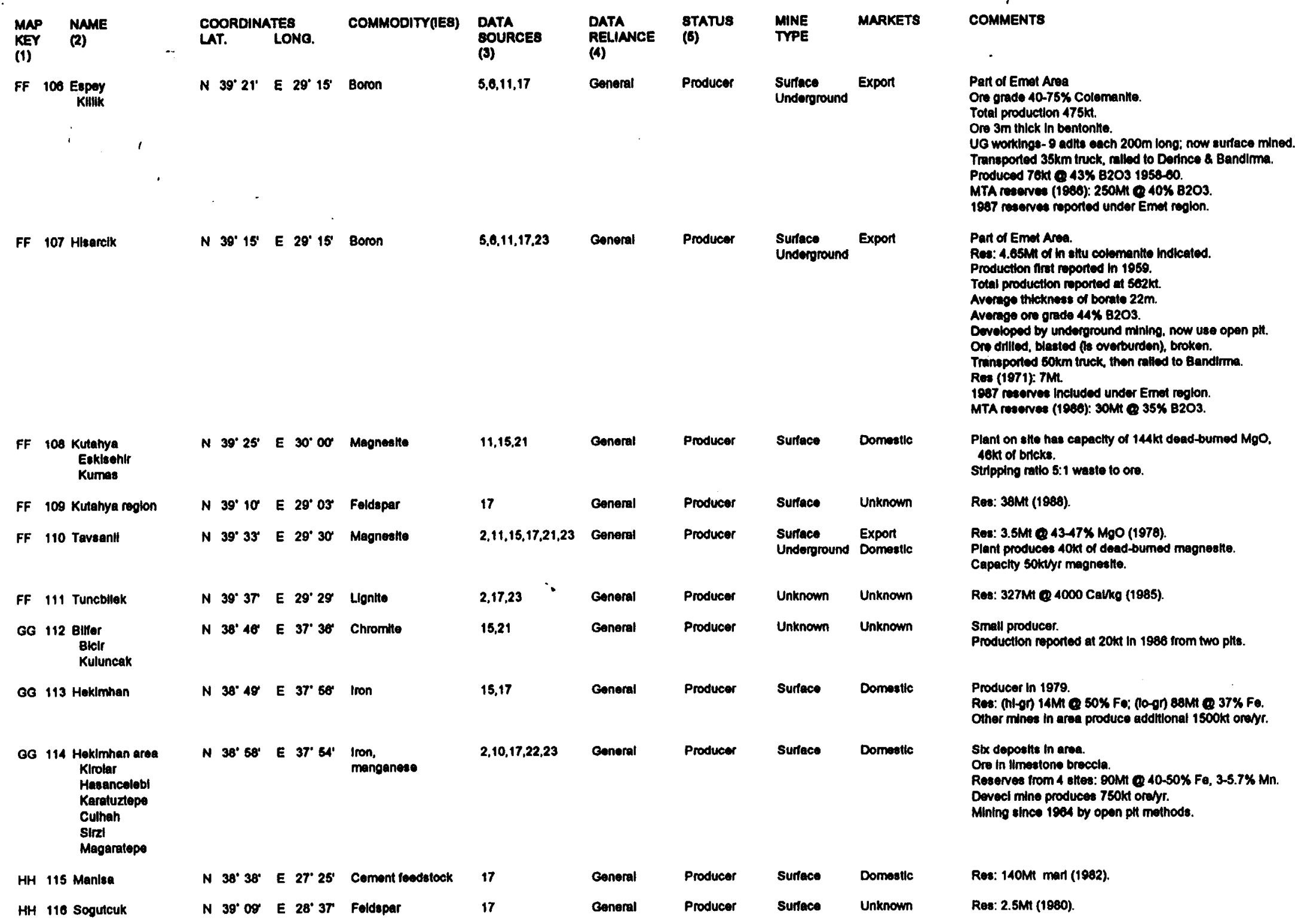


APPENDIX A: PRODUCING AND DEVELOPINO MINERNL PROPERTES IN TURKEY

\begin{tabular}{|c|c|c|c|c|c|c|c|c|c|c|c|}
\hline $\begin{array}{l}\text { MAP } \\
\text { (1) }\end{array}$ & $\begin{array}{l}\text { NAME } \\
\text { (2) }\end{array}$ & $\because$ & $\begin{array}{l}\text { COORDIN } \\
\text { LAT. }\end{array}$ & $\begin{array}{l}\text { ATES } \\
\text { LONO. }\end{array}$ & COMMODITY(RE) & $\begin{array}{l}\text { DATA } \\
\text { SOURCES } \\
\text { (j) }\end{array}$ & $\begin{array}{l}\text { DATA } \\
\text { RELIANCE } \\
\text { (4) }\end{array}$ & $\begin{array}{l}\text { 8TATUS } \\
(6)\end{array}$ & MINE & MARKETS & COMMENTS \\
\hline HH & 117 Soma & & $N 39^{\circ} 10^{\circ}$ & E $27^{\circ} 36^{\prime}$ & Llante & 2.17 & Ceneral & Producer & $\begin{array}{l}\text { Surtace } \\
\text { Underground }\end{array}$ & Unknown & Res: $44 M$ (sum): COMH (UG) (1982). \\
\hline HH & 118 , Soma & 1 & $N 39^{\circ} 09^{\circ}$ & E $27^{*} 30^{\circ}$ & Cement foedstock & 17 & Goneral & Producer & Surface & Domestle & Res: 158MH IImestone/marl (1982). \\
\hline " & 119 Sekeroba & & $N 37^{4}+7$ & $E 36^{\circ} \mathbf{4 3 ^ { \prime }}$ & Bartho & 21 & General & Producer & Underground & Unknown & Minn output: 15kt bartte. \\
\hline JJ & 120 Mazldag & & N. $37^{\circ} 31^{\circ}$ & E $40^{\circ} 30^{\circ}$ & $\begin{array}{l}\text { Phosphate. } \\
\text { uranlum }\end{array}$ & $10,11,15.17,21,23$ & Goneral & Producer (7) & Surface & Domentlc & 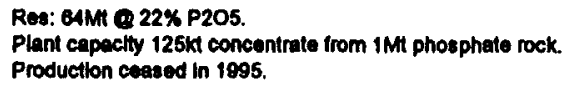 \\
\hline KK & 121 Kandak & & $N 37^{\circ}$ O4. & E 28. 32' & Chromile & 15 & General & Producer & Underground & Unknown & Small producer. \\
\hline KK & 122 Koycegiz & & $N 36^{\circ} 5 r$ & E $28^{\prime} 40^{\circ}$ & Chromite & 15 & General & Producar & $\begin{array}{l}\text { Surface } \\
\text { Underground }\end{array}$ & Unknown & Small producer alnce the $1970 \mathrm{~s}$. \\
\hline KK & 123 Millas & & $N 37^{\circ} 18^{\circ}$ & E $27^{\prime} A T$ & Foldepar & 21 & General & Producer & Surface & Unknown & Annual output: $120 \mathrm{kt}$ Na-teldspar. \\
\hline KK & 124 Millas & & $N 37^{\circ} 18^{\prime}$ & E $27^{\circ} 47$ & $\begin{array}{l}\text { Emory. } \\
\text { Iltgnte }\end{array}$ & 17,21 & Goneral & Producar & $\begin{array}{l}\text { Surtace } \\
\text { Underground }\end{array}$ & Domettlc & 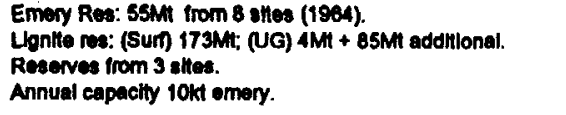 \\
\hline KK & 125 Millas & & $N 37^{\circ} 18^{\circ}$ & E $27^{\circ} 48^{\prime}$ & Bauxthe & 17,21 & General & Producer & $\begin{array}{l}\text { Surtace } \\
\text { Underground }\end{array}$ & $\begin{array}{l}\text { Domestic } \\
\text { Expont }\end{array}$ & 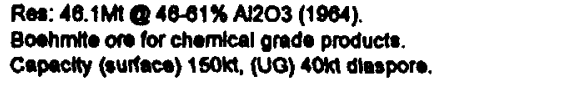 \\
\hline KK & 126 Uckopru & & $N 37^{\prime} 16^{\prime}$ & E $28^{\circ} 10^{\circ}$ & Chromile & $2,15,21$ & General & Producer & Underground & Domestic & $\begin{array}{l}\text { Produced in the } 1950 \text { s. } \\
\text { Roserves at of } 190090 \mathrm{kt} \text { ore. } \\
\text { Production reported to be } 70 \mathrm{kt} \text { in } 1988 . \\
\text { Foede Antalya emotter. }\end{array}$ \\
\hline KK & 127 Yatagan & & $N 37^{\prime} 20$ & E $28^{\circ} 09$ & $\begin{array}{l}\text { Emery, } \\
\text { llgnite }\end{array}$ & 17 & General & Producor & $\begin{array}{l}\text { Surnace } \\
\text { Underground }\end{array}$ & Unknown & $\begin{array}{l}\text { Emery Res: IMt from } 13 \text { sthes (1964). } \\
\text { Coal res: (Surn to9Mt; (UG) 10Mt + 130Mt additional. } \\
\text { Producing in 1969. }\end{array}$ \\
\hline KK & 128 Yatagan & & $N 37^{\circ} 20^{\circ}$ & E $28^{\circ} 09^{\circ}$ & Calctite & 21 & General & Producer & Surfaco & Expont & Capactiy 100kty r calche. \\
\hline LL & 129 Mus & & $N 38^{\prime} 44^{\prime}$ & E $41^{\circ} 30^{\circ}$ & Bartio & 21 & General & Producer & $\begin{array}{l}\text { Surface } \\
\text { Undenground }\end{array}$ & Unknown & Annual output 120kt bartle. \\
\hline MM & 130 Gubehir & & $N 38^{\circ} 45^{\prime}$ & E $34^{\prime} 38^{\circ}$ & Salt & 17 & General & Producer & Surfaco & Domestic & Res: 2.4Bt @ 93\% NaCl (1980), \\
\hline MM & 131 Salonda & & $N 38^{\circ} 50^{\prime}$ & E $34^{*} 32^{\prime}$ & Bartic & 21 & Genenal & Producor & Surface & Unknown & Reportod to produce 10kt in 1986. \\
\hline NN & 132 Ulubey & & $N 40^{\circ} 51^{\prime}$ & E $37^{\circ} 46^{\prime}$ & Kaolin & 17 & General & Producar & Surface & Domestle & $\begin{array}{l}\text { Res: } 2.3 \mathrm{MMt} \text { 17-23\% A12O3 (1986). } \\
\text { Ueed in papor Induatry. }\end{array}$ \\
\hline NN & 133 Unyo & & $N \$ 1088^{\circ}$ & E $37 \%$ & Bentontie & 17,21 & General & Producer & Surface & Unknown & $\begin{array}{l}\text { Res: } 2.5 \mathrm{Mt}(1977) \text {. } \\
\text { Annual production: 85ki bentonthe from } 3 \text { sthes. }\end{array}$ \\
\hline$\infty$ & 134 Cayell & & $N 1^{\circ} 04^{\prime}$ & $E 40^{\circ} 48^{\prime}$ & $\begin{array}{l}\text { Znc, copper } \\
\text { silver, gold }\end{array}$ & $10,11,15$ & General & Producer & Underground & Export & 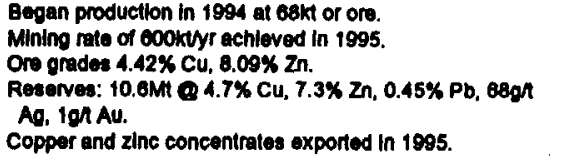 \\
\hline
\end{tabular}


APPENOIX A: PRODUCING AND DEVELOPINO MINERAL PROPERTIES IN TURKEY

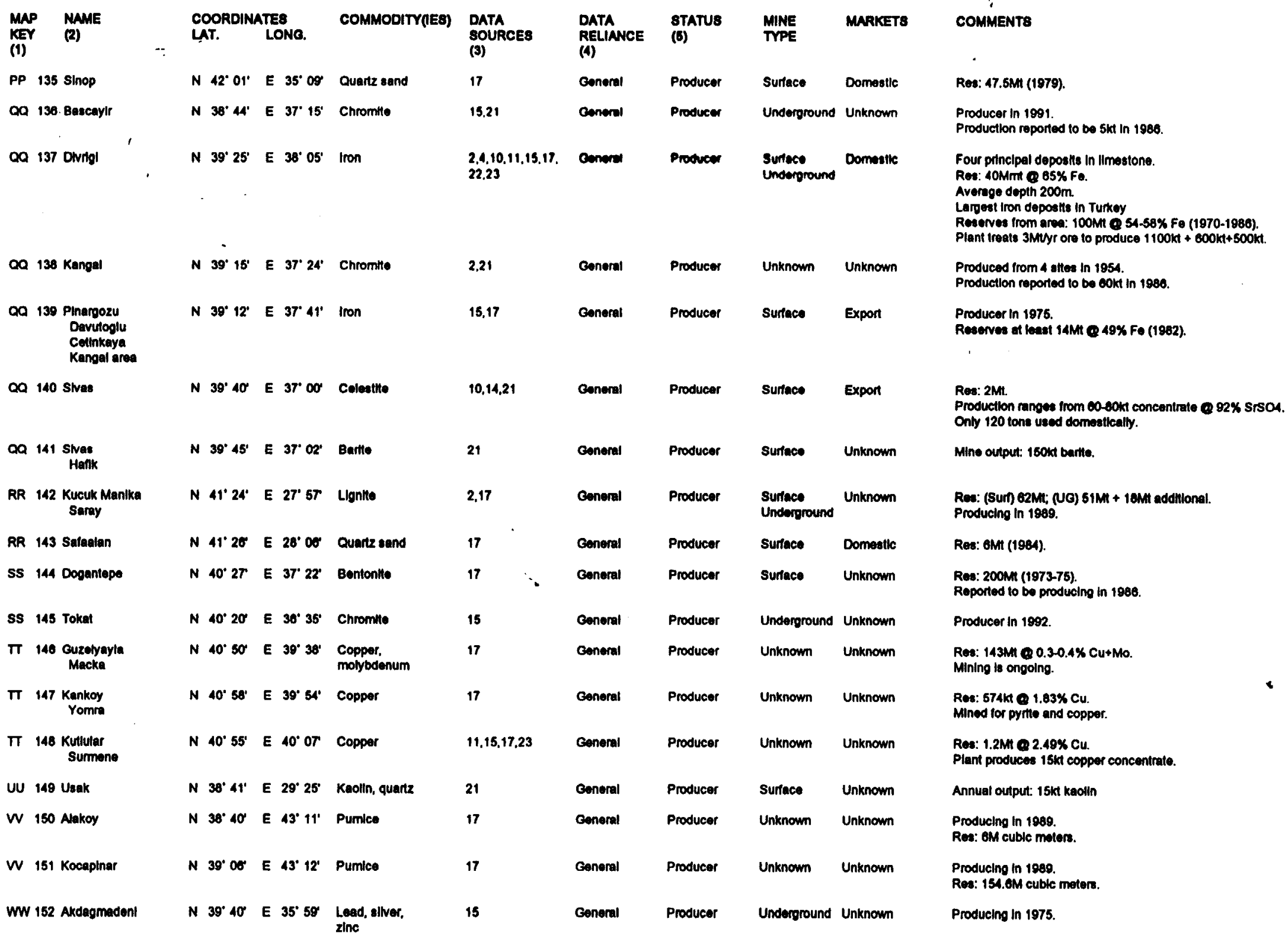


APPENDIX A PRODUCINO AND DEVELOPINO MINERAL PROPERTIES IN TURKEY

\begin{tabular}{|c|c|c|c|c|c|c|c|c|c|c|c|}
\hline $\begin{array}{l}\text { MAP } \\
\text { (1) }\end{array}$ & $\begin{array}{l}\text { NAME } \\
\text { (2) }\end{array}$ & $\because$ & $\begin{array}{l}\text { COORDINA } \\
\text { LAT. }\end{array}$ & $\begin{array}{l}\text { ITES } \\
\text { LONO. }\end{array}$ & COMMODITY(RES) & $\begin{array}{l}\text { DATA } \\
\text { SOURCES } \\
\text { (9) }\end{array}$ & $\begin{array}{l}\text { OATA } \\
\text { RELIANCE } \\
\text { (A) }\end{array}$ & $\begin{array}{l}\text { 8TATUS } \\
\text { (6) }\end{array}$ & $\begin{array}{l}\text { MINE } \\
\text { TYPE }\end{array}$ & MARKET8 & COMMENTS \\
\hline ww & 153 Saray & & N $39^{\circ} 42^{\prime}$ & E $34 \cdot 40$ & Coment foodatock & 17 & Gonoral & Producer & Surface & Domeatic & $\begin{array}{l}\text { Res: soMt IImostone/clay/mart (1984). } \\
\text { Plant produces 2Mt coment annually from phis in } \\
\text { Conum, Sivas, and Yozgat countles. }\end{array}$ \\
\hline$w w$ & $154^{\prime}$ Sefaatll & 1 & N $39^{\circ} 49^{\circ}$ & E $35^{*} 11^{\prime}$ & Quartzine & 17 & Goneral & Producer & Surtace & Domeatlc & Res: $2.88 t$ (1986). \\
\hline $\mathbf{w} \mathbf{w}$ & 155 Sekll & . & N $39^{\circ} 44^{\prime}$ & E $34^{\circ} 15^{\prime}$ & Salt & 2,17 & Gonoral & Producer & Surface & Domestlc & 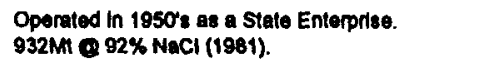 \\
\hline$x \mathbf{x}$ & 156 Alapll & & $N 41^{\circ} 11^{\prime}$ & E $31^{\circ} \mathbf{2 3}$ & Quartzte & 17 & Goneral & Producer & Surface & Domestlc & Res: 105Mt (1980). \\
\hline$x x \quad 1$ & 157 Amasra & & $N 41^{\circ} 44^{\circ}$ & E $32^{\circ} 23^{\prime}$ & Coal & 2.17 & Gonoral & Producer & Underground & Unknown & Res: 287 Mh to depth of $1000 \mathrm{~m}$ (1980). \\
\hline$x \times \quad 1$ & 158 Amutcuk & & $N 41^{\circ} 22^{\prime}$ & E $31^{*} 35^{*}$ & Coal & 2,17 & General & Producer & Underground & Unknown & $\begin{array}{l}\text { Res: } 91 \mathrm{Mm} \text { to depth of } 1000 \mathrm{~m} \text { (1986). } \\
1955 \text { county reserve } 18 \mathrm{st} \text {. }\end{array}$ \\
\hline $\mathbf{x} x$ & 159 Eflant & & N $41^{\circ} 26^{\circ}$ & E $32^{\circ} 57$ & Quartzno & 17 & General & Producer & Surface & Domestlc & Res: 124Mt (1982). \\
\hline$x x \quad 1$ & 160 Karadon & & $N 41^{\circ} 30^{\circ}$ & E $31^{\prime} 32^{\prime}$ & Coal & 17 & Genoral & Producer & Underground & Unknown & Res: 474 Mt to depth of $1200 m$ (1980). \\
\hline$x \times 1$ & 101 Kozlu & & $N 41^{\circ} 20^{\circ}$ & E $31^{\circ} 40^{\circ}$ & Coal & 2,17 & Gonomal & Producer & Underground & Unknown & $\begin{array}{l}\text { Res: } 278 \mathrm{Mm} \text { to depth of } 1200 \mathrm{~m} \text { (1980). } \\
\text { Mined by E.K.I. }\end{array}$ \\
\hline$x \times 1$ & 162 Sopcakoy & & $N 41^{\circ} 25^{\circ}$ & E $31^{\circ} 60^{\circ}$ & Quartzke & 17 & General & Producer & Surface & Domestle & Res: 500MH (1978). \\
\hline$x \times 1$ & 163 Uzulmez & & $N 41^{\circ} 2 T$ & E $31^{\circ} 47$ & Coal & 17 & Goneral & Producer & Underground & Unknown & Res: 244 Mit to depth of $1200 \mathrm{~m}$ (1980). \\
\hline & $\begin{array}{l}\text { (1) Represe } \\
\text { (2) Due to: } \\
\text { (3) Compel } \\
\text { (4) General } \\
\text { (5) Becaus } \\
\text { (0) Uludag } \\
\text { (7) Produet }\end{array}$ & $\begin{array}{l}\text { A - deno } \\
\text { se of the } \\
\text { o mine re } \\
\text { cllon at }\end{array}$ & 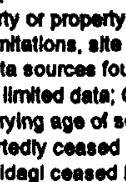 & $\begin{array}{l}\text { grouping as } \\
\text { names do no } \\
\text { und in Appene } \\
\text { Confirmed - d } \\
\text { ource Informe } \\
\text { production in } \\
\text { in } 1995 \text { due tc }\end{array}$ & 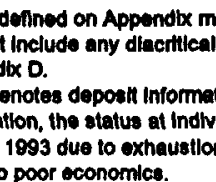 & 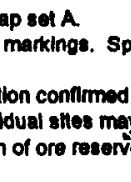 & $\begin{array}{l}\text { veral source: } \\
\text { ganiment. } \\
\text { gani mine kd }\end{array}$ & some proc & or stocks. & & \\
\hline
\end{tabular}




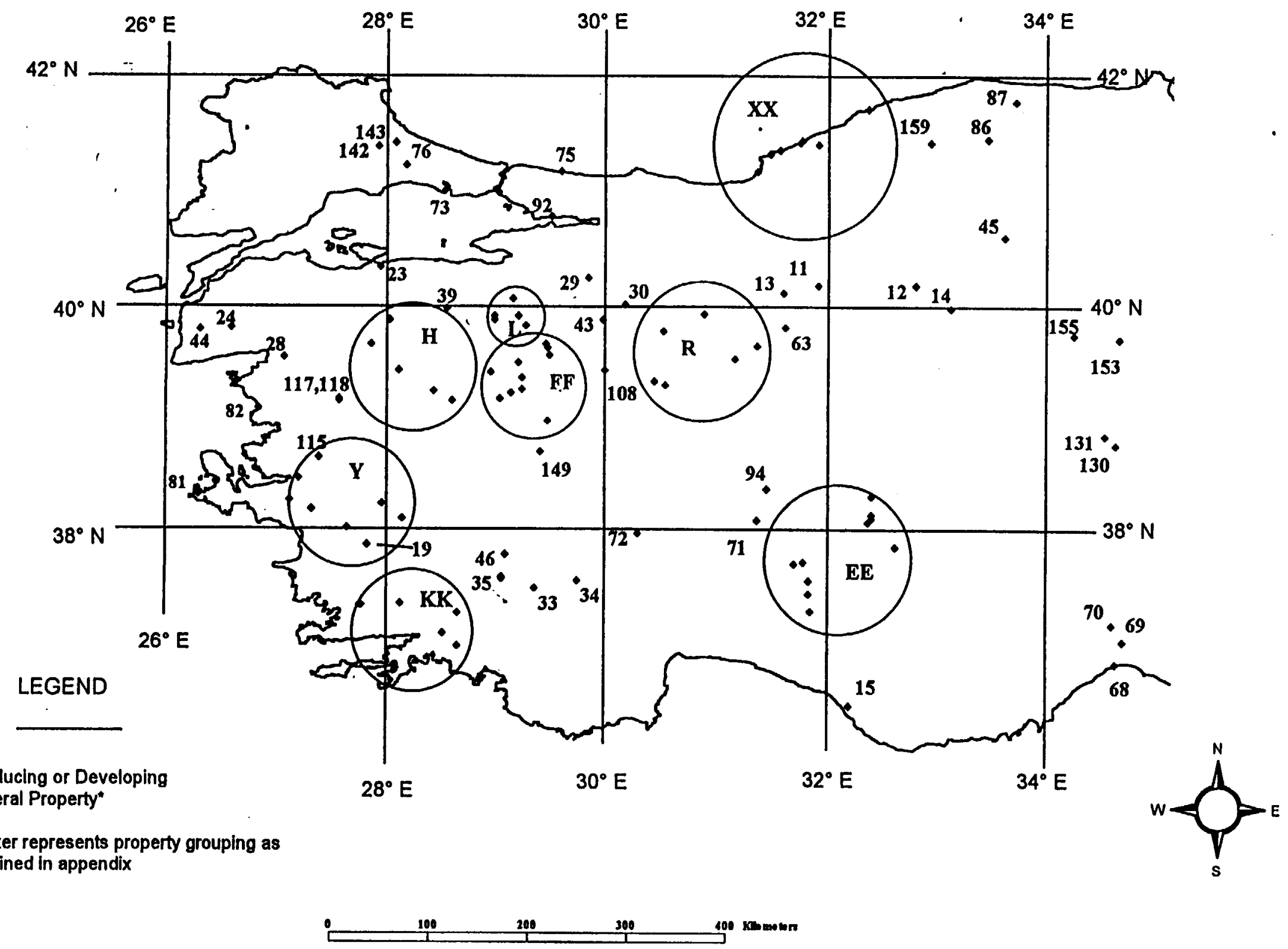

APPENDIX MAP A-1: PRODUCING MINERAL PROPERTIES OF WESTERN TURKEY 


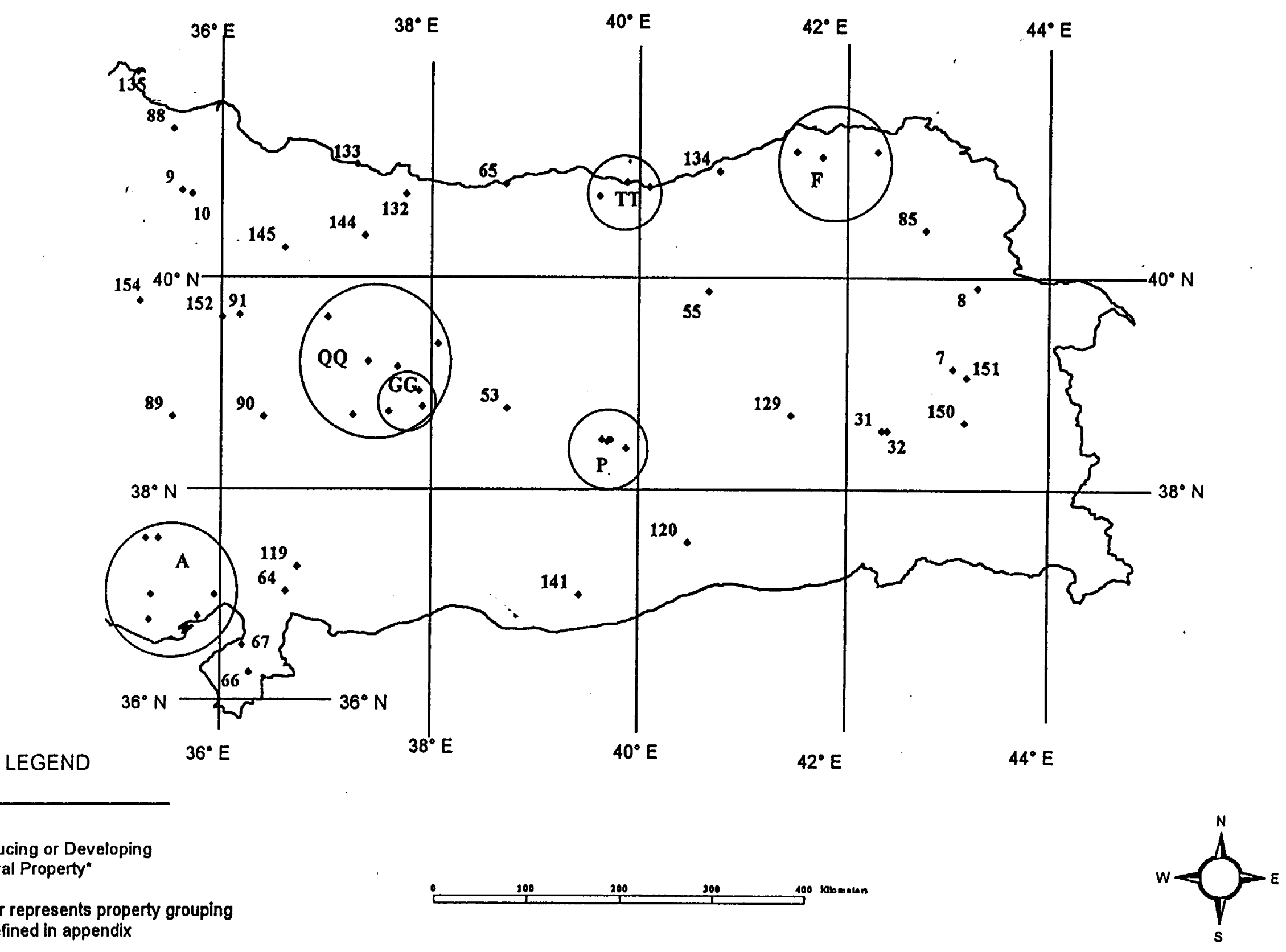

APPENDIX MAP A-2: PRODUCING MINERAL PROPERTIES OF EASTERN TURKEY 
APPENOIX B: PAST PRODUCING MINERAL PROPERTEE IN TURKEY

\begin{tabular}{|c|c|c|c|c|c|c|c|c|c|c|c|}
\hline$\underset{\mathbf{K E}}{\mathrm{MA}}$ & & $\begin{array}{l}\text { NAME } \\
\text { (2) }\end{array}$ & $\begin{array}{l}\text { COORDINA } \\
\text { UAT. }\end{array}$ & $\begin{array}{l}\text { ATE8 } \\
\text { LONO. }\end{array}$ & COMMODITY(EES) & $\begin{array}{l}\text { DATA } \\
\text { BOURCES } \\
\text { (3) }\end{array}$ & $\begin{array}{l}\text { DATA } \\
\text { RELLANCE } \\
\text { (4) }\end{array}$ & 8TATUS & $\begin{array}{l}\text { MINE } \\
\text { TYPE }\end{array}$ & MARKETS & COMMENTS \\
\hline A & 1 & 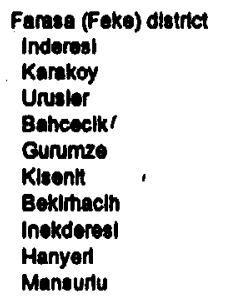 & $N 37: 50$ & E $35^{\circ} 40^{\circ}$ & Iron & 2 & Goneral & Past producer & Surface & Unknown & 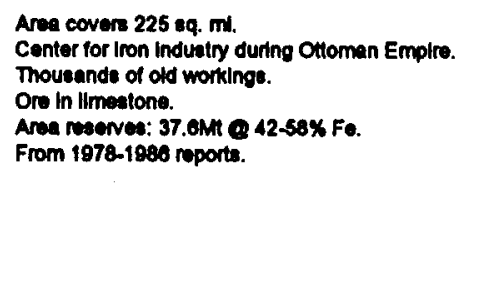 \\
\hline $\mathbf{A}$ & 2 & KIrec & $N 38^{\circ}$ Or & E $30^{\circ} 18^{\prime}$ & Iron & 2 & Ceneral & Peat producer & surtace & Unknown & Area mined in the past. \\
\hline B & 3 & Bakinl Movkll & $N 30^{\circ} \mathbf{2 0}$ & E $30^{\circ}$ or & Coppor & 2 & General & Past producer & Unknown & Unknown & Old working vialble from velin in limestons. \\
\hline B & 4 & Gazllgol & $N 39^{\circ} 00^{\circ}$ & E $30^{\circ} 27$ & Magnealte & 2 & Genenal & Pant producer & Unknown & Unknown & Produced 1085 tons in 1954. \\
\hline B & 5 & KIrka & N $36^{\circ} 44^{\prime \prime}$ & $E 30^{\circ} 14^{\circ}$ & Manganoese & 2 & Coneral & Past producer & Unknown & Unknown & Produced 125 tons in 1951. \\
\hline c & e & Cottok & $N 41^{*} 13^{\circ}$ & E $35^{\circ} 21^{\prime}$ & LIgnne & 2 & General & Past producer & Unknown & Unknown & Res: $7 \mathrm{MI} \& 6265 \mathrm{Cal} / \mathrm{kg}$. \\
\hline c & 7 & Gumusheclkoy & $N 40^{\circ} 53^{\prime}$ & E $35^{\circ} 14^{\prime \prime}$ & Lead, sllver & 2 & Goneral & Past producer & Underground & Unknown & $\begin{array}{l}\text { Oro in limestone and andeste to depth } \\
\text { o } 250 \mathrm{~m} \text {. } \\
\text { Ore deplated, 200,000 lons romoved. }\end{array}$ \\
\hline c & 8 & Merzifion & N $40^{\circ} 53^{\circ}$ & E $35^{\circ} 29^{\prime}$ & Copper & 2 & Goneral & Pest producer & Undorground & Unknown & $\begin{array}{l}\text { Soveral tunnela dituon about } 1870 \text {. } \\
\text { All but ono caved. }\end{array}$ \\
\hline D & 9 & Bolkardag & N 39' 21' & E $33^{\circ} 26^{\circ}$ & Iron & 2 & General & Past producer & Unknown & Unknown & $\begin{array}{l}\text { Magnethe in limeotone wth thleknets of } 20 \mathrm{~m} \text {. } \\
\text { Res:4Mmt } 800-05 \% \text { fo (1983). } \\
\text { Produced } 1953+\text {. }\end{array}$ \\
\hline D & 10 & Camilsagir & N $39^{\circ} 20^{\circ}$ & E $33^{\circ} 25^{\prime}$ & Iron & 2.17 & General & Past producer & Unknown & Unknown & $\begin{array}{l}\text { Oporated in } 1955 \text {. } \\
\text { Res: 0.2MmX } \Phi \text { 00-05\% Fo (1903). }\end{array}$ \\
\hline D & 11 & CayinI & N $39^{\circ} 36^{\prime}$ & E $32^{\circ} 36^{\circ}$ & Manganese & 2,17 & General & Past producer & Unknown & Unknown & $\begin{array}{l}\text { Produced 23.8kt } \\
\text { Res: } 150 \mathrm{kt} \text { \& 19.45\% } \mathrm{Mn} \text { (1951). }\end{array}$ \\
\hline D & 12 & $\begin{array}{l}\text { Colobl } \\
\text { Dominel } \\
\text { Agapinar }\end{array}$ & N $39^{\circ} 28^{\prime}$ & E 33' 32' & Iron & 22 & Goneral & Past producer & Surface & Unknown & Mining carrled out in 1963-04, producing 17.5kt c \\
\hline D & 13 & Denek-Keskla & $N 30^{\circ} 40^{\circ}$ & E $33^{\circ} 36^{\circ}$ & Lead, atwer & 2 & General & Past producer & Underground & Unknown & 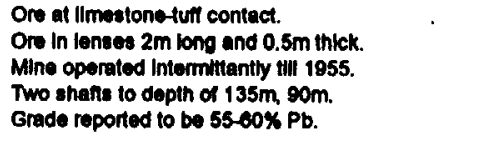 \\
\hline D & 14 & Goztepe & $N 40^{\circ} 08^{\circ}$ & E $33^{\circ} 46^{\prime}$ & Manganese & 2 & General & Paet producer & Unknown & Unknown & $\begin{array}{l}\text { Produced 38.7kt between 1949-1955. } \\
\text { 1950 resentes 50kt @ 38-40\% Mn. }\end{array}$ \\
\hline D & 15 & Kesikkopnu & N $39^{\circ} 23^{\prime}$ & E $33^{\circ} 2 \sigma^{\circ}$ & $\begin{array}{l}\text { Iron, } \\
\text { manganese }\end{array}$ & 2.17 & General & Past producer & Surface & Unknown & $\begin{array}{l}\text { Ore assayling } 50 \% \text { Fo and to\% Mn. } \\
\text { Quarny lace } 25 \mathrm{~m} \mathrm{hlgh} \text {. } \\
\text { Area roserves: } 11.5 \mathrm{Mt} \text { 32-54\% Fo (1983). }\end{array}$ \\
\hline
\end{tabular}


APPENDIX B: PAST PRODUCINO MINERAL PROPERTES IN TURKEY

\begin{tabular}{|c|c|c|c|c|c|c|c|c|c|c|c|c|}
\hline $\begin{array}{l}M A \\
\text { (1) }\end{array}$ & & $\begin{array}{l}\text { NAME } \\
\text { (2) }\end{array}$ & $\because$ & $\begin{array}{l}\text { COORDINA } \\
\text { LAT. }\end{array}$ & $\begin{array}{l}\text { ATE8 } \\
\text { LONO. }\end{array}$ & COMMODITY(RE) & $\begin{array}{l}\text { DATA } \\
\text { SOURCEB } \\
\text { (J) }\end{array}$ & $\begin{array}{l}\text { DATA } \\
\text { RELIANCE } \\
\text { (4) }\end{array}$ & STATUS & $\begin{array}{l}\text { MINE } \\
\text { TYPE }\end{array}$ & MARKET8 & COMMENTS \\
\hline 0 & 16 & Kllovill & & N 39' $52^{\prime}$ & E $33^{\circ} 40^{\circ}$ & Molybdonum & 2 & Goneral & Past producer & Unknown & Unknown & $\begin{array}{l}\text { Worked in the 1930's, neterves oxhaustod. } \\
\text { Ore in fractures of grantite. }\end{array}$ \\
\hline D & 17. & Killelar , & 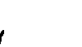 & N 39' $33^{\prime}$ & E 33' 22' & Iron & 2 & Coneral & Past producer & Unknown & Unknown & Production for the partod 1947-1954. \\
\hline 0 & 18 & Killinclar & ' & N $30^{\circ} 53^{\prime \prime}$ & E $33^{\circ} 22^{\circ}$ & Manganose & 2 & Goneral & Past producer & Unknown & $\begin{array}{l}\text { Domoatle } \\
\text { Export }\end{array}$ & $\begin{array}{l}\text { Sold 2/3 to Kanabuk plant, } 1 / 3 \text { expontod. } \\
1950 \text { meserves 10-5okt Q } 47 \% \mathrm{Mn} \text {. }\end{array}$ \\
\hline D & 19 & Kinkkala & & N $39^{\circ} 50^{\circ}$ & E $33^{\circ} 31^{\prime}$ & Lead, zine & 2 & Goneral & Past producer & Underground & Unknown & $\begin{array}{l}\text { Grade reported to be } 18 \% \mathrm{~Pb} \text { and } 22 \% \mathrm{Zn} \text {. } \\
\text { Produced in } 1854 \text {. }\end{array}$ \\
\hline D & 20 & Tilki & & $N 40^{\circ} 16^{\circ}$ & E $33^{\circ} 29^{\prime \prime}$ & Manganeses & 2 & General & Pest producor & Unknown & Domestle & $\begin{array}{l}\text { Two deposile discovered in } 1941 \text {. } \\
\text { Up to 8kt mined by 1946, sold lo Karabuk plant. } \\
\text { Depoatte of sedimentary orlgin in serpentine. }\end{array}$ \\
\hline E & 21 & Adrasan & & N $36^{\prime} 19$ & E $30^{\circ} 27$ & Chromne & 2 & General & Past producer & Unknown & Unknown & $\begin{array}{l}\text { Produced in the 1950's. } \\
1955 \text { county resonves } 525 \mathrm{kt} \text { 38-10\% Cr2O3. }\end{array}$ \\
\hline $\mathbf{E}$ & 22 & Agva & & N $30^{\circ} 33^{\prime}$ & E $30^{\circ} 34^{\circ}$ & Chromtite & 2 & General & Past producor & Unknown & Unknown & $\begin{array}{l}\text { Produced in the } 1950 \mathrm{~s} \text {. } \\
1955 \text { county reserves } 525 \mathrm{kt} \text { o } 30-40 \% \mathrm{Cr} O 3 .\end{array}$ \\
\hline E & 23 & Kamalar & & $N 38^{\circ} 19^{\circ}$ & E $32^{\circ} 21^{\circ}$ & Lead & 2 & General & Past producer & Underground & Unknown & $\begin{array}{l}\text { Ore occurs in a dome od } 90 \times 40 \mathrm{~m} \text {. } \\
\text { Worked pritor to } 1912 \text {. }\end{array}$ \\
\hline $\mathbf{E}$ & 24 & Kumluce & & N 30' $22^{\prime}$ & E $30^{\circ} 17$ & Chromine & 2 & General & Past producer & Unknown & Unknown & $\begin{array}{l}\text { Produced in the 1950's. } \\
1855 \text { county rosenves } 211 \mathrm{kt} \text {. }\end{array}$ \\
\hline E & 25 & Parallmusa & & N $30^{\circ} 25^{\circ}$ & E $30^{\circ} 20^{\circ}$ & Manganese & 2 & General & Past producer & Unknown & Unknown & Produced 4083 tons in 1952. \\
\hline E & 20 & Toklrova & & N 36. $30^{\circ}$ & E $30^{\circ} 32$ & Chromite & 2 & General & Past producer & Unknown & Unknown & $\begin{array}{l}\text { Produced In the 1950's. } \\
1955 \text { county roserves } 525 \mathrm{KT} 36-10 \% \mathrm{Cr} 2 \mathrm{O} \text {. }\end{array}$ \\
\hline E & 27 & Yukan & & N $30^{\circ} 33^{\prime}$ & E $32^{\circ} 01^{\circ}$ & Iron & 2 & General & Past producer & Surrace & Unknown & Conc. of limonite noat sesaying 48.47\% Fo. \\
\hline $\mathbf{F}$ & 28 & Cavdar & & N $37^{\circ} 35^{\prime}$ & E $27^{\circ} 39^{\circ}$ & Iron & $2,17,22$ & Goneral & Past producer & Surface & Domostle & 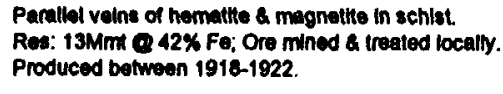 \\
\hline $\mathbf{F}$ & 29 & Kayacik & & $N 37^{\circ} 55^{\circ}$ & E $27^{\circ} 50^{\circ}$ & Lead & 2 & General & Paut producer & Unknown & Unknown & Prod. 82 tons in 19 M8. \\
\hline $\mathbf{F}$ & 30 & Sobuca & & N $37^{\circ} 45^{\circ}$ & E $27^{\circ} 42^{\prime}$ & Arsenic, gold & 2 & General & Paat producer & Unknown & Unknown & Old worklings reported. \\
\hline G & 31 & Adavifan & & N $39^{\circ} 38^{\prime}$ & E $28^{\circ} 45^{\circ}$ & Chromito & 2 & General & Past producer & Unknown & Unknown & $\begin{array}{l}\text { Produced In the } 1950 \text { s. } \\
\text { County roserves } 45,000 \text { tons } 35-46 \% \text { Cr203. }\end{array}$ \\
\hline 6 & 32 & Ayozment & & N $39^{\circ} 18^{\prime}$ & E $26^{\circ} 54^{\prime}$ & Iron & $2,17,22$ & General & Past producer & Underground & Unknown & 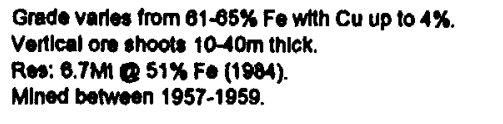 \\
\hline 0 & 33 & Balya & & N $39^{\circ} 45^{\prime}$ & E $27^{\prime} 35^{\prime}$ & $\begin{array}{l}\text { Lead, zlnc } \\
\text { copper }\end{array}$ & 2 & Goneral & Past producer & $\begin{array}{l}\text { Surface } \\
\text { Undorgiound }\end{array}$ & Unknown & 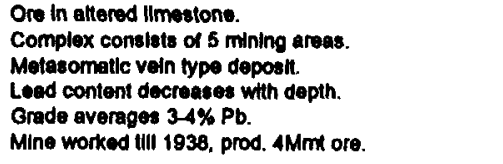 \\
\hline
\end{tabular}


APPENDIX B: PAST PRODUCINO MINERAL PROPERTES IN TURKEY

\begin{tabular}{|c|c|c|c|c|c|c|c|c|c|c|c|}
\hline \multicolumn{2}{|c|}{$\begin{array}{l}\operatorname{MAP} \\
\mathbf{K E Y} \\
\text { (1) }\end{array}$} & \multirow{2}{*}{$\begin{array}{l}\text { NMME } \\
\text { (2) } \\
\text { Cakallar }\end{array}$} & \multicolumn{2}{|c|}{$\begin{array}{l}\text { COORDINATES } \\
\text { LAT. LONO. }\end{array}$} & COMMODITY(IES) & $\begin{array}{l}\text { DATA } \\
\text { 8OURCEB } \\
\text { (3) }\end{array}$ & \multirow{2}{*}{$\begin{array}{l}\text { DATA } \\
\text { RELIANCE } \\
\text { (d) } \\
\text { Goneral }\end{array}$} & BTATUS & $\begin{array}{l}\text { MINE } \\
\text { TPEE }\end{array}$ & MARKETB & \multirow{2}{*}{$\begin{array}{l}\text { COMMENTS } \\
\text { In 1951, produced sootons. }\end{array}$} \\
\hline 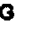 & 34 & & N $39^{\circ} 42^{\circ}$ & E 2734 & Manganeso & 2 & & Past producor & Unknown & Unknown & \\
\hline o & 35 & Cattleak & $N 39^{\circ} 30^{\circ}$ & E $28^{\circ} 66^{\circ}$ & Manganese & 2 & Coneral & Pest producer & Unknown & Unknown & $\begin{array}{l}\text { Throe dopostlta aseaying 33\% -50\% Mn. } \\
\text { Res: 10-30kt. }\end{array}$ \\
\hline $\mathbf{o}$ & 36 & Carmin & $N 39^{\circ} 42^{\circ}$ & E $27^{\circ} 24^{\circ}$ & Iron & 2 & General & Past producer & Unknown & Unknown & $\begin{array}{l}\text { Res: } 32.000 \text { lone } 963.5 \% \text { Fo (1982). } \\
\text { Produced in } 1953 \text {. }\end{array}$ \\
\hline o & 37 & Demiciler & N $39^{\circ} 30^{\circ}$ & E $28^{\prime} 53^{\prime}$ & Chromite & 2 & Goneral & Past producor & Unknown & Unknown & $\begin{array}{l}\text { Produced in the } 1950 \% \text {. } \\
\text { County meserves } 15,000 \text { tone } 35-10 \% \text { Cr2O3. }\end{array}$ \\
\hline $\mathbf{0}$ & 38 & Demirkepl & $N 39^{\circ} 48^{\circ}$ & E $20^{\circ} \infty 0^{\circ}$ & Anilmony & 2.17 & Goneral & Past producer & Unknown & Unknown & $\begin{array}{l}\text { Mine reported to be exhausted. } \\
\text { Res: } 11 k t \text { e } 1.62 \% \text { Sb (1972). }\end{array}$ \\
\hline 6 & 39 & $\begin{array}{l}\text { Hayran area } \\
\text { Eymir } \\
\text { Buyuk Eymir }\end{array}$ & $\mathbf{N} 39^{\prime} 30^{\prime \prime}$ & E $27^{\circ} 15^{\prime}$ & Iron & $2,16,17,22,23$ & Goneral & Past producer & Surtace & Unknown & 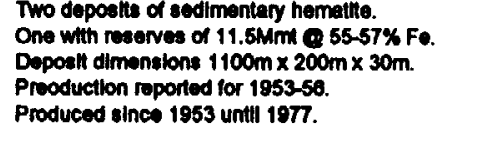 \\
\hline $\mathbf{G}$ & 10 & Hopenlar & N $39^{\circ} 36^{\circ}$ & E $28^{\circ} 58^{\prime}$ & Manganese & 2 & General & Paat producer & Unknown & Unknown & $\begin{array}{l}\text { Dapoall in voins of Paleozote schlats. } \\
\text { Worked In } 1918-49 \text { ylolding } 1385 \text { tons. }\end{array}$ \\
\hline $\mathbf{G}$ & 41 & Kalabak & N $39^{\circ} 36^{\circ}$ & E $27^{\circ}$ OT & Lead, zine & 2 & Goneral & Past producer & Unknown & Unknown & Prod. 23 lons in 1953,53 tone in 1954. \\
\hline $\mathbf{G}$ & 42 & Kalburcu & N 39. $34^{\circ}$ & E $28^{\circ} 09^{\circ}$ & Manganeas & 2 & Gonomal & Past producer & Unknown & Unknown & In 1948, produced 45 tone $45.4 \% \mathrm{Mn}$. \\
\hline $\mathbf{G}$ & 43 & Kocadag & N $39^{\circ} 30^{\circ}$ & E $27^{\circ} 08^{\prime}$ & Iron & 2 & Goneral & Past producer & Underground & Unknown & Deposil worked In 1953. \\
\hline G & 44 & Koncu & N $38^{\circ} 28^{\circ}$ & E $27^{\prime} 22^{\circ}$ & Antimony & 2,17 & General & Past producer & Unknown & Unknown & $\begin{array}{l}\text { Small deposth worked 1945-1948. } \\
\text { Res: } 332 \mathrm{kt} Q 0.05 \% \text { Sb (1973). }\end{array}$ \\
\hline $\mathbf{G}$ & 46 & Purinillk & N $38^{\circ} 34^{\prime}$ & E $20^{\circ} 44^{\prime}$ & Iron & 2 & General & Past producer & Unknown & Unknown & 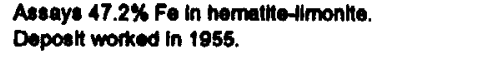 \\
\hline $\mathbf{0}$ & 10 & $\begin{array}{l}\text { Samal } \\
\text { Yagcllar }\end{array}$ & N $30^{\circ} 40^{\circ}$ & E $27^{\circ} 53^{\circ}$ & Iron & $2,15,17,22$ & Goneral & Past producer & Surface & Unknown & 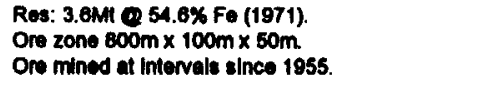 \\
\hline $\mathbf{G}$ & 47 & Samic & $N 39^{\circ} 37$ & E $28^{\prime} 2 r$ & Manganese & 2 & Goneral & Past producer & Unknown & Unknown & Produced 20 tons in 1954. \\
\hline $\mathbf{Q}$ & 40 & $\begin{array}{l}\text { Somimage } \\
\text { Dursunbey }\end{array}$ & $\mathbf{N} 39^{\circ} 35^{\circ}$ & E $28^{\circ} 33^{\prime}$ & Chromite & 2,17 & Ganeral & Paut producor & Unknown & Unknown & $\begin{array}{l}\text { Produced in the 1950s. } \\
\text { Ren: } 64.7 \mathrm{kt} \& 20-25 \% \mathrm{Cr} O 3 \text { (1980). }\end{array}$ \\
\hline 0 & 48 & Seytandere & N $39^{\circ} 56^{\circ}$ & E $27^{\circ} 25^{\circ}$ & Load & 2 & Goneral & Past producer & Unknown & Unknown & As of 1957, ylolded 950 tons. \\
\hline $\mathbf{0}$ & 50 & $\begin{array}{l}\text { Susurfuk } \\
\text { Sultancaylr } \\
\text { Azlzlye }\end{array}$ & $N 39^{\prime} 50^{\circ}$ & E 28.09 & Boron & $5,6,15$ & Goneral & Past producor & Underground & Unknown & 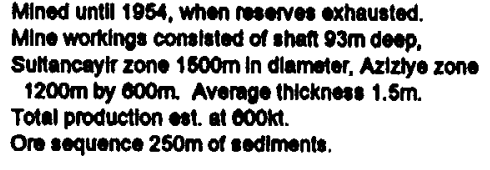 \\
\hline $\mathbf{G}$ & 51 & atilsu & N $39^{\circ} 24^{\prime \prime}$ & $27^{\prime} 55^{n}$ & Antimony & 2 & leral & producer & wn & own & Mine reported to be exhausted. \\
\hline
\end{tabular}


APPENDIX B: PAST PRODUCINO MINERAL PROPERTES IN TURKEY

\begin{tabular}{|c|c|c|c|c|c|c|c|c|c|c|c|}
\hline $\begin{array}{l}\text { MA } \\
\text { KE } \\
(1)\end{array}$ & & $\begin{array}{l}\text { NAME } \\
\text { (2) }\end{array}$ & $\begin{array}{l}\text { COORDIMA } \\
\text { LAT. }\end{array}$ & $\begin{array}{l}\text { TES } \\
\text { LONO. }\end{array}$ & COMMODITY(IEB) & $\begin{array}{l}\text { DATA } \\
\text { SOURCEB } \\
\text { (Э) }\end{array}$ & $\begin{array}{l}\text { DATA } \\
\text { RELIANCE } \\
\text { (4) }\end{array}$ & STATUS & $\begin{array}{l}\text { MiNE } \\
\text { TYPE }\end{array}$ & MARKET8 & COMMENTS \\
\hline 0 & 52 & Tomasallk & N $39^{\circ} 33^{\prime}$ & E $27^{\circ} 06^{\circ}$ & Manganeses & 2 & Goneral & Past producer & Unknown & Unknown & $\begin{array}{l}\text { Worked bolore Wond War I. } \\
\text { Res: \&-10k1 \& 40\% Mn. }\end{array}$ \\
\hline $\mathbf{G}$ & 53 & $\begin{array}{l}\text { Turfular } \\
\text { Arap Deroti } \\
\text { Olkenll } \\
\text { Mezttler }\end{array}$ & N $39^{\circ} 29$ & $E 28^{\circ} 18^{\prime}$ & $\begin{array}{l}\text { Manganese, } \\
\text { Iron }\end{array}$ & 2,17 & General & Past producer & Surface & Unknown & $\begin{array}{l}\text { Produced } 2600 \text { tons in } 1941 \text { in andesllo. } \\
\text { Ore in velna } 0.1-1 \mathrm{~m} \text { thlek, } 150 \mathrm{~m} \text { long, and } 50 \mathrm{~m} \text { whd } \\
\text { Res: } 692 \mathrm{kt} @ 19.73 \% \mathrm{FQ}, 13.7 \% \mathrm{Mn} \text { (1989). }\end{array}$ \\
\hline G & S1 & Yasyert & N $39^{\circ} 38^{\circ}$ & E $27^{\circ} 03^{\circ}$ & Manganese & 2 & Coneral & Past producer & Unknown & Unknown & Produced 18 tona in 1954. \\
\hline $\mathbf{G}$ & 55 & $\begin{array}{l}\text { Yonice } \\
\text { Kayapa }\end{array}$ & $N \quad 39^{\circ} 34^{\prime}$ & E $26^{\circ} 05^{\prime}$ & Antumony & 2.17 & General & Past producer & Unknown & Unknown & 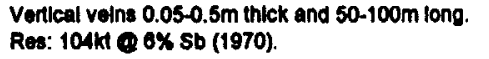 \\
\hline$H$ & 56 & Bozuyuk & N $39^{\circ} 54^{\prime}$ & E $30^{\circ} 03^{\circ}$ & Chromite & 2 & Coneral & Past producer & Unknown & Unknown & Produced in the 1950's. \\
\hline H & 57 & Dudas & $N 40^{\circ} 01^{\circ}$ & E $30^{\circ} 14^{\prime}$ & Antlmony & 2 & General & Past producer & Unknown & Unknown & Mine reopened in 1857. \\
\hline$H$ & 58 & $\begin{array}{l}\text { Gude } \\
\text { Sorgunlu Gol } \\
\text { Yukan Komurcu } \\
\text { Akcakmak Bozca } \\
\text { Doglimen Bogazl }\end{array}$ & $\mathrm{N} 40^{\circ} 00^{\circ}$ & E 29' 54' & Manganese & 2 & Goneral & Past producer & Surface & Unknown & $\begin{array}{l}\text { Ditinct composed of a deposits. } \\
\text { Producod } 5300 \text { tons } 1952-53 \text {. } \\
\text { Assays } 18 \%-10 \% \text { Mn. } \\
\text { Ore in lenses of groen schlst and quarizite. }\end{array}$ \\
\hline H & 59 & Inhlsir & $N 40^{\circ} 03^{\circ}$ & E $30^{\circ} 23^{\circ}$ & Tin & 2 & General & Past producer & Unknown & Unknown & $\begin{array}{l}\text { Historical recovery reported. } \\
\text { Tn assoclated whith grantites. }\end{array}$ \\
\hline$H$ & 60 & Kure & $N 40^{\circ} 05^{\circ}$ & E $30^{\circ} 10^{\prime}$ & LIgnito & 2 & General & Past producar & Unknown & Unknown & $\begin{array}{l}\text { Res: } 3 \mathrm{MH} Q 4050 \text { Calkg. } \\
1955 \text { county reserves } 3.1 \mathrm{Mt} \text {. }\end{array}$ \\
\hline H & 61 & Kuzthdilx & $N 39^{\circ} 54^{\prime}$ & E 29, 56" & Chnomile & 2 & General & Past producer & Unknown & Unknown & Produced in the 1950's. \\
\hline H & 02 & Minalgazi & $N 40^{\circ} 01^{\prime}$ & E $30^{\circ} 34^{\circ}$ & Tin & 2 & General & Past producer & Unknown & Unknown & Historical recovery reported. \\
\hline H & 63 & Nernll & $N 39^{\circ} 43^{\circ}$ & E $30^{\circ} 14^{\prime}$ & Meerachaum & 2 & Goneral & Past producer & Surtace & Domestic & Production from one sithe. \\
\hline H & a & Yakan & $N 39^{\circ} 54^{\circ}$ & E $29^{\circ} 54^{\circ}$ & Chromite & 2 & General & Past producer & Unknown & Unknown & Produced in the 1950 's. \\
\hline H & 05 & $\begin{array}{l}\text { Yarhlsar } \\
\text { Bahceclk } \\
\text { Hasandere } \\
\text { Elmabahce }\end{array}$ & N $10^{\circ} 08^{\prime}$ & E $29^{\circ} 45^{\circ}$ & Menganese & 2 & General & Past producer & Surface & Unknown & Produced 600 tons betwoen 1951-54. \\
\hline 1 & $\theta$ & Sacmallpinar & $N 40^{\circ} 44^{\circ}$ & E $30^{\circ} \mathrm{67}$ & Llonthe & 2 & Goneral & Past producer & Unknown & Unknown & $\begin{array}{l}\text { Res: } 1.5 \mathrm{SMt} Q 4012 \text { Calkg. } \\
1955 \text { county reserves } 25 \mathrm{M} \text {. }\end{array}$ \\
\hline J & 67 & Baalangle & N $37^{\circ} 25^{\prime}$ & E $29^{\circ} 45^{\circ}$ & Chromite & 2 & General & Past producor & Unknown & Unknown & $\begin{array}{l}\text { Produced in the } 1950 \text { 's. } \\
\text { 1955 county reserves } 525 \mathrm{Kt} \text { 30-10\% } \mathrm{Cr} 2 \mathrm{O} \text {. }\end{array}$ \\
\hline J & 68 & Beykoy & N $37^{\circ} 39^{\prime}$ & E $29^{\circ} 51^{\prime}$ & Chromite & 2 & General & Past producer & Unknown & Unknown & $\begin{array}{l}\text { Produced In the 1950's. } \\
1955 \text { county reserves } 525 \mathrm{kt} \text { o } 30-10 \% \mathrm{Cr} 2 \mathrm{O} 3 \text {. }\end{array}$ \\
\hline J & 69 & Corten & N $37^{\circ} 01^{\prime}$ & E $29^{\circ} 32$ & Chromite & 2 & General & Past producer & Unknown & Unknown & $\begin{array}{l}\text { Produced in the } 1950 \% \text {. } \\
1955 \text { county rosorves } 525 \mathrm{Kt} Q 38-10 \% \mathrm{Cr} 2 \mathrm{O} \text {. }\end{array}$ \\
\hline J & 70 & Gokarik & $37^{\circ} 25^{\prime}$ & E $29^{\circ} 45^{\prime}$ & Chromilte & 2 & neral & Past producer & Unknown & Unknown & $\begin{array}{l}\text { Produced in the } 19508 \text {. } \\
\text { 1955 county resenves } 525 \mathrm{kt} Q 38-10 \% \mathrm{Cr} 203 \text {. }\end{array}$ \\
\hline
\end{tabular}


APPENDIX B: PAST PRODUCING MINERN PROPERTEB IN TURKEY

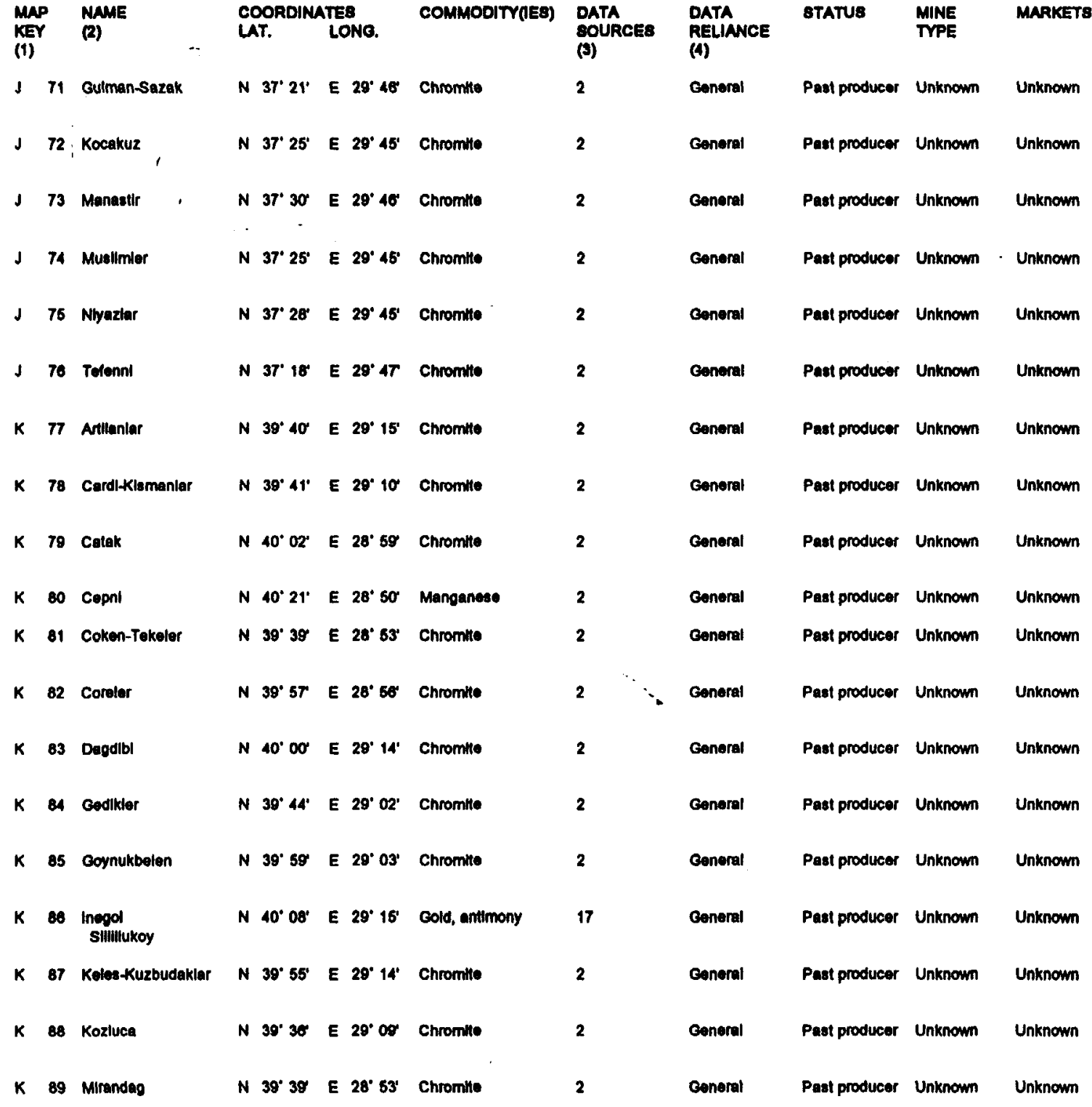

COMMENTS

$\cdot$

Producod in the 1950\%.

1955 county reserves 525kt 8 36-10\% Cro3.

Produced in the 1950's.

1955 county roserves 525kt $36-10 \% \mathrm{Cr} 203$

Produced in the $1950^{\circ}$

1955 county resorves 525kt $30-40 \% \mathrm{Cr} 2 \mathrm{O}$

Produced in the 1950

1955 county resorves 525kt $180-10 \% \mathrm{Cr} 203$

Produced in the 1950's.

1955 county roserves 525kt $36-40 \%$ Cr2O3.

Produced in the 1950".

1955 county romenves 525kt a 38-10\% Cr2O3.

Produced in the 1950:.

County resenves 340kt $38-18 \%$ Cros

Produced in the 1950 .

County resones 340k1 8 38-48\% Cr2O3.

Produced in the 1950'.

County reserves 340kt $38-48 \% \mathrm{Cr} 2 \mathrm{O}$

Produced 480 tons in 1949.

Produced in the $1950^{\circ}$

County reservos 340kt 38-48\% CrzOs.

Produced In the $1950 \%$

County reserves 340kt 38-48\% CrzO3.

Produced in the 1950".

County resenves 340ki 8 38-48\% Cr2O3.

Produced in the 1950's from two sittes. County resenves 3HOkt $38-18 \%$ Cr2O

Produced in the 1950's.

county reserves 340kt $38-48 \%$ Cro3.

Rex: 15kt @ 0.7-28gh Au, 6.5\% Sb County resenes 340kt @38-48\% $\mathrm{Cr} 2 \mathrm{O}$

Produced in the $1950^{\circ}$

County resonves 340kt Q 30-40\% Cr2O3.

Produced in the 1950\%.

County resenves 340kt 38-48\% Cr2O3. 
APPENDIX B: PAST PRODUCINO MINERUL PROPERTIES IN TURKEY

\begin{tabular}{|c|c|c|c|c|c|c|c|c|c|c|c|}
\hline $\begin{array}{l}\text { MN } \\
\text { KEY } \\
(1)\end{array}$ & & $\begin{array}{l}\text { NAME } \\
\text { (R) }\end{array}$ & $\begin{array}{l}\text { COORDIN } \\
\text { LAT. }\end{array}$ & $\begin{array}{l}\text { ATES } \\
\text { LONO. }\end{array}$ & COMMODITY(DES) & $\begin{array}{l}\text { DATA } \\
\text { SOURCES } \\
\text { (3) }\end{array}$ & $\begin{array}{l}\text { DATA } \\
\text { RELIANCE } \\
\text { (4) }\end{array}$ & STATUS & $\begin{array}{l}\text { MINE } \\
\text { TYPE }\end{array}$ & MARKETS & COMMENT8 \\
\hline K & 80 & Orthanell & N $39^{\prime} 42^{\circ}$ & $E 28^{\circ} 50^{\circ}$ & Chromite & 2,17 & Goneral & Past producer & Unknown & Unknown & $\begin{array}{l}\text { Produced in the 1950's. } \\
\text { Res: } 1 \mathrm{MIt} \text { Q 10-48\% Cr2O3 (1974). }\end{array}$ \\
\hline K & 91 & Othanell-Kinik & $N 39^{\circ} 42^{\prime}$ & E $28^{\circ} 50^{\circ}$ & Chromite & 2 & Goneral & Past producer & Unknown & Unknown & $\begin{array}{l}\text { Produced in the } 1950 \text { '. } \\
\text { County reserves } 340 \mathrm{O} \text {. } 38-18 \% \mathrm{Cr} 203 \text {. }\end{array}$ \\
\hline K & 92 & Omanelt-Mirandagl & N $39^{\circ} 40^{\circ}$ & E $28^{\circ} 55^{\prime}$ & Chromte & 2 & General & Past producer & Unknown & Unknown & $\begin{array}{l}\text { Produced in the } 1950 \text { s. } \\
\text { County meserves } 340 \mathrm{kt} \text { 38-48\% Crio3. }\end{array}$ \\
\hline $\mathrm{k}$ & 93 & Pirasallk & $N 39^{\circ} 36^{\circ}$ & E $28^{\circ} 18^{\circ}$ & Chromito & 2 & Coneral & Past producer & Unknown & Unknown & $\begin{array}{l}\text { Produced in the 1950\%. } \\
\text { County roeerves 340kt } 38-48 \% \text { Cr2O3. }\end{array}$ \\
\hline k & 94 & Pirlbeyler & N $39^{\circ} 38^{\prime}$ & E $28^{\circ} 48^{\circ}$ & Chromn: & 2 & General & Past producer & Unknown & Unknown & $\begin{array}{l}\text { Produced in the 1950's. } \\
\text { County reserves 340kt } 30-40 \% \text { crzos. }\end{array}$ \\
\hline$k$ & 95 & Topuk & N $39^{\circ} 59^{\prime}$ & $E 29^{\circ} 00^{\circ}$ & Chromite & 2 & General & Past producer & Unknown & Unknown & 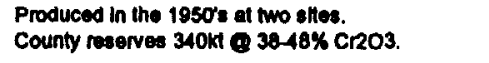 \\
\hline$k$ & 96 & Topuk & N $39^{\circ} 69^{\circ}$ & E 29. 00 & Magneallo & 2,22 & General & Past producer & Unknown & Unknown & $\begin{array}{l}\text { two depoetla; one with } 30,000 \text { tona of reserves } 9 \\
44.6 \% \mathrm{MgO} \text { and one with } 10,000 \text { ions. } \\
\text { Production of } 10 \mathrm{kt} \text { reported in } 1986 \text {. }\end{array}$ \\
\hline k & 97 & Yakuplar & $N 39^{\circ} 45^{\circ}$ & E $29^{\circ} 03$ & Chromite & 2 & Ceneral & Patt producer & Unknown & Unknown & $\begin{array}{l}\text { Produced in the 1950). } \\
\text { County reserves 340kt } 9 \text { 38-48\% Cr2O3. }\end{array}$ \\
\hline L & 98 & Balcllar & $N 40^{\circ} 10^{\circ}$ & E $20^{\circ} 51^{\circ}$ & $\begin{array}{l}\text { Copper, lead, } \\
\text { zine }\end{array}$ & 2 & General & Past producer & Unknown & Unknown & $\begin{array}{l}\text { Quartz volns in dacthe tum. } \\
22.18 \% \text { Cu from mine dumps. }\end{array}$ \\
\hline L & $\boldsymbol{9}$ & Camyun & $N 40^{\circ} 17^{\prime}$ & E $20^{\circ} 50^{\prime}$ & $\begin{array}{l}\text { Copper. } \\
\text { molybdenum }\end{array}$ & 2 & Ceneral & Past producer & Underground & Unknown & $\begin{array}{l}\text { Old workings largely caved. } \\
\text { Dumpe contaln } 2000 \text { tons } 0.31 \% \mathrm{Cu} \text {. }\end{array}$ \\
\hline $\mathbf{L}$ & 100 & Hacibayram Sayasl & $N 10^{\circ} 02^{\circ}$ & E $27^{\circ} 03^{\circ}$ & $\begin{array}{l}\text { Copper, laed, } \\
\text { zinc }\end{array}$ & 2 & General & Past producer & Underground & Unknown & Flve caved tunnels reported. \\
\hline L & 101 & Karapinar & N $39^{\circ} 69^{\circ}$ & E $20^{\circ} 40^{\circ}$ & Copper & 2 & General & Past producer & Unknown & Unknown & $\begin{array}{l}\text { Ore In velns in schists. } \\
\text { Assays rango from 2-20\% Cu. }\end{array}$ \\
\hline L & 102 & Kucukkuyu & $\mathbf{N} 38^{\circ} 3^{*}$ & E $20^{\circ} 36^{\circ}$ & Iron & 2 & General & Past producer & Surface & Unknown & $\begin{array}{l}\text { Three layers: bottom: } 260 \mathrm{kt} Q 48-52 \% \text { Fo, } 3-10 \mathrm{~m} \\
\text { thlek; mid: Assays } 67 \% \text { Fo, and lop: 500kt } \\
\text { 61.68\% Fa up lo } 28 \mathrm{~m} \text { thick. }\end{array}$ \\
\hline $\mathbf{L}$ & 103 & Sarkaya & $N 40^{\circ} 11^{\prime}$ & E $27^{\circ} 13^{\prime}$ & Asente & 2 & Goneral & Past producer & Unknown & Unknown & Small production primarly for local use 1953-55. \\
\hline $\mathbf{L}$ & 104 & Yuvalar & N $40^{\circ} 03^{\prime}$ & E $27^{\circ} 04^{\prime \prime}$ & Graphlie & 2 & General & Past producer & Unknown & Unknown & Produced 700 tons In 1951. \\
\hline M & 105 & Tuhlkoy & $N 40^{\circ} 45^{\prime}$ & $E 33^{\prime} 4 T$ & Copper & 2 & General & Past producer & Surface & Unknown & Small workinge vasible. \\
\hline M & 108 & Unay & N $40^{\circ} 43^{\prime}$ & E $33^{\circ} 43^{\prime \prime}$ & Copper & 2 & General & Patt producer & Surface & Unknown & Imogular deposit with caved workings. \\
\hline $\mathbf{N}$ & 107 & Abuhemsin & $N 40^{\circ} 1 T$ & E $35^{\circ} 00^{\circ}$ & Manganese & 2 & General & Past producer & Unknown & Unknown & $\begin{array}{l}\text { Produced } 2847 \text { lons between 1949-1953, } \\
\text { Ore in slock with reserves of } 2000 \text { tons } 45 \% \text { Mn. }\end{array}$ \\
\hline $\mathbf{N}$ & 108 & $\begin{array}{l}\text { Alpagol } \\
\text { Zanbal }\end{array}$ & $N 40^{\circ} 20^{\circ}$ & $E 34^{\circ} 51^{\circ}$ & Llgnne & 2 & Cenenal & Past producer & Unknown & Unknown & $\begin{array}{l}\text { Res: 22MM. } \\
\text { 1955 county reserves 23Mt. }\end{array}$ \\
\hline N & 109 & Boglevan & $N 41^{\prime} 26^{\prime}$ & E $41^{\prime} 36^{\circ}$ & Copper, zinc & 2 & General & Past producer & Unknown & Unknown & Worked pritor to WWI. \\
\hline
\end{tabular}


APPENDIX B: PAST PRODUCINO MINERUL PROPERTES IN TURKEY

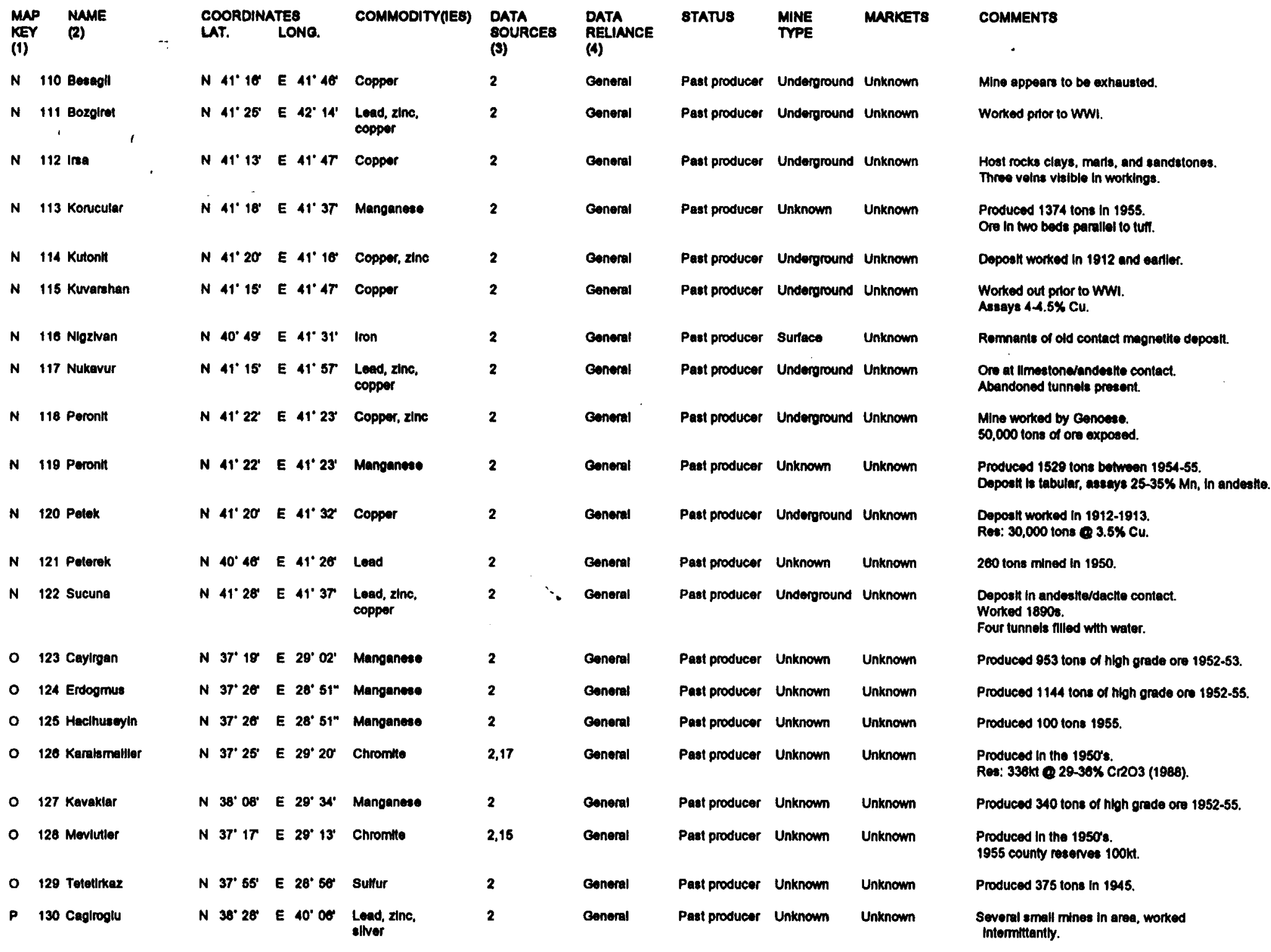


APPENOIX B: PAST PRODUCINO MINERAL PROPERTIES IN TURKEY

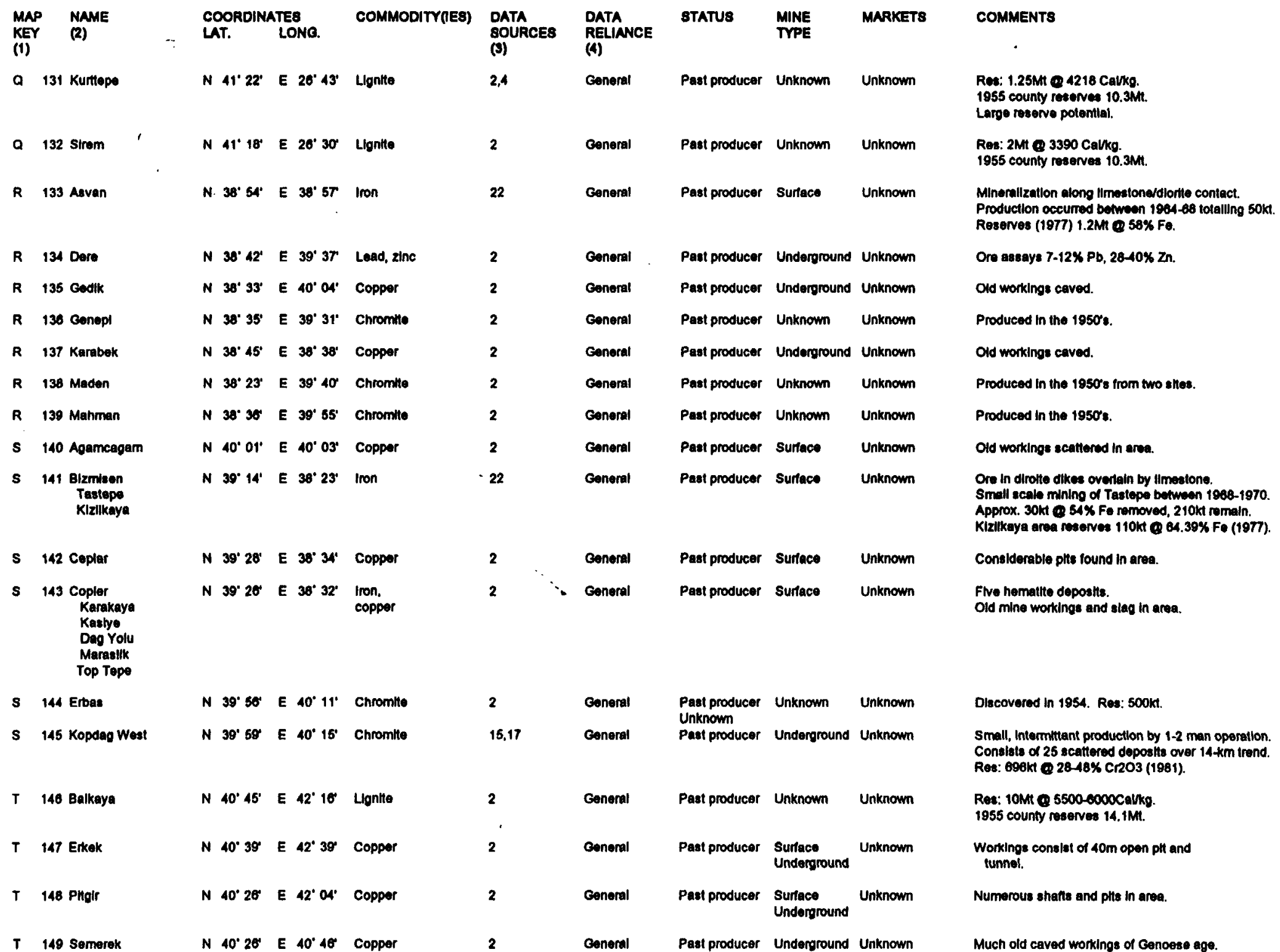


APPENDIX B: PAST PRODUGING MINERNL PROPERTES IN TURKEY

\begin{tabular}{|c|c|c|c|c|c|c|c|c|c|c|c|}
\hline $\begin{array}{l}\text { MNF } \\
\text { KEY } \\
\text { (1) }\end{array}$ & & $\begin{array}{l}\text { NAME } \\
\text { (R) }\end{array}$ & $\begin{array}{l}\text { COORDIN } \\
\text { LAT. }\end{array}$ & $\begin{array}{l}\text { ATES } \\
\text { LONO. }\end{array}$ & COMMODITY(DES) & $\begin{array}{l}\text { DATA } \\
\text { SOURCES } \\
\text { (3) }\end{array}$ & $\begin{array}{l}\text { DATA } \\
\text { RELIANCE } \\
\text { (A) }\end{array}$ & BTATUS & $\underset{\text { TMPE }}{\text { MINE }}$ & MARKET8 & $\begin{array}{c}\text { COMMENTS } \\
.\end{array}$ \\
\hline $\mathbf{u}$ & 150 & Bahlyar & N $39^{\circ} 57$ & $E 31^{\circ} 4 \sigma$ & Chromte & 2 & Gonerat & Pael producor & Unknown & Unknown & $\begin{array}{l}\text { Produced in the 1950). } \\
\text { 1955 county roserves solk \& } 34-44 \% \text { CrzO3. }\end{array}$ \\
\hline$u$ & 151; & ; Basoren , & $N 39^{\circ} 50^{\circ}$ & E $30^{\circ} 58^{\prime}$ & Megneallo & 2 & Canoment & Peor producar & Unknown & Uninown & $\begin{array}{l}\text { Two deposils of undetermined tonnage } 18 \text { 46-48\% } \\
\text { Mgo. Produced } 280 \text { tons botween } 1950-51 \text {. }\end{array}$ \\
\hline $\mathbf{u}$ & 152 & 2 Besoren & N 39' 58' & E $30^{\circ} 58^{\circ}$ & Chromke & 2 & Comenes & Pow proover & Unknown & Unknown & $\begin{array}{l}\text { Produced in the 1950's. } \\
\text { 1955 county resenves sook } 3444 \% \mathrm{Cr} 203 \text {. }\end{array}$ \\
\hline $\mathbf{u}$ & 153 & Basoren & N $39^{\circ} 58^{\circ}$ & E $30^{\circ} 58^{\prime}$ & Moonchoum & 2 & Coneral & Peet prooveren & surtace & Domestic & Production from two alles. \\
\hline u & 154 & I Dagkuplu & $N 39^{\circ} 59$ & $E 30^{\circ} 41^{\circ}$ & Chromte & 2 & Ganaral & Peal producar & Unknown & Unknown & 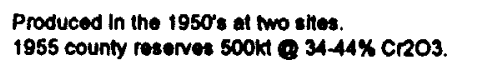 \\
\hline$u$ & 155 & 5 Dagkuptu-Gollnmeze & $N 39^{\circ} 59^{\circ}$ & E $30^{\circ} 41^{\prime}$ & Chromtte & 2 & General & Pasi producer & Unknown & Unknown & $\begin{array}{l}\text { Produced in the 1950: } \\
\text { 1955 county reserves 500k1 } 9 \text { 34-44\% Cro3. }\end{array}$ \\
\hline $\mathbf{u}$ & 156 & Eaklaehir & $N 39^{\circ} 46^{\circ}$ & E $30^{\circ} 32^{\prime}$ & Chromito & 2 & General & Past producer & Unknown & Unknown & $\begin{array}{l}\text { Produced in the } 1950 \text { : } \\
\text { 1955 county reserves 500k } 34-44 \% \text { Cr203. }\end{array}$ \\
\hline u & 157 & Gezigor & N $39^{\circ} 50^{\circ}$ & $E 30^{\circ} 53^{\circ}$ & Meorechaum & 2 & General & Past producer & Surtace & Domestle & Production from one silte. \\
\hline$u$ & 158 & Cokesoglu & $N 39^{\circ} 44^{\circ}$ & E $30^{\circ} 53^{\circ}$ & Meerschaum & 2,17 & Goneral & Pant producer & Surface & Domestle & $\begin{array}{l}\text { Production from five elles. } \\
\text { Res: sokt (1982). }\end{array}$ \\
\hline$u$ & 159 & Gunduzler & N $39^{\circ} 52^{\prime}$ & E $30^{\circ} 49^{\prime}$ & $\begin{array}{l}\text { Magnealls, } \\
\text { chromite }\end{array}$ & 2 & General & Past producer & Unknown & Unknown & $\begin{array}{l}\text { Elght emall deposits. } \\
\text { Produced 2.150 tons betweon 1949-51. }\end{array}$ \\
\hline$u$ & 100 & Gunduzier & N $39^{\circ} 52^{\prime}$ & E $30^{\circ} 49^{\circ}$ & Chromite & 2 & General & Past producar & Unknown & Unknown & $\begin{array}{l}\text { Produced in the } 1950 \text { s at alx shes. } \\
1955 \text { county reserves } 500 \mathrm{kt} Q 34-44 \mathrm{Cr} O 3 .\end{array}$ \\
\hline $\mathbf{u}$ & 101 & Halloglan & N $39^{\circ} 49^{\circ}$ & E $31^{\circ} 40^{\circ}$ & Chromnt & 2 & General & Past producer & Unknown & Unknown & 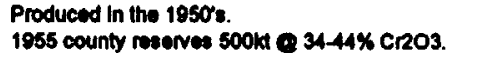 \\
\hline $\mathbf{u}$ & 162 & Imeohir-Karalokal & $N 39^{\circ} 41^{\prime}$ & $E 30^{\circ} 4 T$ & Meorschaum & 2,17 & Goneral & Past producer & Surface & Domentle & $\begin{array}{l}\text { Production from olgh stice. } \\
\text { Res: 465kt (1982). }\end{array}$ \\
\hline$u$ & 183 & 3 Kanabaylt & $N 39^{\circ} 5 r$ & E $30^{\circ}$ O4' & Chromite & 2 & Goneral & Past producor & Unknown & Unknown & $\begin{array}{l}\text { Produced in the 1950's. } \\
1955 \text { county reserves } 500 \mathrm{kt} \text { Q } 34-44 \% \mathrm{Cr} 2 \mathrm{O} .\end{array}$ \\
\hline$u$ & 104 & Kavak & $N 39^{\circ} 35^{\prime}$ & E $31^{\circ} 40^{\circ}$ & Chromite & 2,15 & General & Past producer & Unknown & Unknown & $\begin{array}{l}\text { Producod in the 1950's. } \\
1955 \text { county reserven } 500 \mathrm{kt} \text { 34.44\% Cr2O3. }\end{array}$ \\
\hline u & 185 & KIzllesoren & N $39^{\prime} 38^{\prime}$ & E $31^{\circ} 23^{\circ}$ & Manganese & 2 & Goneral & Past producer & Unknown & Unknown & Produced 1440 tons between $1945-1950$. \\
\hline u & 160 & Lactn & $N 40^{\circ} 03^{\prime}$ & E $30^{\circ} 47$ & Chromito & 2 & General & Past producer & Unknown & Unknown & $\begin{array}{l}\text { Produced in the 1950's. } \\
\text { 1955 county resenes 50okt e } 34-14 \% \mathrm{Cr} 203 \text {. }\end{array}$ \\
\hline u & 167 & Margl & N 39' $55^{\circ}$ & E 30 54 & Iron & 2 & Goneral & Past producer & Unknown & Unknown & Operated In 1955. \\
\hline 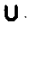 & 188 & Margi & N $39^{\circ} 56^{\prime}$ & E $30^{\circ} 50^{\circ}$ & Meerschaum & 2,17 & General & Past producer & Surface & Domostlc & $\begin{array}{l}\text { Production from soven alles. } \\
\text { Res: 803kt (1982). }\end{array}$ \\
\hline$u$ & 169 & Mlhallecik & N 39' 52' & E $31^{\circ} 30^{\circ}$ & Asbentos & $2,17,22$ & Goneral & Paat producer & Surface & Domestle & $\begin{array}{l}\text { Production from five sittes in 1986; produced for } \\
\text { Res: } 1 \text { Mt (1987). }\end{array}$ \\
\hline
\end{tabular}


APPENDIX B: PAST PRODUCINO MINERNL PROPERTES IN TURKEY

\begin{tabular}{|c|c|c|c|c|c|c|c|c|c|c|c|}
\hline $\begin{array}{l}\text { MAP } \\
\text { (1) }\end{array}$ & & $\begin{array}{l}\text { NAME } \\
\text { (2) } 1\end{array}$ & $\begin{array}{l}\text { COORDIN } \\
\text { LAT. }\end{array}$ & $\begin{array}{l}\text { ATES } \\
\text { LONO. }\end{array}$ & COMMODITY(IES) & $\begin{array}{l}\text { DATA } \\
\text { SOURCES } \\
\text { (3) }\end{array}$ & $\begin{array}{l}\text { DATA } \\
\text { RELIANCE } \\
\text { (A) }\end{array}$ & STATUS & $\begin{array}{l}\text { MINE } \\
\text { TYPE }\end{array}$ & MARKET8 & $\begin{array}{c}\text { COMMENTS } \\
.\end{array}$ \\
\hline u & 170 & Omerkoy & $N 39^{\circ} 80^{\circ}$ & E $31^{\circ} 30$ & Magnesite & 2 & General & Past producer & Unknown & Unknown & Produced 59 lons in 1945. \\
\hline$u$ & 179 & Sazak & N $39^{\circ} 48^{\circ}$ & E $31^{\circ} 36^{\prime}$ & Chromite & 2,15 & General & Past producer & Unknown & Unknown & $\begin{array}{l}\text { Produced in the } 1950 \text {. } \\
1955 \text { county reserves } 500 \mathrm{kt} \& 34-44 \times \mathrm{Cr} 203 \text {. }\end{array}$ \\
\hline u & 172 & Sepetcl & N $39^{\circ} 50^{\circ}$ & E $30^{\circ} \mathbf{5 0}$ & Magnealte & 2 & General & Patt producer & Unknown & Unknom & $\begin{array}{l}\text { Two depostle aggregating } 2-5 \text { ktons o } 18.72 \% \text { MgO. } \\
15 \text { lons produced in } 1947 \text {. }\end{array}$ \\
\hline u & 173 & Sepetel-Tastepe & N $39^{\circ} 60^{\circ}$ & E $30^{\circ} 50^{\circ}$ & Chromte & 2 & General & Paat producer & Unknown & Unknown & 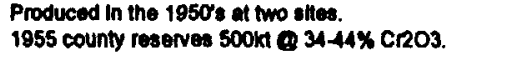 \\
\hline U & 174 & Sogutcuk & N $39^{\circ} 54^{\circ}$ & E $30^{\circ} 59^{\circ}$ & Moorschaum & 2.17 & General & Past producer & Surface & Domestlc & $\begin{array}{l}\text { Production from atght sties. } \\
\text { Ros: 56.68vit (1982). }\end{array}$ \\
\hline v & 175 & Burunsuziar & N $30^{\circ} 57$ & E 38' $60^{\circ}$ & Manganeses & $2,4,17$ & General & Past producer & Unknown & Unknown & $\begin{array}{l}\text { Produced } 1044 \text { tons between 1951-1952. } \\
\text { Produced in } 1973 \text {. } \\
\text { Res: } 17 \mathrm{kt} \text { \& } 35 \% \text { Mn (1984). }\end{array}$ \\
\hline w & 176 & Agll & $N 40^{\circ} 55^{\circ}$ & E 30' 54' & Copper & 2 & Goneral & Pest producer & Underground & Unknown & Old stte. \\
\hline $\mathbf{w}$ & 177 & Boynuyolu & $N 40^{\circ} 4 r$ & E 38' 51' & Iron & 2 & General & Past producer & Underground & Unknown & Eight old tunnels warked in Gonoese times. \\
\hline w & 178 & Duroglu & $N 40^{\circ} 65^{\prime}$ & E $38^{\circ} 31^{\circ}$ & $\begin{array}{l}\text { Coppor, load, } \\
\text { zinc }\end{array}$ & 2 & Goneral & Past producor & Underground & Unknown & $\begin{array}{l}\text { Old workinge and dumps assaying } 4.17 \% \\
\mathrm{~Pb} \text { and } 2.77 \% \mathrm{Cu} \text {. }\end{array}$ \\
\hline $\mathbf{w}$ & 179 & Esell & $N 40^{\circ}$ 64' & E $38^{\circ} 57$ & $\begin{array}{l}\text { Copper, loed, } \\
\text { zinc }\end{array}$ & 2 & Coneral & Past producer & Underground & Unknown & $\begin{array}{l}\text { Fwe drithe and Inclined shants dotected. } \\
\text { Worked by the Genoese and belore WWI. }\end{array}$ \\
\hline w & 180 & Gect Araca & $N 40^{\circ} 46^{\circ}$ & $E 3^{\circ} 33^{\prime}$ & Copper & 2 & Ganeral & Past producer & Underground & Unknown & Abandoned mine in gabbro. \\
\hline w & 181 & Golevard & $N 40^{\circ} 5 r$ & E $38^{\prime} 43^{\circ}$ & Iron & 2 & General & Paal producer & Surface & Unknown & Evidence of old worklngs. \\
\hline w & 182 & Girlak & $N 40^{\circ} 50^{\circ}$ & E $30^{\circ} 40^{\circ}$ & $\begin{array}{l}\text { Lead, zinc. } \\
\text { coppor }\end{array}$ & 2 & Boneral & Past producer & Undorground & Unknown & $\begin{array}{l}\text { Assay ylelds } 0.74 \% \mathrm{~Pb}, 4.92 \% \mathrm{Zn}, 3.09 \% \\
\mathrm{Cu}, 4.13 \text { oz } \mathrm{AO} \text { and } 0.034 \text { oz Au. }\end{array}$ \\
\hline w & 183 & Gumusluk Masat & $N 40^{\circ} 5 T$ & E $38^{\circ} 44^{\prime}$ & $\begin{array}{l}\text { Copper, lead. } \\
\text { zine }\end{array}$ & 2 & General & Pasel producor & Underground & Unknown & $\begin{array}{l}\text { 40-80cm thlck voln in andoesto. } \\
\text { Old workings prosent. } \\
\text { Aasay la } 37.61 \% \mathrm{~Pb}, 23.2 \% \mathrm{Zn}, 1.16 \% \mathrm{Cu}\end{array}$ \\
\hline $\mathbf{w}$ & 184 & Inkoy & $N 40^{\circ} 69^{\circ}$ & E $38^{\circ} 51^{\prime}$ & $\begin{array}{l}\text { Copper, load, } \\
\text { zinc }\end{array}$ & 2 & Ceneral & Pasi producor & Underground & Unknown & Four old lunnols in andestie. \\
\hline w & 185 & Ierall & $N 40^{\circ} 65^{\prime}$ & E $38^{\circ} 54^{\prime}$ & Copper & 2 & Coneral & Past producer & Underground & Unknown & $\begin{array}{l}\text { Worked pror to WWI. } \\
\text { Ten potential depostis in area. }\end{array}$ \\
\hline w & 186 & Kan & $N 40^{\circ} 59^{\circ}$ & E $38^{\circ} 61^{\prime}$ & Copper, zlne & 2 & Ganeral & Past producer & Underground & Unknown & $\begin{array}{l}\text { Caved workings and slag plles visible. } \\
\text { Assay of ore } 0.25 \% \mathrm{~Pb}, 5.01 \% \mathrm{Zn}, 1.46 \% \\
\text { Cu and } 25.75 \% \mathrm{Fe} \text {. }\end{array}$ \\
\hline w & & $\begin{array}{l}\text { Karabork } \\
\text { Cocendere } \\
\text { Pelldo } \\
\text { Glinak }\end{array}$ & N $40^{\circ} 52^{\prime}$ & E $39^{\circ} 04^{*}$ & Copper & 2 & General & Past producer & Underground & Unknown & $\begin{array}{l}\text { Deposks worked by the Genoose. } \\
\text { All ahans and ditte filled with water. }\end{array}$ \\
\hline $\mathbf{w}$ & 188 & aragol & $N 40^{\circ} 54^{\prime}$ & E & $\begin{array}{l}\text { Lead, zinc, } \\
\text { copper }\end{array}$ & 2 & General & Past producer & Inderground & Unknown & $\begin{array}{l}\text { Multiple velns worked In } 1900 \mathrm{~s} \text {. } \\
\text { Asary } 11.01 \% \text { Pb, } 25.9 \% \mathrm{Zn}, 3.85 \% \mathrm{Cu} \\
5.05 \text { oz Ag, and } 0.038 \text { oz Au. }\end{array}$ \\
\hline
\end{tabular}


APPENDIX B: PAST PRODUCINO MINERAL PROPERTES IN TURKEY

\begin{tabular}{|c|c|c|c|c|c|c|c|c|c|c|c|c|}
\hline $\begin{array}{l}\text { MAP } \\
\text { (1) }\end{array}$ & & $\begin{array}{l}\text { NAME } \\
\text { (2) }\end{array}$ & $\begin{array}{l}\text { COORDIN } \\
\text { LAT. }\end{array}$ & $\begin{array}{l}\text { ATES } \\
\text { LO }\end{array}$ & 8 SNO. & COMMODITY(NES) & $\begin{array}{l}\text { DATA } \\
\text { BOURCES } \\
\text { (3) }\end{array}$ & $\begin{array}{l}\text { DATA } \\
\text { RELIANCE } \\
\text { (4) }\end{array}$ & BTATUS & $\underset{\text { MINE }}{\text { MINE }}$ & MARKETS & COMMENTS \\
\hline $\mathbf{w}$ & 189 & Karakaya & $\mathrm{N} 40^{\circ} 69^{\circ}$ & $\mathbf{E}$ & $39^{\circ} 12^{\prime \prime}$ & $\begin{array}{l}\text { Copper, leand, } \\
\text { zine }\end{array}$ & 2 & Goneral & Past producor & Undenground & Unknown & Numorous old workings. \\
\hline w & 180 & Karavaclk & $N 41^{\circ} \infty 0^{\circ}$ & $\mathbf{E}$ & $30^{\circ} 60^{\circ}$ & Iron & 2 & Goneral & Past producer & Surface & Unknown & Evidence of old workinge. \\
\hline $\mathbf{w}$ & 191 & Kastarita & N 40. $83^{\prime}$ & E & $\mathbf{3 6}^{\circ} \mathbf{3 4}$ & $\begin{array}{l}\text { Copper, lead, } \\
\text { zine }\end{array}$ & 2 & Conoral & Paut producer & Underground & Unknown & $\begin{array}{l}\text { Operated by the Rusalane in } 1885 \text {. } \\
\text { Working conelat of } 3 \mathrm{~m} \text { inclined enan, } 25 \mathrm{~m} \\
\text { tunnet. }\end{array}$ \\
\hline w & 192 & Kayabaal & $N 40^{\circ} 53^{\prime}$ & $\mathbf{E}$ & $38^{\prime} \mathbf{3 0}$ & $\begin{array}{l}\text { Copper, lead, } \\
\text { zine }\end{array}$ & 2 & Goneral & Past producer & Unknown & Unknown & Old worklnge reported. \\
\hline w & 193 & Kolele & $N 40^{\circ} 58^{\circ}$ & $\mathbf{E}$ & $38^{\circ} 58^{\prime \prime}$ & Copper & 2 & Goneral & Paal producer & Underground & Unknown & Float ore high in copper from old workings. \\
\hline w & 194 & KIran & $N 41^{\circ}-0^{\prime \prime}$ & $\mathbf{E}$ & $30^{\circ} 48$ & Iron & 2 & General & Past producer & Surnace & Unknown & Evidence of old workings. \\
\hline w & 195 & Klricak & $N 40^{\circ} 50^{\circ}$ & $\mathbf{E}$ & $3^{\circ} 34^{\prime \prime}$ & $\begin{array}{l}\text { Copper, lead, } \\
\text { zlne }\end{array}$ & 2 & General & Past producer & $\begin{array}{l}\text { Surtace } \\
\text { Underground }\end{array}$ & Unknown & $\begin{array}{l}20 \mathrm{~cm} \text { hydrothermal veln in andesite. } \\
\text { Wonkinge conelat of open ph, } 30 \mathrm{~m} \text { tunnel, } \\
\text { and } 3 \mathrm{~m} \text { inclined shaft. }\end{array}$ \\
\hline $\mathbf{w}$ & 190 & KIzllolma Oncbey & $N 10^{\circ} 26^{\circ}$ & $\boldsymbol{E}$ & $37^{\circ} \mathbf{6 8}$ & $\begin{array}{l}\text { Lead, zinc, } \\
\text { copper }\end{array}$ & 2 & General & Past producer & Underground & Unknown & $\begin{array}{l}\text { Two deposits roported in andestlo. } \\
\text { Workings consilot of } 4 \text { tunnels a adits. }\end{array}$ \\
\hline $\mathbf{w}$ & 197 & Kizlletma & $N 40^{\circ} 20^{\circ}$ & $\mathbf{E}$ & $37^{\circ} 66^{\circ}$ & $\begin{array}{l}\text { Copper, lead, } \\
\text { zine }\end{array}$ & 2 & General & Past producer & Underground & Unknown & $\begin{array}{l}\text { Doveloped betore WWII. } \\
\text { Incllned thef conatructed to mine } 40 \mathrm{~cm} \text { veln. }\end{array}$ \\
\hline w & 198 & Koruktepe & $N 40^{\circ} 49^{\circ}$ & $\mathbf{E}$ & $38^{\prime} 14^{\prime}$ & Copper & 2 & General & Past producer & Unknown & Unknown & Abandoned mine reported at thls location. \\
\hline w & 199 & Lahanos & N $40^{\circ} 55^{\circ}$ & $\mathbf{E}$ & $38^{\circ} 45^{\circ}$ & $\begin{array}{l}\text { Copper, lead, } \\
\text { zine, gold, } \\
\text { REO }\end{array}$ & 2.15 & General & Past producer & Underpround & Unknown & $\begin{array}{l}\text { Two groups of multiple depostis. } \\
\text { Old worklings visible. }\end{array}$ \\
\hline $\mathbf{w}$ & 200 & Madendere & $N 40^{\circ} 44^{\circ}$ & $\mathbf{E}$ & $38^{\circ} 45^{\prime}$ & Iron & 2 & General & Pasl producar & Surtace & Unknown & Evidence of dd worklngs. \\
\hline w & 201 & Osman KIran & $N 40^{\circ} 57$ & $\mathbf{E}$ & $38^{\circ} 44^{\prime}$ & Copper & 2 & Goneral & Past producer & Underground & Unknown & $\begin{array}{l}\text { Three ore plpes in andestto. } \\
\text { Slag in area up to } 400,000 \text { tons. }\end{array}$ \\
\hline $\mathbf{w}$ & 202 & Sadl & $N 41^{\circ} 02^{\prime}$ & $\mathbf{E}$ & $39^{\circ} \infty 0^{\prime}$ & $\begin{array}{l}\text { Load, zinc. } \\
\text { copper }\end{array}$ & 2 & Goneral & Past producer & Underground & Unknown & $\begin{array}{l}\text { Dleseminalod coppor in thyolte/andestte. } \\
\text { Aesay of } 83.4 \% \mathrm{~Pb}, 1.02 \% \mathrm{Zn}, 16.62 \% \mathrm{Cu} \text {. }\end{array}$ \\
\hline $\mathbf{W}$ & 203 & Sarpkaya & $\mathrm{N} 40^{\circ} 53^{\circ}$ & $\mathbf{E}$ & $38.2 T$ & Copper, lead & 2 & General & Past producer & Underground & Unknown & $\begin{array}{l}\text { Remalns of soven funnols. } \\
\text { Slag assays } 2.17 \% \mathrm{~Pb} \text { and } 0.36 \% \mathrm{Cu} \text {. }\end{array}$ \\
\hline w & 204 & Soku & N $40^{\circ} 52^{\prime}$ & $\mathbf{E}$ & $38^{\circ} 34^{4}$ & Coppor & 2 & Goneral & Pant producer & Underground & Unknown & $\begin{array}{l}\text { Throe depostits in andeallo. } \\
\text { Old workings and aleg dumps visible. }\end{array}$ \\
\hline w & 205 & Sogullendlyeyla & $N 41^{\circ} 02^{\circ}$ & $\mathbf{E}$ & $39^{\circ} 00^{\circ}$ & Iron & 2 & General & Past producer & Surface & Unknown & Worked prior to WWI. \\
\hline $\mathbf{W}$ & 208 & Tahtall & $N 40^{\circ} 4 r$ & $\mathbf{E}$ & $38.3 T^{\prime}$ & $\begin{array}{l}\text { Copper, load, } \\
\text { zlnc }\end{array}$ & 2 & General & Past producer & Undorground & Unknown & All workings caved. \\
\hline w & 207 & Ywolnclk & $N 40^{\circ} 40^{\circ}$ & $\mathbf{E}$ & $30^{\circ} \mathbf{3 0}$ & $\begin{array}{l}\text { Copper, load, } \\
\text { zlnc }\end{array}$ & 2 & Gonoral & Past producer & Underground & Unknown & $\begin{array}{l}\text { All workings caved. } \\
\text { Four mlnes in area. }\end{array}$ \\
\hline$x$ & 208 & Aylbell & $N \quad 40^{\circ} 36^{*}$ & $\mathbf{E}$ & $38^{\circ} 51^{\prime}$ & Iron & 2 & General & ast producer & urface & nown & t10 \\
\hline
\end{tabular}


APPENDIX B: PAST PRODUCINO MINERAL PROPERTIES IN TURKEY

\begin{tabular}{|c|c|c|c|c|c|c|c|c|c|c|c|}
\hline $\begin{array}{l}\text { MAF } \\
\text { KEY } \\
\text { (1) }\end{array}$ & & $\begin{array}{l}\text { NAME } \\
\text { (2) }\end{array}$ & $\begin{array}{l}\text { COORDIN } \\
\text { LAT. }\end{array}$ & $\begin{array}{l}\text { ITES } \\
\text { LONO. }\end{array}$ & COMMODITY(IES) & $\begin{array}{l}\text { DATA } \\
\text { SOURCES } \\
\text { (j) }\end{array}$ & $\begin{array}{l}\text { DATA } \\
\text { RELIANCE } \\
\text { (4) }\end{array}$ & STATUS & $\begin{array}{l}\text { MINE } \\
\text { TYPE }\end{array}$ & MARKETS & COMMENTS \\
\hline$x$ & 209 & 9 Beylartasl & N $10^{\circ} 32^{\prime}$ & E $38^{\circ} 54^{\circ}$ & Iron & 2 & General & Past producer & Surface & Unknown & Old workings and noat found In area. \\
\hline$x$ & 210 & D Cayir Cukur & $N 40^{\circ} 40^{\circ}$ & E $39^{\circ} 05^{\prime}$ & Copper & 2 & Gonoral & Pant producer & Underground & Unknown & $\begin{array}{l}\text { Workings consibl of } 5 \text { tunnels and several } \\
\text { shafts. }\end{array}$ \\
\hline $\mathbf{x}$ & 211 & 1 Dandi & $N 40^{\circ} 48^{\circ}$ & E 38. 5A" & Copper & 2 & Genural & Past producer & Underground & Unknown & Old workinge vialble. \\
\hline$x$ & 212 & 2 Demiclkkaya & N. $40^{\circ} 30^{\circ}$ & E $38^{\circ} 51^{\circ}$ & Iron & 2 & Goneral & Paut producer & surface & Unknown & Old workinge and outcrops found in area. \\
\hline $\mathbf{x}$ & 213 & 3 Deregozu & $N 40^{\prime} 52^{\prime}$ & E $39^{\circ} 05^{i}$ & Copper & 2 & Goneral & Past producer & Underground & Unknown & Extenetve workinge vielble. \\
\hline$x$ & 214 & 4 HazIne/KInk Pavll & $N 40^{\circ} 2 T$ & E $39^{\circ} 29^{\circ}$ & Lead, sllver & 2 & Gonoral & Paat producor & Unknown & Unknown & 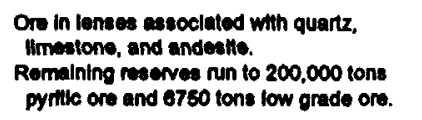 \\
\hline $\mathbf{x}$ & 215 & & $N 40^{\circ} 13$ & E $39^{\prime} 30^{\circ}$ & Copper & 2 & Coneral & Past producer & Undemground & Unknown & $\begin{array}{l}\text { Depoalt occuns in andesho cutting grante. } \\
\text { Workings consist of } 4 \text { shafts and } 4 \text { tunnels. } \\
\text { All workings are caved. }\end{array}$ \\
\hline $\mathbf{x}$ & 216 & 6 Karacukur & $N 40^{\circ} 40^{\circ}$ & E $39^{\circ} 10$ & Copper & 2 & General & Past producer & Underground & Unknown & OAd workings all caved. \\
\hline$x$ & 217 & 7 Kizll Ala & $N 40^{\circ} 47$ & E $39^{\circ} 03^{\circ}$ & Coppor & 2 & Gonoral & Past producer & Underground & Unknown & FWe tunnels are caved. \\
\hline $\mathbf{x}$ & 218 & 8 KIzllkaya & $N 40^{\prime} 32$ & E $38^{\circ} 53^{\prime}$ & Iron & 2 & Ceneral & Pasal producer & Surtace & Uniknown & Old hemalte workings found in area. \\
\hline$x$ & 219 & Kuru Maden & $N 40^{\circ} 51^{\circ}$ & E $39^{\circ} 03^{\circ}$ & $\begin{array}{l}\text { Copper, load, } \\
\text { zinc }\end{array}$ & 2 & Goneral & Past producer & Underground & Unknown & $\begin{array}{l}\text { Ore in chalk and andesile. } \\
\text { Some workings accoselble. }\end{array}$ \\
\hline$x$ & 220 & o Livine & $N 40^{\circ} 2 T$ & E $39^{\circ} 21^{\circ}$ & Copper & 2 & General & Past producer & Underground & Unknown & $\begin{array}{l}\text { Old mine in gnelss or andestite. } \\
\text { Wonkings cover } 200 \mathrm{~m} \times 300 \mathrm{~m} \text {. }\end{array}$ \\
\hline$x$ & 221 & Maden & $N 40^{\circ} 11^{\circ}$ & E $40^{\circ} 22^{\prime}$ & Copper & 2 & General & Past producer & Underground & Unknown & $\begin{array}{l}\text { Mine dumpe contain } 10,000 \mathrm{cu} \text { m materlal. } \\
\text { Water from caved tunnels } \mathrm{hl} \text { in Cu. }\end{array}$ \\
\hline$x$ & 222 & 2 Maden KIranI & $N 40^{\circ} 47$ & E $39^{\circ} 52^{\prime}$ & Copper & 2 & Goneral & Past producer & Underground & Unknown & Sbx tunnets ditven in the past in andeshte. \\
\hline$x$ & 223 & 3 Madenhanlar & $N 40^{\circ} 11^{\circ}$ & E $10^{\circ} 25^{\circ}$ & Copper & 2 & Conoral & Pant producer & Underground & Unknown & Old workings visible. \\
\hline$x$ & 224 & 4 Nikola & $N 40^{\circ} 49^{\circ}$ & E $39^{\circ} 64^{\prime}$ & $\begin{array}{l}\text { Copper, load, } \\
\text { zine }\end{array}$ & 2 & General & Past producer & Undorground & Unknown & Astay of $2.00 \% \mathrm{~Pb}, 1.17 \% \mathrm{Zn}, 2.18 \% \mathrm{Cu}$. \\
\hline$x$ & 225 & 5 Soku & $N 40^{\circ} 52^{\circ}$ & E $38^{\circ} 33^{\circ}$ & Iron & 2 & General & Past producer & Surface & Unknown & Old magnette-hermattle workings. \\
\hline$x$ & 220 & O Tasilea & $N 40^{\circ} 43^{\prime}$ & E $39^{\circ} 03^{\prime}$ & Copper & 2 & General & Past producer & Underground & Unknown & Extensive workings vis|ble, Inaccessible. \\
\hline$x$ & 227 & 7 Terekls & $N 40^{\circ} 50^{\circ}$ & E $38^{\circ} 55^{\circ}$ & $\begin{array}{l}\text { Lead, zinc, } \\
\text { copper, altuer }\end{array}$ & 2 & Coneral & Past producer & Undorground & Unknown & $\begin{array}{l}\text { Developed by Gancose and reopened } \\
\text { prlor to WW. } \\
\text { Assey is } 14.09 \% \mathrm{~Pb}, 19.22 \% \mathrm{Zn}, 0.91 \% \mathrm{Cu} \\
5.09 \text { oz A0.0.03 oz Au. }\end{array}$ \\
\hline$x$ & 228 & 8 Tonan Madon & $N 40^{\circ} 40^{\circ}$ & E $39^{\circ} 21^{\prime}$ & Copper & 2 & General & Past producer & Underground & Unknown & Thres tunnels are caved. \\
\hline$\gamma$ & 229 & 9 Gokdore & $N 36^{\circ} 52^{\circ}$ & E $36^{\circ} 12^{\prime}$ & Chromtle & 2 & General & Past producer & Unknown & Unknown & $\begin{array}{l}\text { Produced In the } 1950^{\circ} \text { from two siltes. } \\
1955 \text { county roserves } 250 \mathrm{kt} \text {. }\end{array}$ \\
\hline
\end{tabular}


APPENDIX B: PAST PROOUCINO MINERAL PROPERTES IN TURKEY

\begin{tabular}{|c|c|c|c|c|c|c|c|c|c|c|c|c|}
\hline $\begin{array}{l}\text { MAP } \\
\text { KEY } \\
\text { (1) }\end{array}$ & & $\begin{array}{l}\text { MAME } \\
\text { (2) }\end{array}$ & -. & $\begin{array}{l}\text { COORDINA } \\
\text { LAT. }\end{array}$ & $\begin{array}{l}\text { ATES } \\
\text { LONO. }\end{array}$ & COMMODITY(IE8) & $\begin{array}{l}\text { DATA } \\
\text { BOURCES } \\
\text { (3) }\end{array}$ & $\begin{array}{l}\text { DATA } \\
\text { RELIANCE } \\
\text { (4) }\end{array}$ & BTATUS & $\begin{array}{l}\text { MINE } \\
\text { TYPE }\end{array}$ & MARKETS & $\begin{array}{c}\text { COMMENTS } \\
.\end{array}$ \\
\hline $\mathbf{r}$ & & 0 Guvenis & & N $30^{\circ} 20^{\prime}$ & E $30^{\circ} 00$ & Chromtto & 2 & General & Past producer & Unknown & Unknown & $\begin{array}{l}\text { Produced in the } 1950 \text { s from two stites. } \\
\text { 1955 county reserves } 250 \mathrm{kt} \text {. }\end{array}$ \\
\hline Y & 231 & 4 , Kucukger , & & N $30^{\circ} \mathbf{2 0}$ & E $30^{\circ} 00^{\circ}$ & Chromite & 2 & Goneral & Past producer & Unknown & Unknown & $\begin{array}{l}\text { Produced in the } 1950 \text { 's irom two siltes. } \\
1955 \text { county reserves } 250 \mathrm{kt} \text {. }\end{array}$ \\
\hline $\mathbf{Y}$ & 232 & 2 Kuyuluk & ' & N $30^{+}$ & E $35^{\circ} 4 T$ & Chromto & 2 & General & Past producer & Unknown & Unknown & $\begin{array}{l}\text { Produced in the 1950's. } \\
1955 \text { county reserves } 250 \mathrm{kt} \text {. }\end{array}$ \\
\hline $\mathbf{Y}$ & & $\begin{array}{l}3 \text { Payas } \\
\text { lekendorun }\end{array}$ & & $N 30^{\prime} 37$ & E $36^{\circ} 12^{i}$ & Iron & 22 & Gonoral & Past producer & Surface & Unknown & Sodimentary doposth wth $0.1 \mathrm{Mt} \Theta 32.3 \% \mathrm{Fe}$. \\
\hline $\mathbf{Y}$ & 234 & Sogukoluk & & N $30^{\circ} 20^{\circ}$ & E $30^{\circ} \mathbf{0 0}$ & Chromtie & 2 & Coneral & Past producer & Unknown & Unknown & $\begin{array}{l}\text { Produced In the } 1950 \mathrm{~s} \text { from three ellos. } \\
\text { 1955 county reserves } 250 \mathrm{kt} \text {. }\end{array}$ \\
\hline $\mathbf{Y}$ & 235 & 5 Yenikoy & & $N 36^{\circ} 19^{\circ}$ & E $35^{\circ} 47$ & Chromite & 2 & General & Past producer & Unknown & Unknown & $\begin{array}{l}\text { Produced in the 1950: } \\
1955 \text { county reserves } 250 k \text {. }\end{array}$ \\
\hline $\mathbf{z}$ & 236 & Buyukecell & & $N 36^{\circ} 11^{\circ}$ & E $33^{\circ} 35^{\circ}$ & Iron & 22 & Gonerat & Paat producer & Underground & Unknown & 1977 roserves indleate $2.4 \mathrm{MH}$ ore. \\
\hline $\mathbf{z}$ & 237 & Caglallk & & $N 30^{\circ} 11^{\circ}$ & E $33^{\circ} 40^{\circ}$ & Iron & 2 & Genomal & Peat producer & Surtace & Unknown & Old worting in limestone assaying $48 \%$ Fe. \\
\hline $\mathbf{z}$ & 238 & Meraln & & $N 36^{\circ} \mathbf{4 0 ^ { \circ }}$ & E $34^{\circ} 30^{\circ}$ & Chromine & 2,15 & General & Past producer & Unknown & Unknown & 1955 county reserves $\pi / \mathrm{k}$. \\
\hline $\mathbf{z}$ & 239 & Karalsail & & $N 36^{\circ} 08^{\circ}$ & E $32^{\circ} 59^{\circ}$ & Chromte & 2 & General & Past producer & Unknown & Unknown & 1955 county reserves 50-00kt. \\
\hline $\mathbf{z}$ & 240 & Melloc & & $N 36^{\circ} 04^{\circ}$ & E $32^{\circ} 42^{\circ}$ & Iron & 22 & General & Past producer & Underground & Unknown & Ore replacement in limesions. \\
\hline $\mathbf{z}$ & 241 & Onakonue & & N 36'05' & $E 32^{\circ} 4 T$ & $\begin{array}{l}\text { Load, zinc, } \\
\text { coppor, siver }\end{array}$ & 2 & General & Past producer & Underground & Unknown & 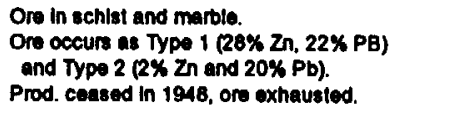 \\
\hline $\mathbf{z}$ & 242 & Osmanlye & & N 36' $20^{\circ}$ & E $33^{\circ} \mathbf{0 0}$ & Chromito & 2 & Goneral & Pest producer & Unknown & Unknown & 1955 county resenves $50-60 \mathrm{kt}$. \\
\hline M & 243 & Yankkaya & & $N 30^{\circ} 28^{\circ}$ & E $3^{\circ} 05^{\prime}$ & Llontie & 2 & Goneral & Past producor & Unknown & Unknown & Res: 5Mt. \\
\hline M & 244 & Yenisarovasi & & N 38, 04, & E 31' 23' & Iron & 2 & Goneral & Past producer & Surface & Unknown & Fomuginous sand dopostt worked in 1951. \\
\hline BB & 245 & Agacll & & N $41^{\circ} 16^{\circ}$ & E $28^{\circ} 52^{\prime}$ & Lignile & 2 & Goneral & Past producer & Unknown & Unknown & $\begin{array}{l}\text { Res: } 18.2 \mathrm{Mt} \text { Q } 2784 \mathrm{CaUkg} \\
1955 \text { county reserves } 17.2 \mathrm{Mm} \text {. }\end{array}$ \\
\hline BB & 240 & Akviran & & $N 41^{\circ} 11^{\prime}$ & E $28^{\circ} 20^{\circ}$ & Manganese & 2 & Goneral & Past producer & Unknown & Unknown & $\begin{array}{l}\text { Produced 32kt between 1952-1955. } \\
\text { Reported lo be of low grade, seolmentary orlgin. }\end{array}$ \\
\hline B日 & 247 & Camilmanl & & $\mathbf{N} 40^{\circ} 53^{*}$ & E $29^{\circ} 05^{\prime}$ & Copper & 2 & Goneral & Past producer & Surface & Unknown & $\begin{array}{l}\text { Open pts assaying } 3.3-0.2 \% \text { Cu. } \\
\text { Dopostl in ellietiled oruptive rocks. }\end{array}$ \\
\hline BB & 248 & Intranca & & $N 41^{\circ} 25^{\prime}$ & E $28^{\circ} 11^{\prime}$ & Manganate & 2 & General & Past producor & Surface & Domestle & $\begin{array}{l}\text { Overlath by } 3 \mathrm{~m} \text { of shale and sandstone. } \\
\text { Ros: 6Mtt } 32.5 \% \mathrm{Mn} \text {. } \\
\text { Productlon } 1955-1956 \text {, of aboun } 10,000 \text { tons. } \\
\text { Sold locally to battery manurecturers. }\end{array}$ \\
\hline BB & & Podima & & $N 41^{\circ} 27$ & E $28^{\circ} 20^{\circ}$ & nesese & 2 & a & oducer & ace & wn & Depostt of sedimentary origin. \\
\hline
\end{tabular}


APPENDIX B: PAST PRODUCINO MINERAL PROPERTIES IN TURKEY

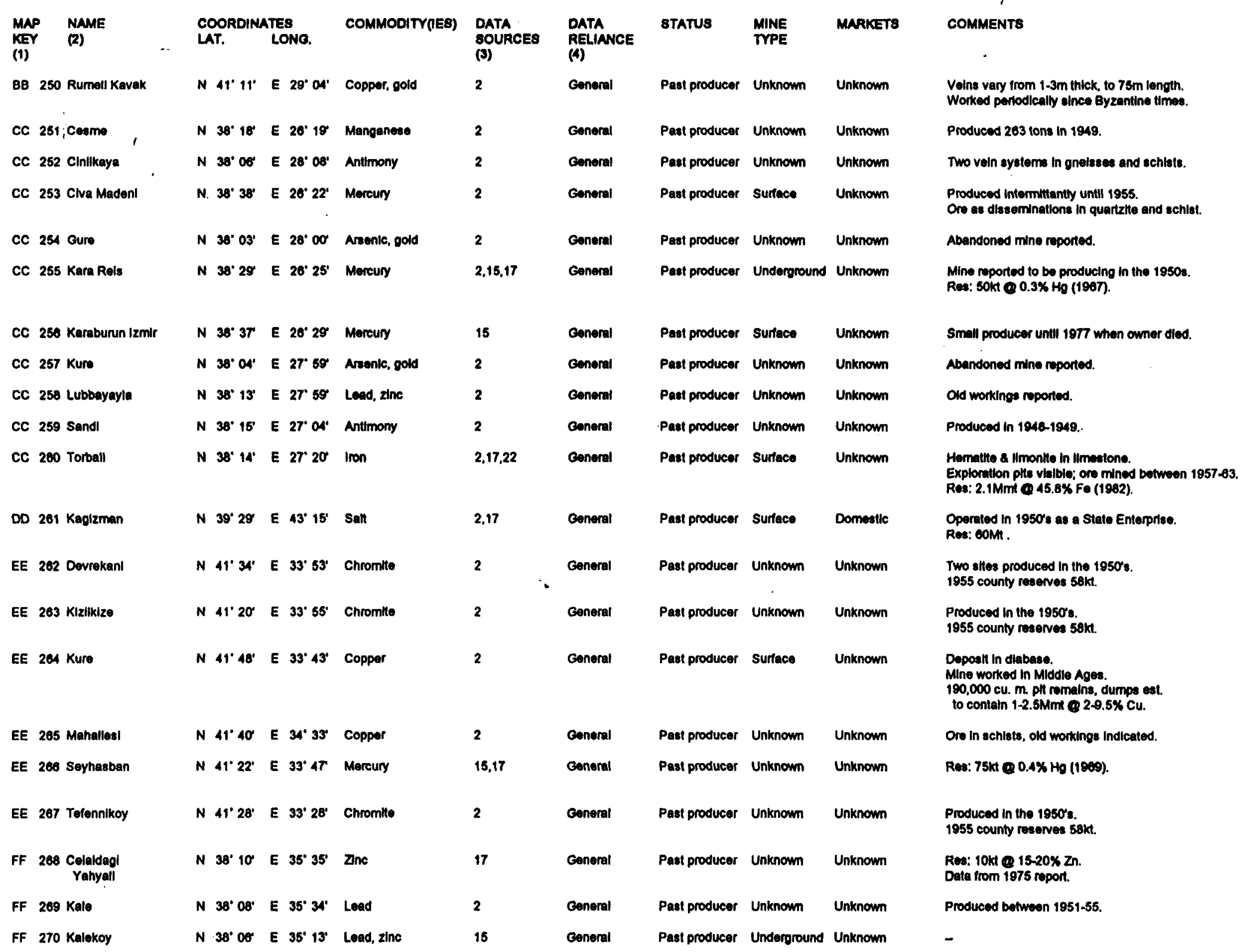


APPENDIX B: PAST PRODUCINO MINERUL PROPERTIES IN TURKEY

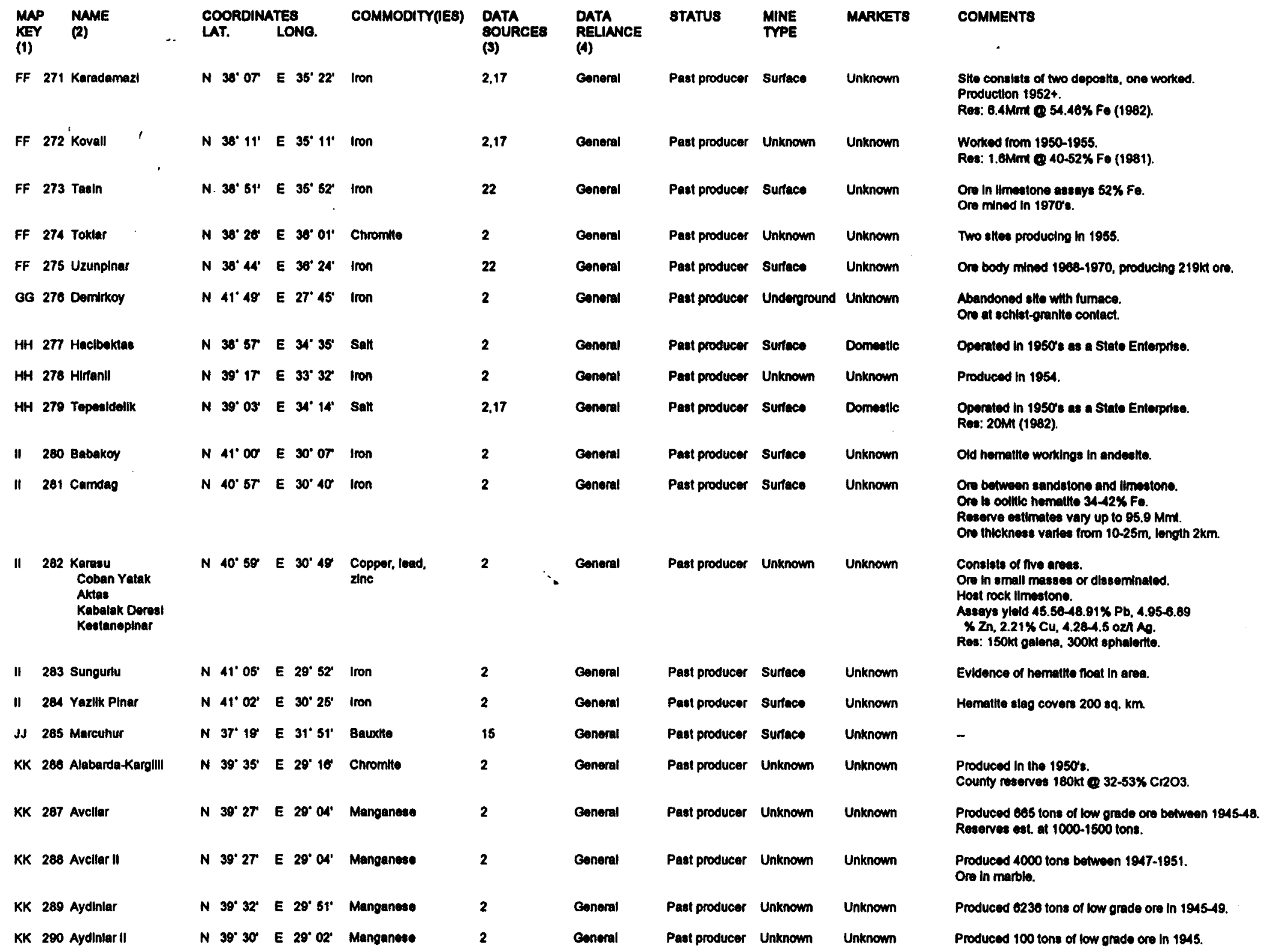


APPENDIX B: PAST PRODUCINO MINERAL PROPERTIES IN TURKEY

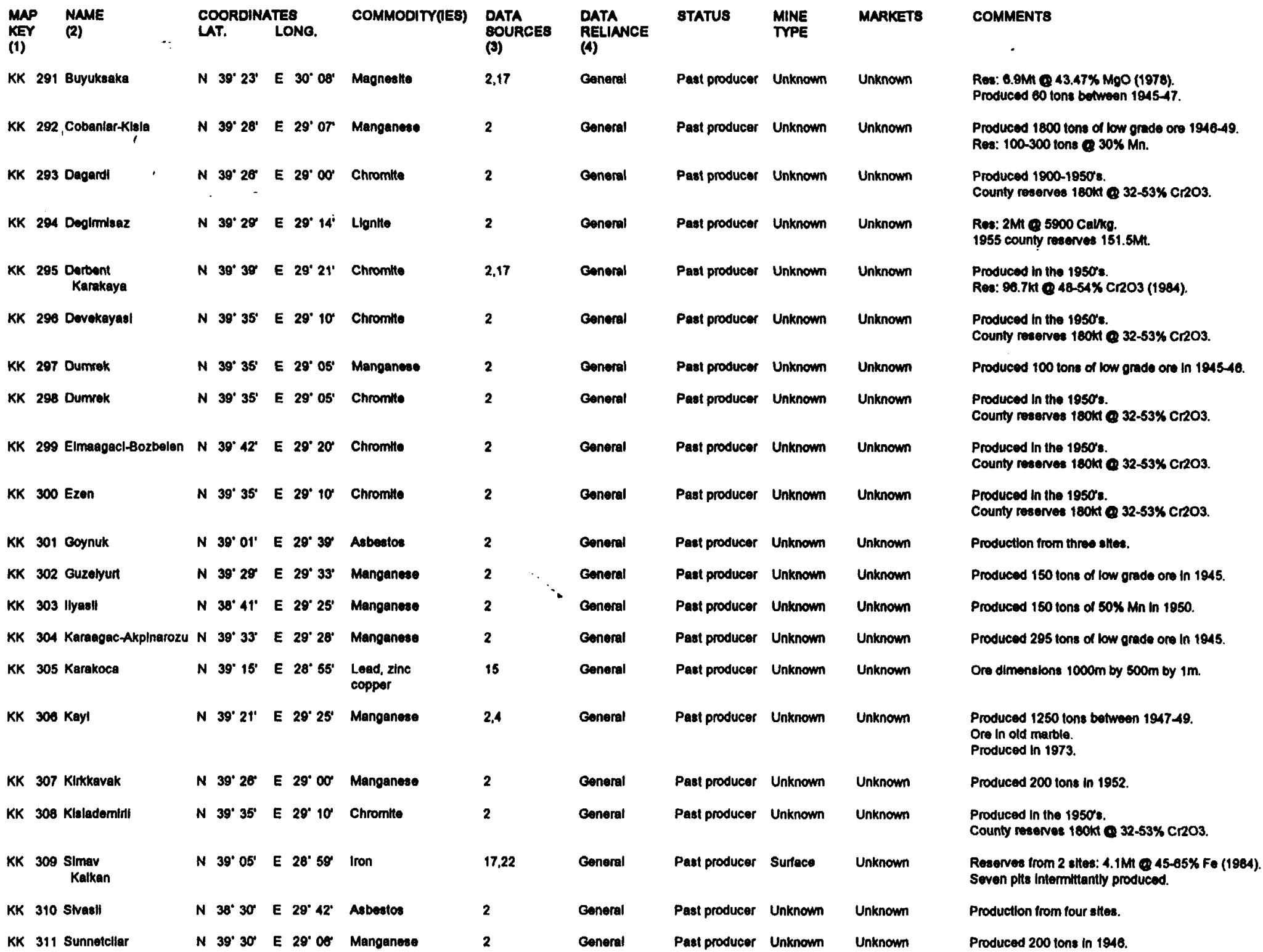


APPENOIX B: PAST PRODUCINO MINERAL PROPERTIES IN TURKEY

\begin{tabular}{|c|c|c|c|c|c|c|c|c|c|c|c|}
\hline $\begin{array}{l}\text { MAP } \\
\text { KEY } \\
\text { (1) }\end{array}$ & & $\begin{array}{l}\text { MME } \\
\text { (2) }\end{array}$ & $\begin{array}{l}\text { COORDINA } \\
\text { LAT. }\end{array}$ & $\begin{array}{l}\text { ATE8 } \\
\text { LONO. }\end{array}$ & COMMODITY(IE8) & $\begin{array}{l}\text { DATA } \\
\text { 8OURCES } \\
\text { (3) }\end{array}$ & $\begin{array}{l}\text { DATA } \\
\text { RELIANCE } \\
\text { (4) }\end{array}$ & 8TATU8 & $\begin{array}{l}\text { MINE } \\
\text { TrPE }\end{array}$ & MARKET8 & $\begin{array}{c}\text { COMMENTS } \\
\text {. }\end{array}$ \\
\hline KK & 312 & Tastlon & $N 39^{\circ} 04^{\circ}$ & E $29^{\circ}$ or & Chromito & 2 & General & Past producer & Unknown & Unknown & $\begin{array}{l}\text { Produced in the 1950s. } \\
\text { County reserves 180kt e 32-53\% Cr2O3. }\end{array}$ \\
\hline KK & 313 & , Yaylaclk , & N $39^{\circ} 35^{\circ}$ & E 20'35' & Chromite & 2 & Goneral & Pael producor & Unknown & Unknown & $\begin{array}{l}\text { Produced in the 1950s: } \\
\text { County resenves 180kt } 32-53 \% \text { Cr2O3. }\end{array}$ \\
\hline LL & 314 & Callkoy & N $37 T^{\prime} 37$ & E $30^{\circ} 36^{\circ}$ & Manganese & 2 & Goneral & Pant producer & Unknown & Unknown & Produced 310 tons in 1954. \\
\hline $\mathbf{L L}$ & & $\begin{array}{l}\text { Hasan Colebl } \\
\text { Hassan Colobl } \\
\text { Devecl }\end{array}$ & $N 38^{\circ} 50^{\circ}$ & E $37^{\circ} 54^{\circ}$ & Iron & $15,17,22$ & Goneral & Past producer & Surface & Unknown & $\begin{array}{l}\text { Res: 005Mt } 15 \% \text { Fo (1974). } \\
\text { Mining by open plt since 1981, total productlon till } 1977 \text { 1M }\end{array}$ \\
\hline $\mathbf{L L}$ & 316 & Kulancak & N 38' 34' & E $37^{\circ} 30^{\circ}$ & Lead & 2 & Ceneral & Past producer & Unknown & Unknown & Produced 1078 lons in 1954-55. \\
\hline MM & 317 & Karadun & $N 37^{\circ} 40^{\circ}$ & E $36^{\circ} 39^{\circ}$ & Iron & 2 & Goneral & Past producar & Unknown & Unknown & indicallons of old workings. \\
\hline MM & 1310 & Kaylis & $N 30^{\circ} 10$ & E $30^{\circ} 5 r$ & Copper & 2 & Goneral & Past producer & Underground & Unknown & Tunnels caved. \\
\hline MM & 319 & Manisa & $N 36^{\circ} 30^{\circ}$ & E $27^{\circ} 20^{\circ}$ & Manganese & 2 & General & Past prosucer & Unknown & Unknown & Produced 600 tons pritor to 1952. \\
\hline MM & 1320 & Suleyman!I & N $37^{\prime} 54^{\circ}$ & E $30^{\circ} \mathbf{6 0}$ & Iron & 2 & General & Past producar & Unknown & Unknown & Old workings. \\
\hline NN & 321 & Besparmak & N $37^{\circ} 28^{\circ}$ & E $27^{\circ} 36^{\prime}$ & Iron & 2 & Goneral & Pest producer & Surtace & Exported & $\begin{array}{l}\text { Specular Iron in gnotse. } \\
\text { Ore ehlpped to Garmany in } 1956 .\end{array}$ \\
\hline NN & 322 & Bezkeso-Dalaman & $N 36^{\circ} 44^{\prime}$ & $E 28^{\prime} 4 r$ & Chromine & 2.17 & Goneral & Past producar & Unknown & Unknown & 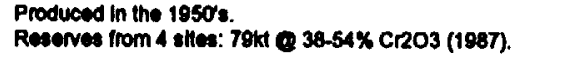 \\
\hline NN & 323 & Caldeg & $N 30^{\circ} 49^{\circ}$ & E $20^{\circ} 10^{\circ}$ & Manganese & 2 & Goneral & Past producer & Unknown & Unknown & Produced 1100 tons in 1955. \\
\hline NN & 324 & Cayhlear & $N \quad 36^{\prime} 58^{\prime}$ & E $28^{\circ} 52^{\prime}$ & Chromite & 2 & General & Past producer & Unknown & Unknown & 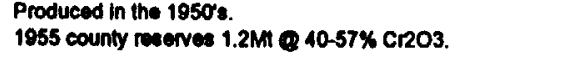 \\
\hline NN & 325 & Cenger & $N 36^{\circ} 46^{\circ}$ & E $28^{\circ} 00^{\circ}$ & Chromitto & 2 & Goneral & Past producer & Unknown & Unknown & $\begin{array}{l}\text { Produced in the 1950's. } \\
1955 \text { county roserves } 1.2 \mathrm{Mt} C 40-57 \% \mathrm{Cr} 2 \mathrm{O} .\end{array}$ \\
\hline NN & 320 & Elclk-Slradero & N $36^{\circ} 50^{\circ}$ & E $29^{\circ} 05^{t}$ & Chromte & 2 & General & Past producer & Unknown & Unknown & 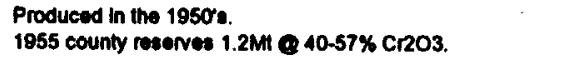 \\
\hline NN & 327 & Eldirek-Gunlukbasl & $N 38^{\circ} 41^{\circ}$ & E $29^{\circ} 12^{\circ}$ & Chromitte & 2 & General & Past producer & Unknown & Unknown & $\begin{array}{l}\text { Produced in the 1950's. } \\
1955 \text { county roservos } 1.2 \mathrm{Mt} \text { 10-57\% cr2O3. }\end{array}$ \\
\hline NN & 328 & Cocok & $N 30^{\circ} 44^{\circ}$ & E $28^{\circ} 58^{\prime}$ & Magnestio & 2 & General & Past producer & Unknown & Unknown & Reported to be exhausted. \\
\hline NN & 329 & Cocak-Bonguc & $N 30^{\circ} \mathbf{4 4 ^ { \prime }}$ & E $28^{\circ} 58^{\circ}$ & Chromite & 2 & Goneral & Pall producer & Unknown & Unknown & $\begin{array}{l}\text { Producend in the 1950s. } \\
1855 \text { county noserves 1.2Mt Q } 40-57 \% \mathrm{Cr} 2 \mathrm{O} \text {. }\end{array}$ \\
\hline NN & 330 & cocek-Inilice & N $36^{\circ} 45^{\prime}$ & E $29^{\circ} 01^{\circ}$ & Chromito & 2 & Goneral & Pacl producer & Unknown & Unknown & $\begin{array}{l}\text { Produced in the 1950s. } \\
\text { I855 county resenven } 1.2 \mathrm{M} \text { Q } 40-57 \% \mathrm{Cr} 203 .\end{array}$ \\
\hline NN & 331 & Cocek-Kurtgedlgl & $N 36^{\circ} 44^{\prime}$ & E $28^{\circ} 58^{\prime}$ & Chromile & 2 & General & Past producer & Unknown & Unknown & 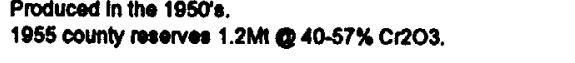 \\
\hline NN & 3 & Cokcoovac & $N 36^{\circ} 47^{\prime}$ & E $28^{\circ} 69^{\circ}$ & Menganese & 2,17 & General & Past producer & Unknown & Unknown & $\begin{array}{l}\text { Produced Intermittantly unth } 1935 . \\
\text { Res: 94kt Q 32\% Mn (1988). }\end{array}$ \\
\hline
\end{tabular}


APPENDIX B: PAST PRODUCINO MINERAL PROPERTES IN TURKEY

\begin{tabular}{|c|c|c|c|c|c|c|c|c|c|c|c|}
\hline $\begin{array}{l}\text { MAP } \\
\text { (1) }\end{array}$ & & $\begin{array}{l}\text { NAME } \\
\text { (R) }\end{array}$ & $\begin{array}{l}\text { COORDIN } \\
\text { LAT. }\end{array}$ & $\begin{array}{l}\text { ATES } \\
\text { LONO. }\end{array}$ & COMMODITY(PES) & $\begin{array}{l}\text { DATA } \\
\text { BOURCES } \\
\text { (3) }\end{array}$ & $\begin{array}{l}\text { DATA } \\
\text { RELANCE } \\
\text { (4) }\end{array}$ & STATU8 & $\begin{array}{l}\text { MINE } \\
\text { TYPE }\end{array}$ & MARKETS & COMMENTS \\
\hline NN & 333 & Golenye & $N 30^{\circ} 4 \mathrm{r}$ & $E 20^{\circ} 13^{\circ}$ & Manganese & 2 & Goneral & Past producer & Unknown & Unknomn & Produced 1500 tona in 1953. \\
\hline NN & 334 & $\begin{array}{l}\text { Gumiukbasi } \\
\text { Fothlye }\end{array}$ & N $30^{\circ} 39$ & E $29^{\circ} 00^{\circ}$ & Chromite & 2.17 & General & Past producer & Unknown & Unknown & $\begin{array}{l}\text { Produced in the 1950) } \\
\text { Reserves from } 2 \text { sthes: } 102 \mathrm{kt} \text { 30-10\% Cr2O3 (1981). }\end{array}$ \\
\hline NN & 335 & 5 Karacaome & $N 30^{\circ} 50$ & E 29:00 & Manganease & 2 & General & Pasl producer & Unknown & Unknown & Produced 2820 tons in 1952-53. \\
\hline NN & 336 & Karaturum & N. $36^{\circ} 50^{\circ}$ & E $29^{\circ} 00^{\circ}$ & Chromtlo & 2 & Gonoral & Past producer & Unknown & Unknown & $\begin{array}{l}\text { Produced in the 1950's. } \\
\text { 1955 county reserves } 1.2 \mathrm{MI} \text { 40.57\% Cr2O3. }\end{array}$ \\
\hline NN & 337 & Komlklor & $N 30^{\circ} 50^{\circ}$ & $E 29^{\circ} \infty 0^{\circ}$ & Chromine & 2 & Goneral & Past producer & Unknown & Unknown & $\begin{array}{l}\text { Producod in the } 1950 \text { 's. } \\
1955 \text { county reserves } 1.2 \mathrm{Mt} \text { 40-57\% } \mathrm{Cr} 203 \text {. }\end{array}$ \\
\hline NN & 338 & Kargltoca & $N 30^{\circ} 42^{\prime}$ & E $29^{\circ} 05^{\prime}$ & Chromllo & 2 & Goneral & Pest producer & Unknown & Unknown & $\begin{array}{l}\text { Produced in the } 1950 \text { 's. } \\
1955 \text { county reserves } 1.2 \mathrm{M} \text { 40-57\% Cr2O3. }\end{array}$ \\
\hline NN & 339 & KI211kaya & $N 30^{\circ} 49$ & $E 28^{\circ} 35^{\circ}$ & Chromite & 2 & General & Past producer & Unknown & Unknown & $\begin{array}{l}\text { Produced in the } 1950 \mathrm{~s} \text {. } \\
1955 \text { county reserves } 1.2 \mathrm{MM} \text { \& } 40-57 \% \mathrm{Cr} 203 \text {. }\end{array}$ \\
\hline NN & 340 & KIzilkaya-Kopez & $N 30^{\circ} 48^{\circ}$ & $E 28^{\circ} 65^{\prime}$ & Chromite & 2 & Goneral & Past producar & Unknown & Unknown & $\begin{array}{l}\text { Produced in the 1950s. } \\
1955 \text { county resenves } 1.2 \mathrm{Mm} \odot 40-57 \% \mathrm{Cr} 203 \text {. }\end{array}$ \\
\hline NN & 342 & Koycegiz-Demirkazlk & $N 36^{\circ} 55^{\prime}$ & $E 28^{\circ} 43^{\prime}$ & Chromite & 2 & General & Past producer & Unknown & Unknown & $\begin{array}{l}\text { Produced in the } 1950^{\prime} \text {. } \\
1855 \text { county reserves } 1.2 \mathrm{Mm} 40-57 \% \mathrm{Cr} 203 \text {. }\end{array}$ \\
\hline NN & 343 & Kuzkavak & $N 30^{\circ} 50^{\circ}$ & E $29^{\circ} 00$ & Chromite & 2 & Goneral & Past producor & Unknown & Unknown & $\begin{array}{l}\text { Produced in the 1950's. } \\
\text { 1965 county resenves } 1.2 \mathrm{Mm} \text { Q } 40-57 \% \mathrm{cr} 203 \text {. }\end{array}$ \\
\hline NN & 344 & Mesebuku & $N 37^{\circ} 10^{\circ}$ & E $28^{\prime} 50^{\circ}$ & Chromtte & 15 & Goneral & Past producer & Unknown & Unknown & Small producer. \\
\hline NN & 345 & Mosowlo & $N 37^{\circ} 28^{\circ}$ & E $20^{\circ} 21^{\prime}$ & Chromtto & 2 & Goneral & Past producer & Unknown & Unknown & $\begin{array}{l}\text { Produced in the 1950's. } \\
\text { 1955 county reserves } 1.2 \mathrm{Mm} \text { Q } 10-57 \% \mathrm{Cr} 203 \text {. }\end{array}$ \\
\hline NN & 340 & & $N 30^{\circ} 49^{\circ}$ & $E 20^{\circ} 10^{\circ}$ & Chromite & 2 & Ceneral & Past producer & Unknown & Unknown & $\begin{array}{l}\text { Produced in the 1950\%s. } \\
\text { 1955 county resenves } 1.2 \mathrm{Mt} \text { 40-57\% Cr2O3. }\end{array}$ \\
\hline NN & 347 & Sakankaya & $N 37^{\circ} \mathbf{2 8}$ & $E 27^{\circ} 30^{\circ}$ & Iron & 17,22 & General & Past producor & Surtace & Unknown & $\begin{array}{l}\text { Red: 3.5Mt e 58X Fo (1981). } \\
\text { Produced during Groek times and 1950-58. }\end{array}$ \\
\hline NN & 348 & Sultanlye & $N 30^{\circ} 60^{\circ}$ & $E 29^{\circ} 00^{\circ}$ & chromile & 2.17 & General & Past producer & Unknown & Unknown & 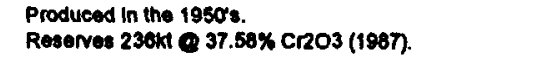 \\
\hline NN & 349 & Toparar & N $36^{\circ} 59$ & E $28^{\circ} 51^{\circ}$ & Chromite & 2 & General & Past producer & Unknown & Unknown & $\begin{array}{l}\text { Produced in the 1950's. } \\
\text { 1955 county reserves } 1.2 \mathrm{Mt} \text { 40-57\% Cr2O3. }\end{array}$ \\
\hline NN & 350 & Uzumia & $N 36^{\circ} 44^{\circ}$ & $E 29^{\circ} 14^{\prime}$ & Chromite & 2 & General & Past producer & Unknown & Unknown & $\begin{array}{l}\text { Produced in the 1950's at two sthos. } \\
1955 \text { county resonves } 1.2 \mathrm{Mm} \text { Q 10-57\% Cr2O3. }\end{array}$ \\
\hline$\infty$ & 352 & : Bolkardag & $N 37^{\circ} 27$ & E $34^{\prime} 37$ & $\begin{array}{l}\text { Lead, zinc, } \\
\text { ollver, gold }\end{array}$ & 2,15 & Goneral & Past producer & Unknown & Unknown & $\begin{array}{l}\text { Res: } 284,000 \text { lons contalning } 15.2 \mathrm{kt} \mathrm{Pb} \text {. } \\
13.2 \mathrm{kt} \mathrm{Zn}, 3 \mathrm{M} \text { oz AO, and } 21.4 \mathrm{~K} \text { oz Au. } \\
\text { Ore in limesione. }\end{array}$ \\
\hline
\end{tabular}


APPENDIX B: PAST PRODUCINO MINERUL PROPERTIES IN TURKEY

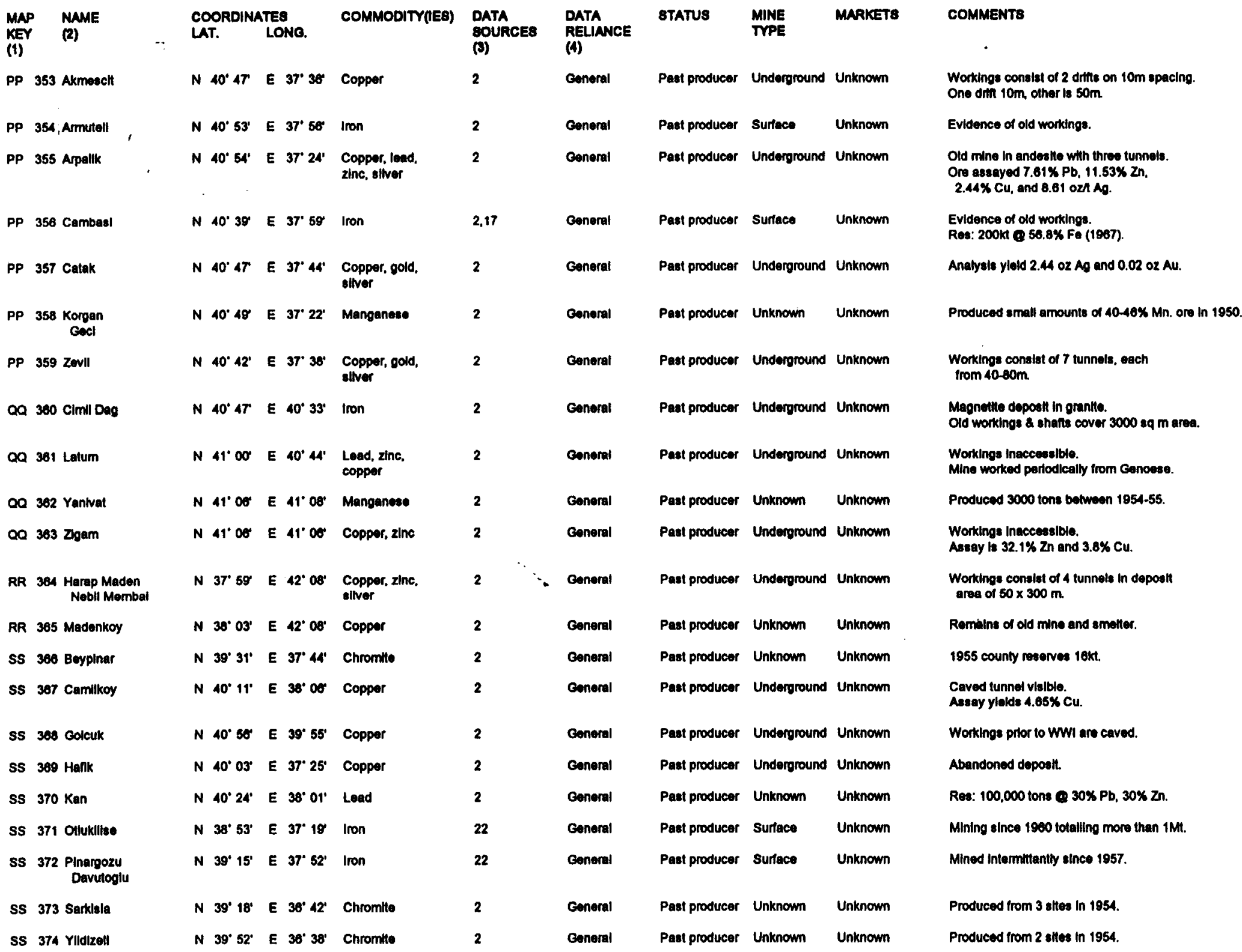


APPENDIX B: PAST PRODUCINO MINERAL PROPERTIES IN TURKEY

\begin{tabular}{|c|c|c|c|c|c|c|c|c|c|c|c|c|}
\hline $\begin{array}{l}\text { MAP } \\
\text { KEY } \\
\text { (1) }\end{array}$ & & $\begin{array}{l}\text { NAME } \\
\text { (2) }\end{array}$ & $\begin{array}{l}\text { COORDINA } \\
\text { LAT. }\end{array}$ & TER & 8 ona. & COMMODITY(DES) & $\begin{array}{l}\text { DATA } \\
\text { BOURCES } \\
\text { (J) }\end{array}$ & $\begin{array}{l}\text { OATA } \\
\text { RELIANCE } \\
\text { (d) }\end{array}$ & status & $\begin{array}{l}\text { MINE } \\
\text { TYPE }\end{array}$ & MARKETS & COMMENTS \\
\hline ss & 375 & Zara & N $39^{\circ} 65^{\circ}$ & E & $37^{\circ} 4 \sigma^{\circ}$ & Anllmony & 2 & Coments & Perl prosucer & Unknown & Untinown & OId workings. \\
\hline$\pi$ & 378 & $\begin{array}{l}\text { Manika } \\
\text { Saray }\end{array}$ & $N 41^{\circ} 23^{\prime}$ & E & $28^{\circ} 08^{\prime}$ & Mangenose & 2 & Camern & Pert moover & Unitiom & Unknown & $\begin{array}{l}\text { In 1954, produced } 15 \text { tons. } \\
\text { Depoest is of sedimentary ongin. }\end{array}$ \\
\hline UU & 377 & Aydogmess & $N 40^{\circ} 08^{\prime}$ & E & $30^{*} 40^{\circ}$ & Chromte & 2 & Onerw & Peet proovere & Unknown & Unknown & 1955 county reserves $20 \mathrm{kt}$. \\
\hline uU & 378 & |blskl & N. $402 \pi$ & $\mathbf{E}$ & $37^{\circ} 03^{\circ}$ & Copper & 2 & Conoral & Part producer & Underground & Unknown & Old workings are caved. \\
\hline UU & 379 & Ortakoy & $N 40^{\circ} 24^{\prime}$ & E & $37^{\circ} 21^{i}$ & Copper & 2 & General & Past producer & Underground & Unknown & Shan now caved. \\
\hline UU & 380 & $\begin{array}{l}\text { Tumal } \\
\text { Ozdemir } \\
\text { Camilca } \\
\text { Elaimis }\end{array}$ & N $40^{\circ} 16^{\circ}$ & $\mathbf{E}$ & $35^{\circ} 52^{\prime}$ & Anlimony & $2,15,17$ & General & Past producer & Underground & Unknown & $\begin{array}{l}\text { Produced 1949-1956. } \\
\text { Ros: } 1.3 M t \text { 3.5\% Sb. } \\
\text { Res: O28kt \& } 4 \% \text { Sb (1983). }\end{array}$ \\
\hline w & 381 & Abyane & $N 40^{\circ} 60^{\circ}$ & $\mathbf{E}$ & $39^{\circ} 54^{\prime}$ & Iron & 2 & General & Past producer & Surface & Unknown & $\begin{array}{l}\text { Slag plles in ovidence. } \\
\text { Magnetlle zone in is } 1 \mathrm{~m} \text { thlck. }\end{array}$ \\
\hline$w$ & 382 & Clcayra & $N 40^{\circ} 58^{\circ}$ & E & $39^{\circ} 54^{\prime}$ & Manganese & 2 & General & Past producer & Unknown & Unknown & $\begin{array}{l}\text { Produced } 109 \text { tons betwen 1952-53. } \\
\text { Ore in contact between limestione \& andesne. }\end{array}$ \\
\hline w & 383 & Kucuk Ayven & $N 40^{\circ} 44^{\circ}$ & $\mathbf{E}$ & $39^{\circ} 50^{\prime \prime}$ & Copper & 2 & General & Past producer & Unknown & Unknown & $\begin{array}{l}\text { Worked by the Genoese. } \\
\text { Ore In Ilmestone. }\end{array}$ \\
\hline$w$ & 3241 & Kuatul-Amenos & $N 40^{\circ} 50^{\circ}$ & $\mathbf{E}$ & $39^{\circ} 36^{\circ}$ & Copper & 2 & General & Past producer & Unknown & Unknown & Old workings grading $34 \% \mathrm{Cu}$. \\
\hline w & 3858 & Pirgl & N $40^{\circ} 53^{\prime}$ & $\mathbf{E}$ & $40^{\circ} 03^{\prime}$ & Mangenese & 2 & General & Past producer & Unknown & Unknown & $\begin{array}{l}\text { Produced } 12.0 \mathrm{kt} \text { between } 1948-1953 \text {. } \\
\text { Ore assoclated with andeste \& llmesione. } \\
\text { Assays range from 45-54\% Mn. }\end{array}$ \\
\hline w & 388 & Uzmesehor & N $40^{\circ} 50^{\circ}$ & $\mathbf{E}$ & $39^{\circ} 60^{\circ}$ & Copper & 2 & General & Past producer & Unknown & Unknown & Worked by the Cenoese. \\
\hline ww & 3871 & Mamies & N $39^{\circ} 14^{\prime}$ & $\mathbf{E}$ & $39^{\circ} 13^{\circ}$ & Copper & 2 & Goneral & Past producer & Unknown & Unknown & Old mine in grantie. \\
\hline ww: & 388 & Venlk & N $39^{\circ} 14^{\circ}$ & $\mathbf{E}$ & $39^{\circ} 13^{\circ}$ & iron & 2 & Goneral & Past producor & Unknown & Unknown & Limonke deposti formerty worked. \\
\hline$x x$ & 389 & Akdagmadenl & N 39' 42' & E & $35^{*}$ 34t & Lead, zlnc & 2 & General & Paut producer & Surface & Unknown & $\begin{array}{l}\text { Oro in crystalline schists and limestones. } \\
\text { Grade aceays } 227.3 \% \mathrm{~Pb}, 33.6 \% \mathrm{Zn} \text {, } \\
2-15 \text { oz1 AQ. }\end{array}$ \\
\hline$x x$ & 390 & Carortl & N $39^{\prime \prime} 33^{\prime}$ & $\mathbf{E}$ & $34^{\prime} \mathbf{4 3}$ & Fluortle & 2 & Genoral & Pant producer & Unknown & Domestle & Produced in 1953, 1955. \\
\hline$x \mathbf{x}$ & 391 & $\begin{array}{l}\text { Clhanpasa } \\
\text { Derbent }\end{array}$ & N $39^{\circ} 69^{\prime}$ & $\mathbf{E}$ & $34^{\circ} 46^{\circ}$ & Manganese & 2 & Genoral & Paut producer & Unknowm & Unknown & Produced 423 tons in 1954. \\
\hline$x x$ & 392 & Davutiu & N $39^{\prime} 43^{\prime}$ & $\mathbf{E}$ & $35^{*} 57$ & Iron & 2 & Genoral & Pest producer & Unknown & Unknown & One of five abandoned mines in area. \\
\hline$x x$ & 393 & Elel & N $39^{\circ} 13^{\prime \prime}$ & $\mathbf{E}$ & $35^{\circ} 43^{\prime}$ & Iron & 2 & General & Past producor & Unknown & Unknown & One of five abandoned mines in area. \\
\hline$x x$ & 3941 & Karapinar Simbuluk & $\mathbf{N} 39^{\circ} 43^{3}$ & E & $35^{\circ} 34^{\prime \prime}$ & Iron & 2.17 & Beneral & Past producer & Unknown & Unknown & $\begin{array}{l}\text { One of five abandoned m/nes in area. } \\
\text { Ros: } 7 \text { MT } 33.39 \% \text { Fe (1978). }\end{array}$ \\
\hline$x x$ & $395 \mathrm{I}-2-3-2-3$ & Kocikalo & N $39^{\circ} 30^{\circ}$ & E & $35^{\circ} 40^{\prime}$ & Iron & 2 & General & Past producer & Unknown & Unknown & One of tive abendoned mines in area. \\
\hline$x \times 3$ & 396 & Yehyasaray & N $39^{\circ} 30^{\circ}$ & $\mathbf{E}$ & $35^{\circ} 40^{\prime}$ & Iron & 2 & General & Past producer & Unknown & Unknown & One of flve abandoned mines in area. \\
\hline
\end{tabular}


APPENDIX B: PAST PRODUCINO MINERAL PROPERTES IN TURKEY

\begin{tabular}{|c|c|c|c|c|c|c|c|c|c|c|c|}
\hline $\begin{array}{l}\text { MAP } \\
\text { KEY } \\
\text { (I) }\end{array}$ & $\begin{array}{l}\text { MAME } \\
\text { (2) }\end{array}$ &.- & $\begin{array}{l}\text { COORDINA } \\
\text { LAT. }\end{array}$ & $\begin{array}{l}\text { ATES } \\
\text { LONO. }\end{array}$ & COMMODITY(IES) & $\begin{array}{l}\text { DATA } \\
\text { 8OURCES } \\
\text { (() }\end{array}$ & $\begin{array}{l}\text { OATA } \\
\text { RELIANCE } \\
\text { (4) }\end{array}$ & 8TATUS & $\underset{\text { TIPE }}{\text { MINE }}$ & MARKETS & COMMENTS \\
\hline$r$ & 397 Alakose & & $N 41^{\circ} 10^{\circ}$ & E $31^{\circ} 44^{\prime}$ & Manganose & 2 & Goneral & Past producer & Unknown & Unknown & Reserves eatimeted at $500-1000$ lons Q 36-50\% Mn. \\
\hline$r r$ & 398 Aydin & & $N 41^{\circ} 10^{\circ}$ & E $31^{\circ} 40^{\circ}$ & Manganese & 2 & Goneral & Past producer & Uniknown & Unknown & Produced 2002 tons in $1947-49$. \\
\hline$r$ & $390^{\prime}$ Ayvatler ' & & $N 41^{\prime} 17$ & E $37^{\circ} 49$ & Manganese & 2 & Goneral & Past producer & Unknown & Unknown & Produced 9420 tons in 1949-53. \\
\hline$r$ & 400 Caglar & & $N 41^{\circ} 1 r$ & $E 31^{\circ} 55^{\prime}$ & Manganose & 2 & Coneral & Past producer & Unknown & Unknown & Produced 3275 tons in 1955. \\
\hline$r r$ & 401 Cubuk & & $\mathrm{N} 41^{\circ} 28^{\circ}$ & E $32 \cdot 30^{\circ}$ & Manganese & 2 & Gonoral & Past producar & Unknown & Unknown & Produced 75kt between 1950-55. \\
\hline$r$ & 402 Cubuklar & & $N 41^{\circ} 28^{\circ}$ & E $32^{\prime} 30$ & Mengenese & 2 & General & Peat producer & Unknown & Unknown & Producad 2561 tons in $1947-49$. \\
\hline$r$ & 403 Esnekoy & & $N 41^{*} 17$ & E $31^{\circ} 40^{\circ}$ & Manganosese & 2 & Genoral & Past producer & Unknown & Unknown & Produced 7117 ions in 1953-55. \\
\hline$r$ & 404 Kepez I & & $N 41^{\circ} 16^{\circ}$ & E $31^{\circ} 28^{\circ}$ & Manganases & 2 & Goneral & Past producer & Unknown & Unknown & Produced 570 tons in 1951. \\
\hline$r$ & 405 Kopez II & & $N 41^{\circ} 16^{\prime}$ & E $31^{\circ} 28^{\circ}$ & Manganese & 2 & Coneral & Past producer & Unknown & Unknown & Producad 2535 tona in 1954-55. \\
\hline$r r$ & 408 Kulah & & $N 41^{*} 13^{\prime}$ & E $31^{\circ} 39^{\circ}$ & Manganeate & 2 & Goneral & Past producer & Unknown & Unknown & Produced 1957 tons in 1951-52. \\
\hline
\end{tabular}




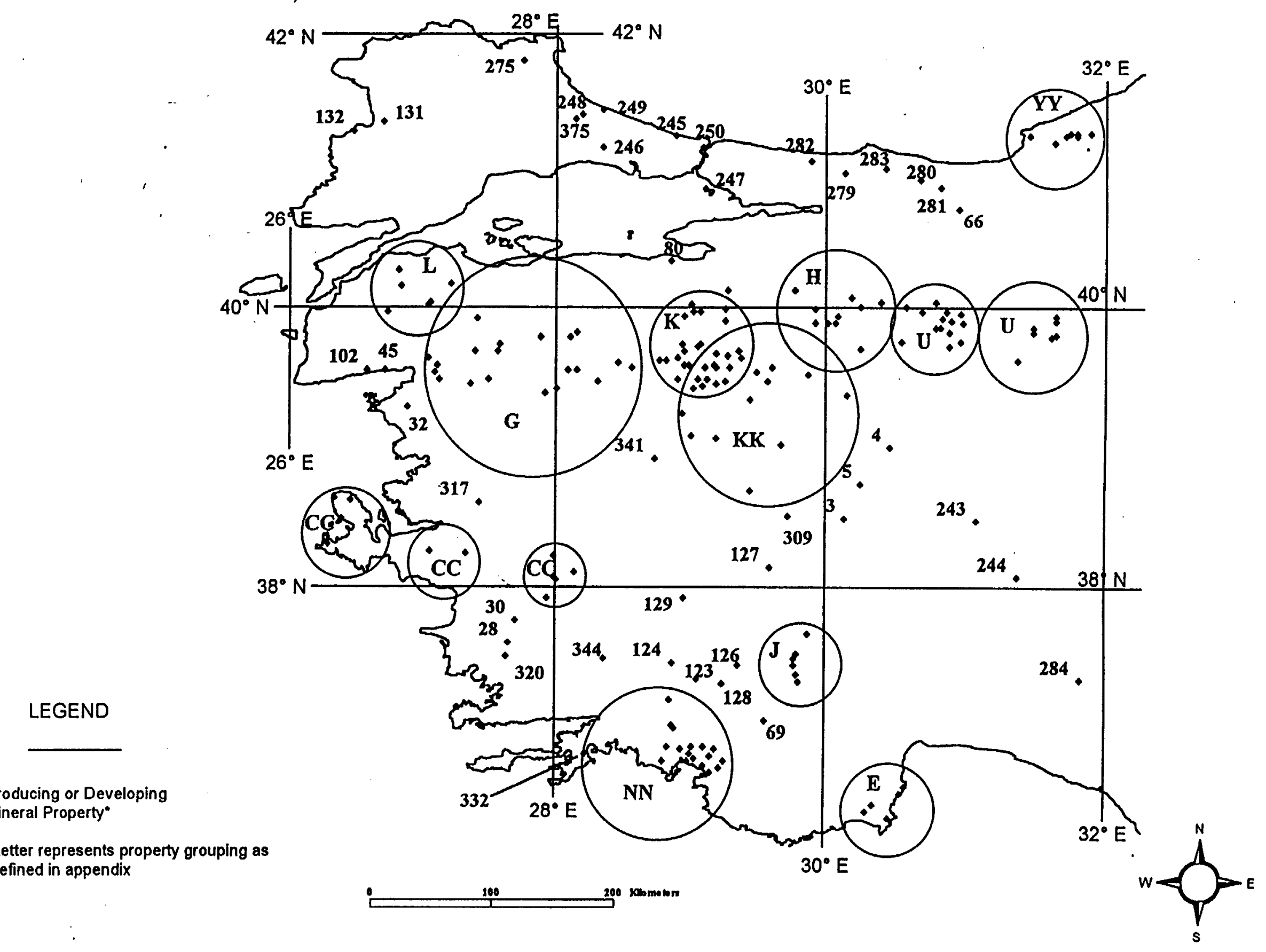

APPENDIX MAP B-1: PAST PRODUCING MINERAL PROPERTIES OF WESTERN TURKEY 


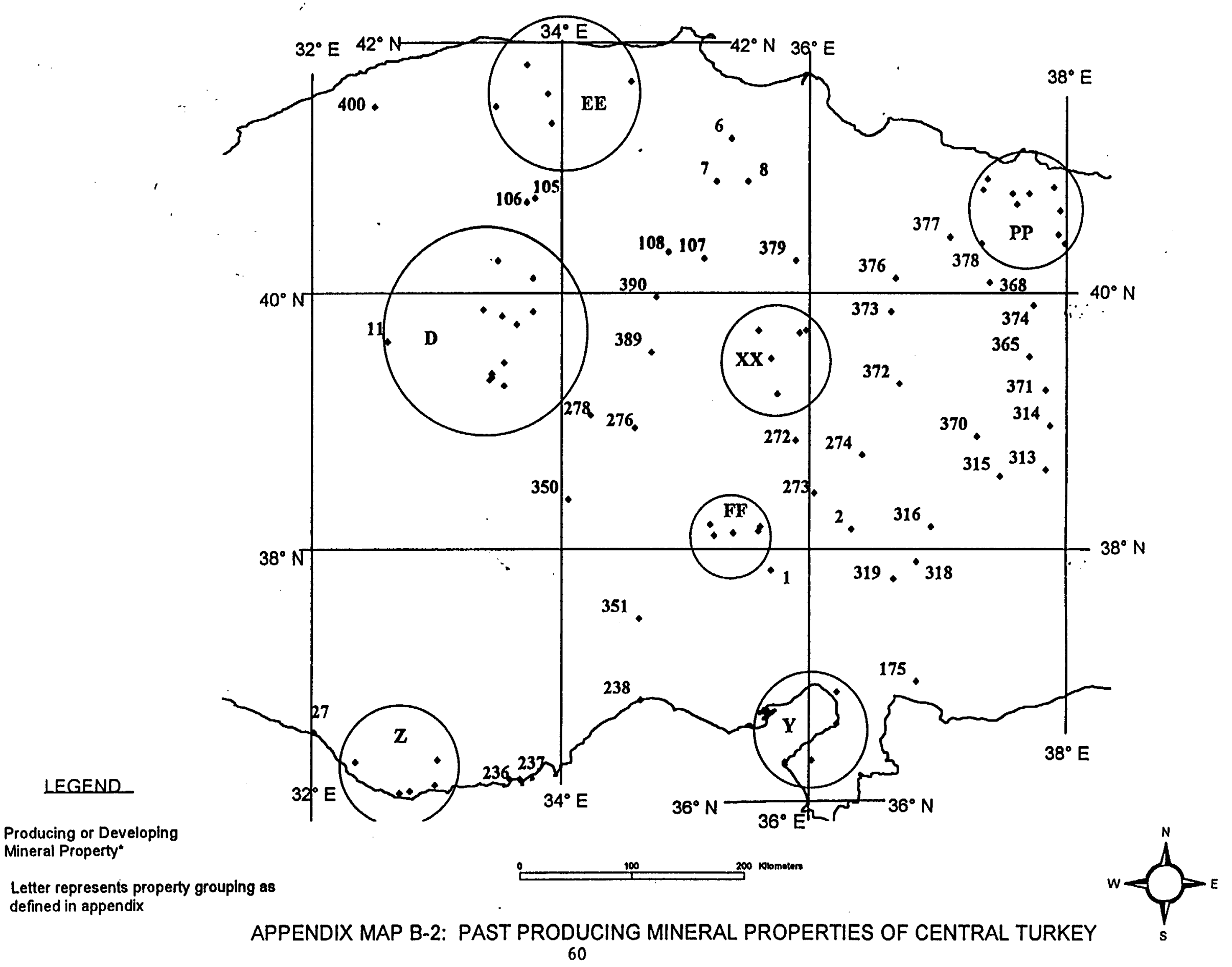






Producing or Developing

- Mineral Property* 
APPENDIX C: PROBPECTS AND UNDEVELOPED MINERNL PROPERTIES IN TURKEY

\begin{tabular}{|c|c|c|c|c|c|c|c|c|c|c|c|}
\hline \multicolumn{2}{|c|}{$\begin{array}{l}\text { MAP } \\
\text { KEY } \\
(1)\end{array}$} & \multirow{2}{*}{$\begin{array}{l}\text { MAME } \\
\text { (2) } \\
\text { Adana }\end{array}$} & \multicolumn{2}{|c|}{$\begin{array}{l}\text { COORDIMATES } \\
\text { LAT. LONO. }\end{array}$} & COMMODITY(IES) & $\begin{array}{l}\text { DATA } \\
\text { 8OURCES } \\
\text { (3) }\end{array}$ & $\begin{array}{l}\text { DATA } \\
\text { RELIANCE - } \\
\text { (4) }\end{array}$ & 8TATUS & $\begin{array}{l}\text { MINE } \\
\text { TYPE }\end{array}$ & MARKETS & COMMENTS \\
\hline A & 1 & & N $37^{\circ} 01$ & E $35^{\circ} 18^{\circ}$ & LImestone & 17 & General & Unknown & Surtace & Domoutlc & Rev: 117Mt (1972). \\
\hline A & 2 . & Belbasl & $N 38^{\circ} 12^{\circ}$ & E 36" 21" & Zne & 17 & General & Deposh & Unknown & Unknown & $\begin{array}{l}\text { Res: } 72 \mathrm{~K} \text { Q 27\% Zn; } 42.0 \mathrm{kt} \odot 8 \% \mathrm{Zn} \text {. } \\
\text { Data from } 1975 \text { repon. }\end{array}$ \\
\hline A & 3 & Dokuztekne & $N 37^{\circ} 01^{\prime}$ & E $35^{\circ} 59^{\circ}$ & Manganese & 17 & General & Deposth & Unknown & Unknown & Res: $76 k 1 @ 16.22 \% \operatorname{Mn}(1981)$. \\
\hline A & 4 & Karsanti arean & N $37^{\circ} 33^{\prime}$ & E 35' 24' & Chromilte & 17 & General & Deposth & Unknown & Unknown & Reserves from 6 shes: $93 \mathrm{MM} @ 5-35 \% \mathrm{Cr} O 3$ \\
\hline A & 5 & Kayadibl & $N 37^{\prime} 2 r$ & E $35^{\circ} 19^{\circ}$ & Lignite & 17 & Ceneral & Deposth & Unknown & Unknown & Ros: 1 MH 5492 Calkg (1960). \\
\hline A & 6 & $\begin{array}{c}\text { Kozan } \\
\text { Horzum }\end{array}$ & $N 372 T$ & E 35.48 & Zne & 17 & Gonoral & Deposalt & Unknown & Unknown & $\begin{array}{l}\text { Ros: } 122.4 \mathrm{kt} 928.15 \% \mathrm{Zn} . \\
\text { Data from } 1983 \text { roport. }\end{array}$ \\
\hline A & 7 & Salmboyll & N 38, $00^{\circ}$ & $E 30^{\circ} 00^{\circ}$ & Iron & 17 & Oonomal & Doposth & Surtace & Unknown & Ret: 3.2Mt Q 52\% Fo (1970) \\
\hline A & 8 & TuranhoyllI & $N 30^{\circ} 10^{\circ}$ & E $30^{\circ} 13^{\prime}$ & Lead, zine & 17 & Gonoral & Depost & Unknown & Unknown & $\begin{array}{l}\text { Ros: sosokt e 5.0X Pb, 13X Zn. } \\
\text { Data from } 1975 \text { roport. }\end{array}$ \\
\hline A & 9 & Tufanhoyll II & $N 36^{\circ} 16^{\circ}$ & $E \mathbf{3 0}^{\circ} \mathbf{1 3}$ & Leed, zinc & 17 & Goneral & Deposil & Unknown & Unknown & $\begin{array}{l}\text { Res: } 49 \mathrm{kt} \text { Q } 0.44 \% \mathrm{~Pb}, 14 \% \mathrm{Zn} \text {. } \\
\text { Data irom } 1975 \text { roport. }\end{array}$ \\
\hline A & 10 & Turenbeyll & N 38' 10 & E $30^{\circ} 13^{\circ}$ & Bauxito & 17 & Gonoral & Dopost & Surface & Unknown & $\begin{array}{l}\text { Res: } 10.75 \mathrm{MH} \text { C 50-52\% A1203 (1905). } \\
\text { Dlaspore ore. }\end{array}$ \\
\hline $\mathbf{B}$ & 11 & Agdilkan & $N 37^{\circ} 44^{\prime}$ & E $30^{\circ} 00^{\circ}$ & Cement reedatock & 17 & Ganoral & Dopostt & Surface & Domestle & Res: 225Mt clay/marl (1962). \\
\hline B & 12 & Collkhan & $N 38^{\circ} 02$ & E $38^{\circ} 15^{\circ}$ & Iron & 17 & General & Deposit & Surface & Unknown & Res: 31Mi @ 28.55\% Fe (magnoltte) (1984). \\
\hline c & 13 & Akhisar & $N 39^{\circ} 05^{\prime}$ & E $30^{\circ} 43^{\circ}$ & Antimony & 2 & Goneral & Doposth & Unknown & Unknown & Doposth assaying 34.45\% Sb. \\
\hline c & 14 & Anzll & $N 30^{\circ} 18^{\circ}$ & $E 30^{\circ} 45^{\circ}$ & Chrormto & 2 & General & Deposin & Unknown & Unknown & Two slles reportedly contain 600 tons. \\
\hline c & 15 & Iscehtsar & N 38' $51^{\prime \prime}$ & E 30' $45^{\prime}$ & Graphite & 17 & General & Unknown & Unknown & Unknown & Res: $30 \mathrm{kt} \odot 1.9-17.9 \% \mathrm{C}$ (1981). \\
\hline c & 16 & Kozoren & N $39^{\circ} 32^{\prime}$ & E $29^{\circ} 4 r$ & Antlmony & 2 & General & Deposit & Unknown & Unknown & Two depostits assaying 27.85\% Sb. \\
\hline D & 17 & Agrt & $N 39^{\circ} 44^{\prime}$ & E 43. 03' & Limestono & 17 & General & Unknown & Surface & Domostle & Res: 100-150Mt (1901). \\
\hline E & 18 & Karalbrahlm & $N 40^{\circ} 32$ & E $38^{\circ} 11^{\circ}$ & Asbentos & 17 & Genoral & Doposth & Surrace & Unknown & Res: 1.4Mt (1985). \\
\hline E & 19 & Ogulbegl & $N 40^{\circ} 51^{\prime}$ & E 35' $41^{\prime}$ & Ltgnite & 17 & General & Depositt & Unknown & Unknown & Ros: 3.4Mt 9574 Calkg (1983). \\
\hline$F$ & 20 & Aktepe & N $40^{\circ} 10^{\circ}$ & E $3^{\circ} 32^{\prime}$ & Manganese & 2 & Coneral & Deposill & Unknown & Unknown & Res: $40 \mathrm{kt} 23.25 \% \mathrm{Mn}$ \\
\hline$F$ & 21 & Ankara & $\mathbf{N} \mathbf{3 9}^{*} \mathbf{5 T}$ & E $32^{\circ} 46^{\prime}$ & Clay & 2 & Genoral & Deposilf & Surface & Domestlc & Slzeable Are clay deposth. \\
\hline $\mathbf{F}$ & 22 & Azmak & $N 40^{\circ} 10^{\circ}$ & E $31^{\circ} 50^{\circ}$ & Gypsum & 17 & Goneral & Doposh & Surface & Unknown & Res: 7.5Mt (1987). \\
\hline $\mathbf{w}$ & 23 & $\begin{array}{l}\text { Beskll } \\
\text { Nazanusagl }\end{array}$ & $N 38^{\circ} 40^{\circ}$ & E 38' 65' & $\begin{array}{l}\text { Gold, allver, } \\
\text { copper }\end{array}$ & 17 & General & Deposil & Unknown & Unknown & $\begin{array}{l}\text { Res: } 49 \mathrm{kt} \text { 2.49n Au, } 4.29 \mathrm{Ag} .2 \times \mathrm{Cu} \text {. } \\
\text { Data from } 1984 \text { ropor. }\end{array}$ \\
\hline $\mathbf{F}$ & 24 & Cubuk & $N 40^{\circ} 15^{\prime}$ & E $33^{\circ} 02^{\prime}$ & Porlito & 17 & Goneral & Unknown & Surface & Unknown & Res: 51Mt (1979). \\
\hline
\end{tabular}


APPENDIX C: PROSPECTS AND UNDEVELOPED MINERAL PROPERTIES IN TURKEY

\begin{tabular}{|c|c|c|c|c|c|c|c|c|c|c|c|}
\hline \multicolumn{2}{|c|}{$\begin{array}{l}\operatorname{MAP} \\
\mathbf{K E Y} \\
(1)\end{array}$} & \multirow{2}{*}{$\begin{array}{l}\text { NAME } \\
\text { (R) } \\
\text { Domitas }\end{array}$} & \multicolumn{2}{|c|}{ COORDINATES } & COMMODITY(1ES) & $\begin{array}{l}\text { DATA } \\
\text { SOURCES } \\
\text { (3) }\end{array}$ & $\begin{array}{l}\text { DATA } \\
\text { RELIANCE } \\
\text { (4) }\end{array}$ & STATUS & $\begin{array}{l}\text { MINE } \\
\text { TYPE }\end{array}$ & MARKETS & COMMENTS \\
\hline $\boldsymbol{F}$ & 25 & & $N 40^{\circ} 23^{\circ}$ & E $33^{*} 23^{*}$ & Bentonite & 17 & General & Deposit & surfece & Unknown & Ret: $75 M$ (1944). \\
\hline $\mathbf{F}$ & 26 & Karaall & N $39^{\circ} 40^{\circ}$ & E $32^{\prime} 5 T$ & Copper & 2 & General & Deposit & Unknown & Unknown & $\begin{array}{l}\text { Anclent depositt in serpentine. } \\
\text { Workinge caved. } 70 \mathrm{kt} \text { of alag. }\end{array}$ \\
\hline$F$ & 27 & Kazan & $N 40^{\circ} 12$ & E $32^{\circ} 41^{\prime}$ & cernent loedstock & 17 & Genoral & Depoill & Surtace & Domestic & Res: 371M IImestone/man (1980). \\
\hline $\boldsymbol{F}$ & 28 & K|z||cahamam & $N 40^{\circ} 28^{\circ}$ & E $32^{\circ} 39^{\circ}$ & Perlite & 17 & General & Unknown & surface & Unknown & Reserves from 3 elles: 43Mt (1972). \\
\hline c & 30 & Agver & $N 30^{\circ} 30^{\circ}$ & E $30^{*} 34^{\circ}$ & $\begin{array}{l}\text { Leand, zinc, } \\
\text { copper, siver }\end{array}$ & 2 & Gonoras & Depostl & Unknown & Unknown & Vein 1-1.4m thlek in achist. \\
\hline 0 & 31 & Aksekl & $\mathrm{N} 37^{\circ} 02^{\circ}$ & E $31^{\circ} 48^{\circ}$ & Bauxito & 17 & Conomal & Deposit & surface & Unknown & $\begin{array}{l}\text { Res: 17.64Mt a 47.60\% N2O3 (1965). } \\
\text { Bochmite ore. }\end{array}$ \\
\hline 0 & 32 & Alanya & N 30' 35 & E $32^{\circ}$ or' & Bauxite & 17 & Goneral & Deposit & surtace & Unknown & $\begin{array}{l}\text { Res: } 4.5 \mathrm{Sm} \text { e } 37-07 \% \text { A2O3 (1904). } \\
\text { Dlaspore ore. }\end{array}$ \\
\hline G & 33 & $\begin{array}{l}\text { Karalar } \\
\text { Gazlpasa }\end{array}$ & $N 30^{\circ} 18^{\circ}$ & E $32^{\circ} 21^{\prime}$ & Bartto & 17 & Genenal & Deposit & Unknown & Unknown & Reserves from 5 attes: 3.5Mt Q 78-98\% BaSO4 (1976). \\
\hline G & 34 & Serik & N 30' $55^{\prime}$ & E $31^{\circ} 0 \theta^{\prime}$ & Cerment loedstock & 17 & Goneral & Depostt & surface & Domestlc & Res: 210Mt clay (1982). \\
\hline G & 35 & Yarpuz & $N 37^{\circ} 00^{\circ}$ & E $31^{\circ} 53^{\prime}$ & Bauxtte & 2 & General & Doposit & Surface & Unknown & $\begin{array}{l}\text { Singla lonsest-2-2.5M, tolal 5-15Mt ore. } \\
\text { Ore gradee 55-60\% A2O3. }\end{array}$ \\
\hline G & 36 & Yulan & N $36^{\prime} 22$ & E $32^{*} 15^{\prime}$ & Lead & 2 & Ganomal & Depostt & Unknown & Unknown & Occurrence. \\
\hline H & 37 & Ardanuc & $N 41^{\circ} 06^{\circ}$ & $E$ 42 04' & Coment foedstock & 17 & General & Deposth & Surface & Domeatle & Res: 18Mt Itmestone (1980). \\
\hline H & 36 & Borcka & $N 11^{\prime} 22^{\circ}$ & $E 41^{\circ} 40^{\circ}$ & Manganese & 17 & General & Deposit & Unknown & Unknown & Reserves from 5 elles: $158 \mathrm{kt} @ 22-12 \% \mathrm{Mn}$ (1981). \\
\hline$H$ & 39 & $\begin{array}{l}\text { Borcka I } \\
\text { Akersen }\end{array}$ & $N 41^{\circ} 11^{\prime}$ & E $41^{\circ} 50^{\circ}$ & $\begin{array}{l}\text { Gold, slver. } \\
\text { copper }\end{array}$ & 17 & Gonoral & Deposit & Unknown & Unknown & $\begin{array}{l}\text { Res: } 682 \mathrm{kt} \text { (289n Ag: } 1.59 \mathrm{AU}, 3.2 \% \mathrm{Cu}(1986) \text {. } \\
\text { Data irom } 1980 \text { report. }\end{array}$ \\
\hline $\mathbf{H}$ & 40 & $\begin{array}{c}\text { Boscka II } \\
\text { Akarsen }\end{array}$ & $N 41^{\prime} 12^{\circ}$ & E $41^{\circ} 50^{\circ}$ & $\begin{array}{l}\text { Copper, alver. } \\
\text { gold }\end{array}$ & 17 & General & Deposit & Unknown & Unknown & 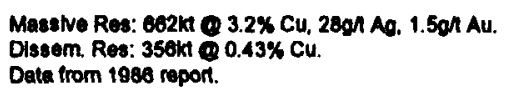 \\
\hline$H$ & 41 & $\begin{array}{l}\text { Boscka } \\
\text { Anayatak }\end{array}$ & N $11^{\prime} 22^{\prime}$ & $E 41^{\circ} 40^{\circ}$ & Copper & 17 & General & Depostt & Unknown & Unknown & $\begin{array}{l}\text { Res: } 20.8 \mathrm{MH} Q 1.32 \% \mathrm{Cu} \text {. } \\
\text { Data from Ettbank report. }\end{array}$ \\
\hline$H$ & 42 & $\begin{array}{l}\text { 8oseka } \\
\text { Cakmakkala }\end{array}$ & $N 41^{\prime} 22^{\circ}$ & $E 41^{\prime} 40^{\circ}$ & Copper & 17 & Goneral & Deposh & Unknown & Unknown & Res: $32.2 \mathrm{Mt} @ 0.98 \% \mathrm{Cu}$. \\
\hline$H$ & 43 & Hendek & $N 41^{\prime} 21^{\prime}$ & E $41^{\circ} 27$ & Coment foodelock & 17 & General & Doposk & Surface & Domestlc & Res: 100MT IImestone (1986). \\
\hline H & 15 & $\begin{array}{l}\text { Hope } \\
\text { Porent }\end{array}$ & $N 41^{\circ} 24^{\prime}$ & E $41^{*} 27$ & $\begin{array}{l}\text { Copper, zinc, } \\
\text { loed }\end{array}$ & 17 & Goneral & Deposth & Unknown & Unknown & $\begin{array}{l}\text { Res: } 1 \text { Mt } 1.4 \% \mathrm{Cu}, 1.0 \% \mathrm{Zn}, 0.3 \% \mathrm{~Pb} \text {. } \\
\text { Data from } 1978 \text { report. }\end{array}$ \\
\hline
\end{tabular}


APPENDIX C: PROSPECTS AND UNDEVELOPED MINERAL PROPERTES IN TURKEY

\begin{tabular}{|c|c|c|c|c|c|c|c|c|c|c|c|}
\hline $\begin{array}{l}\text { MAP } \\
\text { KEY } \\
\text { (1) }\end{array}$ & & $\begin{array}{l}\text { NAME } \\
\text { (2) }\end{array}$ & $\begin{array}{l}\text { COORDIN } \\
\text { LAT. }\end{array}$ & $\begin{array}{l}\text { ATES } \\
\text { LONO. }\end{array}$ & COMMODITY(RES) & $\begin{array}{l}\text { DATA } \\
\text { SOURCES } \\
\text { (3) }\end{array}$ & $\begin{array}{l}\text { DATA } \\
\text { RELIANCE } \\
\text { (4) }\end{array}$ & 8TATus & $\begin{array}{l}\text { MINE } \\
\text { TYPE }\end{array}$ & MARKETS & COMMENTS \\
\hline H & 48 & $\begin{array}{l}\text { Hopa } \\
\text { Slnkot }\end{array}$ & $N 41^{\circ} 25^{\prime}$ & $E 11^{\prime} 30^{\circ}$ & Copper & 17 & Gonoral & Deposin & Unknown & Unknown & Res: $5.1 \mathrm{ML}$ 1.39\% Cu. \\
\hline H & 47 & Inechan, & $N 41^{\circ} 11^{\prime}$ & E $11^{\circ} 50^{\circ}$ & $\begin{array}{l}\text { Copper, load, } \\
\text { zine, siver }\end{array}$ & 17 & Gonemal & Doposk & Unknown & Unknown & 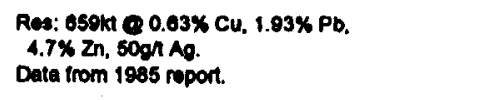 \\
\hline H & 18 & Mastira & N $41^{\circ}-15^{\circ}$ & E $41^{\prime} 20^{\circ}$ & Gold & 11 & General & Doposh & Unknown & Unknown & Drilling ongoing. \\
\hline H & 49 & $\begin{array}{l}\text { Savjat } \\
\text { Moydanclk }\end{array}$ & $N 41^{\circ} 20^{\circ}$ & $E 12^{\circ} 17$ & $\begin{array}{l}\text { Copper, load, } \\
\text { zinc }\end{array}$ & 17 & General & Deposil & Unknown & Unknown & $\begin{array}{l}\text { Res: } 00.204 k \mathrm{KC} 1.30 \% \mathrm{Cu}, 0.38 \% \mathrm{~Pb} \text {, } \\
\text { 3.21\% } \mathrm{Zn} \text {. } \\
\text { Date from } 1973 \text { roport. }\end{array}$ \\
\hline H & 50 & Saveat & $N 41^{\circ} 16^{\prime}$ & $E 42^{\circ} 20^{\circ}$ & Manganewe & 17 & Bonoral & Deposit & Unknown & Unknown & Reserves from 2 sties: 87kt 9 23-31\% Mn (1981). \\
\hline 1 & 51 & Akkuzyaylas! & $N 37^{\circ} 60$ & E $27^{*} 56^{\prime}$ & Lead & 2 & General & Deposit & Unknown & Unknown & oocurrence. \\
\hline 1 & 52 & Gonzilo & $N 37^{\circ} 40^{\circ}$ & E 28' 19 & Graphito & 2 & General & Deposit & Unknown & Unknown & Res: 6000 tons. \\
\hline 1 & 53 & Gumus & $N 37^{\circ} 51^{\circ}$ & E $27^{\prime} 28^{\prime}$ & silver & 2 & General & Deposin & Unknown & Unknown & Occurrence. \\
\hline 1 & 54 & Gurnusdag & $N 37^{\circ} 45^{\circ}$ & E $27^{\circ} 24^{\circ}$ & Emory & 17 & General & Unknown & Unknown & Unknown & Res: 8.7MI (1964). \\
\hline 1 & 55 & Hacllepboloni & $\mathrm{N} 37^{*} 37$ & E $27^{\circ} 66^{\circ}$ & Gold, areente & 2 & Goneral & Dopost & Unknown & Unknown & Occumence. \\
\hline 1 & 50 & Hallaclar & $N 37^{\circ} 42^{\prime}$ & E $27^{\circ} 67$ & Clay & 2 & General & Deposit & Surface & Domeatic & Moderate alze sand clay deposk. \\
\hline 1 & 57 & Karakoy & N $37^{\prime} 55^{\prime}$ & E $27^{\circ} 54^{\circ}$ & Lead & 2 & Coneral & Depoatt & Unknown & Unknown & occurrence. \\
\hline 1 & 58 & Kazandore & $N 373 r$ & E 28' 23' & Oraphite & 2 & Goneral & Depost & Unknown & Unknown & Res: 5000 lons. \\
\hline 1 & 59 & Nalpil & N 3752 & E $27^{\circ} 26^{\circ}$ & Siver & 2 & General & Deposit & Unknown & Unknown & Oecurrence. \\
\hline 1 & 60 & Oren & $N 37^{*} 45^{\prime}$ & E $27^{\circ} 42$ & Aneonle & 2 & Goneral & Doposat & Unknown & Unknown & Depostis of arsenopyrtite and orplment. \\
\hline 1 & 01 & Sahinall & $N 3747$ & E $2 r^{\circ} 5 T$ & Llgntte & 2 & General & Deposh & Unknown & Unknown & $\begin{array}{l}\text { Res: } 1.5 \mathrm{MH} \text {. } \\
\text { 1955 county roserves } 2.75 \mathrm{Mm} \text {. }\end{array}$ \\
\hline 1 & 62 & Torzllor & $N 36^{\circ} 52^{\prime}$ & E $28^{\circ} 50^{\circ}$ & Leed & 2 & Goneral & Deposit & Unknown & Unknown & Oecurrence. \\
\hline I & 4 & Yaylak-Kavacix & $N 37^{\circ} 55^{\prime}$ & E $28^{\circ} 20^{\circ}$ & Antimony & 2 & Genemal & Depositt & Unknown & Unknown & Oecurrence. \\
\hline 1 & 63 & Yaygln & N $37^{\circ} 38^{\prime}$ & E $28^{\circ} 35^{\prime}$ & Emory & 17 & Ganeral & Unknown & Unknown & Unknown & Res: 1.6Mt (1948). \\
\hline J & 65 & $\begin{array}{c}\text { Altinoluk } \\
\text { Edremt }\end{array}$ & $N 39^{\circ} 34^{\circ}$ & $E 26^{\circ} 44^{\prime}$ & $\begin{array}{l}\text { Lead, zinc, } \\
\text { sllver, gold }\end{array}$ & 17 & Genera! & Deposit & Unknown & Unknown & 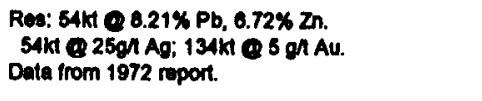 \\
\hline J & 66 & Azizłyo & N $39^{\circ} 30^{\circ}$ & E $28^{\circ} 46^{\circ}$ & Manganese & 2 & Goneral & Depostit & Unknown & Unknown & Eat. reserve of 1000 tons in andeshe. \\
\hline J & 67 & Bakknik & $N 39^{\circ} 17$ & E 20.65 & Copper & 2 & General & Deposh & Unknown & Unknown & Occurmence. \\
\hline J & 68 & Balya & N $39^{\prime} 45^{\prime}$ & E $27^{\circ} 35^{\prime}$ & $\begin{array}{l}\text { Znc, load, } \\
\text { eadmlum, siver }\end{array}$ & 17 & Goneral & Deposin & Unknown & Unknown & 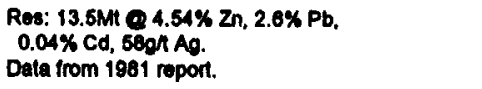 \\
\hline
\end{tabular}


APPENDIX C: PROSPECTS AND UNDEVELOPED MINERML PROPERTIES IN TURKEY

\begin{tabular}{|c|c|c|c|c|c|c|c|c|c|c|c|}
\hline $\begin{array}{l}\operatorname{MaP} \\
\text { (1) }\end{array}$ & & $\begin{array}{l}\text { MAME } \\
\text { (2) }\end{array}$ & $\begin{array}{l}\text { COORDINA } \\
\text { LAT. }\end{array}$ & $\begin{array}{l}\text { TES } \\
\text { LONO. }\end{array}$ & COMMODITY(IES) & $\begin{array}{l}\text { DATA } \\
\text { BOURCES } \\
\text { (9) }\end{array}$ & $\begin{array}{l}\text { DATA } \\
\text { RELIANCE } \\
\text { (A) }\end{array}$ & STATUS & $\begin{array}{l}\text { MINE } \\
\text { TYPE }\end{array}$ & MARKETS & COMMENTS \\
\hline J & 69 & Cemafsar & $N 39^{\circ} 45^{\circ}$ & E $27^{*} 35^{*}$ & Kaolin & 2 & General & Deposit & Surrace & Domestic & Medium size deposit reported. \\
\hline J & 70 & Camlllk & N $39^{\circ} 30^{\circ}$ & E $27^{\circ} 11^{\circ}$ & $\begin{array}{l}\text { Copper, load, } \\
\text { zine }\end{array}$ & 2 & Beneral & Daposit & Unknown & Unknown & Occurrence. \\
\hline J & 71 & Davutlar . & N $\mathbf{3 9}^{\circ} \mathbf{2 8}$ & E $28^{\circ} 18^{\circ}$ & $\begin{array}{l}\text { Lead, zinc, } \\
\text { copper, gold, } \\
\text { siver, barte }\end{array}$ & 2 & Goneral & Deposh & Unknown & Unknown & $\begin{array}{l}\text { Res: } 125.000 \text { tons } 807 \% \mathrm{~Pb}, 1 \% \mathrm{Zn} \text {. } \\
\text { and } 15021 \mathrm{Ag} \text {. }\end{array}$ \\
\hline J & 72 & $\begin{array}{l}\text { Dumenbey } \\
\text { Demirboke }\end{array}$ & N 39' 35' & E $28^{\circ} 30^{\circ}$ & $\begin{array}{l}\text { Lead, zlnc, } \\
\text { copper }\end{array}$ & 17 & Goneral & Deposth & Unknown & Unknown & $\begin{array}{l}\text { Res: } 3.7 \mathrm{Mt} Q 3.91 \% \mathrm{~Pb}, 3.81 \% \mathrm{Zn}, 0.25 \% \mathrm{Cu} \text {. } \\
\text { Deta from } 1975 \text { report. }\end{array}$ \\
\hline J & 73 & $\begin{array}{l}\text { Edremit } \\
\text { Attincluk }\end{array}$ & N $39^{\circ} 30$ & $E 27^{\circ} 15^{\circ}$ & $\begin{array}{l}\text { Gold, sliner, } \\
\text { lead, zince }\end{array}$ & 17 & General & Deposk & Unknown & Unknown & $\begin{array}{l}\text { Res: } 242 \mathrm{kt} Q 2 \operatorname{sgn} \mathrm{Ag}, 5 g h \mathrm{Au}, 8.2 \% \mathrm{~Pb} \text {, } \\
\text { O.7\% } 2 \mathrm{n} \text {. } \\
\text { Data from } 1972 \text { report. }\end{array}$ \\
\hline J & 74 & Fazllica & N $39^{\circ} 3 r$ & E $2 r 09$ & Copper & 2 & Goneral & Deposin & Unknown & Unknown & Deposit about 50m long @ $16.46 \% \mathrm{Cu}$. \\
\hline $\mathbf{J}$ & 75 & $\begin{array}{c}\text { Gaybular } \\
\text { Conen }\end{array}$ & $N 40^{\circ} 02^{\circ}$ & E $27^{\circ} 31^{\circ}$ & Load & 17 & General & Deposh & Unknown & Unknown & $\begin{array}{l}\text { Res: } 15 \mathrm{~K} \text { Q } 10 \% \text { Pb. } \\
\text { Data from } 1970 \text { roport. }\end{array}$ \\
\hline J & 76 & IVrindI & N $39^{\circ} 34^{\circ}$ & E $2 r^{\prime} 2 g^{\circ}$ & Koollin & 17 & Goneral & Deposkl & Surtace & Unknown & Res: 1.6Mt C20-31\% A12O3 (1978). \\
\hline $\mathbf{J}$ & 77 & Kalabak & N $39^{\circ} 36^{\circ}$ & E $2 r^{\circ}$ or & Iron & 2 & Coneral & Deposin & Unknown & Unknown & Small lenses of megnotke in martie. \\
\hline J & 78 & Karaggac & $N 39^{\circ} 17$ & E $28^{\circ} 09^{\circ}$ & Manganeseo & 2 & Cenoral & Dopoek & Unknown & Unknown & $\begin{array}{l}\text { Two depostle with res. of } 41 \mathrm{kt} \text { 25-50\% Mn. } \\
\text { Ore in old sehists. }\end{array}$ \\
\hline J & 79 & KInik & N $39^{\circ} 35^{\circ}$ & E $27^{\circ} 33^{\prime}$ & Antimony & 2 & General & Deposil & Unknown & Unknown & Ore assays $60 \%$ Sb. \\
\hline J & 80 & Kucukler & N $39^{\circ} 33^{\prime}$ & E $28^{\circ} 17$ & Boron & 5,6 & General & Deposil & Unknown & Unknown & Colemantie deposti wth ilmited production. \\
\hline J & 81 & $\begin{array}{l}\text { Kulat } \\
\text { Dursenbey }\end{array}$ & N $39^{\circ} 53^{\prime}$ & E $20^{\circ} 03^{\prime}$ & Lead, zlnc & 17 & General & Deposh & Unknown & Unknown & $\begin{array}{l}\text { Res: } 252 \mathrm{kt} Q 4.4 \% \mathrm{~Pb}, 4.28 \% \mathrm{Zn} . \\
\text { Data from } 1977 \text { roport. }\end{array}$ \\
\hline J & 82 & Kumluca & N $39^{\circ} 31^{\circ}$ & E $28^{\prime} 22^{\prime}$ & Mice & 2 & General & Deposil & Surrace & Unknown & Small occurrence. \\
\hline J & 83 & Illes & $N 38^{*} 35^{\prime}$ & E 20.55' & Copper & 2 & General & Deposit & Unknown & Unknown & Occurrence. \\
\hline J & 84 & Marmara & N $40^{\circ} 15^{\circ}$ & E $27^{\circ} 35^{\circ}$ & Marble & 17 & General & Unknown & Surface & Domestle & Ros: $400 \mathrm{M}$ cuble motors (1970). \\
\hline J & 85 & Narh area & $N 39^{\circ} 41^{\prime}$ & E $27^{\circ} 42^{\prime}$ & Loed, zinc & 2 & Goneral & Deposh & Unknown & Unknown & $\begin{array}{l}\text { Assoclated with voleanles. } \\
\text { Avg. assay is } 27.7 \mathrm{Q} \text { Q } 0.06 \% \mathrm{~Pb} \text {. } \\
0.835 \text { oz Ag. } 0.116 \text { oz Au. }\end{array}$ \\
\hline J & 86 & Poyraclk & N $39^{\circ} 31^{\prime}$ & E $28^{\circ} 48^{\circ}$ & Manganese & 2 & Goneral & Deposil & Unknown & Unknown & Reserves est. at 100 tons. \\
\hline J & 87 & Rahnl Merasl & $N 40^{\circ} 24^{\prime}$ & E $27^{\circ} 48^{\circ}$ & $\begin{array}{l}\text { Talc, } \\
\text { asbestos }\end{array}$ & 2 & Genaral & Deposin & Unknown & Unknown & Reserves estimated at 1000 lons. \\
\hline J & 68 & Sarkaya & N $39^{\circ} 26$ & E $27^{\circ} 53^{\prime}$ & sunur & 2 & Goneral & Deposil & Unknown & Unknown & Occurrence. \\
\hline J & 89 & Sarinc Kozcaglz & N $39^{\circ} 33^{\circ}$ & E 2708 & $\begin{array}{l}\text { Zne, load, } \\
\text { copper }\end{array}$ & 2 & Coneral & Depoen & Unknown & Unknown & $\begin{array}{l}\text { Reforence point Havran. } \\
\text { Prod. sm amounts in } 1955 .\end{array}$ \\
\hline J & 90 & Savastepe & N $39^{\prime} 22^{\prime}$ & E $27^{\circ} 40^{\circ}$ & Perlite & 17 & General & Unknown & Surface & Unknown & Res: 26Mt (1976). \\
\hline
\end{tabular}


APPENDIX C: PROSPECTS AND UNDEVELOPED MINERAL PROPERTES IN TURKEY

\begin{tabular}{|c|c|c|c|c|c|c|c|c|c|c|c|}
\hline $\begin{array}{l}\text { MAP } \\
\text { (1) }\end{array}$ & & $\begin{array}{l}\text { NAME } \\
\text { (2) }\end{array}$ & $\begin{array}{l}\text { COORDINA } \\
\text { LAT. }\end{array}$ & $\begin{array}{l}\text { TES } \\
\text { LONO. }\end{array}$ & COMMODITY(IES) & $\begin{array}{l}\text { DATA } \\
\text { 8OURCE8 } \\
\text { (3) }\end{array}$ & $\begin{array}{l}\text { DATA } \\
\text { RELIANCE } \\
\text { (4) }\end{array}$ & 8TATU8 & $\underset{\text { MMPE }}{\text { MMNE }}$ & MARKETS & $\begin{array}{c}\text { COMMENTS } \\
.\end{array}$ \\
\hline J & 91 & Sogucak & N $39^{\circ} 30^{\circ}$ & E $27^{\circ} 40^{\circ}$ & Moreury & 17 & General & Depost & Unknown & Unknown & Ret: $113 \mathrm{kt} \odot 0.71 \% \mathrm{Hg}$ (1976). \\
\hline J & 92 & Susurtuk & $N 39^{\circ} 54^{\prime \prime}$ & $E 28^{\circ} 10^{\circ}$ & Gypaum & 17 & Conoral & Doposit & Surface & Unknown & Res: 50MAt (1961). \\
\hline J & $93^{\prime}$ & Yasyeri ' & N $39^{\prime} 39^{\circ}$ & E $27^{*} 03^{\prime}$ & Iron & 2 & Coneral & Deposit & Unknown & Unknown & $\begin{array}{l}\text { Small lonees of magnotte in marble. } \\
\text { Res: } 11.000 \text { tons } 57.10 \% \text { Fo. }\end{array}$ \\
\hline J & 94 & Yellea & N $39^{\circ} 33^{\prime}$ & E $26^{\circ} 14^{\prime}$ & Kaolin & 2 & Ceneral & Deposit & Surface & Domestic & Depositt requires study. \\
\hline J & 95 & YHIIz & $N 39^{\circ} 4 r$ & $E 28^{*} 11^{*}$ & $\begin{array}{l}\text { Leed, zinc. } \\
\text { copper, gold, } \\
\text { slver }\end{array}$ & 2 & Goneral & Deposth & Unknown & Unknown & $\begin{array}{l}\text { Assayod at } 0.54 \% \mathrm{~Pb}, 0.41 \% \mathrm{Zn} \text {, } \\
4.05 \% \mathrm{Cu}, 0.7 \text { Oz/ Ag, and trace Au. }\end{array}$ \\
\hline k & 90 & Akcasu & $N 40^{\circ} 00^{\circ}$ & $E 30^{*} 18^{*}$ & Tn & 2 & Goneral & Deposit & Unknown & Unknown & Tn discovered in the area in the 1950 s. \\
\hline $\mathbf{K}$ & 97 & Bahce Sultan & N $10^{\circ} 03^{\circ}$ & E $29^{\circ} 46^{\circ}$ & Manganese & 2 & General & Depoent & Unknown & Unknown & Res: 1000 tons $12.75 \times \mathrm{Mn}$. \\
\hline K & 98 & Koyuntu & $N \quad 10^{\circ} 05^{\circ}$ & E $30^{\prime} 22$ & Tn & 2 & General & Depoett & Unknown & Unknown & Nin discovered in the area in the $1950 \mathrm{~s}$. \\
\hline $\mathbf{L}$ & 99 & Alkiran & $N 39^{\circ} 02^{\prime}$ & E $40^{\circ} 41^{\prime}$ & Cement foodstock & 17 & Beneral & Depoefl & surrace & Domestic & Res: 100Mi ilmestons (1983). \\
\hline $\mathbf{L}$ & 100 & Avnlk & $N 38^{\circ} 30^{\circ}$ & $E 40^{\circ} 20^{\circ}$ & $\begin{array}{l}\text { Iron, } \\
\text { phosphate }\end{array}$ & 2,17 & Gonoral & Depostit & Unknown & Unknown & 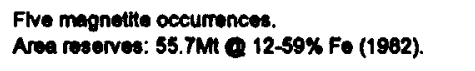 \\
\hline $\mathbf{L}$ & & $\begin{array}{l}\text { Cobancosme } \\
\text { Canc }\end{array}$ & $N 38^{\circ} 33^{\prime}$ & E $10^{\circ} 17$ & Lead, zinc & 17 & Genaral & Daposil & Unknown & Unknown & $\begin{array}{l}\text { Rea: } 21.6 \times 1915 \%(P b+2 n) \\
\text { Data from } 1978 \text { report. }\end{array}$ \\
\hline $\mathbf{L}$ & 102 & Illealer & N 39'02' & $E 40^{\circ} 42^{\prime}$ & Cement feedatock & 17 & Goneral & Doposth & surrace & Domeatle & Res: 25MM IImestone (1983). \\
\hline $\mathbf{L}$ & 103 & Ktol & N $39^{\circ} 19^{\circ}$ & E $10^{\circ} 21^{\circ}$ & Iron & 2 & Boneral & Deposit & Unknown & Unknown & Depoeft of large extent aseaying $50.5 \% \mathrm{Fo}$. \\
\hline M & 104 & Adlicevaz & $N 36^{\circ} 47$ & $E 42^{\circ} 43^{\circ}$ & Perlite & 17 & Gonoral & Unknown & Surtace & Unknown & Res: STOMt (1977). \\
\hline M & 105 & Bahcedere & $N 38^{\circ} 40^{\circ}$ & E $42^{\circ} 36^{\prime}$ & Cement reedatock & 17 & Gonoral & Doposh & Surrace & Domestle & Ros: $900 \mathrm{~m}$ Ummeclone/man/clay (1979). \\
\hline M & 100 & Karakol & N 36' $55^{\circ}$ & E $42^{\circ} 37$ & Clay & 2 & General & Dopostt & Surface & Domestle & Moderate size fullors eanth deposit. \\
\hline M & 107 & Tatvan & $N 36^{\circ} 30^{\circ}$ & $E 42^{\circ} 10^{\circ}$ & Pertite & 17 & General & Unknown & Surtace & Unknown & Res: 370Mt (1977). \\
\hline M & 108 & Unalal & $N 38^{\prime} 17$ & E $42^{\circ} 03^{\prime}$ & Iron & 17 & Gonoral & Depostt & Unknown & Unknown & Reserves trom 2 sttes: $3.1 \mathrm{Mt}$ 15.5\% Fo (1983). \\
\hline M & 109 & $\begin{array}{l}\text { Zzan } \\
\text { Markoz }\end{array}$ & $N 38^{\circ} 15^{\circ}$ & E $41^{\circ} 54^{\prime}$ & Lead, zine & 17 & General & Deposk & Unknown & Unknown & $\begin{array}{l}\text { Res: 6-9kt } 912.7 \% \text { Pb. } 34.4 \% \mathrm{Zn} . \\
\text { Data from } 1975 \text { roport. }\end{array}$ \\
\hline $\mathbf{N}$ & 110 & Bolu & $N 40^{\circ} 44^{\circ}$ & E $31^{*} 3 r$ & Coment feedstock & 17 & Gonoral & Deposit & Surlace & Domealle & Res: 280 cuble motors Ilmestone (1909). \\
\hline $\mathbf{N}$ & 111 & Cayviran plateau & $N 40^{\circ} 48^{\circ}$ & E $32^{\circ} 19^{\circ}$ & Manganese & 17 & Coneral & Deposit & Unknown & Unknown & Res: $105 k \propto 19.03 \%$ Mn (1962). \\
\hline $\mathbf{N}$ & 112 & Mengen & $N 40^{\circ} 50^{\circ}$ & E $32^{\circ}$ on' & Llgnite & 17 & General & Doposit & Unknown & Unknown & Res: $78 M I C 4755$ Calkg (1963). \\
\hline N & 113 & Morkenilor & $N 40^{+} 52$ & E $31^{\circ} 48^{\circ}$ & Lignite & 17 & Coneral & Doposin & Unknown & Unknown & Res: $21 \mathrm{MT}$ Q 4100 Calkgg (1983). \\
\hline o & 114 & Akviran & $N 37^{\prime} 25^{\prime}$ & E $30^{\circ} 05^{\circ}$ & Lignte & 2 & Genoral & Deposit & Unknown & Unknown & $\begin{array}{l}\text { Res: } 2.5 \mathrm{MM} \text {. } \\
1955 \text { county resenves } 2.52 \mathrm{MH} \text {. }\end{array}$ \\
\hline 0 & 115 & 5 Kavaklar & $N 38^{\circ} 08^{\prime}$ & E $29^{\circ} 34^{\circ}$ & Manganese & 17 & Gonoral & Depositt & Unknown & Unknown & Res: $160 \mathrm{kt}$ 25.83\% Mn (1982). \\
\hline
\end{tabular}




\section{APPENDIX C: PROSPECTS AND UNDEVELOPED MINERAL PROPERTIES IN TURKEY}

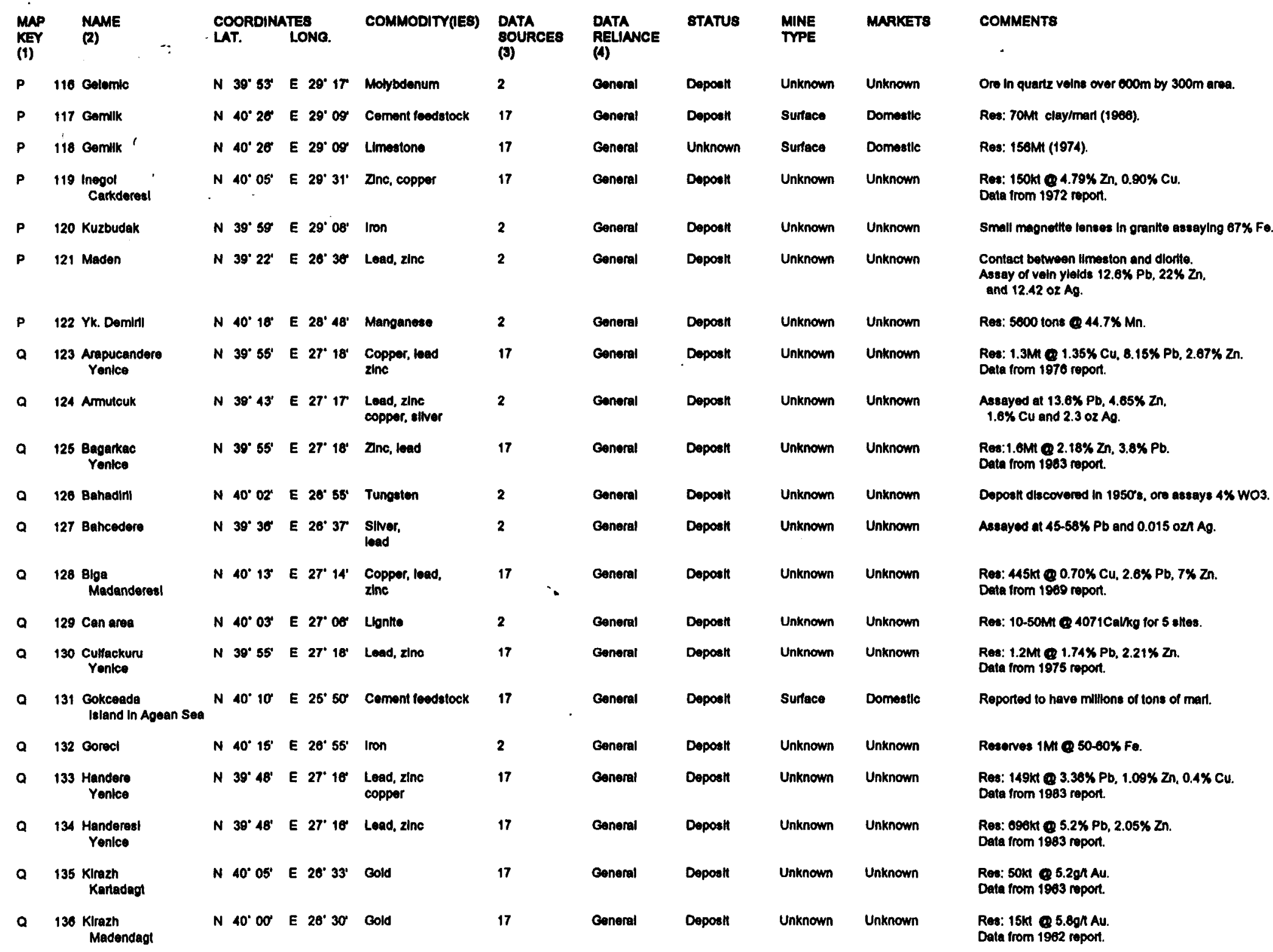


APPENDIX C: PROSPECTS AND UNDEVELOPED MINERNL PROPERTES IN TURKEY

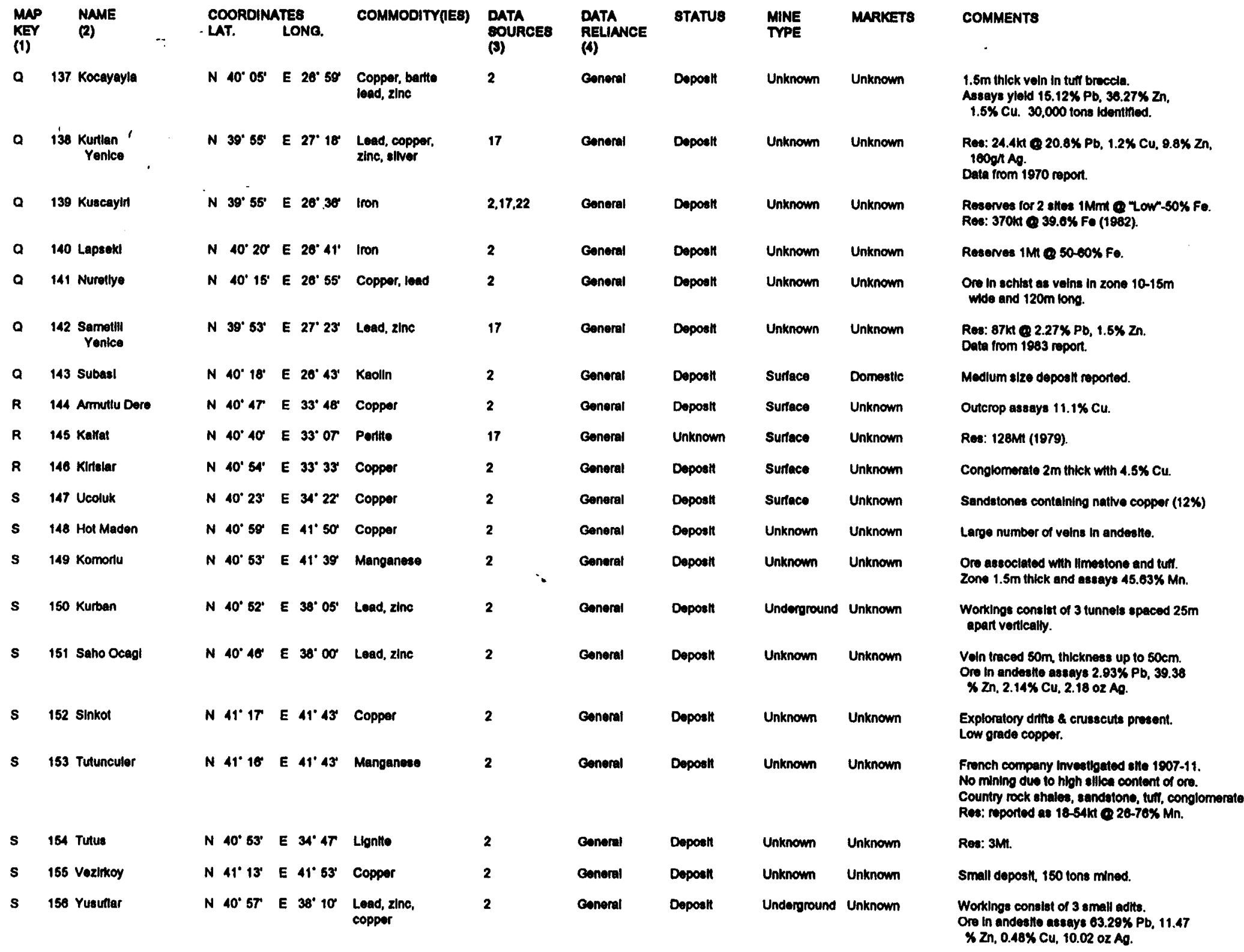


APPENDIX C: PROSPECTS AND UNDEVELOPED MINERNL PROPERTIES IN TURKEY

\begin{tabular}{|c|c|c|c|c|c|c|c|c|c|c|c|}
\hline $\begin{array}{l}\text { MAP } \\
\text { KEY } \\
\text { (1) }\end{array}$ & & $\begin{array}{l}\text { NAME } \\
\text { (2) }\end{array}$ & $\begin{array}{l}\text { COORDIN } \\
\text { UAT. }\end{array}$ & $\begin{array}{l}\text { ATE8 } \\
\text { LONO. }\end{array}$ & COMMODITY(RES) & $\begin{array}{l}\text { DATA } \\
\text { 8OURCES } \\
\text { (3) }\end{array}$ & $\begin{array}{l}\text { DATA } \\
\text { RELIANCE } \\
\text { (A) }\end{array}$ & 8TaTus & $\begin{array}{l}\text { MINE } \\
\text { TPEE }\end{array}$ & MARKETS & COMMENTS \\
\hline $\mathbf{T}$ & 157 & $\begin{array}{l}\text { Babadag } \\
\text { Tavas }\end{array}$ & N $377^{\prime} 48^{\prime}$ & E 28. 52' & Copper & 17 & caneres & Domoon & Uninown & Unknown & $\begin{array}{l}\text { Rer: } 300 k+19.8 \times \mathrm{Cu} . \\
\text { Date from } 1969 \text { report. }\end{array}$ \\
\hline$T$ & 156 & Cambasi & N $37^{\circ} 46^{\circ}$ & E $29^{\circ} 32^{\prime}$ & Sultur & 2 & omerest & Deoosen & Untrown & Unknown & Two deposins, one conteining 10k1 probable ore. \\
\hline $\mathbf{T}$ & 159 & Cumali & N $37^{\prime} 45^{\prime}$ & E $29^{\circ} 35^{\circ}$ & Magnestito & 2 & anerer & Depoen & Unknown & Unknown & Deposth of 150,000 tona $440 \%$ MgO. \\
\hline $\mathbf{T}$ & 100 & Donizil & N $37^{\prime} 46^{\prime}$ & E $29^{\circ} 00^{\circ}$ & Clay & 2 & Goneral & Dopoen & Surface & Domestlc & Moderate size fullers earth depostt. \\
\hline $\mathbf{T}$ & 181 & Kocak & N 30' $20^{\circ}$ & E $29^{\circ} 46^{\circ}$ & Copper & 2 & Goneral & Dopoen & Unknown & Unknown & Voin 20-25cm thlek easaying 8.57\% Cu. \\
\hline u & 162 & Engant & N 36" it & E $39^{\circ} 46^{\circ}$ & Coment roedstock & 17 & Genoral & Depoall & Surface & Domestle & Res: 522MM IImestone/clay (1981). \\
\hline u & 163 & $\begin{array}{l}\text { Kursunhu } \\
\text { Dlelc }\end{array}$ & N 30' 27 & E $40^{\circ}$ or & Lead, zine & 17 & Gonoral & Depoall & Unknown & Unknown & $\begin{array}{l}\text { Res: } 24.6 k t \text { e } 3-35 \% \text { Pb+Zn. } \\
\text { Dala from } 1978 \text { report. }\end{array}$ \\
\hline v & 164 & Hianafl & N $40^{\circ} 43^{\prime}$ & E $20^{\circ} 13^{\circ}$ & Bentontive & 17 & Genoral & Depost & Surraco & Unknown & Res: 150Mi (1907). \\
\hline $\mathbf{v}$ & 165 & Lalapasa & $N 41^{\circ} 50^{\circ}$ & E $20^{\circ} 44^{\circ}$ & Cement foedstock & 17 & Goneral & Depoent & Surface & Domestle & Res: 39Mit limestone/clay (1985). \\
\hline $\mathbf{v}$ & 160 & Uzunkapru area & N $41^{\circ} 08^{\prime}$ & E $20^{\circ} 40^{\circ}$ & Llgnilte & 2 & General & Depoett & Unknown & Unknown & Res: 20-30Mt. \\
\hline w & 167 & Anayatak & $N 36^{\circ} 32^{\circ}$ & E $39^{\circ} 40^{\circ}$ & Copper & $\$ 7$ & Goneral & Depoent & Unknown & Unknown & $\begin{array}{l}\text { Ree: } 14.6 \mathrm{MI} \& 1.39 \% \mathrm{Cu} . \\
\text { Data from } 1978 \text { repon. }\end{array}$ \\
\hline w & 23 & $\begin{array}{l}\text { Baskil } \\
\text { Nazanusagl }\end{array}$ & $\mathbf{N} 30^{\circ} 40^{\circ}$ & E 38' 35" & $\begin{array}{l}\text { Gold, sllver, } \\
\text { copper }\end{array}$ & 17 & Gonoral & Doponit & Unknown & Unknown & 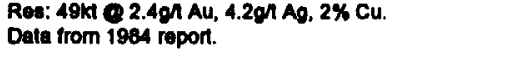 \\
\hline w & 160 & $\begin{array}{l}\text { Hacan } \\
\text { Erganl }\end{array}$ & $N 38^{\circ} 40^{\circ}$ & E $39^{\circ} 41^{\prime}$ & Copper & 17 & Goneral & Deposit & Unknown & Unknown & $\begin{array}{l}\text { Res: 10k1 } 1.69 \% \mathrm{Cu} \text {. } \\
\text { Dath from } 1978 \text { roport. }\end{array}$ \\
\hline w & 169 & $\begin{array}{l}\text { Nalllzlyaret } \\
\text { Kaban }\end{array}$ & $N \quad 38^{\circ} 48^{\prime}$ & E $38^{\circ} 45^{\circ}$ & Copper & 17 & Goneral & Deposit & Unknown & Unknown & $\begin{array}{l}\text { Res: } 1.5 \mathrm{MH} \text { Q } 0.82 \% \mathrm{Cu} . \\
\text { Dath from } 1979 \text { roport. }\end{array}$ \\
\hline w & 170 & Soganil & $N 30^{\circ} 46^{\circ}$ & E $38^{\circ} 46^{\circ}$ & Tungsten & 17 & Goneral & Deposth & Unknown & Unknown & Res: $255 k$ C 0.6\% WO3 (1958). \\
\hline w & 171 & $\begin{array}{l}\text { Wolas } \\
\text { Erganl }\end{array}$ & N $30^{\circ} 32^{\prime}$ & E $39^{\circ} 40^{\circ}$ & Copper & 17 & Goneral & Deposit & Unknown & Unknown & $\begin{array}{l}\text { Res: } 2901 \mathrm{kt} 92.5 \% \mathrm{Cu} . \\
\text { Data from } 1978 \text { report. }\end{array}$ \\
\hline$x$ & 172 & Aravans & N 39. $59^{\circ}$ & $E 40^{\circ} 12$ & Megneatio & 17 & Goneral & Depost & Unknown & Unknown & Res: :MI Q 44.40\% MgO (1988). \\
\hline$x$ & 173 & Gomeck & $N 40^{\circ} 00^{\circ}$ & E $42^{\circ} 05^{\prime \prime}$ & Lead, zlne & 2 & General & Dopost & Unknown & Unknown & Occurmonce. \\
\hline$x$ & 174 & & $N 39^{\circ} 28^{\circ}$ & E $38^{\circ} 34^{\circ}$ & Iron & 17 & Coneral & Deposin & Surface & Unknown & Resenves for 4 sthes: 1.1MI Q 51-G4\% Fo (1971-1983) \\
\hline$x$ & 175 & & N 39" 28, & E 38' 34' & Manganease & 17 & Goneral & Depostl & Unknown & Unknown & Res: 174kt $46 \%$ Mn (1976). \\
\hline$x$ & 170 & & $N 39^{\circ} 20^{\circ}$ & E $30^{\circ} 34^{\prime}$ & Aebertos & 17 & General & Doposh & Surface & Unknown & Res: 3Mt (1980). \\
\hline$x$ & 177 & Kemallye & N $39^{\circ} 10^{\circ}$ & E 38' 29 & Iron & 17 & General & Doposit & Surface & Unknown & Resenves for 3 sttes: $24.5 \mathrm{ML}$ \& 40-55\% Fo (1985). \\
\hline$x$ & 178 & Mollakoy & N $39^{\circ} 39^{\circ}$ & E $39^{\circ} 35^{\prime}$ & Pontlte & 17 & General & Unknown & Surface & Unknown & Res: 71Mt (1977). \\
\hline $\mathbf{Y}$ & 179 & Allelkrek & N 40, $03^{\prime}$ & E $42^{+} 18^{\prime}$ & Lignile & 2 & General & Deposit & Unknown & Unknown & $\begin{array}{l}\text { Res: } 2 \mathrm{MM} \text {. } \\
1955 \text { county resenves } 14.1 \mathrm{Mt} .\end{array}$ \\
\hline
\end{tabular}




\section{APPENDIX C: PROBPECTB AND UNDEVELOPED MINERNL PROPERTES IN TURKEY}

\begin{tabular}{|c|c|c|c|c|c|c|c|c|c|c|c|}
\hline $\begin{array}{l}\operatorname{MAP} \\
\text { (1) }\end{array}$ & & $\begin{array}{l}\text { NAME } \\
\text { (2) }\end{array}$ & $\begin{array}{l}\text { COORDINA } \\
\text { LAT. }\end{array}$ & $\begin{array}{l}\text { ATES } \\
\text { LONO. }\end{array}$ & COMMODITY(IES) & $\begin{array}{l}\text { DATA } \\
\text { BOURCE8 } \\
\text { (3) }\end{array}$ & $\begin{array}{l}\text { DATA } \\
\text { RELIANCE } \\
\text { (4) }\end{array}$ & BTATUS & $\begin{array}{l}\text { MINE } \\
\text { TYPE }\end{array}$ & MARKETS & COMMENTS \\
\hline $\mathbf{Y}$ & 180 & Askale & $N 39^{\circ} 55^{\circ}$ & $E 40^{\circ} 42^{\prime}$ & Gypsum & 17 & General & Daposit & Surtace & Unknown & Res: 20Mt (1968). \\
\hline $\mathbf{Y}$ & 1811 & Hizlr Ilyes & $N 40^{\circ} 00^{\circ}$ & $E 42^{\circ} 16^{\circ}$ & Lead & 2 & Goneral & Dopostt & Unknown & Unknown & Oocumrence. \\
\hline $\mathbf{Y}$ & 182 & Kagdarlc' & N $39^{\circ} 50^{\circ}$ & E $40^{\circ} 47$ & Coment roedatock & 17 & Ganeral & Deposth & surface & Domettic & Res: 12Mt Hmestone (1907). \\
\hline $\mathrm{Y}$ & 183 & $\begin{array}{l}\text { Kavurmacukułu } \\
\text { Kukunlu }\end{array}$ & N 39' $51^{\prime}$ & $E 10^{\circ} 30^{\circ}$ & Llonkte & 2.17 & General & Depostit & Unknown & Unknown & $\begin{array}{l}\text { Res: } 1.3 \mathrm{MH} \text { @ } 4500 \mathrm{Calkkg} \text { (1902). } \\
\text { 1955 county reserves 14.1M. }\end{array}$ \\
\hline Y & 184 & Klgani & $N 40^{\circ} 21^{\circ}$ & E $41 \cdot 5 r$ & Lead & 2 & Coneral & Depoent & Unknown & Unknown & Occumence. \\
\hline $\mathbf{r}$ & 185 & Meschll & N 30'33' & E $41^{\prime} 25^{\circ}$ & Comont readatock & 17 & Ganeral & Depoill & Surface & Domeatle & Res: 30Mt IImestone (1967). \\
\hline Y & 180 & Onu & N $40^{\circ} 33$ & E $41^{\circ} \mathbf{5 0}$ & Salt & 2.17 & cenoral & Deposill & surface & Domestic & Ree: 300Mt. \\
\hline Y & 429 & Paainlor & N $39^{\circ} 69^{\circ}$ & E $41^{\circ} 41^{\circ}$ & Porllte & 17 & Gonoral & Unknown & Surtace & Unknown & Res: 387Mt (1975). \\
\hline $\mathbf{r}$ & 187 & Sutkan: & $N 40^{\circ} 20^{\circ}$ & E $41^{\prime} 55^{\prime}$ & Lugnhe & 17 & Genoral & Deposth & Unknown & Unknown & Res: $7.3 \mathrm{MMT}$ Q $4200 \mathrm{Calkg}$ (1994). \\
\hline$Y$ & 188 & Tortum & $N 10^{\circ} 19$ & E $41^{\circ} 33$ & Dlatomite & 17 & General & Daposh & Surface & Unknown & Res: SOMt (1984). \\
\hline $\mathbf{Y}$ & 189 & $\begin{array}{l}\text { Uiutes } \\
\text { laplr }\end{array}$ & $N 40^{\circ} 29^{\circ}$ & E $41^{\circ} \infty 0^{\circ}$ & Copper & 17 & General & Depositt & Unknown & Unknown & $\begin{array}{l}\text { Res: } 200 \mathrm{Mt} \text { Q } 0.2 \% \mathrm{Cu} . \\
\text { Data from } 1974 \text { report. }\end{array}$ \\
\hline $\mathbf{z}$ & 190 & Ballik & $N 39^{\circ} 50^{\circ}$ & E $30^{\circ} 45^{\prime}$ & Magneatie & 17 & General & Doposin & Unknown & Unknown & Res: $12.3 \mathrm{Mm}$ trom 2 athet $147 \% \mathrm{MgO}$ (1984). \\
\hline $\mathbf{z}$ & 191 & Boylikanir & $N 39^{\circ} 42^{\prime}$ & E $31^{\circ} 13^{\circ}$ & Bartite & 17 & General & Deposit & Unknown & Unknown & Resenves: 9.4MH @ 31X BaSO4 (1980). \\
\hline $\mathbf{z}$ & 192 & Boyllkahir & $N 39^{\circ} 42^{\circ}$ & E $31^{*} 13^{\circ}$ & Thorlum & 17 & Conoral & Deposh & Unknown & Unknown & Ree: 380kt. \\
\hline $\mathbf{z}$ & 193 & Dutluca & $N 39^{\circ} 52^{\prime \prime}$ & E $30^{\circ} 48^{\circ}$ & Magnealte & 17 & Genoral & Deposit & Unknown & Unknown & Ret: $12 \mathrm{Mt}$ Q $46.8 \% \mathrm{MgO}$ (1982). \\
\hline $\mathbf{z}$ & 194 & Karacam & N $39^{\circ} 39^{\circ}$ & E $31^{\circ} 30^{\circ}$ & Iron & 17 & General & Depostl & Surface & Unknown & 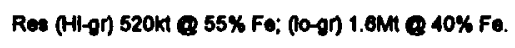 \\
\hline$z$ & 195 & Kizhlcaoren & $N 39^{\circ} 40^{\circ}$ & E $31^{\circ} 30^{\circ}$ & $\begin{array}{l}\text { Rare carthe, } \\
\text { fluorine, bartite }\end{array}$ & 15 & General & Deposit & Unknown & Unknown & 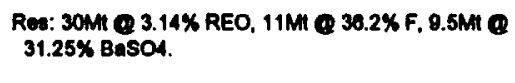 \\
\hline $\mathbf{z}$ & 100 & Minalleclk & N $30^{\circ} 62^{\circ}$ & E $31^{\circ} 30^{\circ}$ & Kaolin & 17 & Genoral & Deposit & Surtace & Unknown & Ret: 3.3Mt \& 20-33\% A2O3 (1979). \\
\hline $\mathbf{z}$ & 197 & Sazak & N $39^{\circ} 46^{\prime}$ & E $31^{\circ} 30^{\prime}$ & Talc & 2 & General & Dapoell & Unknown & Unknown & Deposth of moderate size. \\
\hline $\mathbf{z}$ & 198 & Sazak & $N 39^{\circ} 46^{\circ}$ & E 31" 30 & Clay & 2 & Gonomal & Depostit & Surtace & Domestic & Sizeable fullers earth deposit. \\
\hline $\mathbf{z}$ & 199 & $\begin{array}{l}\text { Yukart Kuzfindik } \\
\text { Yukarl Kartal }\end{array}$ & N $39^{\circ} \mathbf{4 0}$ & $E 30^{\circ} 17$ & Magnealte & 2,17 & General & Deposit & Surtace & Unknown & $\begin{array}{l}\text { Stx deposins reponted in area. } \\
\text { Res: } 519 \mathrm{Mit} \text { e } 47.63 \% \mathrm{MgO} \text { (1981). }\end{array}$ \\
\hline AA & $200 \mathrm{I}$ & Islahlye & N $37^{\circ} 03^{\circ}$ & $E 30^{\circ} 30^{\circ}$ & Iron & 17 & Goneral & Dopost & Surtace & Unknown & Reserves from 2 sttes: somit 2 21-36\% Fo (1974). \\
\hline M & 2011 & KIIIs & $N 36^{\circ} 44^{\prime}$ & E $37^{\circ} 05^{\prime}$ & Manganeres & 17 & Gonomal & Doposit & Unknown & Unknown & Rosenves from 4 attes: 308kt C 12.53\% Mn (1984). \\
\hline MA & 202 & $\begin{array}{l}\text { Kozcaglz } \\
\text { lastahlye }\end{array}$ & N $37^{\circ} 03$ & E $30^{\circ} 30^{\circ}$ & $\begin{array}{l}\text { Iron, } \\
\text { bauxtho }\end{array}$ & 2,17 & Genoral & Doposit & Surface & Unknown & 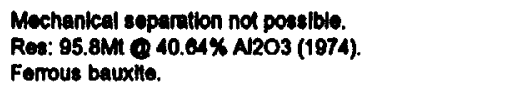 \\
\hline BB & 203 & $\begin{array}{l}\text { Akkoy } \\
\text { Markez }\end{array}$ & $N 40^{\circ} 50^{\circ}$ & E $38^{\circ} 40^{\circ}$ & Znc, copper & 17 & General & Deposit & Unknown & Unknown & $\begin{array}{l}\text { Res: } 1.9 \mathrm{Mt} \text { Q } 2.80 \% \mathrm{Zn}, 0.47 \% \mathrm{Cu} \text {. } \\
\text { Data lrom } 1972 \text { report. }\end{array}$ \\
\hline
\end{tabular}


APPENDIX C: PROSPECTS AND UNDEVELOPED MINERNL PROPERTIES IN TURKEY

\begin{tabular}{|c|c|c|c|c|c|c|c|c|c|c|}
\hline $\begin{array}{l}\text { MAP } \\
\text { (1) }\end{array}$ & $\begin{array}{l}\text { NAME } \\
\text { (2) }\end{array}$ & $\begin{array}{l}\text { COORDIN/ } \\
\text { LAT. }\end{array}$ & $\begin{array}{l}\text { ATES } \\
\text { LONO. }\end{array}$ & COMMODITY(IES) & $\begin{array}{l}\text { DATA } \\
\text { SOURCES } \\
\text { (अ) }\end{array}$ & $\begin{array}{l}\text { DATA } \\
\text { RELIANCE } \\
\text { (4) }\end{array}$ & BTATUS & $\begin{array}{l}\text { MUNE } \\
\text { TYPE }\end{array}$ & MARKETS & COMMENTS \\
\hline BB & $\begin{array}{l}204 \text { Asarclk } \\
\text { Sebenkarahtser }\end{array}$ & $N 40^{\circ} 20^{\circ}$ & E $36^{\circ} 24^{\prime}$ & $\begin{array}{l}\text { Lead, zinc, } \\
\text { copper, sliver }\end{array}$ & 17 & Goneral & Doposth & Unknown & Unknown & $\begin{array}{l}\text { Res: } 1.3 \mathrm{Mm} \propto 3.39 \% \mathrm{~Pb}, 3.94 \times \mathrm{Zn}, 0.40 \% \mathrm{Cu} \text {. } \\
\text { 50.139n Ag. } \\
\text { Data from } 1988 \text { report. }\end{array}$ \\
\hline BB & 205 Avluca & $N 40^{\circ} 40^{\circ}$ & E $30^{\circ} 40^{\circ}$ & Iron & 2 & Goneral & Depostt & Surface & Unknown & Hermathe in clay about $100 \mathrm{~m}$ wido. \\
\hline BB & $\begin{array}{l}200 \text { Calaapagl-8abkink } \\
\text { Esplye }\end{array}$ & $N 40^{\circ} 44^{\prime}$ & E $30^{\circ} 48^{\circ}$ & $\begin{array}{l}\text { Copper, lead. } \\
\text { zinc }\end{array}$ & 2.15 & General & Deposit & $\begin{array}{l}\text { Surface } \\
\text { Underground }\end{array}$ & Unknown & 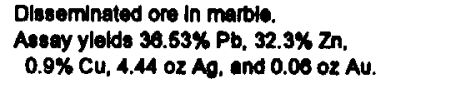 \\
\hline 88 & 207 Clbril & $N 40^{\circ} 54^{\circ}$ & E $30^{\circ} 42^{\prime}$ & Copper & 2 & General & Depostt & Underpround & Unknown & $\begin{array}{l}\text { Wonkings consist of a } 113 \mathrm{~m} \text { funnel and a } \\
\text { series of crosecults. } \\
\text { Res: } 3750 \text { tons. }\end{array}$ \\
\hline BB & 208 Cimide-Slezllk & N $40^{\circ} 54^{\prime}$ & E $30^{\circ} \mathbf{5 r}$ & Copper & 2 & Gonoral & Dopoeft & Unknown & Unknown & Occurnence. \\
\hline BB & 209 Cumanoglu & $N 40^{\circ} 52^{\prime}$ & E $38^{\circ} 17$ & $\begin{array}{l}\text { Copper. toad } \\
\text { zlne }\end{array}$ & 2 & Goneral & Doposit & Unknown & Unknown & Veln $17-30 \mathrm{~cm}$ wide and $27 \mathrm{~m}$ long. \\
\hline 88 & 210 Derell & $N 40^{\circ} 43^{\circ}$ & E $30^{\circ} 2 T$ & Bartte & 17 & General & Dopostit & Unknown & Unknown & Reserves: 2M Q 98\% BesO4 (1974). \\
\hline BB & 211 Deroll & $N 40^{\circ} 45^{\circ}$ & E $36^{\circ} 2 T$ & Comont foodstock & 17 & General & Deposk & Surface & Domesilc & Ros: 30-40MM coment raw materials (1985). \\
\hline BB & 212 Egercan & N $40^{\circ} 52^{\prime}$ & E $38^{\prime} 32^{\prime}$ & $\begin{array}{l}\text { Copper, lead, } \\
\text { zine }\end{array}$ & 2 & Goneral & Doposth & Unknown & Unknown & $\begin{array}{l}\text { Veln in andesilfe. } \\
\text { Assay ylelds 18.77\% } 2 \mathrm{n}, 17.34 \% \mathrm{~Pb} \\
1.08 \% \mathrm{Cu} \text {. }\end{array}$ \\
\hline B8 & 213 Godkll & $N 40^{\circ} 46^{\circ}$ & E $38^{\circ} 18^{\circ}$ & Copper, load & 2 & Genoral & Dopostl & Unknown & Unknown & Veln $20-25 \mathrm{~cm}$ thick in andestite. \\
\hline BB & $\begin{array}{l}214 \text { Harkkoy } \\
\text { Trebolu }\end{array}$ & $N 41^{\circ} 00^{\circ}$ & E $30^{\circ} 40^{\circ}$ & $\begin{array}{l}\text { Copper, zine } \\
\text { load }\end{array}$ & 17 & Genoral & Deposn & Unknown & Unknown & $\begin{array}{l}\text { Res: } 2.2 \mathrm{Mtt} 1.03 \% \mathrm{Cu}, 1.75 \% \mathrm{Zn}, 0.47 \% \mathrm{~Pb} \text {. } \\
\text { Data from } 1980 \text { ropon. }\end{array}$ \\
\hline BB & 215 Hisargeris & $N 40^{\circ} 49^{\prime}$ & E $38^{\circ} 21^{\circ}$ & $\begin{array}{l}\text { Lead, zine, } \\
\text { copper }\end{array}$ & 2 & Gonoral & Deposil & Underground & Unknown & $\begin{array}{l}\text { Three drins on voln at } 5 \mathrm{~m} \text { intervals. } \\
\text { Minerallzation visible for BOm. }\end{array}$ \\
\hline BB & 210 Iklau & $N 40^{\circ} 31^{\prime}$ & E $39^{\circ} 23^{\circ}$ & Copper & 2 & Genoral & Deposh & Unknown & Unknown & Im thlek veln, \\
\hline BB & 217 Ikiau-Karinca & $N 40^{\circ} 31^{\prime}$ & E $39^{\circ} 23^{\prime}$ & $\begin{array}{l}\text { Coppor, load, } \\
\text { zine }\end{array}$ & 2 & General & Deposit & Unknown & Unknown & Voin in granite $13 \mathrm{~cm}$ wde, $200 \mathrm{~m}$ long. \\
\hline BB & 218 Illcaktepesl & $N 40^{\circ} 17$ & $E 3^{\circ} 19^{\circ}$ & $\begin{array}{l}\text { Siver, lead, } \\
\text { zine, coppor, } \\
\text { enllimony }\end{array}$ & 2 & General & Deposit & Unknown & Unknown & Occurrence. \\
\hline BB & $\begin{array}{l}219 \text { lerall } \\
\text { Trebolu }\end{array}$ & $\mathrm{N} 40^{\circ} 55^{\prime}$ & E $30^{\circ} 34^{\circ}$ & Copper & 17 & Goneral & Daposit & Unknown & Unknown & $\begin{array}{l}\text { Res: } 44 k 192.8 \% \mathrm{cu} . \\
\text { Data irom } 1970 \text { roport. }\end{array}$ \\
\hline BB & 220 Karayaylak & N $40^{\circ} 52^{\prime}$ & E $38^{\circ} 17$ & $\begin{array}{l}\text { Coppor. lead, } \\
\text { zinc }\end{array}$ & 2 & Ganeral & Deposit & Unknown & Unknown & Ore in andestis turf. \\
\hline BB & 221 Kizliov & $N 40^{\circ} 41^{\circ}$ & E $38^{\circ} 00^{\circ}$ & $\begin{array}{l}\text { Lead, gold, } \\
\text { silver }\end{array}$ & 2 & General & Deposit & Unknown & Unknown & $\begin{array}{l}\text { Ascay } 1809 \% \mathrm{~Pb}, 0.12 \text { oz Au, and } \\
6.0 \text { oz Ag. }\end{array}$ \\
\hline BB & $\begin{array}{l}222 \text { Kizillkaya } \\
\text { Espiry }\end{array}$ & $N 40^{\circ} 62^{\circ}$ & E $36^{\circ} 63^{\prime}$ & Copper & 15,17 & General & Dopostt & Unknown & Unknown & $\begin{array}{l}\text { Res: } 1.9 \mathrm{Mt} \text { Q 0.8-1.14X Cu. } \\
\text { Dala from } 1970 \text { report. }\end{array}$ \\
\hline
\end{tabular}


APPENDIX C: PROSPECTS AND UNDEVELOPED MINERNL PROPERTIES IN TURKEY

\begin{tabular}{|c|c|c|c|c|c|c|c|c|c|c|c|}
\hline $\begin{array}{l}\text { MNP } \\
\text { (1) }\end{array}$ & & $\begin{array}{l}\text { NAME } \\
\text { (2) }\end{array}$ & $\begin{array}{l}\text { COORDIN } \\
\text { LAT. }\end{array}$ & $\begin{array}{l}\text { ATES } \\
\text { LONO. }\end{array}$ & СOMMODITY(IE8) & $\begin{array}{l}\text { DATA } \\
\text { BOURCES } \\
\text { (3) }\end{array}$ & $\begin{array}{l}\text { DATA } \\
\text { RELIANCE } \\
\text { (A) }\end{array}$ & STATUS & $\begin{array}{l}\text { MINE } \\
\text { TYPE }\end{array}$ & MARKETS & COMMENTS \\
\hline 88 & 223 & $\begin{array}{l}\text { Koprubast } \\
\text { Harsht }\end{array}$ & $N 40^{\circ} 40^{\circ}$ & $E 30^{\circ} 50^{\circ}$ & $\begin{array}{l}\text { Load, zinc, } \\
\text { copper }\end{array}$ & 17 & Conoral & Doposin & Unknown & Unknown & $\begin{array}{l}\text { Res: } 2.3 \mathrm{MMt} Q \mathbf{4} 4.68 \% \mathrm{~Pb}, 0.50 \% \mathrm{Zn}, 0.83 \% \mathrm{Cu} \text {. } \\
\text { Oata from } 1977 \text { report. }\end{array}$ \\
\hline 88 & 224 & $\begin{array}{l}\text { Kuskuntu } \\
\text { Esplyo }\end{array}$ & $N 10^{\circ} 5 T$ & E 38' 54' & Copper & 17 & anoret & Doposin & Unknom & Unknown & $\begin{array}{l}\text { Rea: } 2.7 \mathrm{Mt} 90.87 \% \mathrm{Cu} \\
\text { Dete from } 1983 \text { roport. }\end{array}$ \\
\hline 88 & 225 & Kusituvan ' & $N 40^{\circ} 42^{\circ}$ & E $37^{\circ} 38^{\circ}$ & $\begin{array}{l}\text { Coppor, wes. } \\
\text { zlne }\end{array}$ & 2 & Omeres & Deponin & Untrnown & Unknown & Sevoral ore boaring velns in andesite. \\
\hline BB & 220 & Oren & $N 40^{\circ} 46^{\circ}$ & E $37^{\circ} 40^{\circ}$ & $\begin{array}{l}\text { Coppor, lood. } \\
\text { zlne }\end{array}$ & 2 & Ooneres & Deporin & Unknown & Unknown & $\begin{array}{l}\text { Vein in andestle. } \\
\text { Assay } 5.36 \% \mathrm{~Pb}, 3.09 \% \mathrm{Zn} \text {, and } 0.12 \% \mathrm{Cu} \text {. }\end{array}$ \\
\hline B8 & 227 & $\begin{array}{l}\text { Sermeettin } \\
\text { Bellenclk }\end{array}$ & N $40^{\circ} 56^{\circ}$ & $E 38^{\circ} 15^{\circ}$ & $\begin{array}{l}\text { Copper, load, } \\
\text { zine }\end{array}$ & 17 & Goneral & Deposth & Unknown & Unknown & Ros: $188 \mathrm{kt} @ 2 \% \mathrm{Cu}, 3 \% \mathrm{~Pb}, 4 \% \mathrm{Zn}$. \\
\hline 88 & 228 & Surmenll & $N 40^{\circ} 56^{\circ}$ & E $36^{\circ} 35^{\prime}$ & $\begin{array}{l}\text { Copper, lead. } \\
\text { zine }\end{array}$ & 2 & Genaral & Deposil & Unknown & Unknown & Veln in granitto $13 \mathrm{~cm}$ wide. \\
\hline BB & 220 & Tekmozar & $N 40^{\circ} 5 T$ & E $38^{\circ} 14^{\prime}$ & $\begin{array}{l}\text { Copper, lead, } \\
\text { zinc }\end{array}$ & 2 & Genoral & Dupoeth & Unknown & Unknown & Hyrdothermal veln occurrence. \\
\hline 8B & 230 & $\begin{array}{l}\text { Tekmezar } \\
\text { Bularcak }\end{array}$ & $N 40^{\circ} 30^{\circ}$ & E $30^{\circ} 1 \sigma^{\circ}$ & $\begin{array}{l}\text { Copper, lead. } \\
\text { zine }\end{array}$ & 17 & Conoral & Depostt & Unknown & Unknown & $\begin{array}{l}\text { Res: } 600 \mathrm{kt} 90.72 \% \mathrm{Cu}, 3.75 \% \mathrm{~Pb}, 2.82 \% \mathrm{Zn} \text {. } \\
\text { Data from } 1908 \text { repont. }\end{array}$ \\
\hline BB & 231 & Uzumluk Obusu & N $40^{\circ} 52^{\circ}$ & E $38^{\circ} 17$ & $\begin{array}{l}\text { Lead, zinc. } \\
\text { copper }\end{array}$ & 2 & Ceneral & Depoeth & Unknown & Unknown & $\begin{array}{l}\text { Ore occurs in volme of fractured andestito. } \\
\text { Aasay shows } 28.12 \% \mathrm{~Pb}, 6.45 \% \mathrm{Zn} \text {, } \\
3.57 \text { oz } \mathrm{A0}, 0.02 \text { oz/ Au. }\end{array}$ \\
\hline B8 & 232 & Yakinllk & $N 40^{\circ} 50^{\circ}$ & E $38^{*} 30^{\circ}$ & $\begin{array}{l}\text { Lead, zinc. } \\
\text { copper }\end{array}$ & 2 & General & Doposth & Unknown & Unknown & $\begin{array}{l}\text { Hout rock is andealte. } \\
\text { Sample assaye } 1.59 \% \mathrm{~Pb}, 39.65 \% \mathrm{Zn} \text {, } \\
9.24 \% \mathrm{Cu}, 0.71 \text { oz } \mathrm{Ag} \text {, and } 0.00 \text { or Au. }\end{array}$ \\
\hline 88 & 233 & Yomm & $N 40^{\circ} 58^{\prime}$ & E $39^{\circ} 54^{\prime}$ & Antimony & 2 & Genoral & Doposth & Unknown & Unknown & $1 \mathrm{~m}$ voln assaying $65 \% \mathrm{Sb}$. \\
\hline cc & 234 & Alacayir & $N 40^{\circ} 38^{\circ}$ & E $39^{\circ} 54^{\circ}$ & $\begin{array}{l}\text { Copper, zinc, } \\
\text { ellver }\end{array}$ & 2 & Goneral & Depostt & Unknown & Unknown & $\begin{array}{l}\text { Depost in greywache. } \\
\text { Aseaye yleid 8.9\% Cu, } 12.8 \% \mathrm{~Pb}, 2 \text { oz Ag. }\end{array}$ \\
\hline cc & 235 & Almaclk & $N 40^{\circ} 51^{\prime}$ & E 36' S4' & Copper & 2 & Ganeral & Deposit & Unknown & Unknown & Occurrence yletaling $4.57 \% \mathrm{Cu}$. \\
\hline cc & 230 & Banceclk & $N 40^{\prime} 27$ & E $39^{\circ} 35^{\prime}$ & Comont feedelock & 17 & General & Depositt & Surface & Domestle & Ree: 224MT IImealone/cement rew materials (1985) \\
\hline CC & 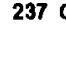 & Ceaylreukur & $N 40^{\circ} 40^{\circ}$ & E $39^{\circ} 05^{\prime}$ & $\begin{array}{l}\text { Coppar, load, } \\
\text { zinc }\end{array}$ & 2 & General & Doposht & Unknown & Unknown & Occurrence. \\
\hline $\mathrm{cc}$ & 238 & Dandekoy & $N 40^{\circ} 48^{\prime}$ & E 38.64 & $\begin{array}{l}\text { Lead, zlnc, } \\
\text { copper, gold, } \\
\text { sliver }\end{array}$ & 2 & Goneral & Deposit & Unknown & Unknown & $\begin{array}{l}\text { Assay le } 72.5 \% \mathrm{~Pb}, 0.5 \% \mathrm{Zn}, 0.39 \text { ozn Au } \\
9.9 \text { oz/ AQ. }\end{array}$ \\
\hline $\mathrm{cc}$ & 2391 & Fol & $N 40^{\circ} 48^{\circ}$ & E $39^{\circ} 17$ & Copper & 2 & General & Doposit & Unknown & Unknown & Slx large velns in andestle. \\
\hline cc & 240 & Gelevera & N $40^{\circ} 36^{\circ}$ & E 36' 51' & Copper & 2 & ceneral & Deposit & Unknown & Unknown & Iron rich ore, sleg dumps up to 15,000 lons. \\
\hline cc & 2411 & Istala & $N 40^{\circ} \mathbf{6 T}$ & E $40^{\circ} \mathrm{iT}$ & $\begin{array}{l}\text { Lead, zinc, } \\
\text { copper }\end{array}$ & 2 & Beneral & Deposit & Unknown & Unknown & Oecurrence. \\
\hline cc & 2421 & Kentes & $N 40^{\circ} 08^{\prime}$ & E $38^{\circ} 66^{\prime \prime}$ & $\begin{array}{l}\text { Load, zlnc, } \\
\text { sllver }\end{array}$ & 2 & Goneral & Deposit & Unknown & Unknown & Hydrothermal vein in andeathe. \\
\hline
\end{tabular}


APPENDIX C: PROSPECTS AND UNDEVELOPED MINERNL PROPERTES IN TURKEY

\begin{tabular}{|c|c|c|c|c|c|c|c|c|c|c|c|}
\hline $\begin{array}{l}\text { MAP } \\
\text { (1) }\end{array}$ & & $\begin{array}{l}\text { MAME } \\
\text { (2) }\end{array}$ & $\begin{array}{l}\text { COORDINA } \\
\text { LAT. }\end{array}$ & $\begin{array}{l}\text { ITES } \\
\text { LONO. }\end{array}$ & COMMODITY(EES) & $\begin{array}{l}\text { DATA } \\
\text { SOURCES } \\
\text { (Э) }\end{array}$ & $\begin{array}{l}\text { OATA } \\
\text { RELIANCE } \\
\text { (4) }\end{array}$ & gTATUS & $\begin{array}{l}\text { MINE } \\
\text { TYPE }\end{array}$ & MARKETS & COMMENTS \\
\hline cc & 243 & Kellope & $N 40^{\circ} 48^{\prime}$ & E 39' or & $\begin{array}{l}\text { Lead, zline, } \\
\text { slver, gold }\end{array}$ & 2 & Gonoral & Deposit & Unknown & Unknown & $\begin{array}{l}\text { Voln in andealte. } \\
\text { Assay is } 13.55 \% \mathrm{~Pb}, 39.25 \% \mathrm{Zn}, 1.99 \text { oz } \\
\mathrm{Ag} \text { and irace of gold. }\end{array}$ \\
\hline cc & 244 & '. & $N 40^{\circ} 30^{\circ}$ & E $39^{\prime} 35^{\prime}$ & $\begin{array}{l}\text { Lead, zinc. } \\
\text { coppor }\end{array}$ & 2 & Gonoral & Doposh & Unknown & Unknown & Flve occurrences reported. \\
\hline cc & 245 & $\begin{array}{l}\text { Okaunuk } \\
\text { Torul }\end{array}$ & N $40^{\circ} 05^{\prime}$ & E $39^{\circ} 50^{\circ}$ & $\begin{array}{l}\text { Copper, lead. } \\
\text { zinc }\end{array}$ & 17 & Genaral & Depostl & Unknown & Unknown & $\begin{array}{l}\text { Res: } 450 \mathrm{kt} \text { e 3.23\% Cu, } 3.84 \% \mathrm{~Pb}, 4.08 \% \mathrm{Zn} \text {. } \\
\text { Data from to71 roport. }\end{array}$ \\
\hline CC & 240 & Swo & $N 40^{\circ} 3 r$ & E $39^{\circ} 18^{\circ}$ & $\begin{array}{l}\text { Copper, load, } \\
\text { zine, sliver }\end{array}$ & 2 & General & Deposin & Unknown & Unknown & $\begin{array}{l}\text { Doposit in andesllo. } \\
\text { Aseny la } 5.18 \% \mathrm{CU}, 15.18 \% \mathrm{~Pb}, 27.15 \% \\
\text { Zn and } 11.0 \text { or AO. }\end{array}$ \\
\hline cc & 247 & Tahnls & N $40^{\circ} \mathbf{2 3}$ & E $39^{\circ} 45^{\prime}$ & Comont foodstock & 17 & Coneral & Daposill & Surface & Domottle & Res: 120Mn IImestone/clay (1988). \\
\hline DD & 248 & Azginillk & $N 36^{\circ} 44^{\circ}$ & E 36' 12' & Iron & 2 & Gonoral & Depoefll & Unknown & Unknown & Res: $3 M M$ Q 34-35\% Fo. \\
\hline DD & 2491 & $\begin{array}{l}\text { Matay } \\
\text { Koseclikkoy } \\
\text { Keseclk }\end{array}$ & $N 36^{\circ} 12^{\prime}$ & E $30^{\circ} 10^{\circ}$ & Gold & 15,17 & Goneral & Dopoen & Unknown & Unknown & $\begin{array}{l}\text { Res: } 450 \mathrm{kt} 9 \text { agh Au. } \\
\text { Data from } 1985 \text { roport. }\end{array}$ \\
\hline DD & 250 & Iskendorun & $N 30^{\circ} 34^{\circ}$ & E $30^{\circ} 10^{\circ}$ & Cement feedatock & 17 & Goneral & Doposth & Surtace & Dometic & Res: 900Mt marl (1982). \\
\hline DD & 251 & $\begin{array}{l}\text { Karayllan } \\
\text { Kintuhan }\end{array}$ & N $36^{\circ} 32^{\prime}$ & E $36^{\prime} 18^{\prime \prime}$ & $\begin{array}{l}\text { Bauxino. } \\
\text { Iron }\end{array}$ & 2.17 & Goneral & Depostt & Surface & Unknown & Res: OMt @ 15-33X Fo; 9-12\% A2O3 (1978). \\
\hline DD & 252 & Kastalkoy & N $30^{\circ} 24^{\circ}$ & E $36^{\circ} 32^{\prime}$ & Iron & 22 & General & Depostt & Surtace & Unknown & Res: $\mathbf{2 M t}$. \\
\hline DD & 253 & Kizildag & N $30^{\circ} 21^{\circ}$ & E 35. 5r & Asbestos & 17 & Coneral & Depositt & Surface & Unknown & Ros: 1.2Mt (1988). \\
\hline DD & 254 & $\begin{array}{l}\text { Payas } \\
\text { IEkenderun }\end{array}$ & $N 36^{\circ} 37$ & E $30^{\circ} 12^{\prime}$ & Iron & 22 & Goneral & Deposth & Surface & Unknown & Sedimentary dopostt wth $6.1 \mathrm{Mt} \in \mathbf{3 2 . 3 \%} \mathrm{Fo}$. \\
\hline DD & $255 \leq$ & Samandag & N 36' or & E $35^{\circ} 30^{\circ}$ & Coment foodelock & 17 & General & Deposh & Surface & Domestle & Ros: 5.78t IImestone/clay/mart (1982). \\
\hline DD & $258 s$ & $\begin{array}{c}\text { Sincan } \\
\text { Payas }\end{array}$ & $N 30^{\circ} 46^{\circ}$ & $E 3^{\circ} 13^{\prime}$ & $\begin{array}{l}\text { Bauxthe. } \\
\text { Iron }\end{array}$ & 2,17 & General & Depostit & Surface & Unknown & 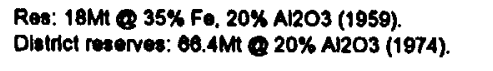 \\
\hline EE & $257 \mathrm{C}$ & Cakall & $N 36^{\circ} 3 r$ & E $33^{\prime} 39^{\circ}$ & Magnealle & 2 & General & Depositt & Unknown & Unknown & Reported deposin of 100,000 tons of magnests. \\
\hline EE & 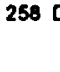 & Dedeler & $N 30^{\circ} 15^{\prime}$ & E $33^{\circ} 37$ & Iron & 2.17 & Genoral & Deposth & Surface & Unknown & $\begin{array}{l}\text { Homathe-limonne depostl covering } 150 \mathrm{~m} \times 1500 \mathrm{~m} \text {. } \\
\text { Res: sookt } 55.9 \% \mathrm{Fo}(1977) \text {. }\end{array}$ \\
\hline EE & 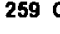 & Gutnar & N $30^{\circ} 39^{\prime}$ & E $34^{\circ} 08^{\prime}$ & Dolomile & 17 & General & Deposin & Surface & Unknown & Res: 253Mt 19-20\% MgO (1973). \\
\hline EE & 260 & $\begin{array}{l}\text { Icel } \\
\text { Adana } \\
\text { Kahramanmaras }\end{array}$ & $N 38^{\circ} 48^{\prime}$ & E $34^{\circ} 38^{\prime}$ & Iron & 19 & General & Deposil & Unknown & Unknown & Res: 30Mt ore Identined In 1978. \\
\hline EE & 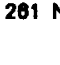 & Nergizillk & $N 37^{\circ} 00^{\circ}$ & E $34^{\circ} \mathbf{4 0}$ & $\begin{array}{l}\text { Copper, } \\
\text { gold, sliver }\end{array}$ & 2 & General & Depositt & Unknown & Unknown & $\begin{array}{l}\text { Expl. Indicales } 29.5 \% \mathrm{Cu}, 0.2302 \mathrm{Au} \text {. } \\
102 \mathrm{Ag} \text {. }\end{array}$ \\
\hline EE & 262 & Orenduzu & N $36^{\circ} 20^{\prime}$ & E $33^{\circ} 25^{\prime}$ & Iron & 17 & Bonoral & Deposit & Surtace & Unknown & 10.9Mt $35 \%$ Fo (1977). \\
\hline EE & $203 \leqslant$ & Sillike & $N 30^{\circ} 22^{\prime}$ & E $33^{\circ} 50^{\circ}$ & Limestone & 17 & General & Enown & face & Domeatle & Res: 550Mt (1973). \\
\hline
\end{tabular}




\section{APPENDIX C: PROSPECTS AND UNDEVELOPED MINERAL PROPERTEES IN TURKEY}

(I)

EE 204 Tasucu

265 Adaler

FF 287 Beyaz Toprak

FF 208 Catalca

FF 209 Catalce

FF 270 Kasbasi-Danik

271 Pasabehce

FF 272 Samandira

FF 273 Sile

FF 274 Yakacll

GG 275 Arkbas!

66276 Burgaz

$$
\text { Odemls }
$$

GG 277 CInUk Ovacll

3G 278 OIKIII

G6 279 Gumussu

GG 280 Kaloclk

60281 Karntylkn

GG 282 Odemis Kure

GG 283 Seydlkoy

GG 284 Turanil

GG 285 Yenlkoy

HH 280 Kaglsman Darphene

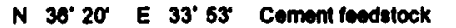

N 40. 53' E 29.03' Kaolln

N 41. O4, E 29.02 Kaoln

N 11'01' E 28' 58' Kaolln

N $41^{\circ} 09^{\circ}$ E 28.2r Quarz eand

N 41' o4' E 29' 34' Copper, slver

DATA DATA

SOURCE

N 41. Or E 29.0\% Clay

N 40, 59' E 29' 13' Copper

N $41^{\circ} 10^{\circ}$ E $29^{\circ} 30^{\circ}$ Thanlum

N $10^{\circ} 65^{\circ}$ E $29^{\circ} 13^{\prime}$ Clay

N 36' 11' E 27' 31' Arentc, gold

N 36' 11' E $27^{\prime} 45^{\circ}$ Tttanlum

N 36. 16 E 26' it Clay

N 39'05' E 20'52 cold

N 38' $05^{\prime}$ E 27' OY Lead, zinc

N 36. 37 E $20^{\circ} 31^{\prime}$ Mercury

N 38' 28' E 27' 10' Siver, gold

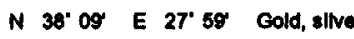

N 39. 32' E 29, 51' Copper

N 39' $1 T$ E $27 \cdot 20^{\circ}$ Bartio

N 38' or E 27' 21" Pentite

N $40^{\circ} 09^{\circ}$ E $43^{\circ}$ or Gold
(4)

General

Gonoral

General

Genoral

General

Gonoral

Gonomal

sTAT

Depoat

Depost

Depost

Depoest

Dopostt

Daport

Depostt

Gonera

Genor

General

Daposit

Depost

Depost

cenena

General

Depost

Deposit

Doposh

Deposit

Depoen

Depost

Deposit

Deposit

Deposth

Deposit

Gonera

Goneral

General

\begin{tabular}{|c|c|c|}
\hline $\begin{array}{l}\text { MINE } \\
\text { TYPE }\end{array}$ & MARKETS & COMMENTS \\
\hline Surtace & Domestic & Res: 200Mt ilmestone (1983). \\
\hline surface & Domeatic & Medium size deposit reported. \\
\hline Surface & Domestle & Ros: 800kt 15-30\% A12O3 (1886). \\
\hline Surtace & Domostic & Good depostl reported. \\
\hline Unknown & Unknown & Res: 304kt $30-32 \%$ Mn (1974). \\
\hline surtace & Domentle & Res: $5.7 \mathrm{Mt}$ (1983). \\
\hline Undemround & Unknown & $\begin{array}{l}\text { Ore in Devonlan chalk. } \\
\text { Nemow velns assaying 1.46-3.55\% Cu, } \\
\text { and 3.12-5.83 ozh AQ. }\end{array}$ \\
\hline Surfaco & Domestle & Moderate size sand clay deposh. \\
\hline Unknown & Unknown & Reported to contaln im tons $2.5 \% \mathrm{Cu}$. \\
\hline Surtace & Unknown & $\begin{array}{l}\text { Beach sands grading 23.4\% magnethe, 32.05\% } \\
\text { Ulmonito. } 18.72 \% \text { zircon. } \\
\text { nO2 contont asseys 14.7\%. }\end{array}$ \\
\hline surface & Domeatle & Large alze fire clay dopoent. \\
\hline Unknown & Unknown & Occurrence. \\
\hline Surtace & Unknown & 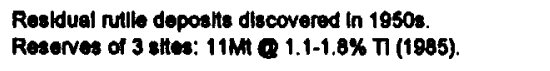 \\
\hline Surface & Domeastic & Moderate size fuller's earth deposit. \\
\hline surtuo & Unknown & 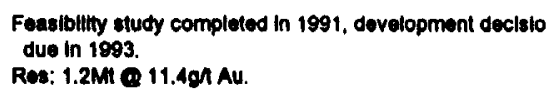 \\
\hline Unknown & Unknown & $\begin{array}{l}\text { Res: 135kt 10\% Pb+Zn. } \\
\text { Data from } 1981 \text { report. }\end{array}$ \\
\hline Unknown & Unknown & Res: $475 k$ K $0.33 \%$ Sb (1981). \\
\hline Unknown & Unknown & 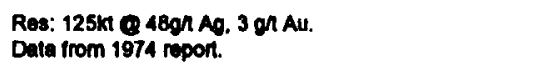 \\
\hline Unknown & Unknown & $\begin{array}{l}\text { Res: } 96 \mathrm{kt} \text { 1.1-8 gh Au, 1-3on Ag. } \\
\text { Data from } 1979 \text { report. }\end{array}$ \\
\hline Unknown & Unknown & Occurrence. \\
\hline Unknown & Unknown & Res: 1Mt. \\
\hline Surface & Unknown & Res: 60Mt (1970). \\
\hline Unknown & Unknown & $\begin{array}{l}\text { Res: } 9 \mathrm{Mm} \mathrm{m} 3 \underset{\mathrm{Q}}{0.1} \mathrm{~g} / \mathrm{m} / \mathrm{m} \text { Au. } \\
\text { Data from } 1955 \text { roport. }\end{array}$ \\
\hline
\end{tabular}


APPENDIX C: PROBPECTE AND UNDEVELOPED MINERNL PROPERTES IN TURKEY

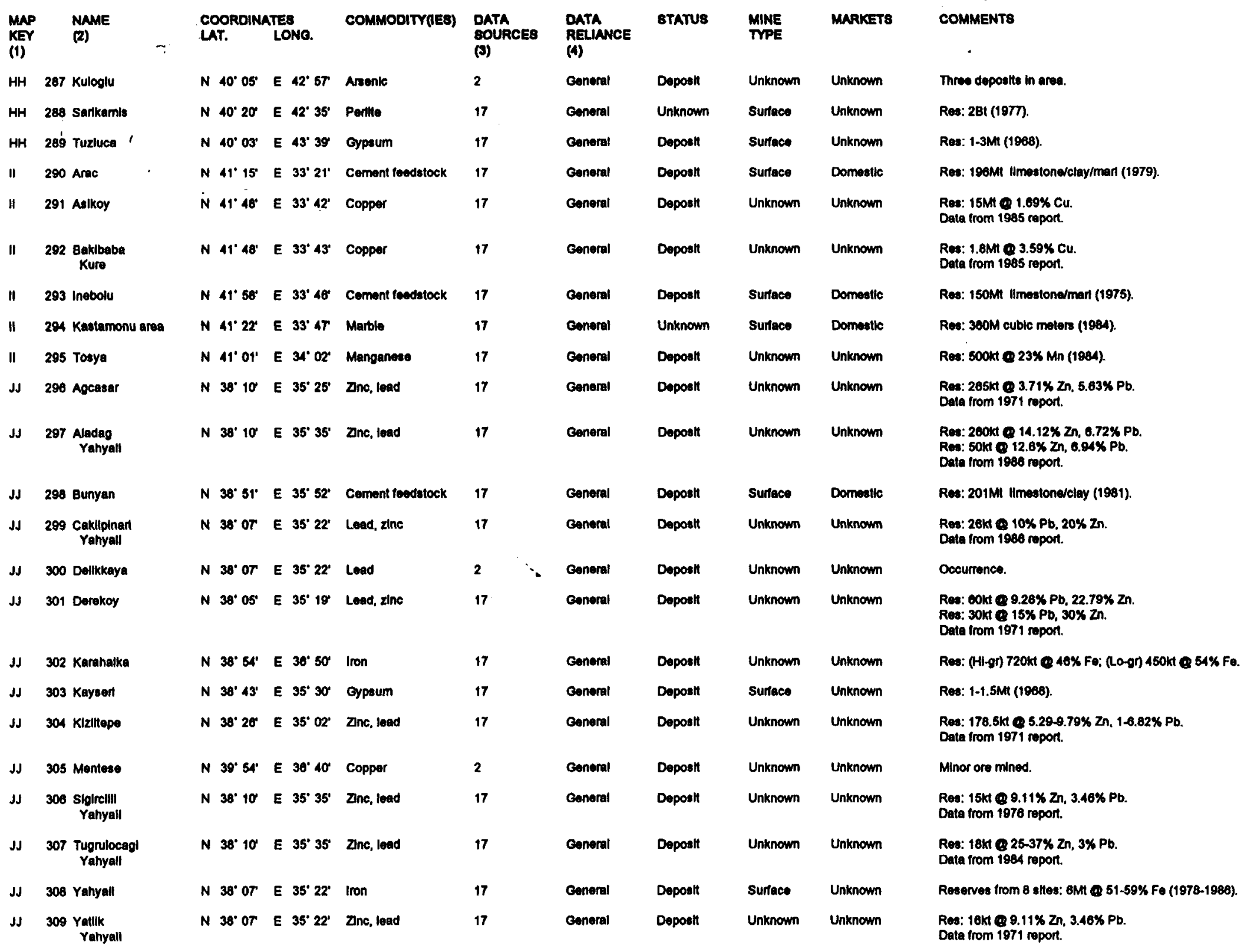


APPENDIX C: PROSPECTS AND UNDEVELOPEO MINERNL PROPERTES IN TURKEY

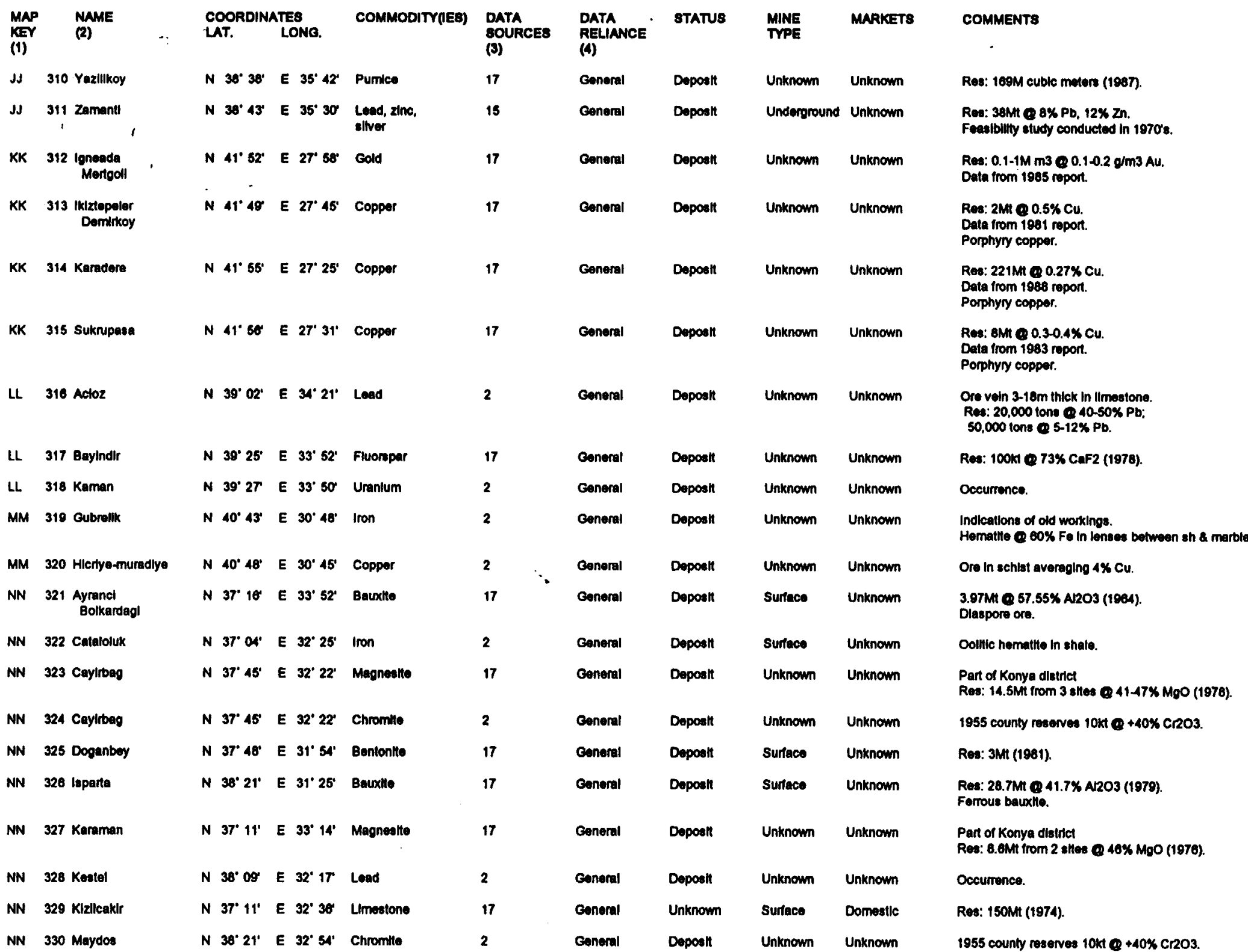


APPENDIX C: PROSPECTS AND UNDEVELOPED MINERAL PROPERTIES IN TURKEY

\begin{tabular}{|c|c|c|c|c|c|c|c|c|c|c|c|}
\hline $\begin{array}{l}\text { MNP } \\
\text { (1) }\end{array}$ & & $\begin{array}{l}\text { NAME } \\
\text { (2) }\end{array}$ & $\begin{array}{l}\text { COORDINA } \\
\text { LAT. }\end{array}$ & $\begin{array}{l}\text { ATES } \\
\text { LONO. }\end{array}$ & COMMODITY(IEE) & $\begin{array}{l}\text { DATA } \\
\text { SOURCES } \\
\text { (9) }\end{array}$ & $\begin{array}{l}\text { DATA } \\
\text { RELIANCE } \\
\text { (4) }\end{array}$ & 8TATU8 & $\begin{array}{l}\text { MINE } \\
\text { TYPE }\end{array}$ & MARKETS & COMMENTS \\
\hline NN & 331 & 1 Meram & N 37. 61' & E 32. 24' & Magneatito & 17 & General & Doposit & Unknown & Unknown & $\begin{array}{l}\text { Part of Konya distriet } \\
\text { Reas: } 62.1 \mathrm{Mi} \text { Q 46.47\% MgO (1975). }\end{array}$ \\
\hline NN & 332 & 2 Saglik & N $37^{\circ} 50^{\circ}$ & E $32^{\circ} 03^{\prime}$ & Bentonke & 17 & Genoral & Dopoett & Surface & Unknown & Res: $2.4 M$ (1981). \\
\hline NN & 333 & 3 Sarllar & N $37^{\circ} 33^{\circ}$ & E $32^{\circ}$ 69 & Limestone & 17 & Genoral & Unknown & Surtace & Domentlc & Res: 410Mt (1974). \\
\hline NN & 334 & Sarloglan & N $37^{\circ}+2^{\circ}$ & E $32^{\circ} 33^{\circ}$ & Lead, bertte & 2 & General & Depostl & Unknown & Unknown & Oceurrence. \\
\hline NN & 335 & 6 Sudur & $N 3 r 1 T$ & E 33' 23' & Chromille & 2 & Genoral & Deposk & Unknown & Unknown & 1955 county resones 10kt $+40 \% \mathrm{Cr} 203$. \\
\hline NN & 336 & 8 Taskent & N $30^{\circ} 55^{\prime}$ & E $32^{\circ} 31^{\circ}$ & Loead & 2 & General & Doposh & Unknown & Unknown & Oecurrence. \\
\hline NN & 337 & 7 Yolmez & N 3702 & E $32^{\circ} 38^{\circ}$ & Lead & 2 & General & Dopoent & Unknown & Unknown & Occurrence. \\
\hline NN & 338 & 8 Youldag & N 37' 33' & E $31^{\circ} 28^{\prime}$ & Llmestone & 17 & General & Unknown & surrace & Domesile & Res: 150Mt (1974). \\
\hline NN & 339 & 9 Yunak & $N 36^{\circ} 49^{\circ}$ & E $31^{\circ} 45^{\prime}$ & Magnoethe & 17 & General & Deposh & Unknown & Unknown & Res: 79.8Mt Q 43-47\% MgO (1978). \\
\hline NN & 340 & Y Yunak & N $38^{\circ} 49^{\circ}$ & E $31^{\circ} 45^{\circ}$ & Moerschaum & 17 & General & Deposh & Surface & Domestic & Res: 121kt (1980). \\
\hline$\infty$ & 341 & 1 Attinas & N 38' 59 & E 29.27 & Kaolln & 17 & General & Depostt & Surtaco & Unknown & Res: $1.2 \mathrm{Mt}$ 20-31\% A2O3 (1982). \\
\hline$\infty$ & 342 & 2 Andiz & N $39^{\circ} 30^{\circ}$ & E $29^{\circ} 55^{\circ}$ & Manganese & 2 & General & Depostt & Unknown & Unknown & Res: 1000 tons $27.72 \%$ Mn. \\
\hline$\infty$ & 343 & 3 Avithor-Kamagac & $N 39^{\circ} 41^{\prime}$ & E $29^{\circ} 42^{\prime}$ & Manganese & 2 & General & Deposit & Unknown & Unknown & Res: 15-20xt roported. \\
\hline$\infty$ & 344 & Avcllar & N 39. $2 T$ & $E 29^{\circ} 04^{\prime}$ & Copper & 2 & General & Deposit & Unknown & Unknown & $\begin{array}{l}\text { Ore assays 25\% Cu, } 0.004 \text { or Au, } 0.9 \text { oz } \\
\text { Ag. }\end{array}$ \\
\hline$\infty$ & 345 & 5 Aydogdu & $N 39^{\circ} 25^{\circ}$ & E 29. 55' & $\begin{array}{l}\text { Load, sllver, } \\
\text { manganese }\end{array}$ & 2 & General & Deposit & Unknown & Unknown & Assay al 0.12 oz/ Ag. \\
\hline$\infty$ & 346 & Aydogdu & N $39^{\circ} 25^{\circ}$ & E $29^{\circ} 55^{\circ}$ & Kaolln & 2 & General & Deposn & Surface & Domestlc & Medlum alze deposth reported. \\
\hline$\infty$ & 347 & Azant & N $39^{\circ} 21^{\circ}$ & E $29^{\circ} 10^{\circ}$ & Copper & 2 & Goneral & Deposit & Unknown & Unknown & Occurrence. \\
\hline$\infty$ & 348 & Batan & N 38' 50 & E 29. 33' & Mercury & 2 & General & Doposit & Unknown & Unknown & Depoefl of 50,000 lons \& $1.67 \% \mathrm{Hg}$ \\
\hline$\infty$ & 349 & Catak & N $39^{\circ} 09^{\prime}$ & E $29^{\circ} 16^{\circ}$ & Iron & 22 & Goneral & Deposth & Surface & Unknown & $\begin{array}{l}\text { Rea: } 6 \text { Mtt } 33-56 \% \text { Fo. } \\
\text { Several amall phits devaloped In 1965, abandoned as hl S. }\end{array}$ \\
\hline$\infty$ & 350 & Comburt & $N 38^{\circ} 58^{\prime}$ & E 29. $2 T$ & Mercury & 2 & Gonoral & Deposit & Unknown & Unknown & Depostl of 140,000 ions. \\
\hline$\infty$ & 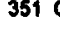 & Cukurviran & N 39' 02 & E $29^{\circ} 25^{\circ}$ & Antlmony & 2 & Gonoral & Depost & Unknown & Unknown & Occurrence. \\
\hline$\infty$ & 352 & Egrigoz & $N 39^{\circ} 24^{\prime}$ & E $29^{\circ} 14^{\prime}$ & Lead & 17 & Ceneral & Deposit & Unknown & Unknown & $\begin{array}{l}\text { Res: } 300 \mathrm{kt} 94.0 \% \mathrm{~Pb} . \\
\text { Data from } 1987 \text { report. }\end{array}$ \\
\hline$\infty$ & 353 & 3 Emet & $N 39^{\prime} 20^{\circ}$ & E $29^{\circ} 15^{\prime}$ & Iron & 17 & General & Deposit & surface & Unknown & Reserves from 6 sthes: 5.5Mt @ 33-61\% Fe (1984). \\
\hline$\infty$ & $354 \mathrm{E}$ & Emet & N 39' $20^{\circ}$ & E 29" $15^{\prime}$ & Coment reedstock & 17 & Coneral & Deposit & Surface & Domestle & Res: 130Mt elay/mart (1979). \\
\hline$\infty$ & 355 & Godiz & N 39. $02^{\prime}$ & E 29, 25' & Llgntie & 17 & General & Deposill & Unknown & Unknown & Res: 23 Mt 5200 Calkg (1976). \\
\hline$\infty$ & 356 & 300012 & N 39'02' & E $29^{\circ} 25^{\prime}$ & Gypsum & 17 & General & Deposh & Surraco & Unknown & Res: 26Mi (1987). \\
\hline
\end{tabular}


APPENDIX C: PROBPECTS AND UNDEVELOPED MINERNL PROPERTIES IN TURKEY

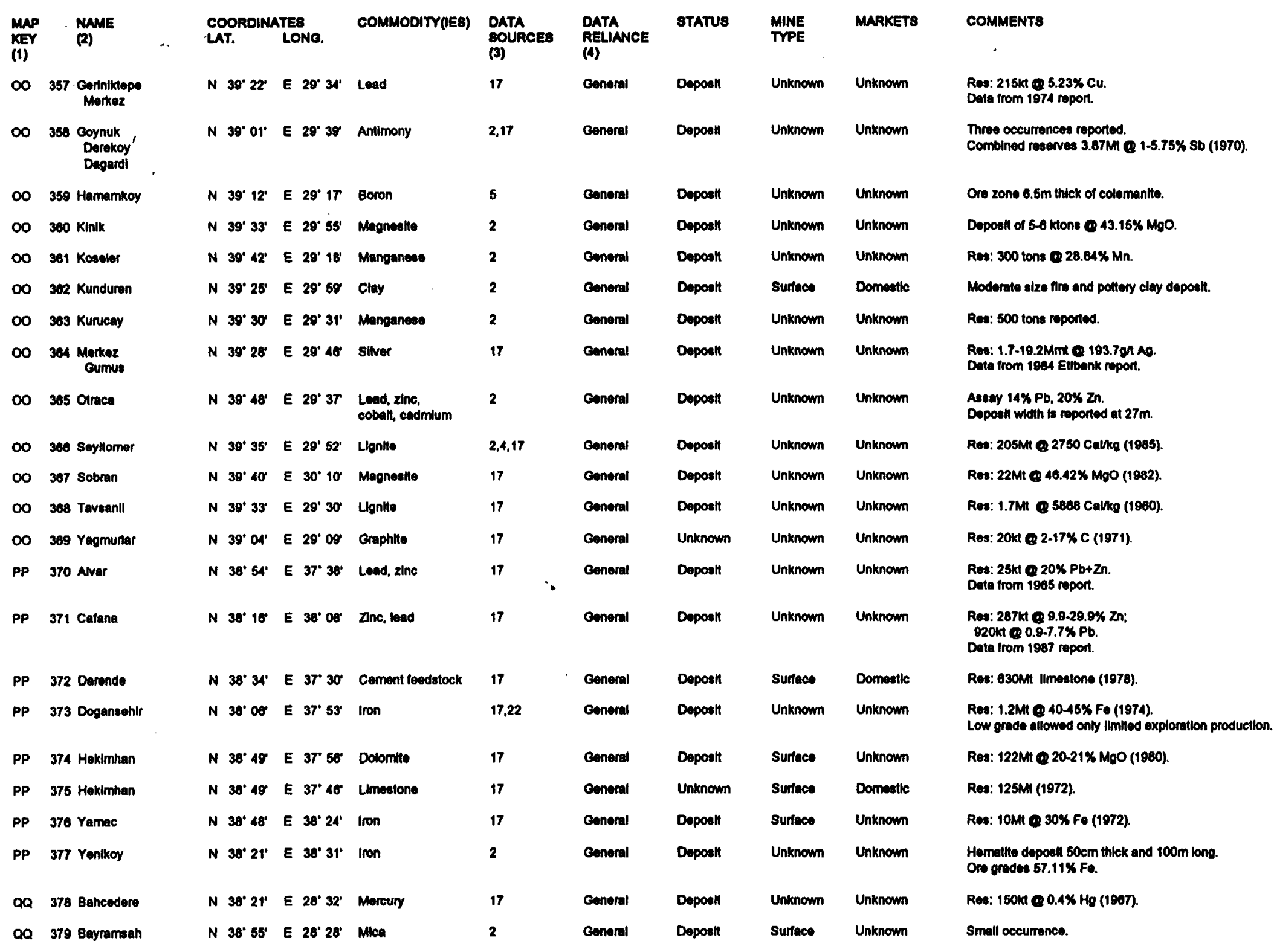




\section{APPENDIX C: PROSPECTS AND UNDEVELOPED MINERAL PROPERTIES IN TURKEY}

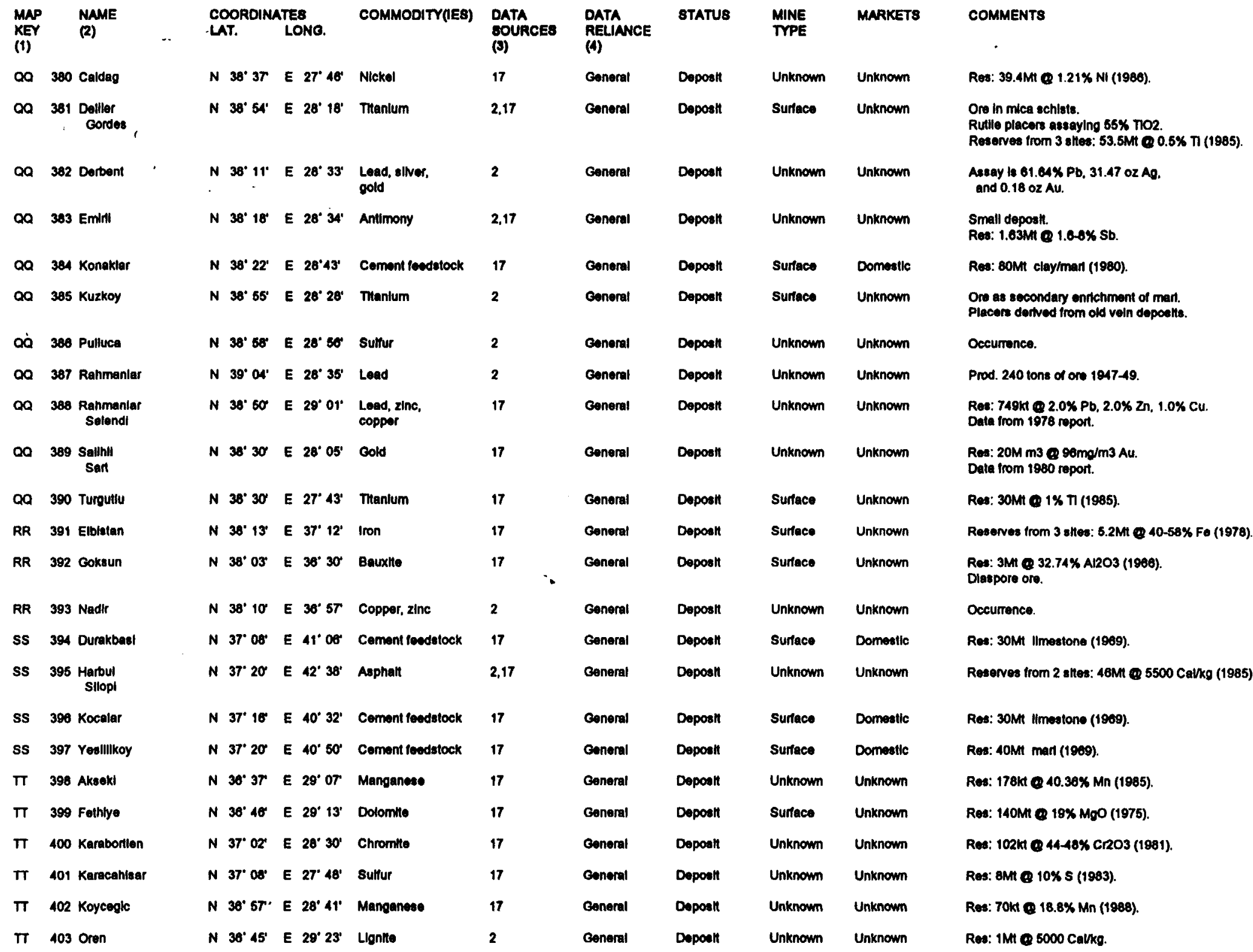


APPENDIX C: PROSPECTS AND UNDEVELOPED MINERAL PROPERTIES IN TURKEY

\begin{tabular}{|c|c|c|c|c|c|c|c|c|c|c|c|}
\hline $\begin{array}{l}\text { MAP } \\
\text { (1) }\end{array}$ & & $\begin{array}{l}\text { NAME } \\
\text { (2) }\end{array}$ & $\begin{array}{l}\text { COORDINA } \\
\text { LAT. }\end{array}$ & $\begin{array}{l}\text { TTES } \\
\text { LONO. }\end{array}$ & COMMODITY(IES) & $\begin{array}{l}\text { DATA } \\
\text { BOURCES } \\
\text { (3) }\end{array}$ & $\begin{array}{l}\text { DATA } \\
\text { RELIANCE } \\
\text { (4) }\end{array}$ & STATuS & $\begin{array}{l}\text { MINE } \\
\text { TYPE }\end{array}$ & MNRKETS & COMMENTS \\
\hline UU & & 4 Mus & $N 38^{\circ} 44^{\circ}$ & E $11^{\circ} 30^{\circ}$ & Bartle & 2,17 & Genoral & Depost & Unknown & Unknown & 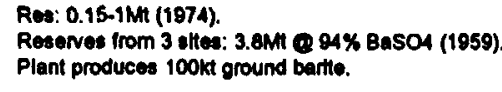 \\
\hline UU & 405 & 5 Mus & $N$ 30 $44^{\circ}$ & E $11^{\circ} 30^{\circ}$ & Coment foedstock & 17 & Gonumal & Deposht & Surface & Domentle & Rea: 2.4 Bt clay/mart (1980). \\
\hline$w$ & 400 & 10 Aclgol & N 38' 33' & E $34^{*} 31^{\prime}$ & Parlke & 17 & Genomal & Unknown & Surface & Unknown & Res: 450Mn (1975). \\
\hline $\mathbf{w}$ & 407 & 7 Dertnkunu & N 36' 23' & E $34^{\circ} 45^{\circ}$ & Porttlo & 17 & General & Unknown & Surfmes & Unknown & Res: 350MAt (1975). \\
\hline w & 408 & 18 Urgup & N 38. $38^{\prime \prime}$ & $E 34^{\circ} 50^{\circ}$ & Coment teedstock & 17 & Genoral & Depositt & Surfaco & Domestle & Res: 30Mt clay/man (1908). \\
\hline ww & 409 & I9 Bolkardgl I & N $37^{\circ} \mathbf{3 3}$ & E $\mathbf{3 4}^{\circ} \mathbf{3 3 ^ { \circ }}$ & $\begin{array}{l}\text { Lead, zinc, } \\
\text { gold, allver }\end{array}$ & 17 & Coneral & Depoett & Unknown & Unknown & 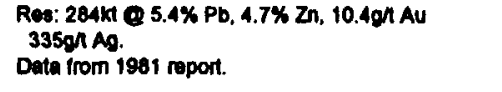 \\
\hline $\mathbf{w} \mathbf{w}$ & & O Bolkerdgl II & N $37^{\circ} 33^{\prime}$ & E $34^{*} 33^{*}$ & $\begin{array}{l}\text { Lead, zinc, } \\
\text { gold, allver }\end{array}$ & 17 & Genoral & Deposth & Unknown & Unknown & $\begin{array}{l}\text { Res: } 152 \mathrm{kt} Q 2.34 \% \mathrm{~Pb}, 1.05 \% \mathrm{Zn}, 3.12 \mathrm{gh} \mathrm{AU} \\
\text { 140g/ Ag. } \\
\text { Data from } 1981 \text { report. }\end{array}$ \\
\hline WW & & 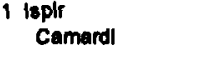 & $N 37^{\circ} 50$ & E $35^{\circ} 00^{\circ}$ & Lead, zine & 97 & Genoral & Deposit & Unknown & Unknown & $\begin{array}{l}\text { Rea: } 29 k \text { e } 6 \% \text { Pb. } 29 \% \text { Zn. } \\
\text { Data irom } 1971 \text { report. }\end{array}$ \\
\hline $\mathbf{w} \mathbf{w}$ & & 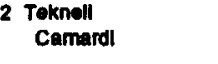 & $\mathrm{N} 37^{*} \mathbf{6 0}$ & E $36^{\circ} 00^{\circ}$ & Lead, zinc & 17 & General & Doposh & Unknown & Unknown & $\begin{array}{l}\text { Res: } 340 \mathrm{kt} \text { ( } 3.6 \% \mathrm{~Pb}, 25 \% \mathrm{Zr} . \\
\text { Data from } 1971 \text { roport. }\end{array}$ \\
\hline$w w$ & 113 & 3 Uluklasla & $\mathbf{N} 38^{\circ} 03^{\circ}$ & $E 3^{\circ} 19^{\circ}$ & Gypoum & 17 & Gonorel & Depoell & Surface & Unknown & Res: 150M1 (1977). \\
\hline ww & & $\begin{array}{l}\text { A Ulukkala } \\
\text { BolkardaglI }\end{array}$ & N $38^{\circ} 03^{\circ}$ & E $34^{\prime} 19^{\prime}$ & $\begin{array}{l}\text { Silver, gold } \\
\text { lead, zinc }\end{array}$ & 15.17 & Goneral & Deposit & Unknown & Unknown & $\begin{array}{l}\text { Res: } 284 k t \text { Q 335gh Ag. 10.4gh Au, } \\
\text { 5.4\% Pb, } 4.7 \% \text { Zn. } \\
\text { Data from } 1981 \text { report. }\end{array}$ \\
\hline ww & & $\begin{array}{l}5 \text { Uluklala } \\
\text { Bokardagi II }\end{array}$ & $N 38^{\circ} 03^{+}$ & E $3^{*} 19^{\prime}$ & $\begin{array}{l}\text { Silver, gold } \\
\text { lead, zinc }\end{array}$ & 17 & General & Deposil & Unknown & Unknown & $\begin{array}{l}\text { Ror: } 152 \mathrm{kt} \text {. } 140 \mathrm{gn} \mathrm{Ag} .3 .12 \mathrm{gn} \mathrm{Au} \text {, } \\
2.3 \% \text { PD, } 1.05 \% \mathrm{Zn} \text {. } \\
\text { Dalla from } 1946 \text { roport. }\end{array}$ \\
\hline$w w$ & 416 & 6 Yapall Agzl Bogazl & $N 37^{\circ} 49^{\circ}$ & E $34^{\circ} 36^{4}$ & Iron & 2 & Goneral & Doposin & Surface & Unknown & $\begin{array}{l}\text { Limonile in limeatione and dlorthe. } \\
\text { Ore dimonsions soom long by } 50 \text { deop. } \\
\text { Res: } 100,000 \text { tons. }\end{array}$ \\
\hline Ww & 417 & $\begin{array}{l}7 \text { Yaylaocaklant } \\
\text { Camardi }\end{array}$ & N $37^{\circ} 50^{\circ}$ & E $35^{\circ} 00^{\circ}$ & Znc, lead & 17 & Coneral & Deposh & Unknown & Unknown & $\begin{array}{l}\text { Res: } 10.5 \mathrm{kt} 930 \% \mathrm{Zn}, 7.7 \% \mathrm{~Pb} \text {. } \\
\text { Dala from } 1971 \text { report. }\end{array}$ \\
\hline$x \mathbf{x}$ & 418 & 8 Akoluk & $N 40^{\circ} 50^{\circ}$ & E $37^{\prime} 42^{\prime}$ & Manganese & 2,17 & Goneral & Deposth & Unknown & Unknown & Resorves 20k Q 36\% Mn (1982). \\
\hline$x \mathbf{x}$ & 119 & o Karakiraz & $N 40^{\circ} 53^{\prime \prime}$ & E $37^{\prime} 56^{\prime}$ & $\begin{array}{l}\text { Copper, lead. } \\
\text { zinc, ellver }\end{array}$ & 2 & General & Depostt & Unknown & Unknown & $\begin{array}{l}\text { Res: } 300 \text { tons } 45.44 \% \text { Pb, } 1.42 \% \text { Zn, } \\
1.6 \% \mathrm{Cu}, 0.30 \text { oz Au, } 2.55 \text { oz Ao. }\end{array}$ \\
\hline$x x$ & 120 & 0 Kirkiraz & $N 40^{\circ} 53^{\prime}$ & E $37^{\circ} 56^{\prime}$ & $\begin{array}{l}\text { Lead, zinc, } \\
\text { copper, gold, } \\
\text { sliver }\end{array}$ & 2 & Goneral & Doposth & Unknown & Unknown & $\begin{array}{l}\text { Res: } 200-300 \text { tons } 45.55 \% \mathrm{~Pb}, 4.42 \% \\
\mathrm{Zn}, 1.6 \% \mathrm{Cu}, 2.55 \text { oz } \mathrm{Aq} \text {, and } 0.36 \text { oz Au. }\end{array}$ \\
\hline$x \mathbf{x}$ & 424 & uk & $N 40^{\circ} 44^{\circ}$ & E $37^{\circ} 01^{\prime}$ & Copper, lead & 2 & oneral & Deposit & Unknown & Unknown & $\begin{array}{l}\text { Volns in volcen lc funt. } \\
\text { Assay is } 1.22 \mathrm{Cu} \text { and } 9.1 \% \mathrm{~Pb} \text {. }\end{array}$ \\
\hline
\end{tabular}


APPENDIX C: PROSPECTS AND UNDEVELOPED MINERNL PROPERTIES IN TURKEY

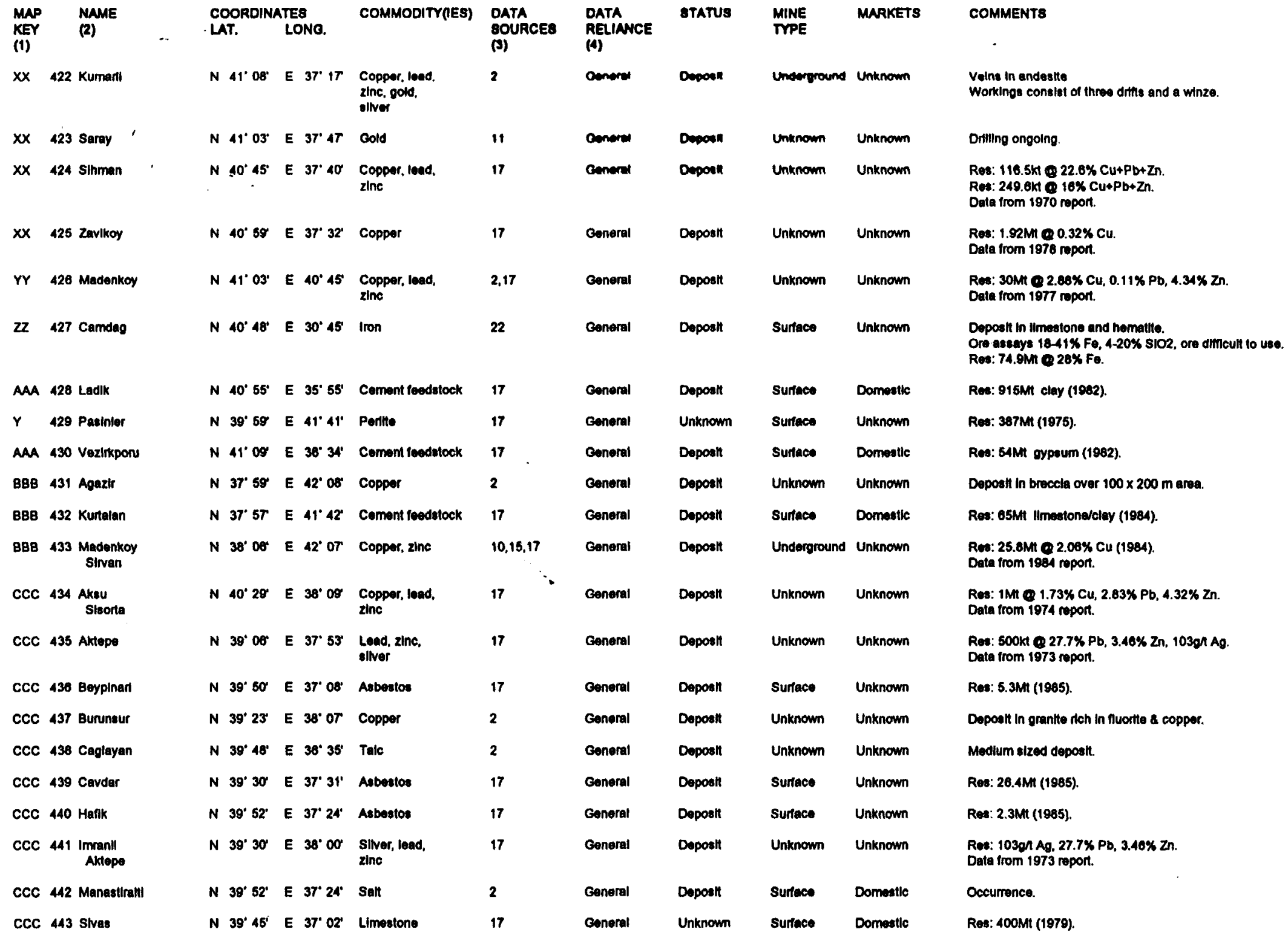


APPENDIX C: PROSPECTS AND UNDEVELOPED MINERNL PROPERTIES IN TURKEY

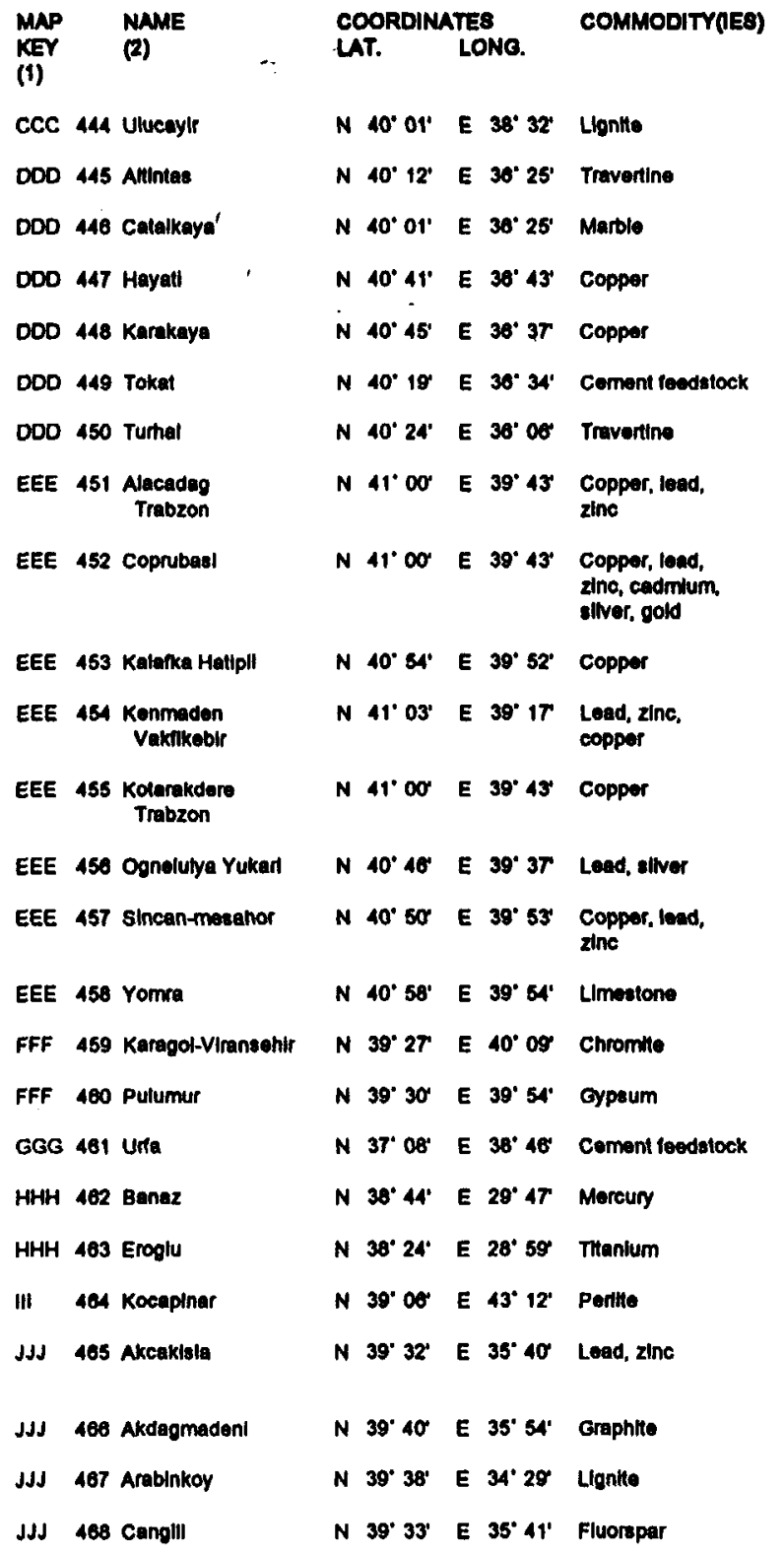

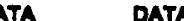

gOURCES RELIANCE

(4)

7

17

17

Goneral

Gonoral

Goneral

Goneral

General

General

General

Gonoral

(1)

Goneral

Goneral

Gonoral

General

coneres

Coneral

Goneral

Gonoral

Genoral

Goneral

Gonoral

Gonoral

General

Ganeral

coneral

General
STATUS

Depostt

Unknown

Deposit

Depoeth

Deposit

Doposit

Deposit

Deposit

Deposit

Doposit

Unknown

Unknown

Unknown Unknown

Dapositt

Unknown

Unknown

Depostt

Unknown Unknown

Depoent

Underground Unknown

Unknown

Surfece Domeatle

Unknown Unknown

Deposit

Deposh

Surface

Unknown

Daposn

Deposh

surrace

Unknown

Unknown Unknown

Depost

Unknown

Surrece

Dapost

Surface

Unknown

Unknown

Unknown

Unknown

Deposts

Unknown

Unknown

Deposit

Unknown Unknown

COMMENTS

Res: 1.39MI 104079 Calkg (1984)

Res: 175M euble moters (1985).

Res: $300 \mathrm{M}$ cubtc metors (1085).

Deposh in sehlat and andestre.

Outcrop $2 \mathrm{~m}$ wido and $50 \mathrm{~m}$ long.

Res: SOMI IImestone (1977).

Res: $190 M$ cuble moters (1965).

Res: $120 \mathrm{kt}$ Q 2.59\% Cu, 9.63\% Pb, 14\% Zn. Data Irom 1971 roport

Undergolng exploretion In 1975.

Occumence.

Ren: 138kt 9 3.6\% Pb, 4.42\% Zn, 0.3\% Cu. Data from 1974 roport.

Res: $900 k \mathrm{kt} 1.28 \% \mathrm{Cu}$

Data from 1976 roport.

Assay to $4.6 \% \mathrm{~Pb}$.

Exploration look place pritor to $\mathrm{WWI}$.

Res: 188Mt (1978)

Two deposfls contalning 20kt $10-41 \%$ Cr2O3.

Res: SOMt (1908).

ReE: 150Mit comont raw matorials/lmestone (1824).

Res: 1.3Mt 0.3\% Ho (1969) trom 8 shes

Res: $12 M$ Q 2\% $\pi$ (1985).

Res: 1.481 (1973).

Veins 0.2-0.5m thlck in limestone. Ore assays 2.53\% Pb. 7.13\% Zn.

Res: 200kt $\odot$ log grade $C$ (1975)

Res: 15-20MH 4700Cainkg

Res: 60kt $72.5 \%$ CaF2 (1970). 
APPENDIX C: PROSPECTS AND UNDEVELOPED MINERAL PROPERTES IN TURKEY

\begin{tabular}{|c|c|c|c|c|c|c|c|c|c|c|c|c|}
\hline $\begin{array}{l}\text { MAP } \\
\text { KEY } \\
\text { (1) }\end{array}$ & & $\begin{array}{l}\text { NAME } \\
\text { (2) }\end{array}$ & $\because$ & $\begin{array}{l}\text { COORDINA } \\
\text { - LAT. }\end{array}$ & $\begin{array}{l}\text { ATES } \\
\text { LONO. }\end{array}$ & COMMODITY(IEB) & $\begin{array}{l}\text { DATA } \\
\text { BOURCES } \\
\text { (B) }\end{array}$ & $\begin{array}{l}\text { DATA } \\
\text { RELIANCE } \\
\text { (4) }\end{array}$ & STATUS & $\begin{array}{l}\text { MINE } \\
\text { TYPE }\end{array}$ & MARKETS & COMMENTS \\
\hline jJJ & 469 & Sefaall & & N $39^{\circ} 31^{\prime}$ & $E 34.46$ & Cerment reodatock & 17 & Goneral & Deposh & Surface & Domestlc & Res: 20MH imestone/mar (1984). \\
\hline JJJ & 470 & Sorgun & & $N 39^{\prime} 49^{\circ}$ & E $35^{\circ} 11^{\prime}$ & Lignite & 17 & Goneral & Deposill & Unknown & Unknown & Res: 13 MI 4928 Calkg (1978). \\
\hline KKK & 471 & Akcaboy' & & N $41^{\circ} 00^{\circ}$ & E $31^{\circ} 52^{\prime}$ & Copper & 2 & General & Depositt & Unknown & Unknown & Veln width $2.5 \mathrm{~m}$, samples assay $7.3 \% \mathrm{Cu}$ \\
\hline KKK & 472 & Bartin & & $N 41^{*} 36^{\circ}$ & E $32^{\circ} 21^{\circ}$ & Clay & 17 & Goneral & Depostl & Surface & Domestlc & Res: 6.8Mt for refractory use. \\
\hline KKK & 473 & Capakdere & & $N 41^{\circ} 45^{\circ}$ & E $32^{\prime} 25^{\prime}$ & Clay & 17 & General & Deposin & Surface & Domestlc & Res: $12 \mathrm{Mt}$ for rofractory use. \\
\hline KKK & 474 & Karadon & & $N 41^{\prime} 28^{\prime}$ & E $31^{\circ} 50^{\circ}$ & Clay & 17 & General & Deposil & Surface & Domestic & Res: 6.1 Mi for rofractory uas. \\
\hline KKK & 475 & Kokaksu & & $N 41^{\circ} 30^{\circ}$ & E $32^{\circ} 05^{\prime}$ & Bauxthe & 2.17 & General & Deposit & Surface & Unknown & $\begin{array}{l}\text { Res: 7.80Mt 9 42\% A2O3 (1981 report) } \\
\text { Ore overtain by sandstone. } \\
\text { Boehmilte ore }\end{array}$ \\
\hline KkK & 178 & Koslu & & $N 41^{\circ} 26^{\prime}$ & E $31^{\circ} 40^{\circ}$ & Clay & 17 & General & Deposil & Surrace & Domestic & Res: 1.4MM for refractory use. \\
\hline KKK & 477 & Kurucasile & & $N 41^{\circ} 50$ & E $32^{\circ} 43^{\prime}$ & Dolomite & 17 & General & Deposin & surface & Unknown & Res: $120 \mathrm{Mt} \odot$ 15-19\% MgO (1981). \\
\hline KK & 478 & Ormanll & & N $41^{\circ} 10^{\circ}$ & E $31^{\circ} 39$ & Dolomite & 17 & Genoral & Deposth & Surface & Unknown & Res: 393Mt \& 16\% MgO (1983). \\
\hline
\end{tabular}

(i) Rapresents property or property grouping as defined on Appendix map set $C$.

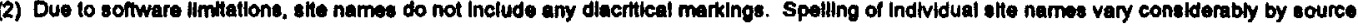

(3) Compete llat of dala sources lound in Appendbx D.

(4) General - denoles ilmited data; Confirmed - denotes deposti informetlon confirmod by several sources. 


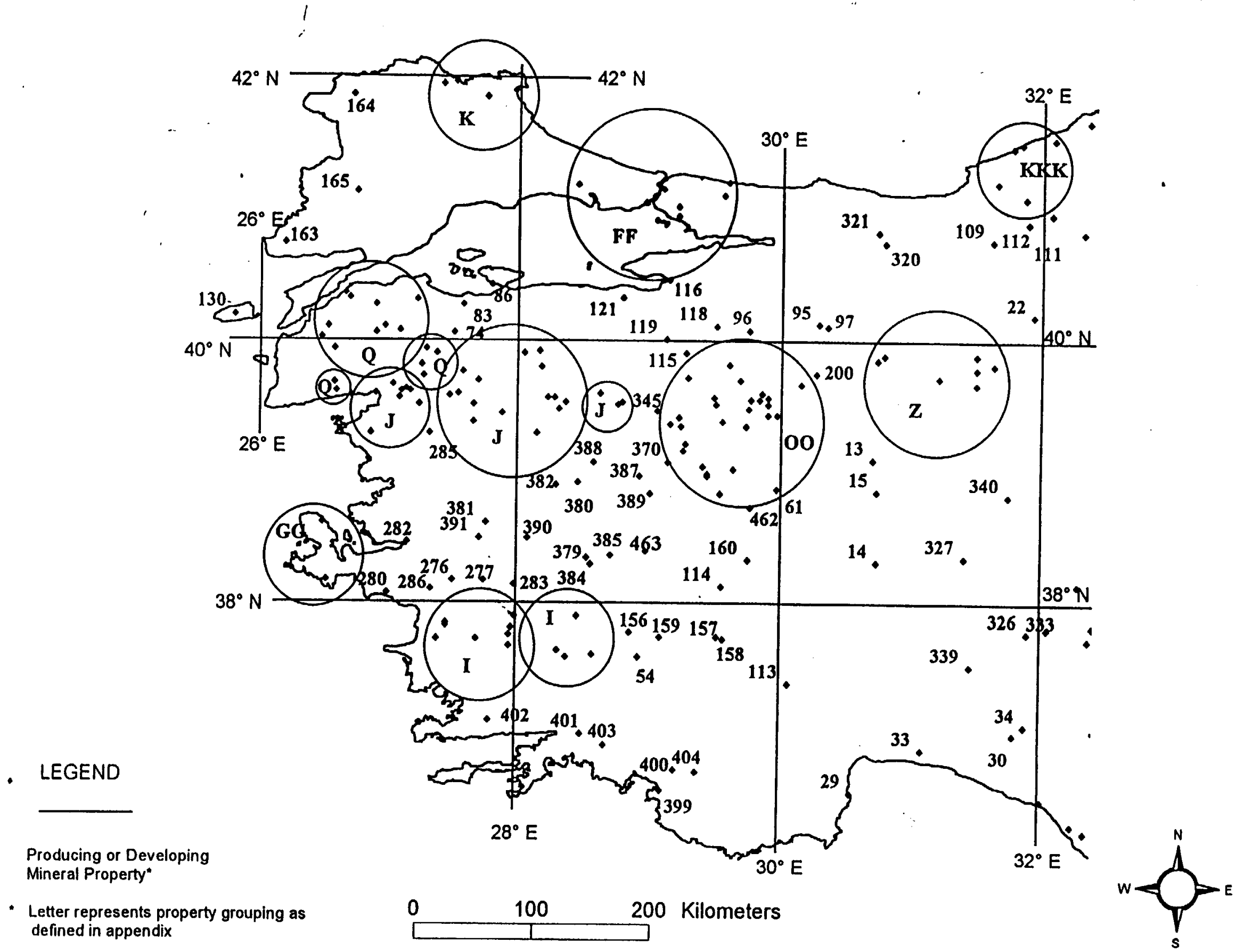

APPENDIX MAP C-1: PROSPECTS AND UNDEVELOPED MINERAL PROPERTIES OF WESTERN TURKEY 


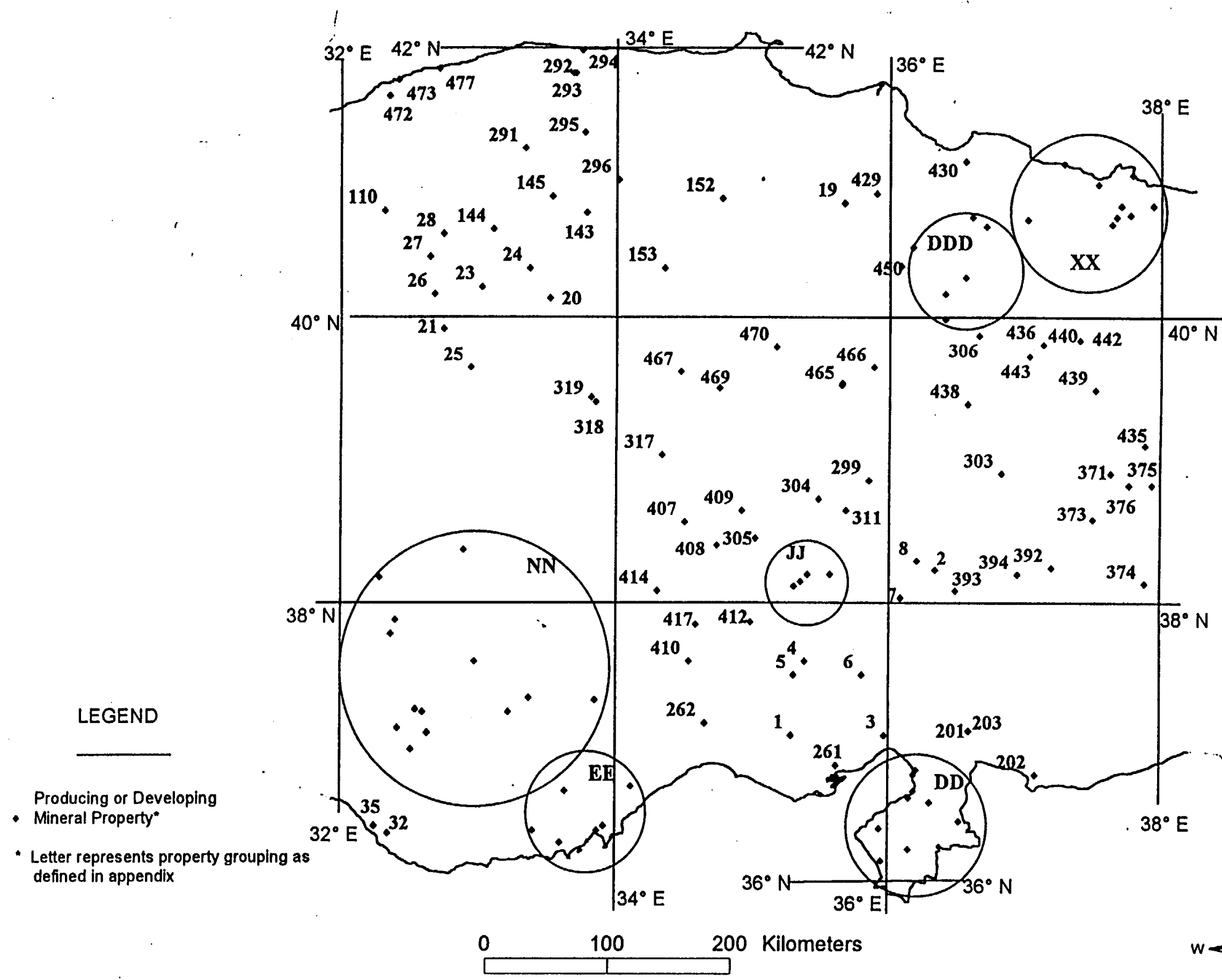

APPENDIX MAP C-2: PROSPECTS AND UNDEVELOPED MINERAL PROPERTIES OF CENTRAL TURKEY 


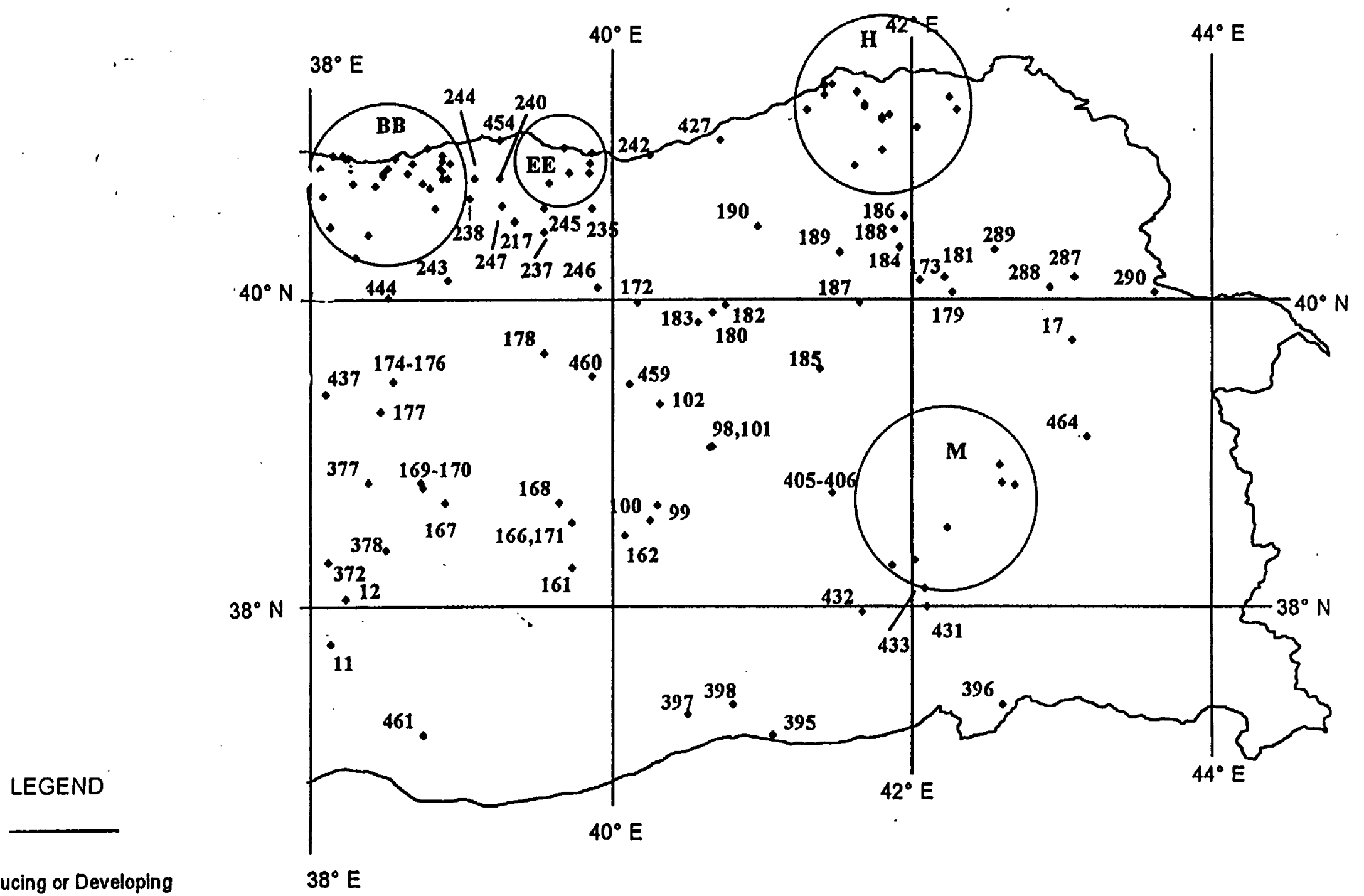

Producing or Developing

- Mineral Property*

- Letter represents property grouping as defined in appendlx

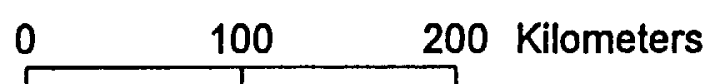

APPENDIX MAP C-3: PROSPECTS AND UNDEVELOPED MINERAL PROPERTIES OF EASTERN TURKEY

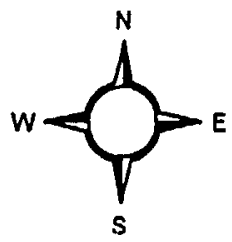




\section{APPENDIX D: PUBLIC SOURCES OF INFORMATION}

1. Report on the Mineral Industry of Egypt. Egyptian Ministry of Finance. Cairo, 1922, $50 \mathrm{pp.}$

2. C. W. Ryan. A Guide to the Known Minerals of Turkey. Mineral Research and Exploration Institute of Turkey. Ankara, $1957,196 \mathrm{pp}$.

3. Turkey, A Country Study. U.S. Library of Congress. Ed. by Paul Pitman III.,1988, pp. 92-231.

4. Area Handbook for the Republic of Turkey. R.F. Nyrop et. al. The American University, 1973, pp. 67-301.

5. Geology and History of Turkey. The Petroleum Exploration Society of Libya. Ed. by A. S. Campbell. 1971, pp. 483-492.

6. Turkish Borates. Scott, 28pp.

7. Z. Ternek. Geological Study of the Region of Kesan-Korudag. $1949.78 \mathrm{pp}$.

8. J.W. Barnes, M.P. Nackowski, and E.H. Bailey. Geology and Ore Deposits of the Sizma-Ladik Mercury District, Turkey. 1969,55 pp.

9. J. S. Whisler. Mineral Development in the Republic of Turkey: Opportunities for Foreign Investment. 1984, 227 pp.

10. H.G. van Oss. U.S. Bureau of Mines. The Mineral Industry of Turkey. Ch. in Minerals Yearbook 1993. P. 79-95.

11. H.G. van Oss. U.S. Bureau of Mines. The Mineral Industry of Turkey. Ch. in Minerals Yearbook 1994. 14 pp.

12. United Nations. Survey of World Iron Ore Resources. New York, 1970,

13. Metall Mining Corporation. Annual Report 1993. p. 17.

14. Mining Journal. June 28, 1991, pp. 494-495.

15 U.S. Geological Survey. Minerals Availability (MAS) database. Nov, 1995.

16. Mining Journal. May 20, 1988, pp. 414-415.

17.

18.

Necdet Ersecen. Known Ore and Mineral Resources of Turkey. Turkish Geological Survey, 1989, 8pp. (Resource estimates may be optimistic.) S. Anac. Etibank's in the Production of Industrial Minerals in Turkey. Presented at Industrial Minerals Conf., Istanbul, Sept. 20-22, 1987. Metal Bulletin Monthly. Jan. 1989, pp. 18-19.

T. Engin. General Geological Setting \& Mineral Resources of Turkey, in Industrial Minerals, Mar. 1988. PP. 5-29. Industrial Minerals Directory. Ed. J. Griffiths, 2nd Ed., 1991.

The Iron Ore Deposits of Europe. Bundesanstalt fur Geowissenschaften und Rohstoffe. 1977. PP. 309-319.

23. U.S. Geological Survey. Mineral Resources Data System (MRDS). 1995. 
; 